\title{
ERZEUGUNG, NACHWEIS UND REAKTIONEN \\ REINER, TEILOXIDIERTER UND SUBSTITUIERTER \\ KOHLENWASSERSTOFFRADIKALE IN DER GASPHASE
}

\author{
Dissertation \\ zur Erlangung des Doktorgrades \\ der Mathematisch-Naturwissenschaftlichen Fakultäten \\ der Georg-August-Universität zu Göttingen
}

vorgelegt von

Jens Wehmeyer

aus Osterode

Göttingen 2002 
D 7

Referent: $\quad$ Prof. Dr. K. Hoyermann

Korreferent: $\quad$ Prof. Dr. M. Buback

Tag der mündlichen Prüfung: 23.04.2002 


\section{Für Katrin}

Klugheit kann eine Gabe der Natur sein;

sie ist Intellekt, der vom Charakter unabbängig ist.

Weisheit ist die Konsequen₹ innerer Tiefe und

bedeutsamer Erfahrungen, die das Leben bereichern;

sie ist der Widerschein einer vielseitigen, gut integrierten Persönlichkeit.

(Aus einem Psychologiebuch, dessen Name längst vergessen wurde.) 
Herrn Prof. Dr. K. Hoyermann danke ich für die Anregung und stete Förderung dieser Arbeit.

Herrn Prof. Dr. I. Morozov danke ich für viele interessante Diskussionen.

Den Mitgliedern der Arbeitsgruppe, namentlich Herrn Dr. T. Beiderhase, Herrn Dr. Uwe Tröger, Herrn Dr. F. Nacke, Herrn Dr. C. Kersten, Herrn M. Fügener, Frau W. Heerdt, Frau G. Gnoyke und Herrn J. Nothdurft danke ich für ihre kollegiale Hilfe. Mein besonderer Dank gilt den Herren M. Hold und T. Zeuch. Ihre Freundschaft und die gute Zusammenarbeit mit ihnen sind für mich prägend gewesen.

Allen Mitarbeitern der Werkstätten unter der Leitung der Herren A. Knorr, V. Meyer und H. Nolte danke ich für ihre Unterstützung beim Bau und bei der Reparatur der Versuchsanordnungen.

Den Herren D. Heinen und W. Noack danke ich für ihre stete und freundschaftliche Hilfsbereitschaft bei Problemen des Laboralltags.

Meinem Zwillingsbruder Carsten danke ich für die Hilfe bei der Bewältigung der Datenverarbeitungsprobleme und für seine ständige Gesprächsbereitschaft.

Meiner Freundin Katrin danke ich für ihre liebevolle Zuwendung in der Endphase der Arbeit und für viele anregende Diskussionen.

Mein Dank gilt meiner Familie, insbesondere meinen Eltern, deren Unterstützung ich mir stets sicher sein konnte. 


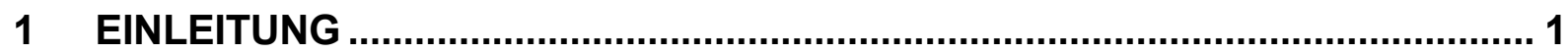

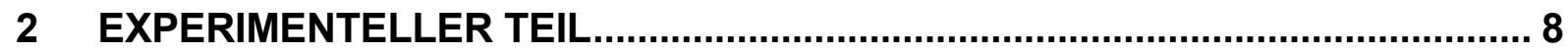

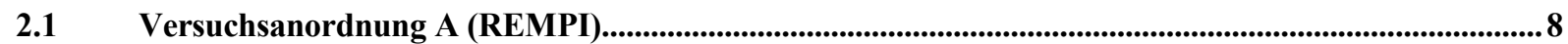

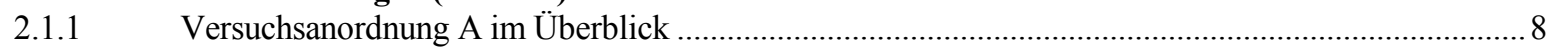

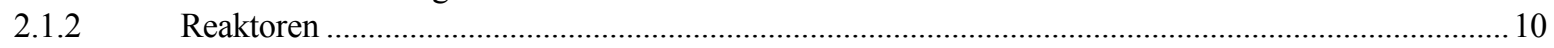

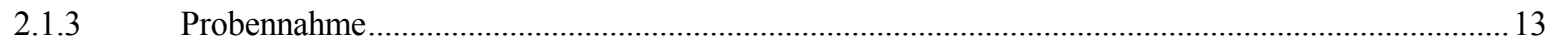

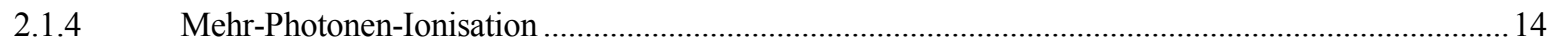

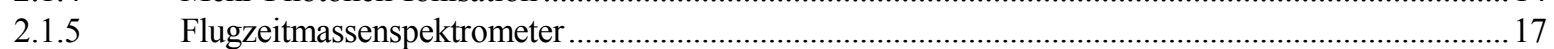

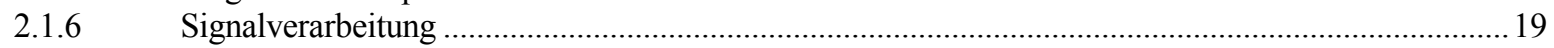

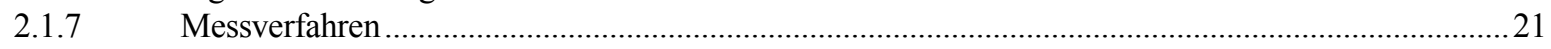

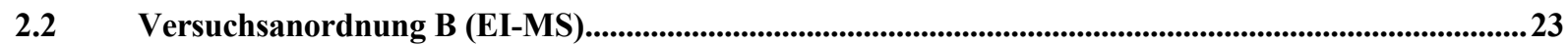

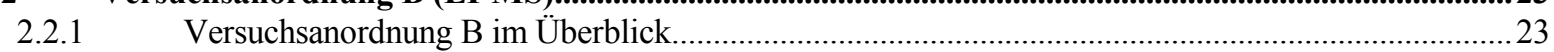

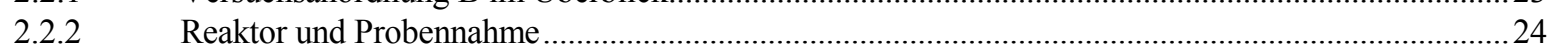

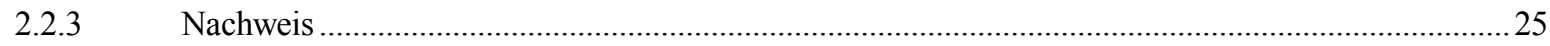

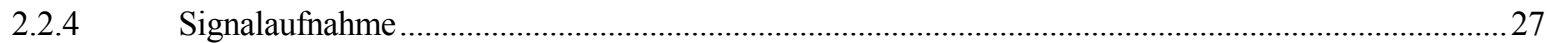

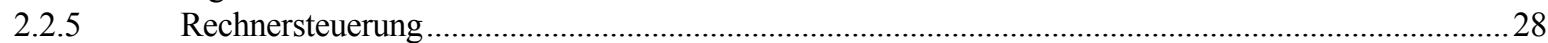

$2.3 \quad$ Versuchsanordnung C (FTIR) .....................................................................................................................30

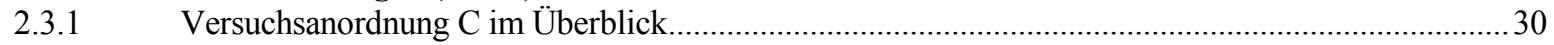

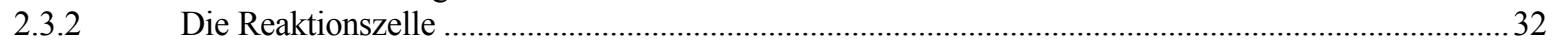

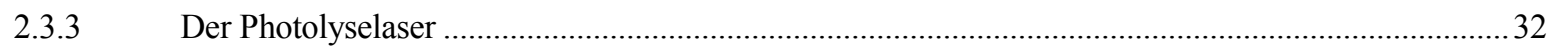

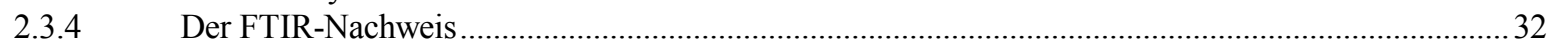

2.4 Methoden zur Auswertung der kinetischen Messungen .................................................................................36

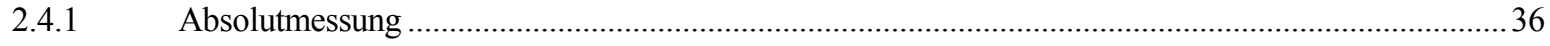

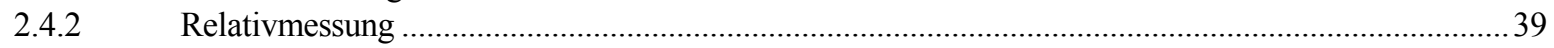

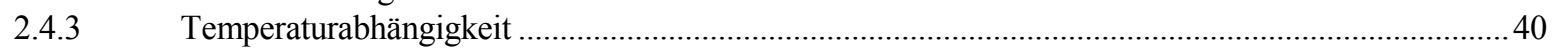

2.5 Reinheit und Dosierung der verwendeten Chemikalien ....................................................................................41

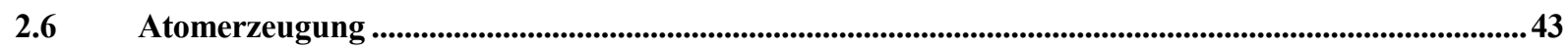

2.7 Ausführung der Messungen ...................................................................................................................................44

$3 \quad$ ERGEBNISSE UND DISKUSSION............................................................. 47

3.1 Erzeugung und Nachweis von Radikalen..............................................................................................4 4

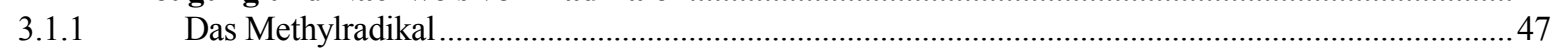

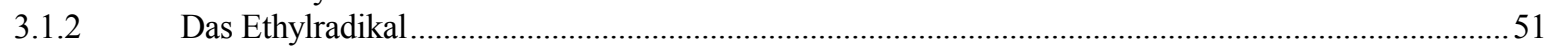

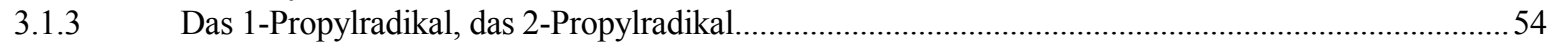

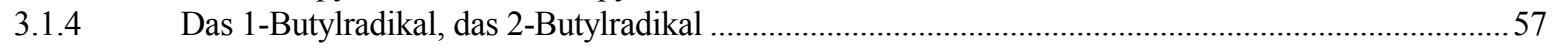

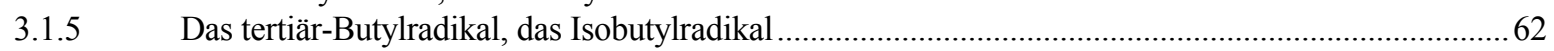

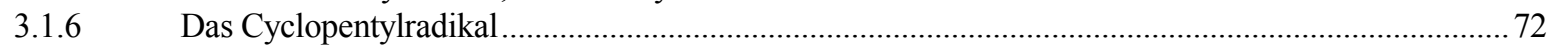

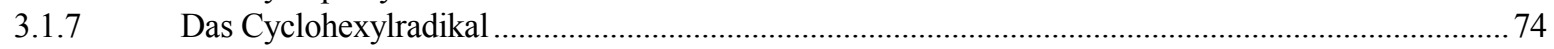

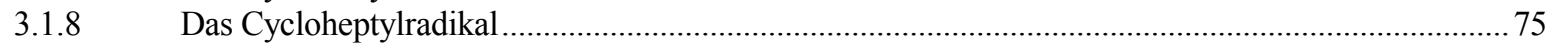

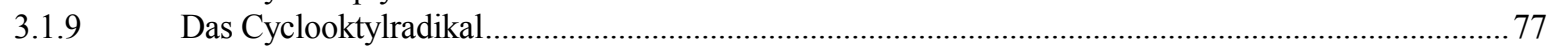

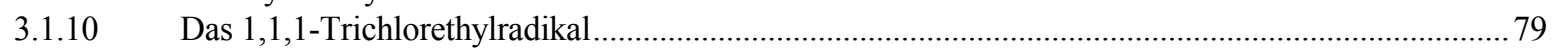

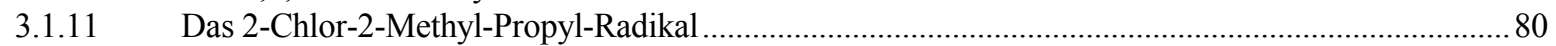

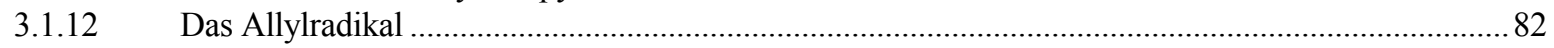

3.1.13 Das Cyclopentadienylradikal, das Fluorocyclopentenylradikal ...................................................... 83

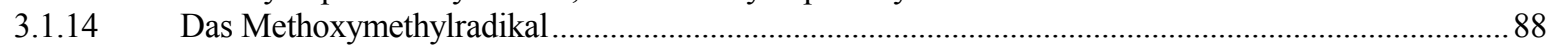


3.2 Reaktionen von Kohlenwasserstoffen mit F-Atomen ...........................................................................................89

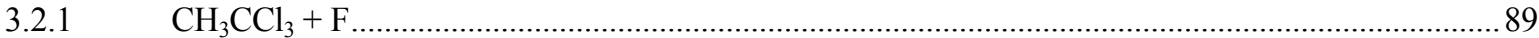

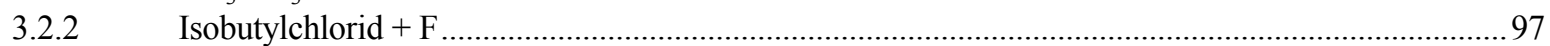

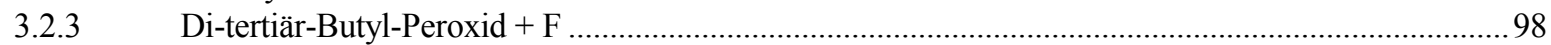

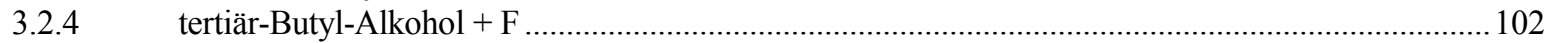

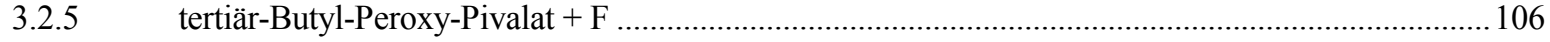

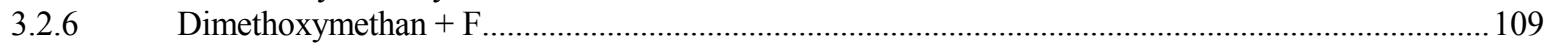

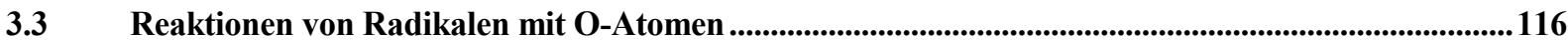

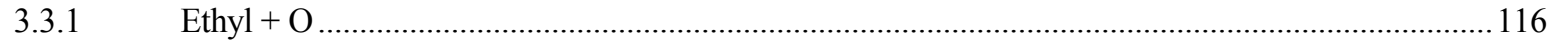

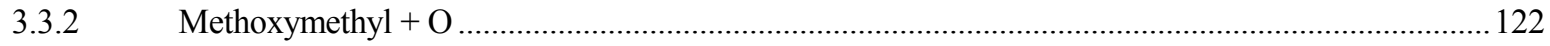

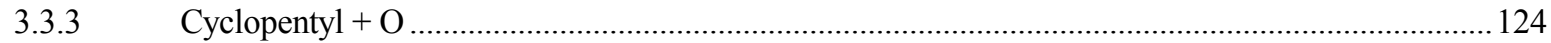

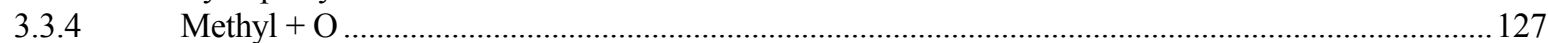

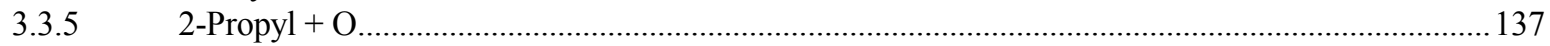

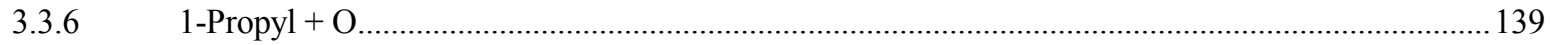

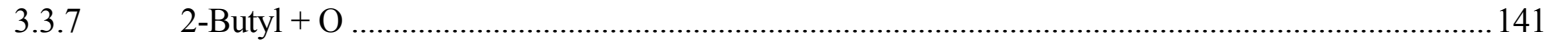

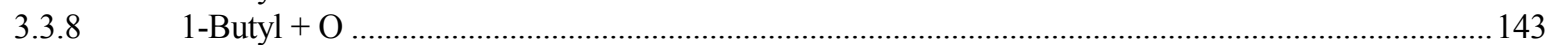

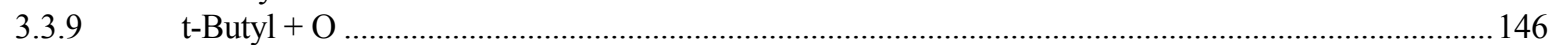

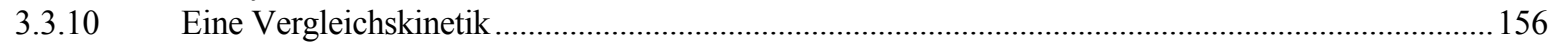

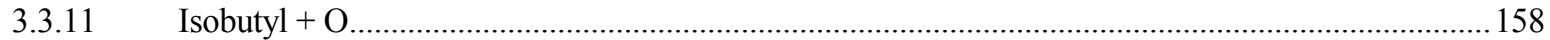

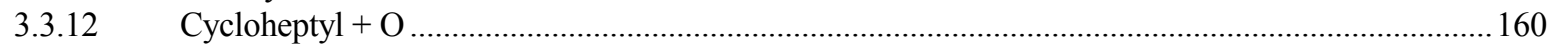

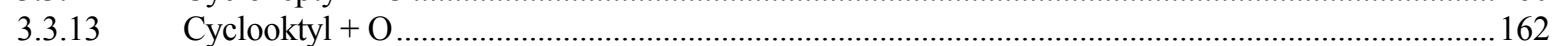

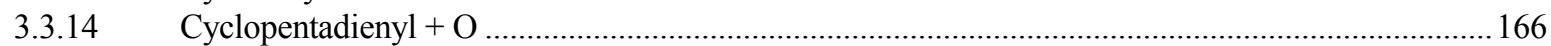

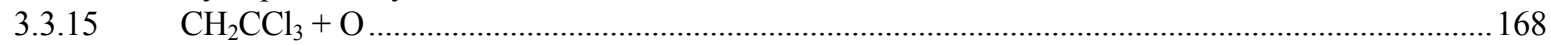

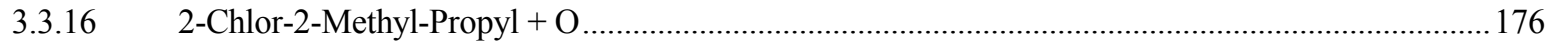

3.3.17 Vergleich der Geschwindigkeiten der Reaktionen Radikal + O................................................. 177

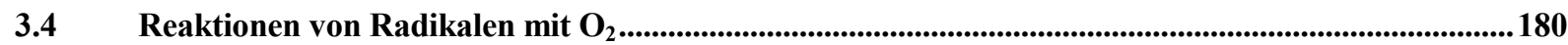

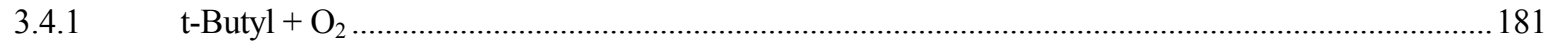

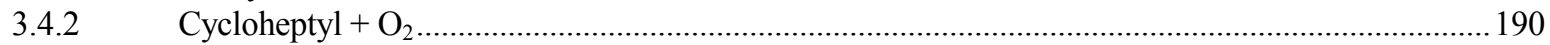

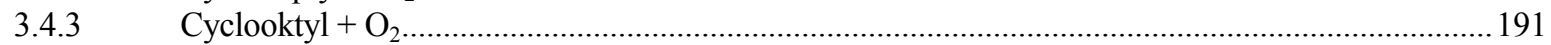

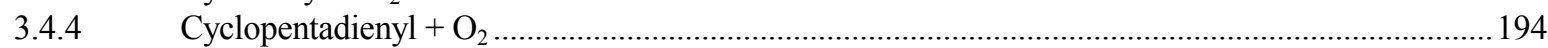

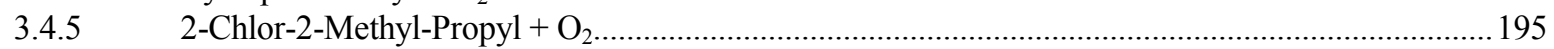

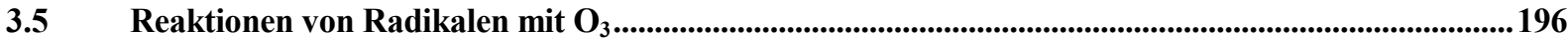

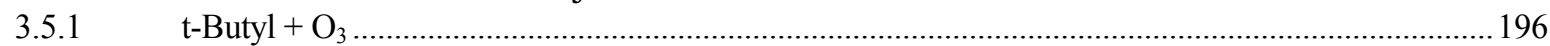

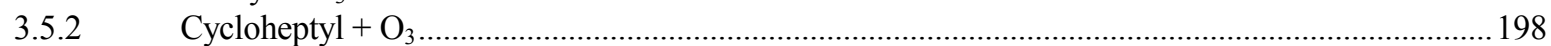

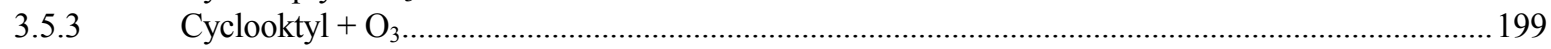

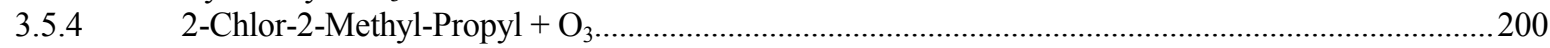

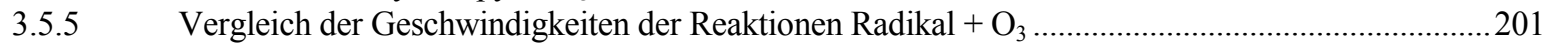

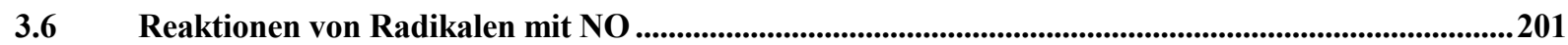

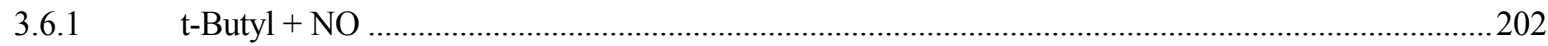

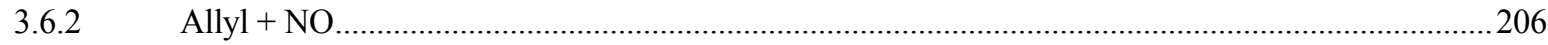

3.7 Reaktionen von Radikalen mit H-Atomen .........................................................................................210

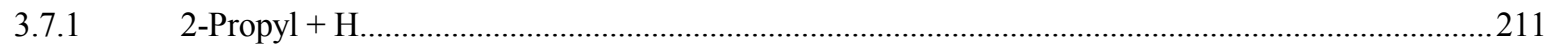

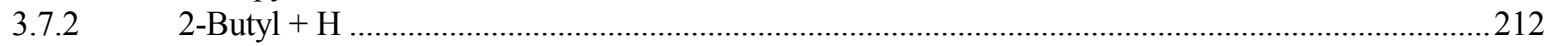

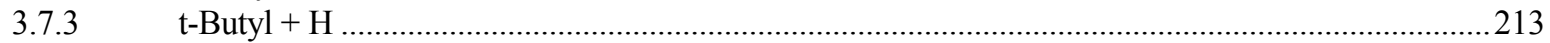

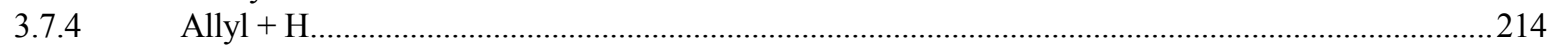

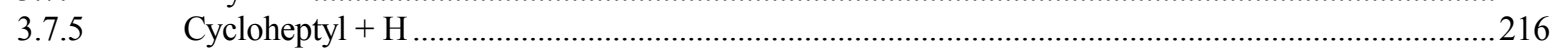

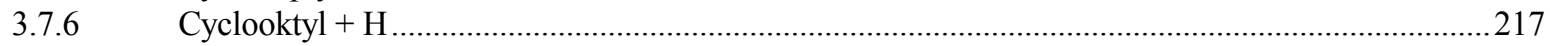

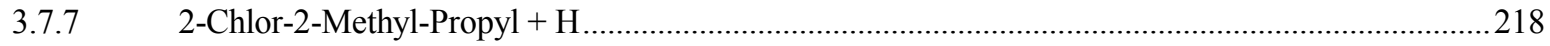

3.7.8 Vergleich der Geschwindigkeiten der Reaktionen Radikal + H.............................................219

4 ZUSAMMENFASSUNG DER ERGEBNISSE............................................... 220

4.1 Erzeugung und Nachweis von Kohlenwasserstoffradikalen..................................................................220 
4.3 Reaktionen von Kohlenwasserstoffradikalen mit O-Atomen ........................................................225

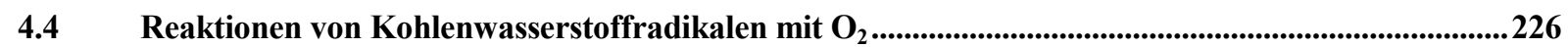

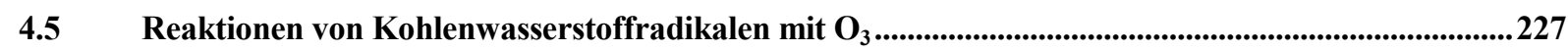

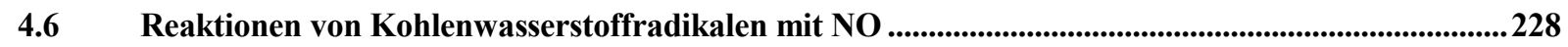

4.7 Reaktionen von Kohlenwasserstoffradikalen mit H-Atomen .......................................................228

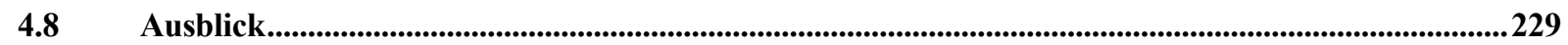

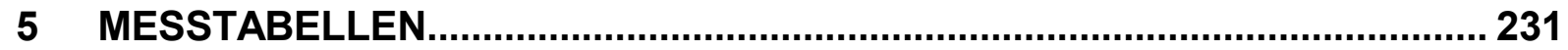

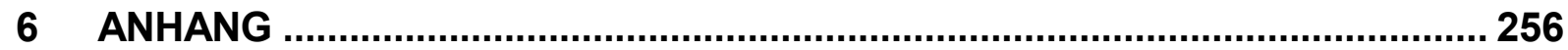

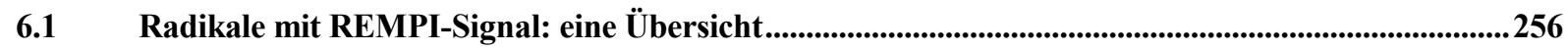

6.2 Verzeichnis der verwendeten Standardbildungsenthalpien.............................................................259

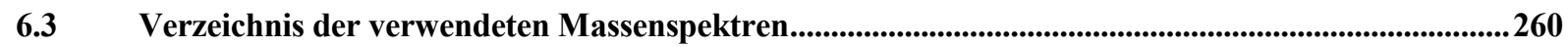

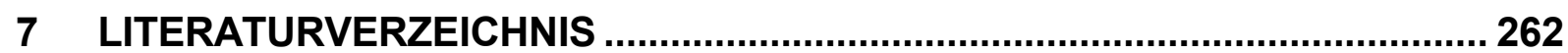

\section{ABBILDUNGSVERZEICHNIS}

Abbildung 1: Temperaturprofil der Erdatmosphäre, [SCH91]

Abbildung 2: Schematische Oxidation von n-Butan im Temperaturbereich 500-850 K, [GRI95]__ 4

Abbildung 3: Versuchsaufbau A im Überblick___ 9

Abbildung 4: Strömungsreaktor 1 Abbildung 5: Strömungsreaktor 2 10

Abbildung 6: Strömungsreaktor 3 Abbildung 7: innerlich verstellbare Sonde___ 12

Abbildung 8: Mechanismen der Photoionisation___ 15

Abbildung 9: Schematische Darstellung eines [2+1]-REMPI-Prozesses und möglicher Konkurrenzmechanismen 16

Abbildung 10: Peakintegration 20

Abbildung 11: Computersteuerung des Versuchsaufbaus A, [NAC98] __ 21

Abbildung 12: Versuchsanordnung B im Überblick___ 24

Abbildung 13: Blockschaltbild zur Rechnerstellung, [NAC94] __ 30

Abbildung 14: Versuchsaufbau C im Überblick, [TRÖ97] __ 31

Abbildung 15: Schematischer Aufbau des FTIR-Spektrometers IFS 66 (intern), [TRÖ97] _ 33

Abbildung 16: Schematischer Aufbau eines Michelson-Interferometers, [TRÖ97] __ 35

Abbildung 17: Sättiger __ 42

Abbildung 18: Flugzeitmassenspektrum $\mathrm{CH}_{3}, \lambda=450,6 \mathrm{~nm}$

Abbildung 19: REMPI-Spektrum $\mathrm{CH}_{3}, \lambda=447-453 \mathrm{~nm} 448$

Abbildung 20: Flugzeitmassenspektrum $\mathrm{CH}_{3}, \lambda=340,8 \overline{\mathrm{nm}} 49$

Abbildung 21: Flugzeitmassenspektrum $C D_{3}, \lambda=340,8 \mathrm{~nm} 49$

Abbildung 22: REMPI-Spektrum $\mathrm{CH}_{3}, \lambda=336-349 \mathrm{~nm} \longrightarrow 50$

Abbildung 23: Flugzeitmassenspektrum $\mathrm{C}_{2} \mathrm{H}_{5}, \lambda = 4 3 0 \longdiv { \mathrm { nm } \_ } 5 1$

Abbildung 24: Flugzeitmassenspektrum $\mathrm{C}_{2} \mathrm{H}_{5}, \lambda=406 \mathrm{~nm} \longrightarrow 52$

Abbildung 25: Flugzeitmassenspektrum $\mathrm{C}_{2} \mathrm{H}_{5}, \lambda=340,8 \mathrm{~nm} 53$

Abbildung 26: REMPI-Spektrum Propan $+F, \lambda=420-462 \mathrm{~nm} 55$ 
Abbildung 27: Flugzeitmassenspektrum $\mathrm{CH}_{3} \mathrm{CD}_{2} \mathrm{CH}_{3}+\mathrm{F}, \lambda=440 \mathrm{~nm}$

Abbildung 28: Flugzeitmassenspektrum $\mathrm{CH}_{3} \mathrm{CD}_{2} \mathrm{CH}_{3}+\mathrm{F}, \lambda=465 \mathrm{~nm}$

Abbildung 29: Flugzeitmassenspektrum $\mathrm{CD}_{3} \mathrm{CH}_{2} \mathrm{CH}_{2} \mathrm{CD}_{3}+\mathrm{F}, \lambda=450 \mathrm{~nm}$

Abbildung 30: Flugzeitmassenspektrum $\mathrm{CD}_{3} \mathrm{CH}_{2} \mathrm{CH}_{2} \mathrm{CD}_{3}+\mathrm{F}, \lambda=450 \mathrm{~nm}$, aufgelöst

Abbildung 31: Flugzeitmassenspektrum $\mathrm{CD}_{3} \mathrm{CH}_{2} \mathrm{CH}_{2} \mathrm{CD}_{3}+\mathrm{F}, \lambda=475 \mathrm{~nm}$, aufgelöst 61

Abbildung 32: Flugzeitmassenspektrum $\mathrm{CD}_{3} \mathrm{CH}_{2} \mathrm{CH}_{2} \mathrm{CD}_{3}+\mathrm{F}, \lambda=475 \mathrm{~nm} \quad 62$

Abbildung 33: Flugzeitmassenspektrum Isobutan $+F, \lambda=500 \mathrm{~nm} \quad 63$

Abbildung 34: Flugzeitmassenspektrum Isobutan $+F, \lambda=500 \mathrm{~nm}$, aufgelöst __ 64

Abbildung 35: Flugzeitmassenspektrum 2-Methylpropan-2-d $+F, \lambda=430 \mathrm{~nm} \longrightarrow 65$

Abbildung 36: Flugzeitmassenspektrum 2-Methylpropan-2-d $+F, \lambda=430 \mathrm{~nm}$, aufgelöst___ 65

Abbildung 37: Flugzeitmassenspektrum 2-Methylpropan-2-d $+F, \lambda=500 \mathrm{~nm}$, aufgelöst $\_66$

Abbildung 38: Flugzeitmassenspektrum 2-Methylpropan-2-d $+F, \lambda=370 \mathrm{~nm}$, aufgelöst___ 66

Abbildung 39: Flugzeitmassenspektrum 2-Methylpropan-2-d $+F, \lambda=400 \mathrm{~nm}$, aufgelöst $\_67$

Abbildung 40: Flugzeitmassenspektrum 2-Methylpropan-2-d $+F, \lambda=410 \mathrm{~nm}$, aufgelöst 167

Abbildung 41: Flugzeitmassenspektrum 2-Methylpropan-2-d $+F, \lambda=440 \mathrm{~nm}$, aufgelöst

Abbildung 42: Flugzeitmassenspektrum 2-Methylpropan-2-d $+F, \lambda=456 \mathrm{~nm}$, aufgelöst

Abbildung 43: Flugzeitmassenspektrum 2-Methylpropan-2-d $+F, \lambda=470 \mathrm{~nm}$, aufgelöst $\_69$

Abbildung 44: Flugzeitmassenspektrum 2-Methylpropan-2-d $+F, \lambda=490 \mathrm{~nm}$, aufgelöst__ 69

Abbildung 45: Flugzeitmassenspektrum 2-Methylpropan-2-d $+F, \lambda=520 \mathrm{~nm}$, aufgelöst__ 70

Abbildung 46: Flugzeitmassenspektrum Isobutan $+F, \lambda=370 \mathrm{~nm} \quad 71$

Abbildung 47: Flugzeitmassenspektrum 2-Methylpropan-2-d $+F, \lambda=370 \mathrm{~nm} \_72$

Abbildung 48: Flugzeitmassenspektrum c-Pentan $+F, \lambda=433 \mathrm{~nm} \_73$

Abbildung 49: Flugzeitmassenspektrum c-Hexan $+F, \lambda=440 \mathrm{~nm} \longrightarrow 74$

Abbildung 50: Flugzeitmassenspektrum c-Heptan $+F, \lambda=440 \mathrm{~nm} \quad 75$

Abbildung 51: Flugzeitmassenspektrum c-Heptan $+F, \lambda=500 \mathrm{~nm} \longrightarrow 76$

Abbildung 52: Wellenlängenscan c-Heptylradikal, $\lambda=420-460 \mathrm{~nm} \longrightarrow 76$

Abbildung 53: Flugzeitmassenspektrum c-Oktan $+F, \lambda=456 \mathrm{~nm} \_77$

Abbildung 54: Wellenlängenscan c-Oktylradikal, $\lambda=443-485 \mathrm{~nm} \longrightarrow 78$

Abbildung 55: Wellenlängenscan c-Oktylradikal, $\lambda=485-530 \mathrm{~nm} \quad 78$

Abbildung 56: Nachweis des Trichlorethylradikals, Versuchsanordnung B_ 79

Abbildung 57: Flugzeitmassenspektrum t-Butylchlorid $+F, \lambda=440 \mathrm{~nm} \longrightarrow 81$

Abbildung 58: Flugzeitmassenspektrum t-Butylchlorid $+F, \lambda=500 \mathrm{~nm} 81$

Abbildung 59: Wellenlängenscan 2-Chlor-2-Methyl-Propyl-Radikal, $\lambda=\overline{420-460 ~ n m ~} 82$

Abbildung 60: REMPI-Spektrum des Allylradikals, $\lambda=485-507 \mathrm{~nm} \_83$

Abbildung 61: Flugzeitmassenspektrum c-Pentadien $+F, \lambda=427 \mathrm{~nm} 85$

Abbildung 62: Flugzeitmassenspektrum c-Pentadien $+F, \lambda=453 \mathrm{~nm} \quad 86$

Abbildung 63: Wellenlängenscan der Signale $m / z=65$ und 83, $C P+\overline{F, 423-460 ~ n m ~} 87$

Abbildung 64: Wellenlängenscan des c-Pentadienylradikals 423-433 nm__ 87

Abbildung 65: Flugzeitmassenspektrum des Methoxymethylradikals, $\lambda=\overline{500 \mathrm{~nm} \_} 88$

Abbildung 66: Massenspektrum $\mathrm{CH}_{3} \mathrm{CCl}_{3} \_90$

Abbildung 67: Isotopenverteilung von Chloratomen in organischen Molekülen___ 91

Abbildung 68: $\mathrm{CH}_{3} \mathrm{CCl}_{3}+\mathrm{F}$ relativ zu $\mathrm{CH}_{2} \mathrm{Cl}_{2}+\mathrm{F}, \mathrm{T}=-20^{\circ} \mathrm{C}$

Abbildung 69: $\mathrm{CH}_{3} \mathrm{CCl}_{3}+\mathrm{F}$ relativ zu $\mathrm{CH}_{2} \mathrm{Cl}_{2}+\mathrm{F}, \mathrm{T}=0^{\circ} \mathrm{C} \longrightarrow 94$

Abbildung 70: $\mathrm{CH}_{3} \mathrm{CCl}_{3}+\mathrm{F}$ relativ zu $\mathrm{CH}_{2} \mathrm{Cl}_{2}+\mathrm{F}, \mathrm{T}=25^{\circ} \mathrm{C} 95$

Abbildung 71: $\mathrm{CH}_{3} \mathrm{CCl}_{3}+\mathrm{F}$ relativ zu $\mathrm{CH}_{2} \mathrm{Cl}_{2}+\mathrm{F}, \mathrm{T}=55^{\circ} \mathrm{C} \longrightarrow 95$

Abbildung 72: $\mathrm{CH}_{3} \mathrm{CCl}_{3}+\mathrm{F}$ relativ zu $\mathrm{CH}_{2} \mathrm{Cl}_{2}+\mathrm{F}, \mathrm{T}=85^{\circ} \mathrm{C} \longrightarrow 96$

Abbildung 73: $\mathrm{CH}_{3} \mathrm{CCl}_{3}+\mathrm{F}$ relativ zu $\mathrm{CH}_{2} \mathrm{Cl}_{2}+\mathrm{F}$, Arrheniusauftragung __ 96

Abbildung 74: Flugzeitmassenspektrum Isobutylchlorid $+F, \lambda=440 \mathrm{~nm} \longrightarrow 97$

Abbildung 75: Flugzeitmassenspektrum DtBP-F/DtBP+F, $\lambda=520 \mathrm{~nm} \longrightarrow 99$

Abbildung 76: Flugzeitmassenspektrum t-Butyl $+/-O, \lambda=520 \mathrm{~nm} \_100$

Abbildung 77: Flugzeitmassenspektrum DtBP+F-O/DtBP+F+O, $\lambda=520 \mathrm{~nm} \_101$

Abbildung 78: Flugzeitmassenspektrum $t-C_{4} H_{9} O D+F+O, \lambda=520 \mathrm{~nm} \_103$

Abbildung 79: Flugzeitmassenspektrum $t-C_{4} H_{9} O D+F, \lambda=440 \mathrm{~nm} \quad 104$

Abbildung 80: Flugzeitmassenspektrum $t-C_{4} H_{9} O D+F+O, \lambda=440 \mathrm{~nm} 105$

Abbildung 81: Flugzeitmassenspektrum tBPP $-F /+F, \lambda=520 \mathrm{~nm} \quad 107$

Abbildung 82: Flugzeitmassenspektrum $t B P P+F-O /+F+O, \lambda = 5 2 \longdiv { n m \_ } 1 0 8$

Abbildung 83: Flugzeitmassenspektrum DMM $+F, \lambda=520 \mathrm{~nm} \_110$

Abbildung 84: Vergleich der Wellenlängenabhängigkeit von $\overline{D M+F(m / z=45) \text { und } M M} 111$ 
Abbildung 85: DMM $+F$ relativ zu $\mathrm{CH}_{4}+F, T=-20^{\circ} \mathrm{C}$

Abbildung 86: $\mathrm{DMM}+\mathrm{F}$ relativ zu $\mathrm{CH}_{4}+\mathrm{F}, \mathrm{T}=0^{\circ} \mathrm{C}$

Abbildung 87: DMM $+F$ relativ zu $\mathrm{CH}_{4}+F, T=25^{\circ} \mathrm{C}$

Abbildung 88: DMM $+F$ relativ zu $\mathrm{CH}_{4}+\mathrm{F}, \mathrm{T}=55^{\circ} \mathrm{C}$

Abbildung 89: DMM $+\mathrm{F}$ relativ zu $\mathrm{CH}_{4}+\mathrm{F}, \mathrm{T}=90^{\circ} \mathrm{C}$

Abbildung 90: DMM $+\mathrm{F}$ relativ zu $\mathrm{CH}_{4}+\mathrm{F}$, Arrheniusauftragung

Abbildung 91: Flugzeitmassenspektrum $\mathrm{C}_{2} \mathrm{H}_{5}-\mathrm{O} /+\mathrm{O}, \lambda=340,8 \mathrm{~nm}$

Abbildung 92: Flugzeitmassenspektrum $C_{2} D_{5}-O /+O, \lambda=340,8$ nm, Darst. 1

Abbildung 93: Flugzeitmassenspektrum $C_{2} D_{5}-O /+O, \lambda=340,8$ nm, Darst. 2

Abbildung 94: $\mathrm{C}_{2} \mathrm{D}_{5}+\mathrm{O}$ relativ zu $\mathrm{C}_{2} \mathrm{H}_{5}+\mathrm{O}, \mathrm{T}=25^{\circ} \mathrm{C}$

Abbildung 95: Methoxymethyl $+O$ relativ zu Ethyl $+O, T=25^{\circ} \mathrm{C}$

Abbildung 96: Cyclopentyl $+O$ relativ zu Ethyl $+O, T=25^{\circ} \mathrm{C}$

Abbildung 97: Cyclopentyl $+O$ relativ zu Methoxymethyl $+O, \overline{T=25^{\circ} \mathrm{C}} 126$

Abbildung 98: Flugzeitmassenspektrum $\mathrm{CH}_{3}-\mathrm{O} /+\mathrm{O}, \lambda=340,8 \mathrm{~nm} \quad 128$

Abbildung 99: Flugzeitmassenspektrum $\mathrm{CD}_{3}-\mathrm{O} /+\mathrm{O}, \lambda=340,8 \mathrm{~nm}$, Darst. $1 \ldots 128$

Abbildung 100: Flugzeitmassenspektrum $C D_{3}-O /+O, \lambda=340,8 \mathrm{~nm}$, Darst. 2

Abbildung 101: $\mathrm{CH}_{3}+\mathrm{O}$ relativ zu $\mathrm{C}_{2} \mathrm{D}_{5}+\mathrm{O}, \mathrm{T}=25^{\circ} \mathrm{C}$

Abbildung 102: Methyl $+O$ relativ zu Methoxymethyl $+\overline{O, T=25^{\circ} \mathrm{C}} 132$

Abbildung 103: Methyl $+O$ relativ zu c-Pentyl $+O, T=25^{\circ} \mathrm{C} \ldots 133$

Abbildung 104: $\mathrm{CD}_{3}+\mathrm{O}$ relativ zu $\mathrm{CH}_{3}+\mathrm{O}, \mathrm{T}=25^{\circ} \mathrm{C} \longrightarrow 136$

Abbildung 105: 2-Propyl $+O$ relativ zu Methoxymethyl $+O, T=25^{\circ} \mathrm{C} \_138$

Abbildung 106: 1-Propyl $+O$ relativ zu 2-Propyl $+O, T=25^{\circ} \mathrm{C} \quad 140$

Abbildung 107: 2-Butyl + O relativ zu Cyclopentyl $+O, T=25^{\circ} \mathrm{C} 142$

Abbildung 108: 1-Butyl + O relativ zu 2-Butyl $+O, T=25^{\circ} \mathrm{C} \longrightarrow 145$

Abbildung 109: FTIR-Spektrum des DtBK_— 146

Abbildung 110: Photolyseprodukte des Dt 1 _ 147

Abbildung 111: Produktanalyse t-Butyl $+O \quad 149$

Abbildung 112: $t$-Butyl $+O$ relativ zu Methoxymethyl $+O, T=25^{\circ} \mathrm{C} \quad 151$

Abbildung 113: $t$-Butyl $+O$ relativ zu Methoxymethyl $+O, T=-30{ }^{\circ} \mathrm{C}$ I 153

Abbildung 114: $t$-Butyl $+O$ relativ zu Methoxymethyl $+O, T=-10^{\circ} \mathrm{C} \longrightarrow 153$

Abbildung 115: $t$-Butyl $+O$ relativ zu Methoxymethyl $+O, T=0{ }^{\circ} \mathrm{C} \quad 154$

Abbildung 116: $t$-Butyl $+O$ relativ zu Methoxymethyl $+O, T=55^{\circ} \mathrm{C} \quad 154$

Abbildung 117: $t$-Butyl $+O$ relativ zu Methoxymethyl $+O, T=90^{\circ} \mathrm{C} \longrightarrow 155$

Abbildung 118: $t$-Butyl $+O$ relativ zu Methoxymethyl $+O$, Arrheniusauftragung ___ 155

Abbildung 119: $t$-Butyl + O relativ zu Allyl $+O, T=25^{\circ} \mathrm{C} \_156$

Abbildung 120: Methoxymethyl $+O$ relativ zu Allyl $+O, T=25^{\circ} \mathrm{C} \quad 157$

Abbildung 121: Isobutyl $+O$ relativ zu t-Butyl $+O, T=25^{\circ} \mathrm{C} \quad 159$

Abbildung 122: c-Heptyl + O relativ zu Methoxymethyl $+O, \overline{T=25^{\circ} \mathrm{C} \_} 161$

Abbildung 123: c-Oktyl + O relativ zu Methoxymethyl $+O, T=25^{\circ} \mathrm{C} \longrightarrow 163$

Abbildung 124: c-Oktyl $+O$ relativ zu Methoxymethyl $+O, T=5^{\circ} \mathrm{C} \longrightarrow 164$

Abbildung 125: c-Oktyl + O relativ zu Methoxymethyl $+O, T=55^{\circ} \mathrm{C} \longrightarrow 164$

Abbildung 126: c-Oktyl + O relativ zu Methoxymethyl $+O, T=90^{\circ} \mathrm{C} \longrightarrow 165$

Abbildung 127: c-Oktyl $+O$ relativ zu Methoxymethyl $+O$, Arrheniusauftragung __ 165

Abbildung 128: Cyclopentadienyl $+O$ relativ zu Methoxymethyl $+O, T=25^{\circ} \mathrm{C} \longrightarrow 167$

Abbildung 129: $\mathrm{CH}_{2} \mathrm{CCl}_{3}+\mathrm{O}$ relativ zu $\mathrm{CHCl}_{2}+\mathrm{O}, \mathrm{T}=25^{\circ} \mathrm{C} \longrightarrow 172$

Abbildung 130: $\mathrm{CH}_{2} \mathrm{CCl}_{3}+\mathrm{O}$ relativ zu $\mathrm{CHCl}_{2}+\mathrm{O}, \mathrm{T}=-20^{\circ} \mathrm{C} \quad 173$

Abbildung 131: $\mathrm{CH}_{2} \mathrm{CCl}_{3}+\mathrm{O}$ relativ zu $\mathrm{CHCl}_{2}+\mathrm{O}, \mathrm{T}=\mathrm{O}^{\circ} \mathrm{C} \longrightarrow 173$

Abbildung 132: $\mathrm{CH}_{2} \mathrm{CCl}_{3}+\mathrm{O}$ relativ zu $\mathrm{CHCl}_{2}+\mathrm{O}, \mathrm{T}=55^{\circ} \mathrm{C} 174$

Abbildung 133: $\mathrm{CH}_{2} \mathrm{CCl}_{3}+\mathrm{O}$ relativ zu $\mathrm{CHCl}_{2}+\mathrm{O}, \mathrm{T}=85^{\circ} \mathrm{C} \longrightarrow 174$

Abbildung 134: $\mathrm{CH}_{2} \mathrm{CCl}_{3}+\mathrm{O}$ relativ zu $\mathrm{CHCl}_{2}+\mathrm{O}$, Arrheniusauftragung 175

Abbildung 135: iso- $\mathrm{C}_{4} \mathrm{H}_{8} \mathrm{Cl}+\mathrm{O}$ relativ zu Methoxymethyl $+\mathrm{O}, \mathrm{T}=25^{\circ} \mathrm{C}$

Abbildung 136: $\lg \{k(R+O)\}$ gegen IP 179

Abbildung 137: Produkte t-Butyl $+\mathrm{O}_{2}, \overline{p=4 \text { mbar___ }} 182$

Abbildung 138: Nachweis Formaldehyd als Produkt von t-Butyl $+\mathrm{O}_{2} \ldots 183$

Abbildung 139: Kalibrierspektrum Formaldehyd, [ZEU02] ___ 184

Abbildung 140: Produkte t-Butyl $+\mathrm{O}_{2}, \mathrm{p}=1 \mathrm{bar} \_185$

Abbildung 141: $t$-Butyl $+\mathrm{O}_{2}$ relativ zu Methoxymethyl $+\mathrm{O}_{2}, \mathrm{~T}=25^{\circ} \mathrm{C}$

Abbildung 142: $t$-Butyl $+\mathrm{O}_{2}$ relativ zu Methoxymethyl $+\mathrm{O}_{2}, \mathrm{~T}=-30^{\circ} \mathrm{C}$

Abbildung 143: $t$-Butyl $+\mathrm{O}_{2}$ relativ zu Methoxymethyl $+\mathrm{O}_{2}, \mathrm{~T}=0^{\circ} \mathrm{C} \longrightarrow 187$

Abbildung 144: $t$-Butyl $+\mathrm{O}_{2}$ relativ zu Methoxymethyl $+\mathrm{O}_{2}, \mathrm{~T}=55^{\circ} \mathrm{C}_{1} 188$ 
Abbildung 145: $t$-Butyl $+\mathrm{O}_{2}$ relativ zu Methoxymethyl $+\mathrm{O}_{2}, \mathrm{~T}=90^{\circ} \mathrm{C}$

Abbildung 146: $t$-Butyl $+\mathrm{O}_{2}$ relativ zu Methoxymethyl $+\mathrm{O}_{2}$, Arrheniusauftragung _ 189

Abbildung 147: c-Heptyl $+\mathrm{O}_{2}$ relativ zu Methoxymethyl $+\mathrm{O}_{2}, \mathrm{~T}=25^{\circ} \mathrm{C} \longrightarrow 190$

Abbildung 148: c-Oktyl $+\mathrm{O}_{2}$ relativ zu Methoxymethyl $+\mathrm{O}_{2}, \mathrm{~T}=25^{\circ} \mathrm{C} \quad 191$

Abbildung 149: c-Oktyl $+\mathrm{O}_{2}$ relativ zu Methoxymethyl $+\mathrm{O}_{2}, \mathrm{~T}=5^{\circ} \mathrm{C} \quad 192$

Abbildung 150: c-Oktyl $+\mathrm{O}_{2}$ relativ zu Methoxymethyl $+\mathrm{O}_{2}, \mathrm{~T}=55^{\circ} \mathrm{C}$

Abbildung 151: c-Oktyl $+\mathrm{O}_{2}$ relativ zu Methoxymethyl $+\mathrm{O}_{2}, \mathrm{~T}=90^{\circ} \mathrm{C}$

Abbildung 152: c-Oktyl $+\mathrm{O}_{2}$ relativ zu Methoxymethyl $+\mathrm{O}_{2}$, Arrheniusauftragung _ 193

Abbildung 153: c-Pentadienyl $+\mathrm{O}_{2}$ relativ zu Ethyl $+\mathrm{O}_{2}, \mathrm{~T}=25^{\circ} \mathrm{C} \quad 194$

Abbildung 154: iso- $\mathrm{C}_{4} \mathrm{H}_{8} \mathrm{Cl}+\mathrm{O}_{2}$ relativ zu Methoxymethyl $+\mathrm{O}_{2}, \mathrm{~T}=\overline{25^{\circ} \mathrm{C} Z} 195$

Abbildung 155: $t$-Butyl $+\mathrm{O}_{3}$ relativ zu Methoxymethyl $+\mathrm{O}_{3}, \mathrm{~T}=25^{\circ} \mathrm{C} \quad 197$

Abbildung 156: c-Heptyl $+\mathrm{O}_{3}$ relativ zu Methoxymethyl $+\mathrm{O}_{3}, \mathrm{~T}=25^{\circ} \mathrm{C} 198$

Abbildung 157: c-Oktyl $+\mathrm{O}_{3}$ relativ zu Methoxymethyl $+\mathrm{O}_{3}, \mathrm{~T}=25^{\circ} \mathrm{C} \quad 199$

Abbildung 158: iso- $\mathrm{C}_{4} \mathrm{H}_{8} \mathrm{Cl}+\mathrm{O}_{3}$ relativ zu Methoxymethyl $+\mathrm{O}_{3}, \mathrm{~T}=25^{\circ} \mathrm{C}$

Abbildung 159: $t$-Butyl $+N O$ relativ zu Methoxymethyl $+N O, T=25^{\circ} \mathrm{C} Z 202$

Abbildung 160: $t$-Butyl $+N O$ relativ zu Methoxymethyl $+N O, T=-30{ }^{\circ} \mathrm{C} \quad 203$

Abbildung 161: $t$-Butyl $+N O$ relativ zu Methoxymethyl $+N O, T=0{ }^{\circ} \mathrm{C} \quad 204$

Abbildung 162: $t$-Butyl $+N O$ relativ zu Methoxymethyl $+N O, T=55^{\circ} \mathrm{C} 204$

Abbildung 163: $t$-Butyl $+N O$ relativ zu Methoxymethyl $+N O, T=90^{\circ} \mathrm{C} Z 205$

Abbildung 164: $t$-Butyl + NO relativ zu Methoxymethyl + NO, Arrheniusauftragung__ 205

Abbildung 165: Allyl $+N O$ relativ zu Methoxymethyl $+N O, T=25^{\circ} \mathrm{C} 206$

Abbildung 166: Allyl + NO relativ zu Methoxymethyl $+N O, T=-30{ }^{\circ} \mathrm{C} 207$

Abbildung 167: Allyl $+\mathrm{NO}$ relativ zu Methoxymethyl $+N O, T=0{ }^{\circ} \mathrm{C} \quad 208$

Abbildung 168: Allyl $+\mathrm{NO}$ relativ zu Methoxymethyl $+N O, T=55^{\circ} \mathrm{C} \quad 208$

Abbildung 169: Allyl $+N O$ relativ zu Methoxymethyl $+N O, T=90^{\circ} \mathrm{C} \quad 209$

Abbildung 170: Allyl + NO relativ zu Methoxymethyl + NO, Arrheniusauftragung _ 209

Abbildung 171: 2-Propyl $+H$ relativ zu Methoxymethyl $+H, T=25^{\circ} \mathrm{C} \quad 212$

Abbildung 172: 2-Butyl + H relativ zu Methoxymethyl $+H, T=25^{\circ} \mathrm{C} \longrightarrow 213$

Abbildung 173: $t$-Butyl + H relativ zu Methoxymethyl $+H, T=25^{\circ} \mathrm{C} \quad 214$

Abbildung 174: Allyl $+\mathrm{H}$ relativ zu Methoxymethyl $+H, T=25^{\circ} \mathrm{C} \quad 215$

Abbildung 175: c-Heptyl + H relativ zu Methoxymethyl $+H, T=2 \overline{5^{\circ} \mathrm{C} Z} 216$

Abbildung 176: c-Oktyl $+\mathrm{H}$ relativ zu Methoxymethyl $+H, T=25^{\circ} \mathrm{C} \longrightarrow 217$

Abbildung 177: iso- $\mathrm{C}_{4} \mathrm{H}_{8} \mathrm{Cl}+\mathrm{H}$ relativ zu Methoxymethyl $+\mathrm{H}, \mathrm{T}=25^{\circ} \mathrm{C}$

\section{TABELLENVERZEICHNIS}

Tabelle 1: Hersteller und Reinheit der verwendeten Chemikalien __ 43

Tabelle 2: Zuordnung von Fragmenten zum Massenspektrum des $\overline{\mathrm{CH}_{3} \mathrm{CCl}_{3} \_} 90$

Tabelle 3: Produktanalyse der Reaktion $\mathrm{CH}_{3} \mathrm{CCl}_{3}+\mathrm{F} \ldots 93$

Tabelle 4: Geschwindigkeitskonstanten ausgewählter Radikale mit O-Atomen___ 158

Tabelle 5: Produktanalyse der Reaktion $\mathrm{CH}_{2} \mathrm{CCl}_{3}+\mathrm{O} \quad 170$

Tabelle 6: Liste der Geschwindigkeitskoeffizienten Radikal +O_ 178

Tabelle 7: Zusammenfassung der Geschwindigkeitskoeffizienten der Reaktionen Radikal $+O \quad 225$

Tabelle 8: Zusammenfassung der Geschwindigkeitskoeffizienten der Reaktionen Radikal $+\mathrm{O}_{2}$

Tabelle 9: Zusammenfassung der Geschwindigkeitskoeffizienten der Reaktionen Radikal $+\mathrm{O}_{3} \quad 227$

Tabelle 10: Zusammenfassung der Geschwindigkeitskoeffizienten der Reaktionen Radikal $+H \quad 228$ 


\section{VERZEICHNIS DER VERWENDETEN ABKÜRZUNGEN}

\begin{tabular}{|c|c|}
\hline$\lambda$ & Wellenlänge \\
\hline$\Delta \mathrm{x}$ & Reaktionsstrecke \\
\hline$\Phi_{\mathrm{X}}$ & Standardvolumenfluss (1,013 bar, 273,15 K) der Komponente X \\
\hline$[\mathrm{X}]_{-\mathrm{Y}}\left([\mathrm{X}]_{+\mathrm{Y}}\right)$ & Konzentration von $\mathrm{X}$ in Abwesenheit (Anwesenheit) von $\mathrm{Y}$ \\
\hline $1.23 \mathrm{E} 4$ & $1.23 \cdot 10^{4}$ (Beispiel) \\
\hline A & Auflösung \\
\hline $\mathrm{A} / \mathrm{D}$ & Analog/Digital \\
\hline Bkg & Untergrund (Background) \\
\hline $\mathrm{CP}$ & Cyclopentadien \\
\hline $\mathrm{CPR} / \mathrm{CP}^{*}$ & Cyclopentadienylradikal \\
\hline DME & Dimethylether $\left(\mathrm{CH}_{3} \mathrm{OCH}_{3}\right)$ \\
\hline DMM & Dimethoxymethan $\left(\mathrm{CH}_{3} \mathrm{OCH}_{2} \mathrm{OCH}_{3}\right)$ \\
\hline DSO & Digitales Speicheroszilloskop \\
\hline DTBE & Di-tertiär-Butyl-Ether $\left(\mathrm{C}_{4} \mathrm{H}_{9} \mathrm{OC}_{4} \mathrm{H}_{9}\right)$ \\
\hline DTBK & Di-tertiär-Butyl-Keton \\
\hline D'TBP & Di-tertiär-Butyl-Peroxid $\left(\mathrm{C}_{4} \mathrm{H}_{9} \mathrm{OOC}_{4} \mathrm{H}_{9}\right)$ \\
\hline $\mathrm{E}$ & Laserenergie, Energie \\
\hline $\mathrm{E}_{\mathrm{A}}$ & Aktivierungsenergie nach Arrhenius \\
\hline EI & Electron Impact, Elektronenstoßionisation \\
\hline est & estimated, rechnerisch abgeschätzt \\
\hline FCPR & Flurocyclopentenylradikal \\
\hline FTIR & Fourier-Transform-Infrarot-Spektrometer \\
\hline GCMS & Gaschromatograph mit angeschlossenem Massenspektrometer \\
\hline $\mathrm{I}_{-\mathrm{X}}\left(\mathrm{I}_{+\mathrm{X}}\right)$ & Intensität auf $\mathrm{m} / \mathrm{z}=\mathrm{n}$ bei Abwesenheit (Anwesenheit) von $\mathrm{X}$ \\
\hline IB & Isobutylradikal \\
\hline IBCR & Isobutylchloridradikal (2-Chlor-2-Methyl-Propyl) \\
\hline IE & Ionisierungsenergie \\
\hline IP & Ionisierungspotenzial \\
\hline $\mathrm{k}(\mathrm{T})$ & Geschwindigkeitskoeffizient bei der Temperatur T \\
\hline $\mathrm{k}_{\mathrm{abs}}$ & absoluter Geschwindigkeitskoeffizient \\
\hline $\mathrm{k}_{\mathrm{B}}$ & Boltzmannkonstante \\
\hline $\mathrm{k}_{\text {ref }}$ & Geschwindigkeitskoeffizient der Referenzreaktion \\
\hline $\mathrm{k}_{\text {rel }}$ & relativer Geschwindigkeitskoeffizient \\
\hline M & Molmasse \\
\hline MCP & Multi Channel Plates (Vielkanalplatten) \\
\hline MSP & Multi Sphere Plate \\
\hline $\mathrm{ME}$ & Methan $\left(\mathrm{CH}_{4}\right)$ \\
\hline MM & Methoxymethylradikal \\
\hline MTBE & Methyl-t-Butyl-Ether $\left(\mathrm{t}-\mathrm{C}_{4} \mathrm{H}_{9} \mathrm{OCH}_{3}\right)$ \\
\hline $\mathrm{m} / \mathrm{z}$ & Masse-Ladungsverhältnis \\
\hline $\mathrm{N}_{\mathrm{A}}$ & Avogadrokonstante \\
\hline $\mathrm{p}$ & Druck \\
\hline $\mathrm{R}$ & Gaskonstante \\
\hline REMPI & Resonance Enhanced Multi Photon Ionization \\
\hline s & Sondenstellung (Reaktionsstrecke) \\
\hline SEV & Sekundärelektronenvervielfacher \\
\hline $\mathrm{t}$ & Reaktionszeit \\
\hline $\mathrm{t}-$ & tertiär \\
\hline $\mathrm{tB}$ & tertiär-Butylradikal \\
\hline $\mathrm{tBAl}$ & t-Butylalkohol, t- $\mathrm{C}_{4} \mathrm{H}_{9} \mathrm{OD}$ \\
\hline $\mathrm{T}$ & Temperatur \\
\hline ТВPР & Tertiär-Butyl-Peroxy-Pivalat $\left(\mathrm{C}_{4} \mathrm{H}_{9}(\mathrm{CO}) \mathrm{OOC}_{4} \mathrm{H}_{9}\right)$ \\
\hline TOF & Time of Flight \\
\hline w.E. & willkürliche Einheiten \\
\hline
\end{tabular}





\section{Einleitung}

Reine, teiloxidierte und substituierte Kohlenwasserstoffradikale spielen in der Atmosphärenchemie und bei Verbrennungsprozessen eine wichtige Rolle. Die Reaktionen dieser Radikale in der Gasphase sind ein aktueller Forschungsschwerpunkt der physikalischen Chemie.

Weltweit werden derzeit mehr als 95\% der von Menschen verbrauchten Energie durch Verbrennungen geliefert [GRI95]. Trotz der kontinuierlichen Suche nach alternativen Energiequellen gibt es wenig Zweifel, dass Verbrennungsprozesse auch in Zukunft sehr bedeutsam sein werden. Gegenstand der Chemie der Verbrennungen sind vor allem die Reaktionsverläufe bei hohen Temperaturen, aber auch die bei mäßigen Temperaturen in "kalten" Zonen von Flammen und in Motoren ablaufenden Prozesse sind von großem Interesse. Bei Temperaturen unter $900 \mathrm{~K}$ ist das Reaktionsverhalten von Alkyl-, Alkoxy und Alkylperoxy-Radikalen bestimmend [BAT94]. Die Erforschung der verschiedenen Reaktionswege und ihrer Kinetik ist von großem wirtschaftlichem und ökologischem Interesse. Die Konstruktion neuer leistungsstarker Motoren mit Zielrichtung auf das treibstoffarme Auto erfordert genaueres Wissen über die im Verbrennungsraum stattfindenden Reaktionen. Dem Fuel Design, der Entwicklung neuer Kraftstoffe, insbesondere für Dieselmotoren, kommt dabei eine Schlüsselrolle zu. Dimethylether und Dimethoxymethan werden als Dieselersatzkraftstoffe diskutiert ([WAL97], [SEH97], [CAT95]). Reaktionen dieser Substanzen werden in der vorliegenden Arbeit untersucht.

Organisches Material wird in großem Maßstab sowohl anthropogen als auch aus natürlichen Quellen in die Atmosphäre freigesetzt. Neben Prozessen in Wolkentröpfchen und an den Oberflächen von Partikeln findet ein erheblicher Teil der aus diesen Stoffen resultierenden Reaktionen direkt in der Gasphase statt [PIL95]. Photochemische Prozesse starten und begleiten diese Reaktionen. Die folgende Abbildung stellt schematisch den Aufbau der Erdatmosphäre einschließlich der in den jeweiligen Höhen herrschenden Druck- und Temperaturverhältnisse dar. 


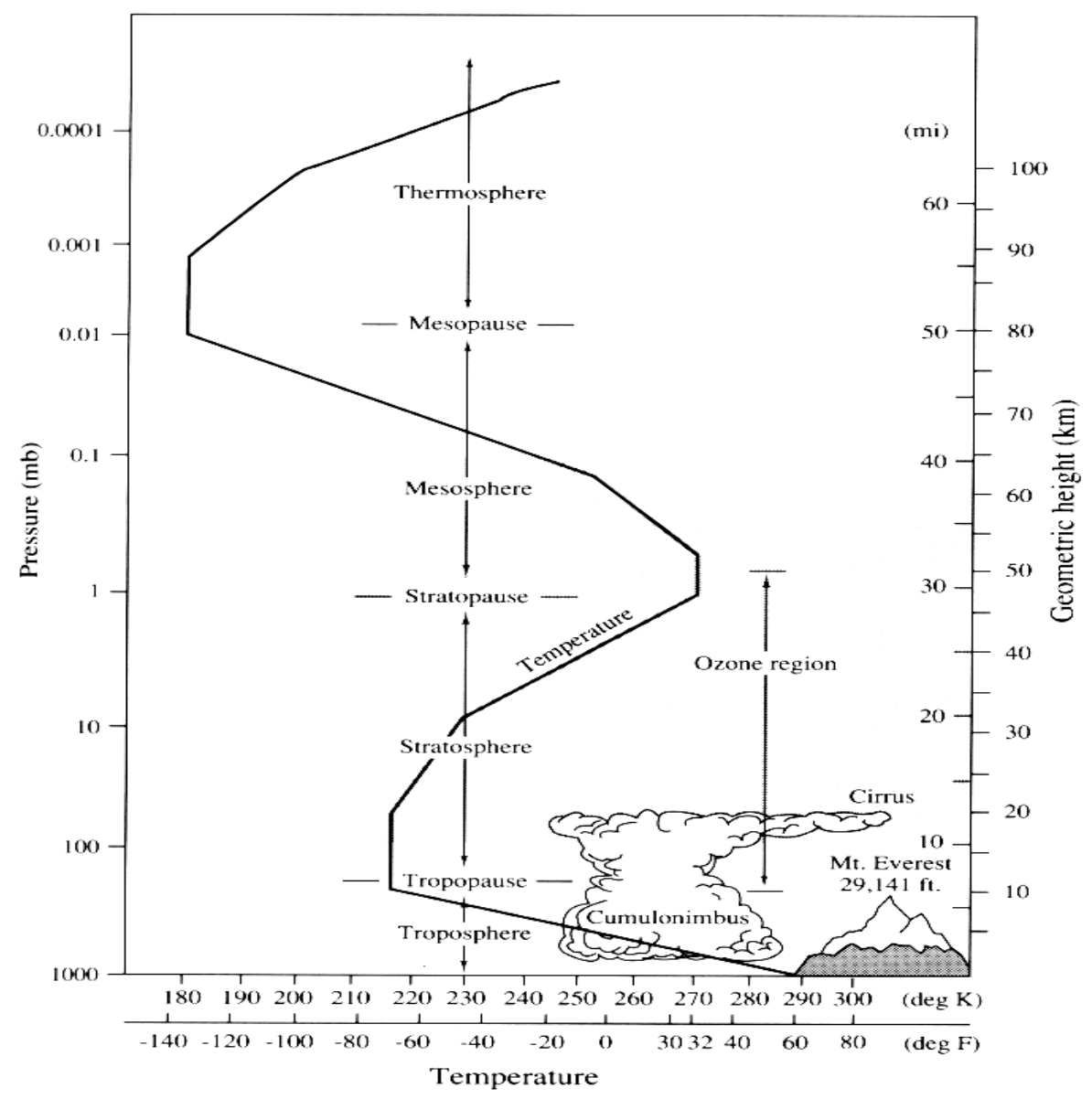

\section{Abbildung 1: Temperaturprofil der Erdatmosphäre, [SCH91]}

Die meisten der in die Atmosphäre freigesetzten Stoffe werden in der Troposphäre abgebaut. Nur besonders inerte Substanzen wie einige Fluorkohlenwasserstoffe (FCKW) gelangen in die Stratosphäre. Das in dieser Arbeit untersuchte Lösungs- und Reinigungsmittel sowie in Polymerisationen verwendete 1,1,1-Trichlorethan [FAL91] wurde im Jahr 1990 in einer Menge von $726 \cdot 10^{6} \mathrm{~kg}$ produziert. Davon gelangen schätzungsweise $705 \cdot 10^{6} \mathrm{~kg}$ in die Atmosphäre. Es wird angenommen, dass ca. 10-15\% die Stratosphäre erreichen [PLA95]. Wegen der möglichen photochemischen Freisetzung von Chloratomen wird dem Trichlorethan ein ozonabbauendes Potenzial zugeschrieben [FAL91]. Durch die FCKW-Halon-Verbotsverordnung von 1991 ist die Verwendung dieser Substanz stark eingeschränkt worden. Die Produktion wird voraussichtlich bis zum Jahr 2005 eingestellt. Trotzdem dürfte eine atmosphärische Wirkung dieser Substanz und der aus ihr abgeleiteten Produkte noch über einige Jahre erhalten bleiben.

Das Reaktionsschema zu Abbauprozessen in der Troposphäre und zu Verbrennungsvorgängen bei gemäßigten Temperaturen ist im Prinzip ähnlich. Es basiert auf radikalischen Kettenreaktionen. Den Kettenstart bildet die Abstraktion eines H-Atoms durch reaktive Spezies wie Sauerstoffatome, 
Wasserstoffatome, Methyl- und vor allem Hydroxylradikale ${ }^{1}$ ([HUC85], [WAR84], [GRI95], [PIL95]):

$$
\mathrm{RH}+\mathrm{O} / \mathrm{H} / \mathrm{CH}_{3} / \mathrm{OH} \rightarrow \mathrm{R}+\mathrm{OH} / \mathrm{H}_{2} / \mathrm{CH}_{4} / \mathrm{H}_{2} \mathrm{O}
$$

Diese Form der chemischen Radikalerzeugung über eine Wasserstoffatomabstraktion ist gleich der in dieser Arbeit überwiegend verwendeten Methode. Abweichend ist, dass Fluoratome als abstrahierende Reagenz eingesetzt werden, da diese Reaktion schneller und dadurch für die folgenden Radikalreaktionen einflussärmer verläuft. Es handelt sich hierbei um eine der am weitesten verbreiteten Methoden zur Gasphasenradikalerzeugung im Labor [NIE97]. Sie ist im Falle äquivalenter H-Atome am Ausgangskohlenwasserstoff eindeutig. Es ist Intention dieser Arbeit, Nachweismethoden für bisher noch nicht untersuchte und auf diesem Wege gebildete Kohlenwasserstoffradikale zu finden. Außerdem sollen bereits bekannte Radikale auf eine neue Art nachgewiesen werden. Für Letzteres sei als Beispiel das Cyclopentadienylradikal genannt.

Bei Substanzen wie Propan oder Butan resultiert die beschriebene Methode zur Radikalerzeugung in einer gleichzeitigen Produktion von zwei verschiedenen Radikalen. Ein weiteres Ziel der vorliegenden Arbeit besteht darin, eines der entstehenden isomeren Radikale spezifisch nachzuweisen, um über die so gewonnene Unterscheidungsfähigkeit das Studium von Reaktionen dieses Radikals zu ermöglichen.

In der Troposphäre und in Verbrennungskammern steht für die durch eine Reaktion gemäß (1) gebildeten Kohlenwasserstoffradikale vor allem molekularer Sauerstoff $\left(\mathrm{O}_{2}\right)$ als Reaktionspartner zur Verfügung. Die Erforschung der Reaktionsklasse Radikal $+\mathbf{O}_{2}$ ist ein weiteres Thema dieser Arbeit. Es gibt folgende Möglichkeiten des Fortgangs der Reaktion: Zum einen kann eine weitere Abstraktion eines H-Atoms am Alkylradikal erfolgen. Dieser Vorgang tritt bei Verbrennungsprozessen insbesondere bei Temperaturen von mehr als $750 \mathrm{~K}$ auf:

$$
\mathrm{R}+\mathrm{O}_{2} \rightarrow \text { Alken }+\mathrm{HO}_{2}
$$

Das $\mathrm{HO}_{2}$-Radikal ist relativ inert und es erfolgt auf diesem Wege nur eine langsame Kettenfortpflanzung [BAT94]. Der zweite Reaktionsweg ist bei niedrigeren Temperaturen bestimmend. Es kommt zur Ausbildung eines Gleichgewichts mit einem $\mathrm{RO}_{2}$-Additionskomplex auf der Produktseite:

\footnotetext{
${ }^{1}$ Solche reaktiven Stoffe werden in der Atmosphäre typischerweise durch photolytische Prozesse in Verbindung mit Sonnenlicht gebildet.
} 


$$
\mathrm{R}+\mathrm{O}_{2} \rightleftharpoons \mathrm{RO}_{2}
$$

Das durch (2b) gebildete Alkyl-Peroxy-Radikal ist in der Lage, eine Vielzahl von Folgereaktionen einzugehen, bei denen als Produkte unter anderem Alkylhydroperoxide, Alkylradikale, Alkoxyradikale, Aldehyde, Epoxide und Alkene sowie als Endprodukte $\mathrm{CO}$ und $\mathrm{CO}_{2}$ auftreten. Des Weiteren entstehen OH-Radikale. Diese bewirken, dass sich je nach Temperatur, Druck und Reaktionskanal eine langsame oder heftige Kettenfortpflanzung ergibt. Die folgende Abbildung stellt die Oxidation von n-Butan im Niedrigtemperaturbereich 500-850 K dar. Das Schema wurde von Griffiths und Barnard aufgestellt und ist typisch für die Oxidation von Alkanen mit vier oder mehr C-Atomen. Die Vielzahl von Reaktionen, die im Gesamtvorgang eine Rolle spielen, wird dabei deutlich:

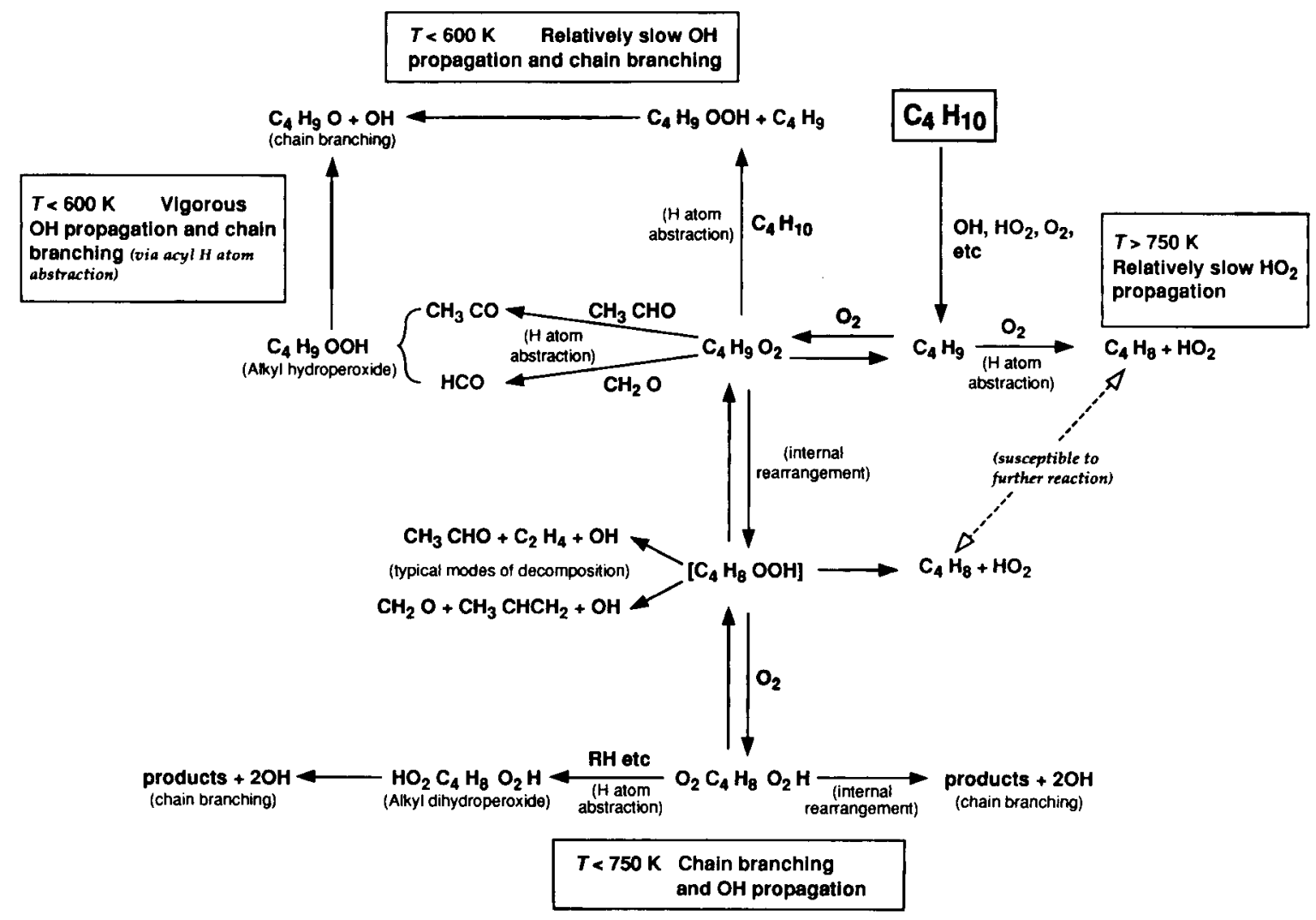

Abbildung 2: Schematische Oxidation von n-Butan im Temperaturbereich 500-850 K, [GRI95]

Steht den aus (1) erzeugten Kohlenwasserstoffradikalen atomarer Sauerstoff (O) als Reaktionspartner zur Verfügung - was bei Verbrennungsprozessen unter kraftstoffarmen Bedingungen von besonderem Interesse ist [HAC02] -, erfolgt diese Reaktion sehr viel schneller als mit molekularem Sauerstoff (vgl. die zugehörigen Geschwindigkeitskoeffizienten an entsprechender Stelle in dieser Arbeit). Die durch Messung und Abschätzung gewonnenen Geschwindigkeitskonstanten unter 
scheiden sich teilweise beachtlich: Für die Reaktion des t-Butylradikals werden z.B. folgende Werte angegeben: $\mathrm{k}\left(\mathrm{t}-\mathrm{C}_{4} \mathrm{H}_{9}+\mathrm{O}\right)=5,2 \cdot 10^{14}$ [WAS80], 1,4·10 14 [WAS00], 1,6 $10^{13}$ [BEN98] $\mathrm{cm}^{3} \cdot \mathrm{mol}^{-1} \cdot \mathrm{s}^{-1}$. Ein weiteres Ziel der Arbeit ist somit, ein in sich konsistentes System von Geschwindigkeitskonstanten für die Klasse der Reaktionen Radikal + $\mathbf{O}$ aufzubauen. Gelingt es dann, dieses System auf eine verlässliche Basis, d.h. in Bezug zu einer allgemein anerkannten und verifizierten absoluten Geschwindigkeitskonstanten einer Vergleichsreaktion zu stellen, kann dieses Vorgehen dazu beitragen, die Kinetik der betrachteten Reaktionsklasse sicher zu charakterisieren. Besonders hervorzuheben ist hierbei die Reaktion $\mathrm{CH}_{3}+\mathrm{O}$. Neben der Tatsache, dass das Ausgangsmolekül Methan den Hauptbestandteil von Erdgas ${ }^{2}$ bildet [FAL91], taucht diese Reaktion bei den Verbrennungsprozessen sehr vieler Kohlenwasserstoffe immer wieder auf. Ferner ist die Erforschung von Reaktionen bisher nicht beachteter Radikale mit Sauerstoffatomen eine Intention dieser Arbeit. Als Beispiel seien die aus c-Heptan, c-Oktan und t-Butylchlorid durch H-Atomabstraktion hervorgehenden Radikale genannt, für die bisher keine experimentellen Werte vorliegen.

\section{Die Geschwindigkeiten der Reaktionen von Kohlenwasserstoffradikalen mit Ozon $\left(\mathrm{O}_{3}\right)$,} Stickstoffmonoxid (NO) und atomarem Wasserstoff $(\mathbf{H})$ gehen in die aktuellen Modellierungen für Verbrennungsprozesse ein. Das gleiche gilt für die Modellrechnungen zu den Vorgängen in der Troposphäre und der Stratosphäre, wo diese reaktiven Gase natürlich und in Form von Luftverschmutzung vorkommen. Da diese Reaktionen bei niedrigen Temperaturen vorwiegend über einen angeregten Additionskomplex verlaufen, sind sie auch für das Studium unimolekularer Prozesse bedeutend. Die vorliegende Arbeit versucht, einen aktiven Beitrag zur Arbeit des Sonderforschungsbereichs SFB 357 "Molekulare Mechanismen Unimolekularer Prozesse" zu leisten, welcher kürzlich in die vierte Förderperiode gegangen ist. Die in dieser Arbeit gewonnenen Erkenntnisse sind über das Teilprojekt A8 "Dynamik chemisch aktivierter teiloxidierter Kohlenwasserstoffe" (Hack/Hoyermann) in den SFB 357 eingebunden.

Organische Peroxide und Persäureester werden zu 90\% in der Kunststoffindustrie als Initiatoren für die radikalische Polymerisation von olefinen Verbindungen wie Vinylchlorid, Ethylen, Styrol und ungesättigten Polyesterharzen verwendet [FAL91]. In diesem Sinne werden auch Di-tertiär-ButylPeroxid (DtBP) und tertiär-Butyl-Peroxy-Pivalat (tBPP) eingesetzt. Die Startreaktion der industriell betriebenen radikalischen Polymerisation ist hierbei der Zerfall der Peroxide:

\footnotetext{
${ }^{2}$ Im Jahr 1988 betrug die weltweite Förderung an Erdgas 1943 Milliarden Kubikmeter [FAL91], mit steigender Tendenz.
} 


$$
\mathrm{R}_{1}-\mathrm{O}-\mathrm{O}-\mathrm{R}_{2} \longrightarrow \mathrm{R}_{1}-\mathrm{O}+\mathrm{R}_{2}-\mathrm{O}
$$

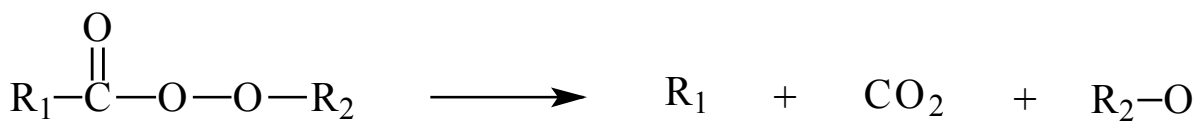

Es entstehen Alkoxy- und Alkylradikale. Sie addieren an die Doppelbindung des Monomers, wodurch ein Monomer-Radikal entsteht. Das Kettenwachstum erfolgt durch Addition weiterer Monomere. In der Startphase der Polymerisation und in Abwesenheit von Diffusionskontrolle ist es vorstellbar, Ergebnisse aus Experimenten in der Gasphase bei mäßigen Drücken ( $p<1$ bar) zur Modellierung heranzuziehen. Die für die Polymerisation relevanten Radikalreaktionen sollten dazu hinreichend charakterisiert sein. Weiterhin sollte die Möglichkeit bestehen, zuverlässige Extrapolationen auf Bedingungen vorzunehmen, die für technische Prozesse relevant sind. Der thermische Zerfall des DtBP und tBPP ist ausgiebig untersucht worden (z.B. [SER94], [AKZ92]). Über andere Reaktionen existieren dagegen nur wenig Daten. Die Untersuchung von Kinetik und Mechanismen des DtBP und des tBPP in homogener Gasphasenreaktion mit Fluor- und mit Sauerstoffatomen war bereits das Ziel des Autors in einer vorhergehenden Staatsexamensarbeit ([WEH97]). Diese Arbeit soll versuchen, die bereits erreichten Erkenntnisse aufzugreifen und mit anderen Methoden zu vertiefen. Damit wird ein Beitrag geleistet zum Europäischen Graduiertenkolleg (EGK) "Microstructural Control In Free-Radical Polymerization", im Speziellen zu dem Forschungsschwerpunkt "Kinetik in homogenen und heterogenen Systemen und in der Gasphase".

Die experimentellen Möglichkeiten der Arbeitsgruppe (Strömungsreaktoren mit EI-MS- und REMPI-MS-Nachweis, als Ergänzung GC-MS-Nachweis zur Endproduktanalyse, stationäre Reaktionszelle mit FTIR-Nachweis) werden konsequent genutzt, um die betrachteten Themen zu bearbeiten. Die sich daraus ergebenden komplementären Methoden erhöhen Erkenntnisgewinn. Hierbei hat sich die Versuchsanordnung Strömungsreaktor mit REMPI-Nachweis durch weitgehende Fragment- und Radikalvorläufer-Signalabwesenheit als besonders geeignet erwiesen, die Kinetik innerhalb einer Reaktionsklasse Radikal $+\mathrm{X}\left(\mathrm{X}=\mathrm{O}, \mathrm{O}_{2}, \mathrm{O}_{3}, \mathrm{NO}, \mathrm{H}\right)$ zu charakterisieren. Die Beschreibung der eingesetzten Versuchsanordnungen erfolgt im Kapitel 2 "Experimenteller Teil".

Im Kapitel 3 "Messergebnisse und Diskussion" werden die erzielten Messergebnisse und ihre Interpretation vorgestellt. Der Vergleich zur Literatur wird gleich im Anschluss zur betrachteten 
Fragestellung durchgeführt, um eine kompakte Darstellung zu gewährleisten. Messergebnisse, Interpretation und Diskussion sind aber deutlich voneinander abgesetzt.

In Kapitel 4 sind die Ergebnisse zusammengefasst.

In Kapitel 5 "Messtabellen" sind die gewonnenen quantitativen Ergebnisse noch einmal in tabellarischer Form angeführt, um eine eventuelle Neuinterpretation der Daten zuzulassen.

Der Anhang (Kapitel 6) listet die verwendeten Standardbildungsenthalpien und Literaturmassenspektren auf. Er enthält außerdem eine neu erstellte Auflistung der REMPI-Signalintensität von Kohlenwasserstoffradikalen. Diese Recherche ist für den Kinetiker als Hilfestellung beim schnellen Auffinden von Referenzradikalen zur Produktuntersuchung und zur Bestimmung von Geschwindigkeitskoeffizienten gedacht.

In Kapitel 7 sind die Hinweise auf die zitierte Literatur zu finden. 


\section{Experimenteller Teil}

Zur Untersuchung der Kinetik und der Produkte homogener Gasphasenreaktionen von Kohlenwasserstoffen, insbesondere der Radikale, haben sich Strömungsreaktoren mit Molekularstrahlprobennahme und anschließendem massenspektrometrischem Nachweis als besonders geeignet erwiesen. Die dabei verwendeten Versuchsanordnungen A (REMPI) und B (EI-MS) wurden schon mehrfach detailliert beschrieben (Versuchsanordnung A: [HEI86], [EDE88], [NAC98]; Versuchsanordnung B: [HOY79], [HOY79A], [HOY79B], [WEH97], [HOL97]). Sie wurden jedoch, auch im Rahmen der Messungen zu dieser Arbeit, mehrfach modifiziert. Die beiden Versuchsanordnungen werden deshalb in den folgenden Abschnitten noch einmal vorgestellt.

Versuchsanordnung C (FTIR) besteht aus einer statischen Zelle mit Photodissoziation zur Radikalund Atomerzeugung und FTIR-Nachweis. Diese Apparatur wurde in dieser Arbeit ausschließlich zur Produktanalyse verwendet. Sie ist bei [TRÖ97] und [KER99] ausführlich erklärt und in dieser Arbeit nur so weit beschrieben, wie es zum Verständnis der durchgeführten Messungen förderlich erscheint.

Die im Rahmen dieser Arbeit mit aufgebaute Versuchsanordnung D (GCMS) wurde einmal zur Endproduktanalyse eingesetzt. Sie ist bei [HOL01] ausführlich dargestellt.

\subsection{Versuchsanordnung A (REMPI)}

\subsubsection{Versuchsanordnung A im Überblick}

Die im folgenden beschriebene Apparatur stellt eine Kombination aus Strömungsreaktor mit kontinuierlicher Probennahme, Mehr-Photonen-Ionisation (MPI) und Flugzeitmassenspektrometer dar. Diese Anordnung hat den Vorteil, dass sich Radikale nach ihrer Entstehung in stationären Reaktionsbedingungen über ihr Massenspektrum direkt nachweisen lassen. Im Gegensatz zu Massenspektrometern mit Elektronenstoßionisation ist hierbei Fragmentbildung gering. Nichtradikalische Teilchen geben nur sehr selten Signale, so dass Überlagerungen häufig vermieden werden. Durch die Wellenlängenabhängigkeit des Ionisierungsprozesses ist in einigen Fällen ein zusätzliches Kriterium eines spezifischen Radikalnachweises gegeben (zweidimensionaler Nachweis). Nachteilig ist, dass nicht jedes Radikal ein Signal zeigt. Für die Untersuchung von Reaktionen, die verschiedene Radikale entstehen lassen, kann dieser Umstand aber auch günstig sein. Im Hinblick auf die ausbleibende Signaltätigkeit nichtradikalischer Teilchen steht dem erwähnten Vorteil die damit verbundene geringere Informationsmenge als Nachteil gegenüber. 
Mit der gewählten Anordnung lassen sich mehrere vollständige Massenspektren in einer Sekunde gewinnen, die allerdings eine gewisse Fehlerquote aufweisen, welches aber durch Mittelwertbildung ausgeglichen werden kann.

Die Apparatur ist sowohl für den qualitativen Radikalnachweis als auch für kinetische Messungen geeignet. Die folgende Abbildung gibt einen Überblick:

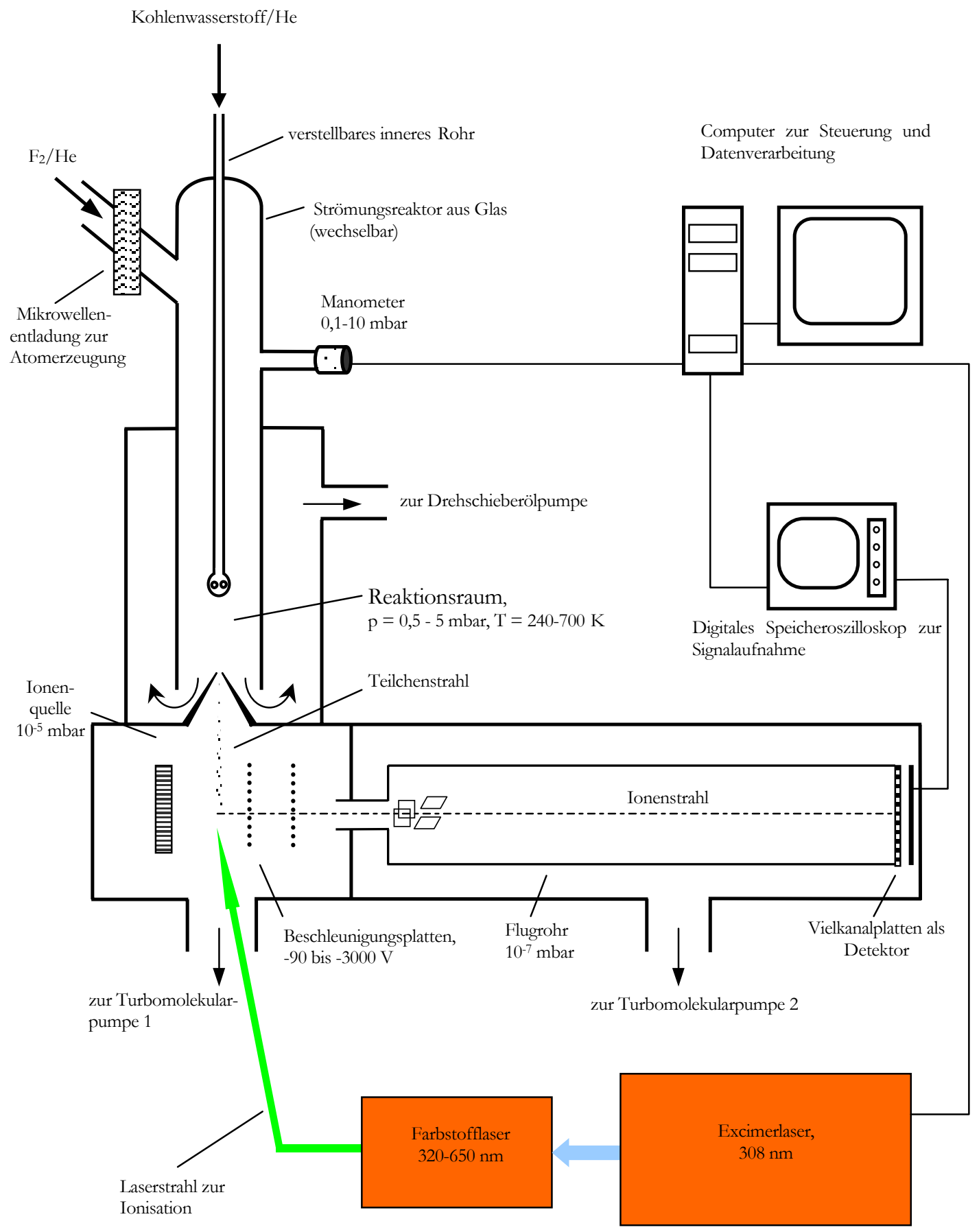

Abbildung 3: Versuchsaufbau A im Überblick 


\subsubsection{Reaktoren}

Schnelle Gasphasenreaktionen lassen sich mit Strömungsreaktoren untersuchen. Die Gase strömen in Richtung Probennahme (Skimmer) und treffen unter hoher Verdünnung mit Inertgas (He oder Ar) in den Reaktionszonen aufeinander. Es bildet sich unter dem Einfluss von Konvektion, Diffusion und chemischer Reaktion ein stationäres Konzentrationsprofil aus. Die Höhe der Gasflüsse, der vorherrschende Druck, die Temperatur und der Durchmesser des Reaktors bestimmen die Strömungsgeschwindigkeiten, wodurch die Verweildauer der Reaktanden und damit die Reaktionszeiten in den Reaktionszonen festgelegt sind. Bei Hoyermann [HOY75] findet sich eine genauere Betrachtung der gegenseitigen Einflüsse der genannten Faktoren.

Die folgenden Abbildungen stellen die zur Verfügung stehenden Reaktoren dar:

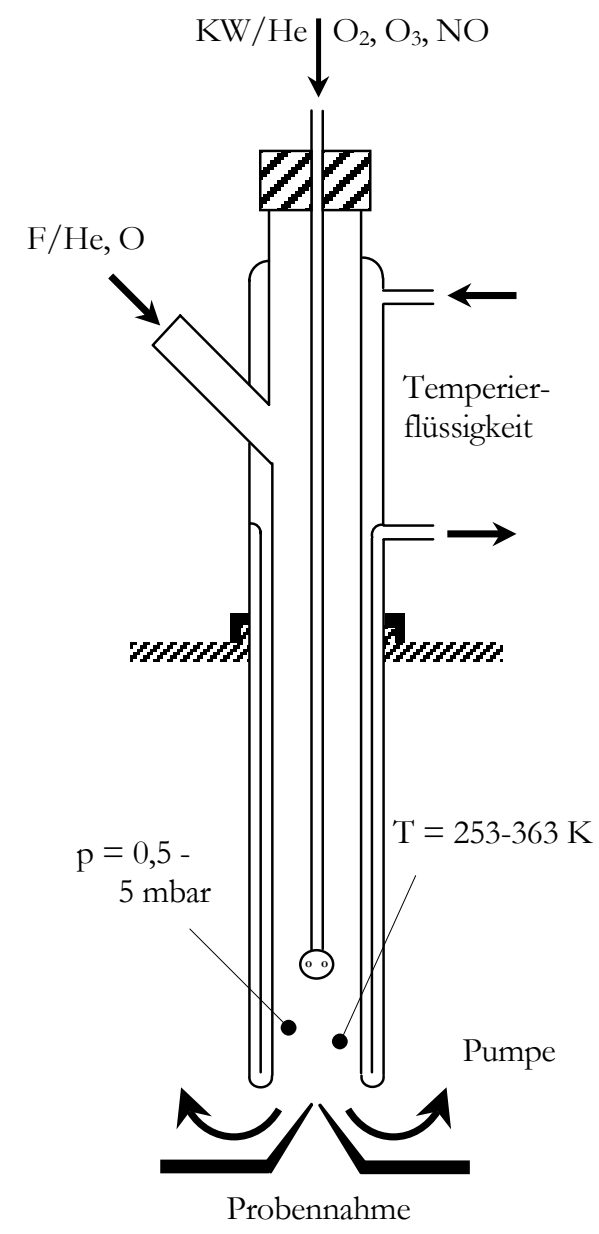

Abbildung 4: Strömungsreaktor 1

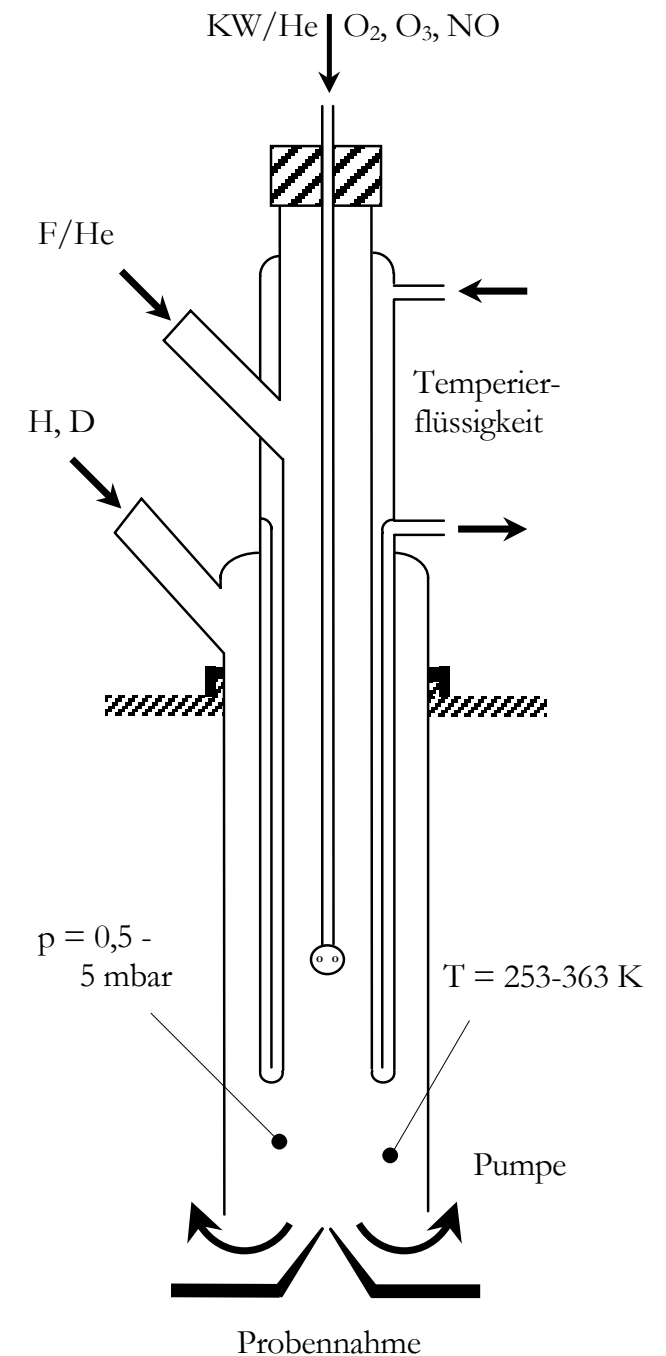

Abbildung 5: Strömungsreaktor 2 
Strömungsreaktor 1 wurde für die meisten Untersuchungen eingesetzt. Er besteht aus zwei zylindrischen Rohren aus Pyrexglas. Der Abstand des inneren Rohrs, der Sonde, zur Probennahme ist von 0-10 cm einstellbar. Die Gesamthöhe des Reaktors beträgt $75 \mathrm{~cm}$, der Innendurchmesser des äußeren Rohrs ist $22 \mathrm{~mm}$, der des inneren Rohrs $3 \mathrm{~mm}$. Für die beispielhaft gewählten Werte

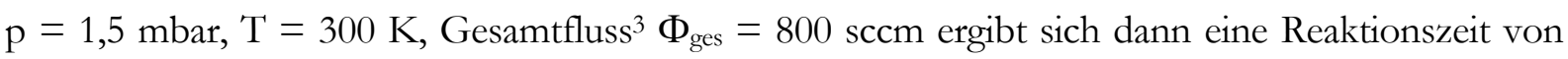
0,38 Millisekunden pro Zentimeter Reaktionsstrecke. Am Ende des inneren Rohrs ist zur besseren Mischung eine Lochkugel mit 0,8 cm Durchmesser zur besseren Mischung der Reaktanden angebracht. Im oberen Bereich ist eine seitliche Öffnung ausgearbeitet, an die ein Quarzglasröhrchen über eine Metallverschraubung angebracht werden kann. Über eine daran montierte elektrodenlose Mikrowellenentladung können die zur Radikalbildung nötigen F-Atome (oder in besonderen Fällen $\mathrm{Cl}$-Atome) aus einem $\mathrm{F}_{2}$-Helium-Gemisch $\left(\mathrm{Cl}_{2}-\mathrm{He}\right.$-Gemisch) erzeugt werden (vgl. Abschnitt 2.6). Über die Wege der übrigen Reaktanden in den Reaktor geben die Abbildungen Auskunft. Außen am Reaktor ist ein Temperiermantel angebracht. Mit einem 1:1 Ethandiol/Wasser Gemisch kann die Temperatur von $-30{ }^{\circ} \mathrm{C}$ bis $+90{ }^{\circ} \mathrm{C}$ eingestellt werden. Zur Aufrechterhaltung des Drucks und zur Herstellung der angestrebten Strömungsrichtung wird eine Drehschieberölpumpe (Leybold, DK 100, Saugleistung $100 \mathrm{~m}^{3} / \mathrm{h}$ ) verwendet. Die Messung des Drucks geschieht mit einem Membranvakuummeter (MKS, Baratron 122A).

Da Wasserstoffmoleküle mit Fluoratomen sehr schnell reagieren ${ }^{5}$, erfordert die Untersuchung der Temperaturabhängigkeit der Reaktionen von Kohlenwasserstoffradikalen mit H-Atomen (oder D-Atomen) eine besondere Konstruktion. Im Rahmen dieser Arbeit ist ein Reaktor gebaut worden, der dies ermöglicht. Strömungsreaktor 2 ist ähnlich aufgebaut wie Strömungsreaktor 1, verfügt außen jedoch über ein drittes Rohr (Innendurchmesser $45 \mathrm{~mm}$ ), welches im oberen Bereich ebenfalls eine seitliche Öffnung hat. Dort kann zur H-Atom-Erzeugung eine zweite Mikrowellenentladung angebracht werden. Die Kohlenwasserstoffradikale werden in einer ersten Reaktionszone im inneren Rohr (Innendurchmesser $20 \mathrm{~mm}$ ) durch schnelle Reaktion von Kohlenwasserstoffmolekülen mit Fluoratomen erzeugt. Die Reaktionsstrecke und damit die Reaktionszeit für diese Radikalbildungsreaktion ist variabel. Die H-Atome treffen weiter unten in einer zweiten Reaktionszone auf die Kohlenwasserstoffradikale, zu einem Zeitpunkt, bei dem die Bildungsreaktion bereits abgeschlossen ist. Für die zweite Reaktion steht eine Strecke von $5 \mathrm{~cm}$ zur Verfügung. Der Temperiermantel befindet sich zwischen dem zweiten und dritten Rohr, wodurch ein isothermes Reaktionsgemisch gewährleistet ist. Es gibt außerdem eine Variante von

\footnotetext{
$31 \mathrm{sccm}=1$ standard cubic centimeter per minute.

${ }^{4}$ Die Saugleistung ist bezogen auf den im Evakuierungsraum (Rezipienten) nötigen Betriebsdruck der Pumpe.

${ }^{5} \mathrm{k}\left(\mathrm{H}_{2}+\mathrm{F} \rightarrow \mathrm{HF}+\mathrm{H}\right)=8,43 \cdot 10^{13} \exp (-500 \mathrm{~K} / \mathrm{T}) \mathrm{cm}^{3} /(\mathrm{mol} \cdot \mathrm{s}), \mathrm{k}(298 \mathrm{~K})=1,58 \cdot 10^{13} \mathrm{~cm}^{3} /(\mathrm{mol} \cdot \mathrm{s})$ [ATK97].
} 
Strömungsreaktor 2 ohne Temperiermantel. Diese als Strömungsreaktor 2a bezeichnete Anordnung hat als Innendurchmesser $38 \mathrm{~mm}$ für das äußere Rohr und $20 \mathrm{~mm}$ für das innere Rohr. Für die zweite Reaktionsstrecke stehen $3 \mathrm{~cm}$ zur Verfügung.

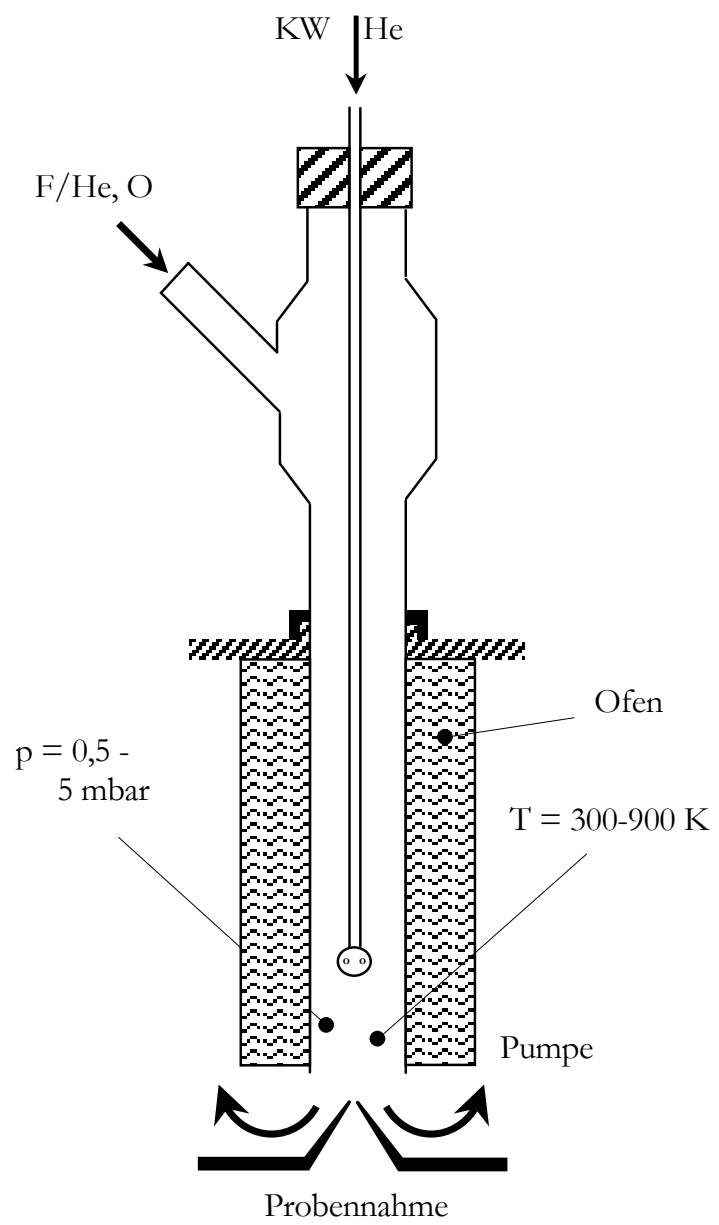

Abbildung 6: Strömungsreaktor 3

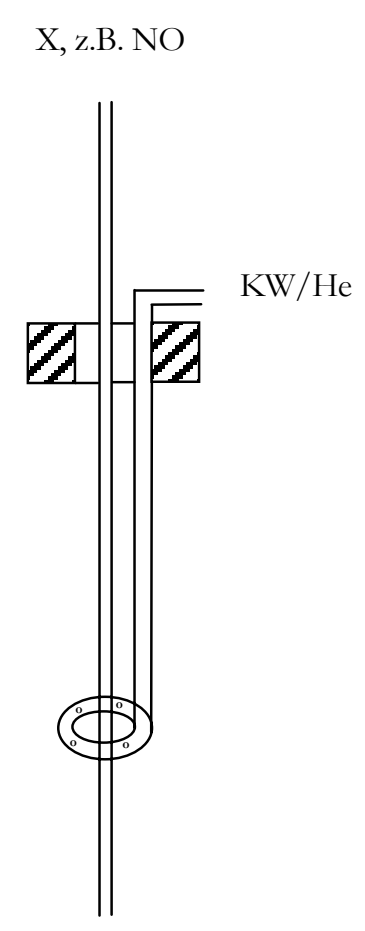

Abbildung 7: innerlich verstellbare Sonde

Strömungsreaktor 3 ist ähnlich aufgebaut wie die bereits beschriebenen. Er verfügt jedoch nicht über einen Temperiermantel, da er zum Einsatz für höhere Temperaturen (300-700 K) gedacht ist. Zur Erreichung dieser Temperaturen ist ein elektrischer Ofen so in die Reaktorhalterung eingelassen, dass ein enger Kontakt zwischen Reaktor und zylindrischem Ofen besteht. Zur Messung und Regelung der Temperatur ist ein Thermoelement in den Ofen eingesetzt. Im Rahmen der Probemessungen ist durch Einbau eines zweiten Thermoelementes in das innere Rohr überprüft worden, dass die eingestellten Ofentemperaturen auch tatsächlich im Reaktor vorherrschen. Fluoratome reagieren mit heißem Glas zu Siliziumfluoriden. Um die Verluste an F-Atomen zu verringern, ist im Bereich des Ofens der Reaktor mit $\mathrm{Al}_{2} \mathrm{O}_{3}$-Keramik ausgekleidet. Aus demselben Grund ist im Rahmen dieser Arbeit ein Reaktor gebaut worden, der im Ofenbereich 
aus Quarzglas (inneres Rohr) bzw. Quarzgut (äußeres Rohr) besteht. Mit diesem Reaktor lassen sich Temperaturen bis zu $900 \mathrm{~K}$ erreichen. Um ein Durchschmelzen der Perbunan- oder Vitondichtungen der Reaktorhalterung vorzubeugen, wurden kühlbare Flansche konstruiert.

Ist der Geschwindigkeitskoeffizient des Radikalreaktionspartners X mit dem Radikalerzeuger F groß (z.B. k(NO + F) = 1,69·1014 $\mathrm{cm}^{3} /(\mathrm{mol} \cdot \mathrm{s})$ [DEM97]), so muss eine getrennte Zufuhr von X gewährleistet sein. Zu diesem Zweck wird eine innerlich verschiebbare Sonde verwendet. Sie ist in allen aufgezeigten Reaktoren einsetzbar. Der Radikalerzeugungsreaktion wird eine bestimmte Sondenstrecke $s_{1}$ vorgegeben, die zum vollständigen Verbrauch von $\mathrm{F}$ ausreichend ist. Die so erzeugten Radikale treffen dann auf $\mathrm{X}$, welches durch ein inneres Rohr zugeführt wird. Der Reaktion Radikal + X steht die Reaktionsstrecke s2, welche ebenfalls variabel ist, zur Verfügung. Das innere Rohr besitzt zur besseren Vermischung seitliche Austrittsöffnungen.

\subsubsection{Probennahme}

Bei einem stationären Reaktionssystem erfolgt eine Probennahme am besten kontinuierlich. Die Konzentrationsverhältnisse zu einer gewählten Reaktionszeit sollen möglichst genau widergegeben werden. Außerdem soll der Nachweis empfindlich sein (hohe Teilchenzahldichte im Nachweisbereich). Eine Molekularstrahlprobennahme hat sich als dazu geeignet erwiesen. Ein Molekularstrabl ist gekennzeichnet durch eine gerichtete Teilchenbewegung bei hoher Geschwindigkeit und niedriger Temperatur (bis zu 1 K) [KNU73]. Zur Ausbildung eines Molekularstrahls muss der Durchmesser der Expansionsdüsenöffnung größer sein als die mittlere freie Weglänge der Teilchen bei den im Reaktor herrschenden Bedingungen. Nach Berechnungen von Hoyermann [HOY79] sind die bei den Messungen dieser Arbeit verwendeten Expansionsdüsen (Skimmer) mit $20 \mathrm{~mm}$ Höhe, einem Innenwinkel $34^{\circ}$ und einem Öffnungsdurchmesser zwischen 0,3 und 0,8 $\mathrm{mm}$ am besten dazu geeignet, Wechselwirkungen des Teilchenstrahls mit den Wänden von Düse und Vakuumkammer zu vermeiden. Die große Druckdifferenz zwischen Reaktor und Nachweiskammer führt zu einer adiabatischen Expansion des Gasgemisches. Die Zahl der Stöße im sich ausbildenden Teilchenstrahl ist stark herabgesetzt. Folgereaktionen werden damit vermieden - die Reaktion wird "eingefroren". Dies ist besonders wichtig für den Nachweis kurzlebiger Spezies.

Da der Strömungsreaktor in eine Halterung mit einem Edelstahlfederbalg (Tombak) eingepasst ist, kann der Molekularstrahl in das Zentrum des Nachweissystems justiert werden.

Zur Herstellung des Druckverhältnisses in der Nachweiskammer $\left(10^{-5}\right.$ mbar $)$ wird eine Turbomolekularpumpe (Leybold LH Turbovac CSV 360, Saugleistung 360 l/s) mit vorgeschalteter Drehschieberölpumpe (Leybold D 30, Saugleistung $30 \mathrm{~m}^{3} / \mathrm{h}$ ) verwendet. Die Druckmessung erfolgt mit einem Ionisationsvakuummeter nach Penning (Leybold PM 41). 


\subsubsection{Mehr-Photonen-Ionisation}

Für den massenspektrometrischen Nachweis ist eine Ionisierung der Teilchen der Probe erforderlich. Materie kann durch die Einwirkung von Licht, dessen Energiegehalt groß genug ist, ionisiert werden. Beträgt das Ionisierungspotenzials eines Moleküls $10 \mathrm{eV}$, so ist für einen Ionisierungsprozess, der über die Aufnahme eines Photons verläuft (Ein-Photonen-AbsorptionsProzess), Licht der Wellenlänge $124 \mathrm{~nm}$, also im schwer zugänglichen Vakuum-UV, erforderlich.

Möglich ist aber auch, dass ein Molekül die zur Ionisierung nötige Energie über die Aufnahme mehrerer Photonen größerer Wellenlänge aufnimmt (Mehr-Photonen-Absorptionsprozess). Allerdings ist die Wahrscheinlichkeit für eine simultane Absorption mehrerer Photonen sehr klein. Erst der Einsatz von gepulstem Laserlicht mit einer hohen Intensität von $10^{6}-10^{9} \mathrm{~W} / \mathrm{cm}^{2}$ macht diesen Prozess bedeutsam [GRO88]. Je mehr Photonen gleichzeitig aufgenommen werden müssen, desto kleiner wird die Ausbeute an Ionen. Die Wahrscheinlichkeit der Ionisation durch die Aufnahme mehrerer Photonen steigt erheblich an, wenn der Prozess resonant über elektronische Molekül-

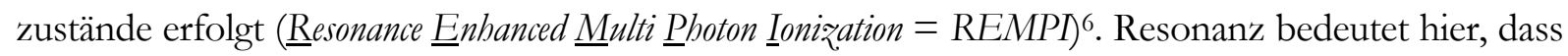
die Wellenlänge des eingestrahlten Lichts auf die Energiedifferenzen zwischen dem Grundzustand und den angeregten Zuständen abgestimmt ist. Die Aufnahme der Photonen muss nicht mehr exakt gleichzeitig erfolgen. Die Ionisation von Radikalen ist häufig erfolgreich, wenn die angeregten Molekülzustände Rydbergzustände sind. Das freie Elektron befindet sich dabei in wasserstoffähnlichen Orbitalen und lässt sich leicht zu höheren Quantenzahlen anregen, wodurch die Ionisierungsgrenze dann erreicht wird. Die Wellenzahlen $\widetilde{v}$, welche die energetische Lage der Rydbergzustände für vertikale Übergänge angeben, werden durch die Rydberg-Formel beschrieben [HUD87]:

$$
\begin{gathered}
\qquad \tilde{v}=\mathrm{IP}-\frac{\mathrm{R}}{\widetilde{\mathrm{n}}^{2}}, \\
\mathrm{IP}=\text { Ionisierungspotenzial (in Wellenzahlen), } \widetilde{\mathrm{n}}^{2}=\mathrm{n}-\delta=\text { effektive Quantenzahl, } \\
\mathrm{n}=\text { Quantenzahl, } \delta=\text { Quantendefekt, } \mathrm{R}=\text { Rydbergkonstante }=109737 \mathrm{~cm}^{-1} .
\end{gathered}
$$

Die Höhe des Quantendefekts hängt davon ab, wie stark das Rydbergorbital den Kern des molekularen Ions räumlich durchdringt. Die Werte von $\delta$ variieren, liegen aber in einem bestimmten Bereich für jeden Übergang: $\delta($ s-Serie $) \approx 0,9-1,2, \delta$ (p-Serie $) \approx 0,4-0,6, \delta(\mathrm{d}-\mathrm{Serie}) \approx 0-0,1$, $\delta(\mathrm{f}-\mathrm{Serie}) \approx 0$ [WEN84]. Für das erste Glied einer Rydbergserie gilt üblicherweise $\mathrm{n}=3$.

\footnotetext{
${ }^{6}$ Die Mehrphotonenspektroskopie hat außerdem zur Gewinnung spektroskopischer Informationen beigetragen. Da hier andere Auswahlregeln gelten als bei der Einphotonspektroskopie, können auch andere angeregte Zustände erreicht werden. Viele neue elektronische Zustände, insbesondere Rydbergzustände, wurden dadurch gefunden.
} 
Die nachfolgende Abbildung zeigt einige Mechanismen zur Photoionisation:

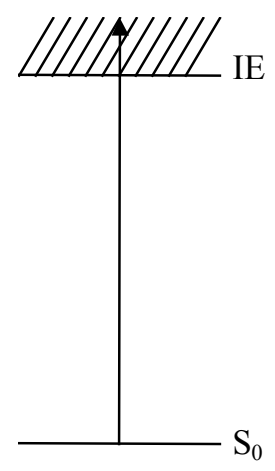

EinphotonenIonisation

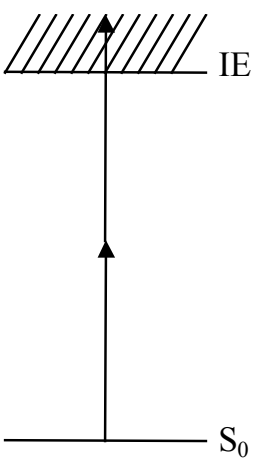

Nicht resonante ZweiphotonenIonisation
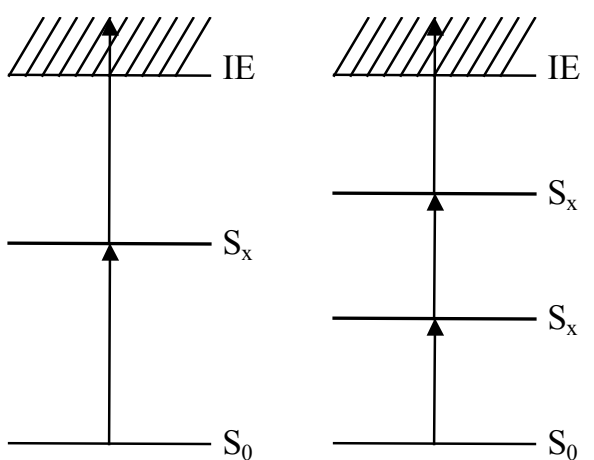

Resonante (1+1)-Ionisation

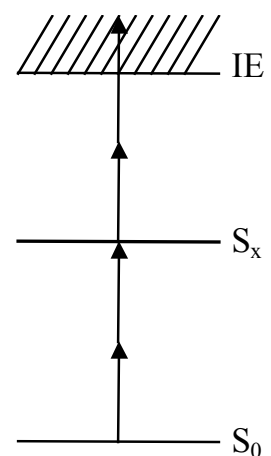

Resonante
$(1+1+1)$-Ionisation
Resonante

$(2+2)$-Ionisation

\section{Abbildung 8: Mechanismen der Photoionisation}

Häufig wird der REMPI-Prozess in der Form "2 + 1" o.ä. angegeben. Die erste Zahl gibt hierbei die zum Erreichen des angeregten Zwischenzustands erforderliche Anzahl an Photonen an. Zwischen dieser Zahl n, der Intensität des Ionensignals I und der Laserenergie E gilt folgender funktionaler Zusammenhang: $\mathrm{I}=\mathrm{E}^{\mathrm{n}}$. Die zweite Zahl gibt die vom Zwischenzustand benötigte Photonenzahl bis zum Ionisationskontinuum an. Die Photonenordnung ist wichtig ist für das Ableiten spektroskopischer Konstanten. Für den im Rahmen dieser Arbeit erforderlichen spezifischen Nachweis von Radikalen ist die Photonenordnung kaum relevant. Es wird daher nur wenig Aufwand betrieben, diese aufzuklären.

Bei höheren Energiedichten des Ionisierungslasers kommt es besonders bei kurzen Wellenlängen zur Fragmentbildung, d.h., das Molekül wird nicht nur als ganzes ionisiert, sondern zerfällt in

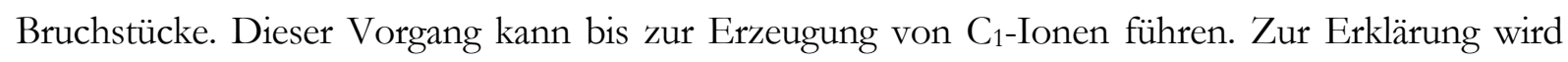
einerseits die Prädissoziation herangezogen, nach der das Molekül nach Aufnahme von Photonen in angeregten Zuständen in neutrale Fragmente zerfällt, die anschließend ionisiert werden. Eine andere Erklärung ist das Ladder-Switching-Modell [BEI91], nach welchem zunächst das Molekül ionisiert wird, um dann nach Aufnahme weiterer Photonen in kleinere Ionen auseinander zu fallen. Das Phänomen der Photofragmentierung kann ein Beitrag zum Teilchennachweis sein, wenn sich ein spezifisches Fragmentierungsmuster ergibt. Es kann aber auch problematisch sein, wenn entschieden werden muss, ob ein Signal von einem Fragment oder dem Produkt eines schnellen Zerfalls im Reaktor hervorgerufen wird. Auch wenn die Vorläufersubstanz eines Radikals ähnlich wie das Radikal selbst fragmentiert, kann dies den spezifischen Nachweis stören. In manchen Fällen 
ist es möglich, den Störeffekt herauszurechnen oder durch Erniedrigung der Laserenergie eine Ionisation der Vorläufersubstanz zu unterbinden.

Dem REMPI-Prozess stehen Konkurrenzprozesse gegenüber: stoßfreie oder stoßinduzierte innere Konversion ${ }^{7}$ und Fluoreszen₹. Die Fluoreszenzlebensdauer liegt in der Größenordnung 10-8-10-9 s. Dem entspricht eine Abklingkonstante von $10^{8}-10^{9} \mathrm{~s}^{-1}$. Die typische Geschwindigkeitskonstante für den Ionisierungsprozess liegt im Bereich $10^{11}-10^{12} \mathrm{~s}^{-1}$ [EDE88] und ist damit deutlich höher, so dass Fluoreszenz als Konkurrenz vernachlässigt werden kann. Die folgende Abbildung gibt eine Übersicht über die Konkurrenzprozesse.

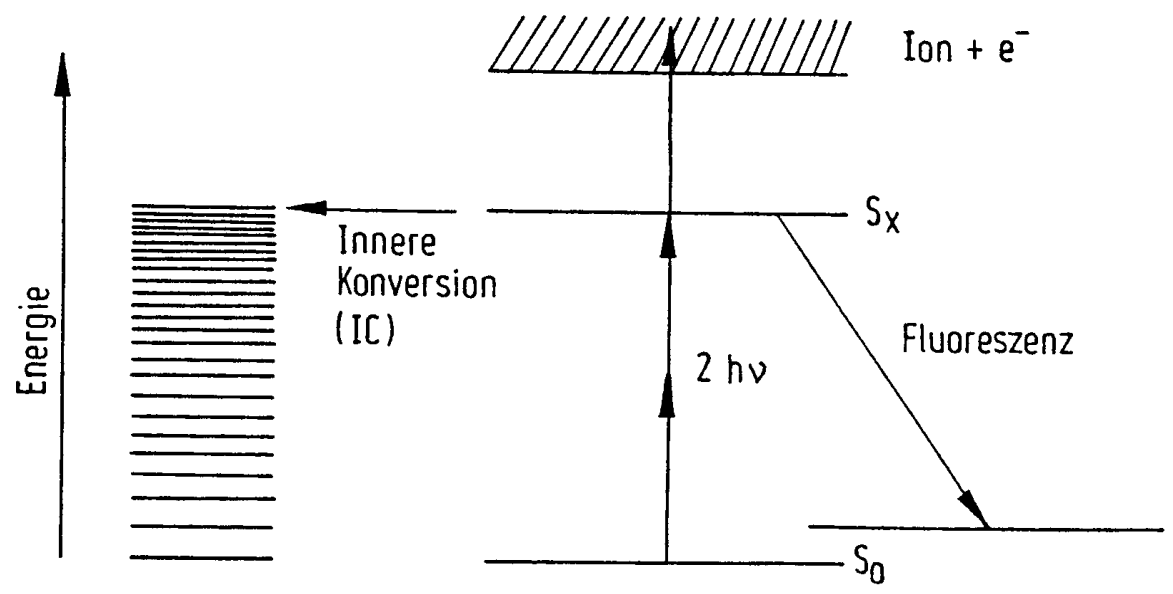

\section{Abbildung 9: Schematische Darstellung eines [2+1]-REMPI-Prozesses und möglicher Konkurrenzmechanismen}

Als Lichtquelle zur Ionisation wird ein durchstimmbarer Farbstofflaser (Lambda Physik, FL 2002) eingesetzt, der von einem gepulsten Excimerlaser (Lambda Physik, LPX 200) gepumpt wird. Der Excimerlaser emittiert Licht der Wellenlänge 308 nm (XeCl-Füllung). Die Pulsdauer beträgt 28 ns, die Pulsfrequenz ist zwischen 1 und $20 \mathrm{~Hz}$, die Intensität zwischen 150 und $400 \mathrm{~mJ}$ regelbar. Für die meisten Messungen wurde als Pulsfrequenz $6 \mathrm{~Hz}$ und als Pulsenergie $300 \mathrm{~mJ}$ gewählt. Die Pulsfrequenz des Farbstofflasers ist gleich der des Pumplasers. Durch den Einsatz verschiedener Farbstofflösungen sind Wellenlängen zwischen 320 und $970 \mathrm{~nm}$ einstellbar. Dieses wird im Laser intern über den Einsatz eines Gitters unter Ausnutzung der Littrow-Bedingung erreicht. Die Neigung des Gitters wird über einen Schrittmotor, der von einem Rechner gesteuert wird, eingestellt. Die Rechnersteuerung macht dabei das weiter unten beschriebene Messverfahren des Wellenlängenscans praktikabel. Die Pulsdauer des Farbstofflasers beträgt 20 ns, die Pulsenergie liegt zwischen 1 und $20 \mathrm{~mJ}$, je nach gewählter Pumpleistung, Art und Zustand des eingesetzten

\footnotetext{
${ }^{7}$ Das ist ein Übergang in höhere Schwingungszustände niedriger liegender elektronischer Zustände.
} 
Farbstoffs. Der aus dem Farbstofflaser austretende Lichtstrahl wird mit Hilfe eines Quarzprismas um $90^{\circ}$ umgelenkt und über eine Quarzplatte in die Ionenquelle eingeblendet. Dort wird er über eine Quarzlinse (Brennweite $\mathrm{f}=40 \mathrm{~mm}$ ) in das Zentrum der Ionenquelle fokussiert, wo er senkrecht auf den Teilchenstrahl trifft. Der nicht absorbierte Teil des Laserlichts wird über eine Quarzplatte ausgekoppelt und trifft auf einen Photomultiplier (Hamamatzu 1P28). Der dort ausgelöste Spannungspuls dient als Triggersignal für die Datenaufnahme. Ein Teil des Laserstrahls wird vor der Ionenquelle über eine Quarzplatte ausgekoppelt und trifft zur Energiemessung auf einen Piezokopf, der mit einem Joulemeter (Gentec ED 200) geeicht wird.

\subsubsection{Flugzeitmassenspektrometer}

Zum Nachweis der erzeugten Ionen wird ein Flugzeitmassenspektrometer verwendet (Eigenbau, Muster: Bendix MA2, Ionenquelle und Detektion modifiziert). Mit der hier verwendeten Anordnung wird pro ionisierenden Laserpuls binnen $40 \mu$ s ein komplettes Massenspektrum erhalten. Die Ionenquelle besteht aus drei Bauteilen: Einem auf Massepotenzial liegenden Edelstahlblock (Pusher), einer ersten Beschleunigungsplatte (Ziehplatte) und einer zweiten Beschleunigungsplatte (Hauptbeschleunigung). Die Beiden Beschleunigungsplatten haben zentral eine runde Öffnung (Durchmesser $10 \mathrm{~mm}$ ), die mit einem hochdurchlässigen Netz bespannt ist. Der Laserstrahl ist so fokussiert und justiert, dass er die Teilchen des Molekularstrahls im räumlichen Zentrum zwischen Pusher und Ziehplatte ionisiert. Das elektrische Potenzial der Ziehplatte ist zwischen -90 und $-1000 \mathrm{~V}$ regelbar. Die positiv geladenen Ionen werden somit in Richtung Ziehplatte beschleunigt. Eine Ziehspannung von $-200 \mathrm{~V}$ hat in bezug auf Signalhöhe und Auflösung die besten Ergebnisse geliefert. Nach Durchtritt durch die Ziehplatte, werden die Ionen in Richtung Beschleunigungsplatte, die auf einem Potenzial von -3000 V liegt, weiter beschleunigt. Jedes einfach geladene $\operatorname{Ion}^{8}$ besitzt eine kinetische Energie von

$$
\begin{gathered}
\mathrm{E}_{\mathrm{kin}}=\frac{1}{2} \mathrm{~m} \cdot \mathrm{v}^{2}=\mathrm{e} \cdot \mathrm{U}, \\
\mathrm{m}=\text { Masse des Ions, } \mathrm{v}=\text { Geschwindigkeit, } \\
\mathrm{e}=\text { Elementarladung, } \mathrm{U}=\text { Beschleunigungspannung. }
\end{gathered}
$$

Da alle Ionen dieselbe Beschleunigungsspannung erfahren, haben sie bei unterschiedlichen Massen gemäß Gleichung (2) unterschiedliche Geschwindigkeiten. Nach Durchtritt durch die Hauptbeschleunigungsplatte fliegen die Ionen unbeschleunigt die $145 \mathrm{~cm}$ lange Driftstrecke s entlang in

\footnotetext{
8 Doppelt ionisierte Teilchen wurden nicht beobachtet. Trotzdem wird an den meisten Stellen dieser Arbeit das in der Massenspektrometrie übliche Masse-Ladungsverhältnis $\mathrm{m} / \mathrm{z}$ ohne die zugehörige Einheit u verwendet.
} 
Richtung Detektor. Aufgrund ihrer unterschiedlichen Geschwindigkeiten benötigen sie auch unterschiedliche Zeiten, die Strecke x zu bewältigen:

$$
\begin{gathered}
\mathrm{t}=\left(\frac{\mathrm{x}}{\sqrt{2 \cdot \mathrm{e} \cdot \mathrm{U}}}\right) \cdot \sqrt{\mathrm{m}}, \\
\mathrm{x}=\text { Driftstrecke, } \mathrm{e}=\text { Ladung des Ions, } \\
\mathrm{U}=\text { Beschleunigungspannung, } \mathrm{m}=\text { Masse des Ions. }
\end{gathered}
$$

Beim verwendeten Aufbau und bei den eingesetzten Substanzen bewegen sich die Flugzeiten in etwa zwischen $8 \mu \mathrm{s}(\mathrm{m}=12 \mathrm{u})$ und $25 \mu \mathrm{s}(\mathrm{m}=100 \mathrm{u})$. Es wurde mit dem 2- $d$-Isobutylradikal $(\mathrm{m}=58 \mathrm{u})$ und dem t-Butylradikal $(\mathrm{m}=57 \mathrm{u})$ bei der Wellenlänge $440 \mathrm{~nm}$ eine Auflösung A von 250 (vollständige Auflösung) ermittelt. Im Flugrohr sind noch jeweils ein Paar vertikale und horizontale Platten sowie eine kreisförmiger Ring (Ionenlinse) angebracht, um den Ionenstrahl auf den Detektor zu justieren. Sie werden mit statischen Spannungen zwischen -2700 V und -3000 V regelbar betrieben.

Als Detektor können zwei Vielkanalplatten (Multi Channel Plates, MCP, Hamamatzu) oder eine Multi Sphere Plate (MSP, EI-Mul Technologies) eingesetzt werden. Beide Systeme sind schnelle Sekundärelektronenvervielfacher, die eine Verstärkung um den Faktor $10^{6}$ erreichen. Bei den MCP ist die erste Platte mit $-2500 \mathrm{~V}$, der Zwischenring mit $-1500 \mathrm{~V}$ und die zweite Platte mit -500 V versorgt. Bei der MSP liegen an der Vorderseite $-3000 \mathrm{~V}$, an der Rückseite $-90 \mathrm{~V}$ an. Die durch die auftreffenden Ionen ausgelöste Elektronenkaskade trifft bei beiden Verstärkersystemen auf einen Edelstahlauffänger, bei dem sie über einen $50 \Omega$ Widerstand abfließt und einen schnellen Spannungspuls auslöst, der über ein digitales Speicheroszilloskop (DSO) weiterverarbeitet wird.

Zwar zeichnet sich die MSP durch eine größere Robustheit und einen günstigeren Preis aus, jedoch wurden für die meisten Messungen zu dieser Arbeit an der Apparatur die MCP eingesetzt. Testmessungen während dieser Arbeit haben gezeigt, dass die Signale der MSP mit einem stärkeren Nachschwingen belastet sind, was sich nicht günstig auf einen konzentrationsgetreuen Nachweis auswirkt und das Auflösungsvermögen mindert.

Zur Erzeugung des benötigten Vakuums (MCP mindestens 5·10-5 Torr, MSP $1 \cdot 10^{-4}$ Torr) ist in der Mitte des Flugrohrs eine Turbomolekularpumpe (Leybold Turbovac 150, Saugleistung 150 1/s)

\footnotetext{
${ }_{9}^{9}$ Die Auflösung A ist definiert als der Quotient $\mathrm{m} / \Delta \mathrm{m}$, wobei $\Delta \mathrm{m}$ die kleinste Massendifferenz ist, die ein Ion der Masse $\mathrm{m}$ von einem anderen haben muss, um noch als getrenntes Signal beobachtbar zu sein. Die Auflösung gibt an, welches die maximale Masse ist, bei der man das gewählte Trennungsmaß vollständige Auflösung, 10\% Tal oder brauchbare Auflösung noch verwirklichen kann. A hängt davon ab, wie scharf man alle Ionen einer bestimmten Massenzahl bündeln kann, um sie noch von Ionen zu unterscheiden, deren Massenzahl nur wenig verschieden ist.
} 
angeflanscht, mit einer Drehschieberölpumpe (Leybold D6, Saugleistung $6 \mathrm{~m}^{3} / \mathrm{h}$ ) als Vorpumpe. Erreicht wird damit ein Vakuum von $10^{-6}-10^{-7}$ Torr. Die Druckmessung erfolgt mit einem Ionisationsvakuummeter nach Penning (Leybold PM 41).

\subsubsection{Signalverarbeitung}

Die Flugzeitunterschiede zweier benachbarter Massen liegen im 100 ns Bereich (z.B. $\mathrm{t}(\mathrm{m} / \mathrm{z}=57)=18,062 \mu \mathrm{s}, \mathrm{t}(\mathrm{m} / \mathrm{z}=58)=18,221 \mu \mathrm{s})$. Daher ist eine schnelle Messelektronik erforderlich, um eine ausreichend aufgelöste Verarbeitung zu gewährleisten. Das am Detektor anfallende Signal wird in ein digitales Speicheroszilloskop (Le Croy DSO 9350 AL) eingelesen. Wie oben schon erwähnt, wird das DSO durch den ionisierenden Laserstrahl mit Hilfe eines Photomultipliers getriggert. Das DSO digitalisiert das eingehende Massenspektrum und visualisiert es auf seinem Bildschirm. Es ist ferner mit einer arithmetischen Mittelwertbildungsfunktion ausgestattet, so dass eine bestimmte Anzahl an Spektren gemittelt und in einem im DSO vorhandenen Speicher abgelegt werden kann. Auch das gemittelte Spektrum kann auf dem DSO-Bildschirm angezeigt und manuell ausgelesen werden. Das Oszilloskop verfügt über eine IEEE488 Schnittstelle (GPIB), über die es mit einer GPIB-Schnittstellenkarte (National Instruments, GPIB-PCII) im zentralen Steuerungsrechner (Intel Pentium, $90 \mathrm{MHz}$ ) kommuniziert. Für die Steuerung der Messabläufe wurde von Nacke ein Programm namens TofShell unter Borland Pascal 7.0 mit Hilfe des objektorientierten Zusatzpakets Turbo Vision geschrieben. Das Programm ermöglicht dem Bediener, die wesentlichen Funktionen der Apparatur zentral steuern zu können und sich wiederholende Messprozeduren automatisch ablaufen zu lassen. Zeitraubendes, ablenkendes und fehlerträchtiges Ablesen und Übertragen der Messwerte entfallen damit weitgehend. Im Laufe der Arbeit wurde dieses Programm vom Autor in Zusammenarbeit mit Hold mehrfach modifiziert und erweitert. So wurde vorher das ganze gemittelte Spektrum (Waveform) byteweise über die GPIBKarte übertragen. Der damit verbundene Verzicht auf das untere Datenbyte führte zu einem Übertragungsfehler von 5\%. Durch Umstellung auf eine wordweise Datenübermittlung ist dieser Fehler nun vollständig ausgelöscht. Eine weitere Verbesserung betrifft die Signalverarbeitung zur weiteren Auswertung. Statt einer Verwendung des Intensitätsmaximums wird nun der Peak numerisch integriert und die Peakfläche als Intensitätsgröße verwendet. Die folgende Abbildung skizziert die Integrationsfläche und einen in die verwendete Trapezregel eingehenden Zeitscheibenbereich der rechten Peakhälfte: 

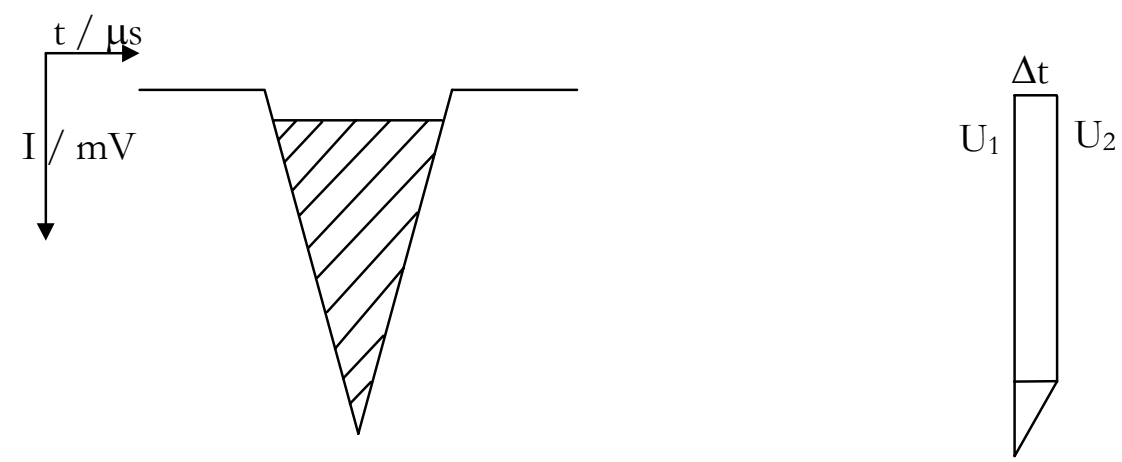

\section{Abbildung 10: Peakintegration}

Es wird zunächst das betragsmäßige Intensitätsmaximum ermittelt. Die Grenzen des Integrationsbereichs werden jeweils links und rechts auf Basis eines Prozentwerts vom Maximum ermittelt, üblicherweise wurden 10\% gewählt. Gemäß des Digitalisierungsrasters des Oszilloskops werden Zeitscheiben eingeteilt, ihre Flächen ermittelt ${ }^{10}$ und addiert. Diese Summe wird als Intensität in der Dimension $\mathrm{mV} \cdot \mu$ s ausgegeben. Unter Ausnutzung der vollen $500 \mathrm{MHz}$ Auflösung des Oszilloskops ergibt sich für einen mittleren am Oszilloskop eingestellten zeitlichen Auswahlbereich zur Mittelwertbildung eine zeitliche Auflösung von 2 ns. Bei einer Peakbreite von 36 ns stehen somit 18 Stützstellen zur Verfügung. In vielen Fällen ist es möglich den Auswahlbereich zur Mittelwertbildung weiter einzugrenzen und somit die Stützstellenzahl weiter zu erhöhen.

Der Steuerungsrechner ist neben der GPIB-Karte auch mit einer A/D-D/A-Karte (Addi Data, PA 310) ausgestattet, die über 16 Eingänge (A/D, 0-10 V), sechs Ausgänge (D/A, 0-10 V) sowie zwei Logiktreiber verfügt. Dadurch ist der Steuerungsrechner in der Lage, mit weiteren externen Geräten zu kommunizieren, insbesondere mit dem Excimerlaser-Kontrollrechner (Commodore PC 10) und dem Farbstofflaser-Kontrollrechner (Tandy TRS 80). Es können die Werte für den Druck im Strömungsreaktor und für die Laserenergie des Farbstofflasers eingelesen werden. Weiterhin können Schaltsignale an einen Mikrowellengenerator und an ein Magnetventil ausgesendet werden. Die folgende Abbildung gibt einen Überblick über die Computersteuerung des Versuchsaufbaus:

\footnotetext{
${ }^{10}$ gemäß Abbildung 10: $A_{\text {Zeitscheibe }}=\mathrm{t} \cdot \mathrm{U}_{2}+1 / 2 \cdot\left(\mathrm{U}_{1}-\mathrm{U}_{2}\right)=1 / 2 \cdot \mathrm{t} \cdot\left(\mathrm{U}_{1}+\mathrm{U}_{2}\right)$
} 


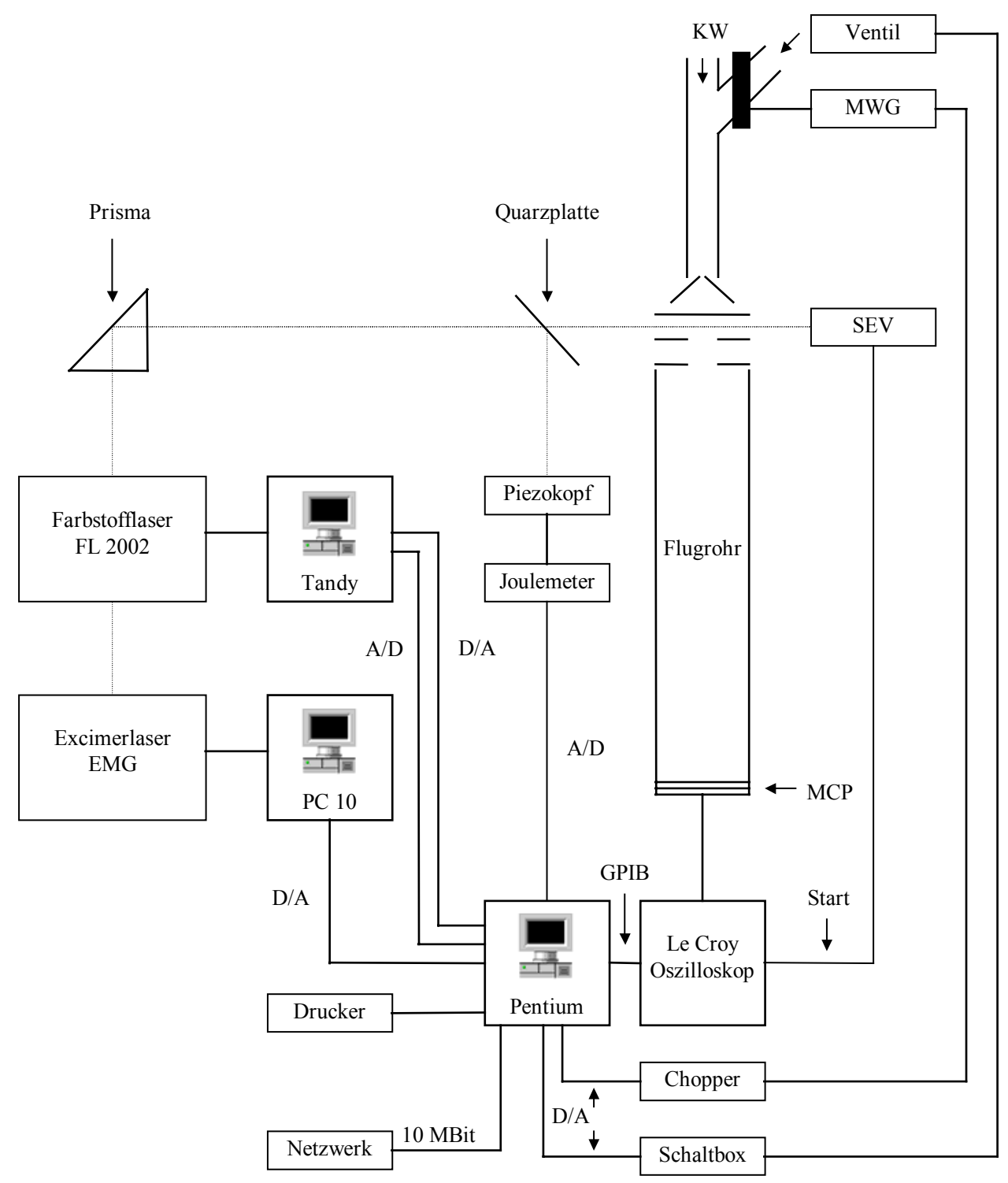

Abbildung 11: Computersteuerung des Versuchsaufbaus A, [NAC98]

\subsubsection{Messverfahren}

In Tof-Shell sind einige Messverfahren vorgesehen, die besonders häufig Anwendung finden. Neben diesen Standardmessroutinen, die im folgenden erläutert werden sollen, hat der Bediener der Apparatur weiterhin die Möglichkeit, die Signale unmittelbar am Oszilloskop mit den Cursorfunktionen manuell auszulesen.

Bei der Waveform to ASCII-Prozedur wird ein im DSO gespeichertes Spektrum, üblicherweise gemittelt, in eine ASCII-Datei auf den Pentium-Rechner überführt. Es wird das abgespeichert, was auf dem Bildschirm des DSO als Sichtbereich ausgewählt wird. Diese Messroutine ist vorgesehen, um ein Einzelphänomen, beispielsweise das REMPI-TOF-Spektrum eines Kohlenwasserstoffradikals festzuhalten. Ähnlich ist die Funktion Screendump. Mit ihr wird das angezeigte Bild des DSO durch einen am Pentium-Rechner angeschlossenen Drucker ausgedruckt. 
In einigen Fällen sind REMPI-Signale von Radikalen wellenlängenselektiv, z.B. beim Methyl- oder Allylradikal. Mit dem automatischen Wellenlängendurchlauf wird die REMPI-Wellenlängenabhängigkeit eines Signals verfolgt. Der Messende gibt dem Programm eine Flugzeit und ein Wellenlängenintervall innerhalb des vom verwendeten Laserfarbstoff möglichen Bereichs vor und setzt den Farbstofflaser auf die Startwellenlänge. Es werden die am Oszilloskop eingestellt Anzahl an Spektren (Sweeps) aufgenommen, gemittelt, abgespeichert und als komplette Waveform an das Programm weitergegeben. Das Programm ermittelt innerhalb einer Umgebung der Flugzeit die Peakfläche (vgl. Abschnitt 2.1.6) und speichert den Wert zugeordnet zur eingestellten Wellenlänge ab. Es setzt den Farbstofflaser in der vom Messenden gewählten Schrittweite (Scanningstep) auf die nächste Wellenlänge und die Prozedur beginnt von vorne. Die Wellenlängen und die zugehörigen Intensitäten befinden sich nach Abschluss der Messungen spaltenweise angeordnet in einer ASCIIDatei. Technisch wird diese Prozedur wie folgt umgesetzt:

Das Programm triggert den Excimerlaser über einen Softwareinterrupt. Die Energie des daraus resultierenden Farbstoff-Laserstrahls wird mit dem Joulemeter gemessen und über einen A/DEingang eingelesen. Gleichzeitig wird das Oszilloskop über den GPIB-Bus abgefragt, ob die vorher eingestellte Zahl an Spektren akkumuliert ist. Ist dies der Fall, so hört das Programm auf, den Excimerlaser zu triggern, überträgt und verarbeitet das gemittelte Spektrum und gibt dem Steuerungsrechner des Farbstofflasers einen Spannungspuls. Dieser vom Laserhersteller mit einem BASIC-Programm ausgerüstete Computer erhöht die Wellenlänge des Farbstofflasers um den Scanning Step, indem ein Schrittmotor das Gitter im Farbstofflaser um einen bestimmten Winkel verändert, und gibt anschließend eine Bestätigung in Form einer positiven Spannung ab. Diese wird über einen Eingang der A/D-Karte des Pentiums eingelesen und das Programm fährt fort, bis die Endwellenlänge erreicht ist.

Für die verwendeten Verfahren der kinetischen Messung sei als Grundlage auf das Kapitel 2.4 hingewiesen. Die folgenden Erläuterungen erklären nur die steuerungstechnischen Zusammenhänge.

Vor Beginn der Absolutmessung erfragt das Programm zwei Flugzeiten und dazugehörige Umgebungen zur Intensitätsmaximumermittlung. Nach Start der Messung wird der Benutzer zur Eingabe der Sondenstrecke s aufgefordert. Dieser Wert und der im Reaktor herrschende Druck werden dem Benutzer auf dem Bildschirm angezeigt und gleichzeitig in einer ASCII-Datei abgespeichert. Das Programm triggert den Excimerlaser über einen Softwareinterrupt. Gleichzeitig wird das Oszilloskop über den GPIB-Bus abgefragt, ob die vorher am DSO eingestellte Zahl an Spektren erreicht ist. Falls ja, hört das Programm auf, den Excimerlaser zu triggern, überträgt und 
verarbeitet das gemittelte Spektrum. Die ermittelten Intensitätsmaxima werden angezeigt. Die Peakfläche wird ermittelt, angezeigt und in die ASCII-Datei abgespeichert. Das Programm stoppt und gibt dem Benutzer die Gelegenheit, die Messung eventuell unter anderen Bedingungen (z.B. plus/minus Reaktionspartner) zu wiederholen oder zu einer anderen Sondenstrecke s überzugehen. Die Prozedur wird fortgesetzt, bis der Benutzer die Messung beendet.

Bei der Relativmessung werden in analoger Weise zur Absolutmessung zwei Massen erfasst. Über einen D/A-Ausgang wird eine 5V-Spannung auf eine Schaltbox gegeben, woraufhin sich ein Magnetventil öffnet und den Zufluss für den Reaktionspartner des Radikals freigibt. Nach einer wählbaren Wartezeit zur Flussstabilisierung triggert das Programm den Excimerlaser, bis die vorher eingestellte Spektrenzahl am Oszilloskop erreicht ist. Dann hört das Programm auf, den Excimerlaser zu triggern, überträgt und verarbeitet das gemittelte Spektrum und schließt das Magnetventil. Danach läuft die gleiche Prozedur ohne Reaktionspartnerzugabe ab. Aus den vorangegangenen Messungen resultiert ein Peakflächenwertepaar, das gesondert abgespeichert und dessen Ergebnis in einer Vorauswertung (lineare Regression) graphisch angezeigt wird. Zur Kontrolle für den Messenden werden Intensitätsmaxima und die Größe der Peakflächen über den Bildschirm ausgegeben. Die Messprozedur kann nun mit einem veränderten Radikalreaktionspartnerfluss wiederholt werden.

\subsection{Versuchsanordnung B (EI-MS)}

\subsubsection{Versuchsanordnung $B$ im Überblick}

Die Versuchsanordnung B stellt eine Kombination aus Strömungsreaktor mit kontinuierlicher Probennahme, Elektronenstoßionisation und Sektorfeldmassenspektrometer dar. Diese Anordnung macht einen der Reaktion zeitlich unmittelbar folgenden und empfindlichen Nachweis möglich. Die Apparatur ist besonders zur Produktanalyse geeignet, da nicht mehr nur vorwiegend Radikale Signale geben wie in Versuchsanordnung A, sondern auch andere Substanzen, die in den Reaktor gegeben werden oder dort gebildet werden. Sie ist aber auch für kinetische Messungen geeignet, was zur Bestimmung der Geschwindigkeit von Radikalbildungsreaktionen genutzt wurde. Die Möglichkeit zur Kinetikmessung kam auch dann zum Tragen, wenn Radikale REMPI-inaktiv waren. Insofern stellt die Versuchsanordnung B eine sehr gute Ergänzung zur Versuchsanordnung A dar.

Durch die Elektronenstoßionisation kommt es zu starker Fragmentierung der nachgewiesenen Teilchen. Die daraus sich ergebenden Fragmentierungsmuster erhöhen den Informationsgrad von Massenspektren. Als nachteilig erweist sich der Effekt der Fragmentierung dadurch, dass er zu Überlagerung der signalgebenden Massen verschiedener Teilchen führt. Dieser Vorgang lässt sich 
bei Versuchsanordnung B durch Verringerung der Ionisierungsenergie bedeutend abmildern. Zwar schwindet die Signalintensität, in vielen Fällen lässt sich aber die Überlagerung dadurch vollständig vermeiden.

Man kann die Versuchsanordnung B systematisch in die drei Baugruppen Reaktor, Probennahmebereich und Nachweisbereich unterteilen. Die folgende Abbildung zeigt den schematischen Aufbau.

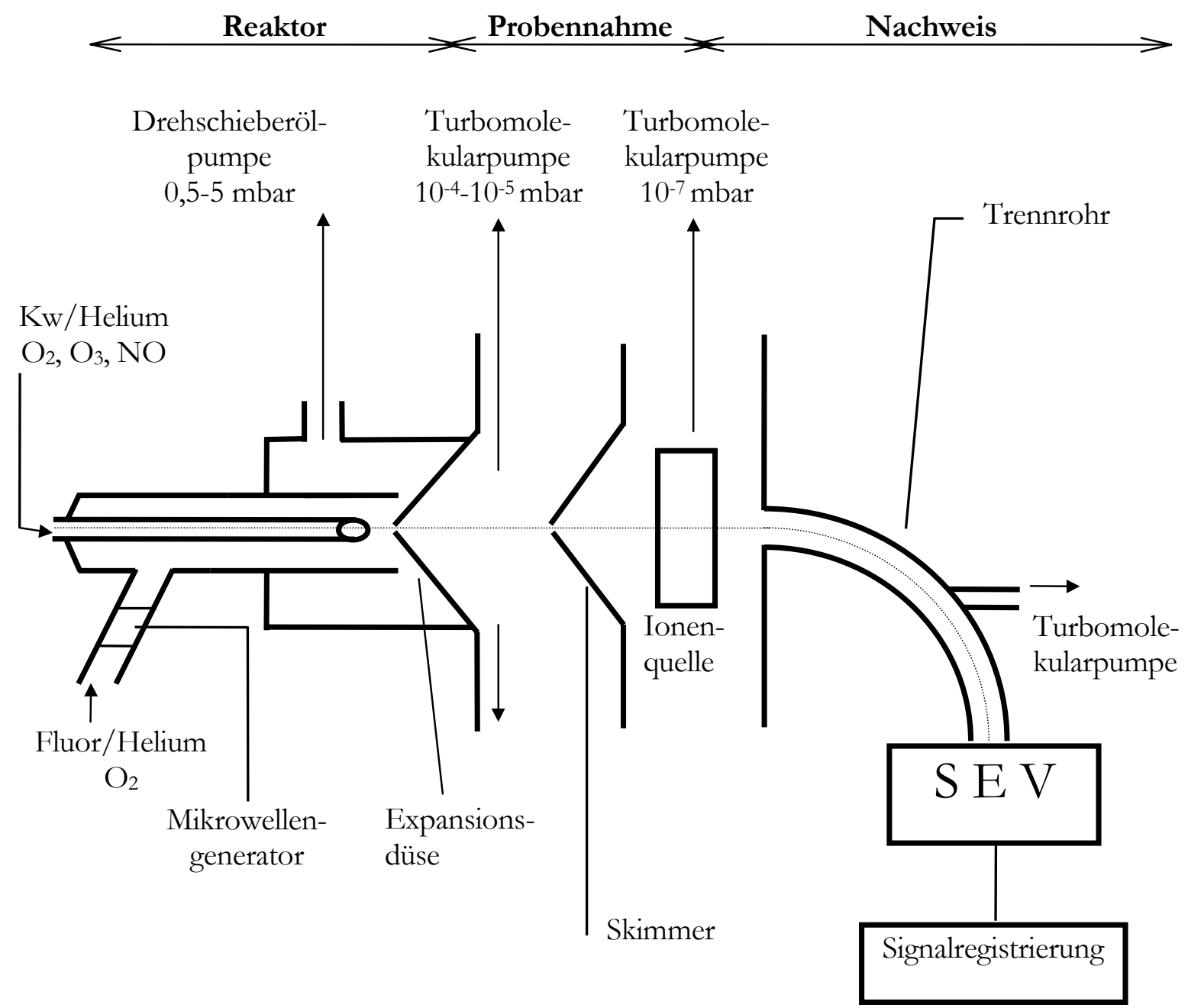

\section{Abbildung 12: Versuchsanordnung B im Überblick}

\subsubsection{Reaktor und Probennahme}

Der verwendete Reaktor entspricht Strömungsreaktor I. Dementsprechend sei auf die in Abschnitt 2.1.2 gemachten Ausführungen verwiesen. Im Unterschied zur Versuchsanordnung A ist der Strömungsreaktor horizontal eingebaut und aus apparaturgeometrischen Gründen $10 \mathrm{~cm}$ länger. Der Druck im Reaktor von 0,5 bis 3 mbar wird durch eine Drehschieberölpumpe (Leybold E 250, 
Saugleistung $250 \mathrm{~m}^{3} / \mathrm{h}$ ) hergestellt. Die Messung des Drucks geschieht mit einem Membranvakuummeter (MKS, Baratron 122A).

Die Probennahme erfolgt doppelstufig. Die Reaktanden gelangen über eine erste Expansionsdüse (Innenwinkel $34^{\circ}$, Öffnungsdurchmesser $0,5 \mathrm{~mm}$ ) in die Zwischenvakuumkammer. Der dort herrschende Druck wird durch eine Turbomolekularpumpe (Leybold Turbovac 450, Saugleistung $450 \mathrm{l} / \mathrm{s}$ ) mit vorgeschalteter Drehschieberölpumpe (Leybold, D 60, Saugvermögen $60 \mathrm{~m}^{3} / \mathrm{h}$ ) hergestellt, die Druckmessung erfolgt mit einem Ionisationsvakuummeter nach Penning (Leybold PM 41). Der sich ausbildende Teilchenstrahl wird mit Hilfe eines Federbalgs auf eine weitere

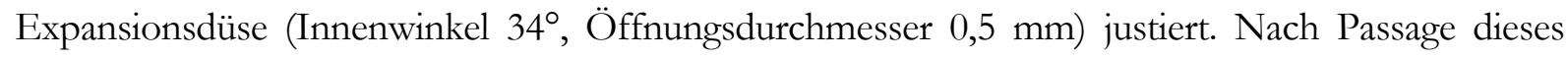
zweiten Skimmers gelangt der Teilchenstrahl in die Ionenquelle des Sektorfeldmassenspektrometers. Über die Eigenschaften von Molekularstrahlen sei auf die in Abschnitt 2.1.3 gemachten Ausführungen verwiesen.

\subsubsection{Nachweis}

Der Nachweis erfolgt unter Verwendung eines Massenspektrometers (Varian MAT CH 5). Es handelt sich hierbei um ein einfach fokussierendes Sektorfeldmassenspektrometer mit kontinuierlicher Elektronenstoßionisation (electron impact, EI). Das Ionenquellengehäuse ist zur besseren Ausbildung des Teilchenstrahls und zur Anpassung an die Evakuierungstechnik vergrößert worden. Die Elektronen werden von einer Glühkathode aus Rheniumdraht, die eine Emission zwischen $3 \mu \mathrm{A}$ und $1 \mathrm{~mA}$ liefert, ausgesandt und treffen nach Beschleunigung senkrecht auf den Teilchenstrahl. Sie werden dabei durch zwei Permanentmagnete auf eine schraubenförmige Trajektorie gebracht, um den Ionisierungsquerschnitt zu erhöhen. Die Ionisierungsenergie lässt sich entweder fest auf $70 \mathrm{eV}$ einstellen oder in einem Bereich zwischen 4,5 eV und $29 \mathrm{eV}$ stufenlos regeln. Durch den Elektronenstoß werden die Moleküle bzw. die Atome positiv ionisiert. Diese Ionen werden dann durch ein elektrisches Feld, welches sich stufenweise bis zu einer Spannung von $3 \mathrm{kV}$ aufbaut, aus dem Teilchenstrahl herausgelöst und beschleunigt. Die Geschwindigkeit, die ein Kation dabei annimmt, unterliegt folgendem Gesetz, welches sich aus dem Energieerhaltungssatz zwischen kinetischer und elektrischer Energie ergibt. Bei vektoriellen Größen sind nur die Beträge angegeben.

$$
\begin{gathered}
\mathrm{v}=\sqrt{\frac{2 \cdot \mathrm{U} \cdot \mathrm{z}}{\mathrm{m}}}, \\
\mathrm{v}=\text { Geschwindigkeit des Kations }, \mathrm{z}=\text { Ladung, } \\
\mathrm{U}=\text { Spannung des elektrischen Feldes, } \mathrm{m}=\text { Masse. }
\end{gathered}
$$


Durch ein elektrooptisches Linsensystem wird der Ionenstrahl auf den Eintrittsspalt des in einem Winkel von $90^{\circ}$ gebogenen Trennrohres gerichtet. Am Trennrohr liegt ein Magnetfeld an, durch das die Ionen auf eine kreisförmige Bahn gebracht werden. Die hier wirkende ablenkende Lorentzkraft ist gegeben durch:

$$
\mathrm{F}_{\mathrm{L}}=\mathrm{z} \cdot \mathrm{B} \cdot \mathrm{v}
$$

$$
\mathrm{F}_{\mathrm{L}}=\text { Lorentzkraft, } \mathrm{B}=\text { magnetische Flussdichte. }
$$

Ihr wirkt die Zentripetalkraft $F_{\text {mech }}$ entgegen:

$$
\begin{gathered}
\mathrm{F}_{\text {mech }}=\frac{\mathrm{m} \cdot \mathrm{v}^{2}}{\mathrm{r}}, \\
\mathrm{r}=\text { Radius der Kreisbahn Gleichung. }
\end{gathered}
$$

(5) und (6) zusammen ergeben:

$$
\mathrm{v}=\frac{\mathrm{z} \cdot \mathrm{B} \cdot \mathrm{r}}{\mathrm{m}}
$$

Aus (4) und (7) folgt die sogenannte massenspektrometrische Grundgleichung:

$$
\frac{\mathrm{m}}{\mathrm{z}}=\frac{\mathrm{r}^{2} \cdot \mathrm{B}^{2}}{2 \cdot \mathrm{U}}
$$

In der Massenspektrometrie organischer Moleküle treten bei Ionisierungsenergien von weniger als $70 \mathrm{eV}$ zwei- oder mehrfach geladene Ionen selten auf. Die Ladung z des Kations kann von daher durch die Elementarladung $e$ ersetzt werden. Durch Umformung ergibt sich:

$$
\mathrm{B}=\frac{1}{\mathrm{r}} \cdot \sqrt{\frac{2 \cdot \mathrm{U}}{\mathrm{e}}} \cdot \sqrt{\mathrm{m}} .
$$

Die Beschleunigungsspannung U und der Ablenkradius r werden konstant gehalten. Gleichung (9) macht dann die Abhängigkeit zwischen der magnetischen Flussdichte und der Masse des abgelenkten Kations deutlich. Durch Variation der Magnetfeldstärke ist es möglich, die interessierende Masse zu erfassen. Das Messen von B geschieht über eine temperaturstabilisierte Hallsonde, welche die zur Magnetfeldstärke proportionale Hallspannung wiedergibt. Diese Hallspannung wird über eine A/D-Karte in den Steuerungsrechner eingelesen. Der Computer rechnet diese Werte mit Hilfe einer Eichkurve in den zugehörigen Massenwert um. Das Einstellen 
des Magnetfeldes geschieht entweder manuell (die Messung wird bei einer bestimmten Masse durchgeführt, Einzelmassenmessung), oder es wird ein vollständiges Massenspektrum in zeitlicher Abfolge aufgenommen, wobei der Magnetstrom kontinuierlich ansteigt (Magnetstromscan).

Die positiv geladenen Ionen gelangen zum Austrittsspalt des Trennrohres, nach dessen Passage ihre Ladung ein elektrisches Signal auslöst (vgl. Abschnitt 2.2.4, Signalaufnahme).

Das Ionisierungspotenzial der meisten organischen Verbindungen liegt zwischen 8 und $15 \mathrm{eV}$ [BEC90]. Da die Energie der Elektronen zur Bindungsspaltung im Molekül ausreicht (C-C-Bindung: 348 kJ/mol = 3,61 eV, C-H-Bindung: 413 kJ/mol = 4,28 eV [PAU67]), kommt es nicht nur zur Ionisierung der Moleküle, sondern die Moleküle fragmentieren auch zu kleineren Einheiten, die ihrerseits wieder geladen sind. Die Verteilung und die Intensität der Fragmentierung ist stoffartspezifisch und kann somit zur Interpretation der Messergebnisse ausgenutzt werden, da für viele stabile Stoffe (nicht aber für Radikale) Spektren katalogisiert sind ([COR75], [MAS74]). Hierbei muss jedoch beachtet werden, dass die Spektren von Apparaturparametern wie etwa Ionenquellentemperatur, Druck, Elektronenemission oder Spaltbreiten abhängig sind und es deshalb zu Abweichungen zwischen den selbst gemessenen Spektren und den Literaturspektren kommen kann. Die Fragmentierung kann so stark sein, dass bei der Masse, bei welcher der sogenannte Molekülpeak oder Mutterpeak erscheint, kein Signal zu beobachten ist. Die Masse, bei der das intensivste Signal liegt, wird als Basispeak bezeichnet. Einige Fragmente sind durch induktive oder mesomere Effekte stabilisiert. Ist deren Masse charakteristisch für den Stoff, so werden sie als Schlüsselfragmente bezeichnet [BEC90]. Die Fragmentierung geht mit der Verkleinerung der Ionisierungsenergie zurück. Beim verwendeten Massenspektrometer kann die Ionisierungsenergie zwischen 4,5 eV und 29,5 eV stufenlos eingestellt werden. Der erwähnte Zusammenhang zwischen Fragmentierung und Ionisierungsenergie ist ein Hilfsmittel, um Aussagen über Produktkanäle zu treffen. So kann zum Beispiel bei Verminderung der Ionisierungsenergie mitunter entschieden werden, ob es sich bei einem Signal bei einer bestimmten Masse um das eines Fragments eines größeren Moleküls oder um einen Mutterpeak eines Moleküls dieser Masse handelt.

\subsubsection{Signalaufnahme}

Der Ionenstrahl trifft nach dem Durchlauf des Trennrohrs durch den Austrittsspalt auf einen Sekundärelektronenvervielfacher (SEV, Kramer), der aus 17 Dynoden aufgebaut ist, die mit einer Spannung zwischen 1,8 und 2,2 kV versorgt werden. Die Ladung eines Ions wird durch den SEV auf etwa $10^{5}-10^{6}$ e zu einem Signalpeak der Halbwertsbreite 20 ns verstärkt. Dieses Signal wird auf eine dreiteilige Baugruppe gegeben, deren erstes Element ein zweikanaliger Vorverstärker ist, der um den Faktor 2300 verstärkt. Das zweite Element ist ein Diskriminator, der zur Verbesserung des 
Signal-Rausch-Verhältnisses beiträgt. Dabei werden nur Signale, die eine Peakhöhe von mehr als $50 \mu \mathrm{V}$ haben, weitergegeben ${ }^{11}$. Alle anderen Signale (Fehlsignale, die von außerhalb oder innerhalb der Apparatur stammen) werden "abgeschnitten" (diskriminiert). Der dritte Teil der Baugruppe ist ein Pulsformer (SSR instruments, Modell 1120), der das Signal in einen genormten Puls der Weite 10 ns und der Höhe $5 \mathrm{~V}$ umformt.

Die genormten Pulse werden dann von einem 85 MHz-Zweikanalzähler (Digital Synchronus Computer) summiert und über Messkarten in einen Personal Computer (80386) eingespeist. Das Signal wird parallel dazu auf einen Vielkanalanalysator (Tracor Northern NS 575 A, 15 MHz) gegeben, so dass es auch optisch auf einem Bildschirm verfolgt werden kann. Der Zweikanalzähler ist von endlicher Bandbreite: Bei Zählungen von mehr als $10^{6}$ pro Sekunde kann es zu Sättigungserscheinungen (pulse pile up) kommen, was die Messung verfälscht. Deshalb wurden die Messungen unterhalb dieses Wertes durchgeführt.

\subsubsection{Rechnersteuerung}

Das Rechnersystem zur Steuerung der Messungen wurde von Nacke installiert und beschrieben [NAC94]. Ein dazugehöriges Steuerungs- und Messprogramm namens WinSiccon wurde von Hold weiterentwickelt und ausführlich dokumentiert [HOL97]. Die Zählwerte des Zweikanalzählers' ${ }^{12}$ werden über eine Schnittstelle (Interface, Appli Data PA 110) eingelesen, auf dem Computerbildschirm angezeigt und auf der Festplatte des Computers gespeichert. Aus den in mehreren Messperioden gezählten Werten wird vom Rechner der arithmetische Mittelwert mit Standardabweichung gebildet und auf dem Computerbildschirm angezeigt. Über eine zweite Schnittstelle (Appli Data PA150) wird der Impuls zum Start einer Zählung an den Zweikanalanalysator gegeben. Die Messdauer wird am Zweikanalzähler eingestellt und kann zwischen 5 und 15 s gewählt werden. Die Zahl der Messperioden wird vom Messprogramm aus eingegeben.

Über ein weiteres Interface (PA 300) wird die Hallspannung digitalisiert eingelesen und auf dem Bildschirm angezeigt. Die Wurzel der Hallspannung UHall ist proportional zur Magnetfeldstärke. Der Rechner ist so programmiert, dass über diese Beziehung sowie eine dazugehörige Eichkurve die Masse, deren zugehöriger Ionenstrahl gerade vom SEV aufgenommen wird, ermittelt wird. Das Programm ist ferner in der Lage, mit dem 1. Logiktreiber der Computerkarte PA 300 ein 5 V Signal an einen Optokoppler auszugeben, um den Mikrowellengenerator zu schalten.

\footnotetext{
${ }^{11}$ Der Wert ist von $50 \mu \mathrm{V}$ bis $500 \mu \mathrm{V}$ verschiebbar.

${ }_{12}$ Neben dem Datenwert DATA werden vom Zweikanalzähler ein ggf. vorher eingelesenes Hintergrundsignal BKG sowie die Summe SUM und die Differenz DIFF aus beiden ausgegeben.
} 
Im Programm sind einige Messroutinen vorgesehen. Dazu gehören die bereits erwähnten Methoden Magnetstromscan und Einzelmassenmessung. Letztere unterteilt sich in Einzelmessung, Doppelmessung mit einem Reaktanden und Doppelmessung mit zwei Reaktanden. Bei der Einzelmessung wird das Signal einer bestimmten Masse aufgenommen und abgespeichert. Bei der Doppelmessung mit einem Reaktanden geschieht dasselbe einmal mit ausgeschaltetem und einmal mit eingeschaltetem Mikrowellengenerator. Diese Messroutine dient vor allem der Bestimmung der Geschwindigkeitskoeffizienten der Reaktionen von Kohlenwasserstoffen mit Fluoratomen (Radikalbildungsreaktionen) in Relativmessung. Daneben ist diese Messroutine auch zur Bestimmung der Kinetik von Reaktionen Radikal $+\mathrm{X}\left(\mathrm{X}=\mathrm{O}_{2}\right.$, NO usw.) in Absolutmessung geeignet. Erneut sei für die beiden Verfahren der kinetischen Messung auf das Kapitel 2.4 hingewiesen. Die Doppelmessung mit zwei Reaktanden wird zur Ermittlung von Geschwindigkeitskonstanten von Reaktionen Radikal + X (X = O, $\mathrm{O}_{2}, \mathrm{H}$ usw.) in Relativmessung eingesetzt. Es wird ein erster Mittelwert ohne Zugabe von X aufgenommen, danach öffnet sich ein Magnetventil und nach einer vom Benutzer eingestellten Pause (meistens 20 s) wird ein zweiter Mittelwert in Anwesenheit von X aufgenommen. Die Radikalbildungsreaktion läuft die ganze Zeit hindurch.

Alle Daten können als ASCII-Datei, in bestimmten Fällen auch als teilausgewertete Dateien besonderen Typs abgespeichert werden. Eine elektronische Bearbeitung der Messergebnisse ist somit möglich. Es wird außerdem eine Messprotokolldatei inklusive der gewählten Messparameter gesichert.

Das nächste Schaubild zeigt im Überblick die Kommunikationsstellen zwischen Apparatur und Rechner. 


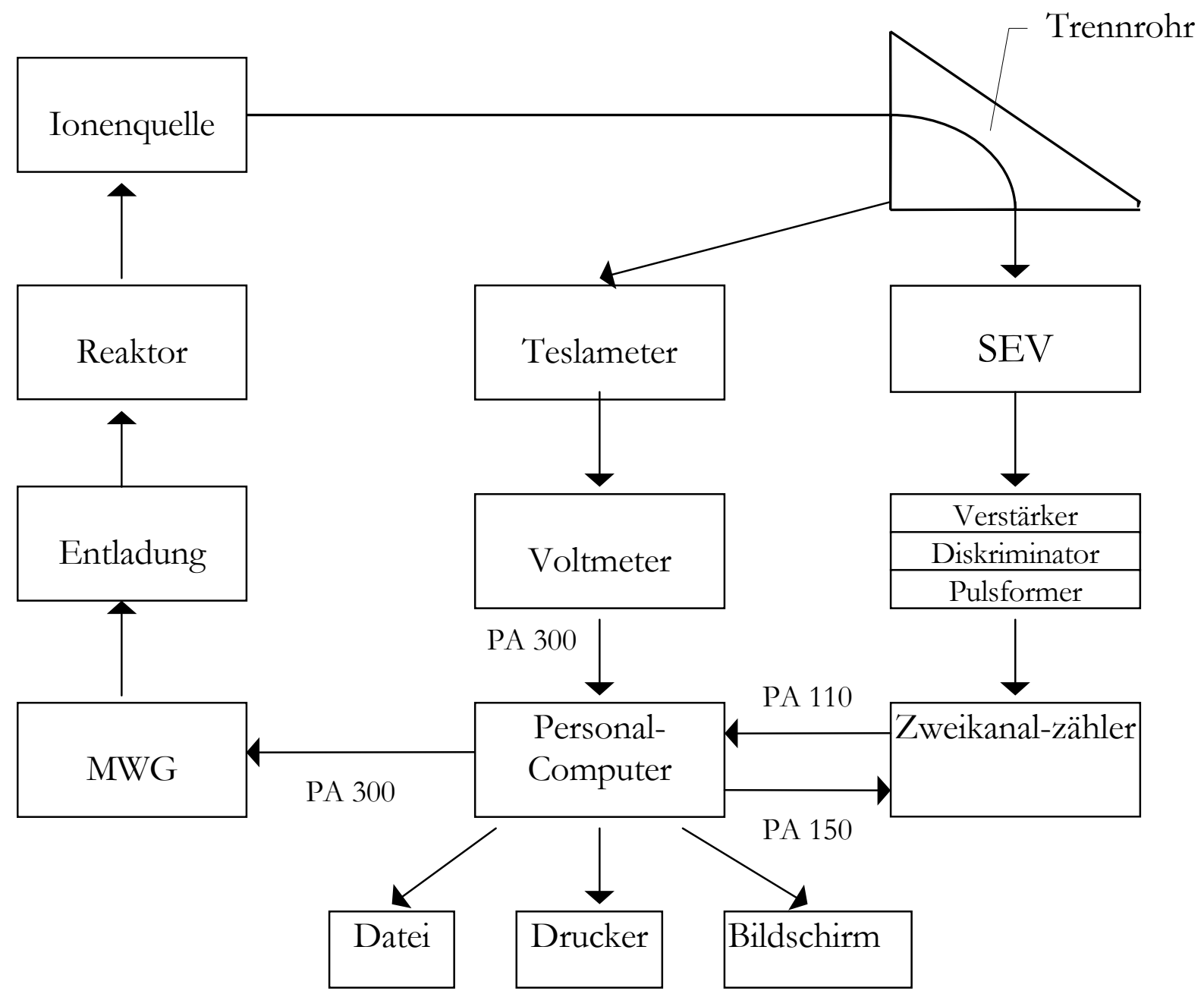

Abbildung 13: Blockschaltbild zur Rechnerstellung, [NAC94]

\subsection{Versuchsanordnung C (FTIR)}

\subsubsection{Versuchsanordnung $\mathrm{C}$ im Überblick}

Versuchsanordnung C besteht aus einer statischen Zelle mit der Möglichkeit von Photodissoziation zur Radikal- und Atomerzeugung und einem Fourier-Transform-Infrarot-Spektrometer (FTIR) als Nachweis. Diese Versuchsanordnung wurde in dieser Arbeit ausschließlich zur Produktanalyse von Radikalreaktionen eingesetzt. Der Vorteil der Apparatur liegt in der selektiven Radikalerzeugung durch Photodissoziation geeigneter Vorläufersubstanzen mittels Laser. Der FTIR-Nachweis der stabilen Edukte und Produkte stellt eine ausgezeichnete Ergänzung zum in den anderen Apparaturen eingesetzten massenspektrometrischen Nachweis dar. Als optische Nachweismethode kann das Gasgemisch ohne Probennahme in situ analysiert werden. Es ist möglich, Reaktionen über einen weiten Druckbereich (1 mbar - 3000 mbar) zu untersuchen, im Gegensatz zur Massenspektrometrie ist keine Druckreduzierung notwendig. Mit Hilfe von Kalibrierungsmessungen ist eine quantitative Produktbestimmung möglich. 
Nachteilig ist, dass ein direkter Radikalnachweis aufgrund der außerhalb der Lebensdauer liegenden Nachweiszeiten nicht möglich ist. Der Photolyseprozess ist oft nicht vollständig selektiv: Zu 5-20\% werden üblicherweise nicht erwünschte Photolyseproduktkanäle eröffnet. Aber nicht erwünschte Photolysekanäle sind tolerabel, wenn ihre Chemie nicht die zu untersuchende Reaktion beeinflusst oder die Störwirkung quantitativ erfasst werden kann. Ein weiterer Nachteil besteht darin, dass verschiedene Edukte und Produkte in gleichen oder nah beieinander liegenden Wellenzahlbereichen absorbieren. Diese Überlagerungseffekte lassen sich jedoch häufig durch eine hohe Auflösung in Verbindung mit Kalibrierungsmessungen unter Ausnutzung des spezifischen wellenzahlabhängigen Absorptionsverlaufs korrigieren.

Versuchsanordnung C ist besteht aus den Baugruppen Reaktionszelle mit einer Spiegelanordnung nach White, dem Photolyselaser, dem FTIR-Nachweissystem sowie einer elektronischen Steuerung, welche die Füllung der Zelle mit Gasen und den Messablauf, der teilweise automatisch abläuft, regelt.

Die nachfolgende Abbildung zeigt Versuchsanordnung C im Überblick:

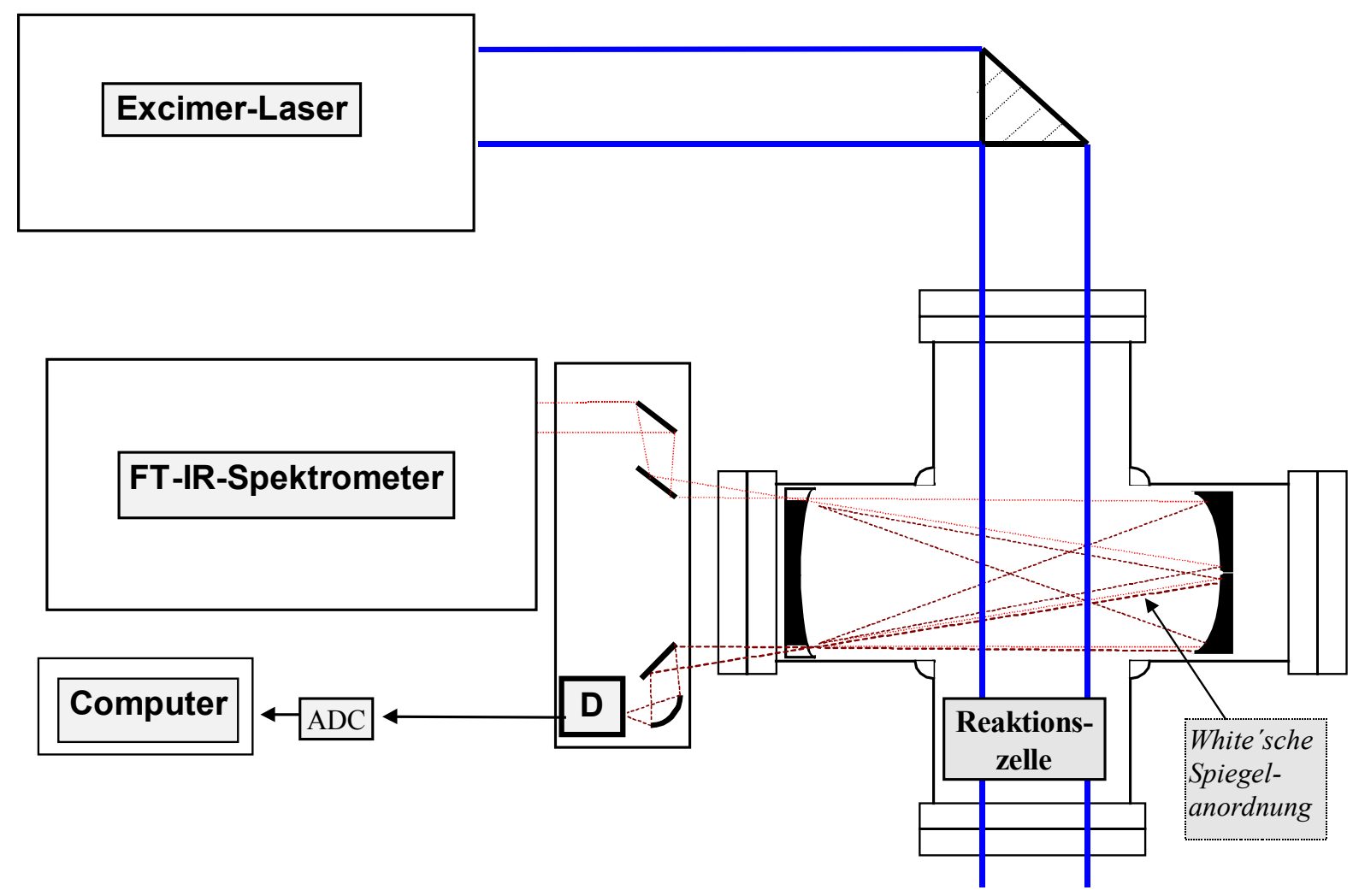

Abbildung 14: Versuchsaufbau C im Überblick, [TRÖ97] 


\subsubsection{Die Reaktionszelle}

Die Reaktionszelle ist eine VA-Stahl-Kugelzelle (VAB Elmshorn, VA 1.4311) mit einem Volumen von 41 und sechs CF 100 Flanschöffnungen. Um die Funktion einer Photolysezelle zu erfüllen, sind zwei gegenüberliegende horizontale Flansche mit Quarzfenstern zum Ein- und Auskoppeln des Excimerlaserstrahls ausgestattet. Der untere Flansch ist zum Evakuieren der Zelle über ein Eckventil mit einer Ölrotationspumpe (Brand, RD 15, Saugvermögen $15 \mathrm{~m}^{3} / \mathrm{h}$ ) verbunden. Vor dem Eckventil sind zellseitig zwei Membranmanometer (MKS, Baratron 122A) mit den Druckmessbereichen 1-100 mbar und 10-1000 mbar angebracht. Am oberen Flansch sind zwei Einlasssysteme angebracht. Bei den in dieser Arbeit durchgeführten Messungen wurde Argon als Spül- und Badgas über diesen Weg in die Zelle geleitet. Um einen effizienten FTIR-Nachweis zu erreichen, ist die Reaktionszelle als eine Multireflektionskugelzelle konstruiert worden. Die übrigen zwei horizontalen Flansche dienen zur Justage der IR-Strahl-Spiegeloptik. Der IR-Strahl wird über einen Parabolspiegel ( $\mathrm{f}=153,7 \mathrm{~mm}$ ) und einen Planspiegel in die Zelle gelenkt, in die er über $\mathrm{KBr}$ Fenster ein- und ausgekoppelt wird. Die Spiegelanordnung in der Zelle besteht aus drei goldbeschichteten Spiegeln, zwei davon stehen dem größeren gegenüber, wobei der Abstand der beiden Seiten gleich der Brennweite der Spiegel ( $f=200 \mathrm{~mm}$ ) ist. Durch diese Whitesche Spiegelanordnung kann bei 40 möglichen Reflektionen ein Lichtweg von 8 m erreicht werden.

\subsubsection{Der Photolyselaser}

Zur Photolyse der Radikalvorläufersubstanzen wird ein Excimerlaser (Lambda Physik, Compex 102) eingesetzt. Die Photolyse wird bei einer Wellenlänge von $\lambda=193 \mathrm{~nm}$ (ArF) durchgeführt. Das Strahlprofil hat Abmessungen von 0,75 $\cdot 2,1 \mathrm{~cm}^{2}$. Die Pulsenergie beträgt 150-200 mJ, die Repetitionsrate wurde auf 1 oder $2 \mathrm{~Hz}$ eingestellt. Der Laserstrahl wird über zwei Umlenkspiegel in die Reaktionszelle eingekoppelt, die Eingangspulsenergie beträgt ca. 60-100 mJ. Nach 100 bis 400 Pulsen wird ein IR-Spektrum des Reaktionsgemisches aufgenommen, falls im Reaktionsmodus gearbeitet wird.

\subsubsection{Der FTIR-Nachweis}

\subsubsection{Der Aufbau des FTIR-Spektrometers}

Zum Nachweis wurde ein FTIR-Spektrometer (Bruker, IFS 66) eingesetzt. Die folgende Abbildung zeigt schematisch den internen Aufbau des Geräts: 


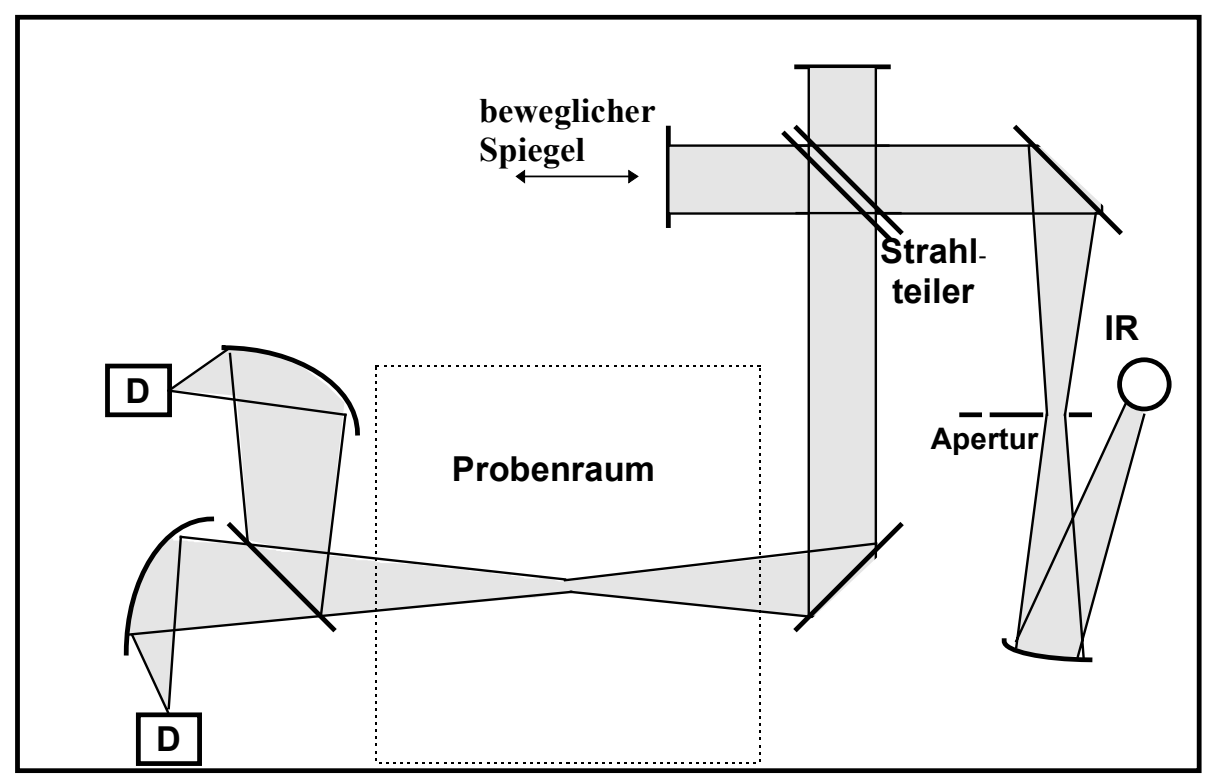

\section{Abbildung 15: Schematischer Aufbau des FTIR-Spektrometers IFS 66 (intern), [TRÖ97]}

Die Baugruppen des IFS 66 sind die IR-Quelle (IR), das Michelson-Interferometer, der interne Probenraum und der Detektor (D). Als IR-Quelle wird ein Globar (Siliziumcarbid) verwendet, der von 400 bis 6000 Wellenzahlen emittiert. Die Funktionsweise des Michelson-Interferometers wird im nächsten Abschnitt beschrieben. Der interne Probenraum bleibt ungenutzt, da - wie oben beschrieben - mit einer extern angebrachten Reaktionszelle gearbeitet wird. Als Detektor dient ein $\operatorname{MCT}(\mathrm{Hg} / \mathrm{Cd} / \mathrm{Te})$ mit einem Wellenzahlenbereich von $\widetilde{v}=560-5000 \mathrm{~cm}^{-1}$ und einer Zeitauflösung von $\Delta \mathrm{t}=5 \mu \mathrm{s}$. Das Detektorsignal wird an einen Analog-Digital-Konverter (ADC) weitergeleitet, von wo es in einen Computer (Intel, Pentium II, $500 \mathrm{MHz}$ ) zur weiteren Auswertung eingelesen wird. Dieser Rechner steuert die Aufnahme des Spektrums und dessen Auswertung mittels einer dafür entwickelten Messsoftware namens Opus (Bruker). Es wird hierbei eine maximale Auflösung von $0,12 \mathrm{~cm}^{-1}$ zugelassen. Die im Rahmen dieser Arbeit aufgenommenen Spektren wurden ausschließlich im Rapid-Scan-Modus gewonnen ${ }^{13}$. Es wird dabei in einem Zug ein vollständiges Interferogramm aufgenommen. Die Messdauer für ein Interferogramm ist durch die Geschwindigkeit des beweglichen Spiegels im Michelson-Interferometer begrenzt. Die Wellenzahlauflösung ist von Aufnahmezeit eines Interferogramms abhängig. Bei einer sehr groben Wellenzahlauflösung von 32 Wellenzahlen können 60 Interferogramme pro Sekunde gewonnen werden, was einer zeitlichen Messdauer von 20 ms entspricht.

\footnotetext{
${ }^{13}$ Der mit dem Spektrometer auch mögliche Step-Scan-Modus wurde nicht verwendet und wird deshalb nicht weiter erläutert.
} 


\subsubsection{Grundlagen der FTIR-Spektroskopie}

Bei der Infrarotspektroskopie wird IR-Licht durch eine Probe geschickt und die dabei transmittierte Lichtintensität gemessen. Für die Konzentrationsbestimmung einer in der Probe befindlichen Stoffkomponente gilt bei einer bestimmten Wellenlänge das Lambert-Beersche Geset\%:

$$
\mathrm{A}=\log \left(\mathrm{I}_{0} / \mathrm{I}\right)=\varepsilon \cdot(\widetilde{\mathrm{v}}) \cdot \mathrm{c} \cdot \mathrm{d},
$$

$\mathrm{A}=$ Absorbanz, $\mathrm{I}_{0}=$ eingestrahlte Lichtintensität, $\mathrm{I}=$ transmittierte Intensität, $\varepsilon=$ molarer dekadischer Absorptionskoeffizient, $\mathrm{c}=$ Konzentration, $\mathrm{d}=$ Schichtdicke, $\mathrm{I} / \mathrm{I}_{0}=$ Transmission.

$\varepsilon$ ist stoffartspezifisch und eine Funktion der Wellenzahl und dient somit zum spezifischen Nachweis einer Substanz. Die IR-Absorption ist auf molekularer Ebene mit der Anregung von Schwingungsniveaus verbunden. Bestimmte funktionelle Gruppen innerhalb eines Moleküls führen dabei zu Absorption in ganz bestimmten Wellenzahlbereichen (Gruppenfrequenzen). Die Art der chemischen Umgebung dieser funktionellen Gruppen führt oft zu einer leichten Verschiebung des Absorptionsbereichs, was in vielen Fällen erst eine Unterscheidung zwischen ähnlichen Substanzen ermöglicht.

Das Lambert-Beersche Gesetz gilt in dieser Form nur für monochromatisches Licht. Die Integration über einen Wellenzahlbereich kann aber sinnvoll sein. Dazu wird die integrale Molabsorptivität B wie folgt definiert:

$$
\mathrm{B}:=\int_{\widetilde{v}}^{\widetilde{v}} \varepsilon(\widetilde{v}) \mathrm{d} \widetilde{v} .
$$

Das Lambert-Beersche Gesetz lautet dann:

$$
\int_{\widetilde{v}}^{\widetilde{v}^{\prime}} \operatorname{Ad} \widetilde{v}=\mathrm{B} \cdot \mathrm{c} \cdot \mathrm{d} .
$$

Hierbei gilt es zu beachten, dass die Absorption in vielen Fällen mit Veränderung von Druck und Temperatur zwar ihre Form, nicht aber Gesamtintensität verändert [HED85].

Die Fourier-Transformations-Technik (FT) führt zur schnellen Realisierung eines vollständigen IRSpektrums über einen weiten Wellenzahlbereich in hoher Wellenzahlauflösung. Kernstück der FTTechnik ist hierbei das Michelson-Interferometer. Die folgende Abbildung stellt ein solches schematisch dar: 


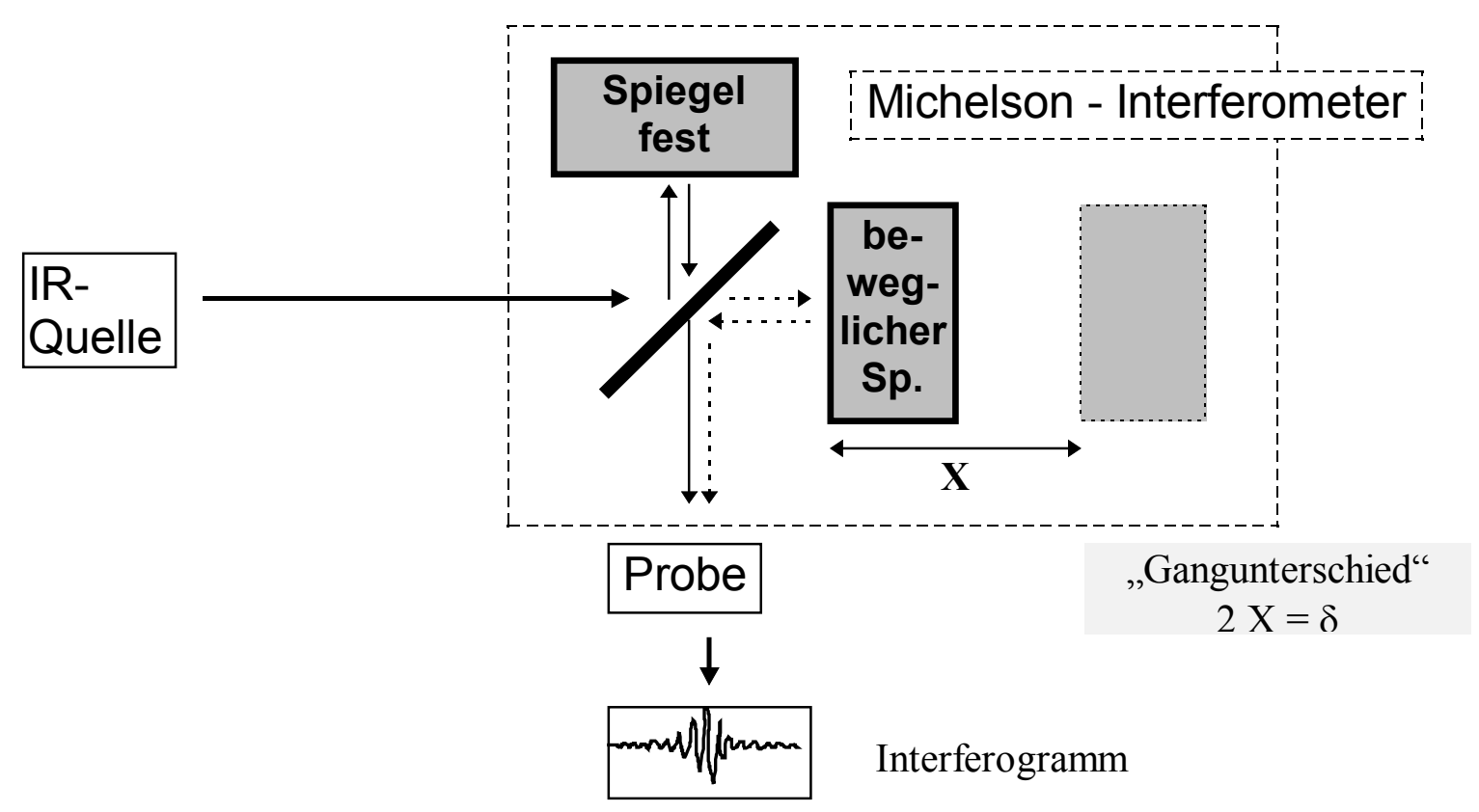

Abbildung 16: Schematischer Aufbau eines Michelson-Interferometers, [TRÖ97]

Die von der Lichtquelle emittierte polychromatische Strahlung fällt im Michelson-Interferometer auf einen Strahlteiler, der die Hälfte des einfallenden Lichts durchlässt und die andere Hälfte reflektiert. Das reflektierte Licht trifft nach einer Strecke L auf einen fest montierten Spiegel und wird dort erneut reflektiert, bis es wieder auf den Strahlteiler trifft. Es hat somit eine Gesamtstrecke 2L durchlaufen. Der andere Teil des Lichts, der am Strahlteiler transmittiert wurde, trifft auf einen um die Strecke x beweglichen Spiegel. Die hier vom Licht zurückgelegte Strecke beträgt 2(L+x). Die beiden Strahlhälften treffen mit der Wegdifferenz $\delta=2 \mathrm{x}$ (Gangunterschied) aufeinander und es kommt zur Interferenz. Der das Interferometer verlassende Lichtstrahl passiert den Probenraum und trifft auf den Detektor. Die Absorption im Probenraum führt zu einem veränderten Interferogramm. Das aufgezeichnete Interferogramm wird im Rechner mit Hilfe des mathematischen Verfahrens der Fouriertransformation in eine frequenzabhängige Intensität gemäß folgender Formel umgewandelt:

$$
\mathrm{B}(\widetilde{v})=2 \int_{0}^{\infty} \mathrm{I}(\delta) \cos (2 \pi \widetilde{v} \delta) \mathrm{d} \delta
$$

Die exakte Lösung ist besonderen Fällen vorbehalten. In der Anwendung wird eine numerisch berechnete Näherungslösung ermittelt. Als Resultat erhält man das in dem üblichen Format dargestellte IR-Spektrum. 


\subsection{Methoden zur Auswertung der kinetischen Messungen}

In diesem Kapitel werden die für die Auswertungen benötigten kinetischen und strömungsmechanischen Grundlagen erläutert.

\subsubsection{Absolutmessung}

Für eine bimolekulare Reaktion $\mathrm{R}+\mathrm{X} \stackrel{\mathrm{k}}{\longrightarrow}$ Prod. wird die zeitliche Änderung der Konzentration von R durch folgende Differentialgleichung beschrieben:

$$
\frac{\mathrm{d}[\mathrm{R}]}{\mathrm{dt}}=-\mathrm{k} \cdot[\mathrm{R}] \cdot[\mathrm{X}]
$$

Trennung der Variablen und Integration führt zu

$$
\int_{[\mathrm{R}]_{0}}^{[\mathrm{R}]} \frac{\mathrm{d}[\mathrm{R}]}{[\mathrm{R}]}=-\mathrm{k} \cdot \int_{\mathrm{t}_{0}}^{\mathrm{t}}[\mathrm{X}] \mathrm{dt} .
$$

Die Kenntnis der Konzentrationen von $\mathrm{X}$ zur Zeit $\mathrm{t}_{0}$ und $\mathrm{t}$ ist Voraussetzung zur Bestimmung von $\mathrm{k}$ unter direkter Anwendung dieser Gleichung. Unter der Annahme, dass $[\mathrm{X}]>>[\mathrm{R}]$ (psendoerste Ordnung) gilt, kann $[\mathrm{X}]$ als konstant angesehen werden. Man erhält dann als Lösung für Gleichung $(15)$ :

$$
\ln \left(\frac{[\mathrm{R}]_{0}}{[\mathrm{R}]}\right)=\mathrm{k} \cdot[\mathrm{X}] \cdot\left(\mathrm{t}-\mathrm{t}_{0}\right)
$$

Die Ionensignale $I_{R}$ sind den Konzentrationen $[R]$ im Reaktor proportional. Die Quotienten $\frac{I_{R_{0}}}{I_{R}}$ und $\frac{[R]_{0}}{[R]}$ sind also gleich. Durch eine Auftragung der linken Seite von Gleichung (16) gegen $\left(t-t_{0}\right)$ ergibt sich eine Ursprungsgerade, aus deren Steigung bei bekannter Konzentration von X der Geschwindigkeitskoeffizient k bestimmt werden kann. Durch Variation der Sondenstrecke s erreicht man verschiedene Reaktionszeiten ( $\left.\mathrm{t}_{1}-\mathrm{t}_{0}\right)$, ( $\left.\mathrm{t}_{2}-\mathrm{t}_{0}\right)$, ( $\left.\mathrm{t}_{3}-\mathrm{t}_{0}\right)$ usw. (vgl. Gl. (20)). Das Produkt $\mathrm{k} \cdot[\mathrm{X}]$ wird als Geschwindigkeitskonstante pseudoerster Ordnung bezeichnet.

Durch Messungen bei verschiedenen Überschusskonzentrationen [X] kann die Richtigkeit der Annahme pseudoerster Ordnung überprüft werden.

Bei der Absolutmessung wird vorausgesetzt, dass eine laminare Strömung mit Poiseuille-Profil vorliegt. Diese Prämisse ist dann erfüllt, wenn die dimensionslose Reynoldşab/Re kleiner als 1160 ist [REN95]. Für eine Rohrströmung ist sie definiert durch: 


$$
\mathrm{R}_{\mathrm{e}}=\frac{\rho \cdot \mathrm{v} \cdot \mathrm{r}}{\eta}
$$

$\rho=$ Dichte, $\eta=$ Viskosität, $\mathrm{v}=$ lineare Strömungsgeschwindigkeit, $\mathrm{r}=$ Radius des Reaktors.

Unter den bei den meisten Messungen eingestellten Bedingungen errechnet sich eine Strömungsgeschwindigkeit von $40 \mathrm{~m} / \mathrm{s}$. Helium als Badgas macht dabei mehr als 90 Vol.\% aller Gase aus. Bei den Druckverhältnissen hat es eine Dichte von $\rho=2,4 \cdot 10^{-7} \mathrm{~g} / \mathrm{cm}^{3}$. Die Viskosität beträgt $\eta=1,96 \cdot 10^{-5} \mathrm{~Pa} \cdot \mathrm{s}$. Es wird ein Rohrradius von $\mathrm{r}=1 \mathrm{~cm}$ angenommen. Die Reynoldszahl errechnet sich dann zu Re $=5$. Es liegt somit eine laminare Strömung vor. Sie bildet sich jedoch erst nach einer gewissen Strecke, der Einlauflänge $L_{e}$, heraus:

$$
\mathrm{L}_{\mathrm{e}}=0,116 \cdot \mathrm{Re} \cdot \mathrm{r}
$$

Unter den genannten Bedingungen ergibt sich dafür ein Wert von $\mathrm{L}_{\mathrm{e}}=0,6 \mathrm{~cm}$.

Die lineare Strömungsgeschwindigkeit v wird wie folgt berechnet:

$$
\begin{gathered}
\mathrm{v}=\frac{\Phi}{\pi \cdot \mathrm{r}^{2}} \cdot \frac{\mathrm{T}}{\mathrm{T}_{0}} \cdot \frac{\mathrm{p}_{0}}{\mathrm{p}}, \\
\Phi=\text { Standard-Gesamtgasfluss }{ }^{14}, \mathrm{r}=\text { Rohrradius, } \\
\mathrm{T}=\text { Temperatur, } \mathrm{p}=\text { Druck, } \mathrm{p}_{0}=101325 \mathrm{~Pa}, \mathrm{~T}_{0}=273,15 \mathrm{~K} .
\end{gathered}
$$

Ist durch geeignete Zumischung eine gleichmäßige Anfangsverteilung in radialer Richtung gegeben, so kann als vereinfachtes Modell eine eindimensionale Strömung angenommen werden. Die Verweilzeit im Strömungsreaktor, errechnet sich dann mit der Reaktionsstrecke s (Sondenstellung) $\mathrm{zu}:$

$$
\mathrm{t}=\frac{\mathrm{s}}{\mathrm{v}}
$$

Die Konzentration der Komponente i im Strömungsreaktor wird gemäß einer Formel, die sich aus dem idealen Gasgesetz ergibt, ermittelt:

$$
\mathrm{c}_{\mathrm{i}}=\frac{\Phi_{\mathrm{i}}}{\Phi} \cdot \frac{\mathrm{p}}{\mathrm{R} \cdot \mathrm{T}}
$$

$\Phi_{\mathrm{i}}=$ Standardgasfluss von i, $\Phi=$ Gesamtfluss, $\mathrm{R}=$ Gaskonstante, $\mathrm{p}=$ Druck, $\mathrm{T}=$ Temperatur.

${ }^{14}$ Standard bedeutet unter $\mathrm{p}_{0}=101325 \mathrm{~Pa}$ und $\mathrm{T}_{0}=273,15 \mathrm{~K}$ Bedingungen. 
In einem Strömungsreaktor beeinflussen sich Konvektion ${ }^{15}$, Diffusion ${ }^{16}$ und zeitliche Änderung der Konzentration durch chemische Reaktion gegenseitig. Bei Vorliegen eines Poiseuilleprofils und unter Vernachlässigung des Temperatur- und Druckgradienten wird dieser Zusammenhang durch die Kontinuitätsgleichung beschrieben [HOW79]:

$$
\begin{gathered}
2\langle\mathrm{v}\rangle\left(1-\frac{\mathrm{r}^{2}}{\mathrm{R}^{2}}\right) \frac{\partial \mathrm{c}}{\partial \mathrm{z}}=\mathrm{D}\left(\frac{\partial^{2} \mathrm{c}}{\partial \mathrm{r}^{2}}+\frac{1}{\mathrm{r}} \frac{\partial \mathrm{c}}{\partial \mathrm{r}}+\frac{\partial^{2} \mathrm{c}}{\partial \mathrm{z}^{2}}\right)+\frac{\partial \mathrm{c}}{\partial \mathrm{t}}, \\
\langle\mathrm{v}\rangle=\text { mittlere Flußgeschwindigkeit }, \mathrm{r}, \mathrm{z}=\text { Zylinderkoordinaten } \\
\mathrm{R}=\text { Reaktorradius, } \mathrm{c}=\text { Konzentration, } \mathrm{D}=\text { binärer Diffusionskoeffizient, } \mathrm{t}=\text { Zeit. }
\end{gathered}
$$

Diese Gleichung geschlossen ist analytisch nicht lösbar ([JOS52], [OGR75]), jedoch existieren Näherungslösungen für einige Sonderfälle. Unter Vernachlässigung radialer Diffusion und von Wandreaktionen erhält man [HOY75]:

$$
\mathrm{k}=\mathrm{k}_{\mathrm{obs}} \cdot\left(1+\frac{\mathrm{D}}{\langle\mathrm{v}\rangle^{2}} \cdot \mathrm{k}_{\mathrm{obs}}\right)
$$

$\mathrm{k}=$ tatsächlicher Geschwindigkeitskoeffizient, $\mathrm{k}_{\mathrm{obs}}=$ gemessener Geschwindigkeitskoeffizient.

Berücksichtigt man radiale Diffusion, so ergibt sich ([TAY53],[TAY54],[ARI56]):

$$
\mathrm{k}=\mathrm{k}_{\mathrm{obs}} \cdot\left(1+\frac{\mathrm{D}}{\langle\mathrm{v}\rangle^{2}} \cdot \mathrm{k}_{\mathrm{obs}}+\frac{\mathrm{R}^{2}}{48 \cdot \mathrm{D}} \cdot \mathrm{k}_{\mathrm{obs}}\right)
$$

Der gemessene Geschwindigkeitskoeffizient stellt also aufgrund des Einflusses der Diffusion eine Untergrenze gegenüber dem tatsächlichen dar. Mit Hilfe von Näherungsformeln lässt sich abschätzen, inwieweit eine Diffusionskorrektur erforderlich ist [WAL61].

Bei sehr schnellen Reaktionen $\left(\mathrm{k}>10^{13} \mathrm{~cm}^{3} \cdot \mathrm{mol}^{-1} \cdot \mathrm{s}^{-1}\right)$ ergeben sich für die Absolutmessung Probleme. Die Radikalkonzentration nimmt unter den Bedingungen pseudoerster Ordnung $([\mathrm{X}]>>[\mathrm{R}])$ sehr schnell ab. Es muss entweder mit sehr geringen Konzentrationen der Edukte gearbeitet werden, was erhebliche Abstriche in der Nachweisempfindlichkeit und Schwierigkeiten bei der Dosierung zur Folge hat. Oder es muss eine extrem kurze Reaktionsstrecke eingestellt

\footnotetext{
15 Stofftransport in axialer Richtung aufgrund der Strömung

16 Stofftransport in axialer und radialer Richtung aufgrund der Molekularbewegung
} 
werden. Dann führen aber schwer quantifizierbare Mischeffekte zu einer Verfälschung des Wertes für die Reaktionszeit.

\subsubsection{Relativmessung}

Die zweite Methode zur Bestimmung von Geschwindigkeitskonstanten ist die Relativmessung. Hierbei wird dem Reaktor eine zweite Substanz $\mathrm{R}^{*}$ zugeführt, die mit dem Reaktanden X reagiert (Referenzreaktion):

$$
\mathrm{R}^{*}+\mathrm{X} \stackrel{\mathrm{k}_{\mathrm{ref}}}{\longrightarrow} \text { Produkte. }
$$

Durch dieselbe Herleitung erhält man die zu (15) analoge Gleichung:

$$
\int_{\left[\mathrm{R}^{*}\right]_{0}}^{\left[\mathrm{R}^{*}\right]} \frac{\mathrm{d}\left[\mathrm{R}^{*}\right]}{\left[\mathrm{R}^{*}\right]}=-\mathrm{k} \cdot \int_{\mathrm{t}_{0}}^{\mathrm{t}}[\mathrm{X}] \mathrm{dt} .
$$

Der Integralausdruck der rechten Seite ist für (15) und (24) gleich, so dass Division der beiden Gleichungen zu folgendem Ausdruck führt:

$$
\frac{\ln \frac{[\mathrm{R}]_{0}}{[\mathrm{R}]}}{\ln \frac{\left[\mathrm{R}^{*}\right]_{0}}{\left[\mathrm{R}^{*}\right]}}=\frac{\mathrm{k}}{\mathrm{k}_{\mathrm{ref}}}=\mathrm{k}_{\mathrm{rel}}
$$

Die gemessenen massenspektrometrischen Intensitäten der Stoffe sind den Konzentrationen proportional, so dass in Gleichung (25) die Konzentration [ ] durch die Intensität $\mathrm{I}_{+\mathrm{x}}$ und die Anfangskonzentration [ ] o durch $\mathrm{I}_{-\mathrm{x}}$, der Intensität in Abwesenheit von X, ersetzt werden können. Eine Auftragung von $\ln \frac{I_{-x}}{I_{+x}}$ gegen $\ln \frac{I_{-x}^{*}}{I_{+x}^{*}}$ ergibt eine Gerade durch den Koordinatenursprung mit der relativen Geschwindigkeitskonstanten $k_{\text {rel }}$ als Steigung. Ist die Geschwindigkeitskonstante der Referenzreaktion kref bekannt, kann daraus der Geschwindigkeitskoeffizient der Hauptreaktion k errechnet werden. Die jeweiligen Intensitäten werden für unterschiedliche Flüsse des Reaktionspartners X ermittelt. Die Reaktionsstrecke ist konstant und möglichst klein gewählt, um Nebenund Folgereaktionen zu vermeiden.

Für die Relativmessung ist es wichtig, dass die Geschwindigkeitskonstanten von Hauptreaktion und Referenzreaktion in derselben Größenordnung liegen. 


\subsubsection{Temperaturabhängigkeit}

Die Temperaturabhängigkeit der Geschwindigkeitskonstanten wird häufig durch die Arrbeniusgleichung beschrieben:

$$
\begin{gathered}
\mathrm{k}(\mathrm{T})=\mathrm{A} \cdot \exp \left(-\frac{\mathrm{E}_{\mathrm{a}}}{\mathrm{R} \cdot \mathrm{T}}\right), \\
\mathrm{T}=\text { Temperatur, } \mathrm{R}=\text { Gaskonstante, } \mathrm{A}=\text { Vorfaktor, } \mathrm{E}_{\mathrm{a}}=\text { Aktivierungsenergie. }
\end{gathered}
$$

Eine andere Darstellung von (26) lautet:

$$
\ln \mathrm{k}(\mathrm{T})=-\frac{\mathrm{E}_{\mathrm{a}}}{\mathrm{R}} \cdot \frac{1}{\mathrm{~T}}+\ln \mathrm{A}
$$

Eine Auftragung von $\ln (\mathrm{k})$ gegen $1 / \mathrm{T}$ ergibt die beiden charakteristischen Parameter $\mathrm{E}_{\mathrm{a}}$ und $\mathrm{A}$. Hierbei wird vorausgesetzt, dass die Aktivierungsenergie und der Vorfaktor selber temperaturunabhängig sind, was für nicht zu komplizierte Reaktionen (idealerweise Elementarreaktionen) in nicht zu großen Temperaturintervallen meistens auch gegeben ist. Der Vorfaktor A hat die Dimension einer Stoßzahl und wird als Geschwindigkeitskoeffizient bei unendlicher hoher Temperatur interpretiert.

Eine genauere Betrachtung mit Hilfe der Theorie des Übergangszustands zeigt, dass der Vorfaktor nicht notwendigerweise temperaturunabhängig sein muss [LAI86]. Es ergibt sich:

$$
\mathrm{k}(\mathrm{T})=\mathrm{a} \cdot \mathrm{T}^{\mathrm{n}} \cdot \exp \left(-\frac{\mathrm{E}_{\mathrm{A}}}{\mathrm{R} \cdot \mathrm{T}}\right)
$$

$\mathrm{n}$ ist hierbei eine reelle Zahl. Nach der Stoßtheorie nimmt $\mathrm{n}$ den Wert $1 / 2$ an. Die Gleichung stellt bei hinreichend großer Aktivierungsenergie nur für große Temperaturintervalle eine wesentliche Veränderung zu (26) dar.

Für Reaktionen mit einer betragsmäßig sehr geringen Aktivierungsenergie nimmt der Exponentialfaktor in (28) einen Wert nahe bei 1 an. Man benutzt zur Beschreibung der Temperaturabhängigkeit einer Reaktion in solchen Fällen deshalb häufig die Formel:

$$
\mathrm{k}(\mathrm{T})=\mathrm{a} \mathrm{T}^{\mathrm{n}}
$$

Eine Auftragung von $\ln (k)$ gegen $\ln (\mathrm{T})$ ergibt dann den Exponenten n. 
Die Stoßzahl einer Reaktion A $+B \rightarrow A B$ lässt sich mit Hilfe der folgenden aus der kinetischen Gastheorie abgeleiteten Formel berechnen:

$$
\begin{gathered}
Z_{\mathrm{AB}}=\mathrm{N}_{\mathrm{A}} \cdot \sigma_{\mathrm{AB}}^{2} \cdot \sqrt{\frac{8 \cdot \mathrm{R} \cdot \mathrm{T}}{\pi \cdot \mu_{\mathrm{AB}}}} \cdot \Omega_{\mathrm{AB}}, \\
\mathrm{N}_{\mathrm{A}}=\text { Avogadrokonstante, } \sigma_{\mathrm{AB}}=\text { Lennard-Jones-Länge, } \\
\mu_{\mathrm{AB}}=\text { reduzierte Molmasse, } \Omega_{\mathrm{AB}}=\text { Stoßintegral. }
\end{gathered}
$$

Für das Stoßintegral kann im Bereich von $0,3 \leq \frac{\mathrm{k}_{\mathrm{B}} \mathrm{T}}{\varepsilon_{\mathrm{AB}}} \leq 500$ nach [TRO77] folgende Näherung verwendet werden:

$$
\begin{gathered}
\Omega_{\mathrm{AB}}=\frac{1}{0.636+0.567 \cdot \lg \left(\frac{\mathrm{k}_{\mathrm{B}} \mathrm{T}}{\varepsilon_{\mathrm{AB}}}\right)}, \\
\mathrm{k}_{\mathrm{B}}=\text { Boltzmannkonstante, } \varepsilon_{\mathrm{AB}}=\text { Lennard-Jones-Potenzial. }
\end{gathered}
$$

\subsection{Reinheit und Dosierung der verwendeten Chemikalien}

Helium bildet bei allen Messungen mit einem Anteil von mehr als 90\% die Hauptkomponente des Gesamtflusses. Die Reaktanden liegen dadurch in großer Verdünnung vor, was verfälschenden Nebenreaktionen entgegenwirkt. Außerdem hat Helium eine hohe Wärmeleitfähigkeit, was für eine schnelle Abfuhr der Reaktionswärme sorgt und zu einer gleichmäßigen und unmittelbaren Annahme der vorgegebenen Temperatur beiträgt (isotherme Reaktionsbedingungen). Die hohe Viskosität Heliums fördert die rasche Ausbildung einer laminaren Strömung im Reaktor.

Die Dosierung der gasförmigen Stoffe erfolgt mit elektronisch geregelten Gasflusskontrollgeräten (Tylan/Millipore/Mykrolis FC 260) oder bei aggressiven Substanzen mit geeichten Feindosierventilen (Hoke Mikromite).

Flüssige Kohlenwasserstoffe werden über einen zweistufigen Sättiger (Eigenbau des Instituts) mit Helium als Trägergas in die Gasphase überführt. Der Volumenfluss des Kohlenwasserstoffs wird unter Annahme des Dampfgleichgewichts im Sättiger unter Verwendung der Partialdrücke errechnet. Der Gesamtdruck im Sättiger beträgt in der Regel 3 bar (absolut). Die anschließende Flussregulierung erfolgt mit geeichten Feindosierventilen. Folgende Abbildung stellt einen Sättiger schematisch dar: 


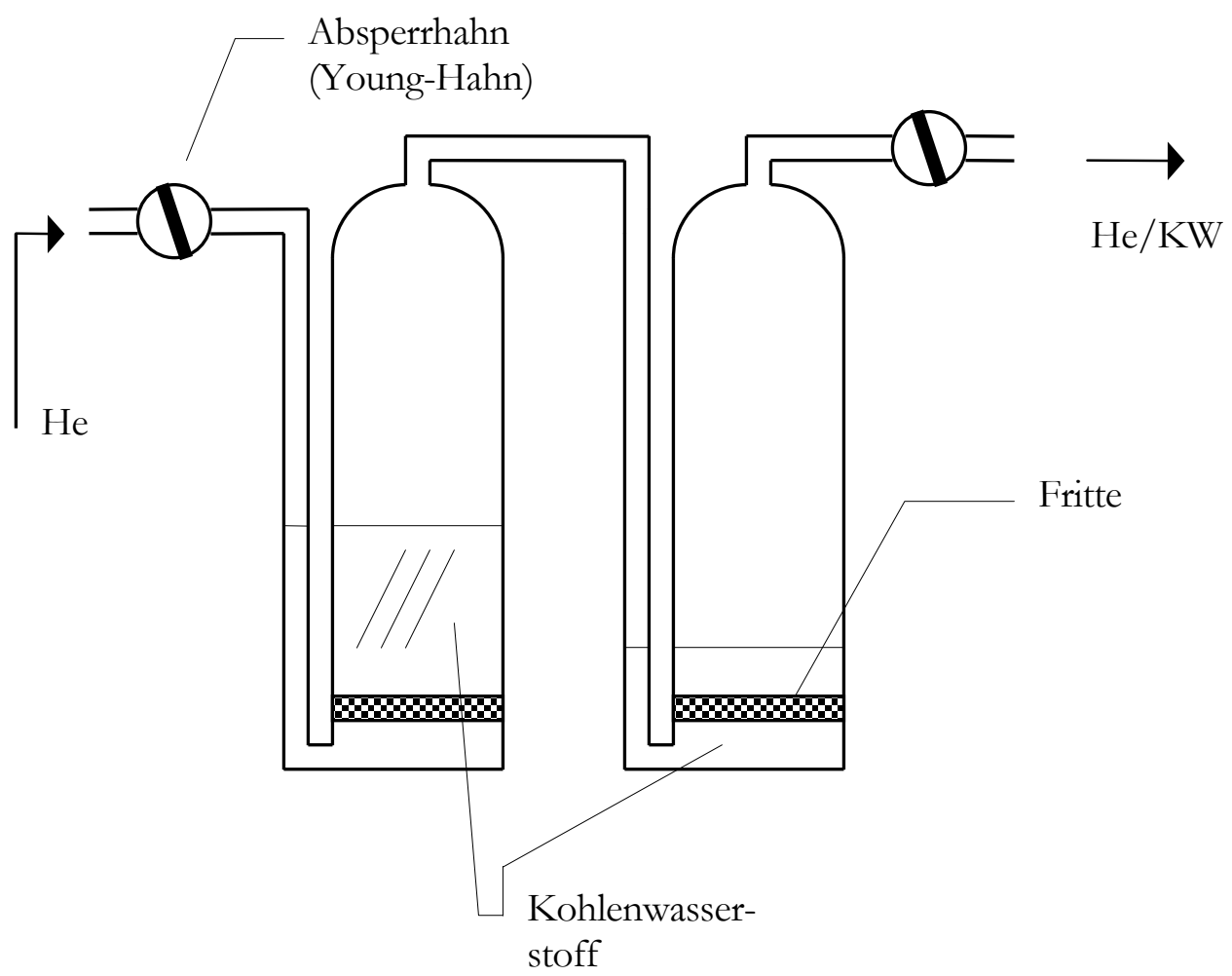

\section{Abbildung 17: Sättiger}

Die folgenden Substanzen wurden ohne weitergehende Reinigung verwendet:

\begin{tabular}{|c|c|c|}
\hline Substanz & Reinheit & Hersteller \\
\hline Helium (He) & $\geq 99,996 \%$ & Messer-Griesheim \\
\hline Argon (Ar) & $\geq 99,998 \%$ & Messer-Griesheim \\
\hline $\begin{array}{c}\text { Fluor }\left(\mathrm{F}_{2}\right) / \mathrm{He} \\
\left(5 \% \text { oder } 0,5 \% \mathrm{~F}_{2} \text {-Anteil }\right)\end{array}$ & $\begin{array}{c}\text { He: } \geq 99,996 \% \\
F_{2}: \geq 98 \%\end{array}$ & Messer-Griesheim \\
\hline Sauerstoff $\left(\mathrm{O}_{2}\right)$ & $\geq 99.995 \%$ & Messer-Griesheim \\
\hline $\begin{array}{c}\mathrm{O}_{2} / \mathrm{Ar} \\
\left(20 \% \mathrm{O}_{2} \text {-Anteil }\right)\end{array}$ & $\begin{array}{l}\text { Arr: } \geq 99,999 \% \\
\mathrm{O}_{2}: \geq 99,995 \%\end{array}$ & Messer-Griesheim \\
\hline Wasserstoff $\left(\mathrm{H}_{2}\right)$ & $\geq 99,5 \%$ & Messer-Griesheim \\
\hline Deuterium $\left(\mathrm{D}_{2}\right)$ & $\geq 99,5 \%$ & Messer-Griesheim \\
\hline Stickstoffmonoxid (NO) & $\geq 99,5 \%$ & Messer-Griesheim \\
\hline Methan $\left(\mathrm{CH}_{4}\right)$ & $\geq 99,95 \%$ & Messer-Griesheim \\
\hline Ethan $\left(\mathrm{C}_{2} \mathrm{H}_{6}\right)$ & $\geq 99,95 \%$ & Messer-Griesheim \\
\hline Propen $\left(\mathrm{C}_{3} \mathrm{H}_{6}\right)$ & $\geq 99 \%$ & Aldrich \\
\hline Propan $\left(\mathrm{C}_{3} \mathrm{H}_{8}\right)$ & $\geq 99 \%$ & Aldrich \\
\hline $\mathrm{CH}_{3} \mathrm{CD}_{2} \mathrm{CH}_{3}$ & $98 \%$ D-Atom & Aldrich \\
\hline Butan $\left(\mathrm{C}_{4} \mathrm{H}_{10}\right)$ & $\geq 99,1 \%$ & Fluka \\
\hline $\mathrm{CD}_{3} \mathrm{CH}_{2} \mathrm{CH}_{2} \mathrm{CD}_{3}$ & $98 \%$ D-Atom & Aldrich \\
\hline Isobutan (2-Methylpropan, $\left.\mathrm{C}_{4} \mathrm{H}_{10}\right)$ & $\geq 99,6 \%$ & Fluka \\
\hline 2-Methylpropan-2- $d,\left(\mathrm{C}_{4} \mathrm{H}_{9} \mathrm{D}\right)$ & $98 \%$ D-Atom & Aldrich \\
\hline Cyclopentan $\left(\mathrm{c}-\mathrm{C}_{5} \mathrm{H}_{10}\right)$ & $\geq 99,5 \%$ & Merck \\
\hline
\end{tabular}




\begin{tabular}{|c|c|c|}
\hline Cyclohexan $\left(\mathrm{c}-\mathrm{C}_{6} \mathrm{H}_{12}\right)$ & $\geq 99,5 \%$ & Merck \\
\hline Cycloheptan $\left(\mathrm{c}-\mathrm{C}_{7} \mathrm{H}_{14}\right)$ & $\geq 99 \%$ & Fluka \\
\hline Cyclooktan $\left(\mathrm{c}-\mathrm{C}_{8} \mathrm{H}_{16}\right)$ & $\geq 99 \%$ & Fluka \\
\hline Trichlorethan $\left(\mathrm{CH}_{3} \mathrm{CCl}_{3}\right)$ & $\geq 99,5 \%$ & Fluka \\
\hline t-Butylchlorid $\left(\mathrm{t}-\mathrm{C}_{4} \mathrm{H}_{9} \mathrm{Cl}\right)$ & $\geq 99 \%$ & Fluka \\
\hline Isobutylchlorid $\left(\mathrm{t}-\mathrm{C}_{4} \mathrm{H}_{8} \mathrm{ClH}\right)$ & $\geq 99 \%$ & Fluka \\
\hline Dicyclopentadien & $\geq 92 \%$ & Fluka \\
\hline DME $\left(\mathrm{CH}_{3} \mathrm{OCH}_{3}\right)$ & $\geq 99 \%$ & Aldrich \\
\hline t-Butylalkohol $\left(\mathrm{C}_{4} \mathrm{H}_{9} \mathrm{OD}\right)$ & $98 \%$ D-Atom & Aldrich \\
\hline MTBE $\left(\mathrm{t}-\mathrm{C}_{4} \mathrm{H}_{9} \mathrm{OCH}_{3}\right)$ & $\geq 99,8 \%$ & Aldrich \\
\hline DMM $\left(\mathrm{CH}_{3} \mathrm{OCH}_{2} \mathrm{OCH}_{3}\right)$ & $\geq 99 \%$ & Fluka \\
\hline DtBK $\left(\mathrm{t}-\mathrm{C}_{4} \mathrm{H}_{9} \mathrm{COt}_{-}-\mathrm{C}_{4} \mathrm{H}_{9}\right)$ & $\geq 98 \%$ & Fluka \\
\hline $\mathrm{DtBP}^{\mathrm{DBPP}}{ }^{17}$ & $\geq 98 \%$ & Merck-Schuchardt \\
\hline & $93-98 \%$ & Akzo-Nobel \\
\hline
\end{tabular}

Tabelle 1: Hersteller und Reinheit der verwendeten Chemikalien

Ozon wurde in einem Ozonisator (Sander) durch eine elektrische Entladung von Sauerstoff hergestellt und durch Adsorption an Kieselgel (Korngröße 2-5 mm) bei $-78{ }^{\circ} \mathrm{C}$ in zwei hintereinander angeordneten Kühlfallen aufgefangen. Überschüssiges $\mathrm{O}_{2}$ wurde mit einer Drehschieberölpumpe entfernt. Durch Entfernung der Kühlung und Zugabe von Badgas (He oder Ar) wird das Ozon freigegeben und anschließend im Badgasstrom über ein Feindosierventil dem Reaktor zugeführt.

Cyclopentadien wurde nach einer Anleitung von Moffett [MOF63] aus Dicyclopentadien destilliert und entweder unmittelbar verwendet oder über kürzere Zeiträume hinweg bei Temperaturen kleiner als $-30{ }^{\circ} \mathrm{C}$ gelagert.

\subsection{Atomerzeugung}

Die in den Reaktionen eingesetzten Fluor-, Sauerstoff- und Wasserstoffatome werden durch eine elektrodenlose Mikrowellenentladung erzeugt. Der dazu eingesetzte Mikrowellengenerator $(\lambda / 4-$ Resonator, Bosch Radarmed) wird mit 50-70 W Ausgangsleistung betrieben. Das Zünden der Mikrowelle wird durch ein schwaches elektrisches Wechselfeld $(15 \mathrm{kV} / \mathrm{cm}, 10 \mathrm{kHz})$ erleichtert. Aus Gasflaschen wird Fluor (5\% oder 0,5\% F2, Rest He), Sauerstoff (rein oder als $\mathrm{Ar} / \mathrm{O}_{2}$-Mischung), Wasserstoff (rein) oder Deuterium (rein) entnommen und nach weiterer Verdünnung mit Helium in das aus Quarzglas bestehende Entladungsröhrchen geleitet, wo die Dissoziation in die Atome erfolgt. Bei Fluor ist die Wand des Entladungsröhrchen mit einer $\mathrm{Al}_{2} \mathrm{O}_{3}-$ Keramik abgeschirmt, um die Bildung von Siliziumfluoriden $\left(\mathrm{SiF}_{4}, \mathrm{SiF}_{3}\right)$ zu verhindern.

${ }^{17}$ Handelsname: Trigonox 25 [AKZ92] 
Eine spontane Rekombination der Atome wird durch die Verdünnung mit Helium erschwert. Außerdem wären die rekombinierten Moleküle stark schwingungsangeregt, was wegen fehlender Relaxationsmöglichkeiten (nur ein Schwingungsfreiheitsgrad und niedriger Druck im System) zur erneuten Spaltung führen würde. Der Dissoziationsgrad von $\mathrm{F}_{2}$ und $\mathrm{O}_{2}$ wurde schon mehrfach durch Messung der Abnahme auf $\mathrm{m} / \mathrm{z}=38$ bzw. $\mathrm{m} / \mathrm{z}=32$ experimentell bestimmt ([BEI91], [NAC94], [HOL97]). Bei $F_{2}$ ist eine Dissoziation von 75-95\%, bei Sauerstoff von 20\% (Sauerstoff allein) bis 50\% (Sauerstoff mit Fluor) ermittelt worden. Diese Werte entsprechen den unterschiedlichen Dissoziationsenergien der Moleküle ${ }^{18}$.

Da die Reaktionen von Fluoratomen mit $\mathrm{H}_{2}\left(\mathrm{k}^{298 \mathrm{~K}}=1,58 \cdot 10^{13} \mathrm{~cm}^{3} \cdot \mathrm{mol}^{-1} \cdot \mathrm{s}^{-1}\right.$, [ATK97] $)$ und $\mathrm{D}_{2}$ $\left(\mathrm{k}^{298 \mathrm{~K}}=8,49 \cdot 10^{12} \mathrm{~cm}^{3} \cdot \mathrm{mol}^{-1} \cdot \mathrm{s}^{-1}\right.$, [COH83]) schnell sind, führt eine gleichzeitige Zugabe dieser Stoffe durch die Entladung zu einer Verfälschung. Eine Erhöhung der Wasserstoffkonzentration würde eine Erniedrigung der Fluoratomkonzentration nach sich ziehen. Aus diesem Grund wurden Wasserstoff- und Deuteriumatome nicht zusammen mit Fluoratomen in einer Entladung erzeugt (vgl. Abschnitt 2.1.2).

\subsection{Ausführung der Messungen}

Die Reaktionen wurden, mit Ausnahme der in Versuchsanordnung C (FTIR) durchgeführten Produktanalysen, in isothermen Strömungsreaktoren durchgeführt. Die Bedingungen wurden so eingestellt, dass eine laminare Strömung mit konstanter Strömungsgeschwindigkeit vorlag (vgl. Abschnitt 2.4.1). Andererseits wurde die Reaktionsstrecke möglichst kurz gehalten, um Folgereaktionen zu vermeiden. Die linearen Strömungsgeschwindigkeiten lagen zwischen 10 und $50 \mathrm{~m} / \mathrm{s}$. Die Messbedingungen im Einzelfall sind in den Auftragungen oder bei den Messtabellen vermerkt.

Der Druck wurde bei allen Messungen so gering wie möglich eingestellt (1-3 mbar). Durch die damit verbundene geringe Teilchenzahldichte sollten Folgereaktionen im Strömungsreaktor vermindert werden. Außerdem wurde so in der Ionenquelle ein hohes Vakuum (10-5-10-7 Torr) erreicht, wodurch Folgereaktionen an dieser Stelle minimiert wurden.

Für die Messungen im Temperaturbereich von 243 bis $363 \mathrm{~K}$ wurde ein Thermostat Julabo, F30 und Lauda, Ultra-Kryomat RUK 90) verwendet, der mit einem Gemisch aus Ethylenglykol/Wasser betrieben wurde. Die aus Perbunan bestehenden Schlauchverbindungen zwischen dem Kryostaten und dem Strömungssystem waren zur besseren thermischen Isolation mit einem Wärmedämmschlauch ummantelt. Die Temperatur wurde am Thermostaten abgelesen. Kontrollmessungen mit

${ }^{18}$ Dissoziationsenergie $\mathrm{F}_{2}: 159 \mathrm{~kJ} / \mathrm{mol}, \mathrm{O}_{2}: 498 \mathrm{~kJ} / \mathrm{mol}$ [CHR88]. 
einem Thermoelement innerhalb der Sonde des Reaktors haben bestätigt, dass die eingestellte Temperatur auch tatsächlich im Reaktor vorherrscht.

Die kinetischen Messungen der Radikale wurden, soweit diese ein REMPI-Signal zeigten, mit der Versuchsanordnung A ausgeführt. Die Radikale wurden durch Reaktion von Kohlenwasserstoffen mit F- oder Cl-Atomen erzeugt. Dabei wurden die Radikale im Kohlenwasserstoffüberschuss erzeugt, so dass Folgereaktionen der Fluoratome mit den Kohlenwasserstoffradikalen vernachlässigbar waren. Es wurde bei allen Radikalen durch Abschalten der F-Atomerzeugung überprüft, dass Überlagerungen durch Fragmentierung nicht auftraten, so dass Maßnahmen wie eine Absenkung der Laserenergie zur Ionisation nicht erforderlich waren. Waren die Reaktionsgeschwindigkeiten der F-Atome mit dem Radikalreaktionspartner X hoch, wurde auf eine getrennte Injektion von X geachtet. Die Radikalkonzentration wurde möglichst niedrig gehalten, um eine Radikalkombinationsreaktion zu unterdrücken. In einigen Fällen wurden Simulationsrechnungen durchgeführt, um das zu überprüfen. Es wurde über 200 bis 800 Spektren gemittelt, um eine Konstanz der Signale bei gleichen Reaktionsbedingungen zu gewährleisten.

Versuchsanordnung B (EI-MS) wurde vorwiegend zur Produktanalyse, zur Bestimmung der Reaktionsgeschwindigkeit von Kohlenwasserstoffen mit F-Atomen und zur Untersuchung der Reaktionen von Radikalen ohne REMPI-Signal eingesetzt. Die Ausführungen zur Radikalerzeugung des letzten Absatzes gelten sinngemäß auch für diese Apparatur. Es wurden Massenspektren im Magnetstromscans unter verschiedenen Reaktionsbedingungen (Untergrund, nur $\mathrm{KW},+\mathrm{F}_{2},+\mathrm{F}$, +X) aufgenommen. Diese wurden bei 200 oder 400 ms Zählzeit pro Massemesspunkt und linearem Massendurchlauf ausgeführt. Die Ionisierungsenergie war kleiner gleich 29,5 eV, um Überlagerungen durch Fragmentierung möglichst niedrig zu halten. Für besondere Betrachtungen der Produktanalyse und für kinetische Messungen wurden die interessierenden Massen manuell angesteuert und blieben fest eingestellt. Es wurde dann pro Messpunkt eine Zählzeit von $10 \mathrm{~s}$ gewählt. 3-10 Messpunkte wurden zu einem Mittelwert verrechnet. Um eine Verfälschung der Signale durch Sättigungseffekte am 85-MHz-Zweikanalzähler auszuschalten, wurde darauf geachtet, dass der Ionenstrom kleiner als $10^{6}$ Zählwerte/s verursachte. Wurde ein nicht vernachlässigbarer Untergrund (Bkg) bei einer Masse festgestellt, wurde die Höhe dieses Untergrunds unter denselben Druck-, Temperatur- und Flussbedingungen quantitativ erfasst und durch das Steuerungsprogramm WinSiccon vom späteren Messwert (Data) automatisch subtrahiert.

Findet eine Reaktion eines Kohlenwasserstoffes z.B. mit F-Atomen gemäß der Reaktionsgleichung $\mathrm{KW}+\mathrm{F} \rightarrow$ Prod. statt, so berechnet sich der Umsatz U des Kohlenwasserstoffs durch die Gleichung: 


$$
\mathrm{U}=\frac{\mathrm{I}_{-\mathrm{F}}-\mathrm{I}_{+\mathrm{F}}}{\mathrm{I}_{-\mathrm{F}}}=1-\frac{\mathrm{I}_{+\mathrm{F}}}{\mathrm{I}_{-\mathrm{F}}} .
$$

Falls diese Umsatzermittlung mit Versuchsanordnung B auf überlagerungsfreien Fragmenten möglich ist, ist diese bei den Auftragungen bzw. Messtabellen angegeben. Ist die Masse eines Radikals durch ein Fragment des Ausgangskohlenwasserstoff überlagert, wie etwa die des Methylradikals $(\mathrm{m} / \mathrm{z}=15)$ durch ein Fragment des Methans, so wurde eine Umsatəkoorrektur für Reaktionen des Radikals mit einer Substanz X durchgeführt. Diese sei für das angesprochene Beispiel erklärt:

$$
\begin{aligned}
& I_{15+F-X}^{\text {korr }}=I_{15+F-X}^{\text {gemessen }}-I_{15-F-X} \cdot \frac{I_{16+F-X}}{I_{16-F-X}}, \\
& I_{15+F+X}^{\text {korr }}=I_{15+F+X}^{\text {gemessen }}-I_{15-F-X} \cdot \frac{I_{16+F-X}}{I_{16-F-X}} .
\end{aligned}
$$

Die Standardabweichungen der Mittelwerte wurden von WinSiccon für ein Konfidenzintervall von 95\% errechnet. Wann immer eine Auftragung gemacht wurde und die daraus resultierenden Parameter zur Angabe von Geschwindigkeitskoeffizienten genutzt wurden, wurde eine Ausgleichsrechnung (lineare Regression) durchgeführt. Die Fehler der Ausgleichsrechnung (Regressionsfehler) bestimmten dann den Fehler der errechneten Größe.

Bei den mit Versuchsanordnung C (FTIR) durchgeführten Produktanalysen wurden zunächst die Vorläufersubstanzen in die Reaktionszelle eingeführt und deren Spektrum (50-100 Mittlungen) aufgenommen. Als Badgas wurde Argon verwendet. Dieses Einkanalspektrum diente als $\mathrm{I}_{0}$-Wert. Anschließend wurde in mehreren Schritten zu je 100-200 Laserpulse photolysiert und jeweils die Spektren aufgenommen. Mit dem ermittelten Spektrum der Vorläufer als $\mathrm{I}_{0}$-Wert wurde aus diesen Spektren die Absorbanz $\log \left(\mathrm{I}_{0} / \mathrm{I}\right)$ errechnet. Störende Absorbanzen von Wasser wurden subtrahiert. Die aus der Photolyse entstandenen Produkte konnten so identifiziert werden. Im nächsten Untersuchungsschritt wurden mögliche Reaktionspartner (z.B. $\mathrm{O}_{2}$ ) der aus der Photolyse produzierten Radikale bzw. die Vorläufersubstanzen der Reaktionspartner (z.B. $\mathrm{SO}_{2}$ zur O-Atomerzeugung) mit in die Zelle eingeführt und anschließend in mehreren Schritten zu 100-200 Laserpulspaketen photolysiert. Erneut diente das Spektrum der Vorläufersubstanzen als I ${ }_{0}$-Wert zur Berechnung der Absorbanz. Störende Absorbanzen von Wasser wurden mittels eines Spektrums von reinem Wasser subtrahiert. Die daraus entstandenen Differenzspektren dienten zur Einschätzung von Reaktionseffekten, zur Ermittlung von Produkten und zur Bewertung von Reaktionskanälen. Wann immer sinnvoll, wurden verschiedene Druckbereiche in der Zelle realisiert. 


\section{Ergebnisse und Diskussion}

\subsection{Erzeugung und Nachweis von Radikalen}

\subsubsection{Das Methylradikal}

Methan ist ein wichtiger Rohstoff für die großtechnische Synthese einer Vielzahl von Stoffen. Der weitaus größte Teil des geförderten Methans wird jedoch als Brennstoff oder Treibstoff genutzt. Der Methangehalt der Luft betrug im Jahr 1991 ca. 1,3 ppm, wobei ein großer Teil biologischen Ursprungs ist. Die Konzentration hat sich seit dem Jahr 1750 in etwa verdoppelt und steigt zur Zeit um 1-2\% pro Jahr [FAL91]. Dem Methan wird pro Molekül eine 20fach stärkere Treibhauswirkung zugeschrieben als dem $\mathrm{CO}_{2}$, welches in einer Konzentration von $350 \mathrm{ppm}$ in der Atmosphäre vorliegt, dessen Anstieg aber weitaus geringer ist [SCH91]. Das meiste atmosphärische Methan wird durch Reaktion mit $\mathrm{OH}$ zur Bildung von Methyl, welches dann weiterreagiert, abgebaut.

Messergebnisse: In der vorliegenden Arbeit wurden Methylradikale über die Reaktion

$$
\begin{gathered}
\mathrm{CH}_{4}+\mathrm{F} \rightarrow \mathrm{CH}_{3}+\mathrm{HF}, \\
\mathrm{k}^{298 \mathrm{~K}=} 4,72 \cdot 10^{13} \mathrm{~cm}^{3} \cdot \mathrm{mol}^{-1} \cdot \mathrm{s}^{-1}[\text { ATK97] }
\end{gathered}
$$

dargestellt. Die folgende Abbildung zeigt ein mit der Versuchsanordnung A (REMPI) aufgenommenes Flugzeitmassenspektrum bei $\lambda=450,6 \mathrm{~nm}$ :

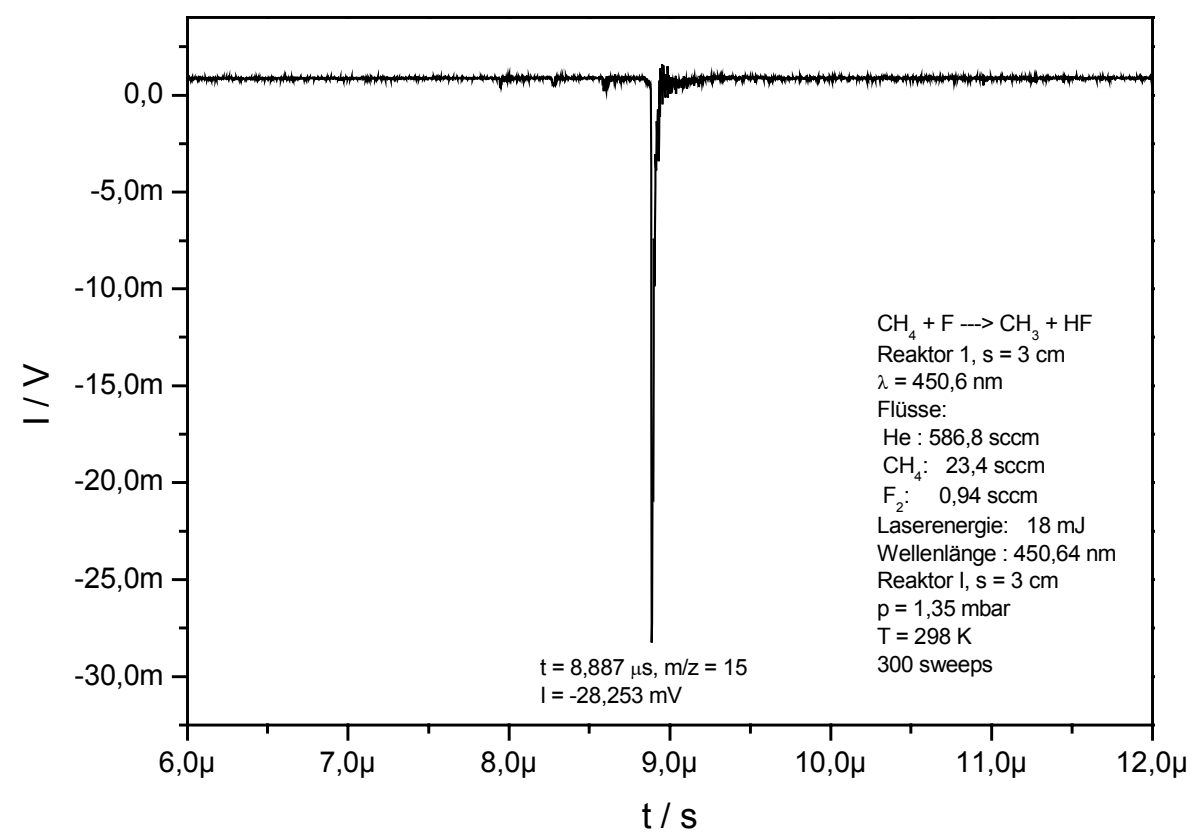

Abbildung 18: Flugzeitmassenspektrum $\mathrm{CH}_{3}, \lambda=450,6 \mathrm{~nm}$ 
Das Methylradikal zeigt in diesem Bereich eine hohe Wellenlängenselektivität. Die nachfolgende Abbildung zeigt ein mit Versuchsanordnung A aufgenommenes REMPI-Spektrum von $\lambda=447$ bis $\lambda=453 \mathrm{~nm}$.

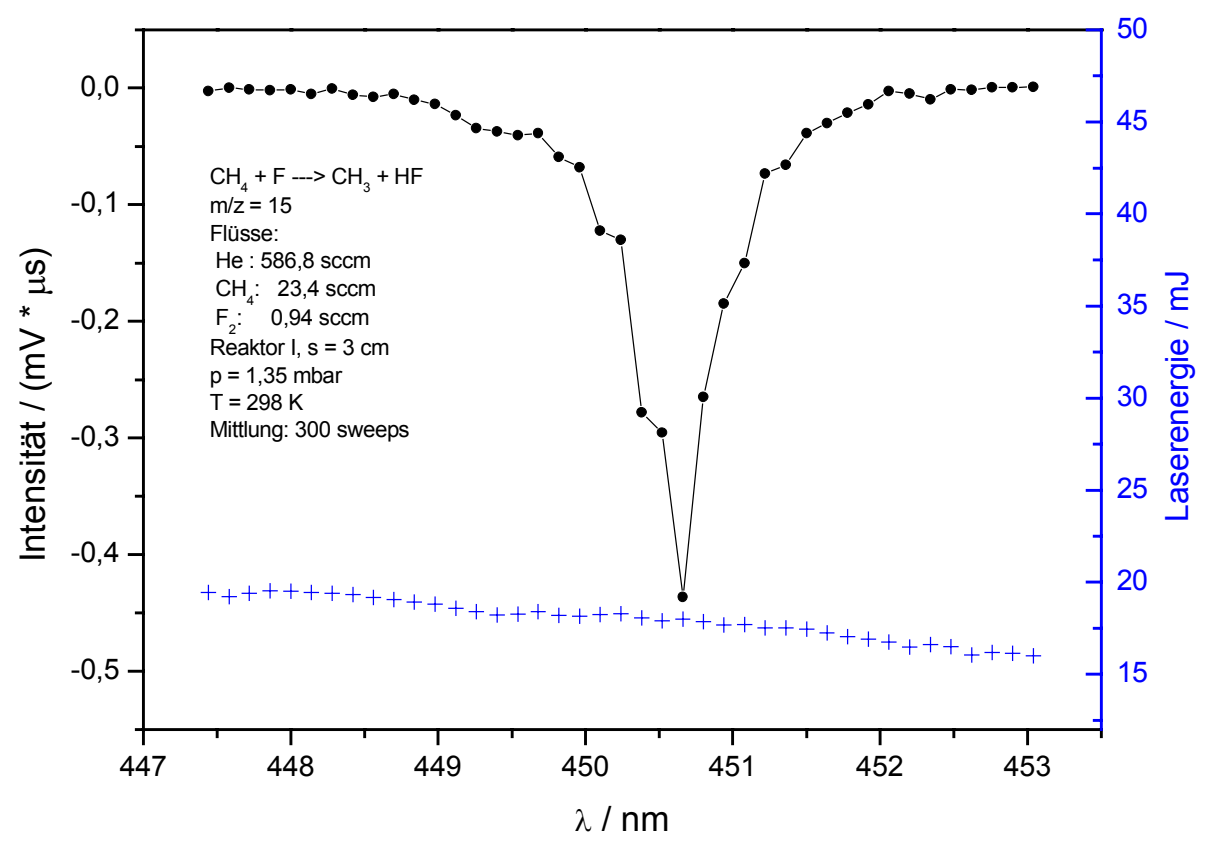

\section{Abbildung 19: REMPI-Spektrum $\mathrm{CH}_{3}, \lambda=447-453 \mathrm{~nm}$}

Diskussion: Das Methylradikal ist eines der meist untersuchten Kohlenwasserstoffradikale. Die Nachweisbarkeit des Methylradikals mittels REMPI (im folgenden REMPI-Aktivität genannt) im Bereich $\lambda=450-451 \mathrm{~nm}$ Wellenlänge wurde von DiGuiseppe, Hudgens und Lin [DIG81] entdeckt. Die Erzeugung des Radikals geschah durch Pyrolyse von Azomethan, Methyljodid, DtBP oder Dimethylsulfoxid. DiGuiseppe et al. berichten, dass im Bereich $\lambda=410-460 \mathrm{~nm} 3$ Photonen gleichzeitig aufgenommen werden und so die Zustände $3 \mathrm{~d}{ }^{2} \mathrm{E}^{\prime}, 4 \mathrm{~d}^{2} \mathrm{~A}^{\prime}{ }_{1}$ und $4 \mathrm{~s}^{2} \mathrm{~A}^{\prime}{ }_{1}$ erreicht werden. Der intensive Nachweis bei $\lambda=450,6$ geht über den $3 \mathrm{~d}{ }^{2} \mathrm{E}$ " Zustand. Absorption eines weiteren Photons führt dann zur Ionisation, es handelt sich also um einen 3+1 Mechanismus ([DIG81], [HUD87]). Auch das $\mathrm{CD}_{3}$-Radikal ist in diesem Bereich REMPI-aktiv, wobei die Isotopenverschiebung ca. $0,5 \mathrm{~nm}$ beträgt [HUD87]. Eigene Messungen bestätigen dies.

Neben diesem 3 Photonen REMPI-Prozess gibt noch 1 und 2 Photonen REMPI-Prozesse, die zu kürzeren Nachweiswellenlängen führen (vgl. Anhang 6.1 Radikale mit REMPI-Signal). Der intensivste Nachweis des Methylradikals überhaupt erfolgt bei $\lambda=333,4 \mathrm{~nm}$.

Messergebnisse: Die folgende Abbildung zeigt ein mit Versuchsanordnung A (REMPI) aufgenommenes Flugzeitmassenspektrum bei $\lambda=340,8 \mathrm{~nm}$ : 


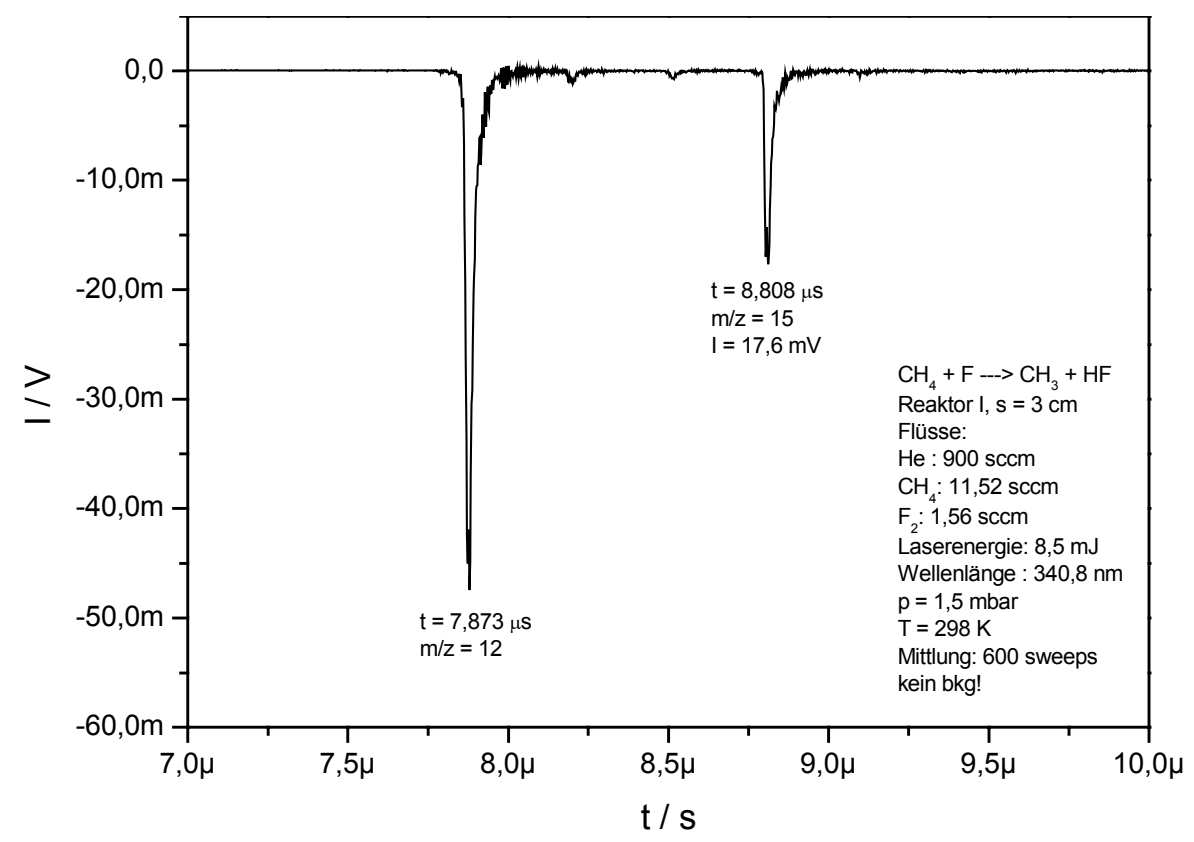

\section{Abbildung 20: Flugzeitmassenspektrum $\mathrm{CH}_{3}, \lambda=340,8 \mathrm{~nm}$}

Nach [HUD87] handelt es sich um einen 2+1 REMPI-Prozess mit dem Übergang $3 \mathrm{p}^{2} \mathrm{~A}_{2}{ }_{2}{ }^{0}$. Es ist eine beachtliche Fragmentierung zu m/z $=12$ zu erkennen. Auch das $\mathrm{CD}_{3}$-Radikal ist in diesem Wellenlängenbereich mit guter Intensität und gleicher Fragmentierung zu m/z $=12$ nachzuweisen, wie die folgende Abbildung zeigt.

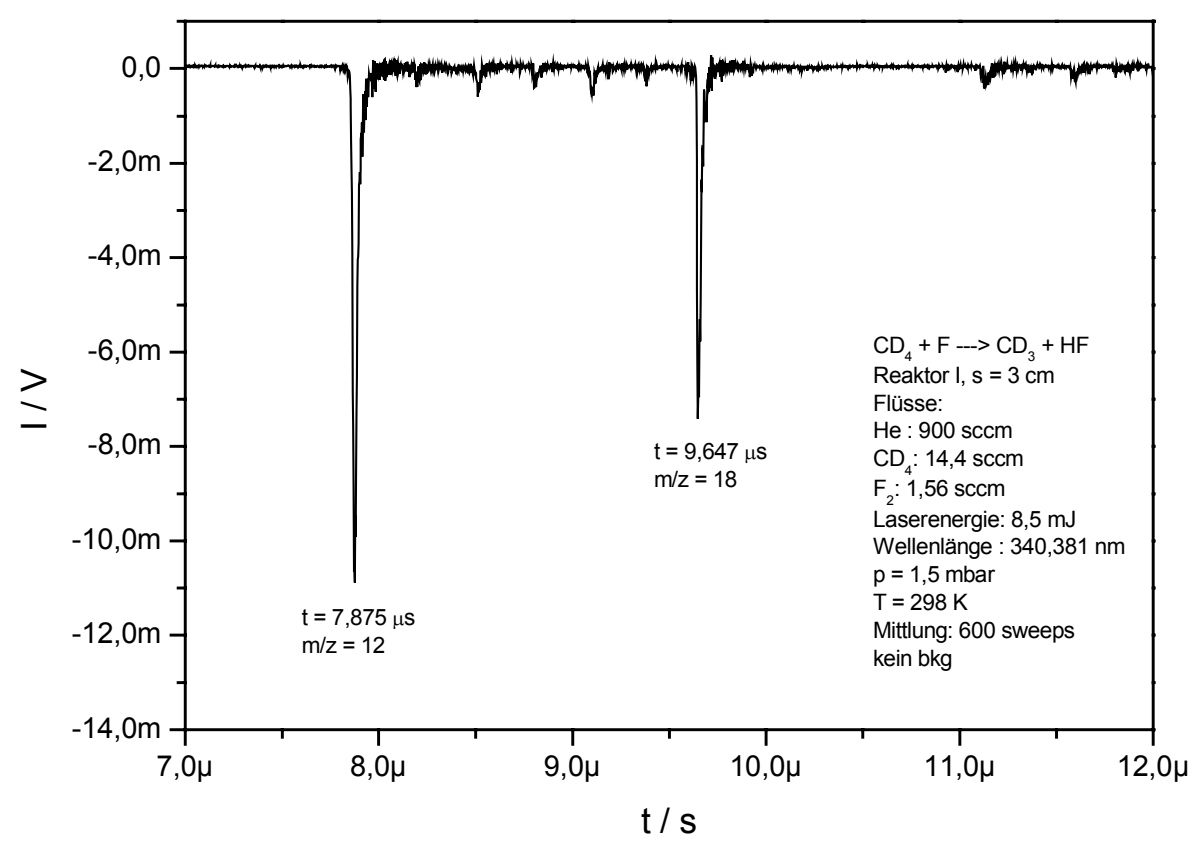

Abbildung 21: Flugzeitmassenspektrum $\mathrm{CD}_{3}, \lambda=340,8 \mathrm{~nm}$

Es wurde außerdem die Wellenlängenabhängigkeit des $\mathrm{CH}_{3}$-Radikals im Bereich $\lambda=336-349 \mathrm{~nm}$ untersucht (Abbildung 22). Die violette Linie zeigt den für den verwendeten Laserfarbstoff paraTerphenyl typischen Energieverlauf. Die Wellenlängenselektivität des Methylradikals ist in diesem Bereich geringer als bei $\lambda=450-451 \mathrm{~nm}$. Von $\lambda=336$ bis $\lambda=342 \mathrm{~nm}$ folgt die Signalintensität von 
$\mathrm{m} / \mathrm{z}=15 \mathrm{im}$ wesentlichen der Laserenergie, geht von da an aber trotz schwindender Laserenergie einem Maximum bei $\lambda=345,5 \mathrm{~nm}$ entgegen. Das Signal zeigt somit einen gewissen charakteristischen Verlauf. Es sei noch angemerkt, dass ab $\lambda=342 \mathrm{~nm}$ die Fragmentierung zu $\mathrm{m} / \mathrm{z}=12$ beträchtlich abnimmt.

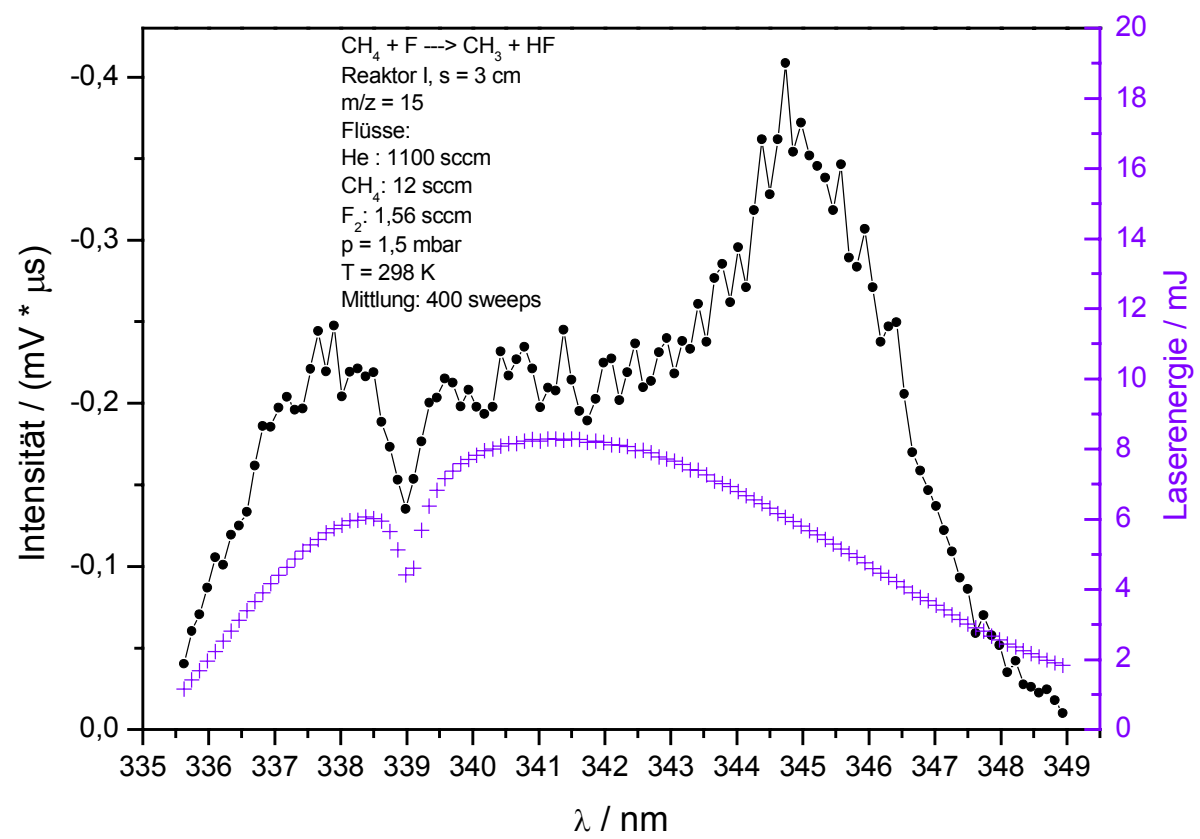

\section{Abbildung 22: REMPI-Spektrum $\mathrm{CH}_{3}, \lambda=336-349 \mathrm{~nm}$}

Diskussion: Der REMPI-Nachweis des Methylradikals im Wellenlängenbereich $\lambda=332-342 \mathrm{~nm}$ wurde von Smyth und Taylor untersucht [SMY85]. Methylradikale wurden dabei in einer Methan/Luft Diffusionsflamme bei einer Temperatur von $1700 \mathrm{~K}$ erzeugt. Der dort in Abhängigkeit von der Wellenlänge skizzierte Verlauf der Signalintensität ist allerdings anders als hier dargestellt: Es werden scharfe Peaks bei $\lambda=333,5 \mathrm{~nm}$ und 340,8 nm gefunden, sonst aber keine Intensitäten. Ein Grund für dieses andersartige Verhalten mag in den dort eingesetzten niedrigen Laserenergien ( $\mathrm{E}=0,1-0,5 \mathrm{~mJ} / \mathrm{Puls})$ liegen. Es wird bei [SMY85] nicht mit einem massenspektrometrischen Nachweis gearbeitet, sondern mit einer Tungsten-Elektrode in der Flamme detektiert. Somit ist keineswegs gewährleistet, dass das gemessene Signal tatsächlich von Methylradikalen stammt, was von den Autoren auch eingeräumt wird. Diese halten es ebenso für möglich, dass das Messsignal von in der Flamme erzeugten C-Atomen hervorgerufen wurde. Diese Schwierigkeiten treten bei dem hier eingesetzten massenspektrometrischen Nachweis nicht auf. Da in der vorliegenden Arbeit nicht primär die spektroskopischen Hintergründe des Nachweises betrachtet werden sollen, sondern es in erster Linie auf einen eindeutigen Nachweis in für kinetische Messungen ausreichender Intensität ankommt, wird diese Frage nicht weiter erörtert. 


\subsubsection{Das Ethylradikal}

Ethan kommt in vielen Erdgasen sowie in Raffineriegasen vor und wird somit in großen Mengen verbrannt. Es wird zur Gewinnung von Ethen durch Pyrolyse und zur Produktion von Acetaldehyd und Essigsäure durch katalytische Oxidation verwendet [FAL91]. Ethan und das daraus durch HAtomabstraktion resultierende Ethylradikal spielen außerdem bei der Verbrennung von Methan (Hauptbestandteil von Erdgas) und bei vielen weiteren Verbrennungsvorgängen als Zwischenprodukt eine zentrale Rolle.

Messergebnisse: In der vorliegenden Arbeit wurden Ethylradikale über die Reaktion

$$
\begin{gathered}
\mathrm{C}_{2} \mathrm{H}_{6}+\mathrm{F} \rightarrow \mathrm{C}_{2} \mathrm{H}_{5}+\mathrm{HF} \\
\mathrm{k}^{298 \mathrm{~K}}=6,00 \cdot 10^{13} \mathrm{~cm}^{3} \cdot \mathrm{mol}^{-1} \cdot \mathrm{s}^{-1}[\mathrm{PEA} 73]
\end{gathered}
$$

erzeugt. Die folgende Abbildung zeigt ein mit der Versuchsanordnung A (REMPI) aufgenommenes Flugzeitmassenspektrum bei $\lambda=430 \mathrm{~nm}$ :

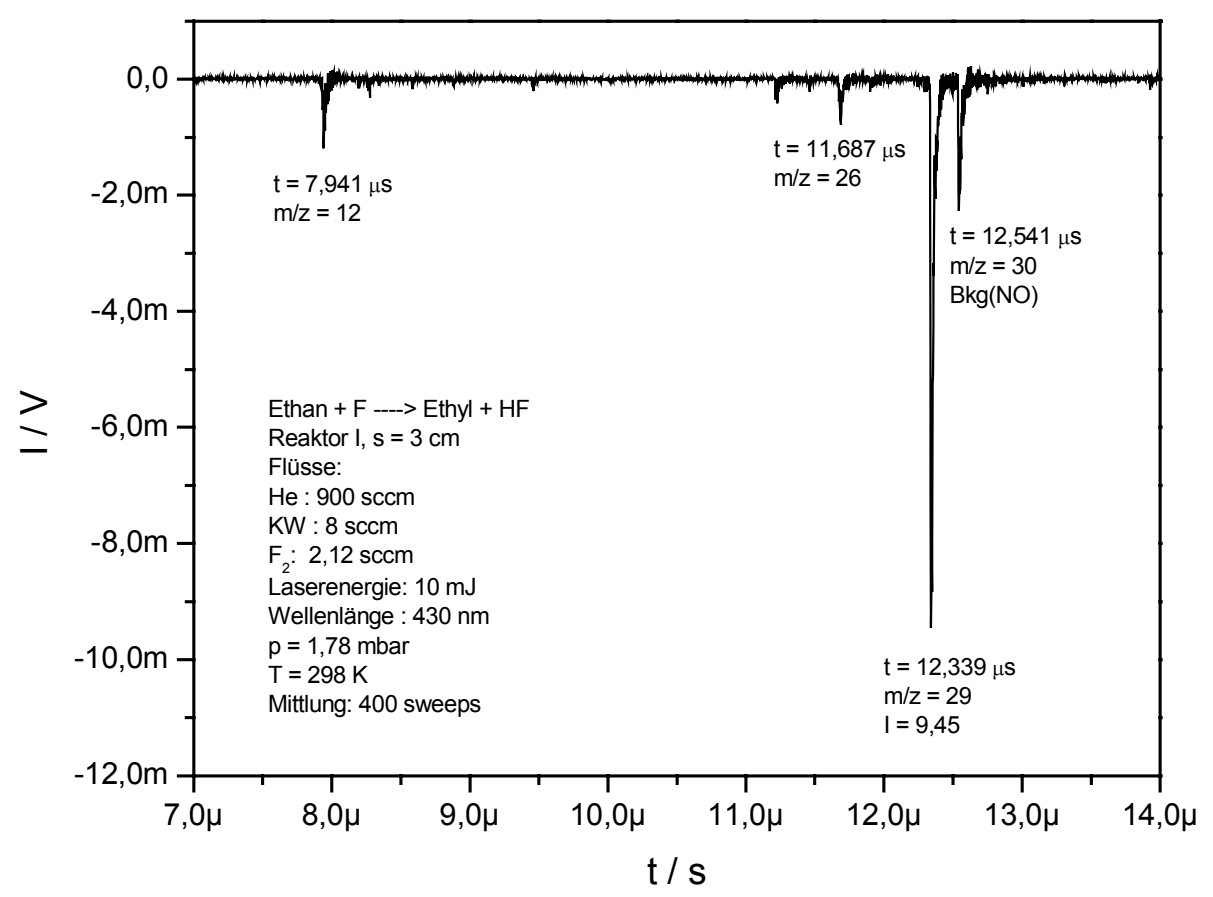

\section{Abbildung 23: Flugzeitmassenspektrum $\mathrm{C}_{2} \mathrm{H}_{5}, \lambda=430 \mathrm{~nm}$}

Das Radikal ist eindeutig auf seiner Muttermasse $\mathrm{m} / \mathrm{z}=29$ nachweisbar. Ein Untergrund vom Radikalvorläufer Ethan ist nicht vorhanden. Das Ethylradikal besitzt bei $\lambda=430 \mathrm{~nm}$ kleinere Fragmente bei $\mathrm{m} / \mathrm{z}=26$ und 12. Testmessungen haben gezeigt, dass das auf $\mathrm{m} / \mathrm{z}=30$ liegende Signal von in der Mikrowellenentladung durch Luftspuren entstandenes NO herrührt. NO ist in 
den Wellenlängenbereichen $\lambda=380-383$, 429-431 und 452-454 nm in geringsten Konzentrationen REMPI-aktiv (vgl. Abschnitt 6.1, Anhang).

Bei $\lambda=406 \mathrm{~nm}$ ist dieses NO-Signal nicht festzustellen. Die folgende Abbildung zeigt ein Flugzeitmassenspektrum des Ethylradikals bei dieser Wellenlänge. Ein Untergrund im Sinne eines Signals durch das Edukt Ethan ist nicht festzustellen. Das Fragmentierungsmuster entspricht demjenigen bei $\lambda=430 \mathrm{~nm}$.

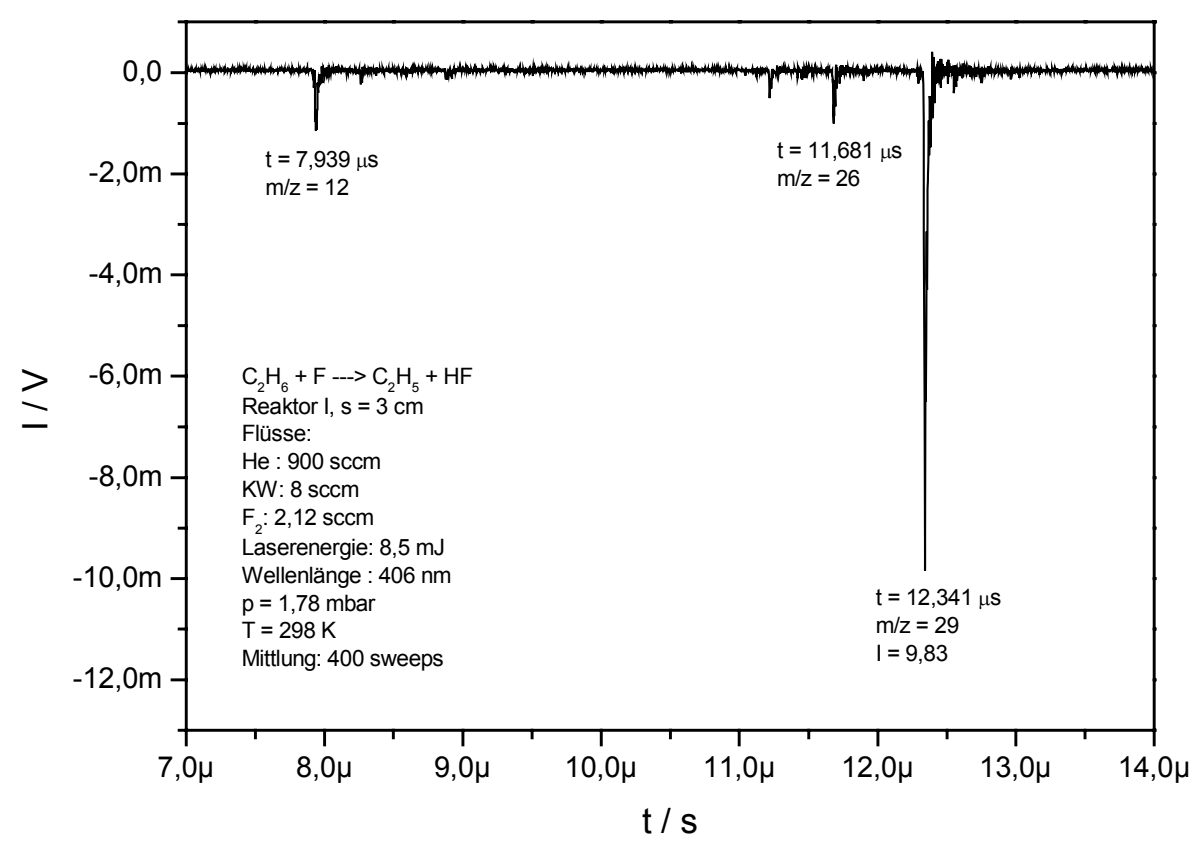

\section{Abbildung 24: Flugzeitmassenspektrum $\mathrm{C}_{2} \mathrm{H}_{5}, \lambda=406 \mathrm{~nm}$}

Auch bei kürzeren Wellenlängen sind Ethylradikale zu detektieren. So zeigt die folgende Abbildung ein Flugzeitmassenspektrum des Ethylradikals bei $\lambda=340,8 \mathrm{~nm}$ (rote Linie). Neben dem Mutterpeak bei $\mathrm{m} / \mathrm{z}=29$ finden sich Fragmente bei $\mathrm{m} / \mathrm{z}=26,24,15$ und besonders 12. Es ist ein kleiner Untergrund durch Ethan bei $\mathrm{m} / \mathrm{z}=12$ zu verzeichnen (schwarze Linie). 


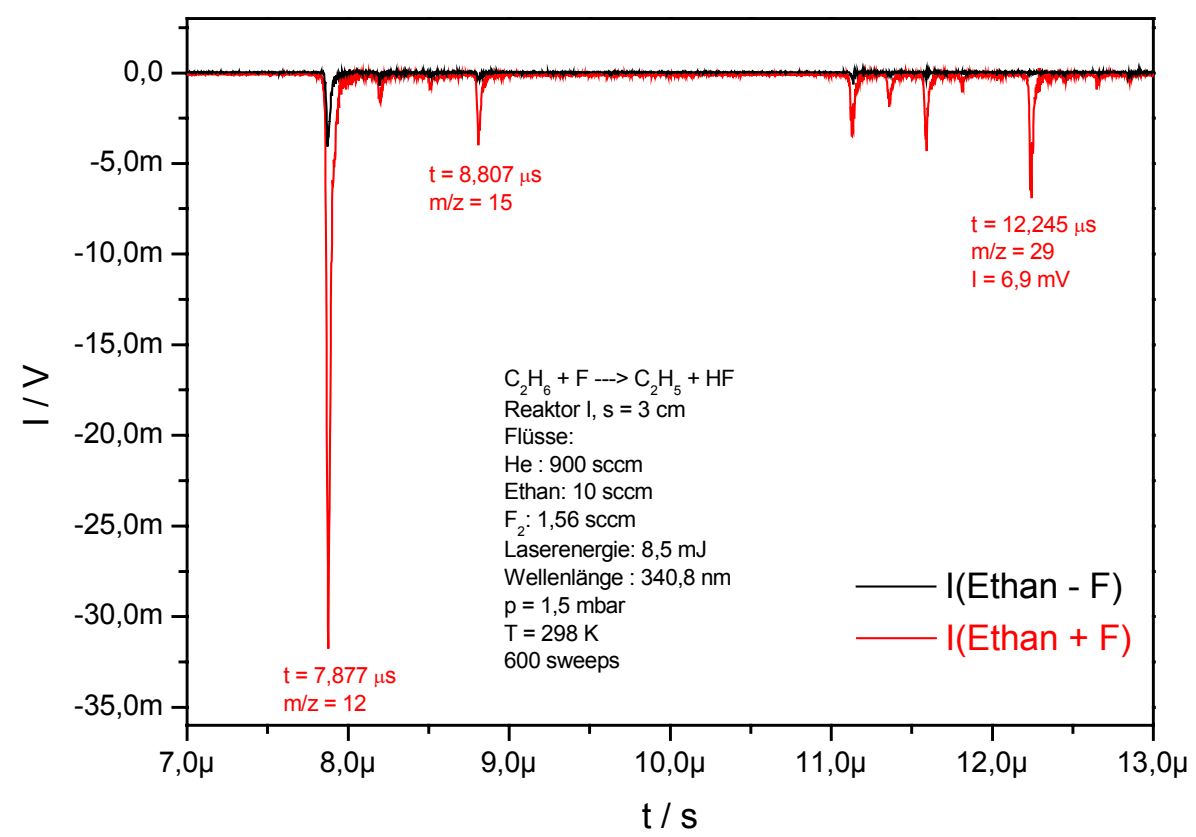

Abbildung 25: Flugzeitmassenspektrum $\mathrm{C}_{2} \mathrm{H}_{5}, \lambda=340,8 \mathrm{~nm}$

Auch das deuterierte Ethylradikal $\mathrm{C}_{2} \mathrm{D}_{5}$ ist bei dieser Wellenlänge signalgebend (vgl. Abschnitt 3.3.1).

Diskussion: Das Ethylradikal gehört zu den am meisten untersuchten Kohlenwasserstoffradikalen in der Gasphasenkinetik. Ein REMPI-Signal des Ethylradikals ist erstmalig von Sappey und Weisshaar im Wellenlängenbereich 398,5-409,5 nm festgestellt und durch einen 2+1 REMPI-Prozess beschrieben worden [SAP87]. Dort wurden Ethylradikale durch Photolyse von $\mathrm{C}_{2} \mathrm{H}_{5} \mathrm{NO}_{2}$ bei $\lambda=193 \mathrm{~nm}$ im Molekularstrahl erzeugt. Die Signalintensität auf der Radikalmuttermasse $\mathrm{m} / \mathrm{z}=29$ war jedoch gering. Das kann dadurch erklärt werden, dass Ethylradikale photolytisch erzeugt wurden, was den Nachteil einer hohen energetischen Anregung dieser Radikale (bot radicals) und eines damit verbundenen schnellen Zerfalls über eine H-Atom Abspaltung mit sich bringt [HUD87], auch wenn in [SAP87] von einer Erzeugung "kalter Radikale" ausgegangen wird. Ein weiterer Grund für die geringe Signalintensität könnte die geringe Energie des Nachweislasers von $2,5 \mathrm{~mJ} /$ Puls sein.

Weitere Untersuchungen zum Nachweis des Ethylradikals mittels REMPI im Wellenlängenbereich $\lambda=397 \mathrm{~nm}$ bis $\lambda=530 \mathrm{~nm}$ sind bei [EDE88], [ROH91] und [NAC98] zu finden. Die genannten Autoren berichten über breite unstrukturierte Banden, deren Intensität im wesentlichen der Laserenergie folgt. Allerdings ist eine Ähnlichkeit im Verlauf zwischen einem REMPI-Spektrum bei [ROH91] und einem Einphotonen-UV-Spektrum bei [WEN84] bezüglich der Intensität von 3pund 3s-Rydbergzuständen durchaus erkennbar. Aus der Ionisierungsenergie des Ethylradikals von 


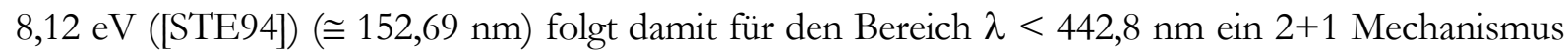
und für größere Wellenlängen ein 2+2 Mechanismus. Im Rahmen dieser Arbeit durchgeführte Messungen zeigen oberhalb dieser Wellenlänge eine deutlich geringer werdende Signalintensität, die darauf aufbauende kinetische Messungen nicht mehr sinnvoll erscheinen lassen.

\subsubsection{Das 1-Propylradikal, das 2-Propylradikal}

Propan wird in Druckgasflaschen als Flüssiggas in Laboren und Haushalten zu Brenn- und Heizzwecken verwendet. Vielfach wird Propan - im allgemeinen zusammen mit Butan - anstelle von Fluorchlorkohlenwasserstoffen als Treibmittel in Sprays eingesetzt [FAL91]. Die aus dem Propan durch Wasserstoffatomabstraktion abgeleiteten Propylradikale tauchen somit bei wirtschaftlich bedeutungsvollen Verbrennungsvorgängen und bei Abbauprozessen in der Atmosphäre auf.

Diskussion: Die Reaktion von Propan mit F-Atomen wurde von Edelbüttel-Einhaus mittels REMPIIonisation und Flugzeitmassenspektrometer im Wellenlängenbereich $\lambda=397-415 \mathrm{~nm}$ untersucht. Dabei wurden Ionensignale der Masse $\mathrm{m} / \mathrm{z}=43$ gefunden. Folgende zwei Reaktionskanäle wurden postuliert:

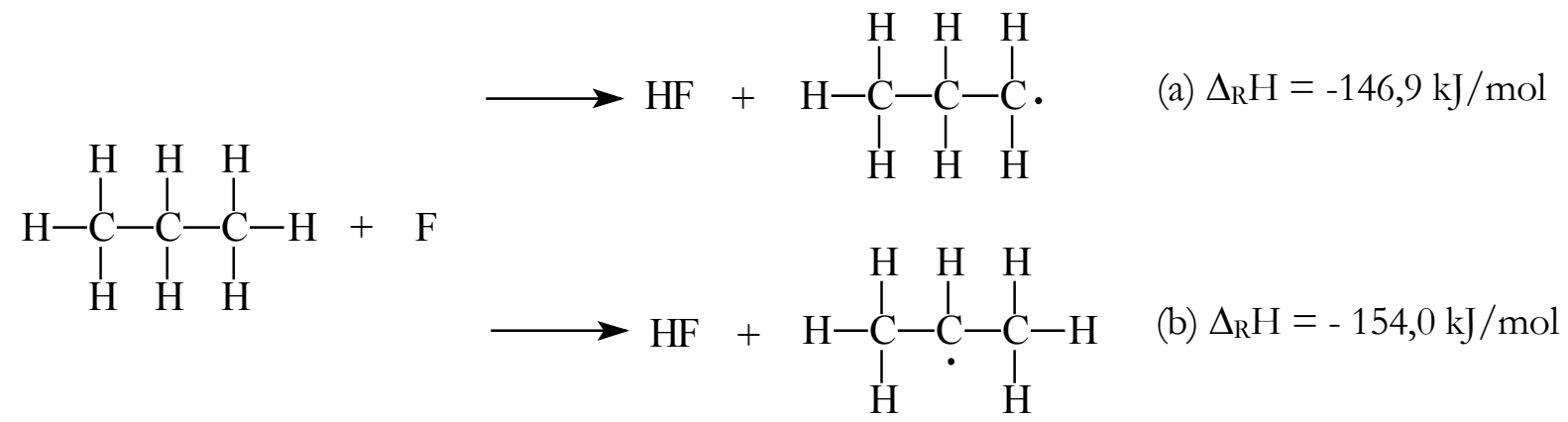

Es wurde von Edelbüttel-Einhaus vermutet, dass beide Kanäle relevant sind. Ein Vergleich der Reaktionsenthalpien sowie gemessener Reaktionsgeschwindigkeiten von Kanal (a) und (b) ${ }^{19}$ stützt diese These. Es konnte aber nicht entschieden werden, ob das gefundene Ionensignal einem der beiden Radikale selektiv zuzuordnen ist. In der vorlegenden Arbeit kann diese Frage nun beantwortet werden. Das Ionisierungspotenzial des 1-Propylradikals (n-Propyl) aus Kanal (a) beträgt 8,09 eV, das des 2-Propylradikals (Isopropyl) aus Kanal (b) 7,37 eV [STE94]. Das eröffnet die Chance, eine Grenzwellenlänge zu finden, bei der nur noch das Radikal mit der geringeren Ionisierungsenergie, also das 2-Propylradikal signalgebend ist, vorausgesetzt beide Reaktionskanäle finden unter den gegebenen Reaktionsbedingungen tatsächlich in ähnlichen Größenordnungen statt. Da das von Edelbüttel-Einhaus beobachtete Signal bei $\mathrm{m} / \mathrm{z}=43$ nicht wellenlängenselektiv

${ }^{19} \mathrm{k}^{298 \mathrm{~K}}(\mathrm{a})=3,5 \cdot 10^{13} \mathrm{~cm}^{3} /(\mathrm{mol} \cdot \mathrm{s}), \mathrm{k}^{298 \mathrm{~K}}(\mathrm{~b})=4,1 \cdot 10^{13} \mathrm{~cm}^{3} /(\mathrm{mol} \cdot \mathrm{s})$, [FET60] 
war, sollte eine Grenzwellenlänge möglichst im langwelligen REMPI-Bereich liegen. Auf der Suche danach wurden für die vorliegende Arbeit REMPI-Spektren von $\lambda=420-530 \mathrm{~nm}$ aufgenommen.

Messergebnisse: Die folgende Abbildung zeigt ein solches im Bereich $\lambda=420-462 \mathrm{~nm}$ :

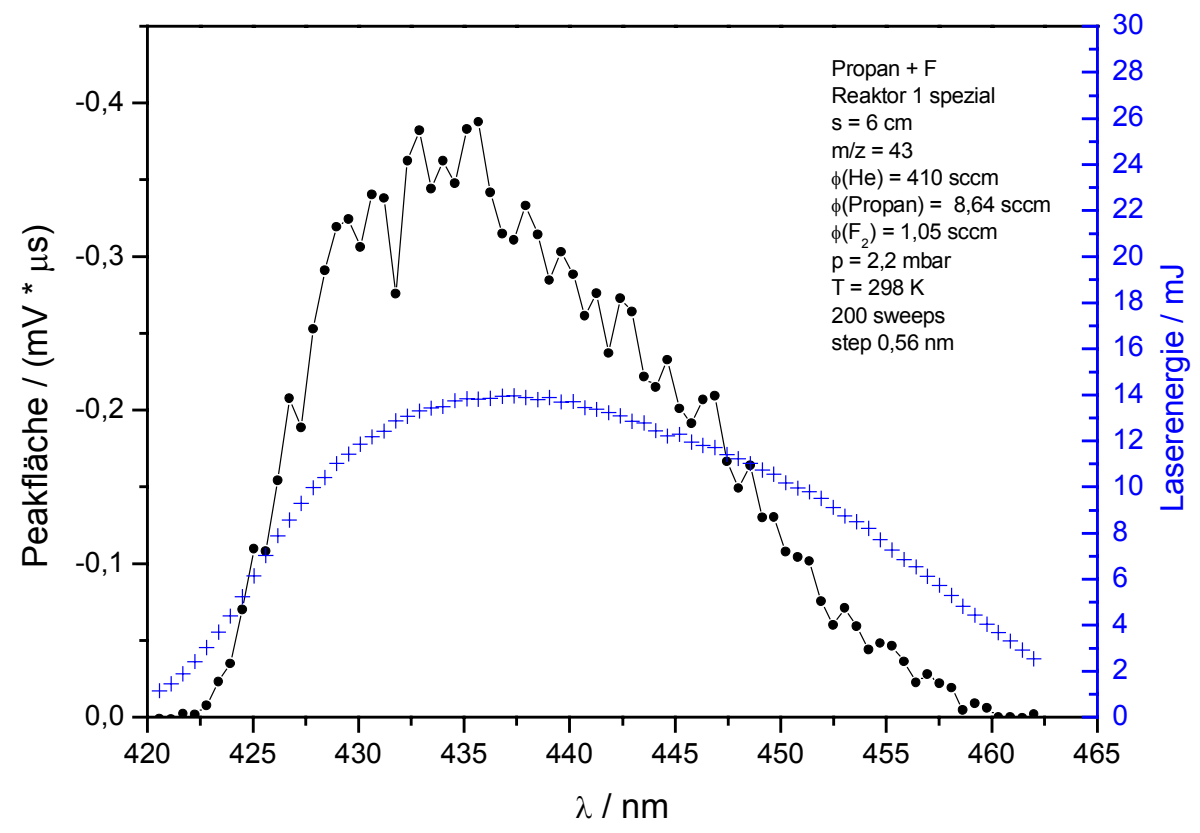

Abbildung 26: REMPI-Spektrum Propan $+F, \lambda=420-462 \mathrm{~nm}$

Ab $\lambda=445 \mathrm{~nm}$ lässt sich ein über den Grad des Absinkens der Laserenergie hinausgehendes Abschwächen des Ionensignals feststellen. Eine Entscheidung, ob in diesem Bereich nur noch eines der beiden Radikale signalgebend ist, lässt sich durch den Einsatz teildeuterierten Propans beantworten. Es wurden Flugzeitmassenspektren von der Reaktion $\mathrm{CH}_{3} \mathrm{CD}_{2} \mathrm{CH}_{3}$ mit F-Atomen bei ausgewählten Wellenlängen in diesem Bereich aufgenommen. Die folgende Abbildung zeigt ein solches bei $\lambda=440 \mathrm{~nm}$ : 


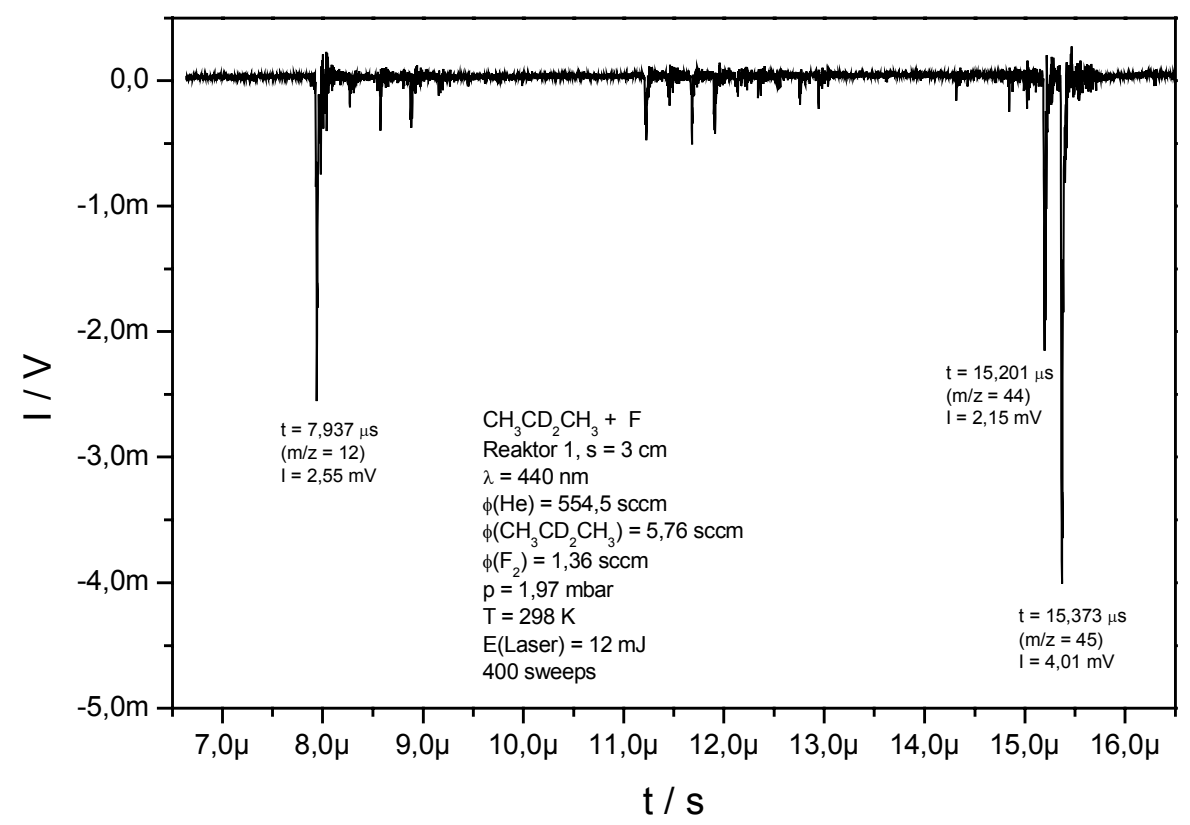

\section{Abbildung 27: Flugzeitmassenspektrum $\mathrm{CH}_{3} \mathrm{CD}_{2} \mathrm{CH}_{3}+\mathrm{F}, \lambda=440 \mathrm{~nm}$}

Bei dieser Wellenlänge sind neben einiger Fragmente die Molekülpeaks sowohl des 1-Propyl- als auch des 2-Propylradikals deutlich erkennbar. Ein Untergrund ist nicht vorhanden, das heißt, Propan selbst gibt keine Signale. Beide Reaktionskanäle der Reaktion Propan + F sind unter den Versuchsbedingungen also existent. Das Signal des 1-Propylradikals ist um den Faktor 2 größer als das des 2-Propylradikals. Hieraus darf jedoch nicht geschlossen werden, dass Kanal (a) um einen solchen Faktor schneller ist als (b), da die spektroskopischen Empfindlichkeiten der Radikale in der Ionenquelle bei dieser Wellenlänge nicht bekannt sind.

Es wurden Flugzeitmassenspektren bei weiteren Wellenlängen aufgenommen. Das Verhältnis der Intensitäten vom Isopropylradikal zum n-Propylradikal wird zunehmend größer, je länger die Wellenlänge wird:

$\mathrm{I}(2$-Propyl $) / \mathrm{I}(1-$ Propyl $)=1(450 \mathrm{~nm}),=2(453 \mathrm{~nm}),=4(456 \mathrm{~nm}),=5(460 \mathrm{~nm})$.

Die folgende Abbildung zeigt ein Flugzeitmassenspektrum bei $\lambda=465 \mathrm{~nm}$ : 


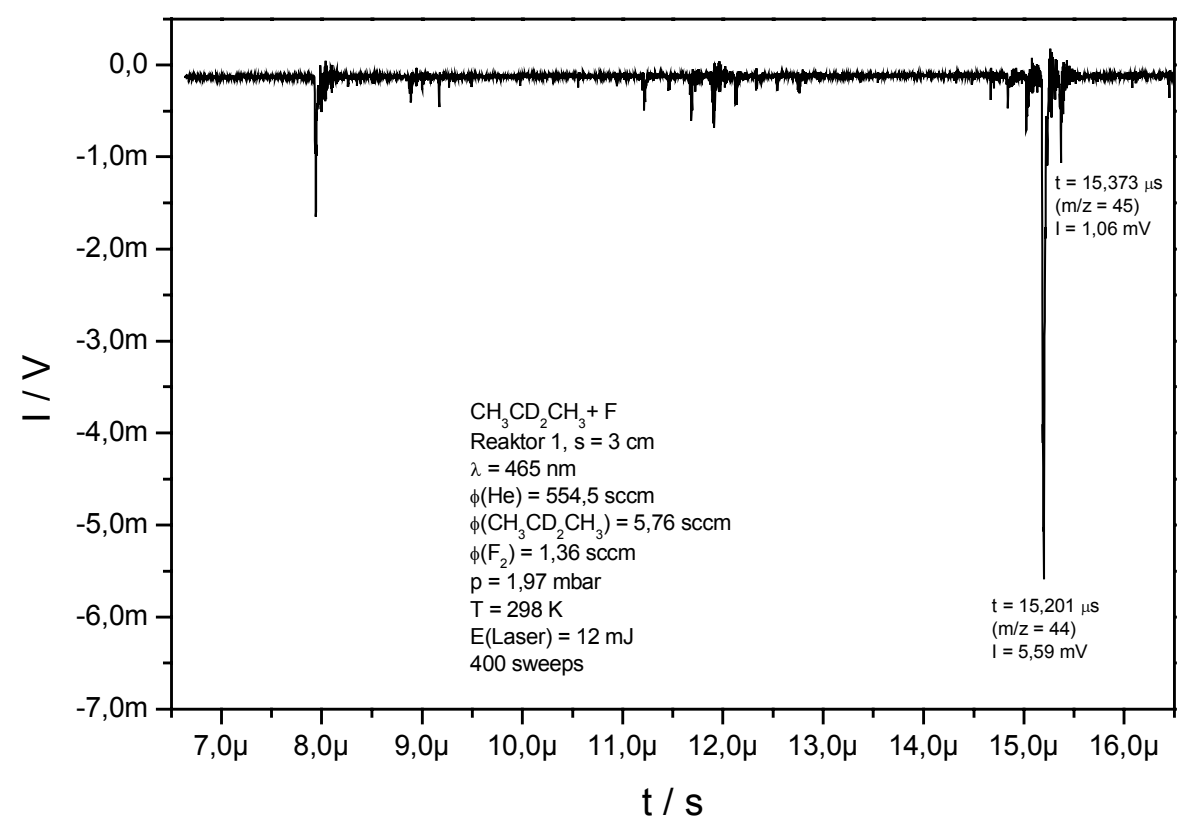

\section{Abbildung 28: Flugzeitmassenspektrum $\mathrm{CH}_{3} \mathrm{CD}_{2} \mathrm{CH}_{3}+\mathrm{F}, \lambda=465 \mathrm{~nm}$}

Die Intensität der Masse $\mathrm{m} / \mathrm{z}=44$ (Isopropyl) übersteigt die von $\mathrm{m} / \mathrm{z}=45$ (n-Propyl) um den Faktor 6. Dabei muss auch noch ein ${ }^{13} \mathrm{C}$-Isotopeneffekt bedacht werden, d.h. ein Teil des Signals von $\mathrm{m} / \mathrm{z}=45$ resultiert aus Isopropyl ${ }^{20}$, welches ${ }^{13} \mathrm{C}$ enthält.

Interpretation: In guter Näherung kann $\lambda=465 \mathrm{~nm}$ als die Grenzwellenlänge bezeichnet werden, bei der nur noch das Isopropylradikal signalgebend ist. Das Verschwinden des Signals des n-Propylradikals mit länger werdender Wellenlänge ist aber nicht scharf, es beginnt bei $\lambda=445 \mathrm{~nm}$ und nähert sich bei $\lambda=460 \mathrm{~nm}$ der Nulllinie.

\subsubsection{Das 1-Butylradikal, das 2-Butylradikal}

n-Butan wird in Druckgasflaschen als Flüssiggas in Laboren und Haushalten zu Brenn- und Heizzwecken verwendet. Vielfach wird n-Butan - im allgemeinen zusammen mit Propan und Isobutan - anstelle von Fluorchlorkohlenwasserstoffen als Treibmittel in Sprays eingesetzt [FAL91]. Die aus dem n-Butan durch H-Atomabstraktion abgeleiteten 1- und 2-Butylradikale tauchen somit bei wirtschaftlich bedeutungsvollen Verbrennungsvorgängen und bei Abbauprozessen in der Atmosphäre auf.

Diskussion: Die Reaktion von n-Butan mit F-Atomen wurde von Edelbüttel-Einhaus [EDE88] mittels REMPI-Ionisation und Flugzeitmassenspektrometer im Wellenlängenbereich

\footnotetext{
${ }^{20}$ Ca. $3 \%$ des Isopropyls enthält ein ${ }^{13} \mathrm{C}$.
} 
$\lambda=397-415 \mathrm{~nm}$ untersucht. Dabei wurden Ionensignale der Masse $\mathrm{m} / \mathrm{z}=57$ gefunden. Folgende zwei Reaktionskanäle wurden vorgeschlagen:<smiles>CCCC</smiles><smiles>CCCC</smiles><smiles>CCCC</smiles>

Die Reaktionsenthalpie für Kanal (a) beträgt $\Delta_{\mathrm{R}} \mathrm{H}^{0} 298 \mathrm{~K}=-150,2 \mathrm{~kJ} / \mathrm{mol}$, die für Kanal (b) $\Delta_{\mathrm{R}} \mathrm{H}^{0}{ }_{298 \mathrm{~K}}=-154,4 \mathrm{~kJ} / \mathrm{mol}$. Die Geschwindigkeitskonstante für Kanal (a) beträgt $\mathrm{k}^{298 \mathrm{~K}}=3,89 \cdot 10^{13}$ $\mathrm{cm}^{3} /(\mathrm{mol} \cdot \mathrm{s})$, die für Kanal (b) $\mathrm{k}^{298 \mathrm{~K}}=4,86 \cdot 10^{13} \mathrm{~cm}^{3} /(\mathrm{mol} \cdot \mathrm{s})$ [FET60]. Es wurde von EdelbüttelEinhaus angenommen, dass beide Kanäle in ähnlichen Größenordnungen stattfinden. Ein Vergleich der Reaktionsenthalpien sowie der Reaktionsgeschwindigkeiten begründet die Vermutung. Es konnte aber nicht entschieden werden, ob das gefundene Ionensignal einem der beiden Radikale selektiv zuzuordnen ist.

Die Reaktion n-Butan $+\mathrm{F}$ und $\mathrm{n}$-Butan $+\mathrm{Cl}$ ist von Rohde anhand des teildeuterierten Butans $\mathrm{CD}_{3} \mathrm{CH}_{2} \mathrm{CH}_{2} \mathrm{CD}_{3}$ bei $\lambda=496 \mathrm{~nm}$ untersucht worden [ROH91]. Für die Reaktion mit F-Atomen wird ein Signalintensitätsverhältnis von (a) : (b) von 0,32 : 0,68 angegeben, für die analoge Reaktion mit Cl-Atomen von $0,06: 0,94$. Allerdings sind die Spektren sehr verrauscht, so dass Zweifel angebracht sind. Die dort unternommene Interpretation des Intensitätsverhältnisses als Kanalverzweigungsverhältnis ist unzulässig, da die spektralen Empfindlichkeiten des 1-Butylradikals und des 2-Butylradikals bei der angegebenen Wellenlänge unbekannt sind und deshalb auch völlig unberücksichtigt bleiben.

Auch Seeba hat die Reaktion n-Butan + F anhand des $\mathrm{CD}_{3} \mathrm{CH}_{2} \mathrm{CH}_{2} \mathrm{CD}_{3}$ untersucht [SEE94]. Laut seinen Angaben ist das Signal der Reaktion des n-Butans mit F-Atomen ausschließlich dem 2-Butyl zuzuordnen. Angaben über spezifische Wellenlängen sowie begründende Daten fehlen aber vollständig. Seine darauf aufbauenden kinetischen Messungen wurden bei $\lambda=450,8 \mathrm{~nm}$ und bei $\lambda=502,5$ nm durchgeführt

Durch die vorliegende Arbeit sollen die durch die vorhergehenden Arbeiten aufgeworfenen Fragen und Zweifel geklärt werden. Das Ionisierungspotenzial des 1-Butylradikals beträgt 8,02 eV, das des 
2-Butylradikals 7,25 eV [STE94]. In ähnlicher Argumentation wie bei den Propylradikalen (vgl. Abschnitt 3.1.3) ergibt sich somit die Chance, eine Grenzwellenlänge zu finden, ab der im wesentlichen nur noch das Radikal mit der geringeren Ionisierungsenergie, hier also das 2Butylradikal, signalgebend ist.

Messergebnisse: Die folgende Abbildung zeigt ein Flugzeitmassenspektrum der Reaktion $\mathrm{CD}_{3} \mathrm{CH}_{2} \mathrm{CH}_{2} \mathrm{CD}_{3}+\mathrm{F}$ bei $\lambda=450 \mathrm{~nm}$ :

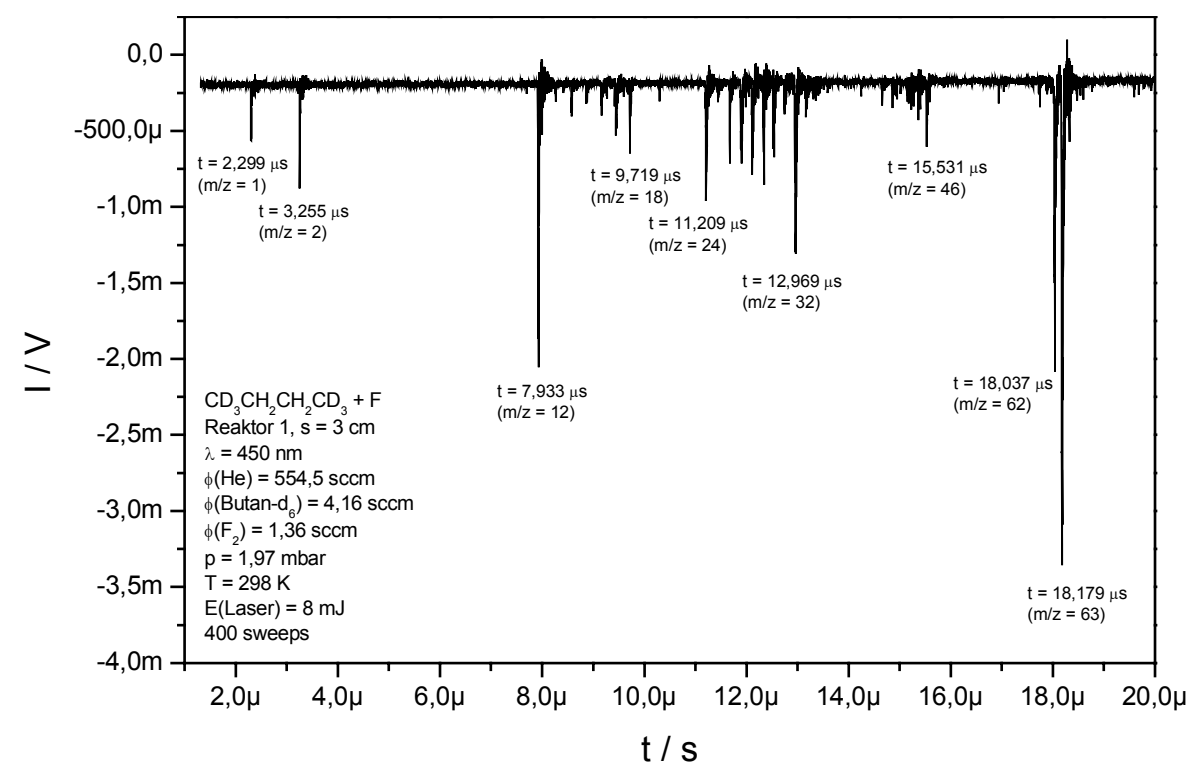

\section{Abbildung 29: Flugzeitmassenspektrum $\mathrm{CD}_{3} \mathrm{CH}_{2} \mathrm{CH}_{2} \mathrm{CD}_{3}+\mathrm{F}, \lambda=450 \mathrm{~nm}$}

Es zeigt sich ein fragmentreiches Spektrum, dessen einzelne Signale Bruchstücken des Moleküls in sinnvoller Weise zugeordnet werden können. Das Spektrum ist untergrundfrei, d.h., ohne das Vorhandensein von F-Atomen sind keine Signale zu beobachten. Zur Beantwortung der oben gestellten Frage sind aber die Signale der Radikalmuttermassen entscheidend. Die folgende Abbildung zeigt deshalb dasselbe Spektrum, aber in diesem Bereich hoch aufgelöst: 


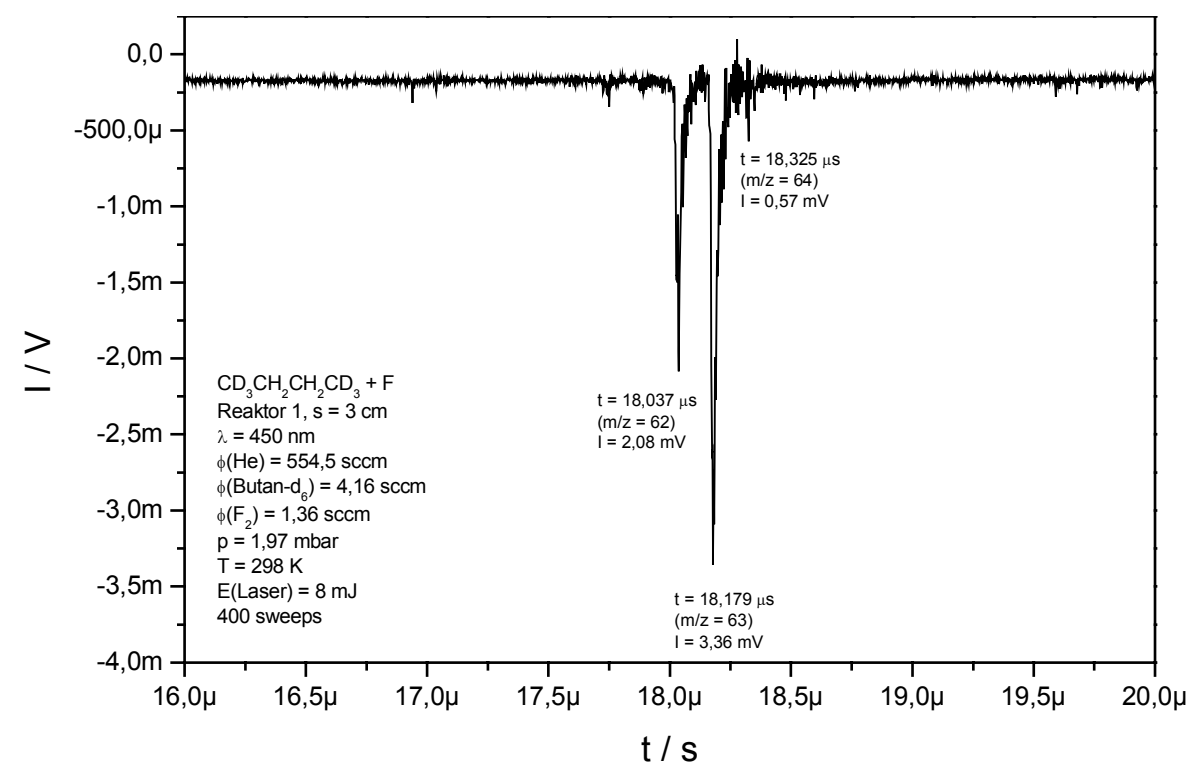

Abbildung 30: Flugzeitmassenspektrum $\mathrm{CD}_{3} \mathrm{CH}_{2} \mathrm{CH}_{2} \mathrm{CD}_{3}+\mathrm{F}, \lambda=450 \mathrm{~nm}$, aufgelöst

Deutlich lässt sich erkennen, dass sowohl das 1-Butylradikal $(\mathrm{m} / \mathrm{z}=62)$ als auch das 2-Butylradikal $(\mathrm{m} / \mathrm{z}=63)$ durch die Reaktion des $\mathrm{n}$-Butans mit F-Atomen gebildet werden und bei $\lambda=450 \mathrm{~nm}$ signalgebend sind. Bei kürzeren Wellenlängen, z.B. bei $430 \mathrm{~nm}$ und $440 \mathrm{~nm}$, sind ähnliche Verhältnisse zu beobachten. Eine wesentliche Verschiebung zu Gunsten des einen oder anderen Radikals ist bei diesen kürzeren Wellenlängen nicht festzustellen. Eine quantitative Aussage hinsichtlich des Kanalverzweigungsverhältnisses von (a) und (b) kann aber aufgrund der nicht bekannten REMPI-Empfindlichkeit des 1-Butyl- und des 2-Butylradikals mit der gewählten Methode nicht gemacht werden. Die oben angegebenen Geschwindigkeitskonstanten stützen jedoch die These, dass die Signalverhältnisse die Kanalverzweigungsverhältnisse zumindest tendenziell richtig widerspiegeln.

Die Aufnahme von Flugzeitmassenspektren bei längeren Wellenlängen ( $\lambda=460$ und $465 \mathrm{~nm})$ zeigt, dass das Signal bei $\mathrm{m} / \mathrm{z}=62 \mathrm{im}$ Vergleich zum Signal bei $\mathrm{m} / \mathrm{z}=63$ drastisch kleiner wird. Die folgende Abbildung zeigt ein im Bereich der Radikalmuttermassen aufgelöstes Spektrum bei $\lambda=475 \mathrm{~nm}:$ 


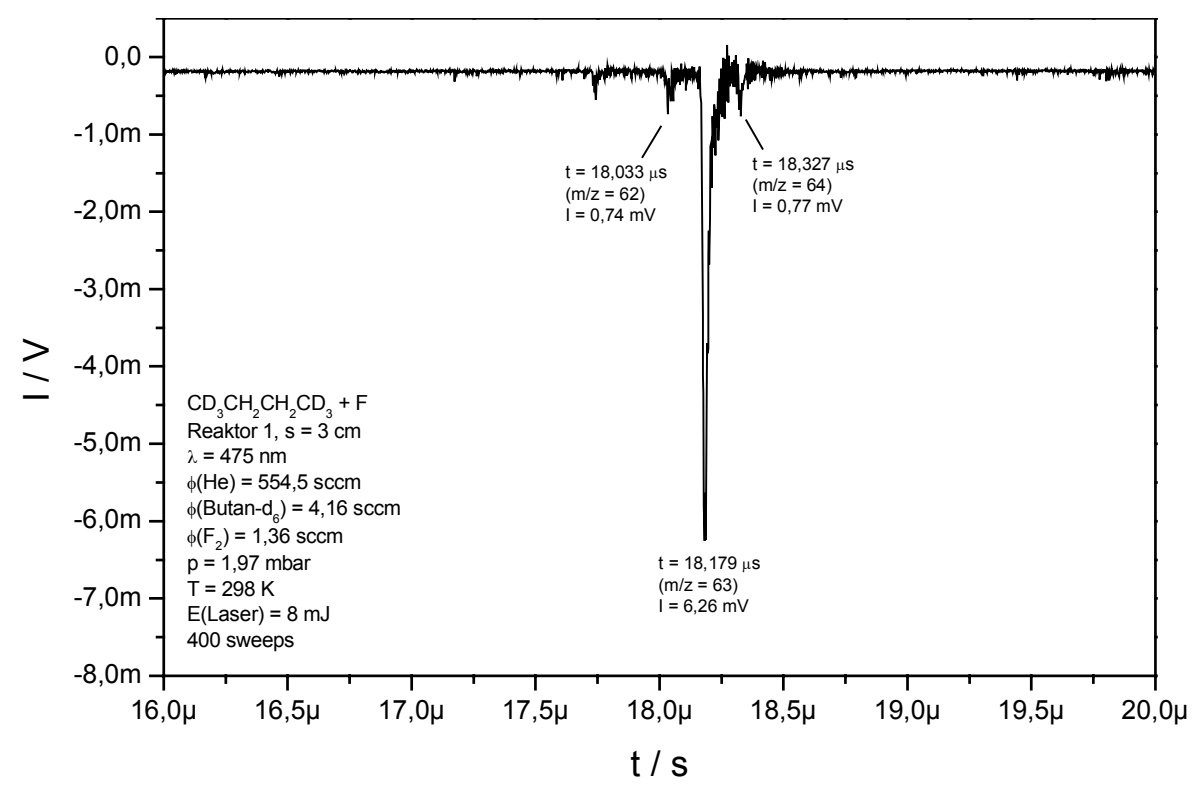

Abbildung 31: Flugzeitmassenspektrum $\mathrm{CD}_{3} \mathrm{CH}_{2} \mathrm{CH}_{2} \mathrm{CD}_{3}+\mathrm{F}, \lambda=475 \mathrm{~nm}$, aufgelöst

Das Signal des 1-Butylradikals $(\mathrm{m} / \mathrm{z}=62)$ ist kaum noch vorhanden. Es bewegt sich in der Größenordnung des ${ }^{13} \mathrm{C}$-Isotopenpeaks vom 2-Butylradikal $(\mathrm{m} / \mathrm{z}=64)$. Das Signal des 2-Butylradikals $(\mathrm{m} / \mathrm{z}=63)$ ist dagegen immer noch signifikant.

Interpretation: Da schon bei $\lambda=465 \mathrm{~nm}$ (Spektrum hier nicht dargestellt) das Signal des 1-Butylradikals kaum relevant ist, soll diese Wellenlänge in akzeptabler Näherung als die Grenzwellenlänge bezeichnet werden, ab der nur noch das 2-Butylradikal signalgebend ist.

Das verwendete n-Butan ist am Molekülrand deuteriert, im Gegensatz zum im Abschnitt 3.1.3 dargestellten Propan, welches mittelständig deuteriert ist. Da in beiden Fällen aber ab eines fest umrissenen Bereichs nur das 2-Alkylradikal mit einem geringeren Ionisierungspotenzial als das 1-Alkylradikal ein Signal hervorruft, kann ein Isotopeneffekt sowohl hinsichtlich der Radikalbildungsreaktion als auch hinsichtlich des Ionisierungsprozesses kaum maßgebend sein.

Diskussion: Die von Seeba durchgeführten kinetischen Messungen bei $\lambda=450,8 \mathrm{~nm}$, welche unter der Annahme der eindeutigen Beziehung zwischen beobachtetem Signal und dem 2-Butylradikal stattgefunden haben, sind unter der Berücksichtigung der hier durchgeführten Messungen als methodisch falsch anzusehen. Inwieweit sich hieraus Fehler für die Ergebnisse hinsichtlich der gemessenen Geschwindigkeitskonstante ergeben, hängt von dem Grad des unterschiedlichen Reaktionsverhalten des 1-Butyl- und des 2-Butylradikals ab. Soweit eigene kinetische Messungen erfolgt sind, wird das an entsprechender Stelle diskutiert. 
Messergebnisse: In folgender Abbildung ist das vollständige Flugzeitmassenspektrum bei $\lambda=475 \mathrm{~nm}$ aufgeführt:

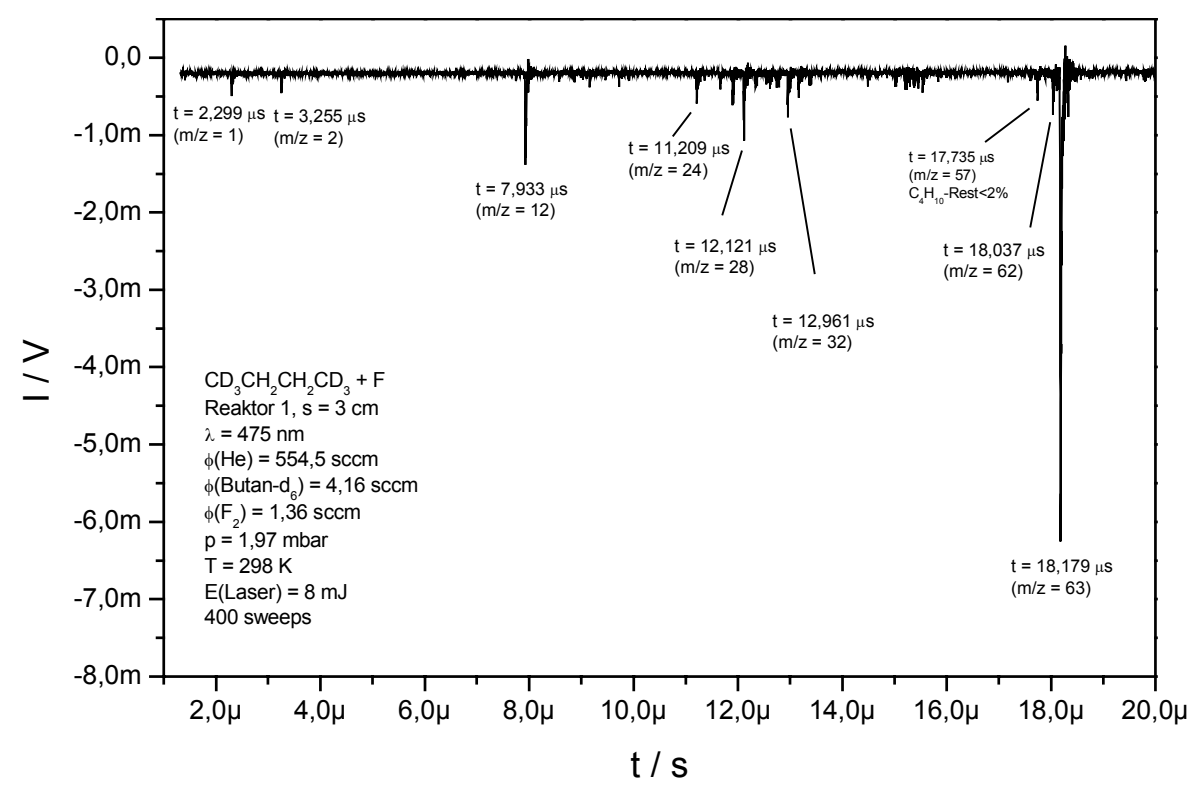

\section{Abbildung 32: Flugzeitmassenspektrum $\mathrm{CD}_{3} \mathrm{CH}_{2} \mathrm{CH}_{2} \mathrm{CD}_{3}+\mathrm{F}, \lambda=475 \mathrm{~nm}$}

Es ist im Vergleich zur Abbildung 29 festzustellen, dass der Fragmentierungsanteil bei längerwelligem Licht zur Ionisierung deutlich abnimmt, was dem üblichen Verhalten von Molekülen in REMPI-Ionenquellen entspricht.

\subsubsection{Das tertiär-Butylradikal, das Isobutylradikal}

Isobutan wird in Druckgasflaschen als Flüssiggas in Laboren und Haushalten zu Brenn- und Heizzwecken verwendet. Vielfach wird Isobutan - im allgemeinen zusammen mit Propan und n-Butan - anstelle von Fluorchlorkohlenwasserstoffen als Treibmittel in Sprays eingesetzt [FAL91]. Die aus dem n-Butan durch Wasserstoffabstraktion hervorgehenden t-Butyl- und Isobutylradikale tauchen somit bei wirtschaftlich bedeutungsvollen Verbrennungsvorgängen und bei Abbauprozessen in der Atmosphäre auf.

Diskussion: Die Reaktion von Isobutan mit F-Atomen wurde von Edelbüttel-Einhaus [EDE88] mittels REMPI-Ionisation im Wellenlängenbereich 397-415 nm untersucht. Es wurde ein Signal der Masse $\mathrm{m} / \mathrm{z}=57$ gefunden, welches dem $\mathrm{C}_{4} \mathrm{H}_{9}$-Radikal zugeordnet wurde. Wegen der Massengleichheit konnte nicht entschieden werden, ob es sich um das t-Butylradikal, um das Isobutylradikal oder eine Mischung aus beiden handelt. Im Rahmen dieser Arbeit wurde diese Fragestellung neu aufgenommen. Durch den Einsatz teildeuterierten Isobutans kann die Frage in eindeutiger Weise beantwortet werden. Das soll im folgenden erläutert werden. 
Die Reaktion von Isobutan mit F-Atomen resultiert in zwei Kanäle:<smiles>CC(C)C(C)C(C)(C)C(C)(C)C</smiles>

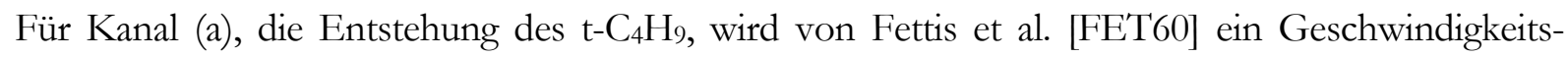
koeffizient von $\mathrm{k}^{298 \mathrm{~K}}=5,75 \cdot 10^{13} \mathrm{~cm}^{3} /(\mathrm{mol} \cdot \mathrm{s})$ angegeben, für Kanal (b), die Entstehung des iso- $\mathrm{C}_{4} \mathrm{H}_{9}, \mathrm{k}^{298 \mathrm{~K}}=4,07 \cdot 10^{13} \mathrm{~cm}^{3} /(\mathrm{mol} \cdot \mathrm{s})$. Demnach entstehen beide Radikale in ähnlichen Größenordnungen, das t-Butylradikal müsste leicht im Überschuss vorliegen.

Messergebnisse: Mit der Versuchsanordnung A (REMPI) wurde ein Flugzeitmassenspektrum bei $\lambda=500 \mathrm{~nm}$ aufgenommen:

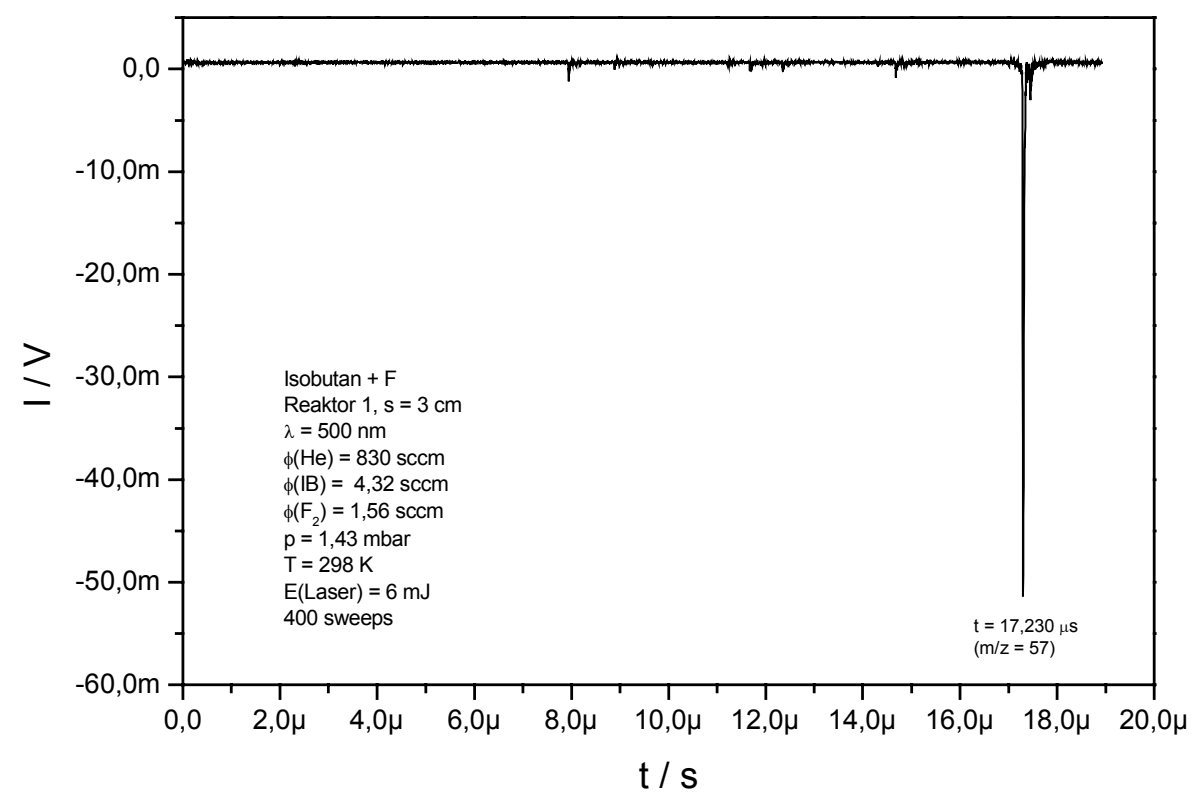

\section{Abbildung 33: Flugzeitmassenspektrum Isobutan $+F, \lambda=500 \mathrm{~nm}$}

Ein Untergrund durch Isobutan wird bei Wellenlängen $\lambda>420 \mathrm{~nm}$ nicht beobachtet, was in Anbetracht eines Ionisierungspotenzials von 10,57 eV [STE94] nicht überrascht. Eine Kalibrierung hat ergeben, dass das Signal einer Masse von $\mathrm{m} / \mathrm{z}=57$ zuzuordnen ist. Das Signal ist von 
$\lambda=370-580 \mathrm{~nm}$ existent, seine Intensität folgt im wesentlichen der Laserenergie. Die nächste Abbildung zeigt dasselbe Spektrum im Bereich der Masse $\mathrm{m} / \mathrm{z}=57$, höher aufgelöst:

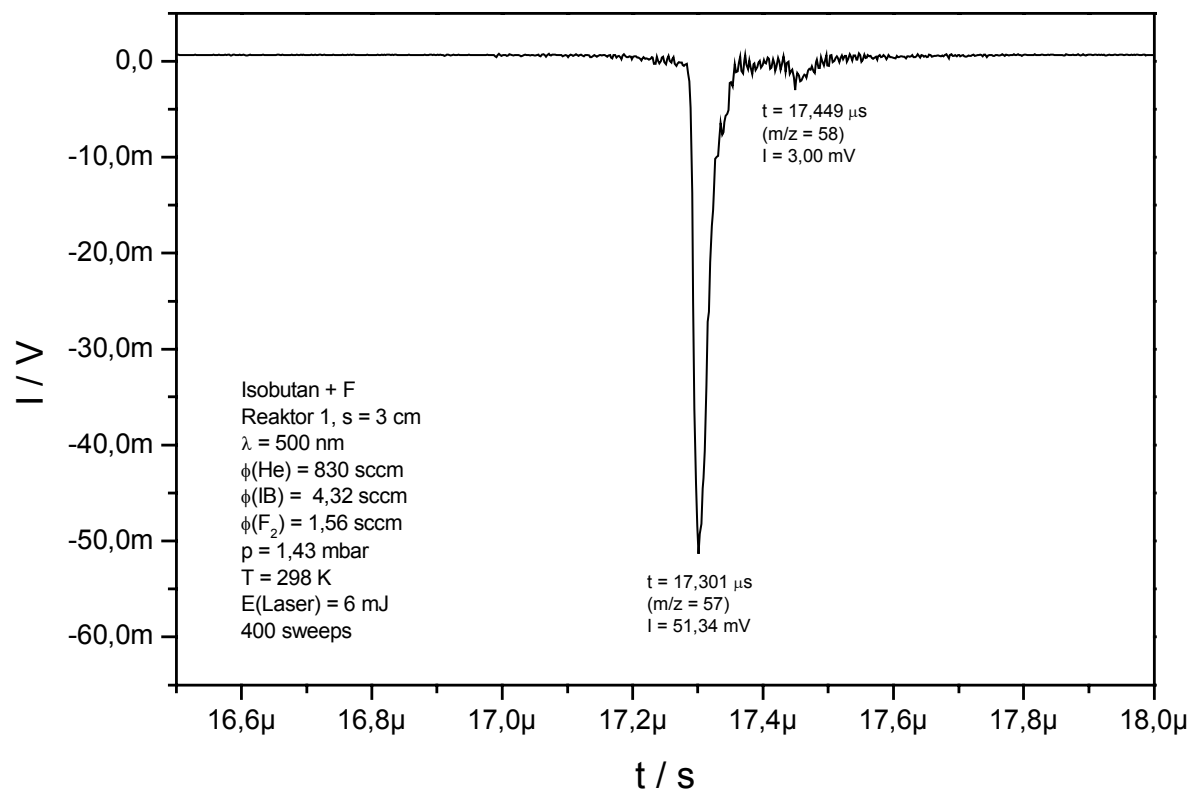

\section{Abbildung 34: Flugzeitmassenspektrum Isobutan $+F, \lambda=500 \mathrm{~nm}$, aufgelöst}

Bei $\mathrm{m} / \mathrm{z}=58$ ist ein Isotopenpeak resultierend aus im Radikal enthaltenes ${ }^{13} \mathrm{C}$ zu beobachten ${ }^{21}$.

Interpretation: Das t-Butylradikal hat ein Ionisierungspotenzial IP $=6,70 \mathrm{eV}$, das Isobutylradikal hat $\mathrm{IP}=7,93 \mathrm{eV}$ [STE94], so dass eine Ionisierung des t-Butylradikals begünstigt erscheint. Setzt man 2-Methyl-Propan-2- $d$ statt Isobutan als Radikalvorläufersubstanz ein, so unterscheiden sich die beiden entstehenden Radikals um $\Delta(\mathrm{m} / \mathrm{z})=1$ :

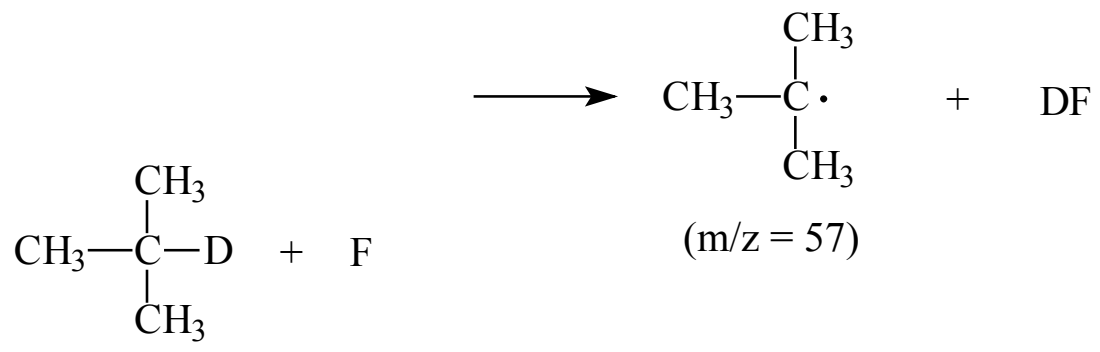<smiles>[2H]C(C)(C)CC</smiles>

${ }^{21}$ Gemäß der natürlichen Zusammensetzung besitzen ca. 3-4\% der t-Butylradikale ein ${ }^{13} \mathrm{C}$-Atom. 
Messergebnisse: Es wurden Flugzeitmassenspektren von $\mathrm{t}-\mathrm{C}_{4} \mathrm{H}_{9} \mathrm{D}+\mathrm{F}$ bei verschiedenen Wellenlängen aufgenommen. Die folgenden Abbildungen geben das Spektrum bei $\lambda=430 \mathrm{~nm}$ wieder, einmal im ganzen und einmal aufgelöst im für den Nachweis interessanten Massenbereich:

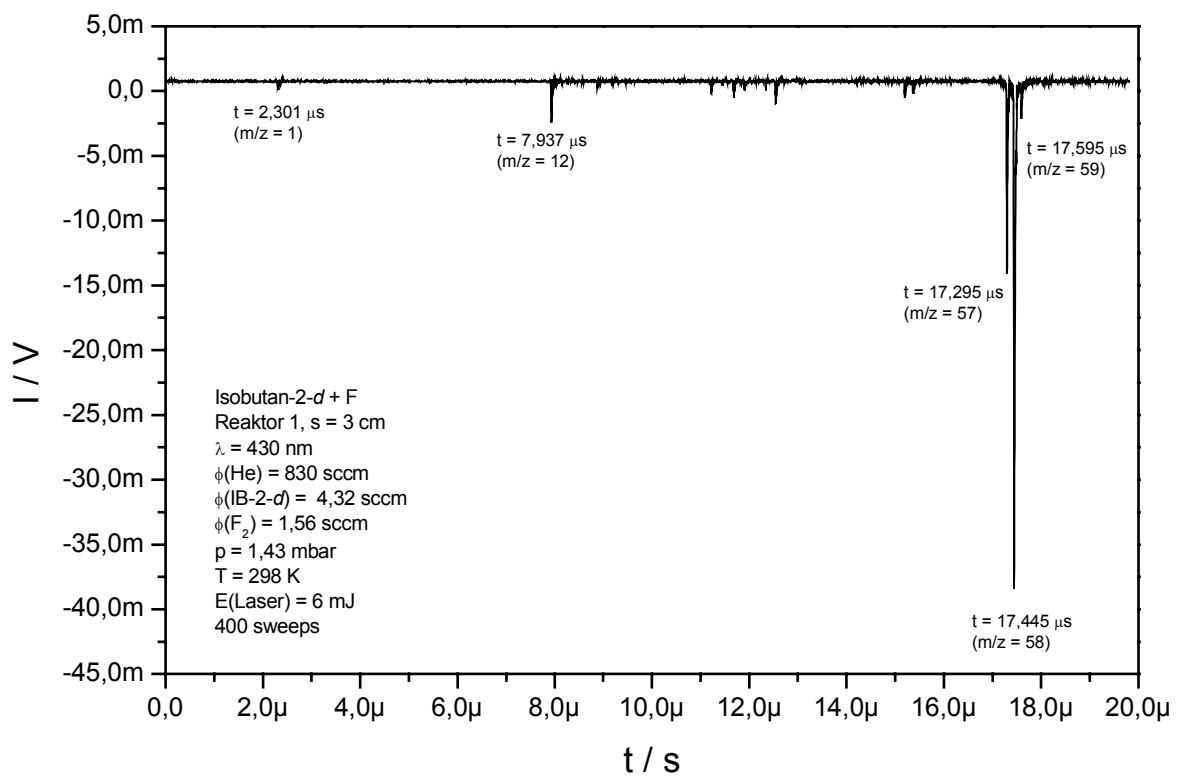

Abbildung 35: Flugzeitmassenspektrum 2-Methylpropan-2-d $+F, \lambda=430 \mathrm{~nm}$

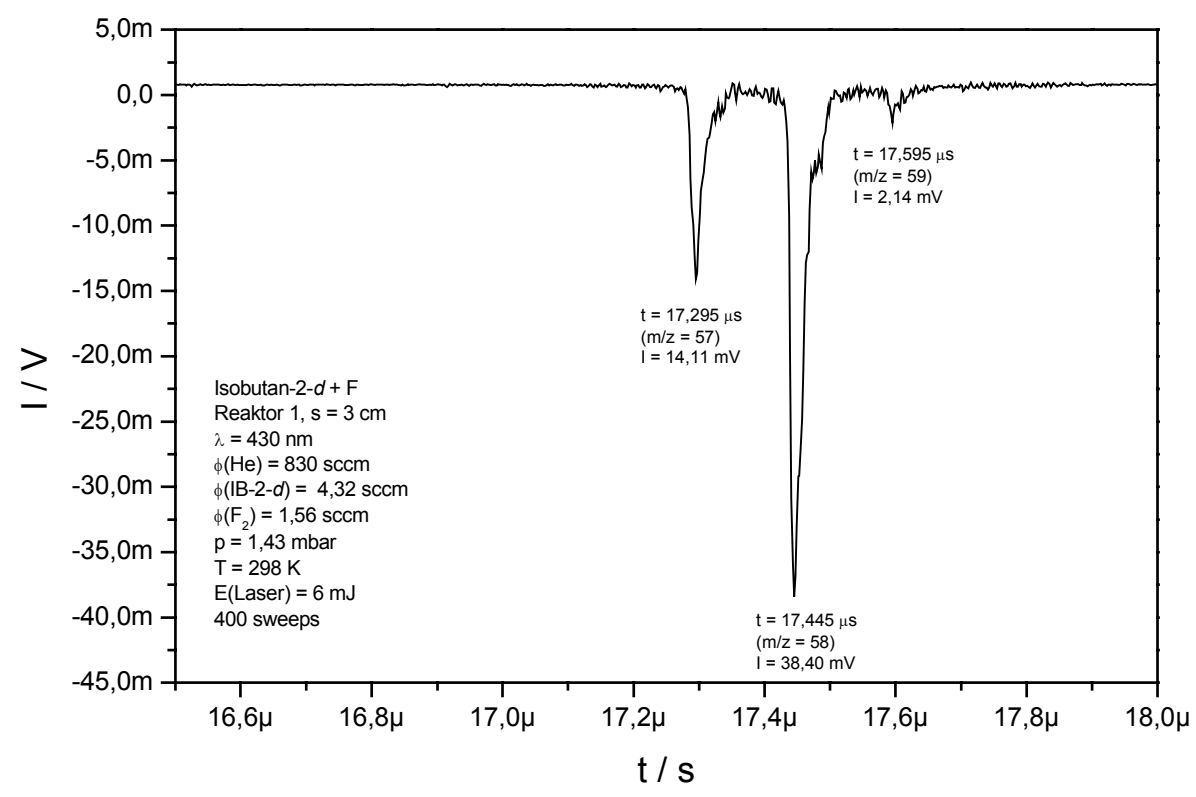

Abbildung 36: Flugzeitmassenspektrum 2-Methylpropan-2- $d+F, \lambda=430 \mathrm{~nm}$, aufgelöst

Interpretation: Beide Radikale sind zu erkennen. Das Signal der Reaktion des nicht deuterierten Isobutans mit F-Atomen ist bei dieser Wellenlänge demnach eine Mischung aus dem t-Butyl- und dem Isobutylradikal. Die Signalstärke des Isobutylradikals ist im Vergleich zu der des t-Butylradikals mindestens um den Faktor 2 größer. Hieraus darf jedoch nicht leichtfertig auf ein entsprechendes 
Kanalverzweigungsverhältnis zu Gunsten Reaktionskanals (b') geschlossen werden, da die spektralen Empfindlichkeiten der entstehenden Radikale nicht bekannt sind.

Messergebnisse: Bei $500 \mathrm{~nm}$ stellt sich das Spektrum wie folgt dar:

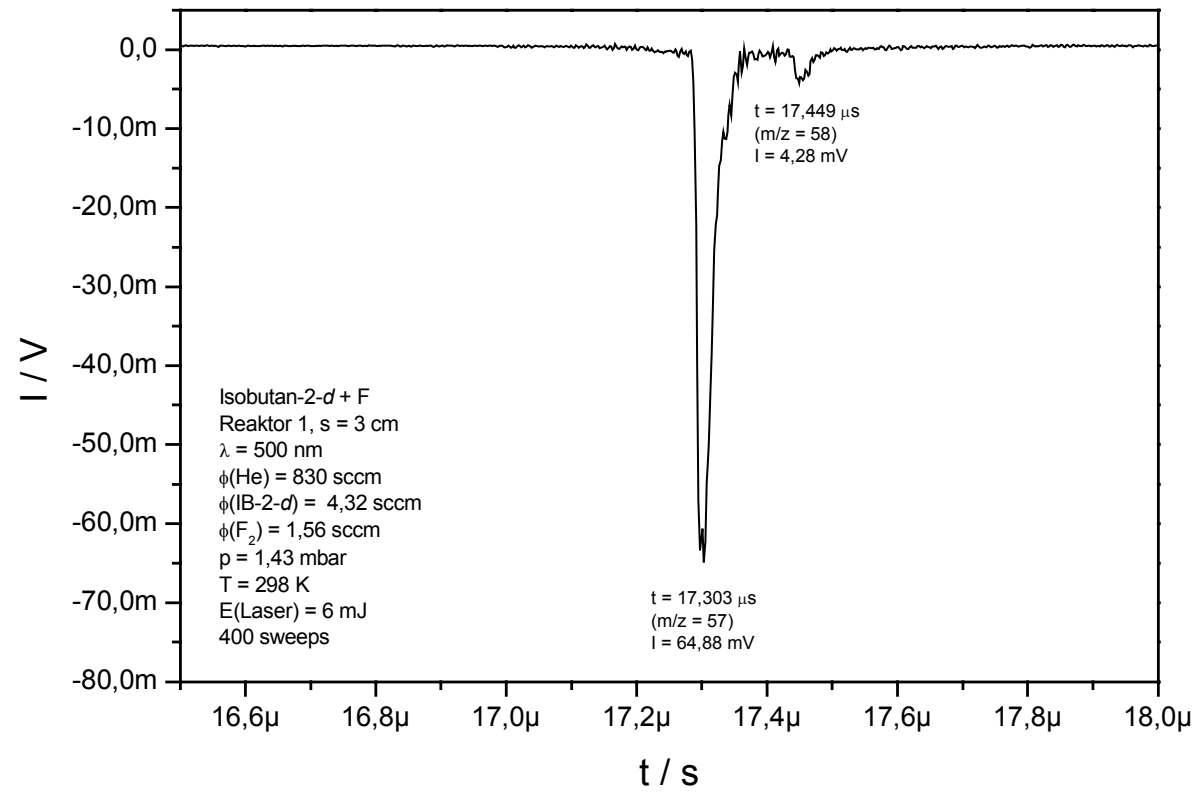

Abbildung 37: Flugzeitmassenspektrum 2-Methylpropan-2-d $+\mathrm{F}, \lambda=500 \mathrm{~nm}$, aufgelöst

Interpretation: Ein Vergleich zu Abbildung 34 zeigt, dass bei dieser Wellenlänge keine Isobutylradikale mehr ionisiert werden.

Messergebnisse: Die folgenden Abbildungen zeigen entsprechende Spektren bei $\lambda=370 \mathrm{~nm}, 400 \mathrm{~nm}$, $410 \mathrm{~nm}, 440 \mathrm{~nm}, 456 \mathrm{~nm}, 470 \mathrm{~nm}, 490 \mathrm{~nm}$ und $520 \mathrm{~nm}$.

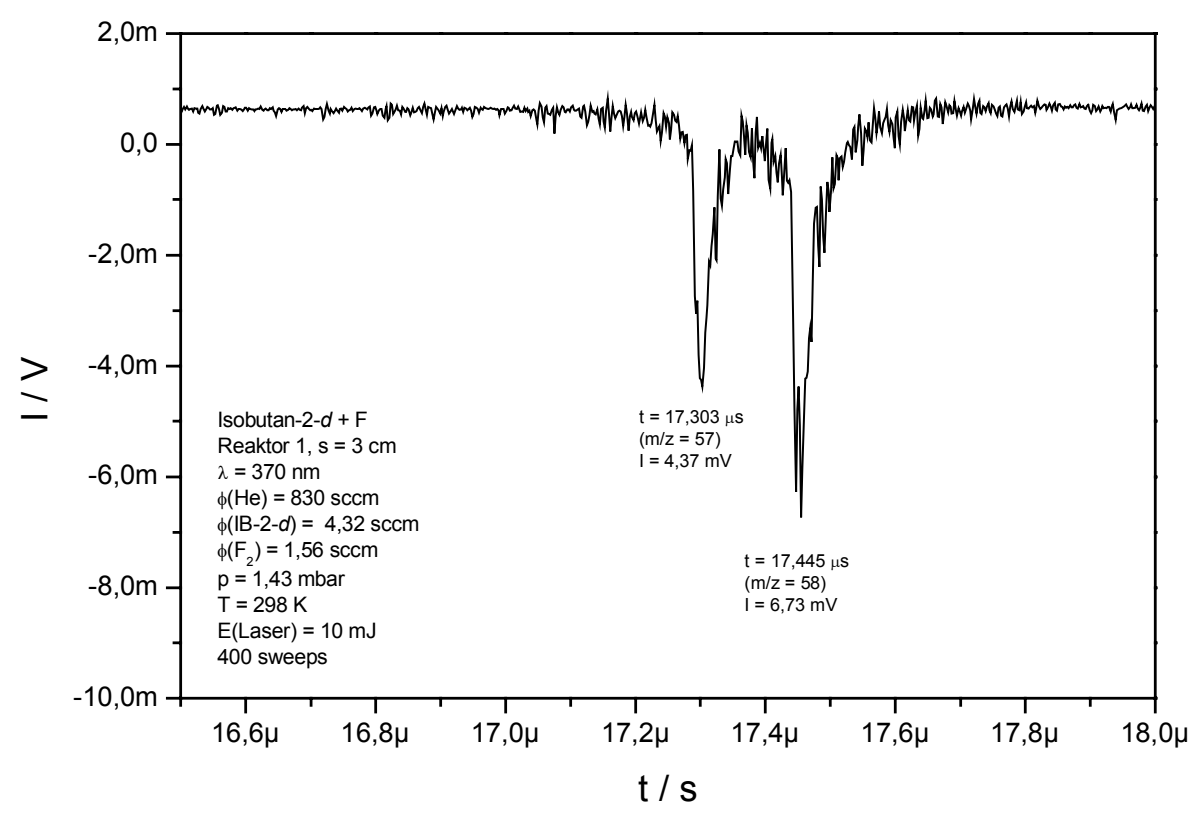

Abbildung 38: Flugzeitmassenspektrum 2-Methylpropan-2- $d+\mathrm{F}, \lambda=370 \mathrm{~nm}$, aufgelöst 


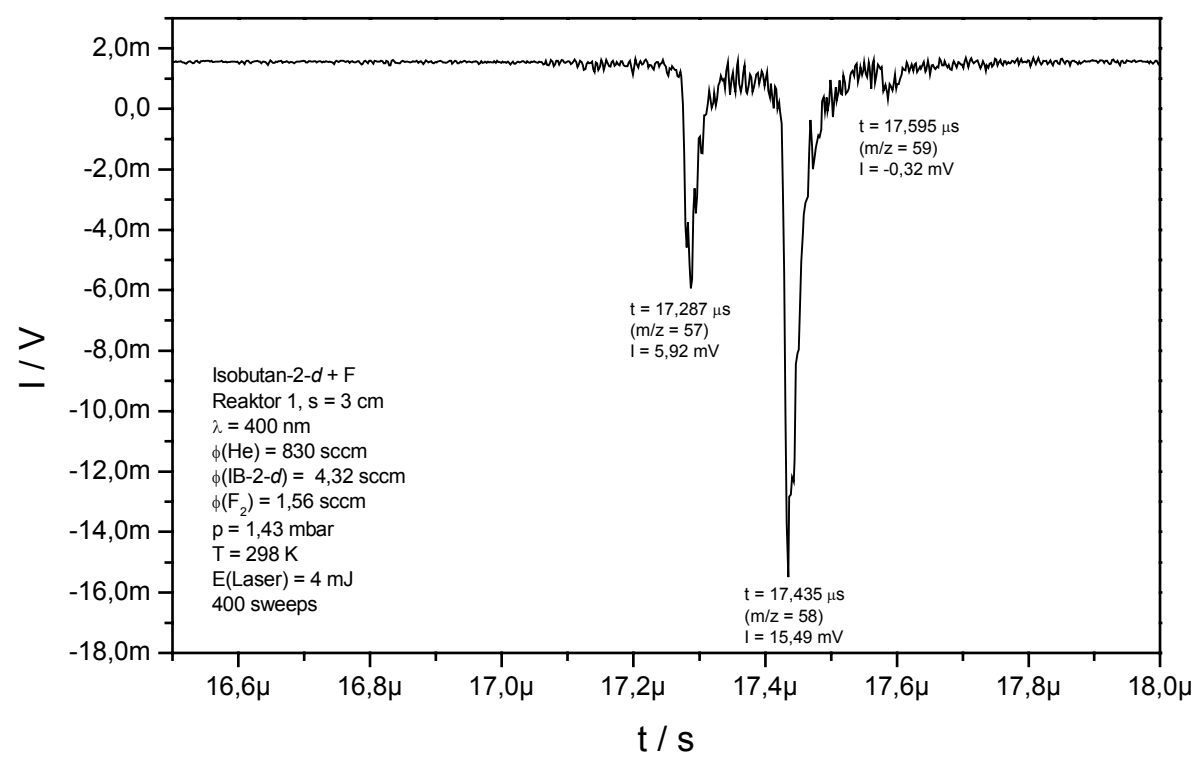

Abbildung 39: Flugzeitmassenspektrum 2-Methylpropan-2-d $+F, \lambda=400 \mathrm{~nm}$, aufgelöst

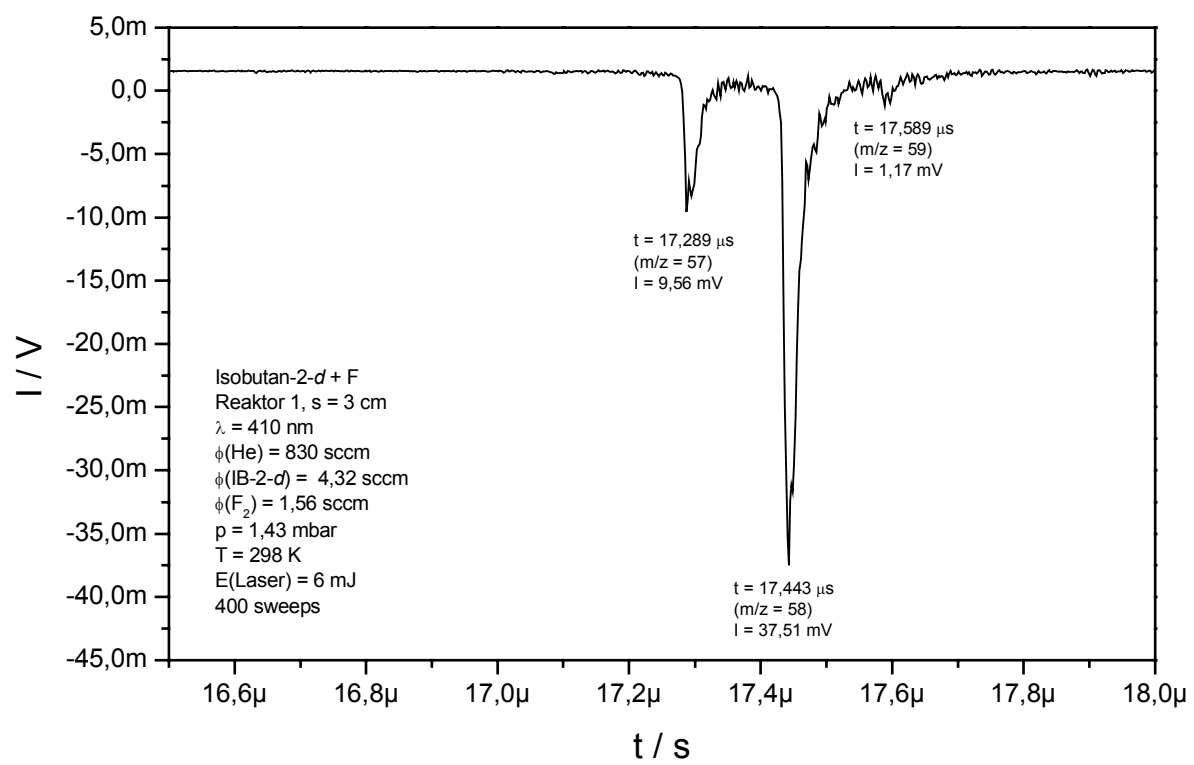

Abbildung 40: Flugzeitmassenspektrum 2-Methylpropan-2- $d+F, \lambda=410 \mathrm{~nm}$, aufgelöst 


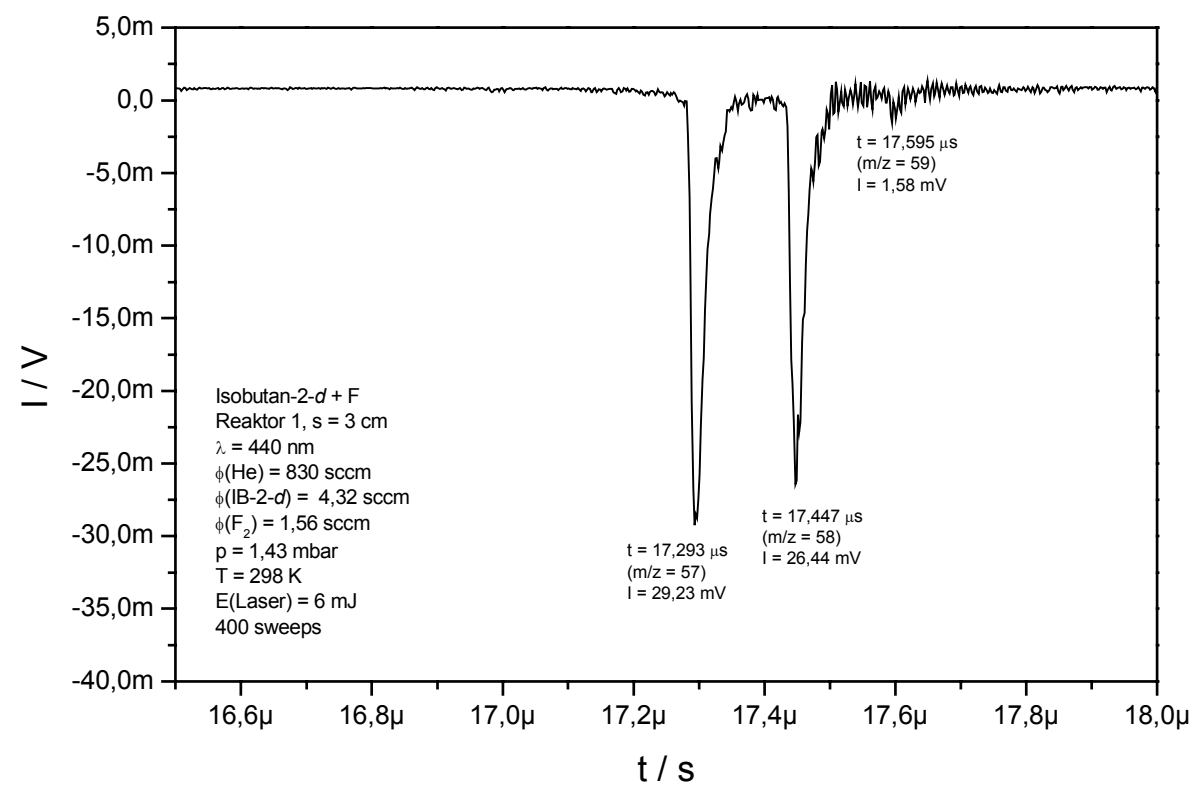

Abbildung 41: Flugzeitmassenspektrum 2-Methylpropan-2-d $+F, \lambda=440 \mathrm{~nm}$, aufgelöst

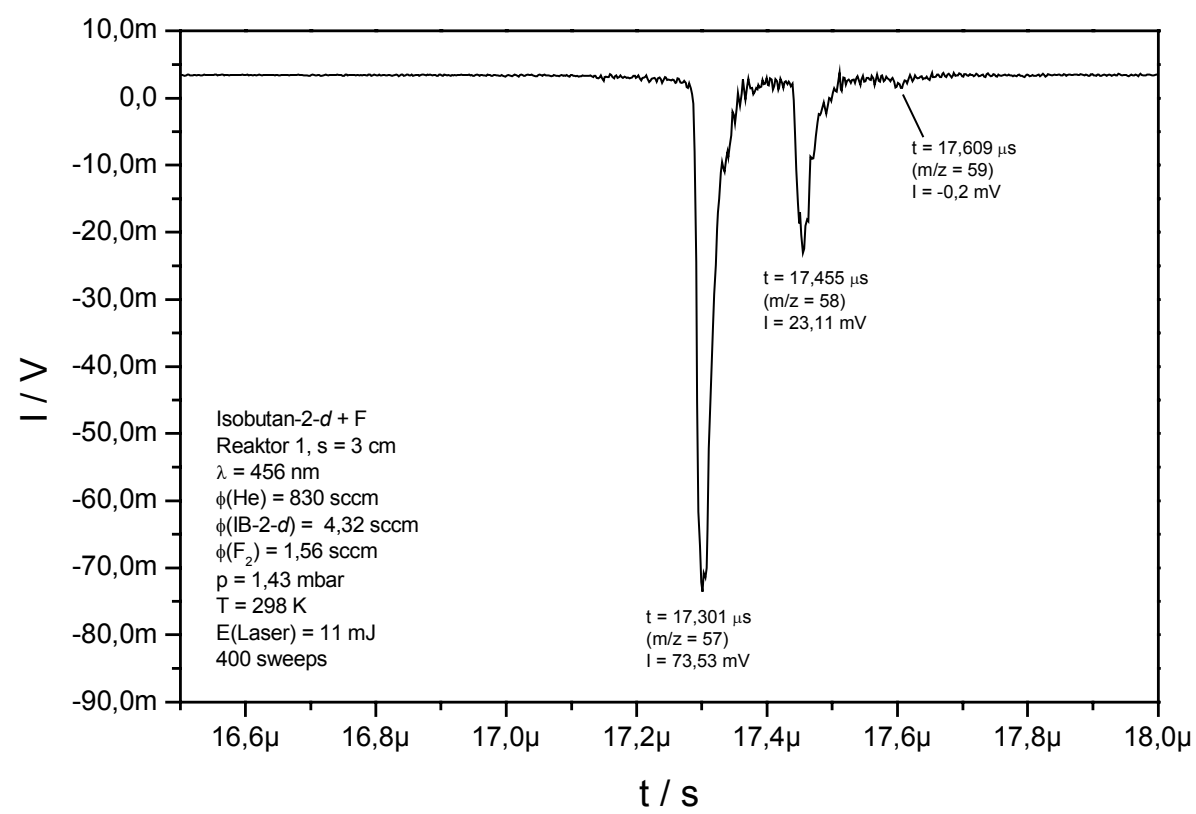

Abbildung 42: Flugzeitmassenspektrum 2-Methylpropan-2- $d+F, \lambda=456 \mathrm{~nm}$, aufgelöst 


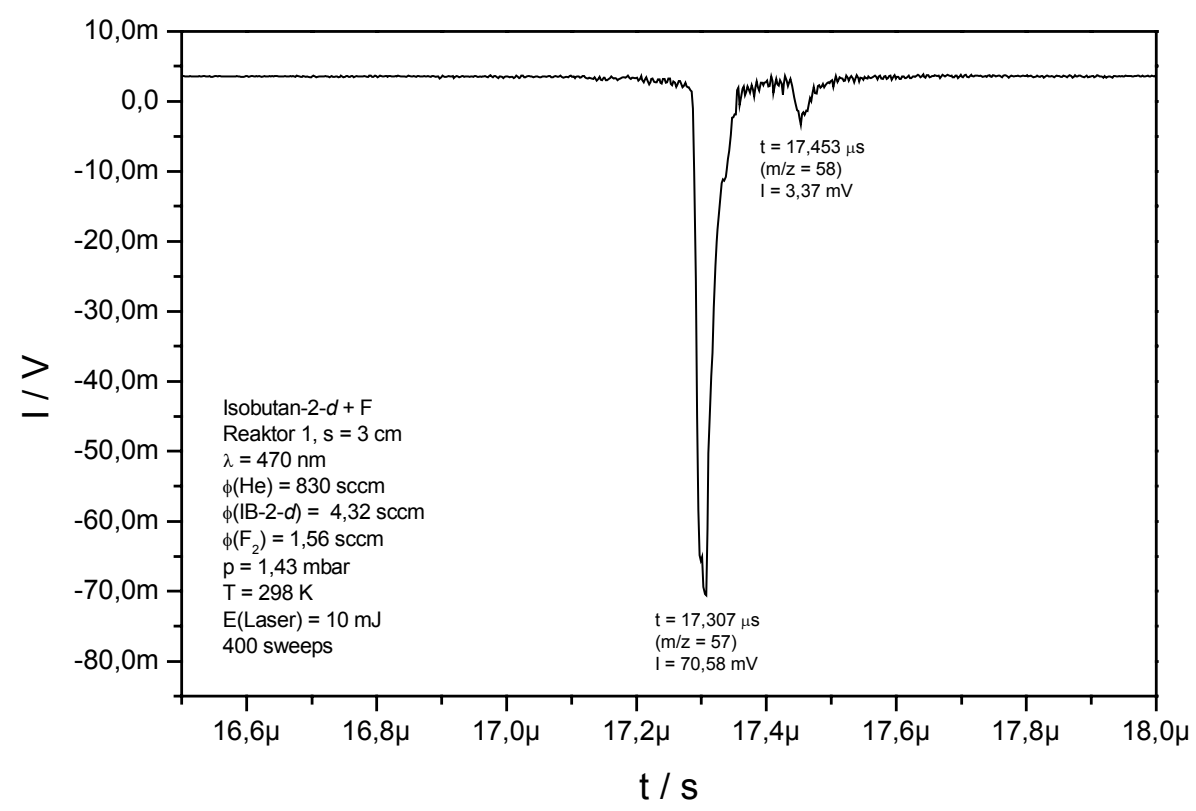

Abbildung 43: Flugzeitmassenspektrum 2-Methylpropan-2- $d+F, \lambda=470 \mathrm{~nm}$, aufgelöst

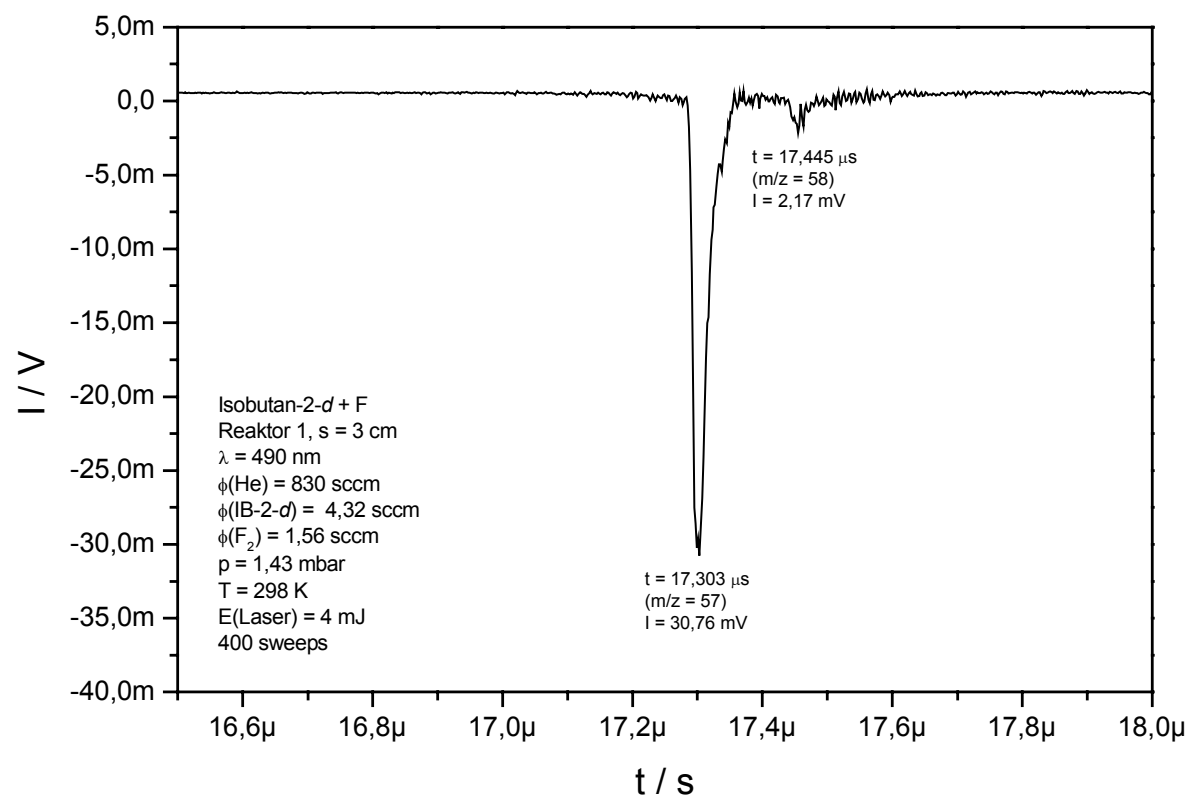

Abbildung 44: Flugzeitmassenspektrum 2-Methylpropan-2-d $+\mathrm{F}, \lambda=490 \mathrm{~nm}$, aufgelöst 


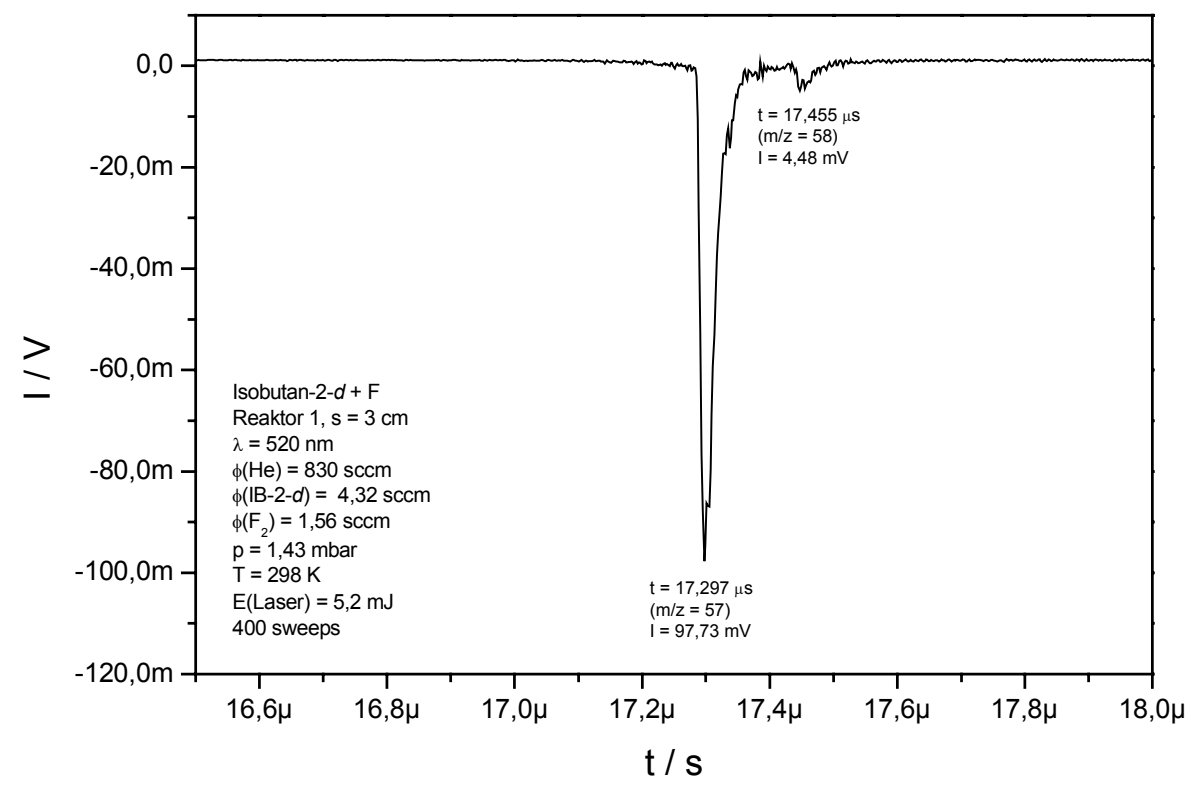

Abbildung 45: Flugzeitmassenspektrum 2-Methylpropan-2- $d+\mathrm{F}, \lambda=520 \mathrm{~nm}$, aufgelöst

Interpretation: Der Vergleich der Spektren bei den verschiedenen Wellenlängen zeigt, dass das Verschwinden des Isobutylradikalsignals mit wachsender Wellenlänge kein absolut diskontinuierlicher Prozess ist, aber bei $470 \mathrm{~nm}$ ist das Signal bei $\mathrm{m} / \mathrm{z}=58$ schon so gering, dass man von einer Schwelle sprechen kann. Jenseits dieser Wellenlänge ist faktisch nur noch das t-Butylradikal signalgebend. Die kinetischen Messungen mit dem $\mathrm{t}-\mathrm{C}_{4} \mathrm{H}_{9}$ wurden im Bereich $\lambda=495-510 \mathrm{~nm}$ durchgeführt.

Diskussion: Dem Ionisierungspotenzial des Isobutylradikals entspricht in einem Einphotonenionisierungsvorgang die Wellenlänge $158 \mathrm{~nm}$, dem des t-Butylradikals $187 \mathrm{~nm}$. Ein DreiphotonenProzess vorausgesetzt wäre die minimale Wellenlänge zur Ionisierung beim iso- $\mathrm{C}_{4} \mathrm{H}_{9} \lambda=474 \mathrm{~nm}$, beim $\mathrm{t}_{-} \mathrm{C}_{4} \mathrm{H}_{9} \lambda=561 \mathrm{~nm}$. Das entspricht den Beobachtungen. Es kann somit von einem 3-Photonen-REMPI-Prozess ausgegangen werden. Ein UV-Spektrum des t-Butylradikals wurde von Wendt und Hunziker aufgenommen [WEN84]. Es zeigen sich recht breite Absorptionsbanden zwischen 200 und $370 \mathrm{~nm}$. Solch breite Banden von Alkylradikalen wurden des öfteren so interpretiert, dass sie Valenzübergänge repräsentieren. Die lokalen Maxima liegen bei $\lambda=333 \mathrm{~nm}$, $253 \mathrm{~nm}$ und $233 \mathrm{~nm}$. Die beiden Autoren interpretieren die Banden als 3s, 3p und 3d Rydbergübergänge. Sie stützen ihre These auf ab initio Rechnungen von Lengsfield et al., welche die Absorptionsmuster annähernd vorausgesagt haben und eine Valenzanregung erst für viel höhere Energien zulassen [LEN84]. Der Rydbergcharakter der beobachteten Banden wird ferner dadurch bestätigt, dass die Rydbergformel (vgl. Abschn. 2.1.4 Mehr-Photonen-Ionisation) mit den gemessenen Absorptionsmaxima vernünftige Werte für die Quantendefekte liefert. Diese Rydberg 
zustände vorausgesetzt, ergibt sich also ein 2+1 REMPI-Prozess. Durch die sehr breiten Absorptionsbanden erklärt sich aber, warum der Ionisationsprozess wenig wellenlängenselektiv ist.

Für das Isobutylradikal liegt kein UV-Absorptionsspektrum vor, so dass eine derartige Argumentation nicht möglich ist. Weil dass Isobutyl ein primäres Radikal ist, dürften entsprechende Rydbergzustände dem Trend einer höheren Ionisierungsenergie folgend jedoch weiter im kurzwelligen Bereich liegen [WEN84].

Messergebnisse: Bei kürzeren Ionisierungswellenlängen weisen beide Radikale ein reiches Fragmentierungsmuster auf. Um dieses zu verdeutlichen, seien noch einmal die vollständigen Flugzeitmassenspektren der Reaktion $\mathrm{C}_{4} \mathrm{H}_{9} \mathrm{H}+\mathrm{F}$ und $\mathrm{C}_{4} \mathrm{H}_{9} \mathrm{D}+\mathrm{F}$ bei $\lambda=370 \mathrm{~nm}$ dargestellt. Da bei dieser Wellenlänge bereits das Isobutan selbst Signale zeigt, ist dieser Hintergrund bei $\mathrm{C}_{4} \mathrm{H}_{9} \mathrm{H}+\mathrm{F}$ farblich unterlegt.

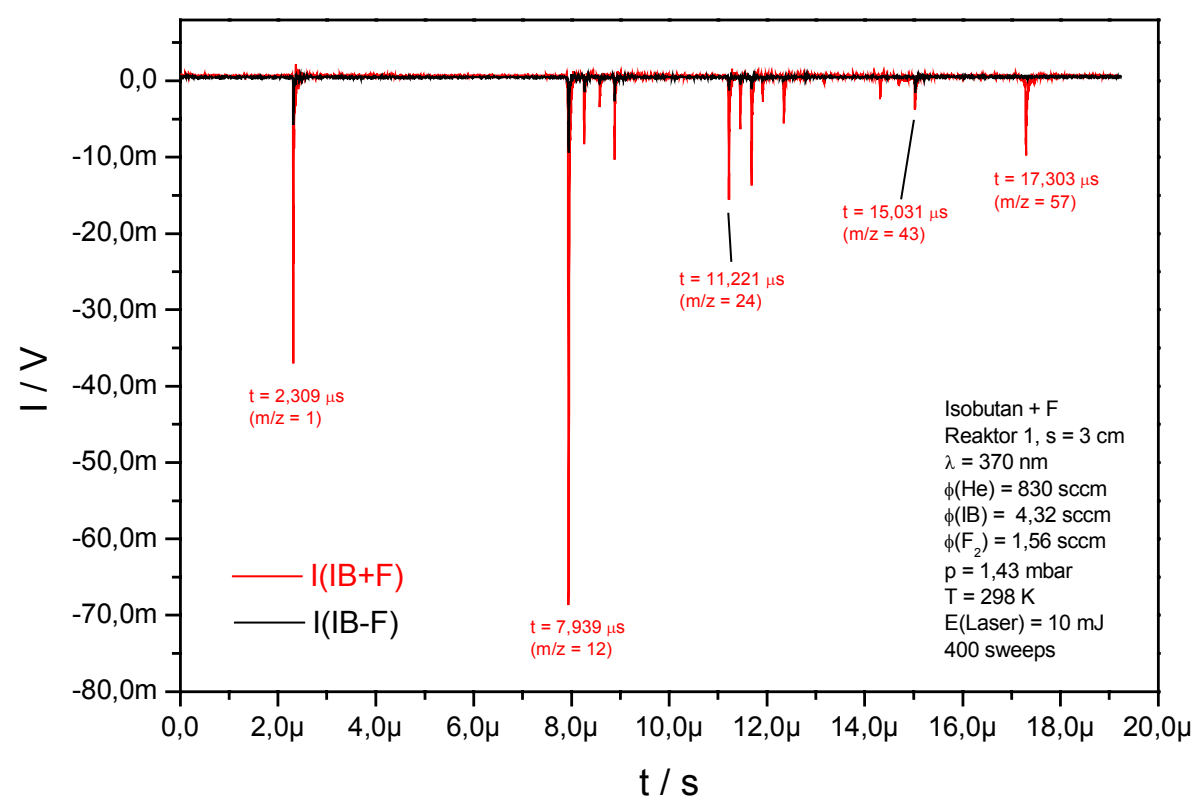

Abbildung 46: Flugzeitmassenspektrum Isobutan $+F, \lambda=370 \mathrm{~nm}$ 


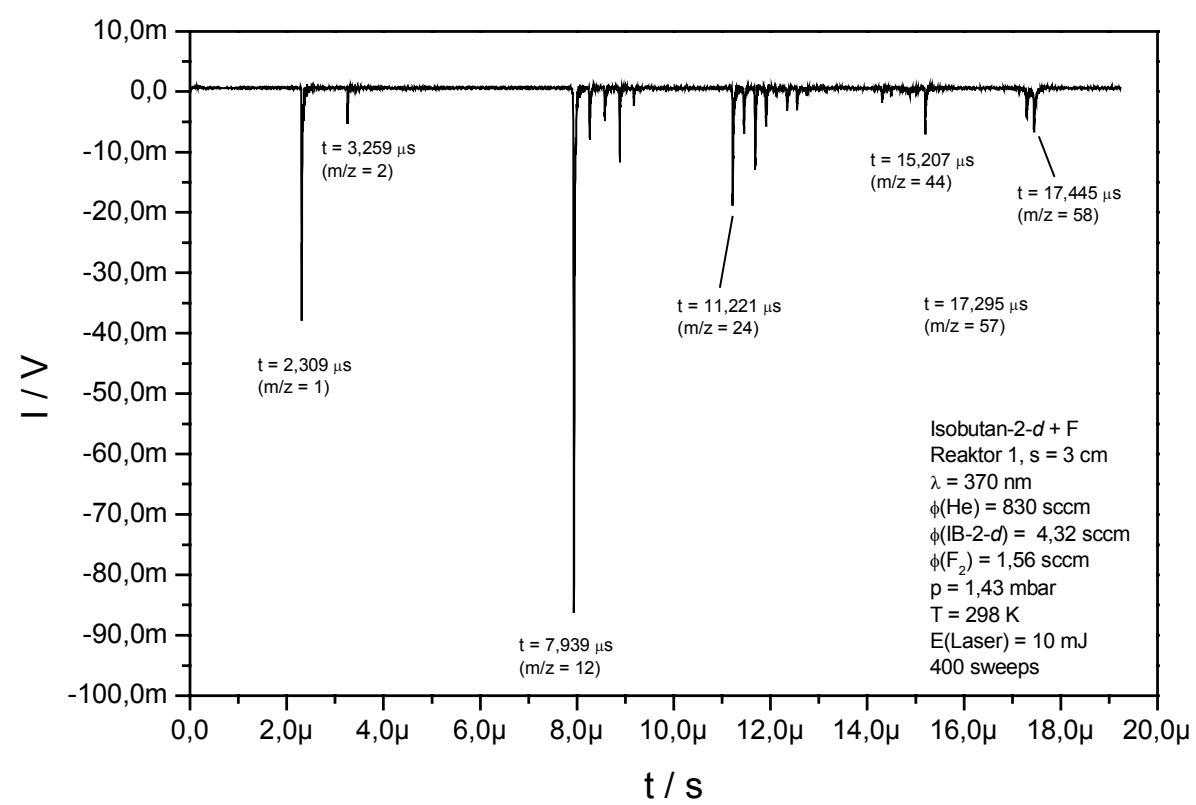

Abbildung 47: Flugzeitmassenspektrum 2-Methylpropan-2- $d+F, \lambda=370 \mathrm{~nm}$

\subsubsection{Das Cyclopentylradikal}

Cyclopentan, Cyclohexan und Cycloheptan sind Bestandteil von Erdöl [FAL91]. Diese Stoffe und die aus ihnen durch H-Atom-Abstraktion abgeleiteten Radikale sind somit an wirtschaftlich bedeutungsvollen Verbrennungsvorgängen beteiligt.

Messergebnisse: c-Pentylradikale wurden in der vorliegenden Arbeit über die Reaktion

$$
\begin{aligned}
& \mathrm{c}-\mathrm{C}_{5} \mathrm{H}_{10}+\mathrm{F} \rightarrow \mathrm{c}^{-} \mathrm{C}_{5} \mathrm{H}_{9}+\mathrm{HF}, \\
& \mathrm{k}^{298 \mathrm{~K}}=1,7 \cdot 10^{14} \mathrm{~cm}^{3} \cdot \mathrm{mol}^{-1} \cdot \mathrm{s}^{-1}[\text { BAR85] }
\end{aligned}
$$

erzeugt. Die folgende Abbildung zeigt ein Flugzeitmassenspektrum bei $\lambda=433 \mathrm{~nm}$. 


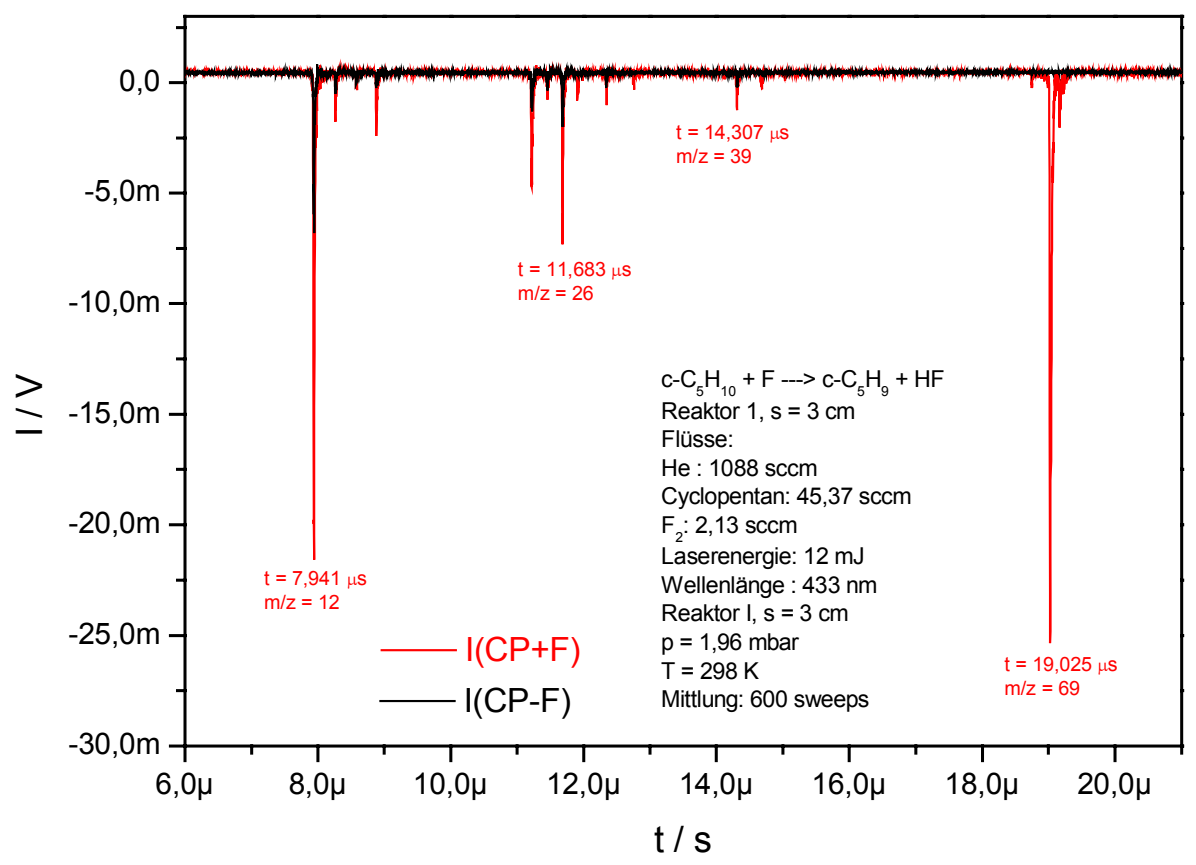

\section{Abbildung 48: Flugzeitmassenspektrum c-Pentan $+F, \lambda=433 \mathrm{~nm}$}

Das Radikal ist in eindeutiger Weise auf seiner Molekülmasse nachzuweisen. Es finden sich beachtliche Fragmente bei $\mathrm{m} / \mathrm{z}=26,24$ und 12, welche für cyclische Kohlenwasserstoffradikale typisch sind.

Diskussion: Der REMPI-Nachweis des Cyclopentylradikals ist erstmals Edelbüttel-Einhaus im Wellenlängenbereich $\lambda=423-463 \mathrm{~nm}$ mit einem Intensitätsmaximum bei $\lambda=437,5 \mathrm{~nm}$ gelungen [EDE88]. Die Radikale wurden über die Reaktion von Cyclopentan mit Chloratomen erzeugt. Weitere Untersuchungen wurden von [ROH91] und [NAC98] im Bereich $\lambda=435-507 \mathrm{~nm}$ bzw. $\lambda=420-460 \mathrm{~nm}$ durchgeführt. Diese erzeugten die c-Pentylradikale über die Reaktion c-Pentan + F. Das MPI-Spektrum zeigt bei den genannten drei Autoren breite, wenig strukturierte Banden im untersuchten Wellenlängenbereich. Mit einer Ionisierungsenergie von IE $=7,21 \mathrm{eV}$ [STE94] werden von $\lambda=420-483 \mathrm{~nm}$ drei, von $\lambda=483-507 \mathrm{~nm}$ vier Photonen zur Ionisierung benötigt. Ein von [EDE88] beobachtetes Fragment bei $\mathrm{m} / \mathrm{z}=67$ wurde bei den Messungen zur vorliegenden Arbeit nicht beobachtet. Da auch [ROH91] und [NAC98] nichts dergleichen erwähnen, ist anzunehmen, dass dieses Fragment auf ein Neben- oder Folgeprodukt infolge des verwendeten $\mathrm{H}$-Atom-Abstraktors $\mathrm{Cl}$ zurückzuführen ist, was von [EDE88] auch vermutet wurde. 


\subsubsection{Das Cyclohexylradikal}

Messergebnisse: c-Hexylradikale wurden über folgende Reaktion erzeugt:

$$
\begin{aligned}
& \mathrm{c}-\mathrm{C}_{6} \mathrm{H}_{12}+\mathrm{F} \rightarrow \mathrm{c}^{-} \mathrm{C}_{6} \mathrm{H}_{11}+\mathrm{HF}, \\
& \mathrm{k}^{298 \mathrm{~K}}=5,7 \cdot 10^{13} \mathrm{~cm}^{3} \cdot \mathrm{mol}^{-1} \cdot \mathrm{s}^{-1}[\text { HEI90]. }
\end{aligned}
$$

Die folgende Abbildung zeigt ein im Rahmen dieser Arbeit aufgenommenes Flugzeitmassenspektrum bei $\lambda=440 \mathrm{~nm}$.

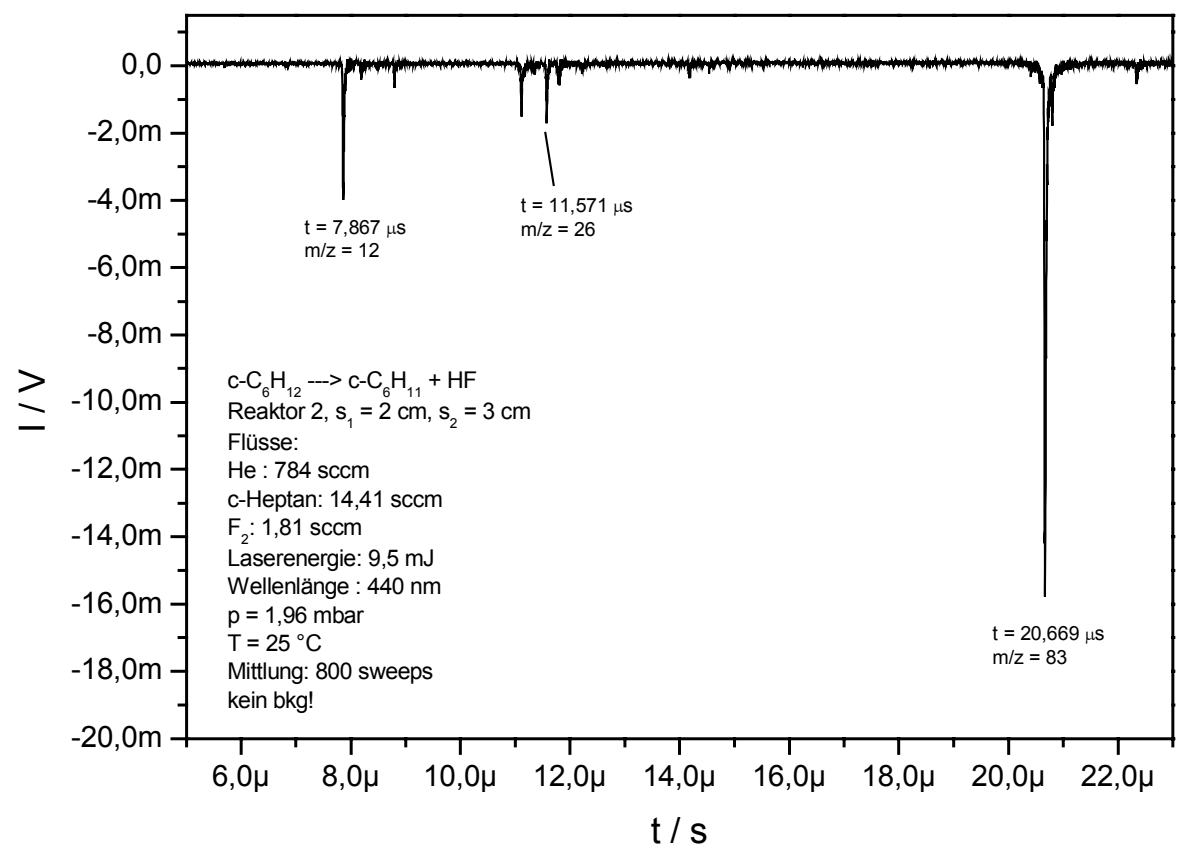

\section{Abbildung 49: Flugzeitmassenspektrum c-Hexan $+F, \lambda=440 \mathrm{~nm}$}

Der Molekülpeak bei $\mathrm{m} / \mathrm{z}=83$ ist am intensivsten, daneben finden sich kleinere Fragmente bei $\mathrm{m} / \mathrm{z}=26,24$ und 12 .

Diskussion: Der REMPI-Nachweis des c-Hexylradikals ist erstmalig Hudgens et al. gelungen [HUD87]. Weitere Untersuchungen zum Nachweis und zu Reaktionen dieses Radikals sind bei [NAC98] zu finden. Dieser erzeugte - wie [HUD87] - c-Hexylradikale über die Reaktion c-Hexan + F. Das Radikal wurde dabei auf seiner Muttermasse $\mathrm{m} / \mathrm{z}=83$ nachgewiesen. Es wurden MPI-Spektren im Bereich $\lambda=420-460 \mathrm{~nm}$ und $\lambda=480-530 \mathrm{~nm}$ aufgenommen. Der Verlauf der Spektren zeigt breite unstrukturierte Banden, deren Intensität im wesentlichen der Energie des Nachweis-Farbstofflasers folgt. Mit einer von [NAC98] angegebenen Ionisierungsenergie von $\mathrm{IE}=6,8 \mathrm{eV}$ werden drei Photonen zur Ionisierung benötigt.

In der vorliegenden Arbeit wird das c-Hexylradikal als eine Referenz für kinetische Messungen verwendet. 


\subsubsection{Das Cycloheptylradikal}

Die Ergebnisse zur Untersuchung des c-Pentyl- und c-Hexylradikals lassen vermuten, dass ein REMPI-Nachweis auch für das c-Heptylradikal möglich ist. Literatur über Erzeugung, Nachweis und Reaktionen dieses Radikals liegen zur Zeit nicht vor.

Messergebnisse: Cycloheptylradikale wurden durch eine Abstraktionsreaktion aus der Reaktion von Cycloheptan mit F-Atomen gemäß folgender Reaktionsgleichung gebildet:

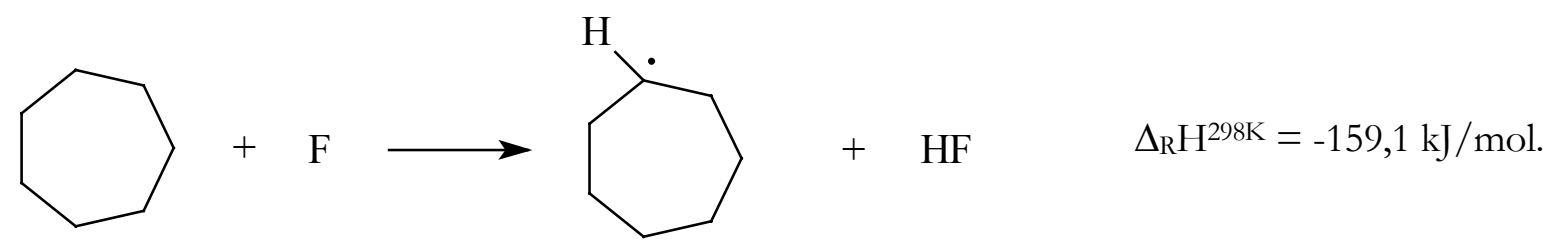

Der Nachweis erfolgte mit Hilfe von Versuchsanordnung A (REMPI) auf der Radikalmuttermasse $\mathrm{m} / \mathrm{z}=97$.

Die folgenden Abbildungen zeigen Flugzeitmassenspektren der Reaktion Cycloheptan $+\mathrm{F}$ bei $\lambda=440 \mathrm{~nm}$ und $\lambda=500 \mathrm{~nm}$. Ein Untergrund durch das Edukt Cycloheptan ist bei diesen Wellenlängen nicht festzustellen.

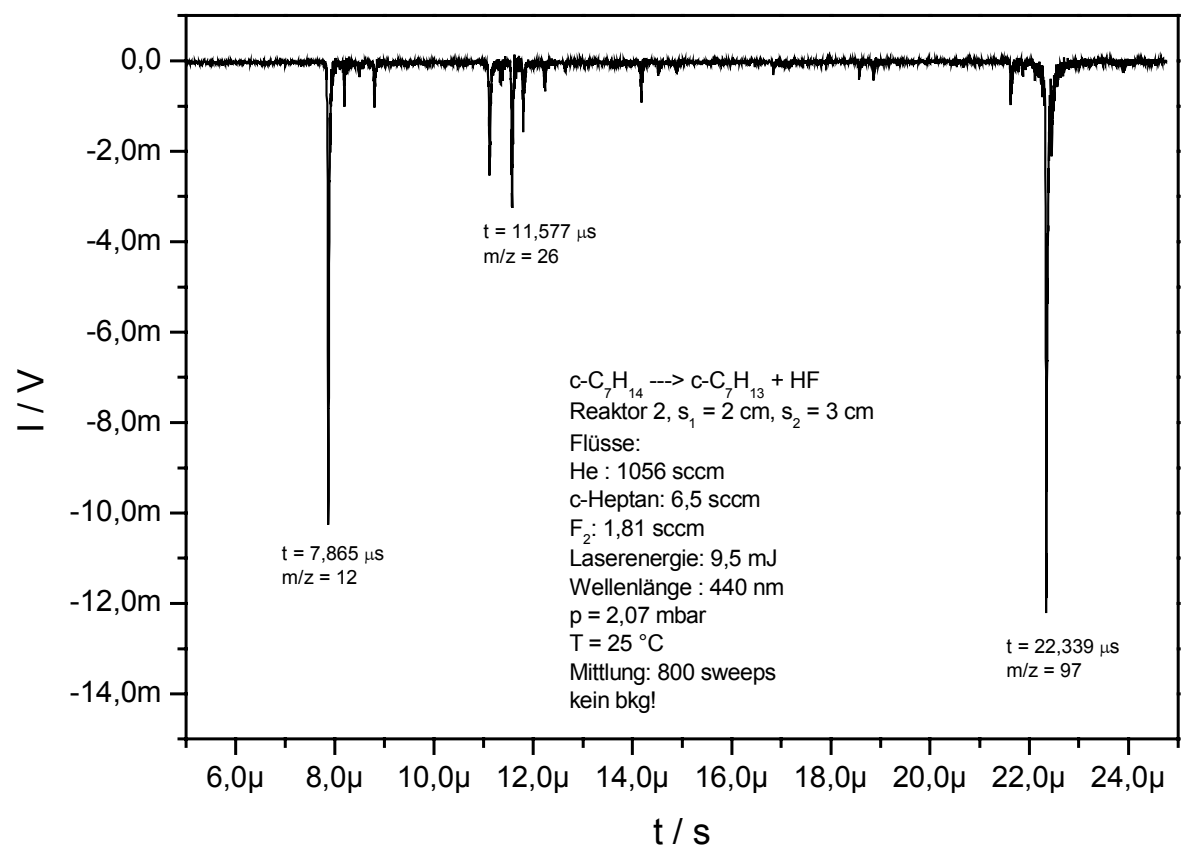

Abbildung 50: Flugzeitmassenspektrum c-Heptan $+F, \lambda=440 \mathrm{~nm}$ 


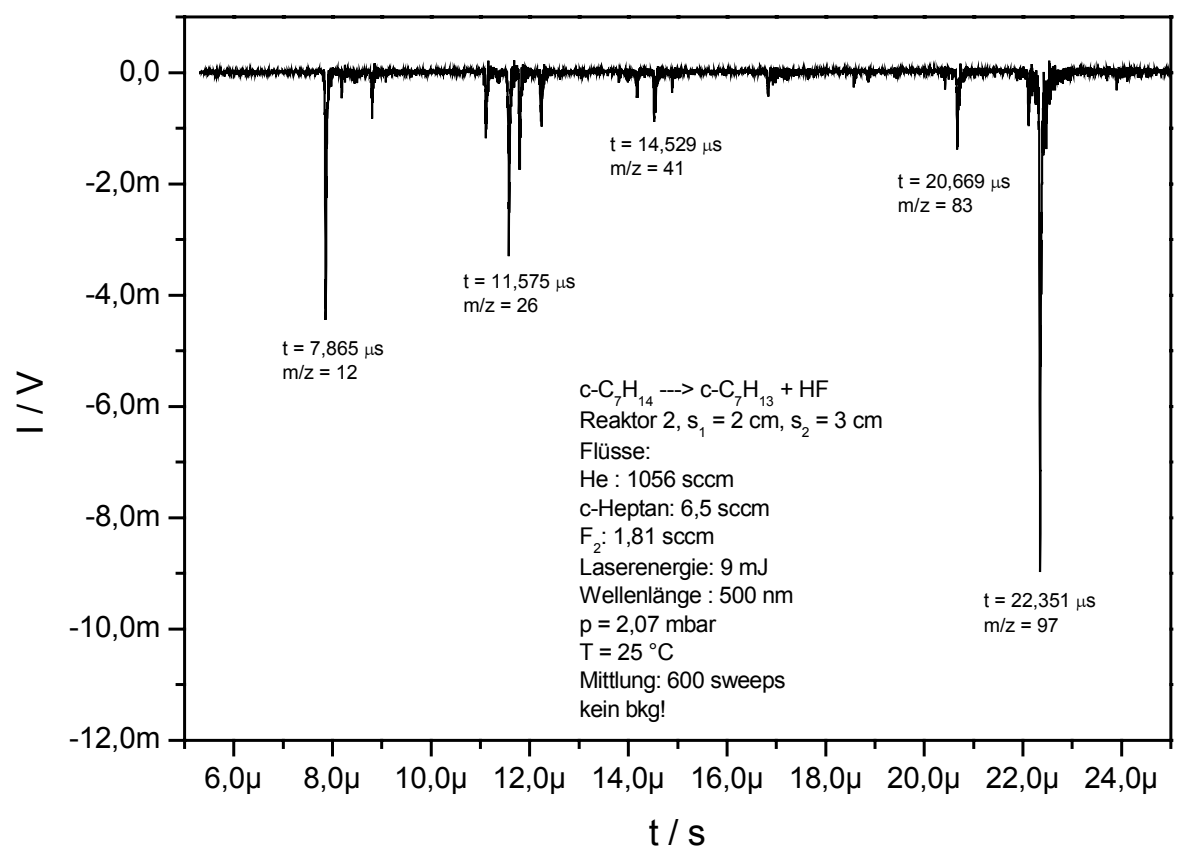

Abbildung 51: Flugzeitmassenspektrum c-Heptan $+F, \lambda=500 \mathrm{~nm}$

Neben dem intensivsten Peak auf der Radikalmuttermasse finden sich Fragmente bei $\mathrm{m} / \mathrm{z}=26,24$ und 12, die bei der höheren Wellenlänge $\lambda=500 \mathrm{~nm}$ im Verhältnis zur Molekülmasse kleiner werden.

Es wurde die Wellenlängenabhängigkeit des c-Heptyl-Signals im Bereich $\lambda=420-460 \mathrm{~nm}$ untersucht, wobei keine besondere Charakteristik erkennbar ist: Das Signal folgt der Energie des Nachweislasers. Die folgende Abbildung verdeutlicht dies.

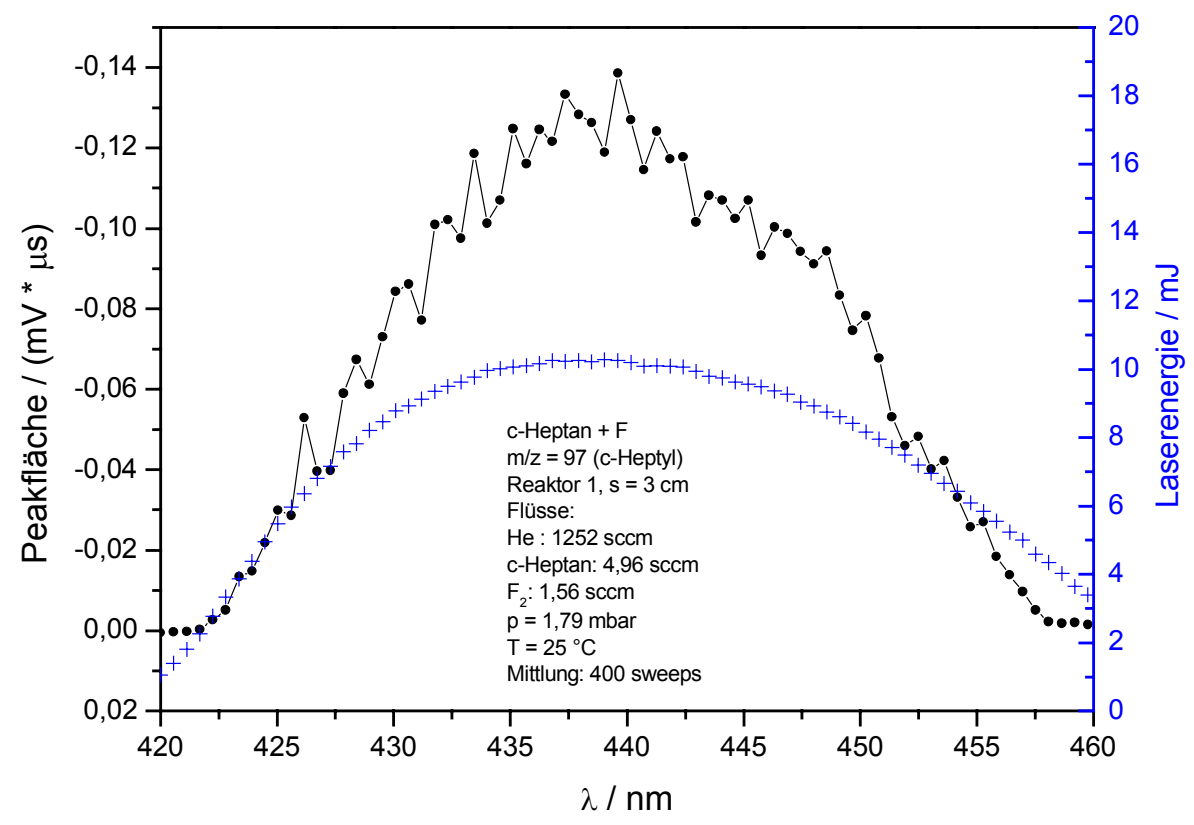

Abbildung 52: Wellenlängenscan c-Heptylradikal, $\lambda=420-460 \mathrm{~nm}$ 


\subsubsection{Das Cyclooktylradikal}

Messergebnisse: Das Cyclooktylradikal wurde aus der Reaktion von Cyclooktan mit F-Atomen dargestellt und mit Hilfe von Versuchsanordnung A (REMPI) auf der Radikalmuttermasse $\mathrm{m} / \mathrm{z}=111$ nachgewiesen. Literatur über Erzeugung, Nachweis und Reaktionen dieses Radikals lag bisher nicht vor. Die folgende Abbildung zeigt ein Flugzeitmassenspektrum der Reaktion Cyclooktan + F bei $\lambda=456 \mathrm{~nm}$. Ein Untergrund durch das Edukt Cyclooktan ist bei dieser Wellenlänge nicht festzustellen.

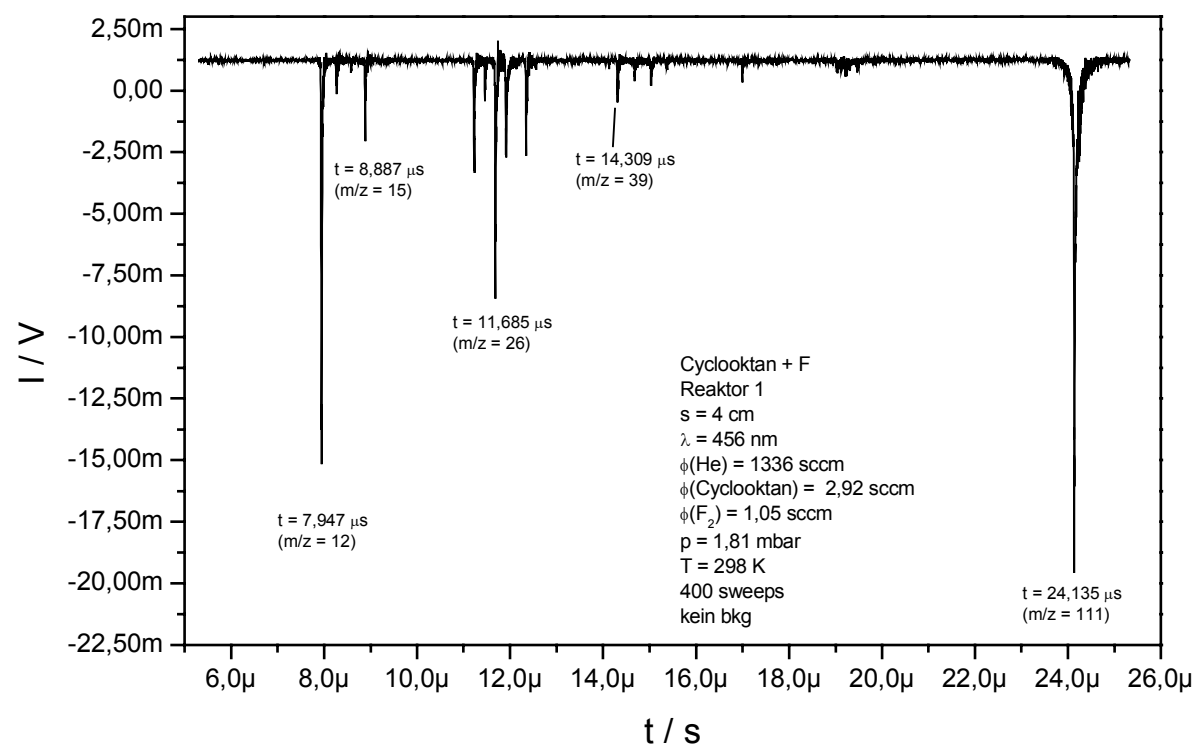

Abbildung 53: Flugzeitmassenspektrum c-Oktan $+F, \lambda=456 \mathrm{~nm}$

Es ist ein reiches Fragmentierungsmuster zu erkennen, welches auch bei längeren Wellenlängen nicht zurückgedrängt werden kann.

Das c-Oktylradikal wird durch eine Abstraktionsreaktion gemäß folgender Reaktionsgleichung gebildet:

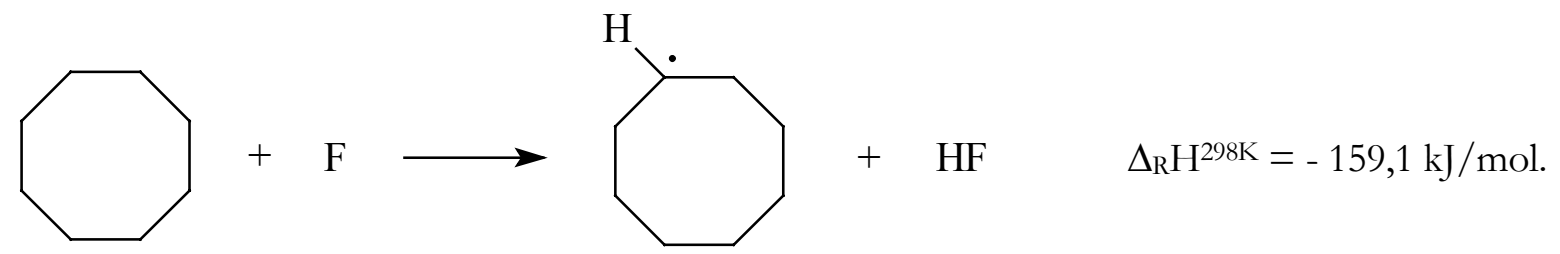

Zur Untersuchung der Wellenlängenabhängigkeit des Nachweises wurden Wellenlängenspektren von $\lambda=443-530 \mathrm{~nm}$ aufgenommen: 


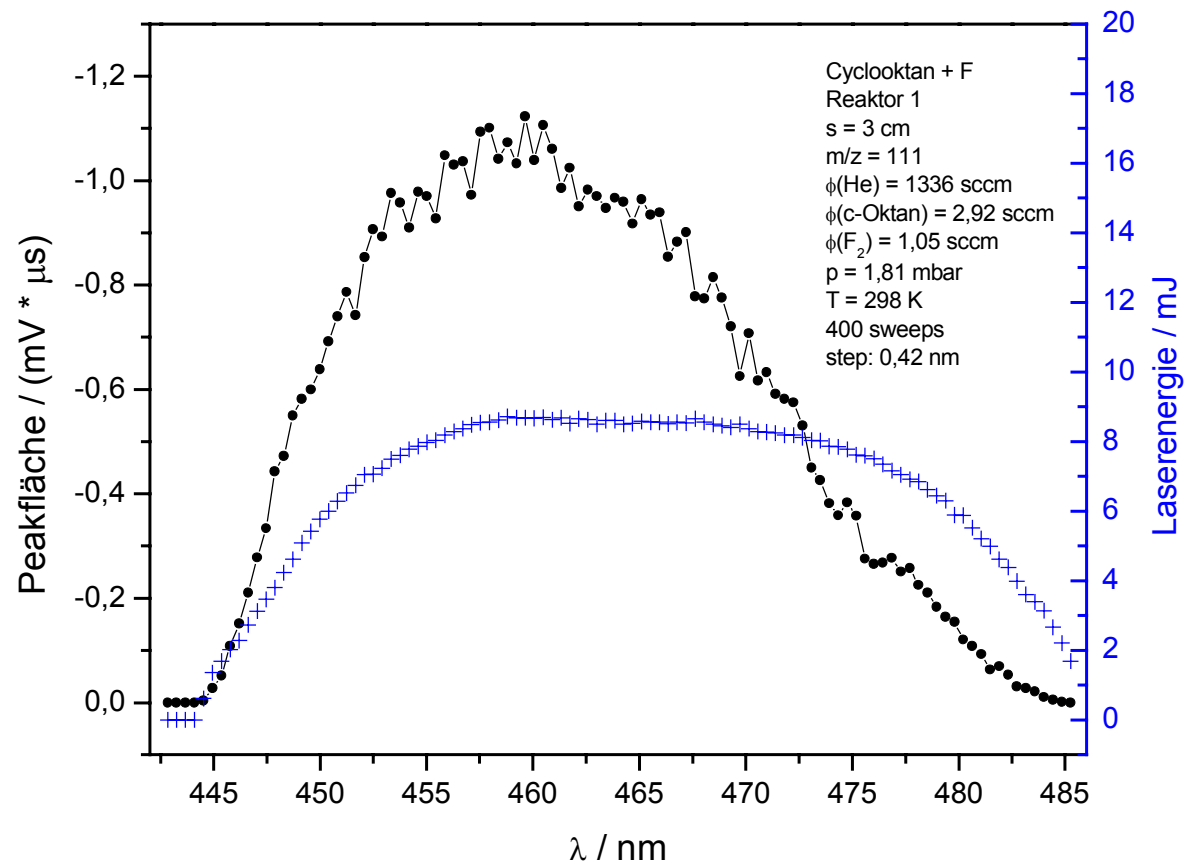

Abbildung 54: Wellenlängenscan c-Oktylradikal, $\lambda=443-485 \mathrm{~nm}$

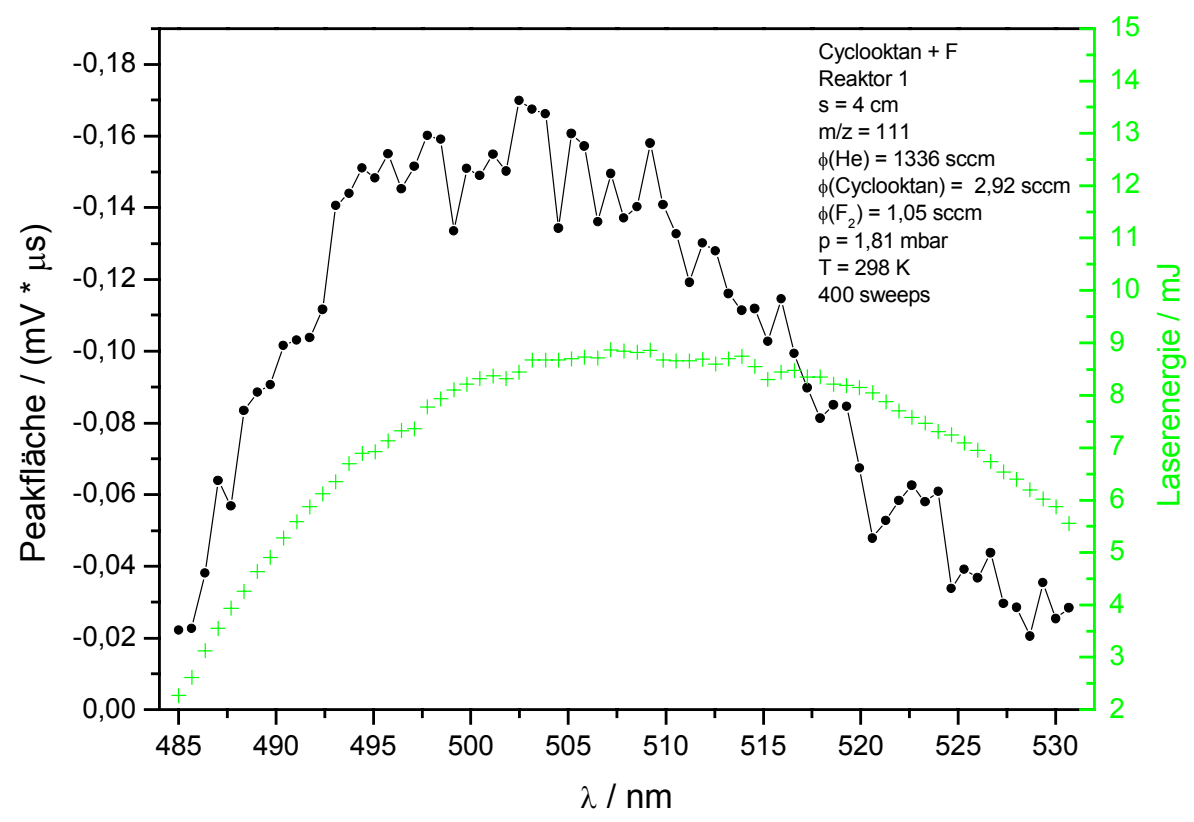

Abbildung 55: Wellenlängenscan c-Oktylradikal, $\lambda=485-530 \mathrm{~nm}$

Bei $\lambda=460 \mathrm{~nm}$ und $468 \mathrm{~nm}$ ist ein gewisser Einbruch der Signalstärke zu beobachten, der nicht mit dem mäßigen Absinken der Laserenergie erklärbar ist. Ansonsten ist keine besondere Struktur erkennbar, die Intensität des Signals folgt im wesentlichen der Laserenergie. 


\subsubsection{Das 1,1,1-Trichlorethylradikal}

Wie in der Einleitung erläutert, wird 1,1,1-Trichlorethan in großen Mengen produziert und in die Atmosphäre freigesetzt. $\mathrm{CH}_{3} \mathrm{CCl}_{3}$ wird dabei vorwiegend mit Hydroxylradikalen zur Bildung von $\mathrm{CH}_{2} \mathrm{CCl}_{3}$ reagieren. In bezug auf diese Reaktion wird dem Trichlorethan eine atmosphärische Lebensdauer von 5,7 \pm 0,7 Jahren zugeschrieben. Das Trichlorethylradikal wird binnen $1 \mu$ s zum zugehörigen Peroxyradikal $\mathrm{CCl}_{3} \mathrm{CH}_{2} \mathrm{O}_{2}$ weiterreagieren. Das weitere Schicksal liegt wahrscheinlich in einer Reaktion mit $\mathrm{NO} z u \mathrm{NO}_{2}$ und dem Trichlorethoxyradikal $\mathrm{CCl}_{3} \mathrm{CH}_{2} \mathrm{O}$ in einem abgeschätzten Zeitraum von weniger als $11 \mathrm{~min}$. Dieses $\mathrm{CCl}_{3} \mathrm{CH}_{2} \mathrm{O}$ geht dann eine Folgereaktion mit $\mathrm{O}_{2}$ ein, welche in einer Erzeugung von $\mathrm{CCl}_{3} \mathrm{CHO}$ mündet, welches durch Reaktion mit $\mathrm{OH}$ und durch Photolyse weiter abgebaut wird [PLA95].

Messergebnisse: Das 1,1,1-Trichlorethylradikal wurde über eine Reaktion von 1,1,1-Trichlorethan mit Fluoratomen erzeugt:

$$
\mathrm{CH}_{3} \mathrm{CCl}_{3}+\mathrm{F} \rightarrow \mathrm{CH}_{2} \mathrm{CCl}_{3}+\mathrm{HF}, \quad \Delta_{\mathrm{R}} \mathrm{H}^{298 \mathrm{~K}}=162,2 \mathrm{~kJ} / \mathrm{mol} .
$$

Neben diesem Reaktionskanal existieren noch weitere (vgl. Abschnitt 3.2.1 $\mathrm{CH}_{3} \mathrm{CCl}_{3}+\mathrm{F}$ ). Das Radikal wurde auf seinen Molekülmassen gemäß seiner natürlichen Isotopenzusammensetzung mit der Versuchsanordnung B (EI-MS) nachgewiesen. In der folgenden Abbildung sind die Zuwächse der Signalintensität auf den Radikalmolekülmassen dargestellt:

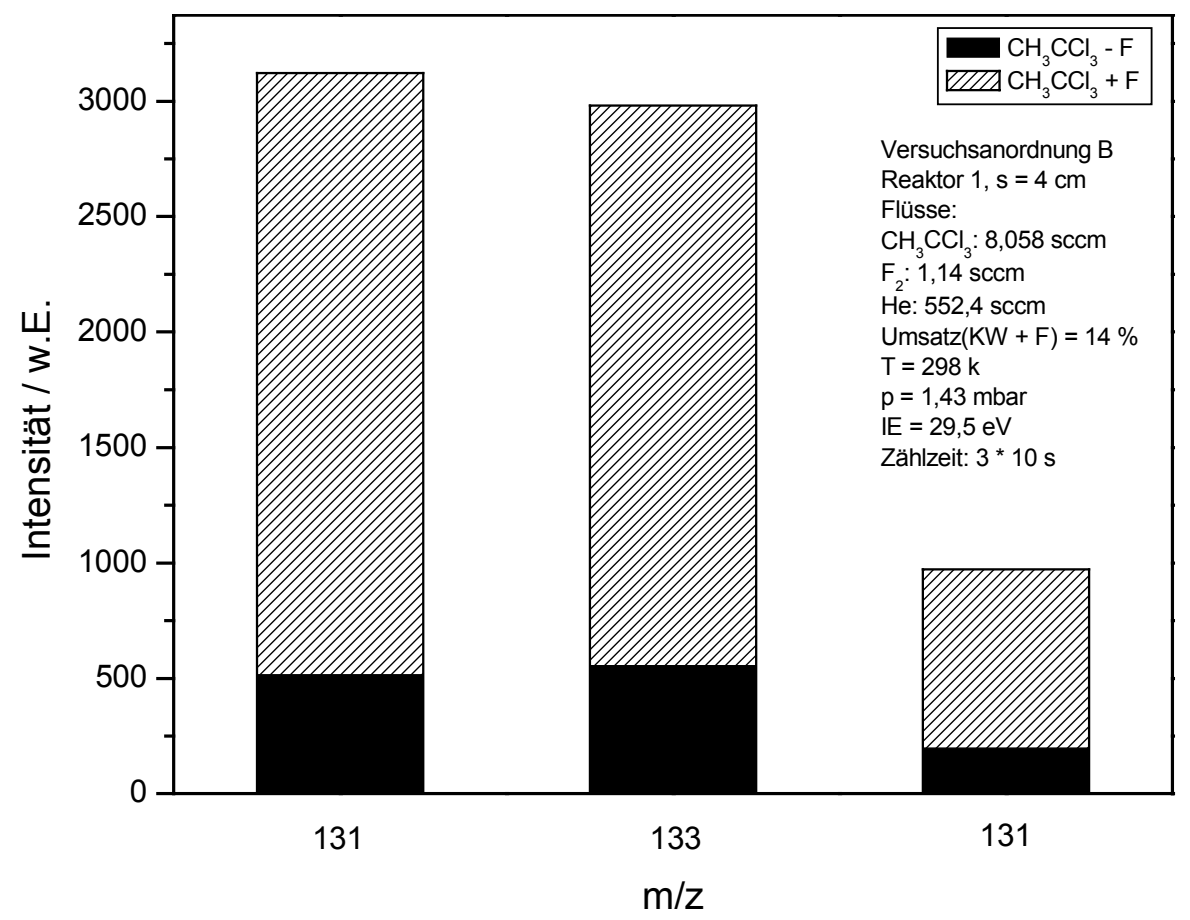

Abbildung 56: Nachweis des Trichlorethylradikals, Versuchsanordnung B 
Ein REMPI-Nachweis des Radikals mit Versuchsanordnung A (REMPI) im Wellenlängenbereich von $\lambda=350-550 \mathrm{~nm}$ gelang trotz intensiver Bemühungen nicht. Für $\lambda<400 \mathrm{~nm}$ zeigen sich beachtliche Fragmentsignale des Trichlorethans. Eine Reaktion mit F-Atomen ist aufgrund der Abnahme der Intensitäten eindeutig zu beobachten. Eine Intensitätszunahme, welche auf ein Radikalsignal hingedeutet hätte, konnte aber nicht detektiert werden.

\subsubsection{Das 2-Chlor-2-Methyl-Propyl-Radikal}

t-Butylchlorid wird bei synthetischen Reaktionen zur Einführung der t-Butylgruppe und als Lösungsmittel eingesetzt [FAL91]. Das aus dem t-Butylchlorid durch H-Abstraktion erhaltene 2-Chlor-2-Methyl-Propyl-Radikal ist dem Isobutylradikal (vgl. Abschnitt 3.1.5) von seiner Struktur her sehr ähnlich. Literatur über Erzeugung, Nachweis und Reaktionen dieses Radikals liegt bisher nicht vor.

Messergebnisse: Das 2-Chlor-2-Methyl-Propyl-Radikal wurde durch die Reaktion von t-Butylchlorid (2-Chlor-2-Methylpropan) mit F Atomen erzeugt:<smiles>CCC(C)(Cl)[I+]I</smiles>

Das Radikal wurde mit Hilfe von Versuchsanordnung A (REMPI) auf seinen Molekülmassen $\mathrm{m} / \mathrm{z}=92$ und 94 nachgewiesen. Da die Abstraktion von H-Atomen nur zu diesem Radikal führen kann, ist der Nachweis eindeutig. Die folgenden Abbildungen zeigen Flugzeitmassenspektren der Reaktion t-Butylchlorid + F bei $\lambda=440 \mathrm{~nm}$ und $\lambda=500 \mathrm{~nm}$. Ein Untergrundsignal vom Edukt t-Butylchlorid ist nicht festzustellen. 


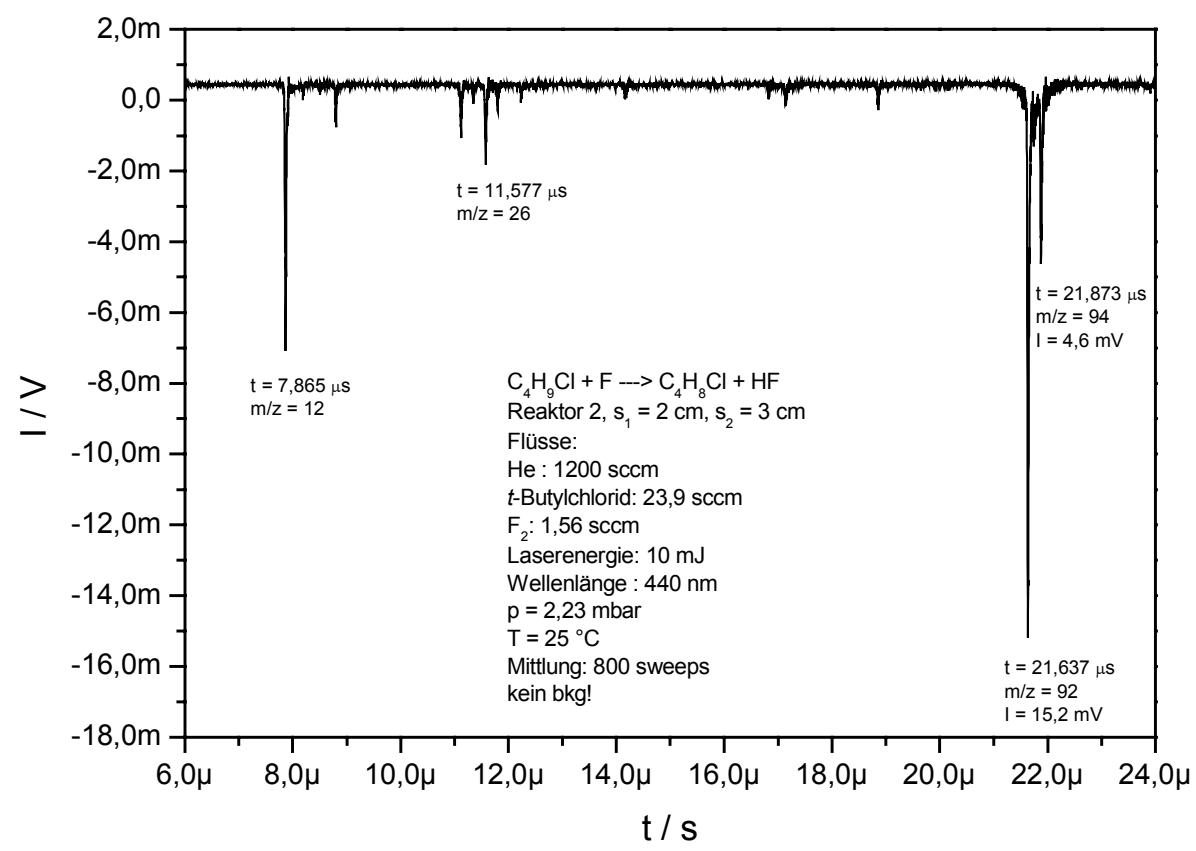

Abbildung 57: Flugzeitmassenspektrum t-Butylchlorid + F, $\lambda=440 \mathrm{~nm}$

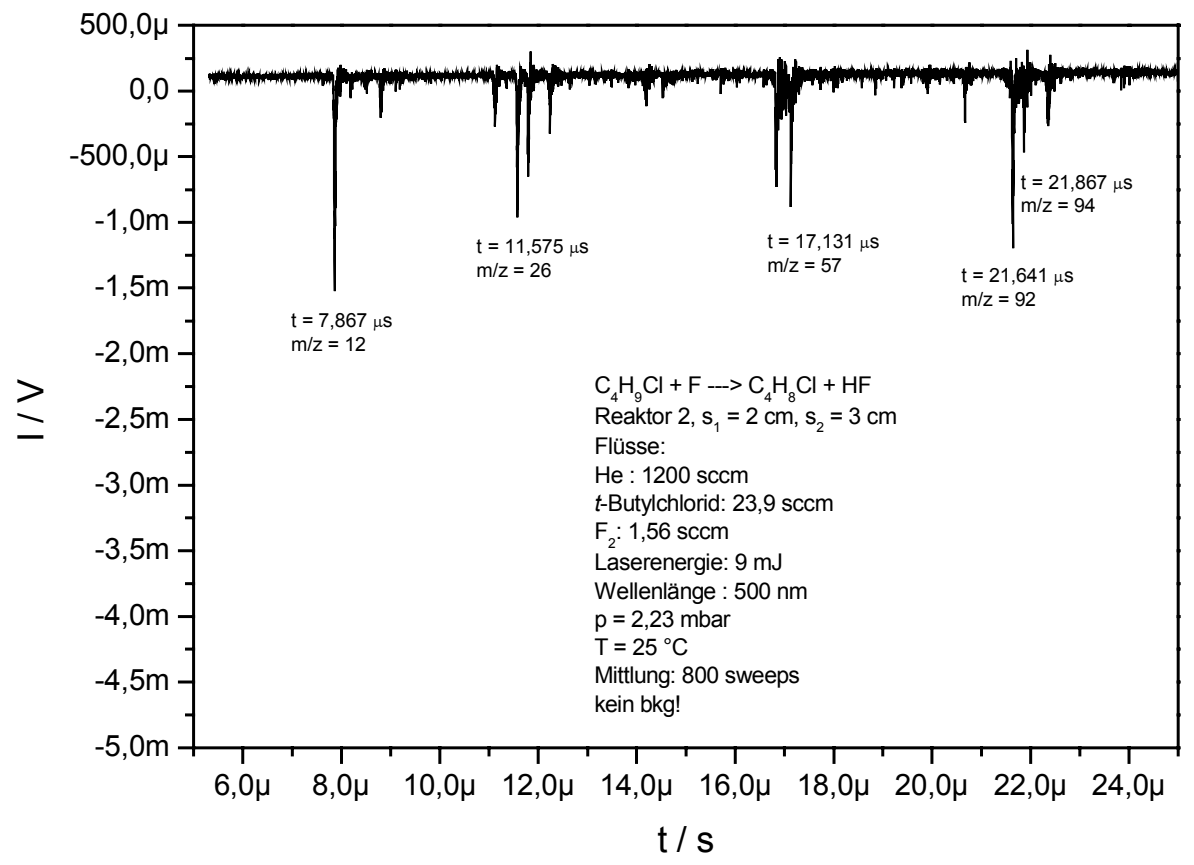

Abbildung 58: Flugzeitmassenspektrum t-Butylchlorid $+F, \lambda=500 \mathrm{~nm}$

Das Verhältnis der Signalintensitäten der Radikalmuttermassen $\mathrm{m} / \mathrm{z}=92$ und 94 zeigt sich in erwarteter Chloratomisotopenverteilung. Es finden sich in kleinerer Intensität Fragmente bei $\mathrm{m} / \mathrm{z}=26,24$ und 12. Bei $\lambda=440 \mathrm{~nm}$ sind die Signalstärken hoch, bei $\lambda=500 \mathrm{~nm}$ nur noch marginal zu verzeichnen. Dieses Verhalten ist ähnlich zum Nachweis des Isobutylradikals, welches bei $\lambda=500 \mathrm{~nm}$ nicht mehr signalgebend ist (vgl. Abschnitt 3.1.5). 
Im Bereich $\lambda=420-460 \mathrm{~nm}$ wurde die Wellenlängenabhängigkeit des 2-Chlor-2-Methyl-PropylRadikals untersucht. Die folgende Abbildung stellt die Intensitäten der beiden Radikalmuttermassen in Abhängigkeit von der Wellenlänge dar. Ein besonderer Zusammenhang ist nicht zu vermerken, die Intensitäten folgen der Energie die Nachweislasers.

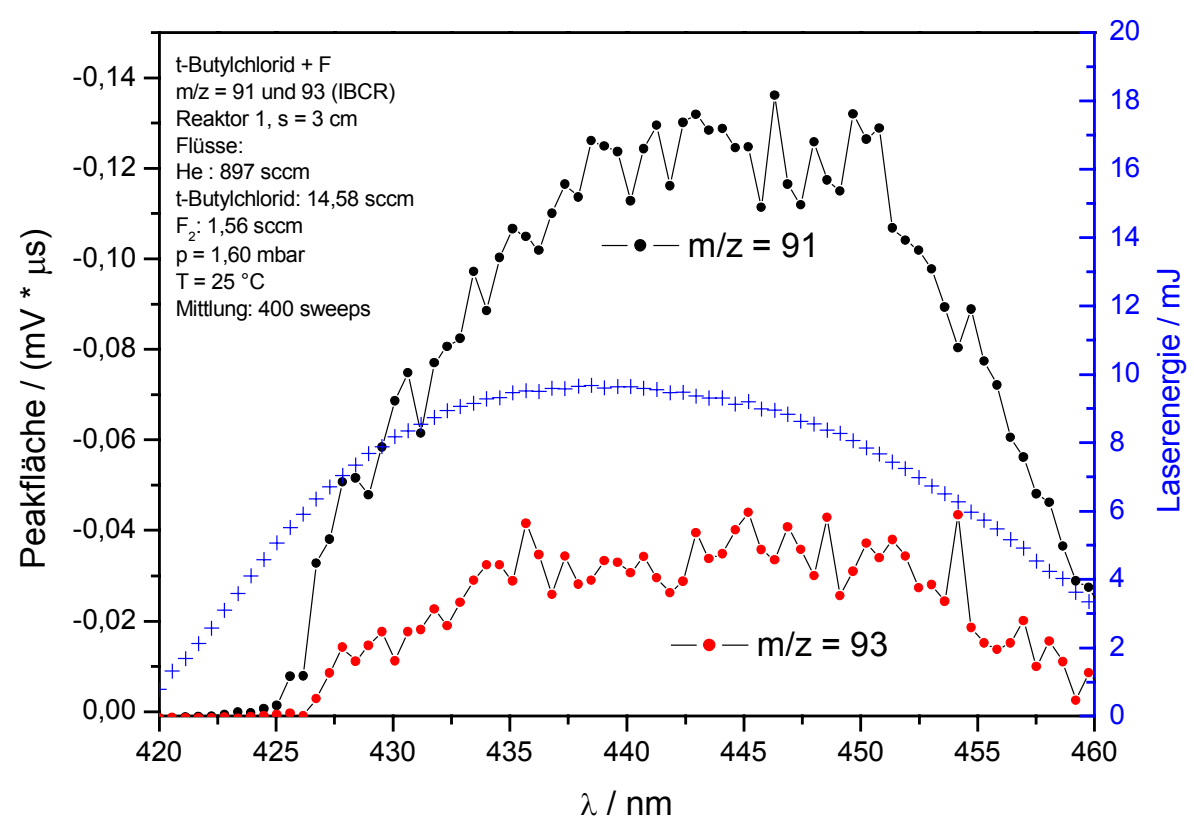

Abbildung 59: Wellenlängenscan 2-Chlor-2-Methyl-Propyl-Radikal, $\lambda=420-460 \mathrm{~nm}$

\subsubsection{Das Allylradikal}

Propen wird zur Benzinherstellung und zur Produktion chemischer Produkte, insbesondere des Polypropylens verwendet [FAL91]. Das formal durch H-Atomabstraktion entstehende Allylradikal hat bei der Pyrolyse und Oxidation von Kohlenwasserstoffen eine große Bedeutung. Es gehört zu den mesomeriestabilisierten Radikalen und ist in hohen Konzentrationen in Flammen und bei Hochtemperaturpyrolysen anwesend ([WES84], [EDE80]).

Messergebnisse: Für die vorliegende Arbeit wurde das Allylradikal über die Reaktion Propen + F erzeugt:

$$
\begin{aligned}
& \mathrm{CH}_{3}-\mathrm{CH}=\mathrm{CH}_{2}+\mathrm{F} \longrightarrow \mathrm{CH}_{2}-\mathrm{CH}-\mathrm{CH}_{2} \\
& \mathrm{k}=2,7 \cdot 10^{13} \mathrm{~cm}^{3} \cdot \mathrm{mol}^{-1} \cdot \mathrm{s}^{-1}[\mathrm{PEA} 73], \Delta_{\mathrm{R}} \mathrm{H}^{298 \mathrm{~K}}=208,8 \mathrm{~kJ} / \mathrm{mol} .
\end{aligned}
$$

Die folgende Abbildung zeigt ein REMPI-Spektrum, welches zwischen $\lambda=485$ und $\lambda=507 \mathrm{~nm}$ aufgenommen wurde. 


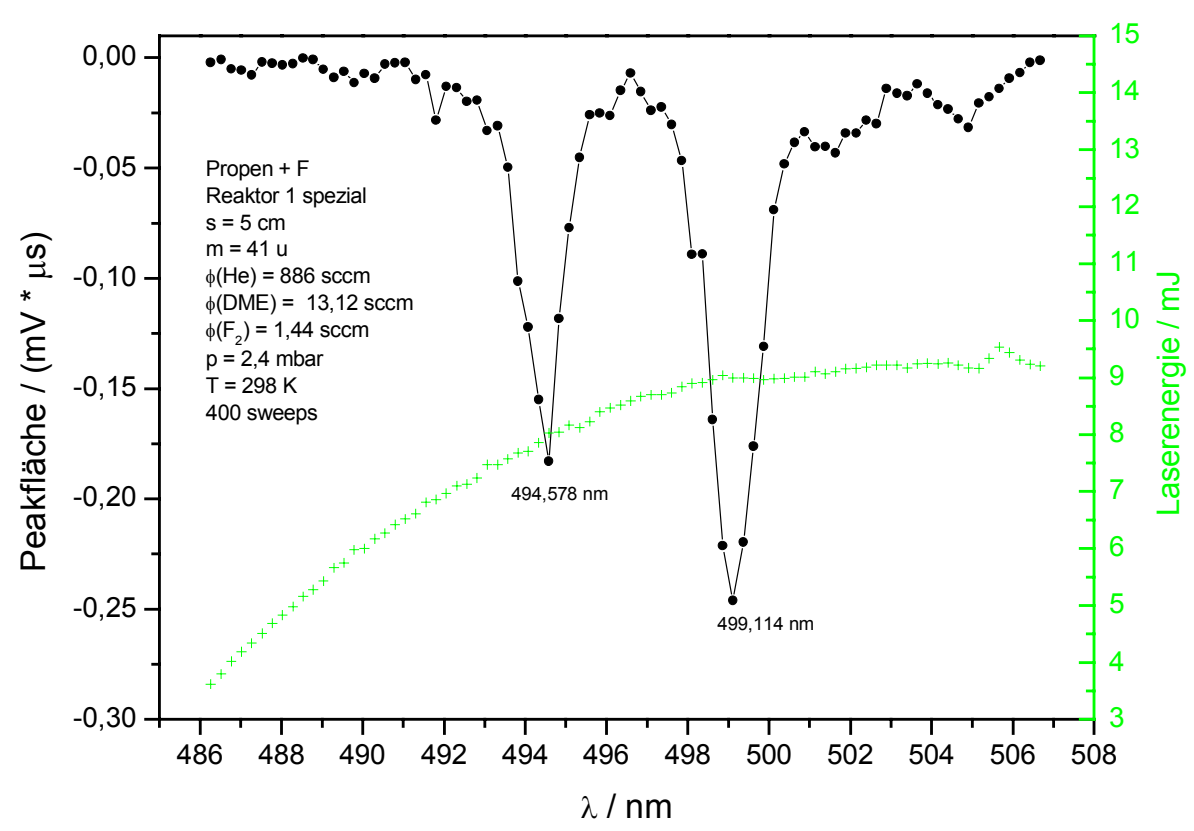

Abbildung 60: REMPI-Spektrum des Allylradikals, $\lambda=485-507 \mathrm{~nm}$

Diskussion: Das Allylradikal ist das einfachste konjugierte Kohlenwasserstoffradikal. Dulcey und Hudgens haben als erste ein REMPI-Spektrum dieses Radikals zwischen $\lambda=480$ und $\lambda=535 \mathrm{~nm}$ aufgenommen [HUD85]. Das Radikal wurde über die Reaktionen Cyclopropan + F, Propen + F und Propen $+\mathrm{Cl}$ erzeugt. Das Ionensignal bei $\mathrm{m} / \mathrm{z}=41$, welches seine größte Intensität bei $\lambda=499,2 \mathrm{~nm}$ hat, wurde dem Allylradikal zugeordnet, weil dieses das einzige thermodynamisch mögliche Produkt einer Abstraktionsreaktion ist. Das in der vorliegenden Arbeit aufgenommene Spektrum zeigt die für das Allylradikal charakteristischen Banden, die auch von Hudgens und Dulcey angegeben werden.

Neben dem Abstraktionskanal existiert noch ein Substitutionskanal, welcher von Heinemann beobachtet wurde [HEI86]:

$$
\mathrm{C}_{3} \mathrm{H}_{6}+\mathrm{F} \rightarrow \mathrm{C}_{2} \mathrm{H}_{3} \mathrm{~F}+\mathrm{CH}_{3}
$$

\subsubsection{Das Cyclopentadienylradikal, das Fluorocyclopentenylradikal}

Cyclopentadien wird als Nebenprodukt aus Koksofengas, Steinkohlenteer, aus Erdölcrackprodukten oder durch katalytische Dehydrierung von Cyclopentan hergestellt. Es findet Verwendung zur Synthese von Terpenen, chlorhaltigen Insektiziden und anderen Produkten.

Polyaromatische Kohlenwasserstoffe (PAH) leisten bei nicht optimaler Verbrennung einen großen Beitrag zur Rußpartikelbildung. Die Bildung des ersten aromatischen Rings ist dabei noch umstritten. Dem Cyclopentadienylradikal, welches während des Verbrennungsgeschehens entsteht, 
wird durch eine Reaktion mit Methylradikalen zur Bildung von Methylcyclopentadienyl und anschließender Benzolbildung unter H-Abspaltung eine Bedeutung bei diesen Vorgängen zugesprochen. Benzol wiederum ist Ausgangsstoff zur Entstehung von PAH. Außerdem wird die Bildung von Naphthalin aus der Selbstreaktion zweier Cyclopentadienylradikale diskutiert [ROY99].

Diskussion: Das Cyclopentadienylradikal (CPR) in der Gasphase wurde bereits mehrfach nachgewiesen. Eine eingehende Untersuchung der Erzeugung des Radikals und dessen Reaktionen ist von Buth durchgeführt worden [BUT93]. Buth erzeugte Cyclopentadienylradikale über die Reaktion Cyclopentadien (CP) + F im isothermen Strömungssystem mit EI-MS Nachweis. Zusätzlich zu diesem Reaktionskanal gibt es Hinweise für weitere Reaktionskanäle. Insbesondere wurde die Entstehung des Fluorocyclopentenylradikals (FCPR) nachgewiesen. Für dieses Radikal existieren zwei Isomere, die massenspektrometrisch nicht unterschieden werden können. Folgende Reaktionen von Cyclopentadien mit F-Atomen wurden von Buth in Erwägung gezogen:
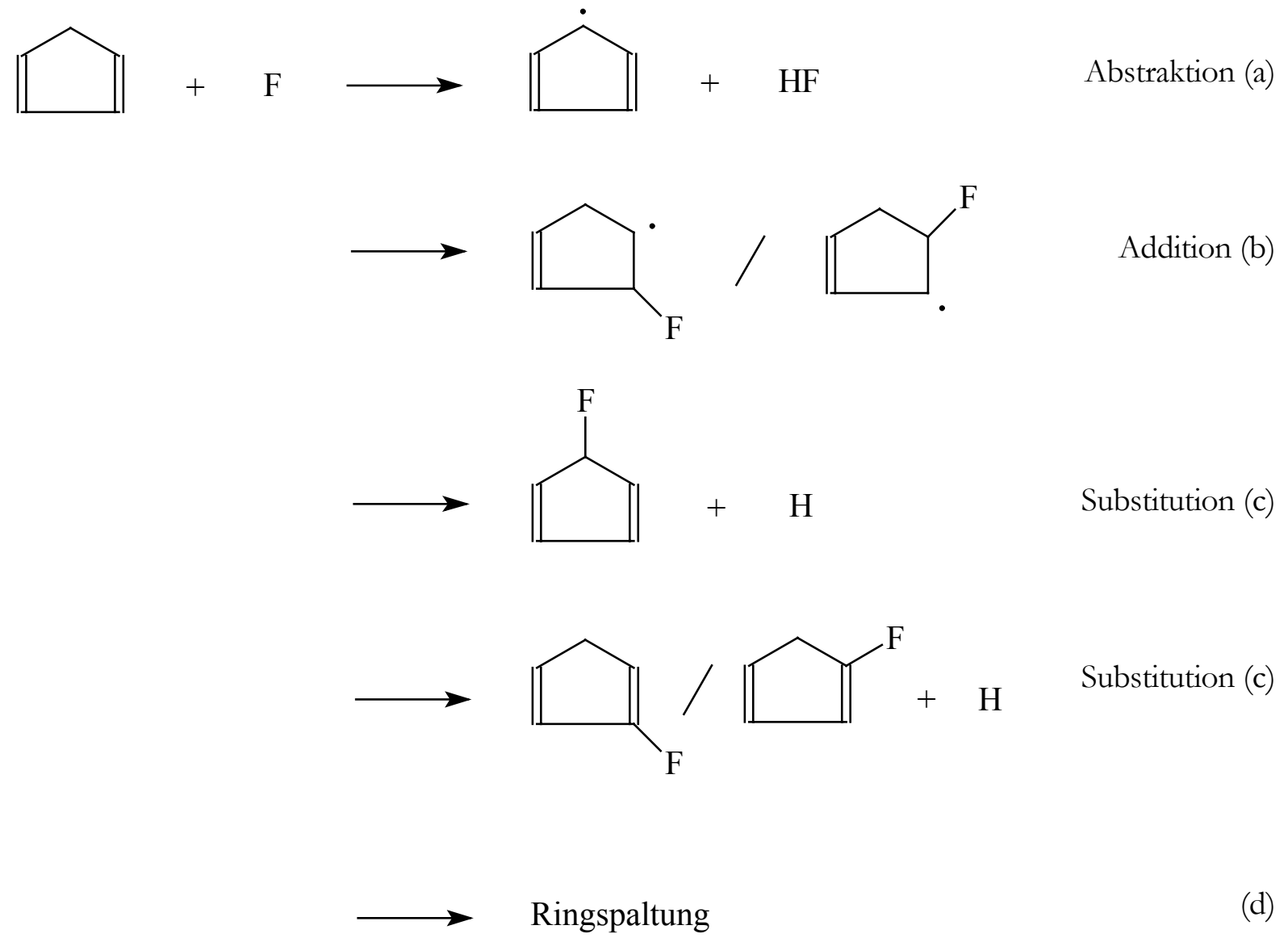

Die Reaktionsenthalpie bei Zimmertemperatur beträgt für Kanal (a) -239,6 kJ/mol und für Kanal (c) $-42,5 \mathrm{~kJ} / \mathrm{mol}$. Für die übrigen Kanäle ist eine Reaktionsenthalpie nicht zu ermitteln, da die Bildungsenthalpien der Produktradikale unbekannt sind. 
Zur Überprüfung und Ergänzung der Ergebnisse von Buth wurde im Rahmen dieser Arbeit die Reaktion CP + F mit Versuchsanordnung A (REMPI) untersucht.

Messergebnisse: Im folgenden ist ein Flugzeitmassenspektrum von CP unter den Bedingungen -F/ $+\mathrm{F}$ bei $\lambda=427 \mathrm{~nm}$ dargestellt:

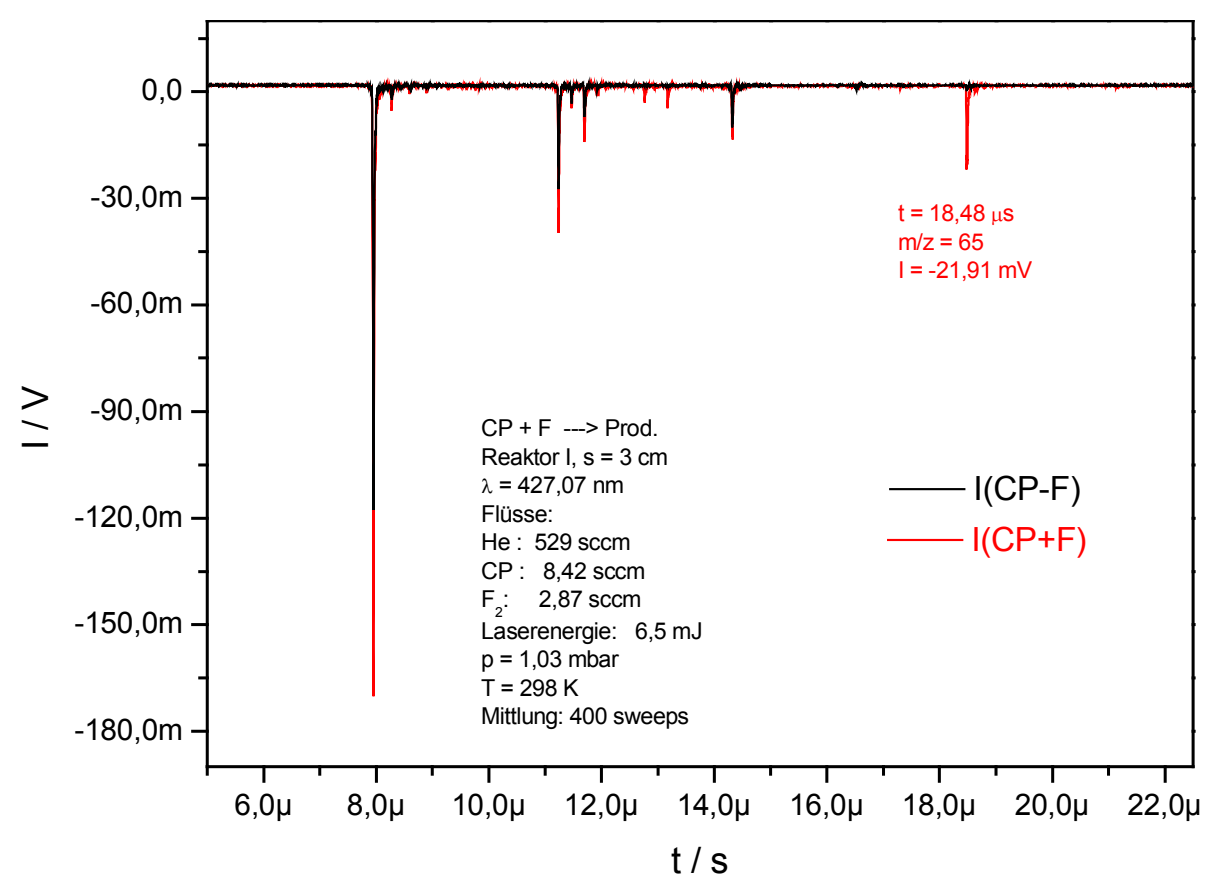

Abbildung 61: Flugzeitmassenspektrum c-Pentadien $+F, \lambda=427 \mathrm{~nm}$

Das Cyclopentadien selbst zeigt bereits eine starke Fragmentierung mit intensiven Ionensignalen. Ein Molekülpeak ist jedoch nicht erkennbar. Das Intensitätsmaximum liegt bei $\mathrm{m} / \mathrm{z}=12$, also der Abspaltung von ${ }^{12} \mathrm{C}^{+}$. Unter der Bedingung $+\mathrm{F}$ ist eine erhebliche Intensitätsverstärkung bei allen Fragmenten auszumachen. Allerdings ist hier ein deutliches untergrundfreies Signal bei $\mathrm{m} / \mathrm{z}=65$ zu beobachten, der Radikalmuttermasse des Cyclopentadienylradikals.

Bei längeren Wellenlängen ist auch ein Ionensignal bei $\mathrm{m} / \mathrm{z}=85 \mathrm{zu}$ verzeichnen, wenn auch in geringerer Intensität. Da laut Reaktionsschema durchaus auch benachbarte Massen durch Produkte belegt sein könnten, wurde eine Masseneichung mit Hilfe des Cyclohexylradikals vorgenommen (Nachweis des Cyclohexylradikals vgl. Abschnitt 3.1.7), welche eine eindeutige Zuordnung des Signals zu m/z $=85$ erbracht hat. Die folgende Abbildung zeigt ein Flugzeitmassenspektrum der Reaktion CP + F bei $\lambda=453 \mathrm{~nm}$ : 


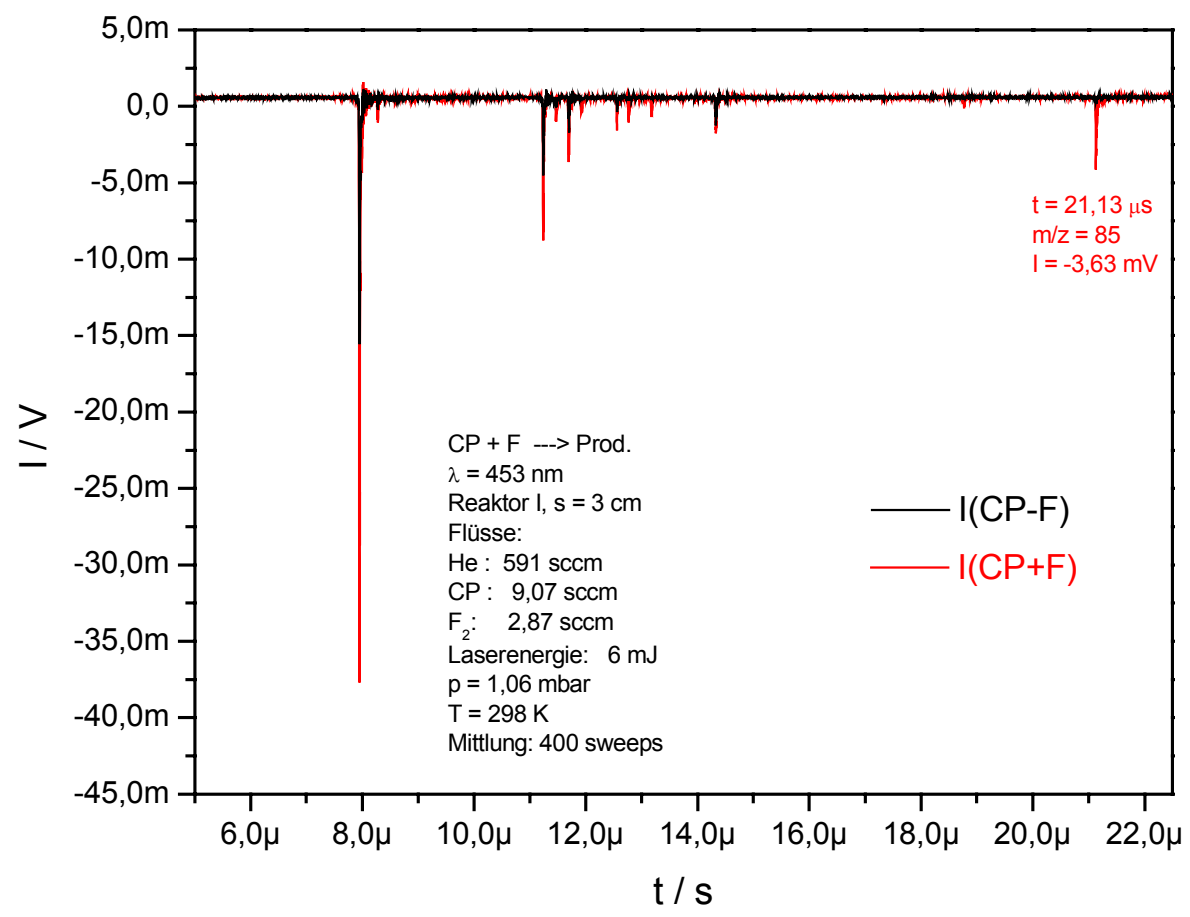

Abbildung 62: Flugzeitmassenspektrum c-Pentadien $+F, \lambda=453 \mathrm{~nm}$

Wie bei $427 \mathrm{~nm}$ ist eine Fragmentierung des Cyclopentadiens deutlich erkennbar, wenn auch bei weitem nicht mehr so stark. Die bei der Reaktion mit F-Atomen entstehenden Produkte fragmentieren auf denselben Massen. Das Ionensignal des Cyclopentadienylradikals bei $\mathrm{m} / \mathrm{z}=65$ ist fast verschwunden. Dagegen ist das Signal des Fluorocyclopentenylradikals untergrundfrei bei $\mathrm{m} / \mathrm{z}=85$ vorhanden.

Im Rahmen des zweidimensionalen Nachweises, den die Versuchsapparatur A (REMPI) bietet, wurde ein Wellenlängenscan der Signale bei $\mathrm{m} / \mathrm{z}=65$ und $85 \mathrm{im}$ Bereich 423 bis $460 \mathrm{~nm}$ mit einer Schrittweite von $0,84 \mathrm{~nm}$ aufgenommen. 


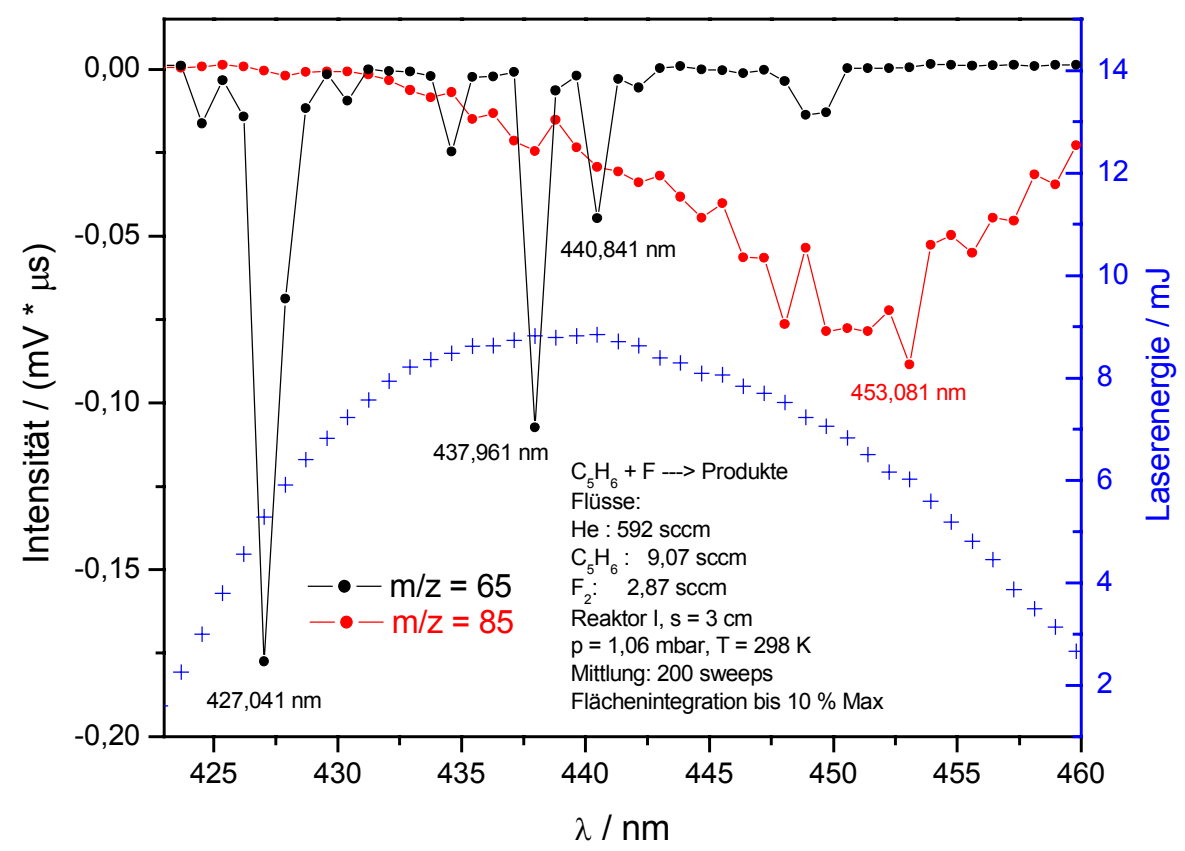

Abbildung 63: Wellenlängenscan der Signale $\mathrm{m} / \mathrm{z}=65$ und 83, CP $+\mathrm{F}, 423-460 \mathrm{~nm}$

Das Cyclopentadienylradikal weist enge Banden mit einer spezifischen Struktur auf. Im Gegensatz dazu ist für das Fluorocyclopentenylradikal keine solch ausgeprägte Struktur zu erkennen.

Im Bereich des Maximums des Signals des CPR $(\mathrm{m} / \mathrm{z}=65)$ wurde ein Wellenlängenscan mit feinerer Schrittweite $(0,14 \mathrm{~nm})$ aufgenommen. Es ist eine sehr charakteristische Wellenlängenabhängigkeit festzustellen:

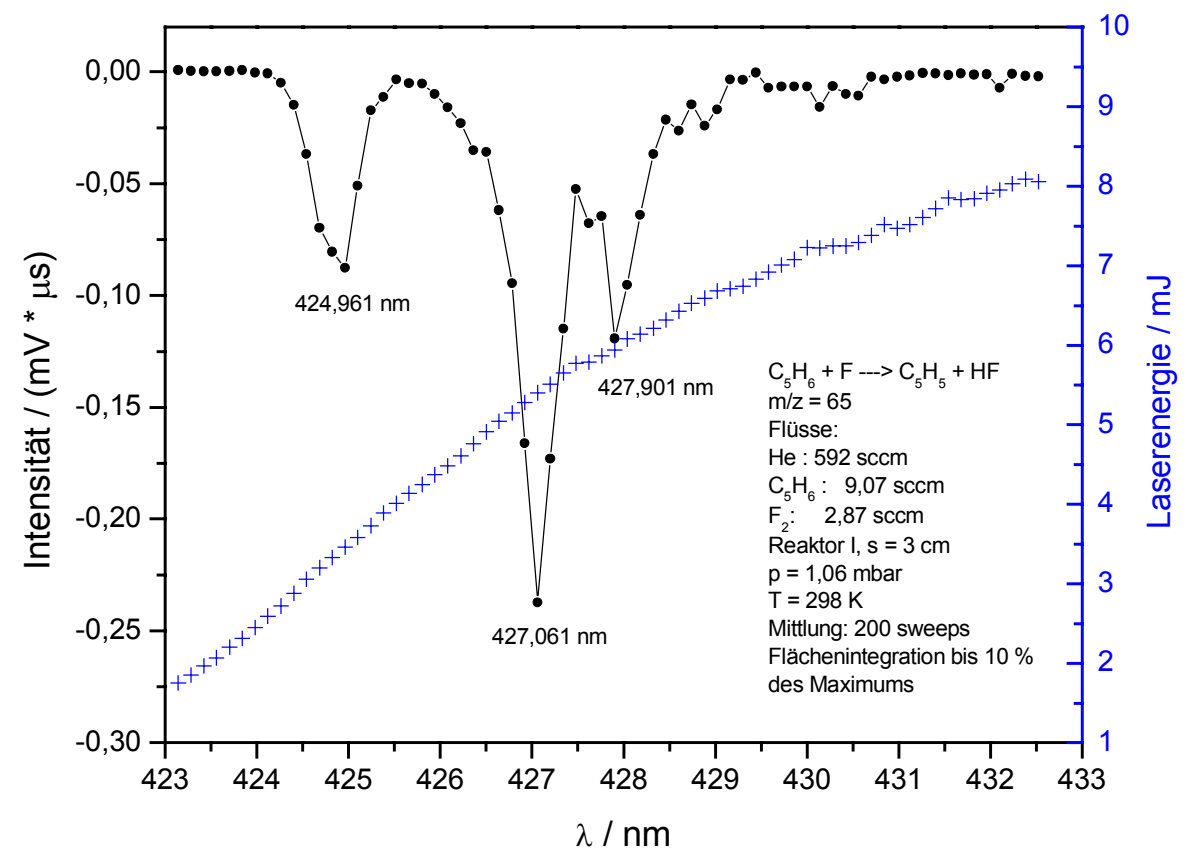

Abbildung 64: Wellenlängenscan des c-Pentadienylradikals 423-433 nm 
Derselbe charakteristische Verlauf des Spektrums wurde für das Cyclopentadienylradikal bei Erzeugung über die Reaktion $\mathrm{CP}+\mathrm{Cl}$ gefunden.

Interpretation: Die von Buth favorisierten Reaktionskanäle (a) und (b) können aufgrund der Messergebnisse bestätigt werden. Für die Kanäle (c), (d) und (e) wurden keine Hinweise gefunden.

\subsubsection{Das Methoxymethylradikal}

Dimethylether, das einfachste Glied in der Reihe der aliphatischen Ether, wird als Ersatz Dieselkraftstoffe diskutiert ([SEH97], [CAT95], [WAL97]). Er besitzt einige Eigenschaften, die ihn für diese Anwendung prädestinieren, vor allem, dass die Verbrennung dieser Substanz frei von Rußpartikeln abläuft. Ferner wird Dimethylether auch als Treibgas für Aerosole eingesetzt [FAL91], was diese Substanz und das durch H-Atomabstraktion resultierende Radikal für chemische Vorgänge in der Atmosphäre interessant macht.

Messergebnisse: Methoxymethylradikale wurden über die Reaktion

$$
\begin{aligned}
& \mathrm{CH}_{3} \mathrm{OCH}_{3}+\mathrm{F} \rightarrow \mathrm{CH}_{3} \mathrm{OCH}_{2}+\mathrm{HF}, \\
& \mathrm{k}^{298 \mathrm{~K}}=1,1 \cdot 10^{14} \mathrm{~cm}^{3} \cdot \mathrm{mol}^{-1} \cdot \mathrm{s}^{-1}[\mathrm{HOY} 96]
\end{aligned}
$$

erzeugt. Das Radikal ist auf seiner Molekülmasse $\mathrm{m} / \mathrm{z}=45$ nachweisbar.

Die folgende Abbildung zeigt ein Flugzeitmassenspektrum des Methoxymethylradikals bei $500 \mathrm{~nm}$ :

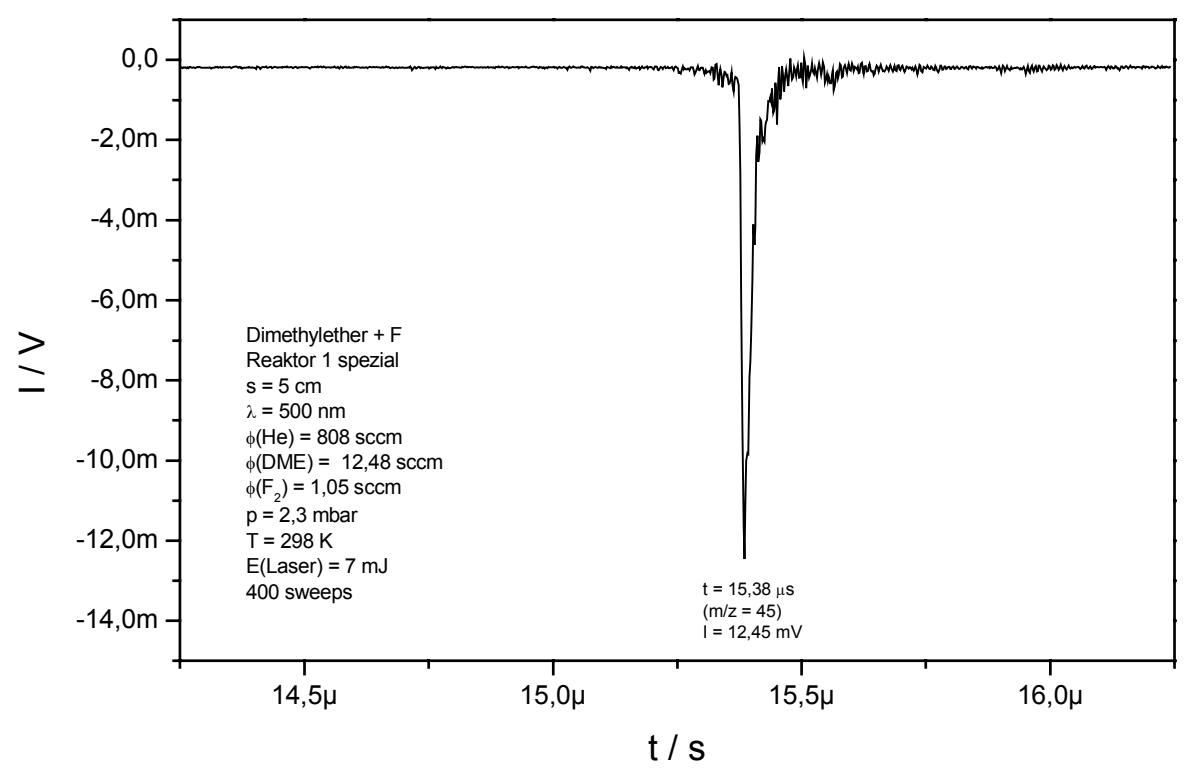

Abbildung 65: Flugzeitmassenspektrum des Methoxymethylradikals, $\lambda=500 \mathrm{~nm}$ 
Diskussion: Die REMPI-Aktivität des Methoxymethylradikals wurde von Nacke gefunden [NAC98]. Es wurden MPI-Spektren von 420-530 nm aufgenommen, wobei sich breite, wenig strukturierte Banden zeigten, deren Intensität der Energie des ionisierenden Farbstofflasers folgten. Eigene Messungen zeigen, dass sich der Nachweisbereich auf $\lambda=360-580 \mathrm{~nm}$ erweitern lässt, bei kürzeren Wellenlängen setzt zunehmend Radikalfragmentierung insbesondere $z u \mathrm{~m} / \mathrm{z}=15$ ein. Im Bereich $\lambda<400 \mathrm{~nm}$ wird auch die Radikalvorläufersubstanz Dimethylether ionisiert, so dass es zu Überlagerungen kommt. Das Methoxymethylradikal hat ein Ionisierungspotenzial von 6,94 eV [STE94]. Beim Ein-Photonen-Mechanismus wäre somit eine Wellenlänge von 178,65 nm zur Ionisation nötig. Unter Berücksichtigung des UV-Absorptionsspektrums des Radikals [LAN95] folgert Nacke einen (2+1)-REMPI-Mechanismus.

\subsection{Reaktionen von Kohlenwasserstoffen mit F-Atomen}

\subsection{1 $\mathrm{CH}_{3} \mathrm{CCl}_{3}+\mathrm{F}$}

Produkte:

Messergebnisse: Die Produkte dieser Reaktion wurden mittels Versuchsanordnung B (EI-MS) bei einer Ionisierungsenergie von 29,5 eV im Magnetstromscan und in Einzelmassenzählung untersucht. Folgende Reaktionskanäle sind denkbar:

$$
\begin{array}{rlll} 
& \longrightarrow & \dot{\mathrm{C}} \mathrm{H}_{2}-\mathrm{CCl}_{3}+\mathrm{HF} & \Delta_{\mathrm{R}} \mathrm{H}^{298 \mathrm{~K}}=-162,2 \mathrm{~kJ} / \mathrm{mol} \text { (a) } \\
\mathrm{CH}_{3}-\mathrm{CCl}_{3}+\mathrm{F} & \longrightarrow \mathrm{CH}_{3}-\mathrm{CCl}_{2} \mathrm{~F}+\dot{\mathrm{Cl}} & \Delta_{\mathrm{R}} \mathrm{H}^{298 \mathrm{~K}}=-146,5 \mathrm{~kJ} / \mathrm{mol} \text { (b) } \\
& \longrightarrow \dot{\mathrm{CH}}_{2}-\mathrm{CCl}_{2} \mathrm{~F}+\mathrm{HCl} & \Delta_{\mathrm{R}} \mathrm{H}^{298 \mathrm{~K}}=-135,2 \mathrm{~kJ} / \mathrm{mol} \text { (c) }
\end{array}
$$

Um zu ermitteln, welche Kanäle relevant sind, wurde zunächst ein Massenspektrum des 1,1,1-Trichlorethans im Magnetstromscanverfahren aufgenommen: 


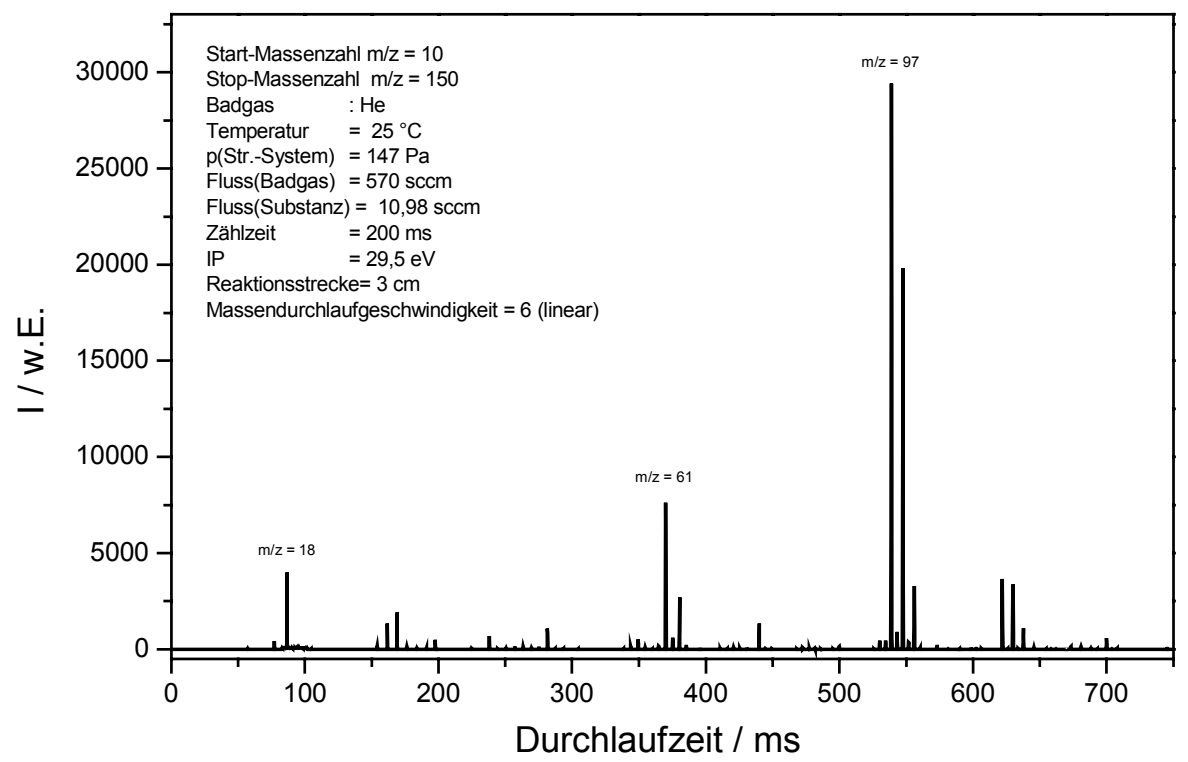

\section{Abbildung 66: Massenspektrum $\mathrm{CH}_{3} \mathrm{CCl}_{3}$}

Ein nennenswerter Untergrund war nur auf den Massen $\mathrm{m} / \mathrm{z}=18$ (Wasser), 28 und 32 (Luft), 57 und 137 (Pumpenöl) zu erkennen, was in den folgenden Betrachtungen aber keine Rolle spielt.

Interpretation: Um die weitere Aufklärung der Reaktion des Trichlorethans mit Fluoratomen zu erleichtern, soll in Tabellenform eine Zuordnung der Massen zu den Fragmenten erfolgen:

\begin{tabular}{|c|c|c|}
\hline $\mathrm{m} / \mathrm{z}$ & Fragment & Anmerkungen \\
\hline 138 & $\left(\mathrm{CH}_{3} \mathrm{CCl}_{3}{ }^{37}\right)^{+}$ & \multirow{4}{*}{$\begin{array}{l}\text { Molekülpeaks (Intensität klein) } \\
\text { (überlagert durch }\left(\mathrm{C}_{2} \mathrm{Cl}_{2}{ }^{35} \mathrm{C}^{37} \mathrm{H}\right)^{+} \text {und } \\
\left.\left(\mathrm{C}_{2} \mathrm{Cl}^{35} \mathrm{Cl}_{2}{ }^{37} \mathrm{H}^{+}\right)\right)\end{array}$} \\
\hline 136 & $\left(\mathrm{CH}_{3} \mathrm{CCl}_{2}{ }^{37} \mathrm{Cl}^{35}\right)^{+}$ & \\
\hline 134 & $\left(\mathrm{CH}_{3} \mathrm{CCl}_{2}{ }^{35} \mathrm{Cl}^{37}\right)^{+}$ & \\
\hline 132 & $\left(\mathrm{CH}_{3} \mathrm{CCl}_{3}{ }^{35}\right)^{+}$ & \\
\hline 130 & $\left(\mathrm{C}_{2} \mathrm{Cl}_{3}{ }^{35} \mathrm{H}\right)^{+}$ & ähnlich hohe Intensität wie 132 \\
\hline 121 & $\left(\mathrm{CCl}_{2}{ }^{37} \mathrm{C}^{35}\right)^{+}$ & \multirow{3}{*}{ Intensität groß } \\
\hline 119 & $\left(\mathrm{CCl}^{37} \mathrm{Cl}_{2}{ }^{35}\right)^{+}$ & \\
\hline 117 & $\left(\mathrm{CCl}_{3}^{35}\right)^{+}$ & \\
\hline 101 & $\left(\mathrm{CH}_{3} \mathrm{CCl}_{2}{ }^{37}\right)^{+}$ & \multirow{3}{*}{$\begin{array}{c}\text { sehr große Intensitäten, } \mathrm{m} / \mathrm{z}=97 \text { zur } \\
\text { Umsatzbestimmung geeignet, } \mathrm{da} \\
\text { überlagerungsfrei }\end{array}$} \\
\hline 99 & $\left(\mathrm{CH}_{3} \mathrm{CCl}^{37} \mathrm{Cl}^{35}\right)^{+}$ & \\
\hline 97 & $\left(\mathrm{CH}_{3} \mathrm{CCl}_{2}{ }^{35}\right)^{+}$ & \\
\hline 96 & $\left(\mathrm{CH}_{2} \mathrm{CCl}_{2}{ }^{35}\right)^{+}$ & \\
\hline 95 & $\left(\mathrm{CHCCl}_{2}{ }^{35}\right)^{+}$ & \\
\hline 64 & $\left(\mathrm{CH}_{3} \mathrm{CCl}^{37}\right)^{+}$ & \\
\hline 63 & $\left(\mathrm{CH}_{2} \mathrm{CCl}^{37}\right)^{+}$ & Intensität groß \\
\hline 62 & $\left(\mathrm{CH}_{3} \mathrm{CCl}^{35}\right)^{+},\left(\mathrm{CHCCl}^{37}\right)^{+}$ & \\
\hline 61 & $\left(\mathrm{CH}_{2} \mathrm{CCl}^{35}\right)^{+}$ & Intensität groß \\
\hline 60 & $\left(\mathrm{CHCCl}^{35}\right)^{+}$ & \\
\hline 38 & $\left(\mathrm{HCl}^{37}\right)^{+}$ & \\
\hline 37 & $\left(\mathrm{Cl}^{37}\right)^{+}$ & \\
\hline 36 & $\left(\mathrm{HCl}^{35}\right)^{+}$ & \\
\hline 35 & $\left(\mathrm{Cl}^{35}\right)^{+}$ & \\
\hline 27 & $\left(\mathrm{C}_{2} \mathrm{H}_{3}\right)^{+}$ & \\
\hline 26 & $\left(\mathrm{C}_{2} \mathrm{H}_{2}\right)^{+}$ & \\
\hline
\end{tabular}

Tabelle 2: Zuordnung von Fragmenten zum Massenspektrum des $\mathrm{CH}_{3} \mathrm{CCl}_{3}$ 
Die nachfolgende Darstellung zeigt die nach der Binomialverteilung errechnete relative Häufigkeit von $\mathrm{Cl}^{35}$ und $\mathrm{Cl}^{37}$ in Molekülen, die Chloratome enthalten:

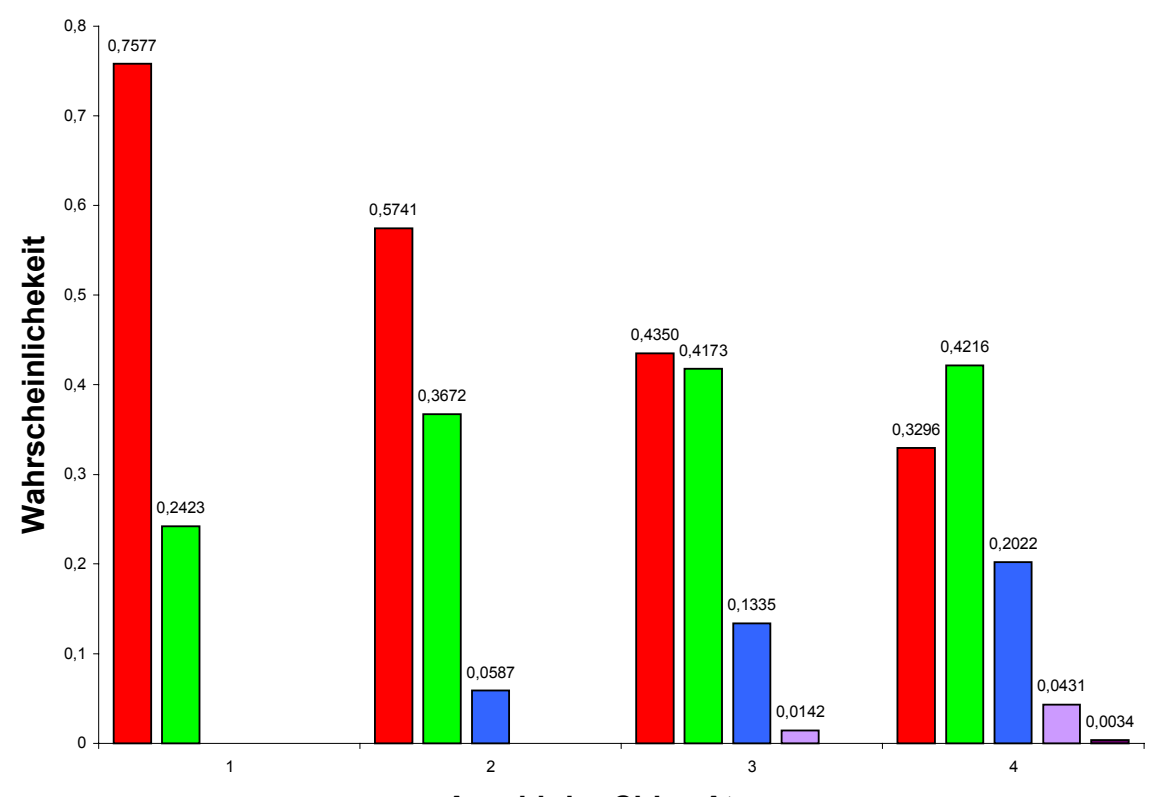

Anzahl der Chlor-Atome

\section{Abbildung 67: Isotopenverteilung von Chloratomen in organischen Molekülen}

Es lässt sich feststellen, dass die gemessenen Intensitätsverhältnisse der entsprechenden Fragmente weitgehend dieser Verteilung entsprechen.

Die für die Reaktion $\mathrm{CH}_{3} \mathrm{CCl}_{3}$ interessanten Massen wurden in Einzelmassenzählung unter den Bedingungen "KW-F", "KW+F" und "KW+F+O" untersucht. Die Ergebnisse sind in Messtabelle 2 zusammengefasst (vgl. Abschnitt 5). Entstehende Produkte lassen sich anhand von Intensitätszunahmen bestimmter Massen erkennen. In der folgenden Tabelle sind die Massen mit wesentlicher Intensitätszunahme aus Messtabelle 2 ausgewählt. Dabei soll nicht jeder Isotopenpeak berücksichtigt werden. Gleichzeitig soll in der Spalte "Interpretation" stichwortartig diskutiert werden, für oder gegen welchen Reaktionskanal der beobachtete Effekt steht. Die hier nicht im Detail diskutierte Reaktion $\mathrm{KW}+\mathrm{F}+\mathrm{O}$ dient als weitere Interpretationshilfe. Die angegebenen Anstiegsfaktoren sind abhängig von der Überlagerung der Massen durch verschiedene Substanzen und deren massenspektrometrischer Empfindlichkeit. Sie dienen lediglich der groben Orientierung. 


\begin{tabular}{|c|c|c|c|c|}
\hline $\mathrm{m} / \mathrm{z}$ & $\begin{array}{l}\mathrm{I}(\mathrm{KW}-\mathrm{F}) \\
/ \text { counts }\end{array}$ & $\begin{array}{c}\mathrm{I}(\mathrm{KW}+\mathrm{F}) \\
/ \text { counts }\end{array}$ & $\begin{array}{c}\text { Anstieg } \\
\text { um } \\
\text { Faktor }\end{array}$ & Interpretation \\
\hline 131 & 251 & 2800 & 11,2 & $\begin{array}{c}\left.\mathrm{CH}_{2} \mathrm{CCl}_{3}{ }^{35}\right)^{+} \text {, massenspektrometrischer Nachweis des } \\
\text { Reaktionskanals (a), Radikalmuttermasse }\end{array}$ \\
\hline 133 & 250 & 2303 & 9,2 & $\left(\mathrm{CH}_{2} \mathrm{Cl}^{37} \mathrm{Cl}_{2}{ }^{35}\right)^{+}, \mathrm{s} . \mathrm{m} / \mathrm{z}=131$ \\
\hline 130 & 5833 & 16170 & 2,8 & $\begin{array}{l}\text { Fragment des Radikals aus Kanal (a), überlagert durch } \\
\text { das Fragment aus dem Ausgangsmolekül } \mathrm{CH}_{3} \mathrm{CCl}_{3}{ }^{35}\end{array}$ \\
\hline 132 & 5957 & 16530 & 2,8 & $\begin{array}{c}\text { Fragment des Radikals aus Kanal (a) (mit } \mathrm{Cl}^{37} \text { im } \\
\text { Radikal), überlagert durch das Signal des } \\
\text { Ausgangsmoleküls } \mathrm{CH}_{3} \mathrm{CCl}_{3}{ }^{35}\end{array}$ \\
\hline 118 & 1477 & 3933 & 2,7 & $\begin{array}{c}\left(\mathrm{CH}_{3} \mathrm{CCl}^{37} \mathrm{C}^{35} \mathrm{~F}\right)^{+} \text {, Produkt des Kanals (b), allerdings } \\
\text { nur in geringem Ausmaß; kaum Abnahme bei } \\
\text { zusätzlicher Zufuhr von O-Atomen: (b) produziert } \\
\text { nicht-radikalisches KW-Produkt }\end{array}$ \\
\hline 116 & 34 & 14330 & 421 & $\begin{array}{c}\left.\mathrm{CH}_{3} \mathrm{CCl}_{2}{ }^{35} \mathrm{~F}\right)^{+} \text {aus }(\mathrm{b}) ;\left(\mathrm{CHCCl}^{37} \mathrm{Cl}^{35} \mathrm{~F}\right)^{+} \text {Fragment aus } \\
\text { (c); hier starke Abnahme bei Bedingung "KW+F+O"; } \\
\text { das stützt die These, dass diese Masse vor allem durch } \\
\text { Kanal (c) bestimmt wird, da dieser radikalisches KW- } \\
\text { Molekül erzeugt }\end{array}$ \\
\hline 115 & 25 & 1443 & 57,7 & $\begin{array}{c}\left.\mathrm{CH}_{2} \mathrm{CCl}_{2}{ }^{35} \mathrm{~F}\right)^{+} \text {, Molekülpeak des radikalischen } \\
\text { Produktes aus Kanal (c); möglich ist auch, dass es sich } \\
\text { teilweise um Fragment aus (b) handelt, aufgrund der } \\
\text { quantitativen Abnahme bei "KW+F+O" aber wohl } \\
\text { nur in geringem Ausmaß }\end{array}$ \\
\hline 114 & 17 & 21130 & 1243 & $\begin{array}{c}\left(\mathrm{CHCCl}_{2}{ }^{35} \mathrm{~F}\right)^{+}, \text {Fragment des Produktes aus }(\mathrm{c})(\mathrm{H}- \\
\text { Atome werden bei } \mathrm{KW} \text { in Ionenquelle leicht } \\
\text { abgespalten })\end{array}$ \\
\hline 103 & 460 & 1014 & 2,2 & $\left(\mathrm{CCl}^{37} \mathrm{Cl}^{35} \mathrm{~F}\right)^{+}$, Fragment aus (c) oder aus (b) \\
\hline 100 & 19000 & 20970 & 1,1 & $\begin{array}{c}\left(\mathrm{CH}_{2} \mathrm{CCl}_{2}{ }^{37}\right)^{+}, \text {Fragment sowohl des Ausgangs-KW als } \\
\text { auch des Radikals aus Kanal (a) }\end{array}$ \\
\hline 98 & 41570 & 58370 & 1,4 & $\left(\mathrm{CH}_{2} \mathrm{CCl}^{37} \mathrm{Cl}^{35}\right)^{+}$, s. $\mathrm{m} / \mathrm{z}=100$ \\
\hline 96 & 21230 & 43570 & 2,1 & $\left(\mathrm{CH}_{2} \mathrm{CCl}_{2}{ }^{35}\right)^{+}$, s. $\mathrm{m} / \mathrm{z}=100$ \\
\hline 95 & 20200 & 27400 & 1,4 & $\left(\mathrm{CHCCl}_{2}{ }^{35}\right)^{+}, \mathrm{s} \cdot \mathrm{m} / \mathrm{z}=100$ \\
\hline 94 & 938 & 1893 & 2,0 & $\left(\mathrm{CCCl}_{2}^{35}\right)^{+}, \mathrm{s} \cdot \mathrm{m} / \mathrm{z}=100$ \\
\hline 85 & 622 & 3613 & 5,8 & $\begin{array}{c}\left(\mathrm{CHCl}^{37} \mathrm{Cl}^{35}\right)^{+} \text {, zunächst Ausgangsmolekülfragment, } \\
\text { aber vor allem Radikalfragment, am ehesten aus (a) } \\
\text { vorstellbar }\end{array}$ \\
\hline 83 & 824 & 2460 & 3,0 & $\left(\mathrm{CHCl}_{2}{ }^{35}\right)^{+}$, s. $\mathrm{m} / \mathrm{z}=85$ \\
\hline 82 & 3723 & 7933 & 2,1 & $\left(\mathrm{CCl}_{2}{ }^{35}\right)^{+}, \mathrm{s} . \mathrm{m} / \mathrm{z}=85 ;\left(\mathrm{CH}_{2} \mathrm{CCl}^{37} \mathrm{~F}\right)^{+}$aus $(\mathrm{c}) \operatorname{oder}(\mathrm{b})$ \\
\hline 81 & 60 & 2603 & 43,4 & $\begin{array}{c}\left(\mathrm{CHCCl}^{37} \mathrm{~F}\right)^{+}, \text {Fragment aus (c); da quantitative } \\
\text { Abnahme bei "KW+F+O" ist Herkunft aus (b) wenig } \\
\text { wahrscheinlich }\end{array}$ \\
\hline 80 & 48 & 14330 & 298,5 & $\left(\mathrm{CH}_{2} \mathrm{CCl}^{35} \mathrm{~F}\right)^{+}, \mathrm{s} . \mathrm{m} / \mathrm{z}=81$ \\
\hline 79 & 264 & 8223 & 31,1 & $\left(\mathrm{CHCCl}^{35} \mathrm{~F}\right)^{+}, \mathrm{s} . \mathrm{m} / \mathrm{z}=81$ \\
\hline 72 & 113 & 4793 & 42,4 & $\begin{array}{c}\left(\mathrm{Cl}^{37} \mathrm{Cl}^{35}\right)^{+} \text {, aus (b); andererseits beachtliche Abnahme } \\
\text { bei "KW+F+O": Teilchen entsteht bei } \\
\text { Fragmentierung von Produkten aus (a) und (c) in der } \\
\text { Ionenquelle }\end{array}$ \\
\hline 70 & 171 & 6810 & 39,8 & $\left(\mathrm{Cl}_{2}^{35}\right)^{+}, \mathrm{s} \cdot \mathrm{m} / \mathrm{z}=72$ \\
\hline 67 & 65 & 2257 & 34,7 & $\left(\mathrm{CCl}^{35} \mathrm{FH}\right)^{+}$, aus (c), s. m/z $=81$ \\
\hline
\end{tabular}




\begin{tabular}{|c|c|c|c|c|}
\hline 64 & 7830 & 9840 & 1,3 & $\begin{array}{l}\left(\mathrm{CH}_{3} \mathrm{CCl}^{37}\right)^{+} \text {, zunächst Ausgangsmolekülfragment, } \\
\text { Steigerung kann aber nur aus (b) heraus erklärt } \\
\text { werden, Ausmaß der Steigerung und die kaum } \\
\text { vorhandene Abnahme bei "KW+F+O" stützen dieses }\end{array}$ \\
\hline 20 & 1223 & 11170 & 9,13 & HF-Bildung \\
\hline \multicolumn{5}{|c|}{ Die folgenden Intensitätszunahmen lassen sich mit Kanal (a), (b) oder (c) nicht mehr erklären: } \\
\hline 149 & - & 2093 & - & $\left(\mathrm{CHFCCl}_{3}{ }^{35}\right)^{+}$ \\
\hline 150 & - & 371 & - & $\begin{array}{l}\left.\mathrm{CH}_{2} \mathrm{FCCl}_{3}{ }^{35}\right)^{+} \text {, es scheint, es gibt noch einen vierten } \\
\text { Reaktionskanal: eine Substitution von } \mathrm{H} \text { durch F. Die } \\
\text { Reaktionsenthalpie errechnet sich zu } \Delta_{\mathrm{R}} \mathrm{H}^{0}{ }_{298 \mathrm{~K}}=-36,3 \\
\mathrm{~kJ} / \mathrm{mol} \text {. Dabei wurde } \Delta_{\mathrm{f}} \mathrm{H}^{0}{ }_{298 \mathrm{~K}}\left(\mathrm{CH}_{2} \mathrm{FCCl}_{3}{ }^{35}\right) \text { von } \\
\text { [STE94] abgeschätzt zu -319,6 KJ/mol. In der } \\
\text { Literatur wurde über diesen Kanal nichts gefunden. }\end{array}$ \\
\hline
\end{tabular}

\section{Tabelle 3: Produktanalyse der Reaktion $\mathrm{CH}_{3} \mathrm{CCl}_{3}+\mathrm{F}$}

Die Reaktionskanäle (a) und (c) können klar nachgewiesen werden. Auch Kanal (b) ist nachzuweisen, allerdings nur in einem kleinen Umfang. Anzeichen für das nicht radikalische Produkt aus Kanal (b) $\mathrm{CH}_{3} \mathrm{CCl}_{2} \mathrm{~F}$ konnten in einer Endproduktanalyse dagegen nicht gefunden werden. Dazu wurde ein Teil des Reaktionsgemisches bei $-190{ }^{\circ} \mathrm{C}$ ausgefroren und einer GCMS-Analyse zugeführt (Versuchsanordnung D). Eine Bestimmung der Kanalverzweigungsverhältnisse ist aufgrund unbekannter massenspektrometrischer Empfindlichkeiten nicht möglich. Eine Kalibrierung diesbezüglich erscheint hoffnungslos, da zwei der drei Kanäle radikalische Kohlenwasserstoffe liefern.

In der Literatur findet sich nur der Reaktionskanal (a) [PLA95], näheres zu dieser Literaturstelle ist in der Diskussion der Reaktionsgeschwindigkeit aufgeführt.

\section{$\underline{\text { Reaktionsgeschwindigkeit: }}$}

Messergebnisse: Die Geschwindigkeit der Reaktion $\mathrm{CH}_{3} \mathrm{CCl}_{3}+\mathrm{F} \rightarrow$ Prod. wurde mit Versuchsanordnung B (EI-MS) relativ zur Geschwindigkeit der Reaktion $\mathrm{CH}_{2} \mathrm{Cl}_{2}+\mathrm{F} \rightarrow$ Prod. im Temperaturbereich -20 bis $+85{ }^{\circ} \mathrm{C}$ untersucht. Die folgenden Abbildungen stellen die Messungen in graphischer Auswertung (vgl. Abschnitt 2.4.2 Relativmessung) dar: 


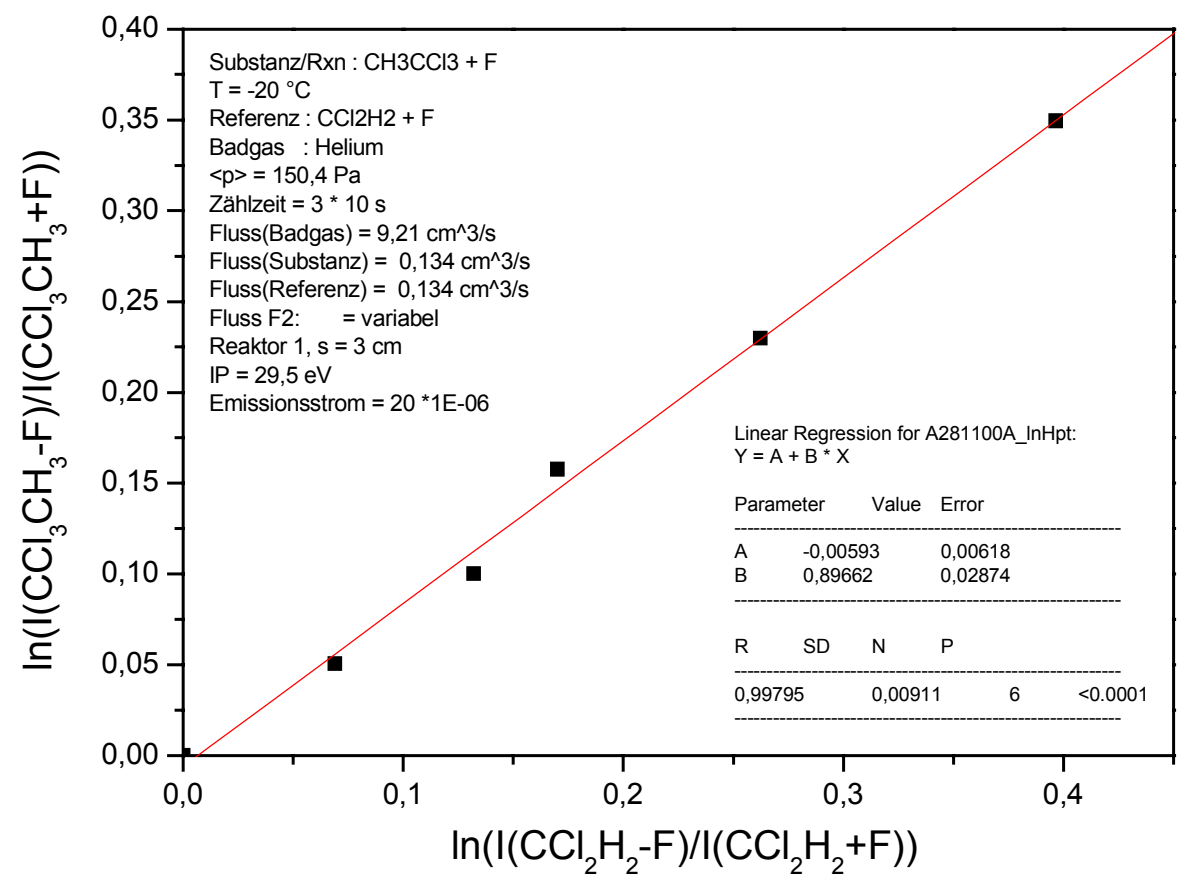

Abbildung 68: $\mathrm{CH}_{3} \mathrm{CCl}_{3}+\mathrm{F}$ relativ zu $\mathrm{CH}_{2} \mathrm{Cl}_{2}+\mathrm{F}, \mathrm{T}=-20^{\circ} \mathrm{C}$

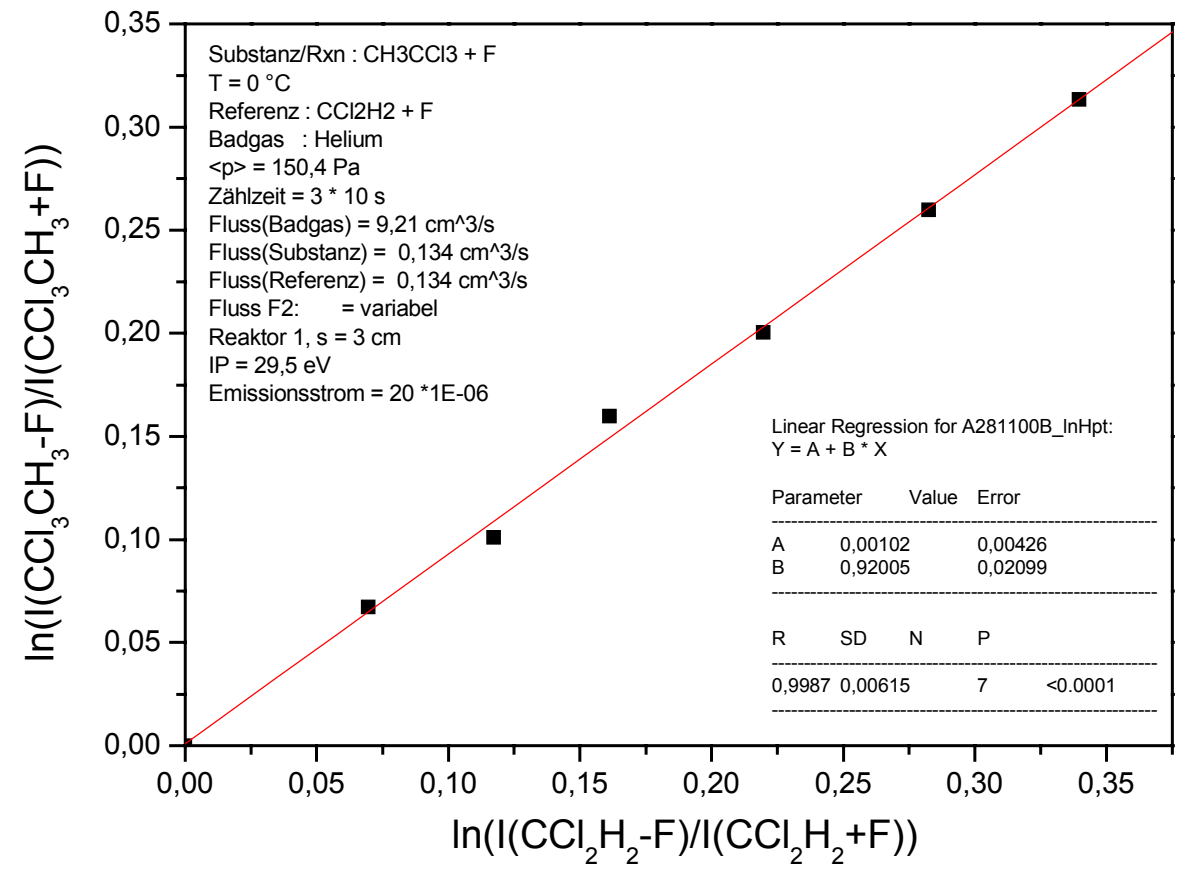

Abbildung 69: $\mathrm{CH}_{3} \mathrm{CCl}_{3}+\mathrm{F}$ relativ zu $\mathrm{CH}_{2} \mathrm{Cl}_{2}+\mathrm{F}, \mathrm{T}=0{ }^{\circ} \mathrm{C}$ 


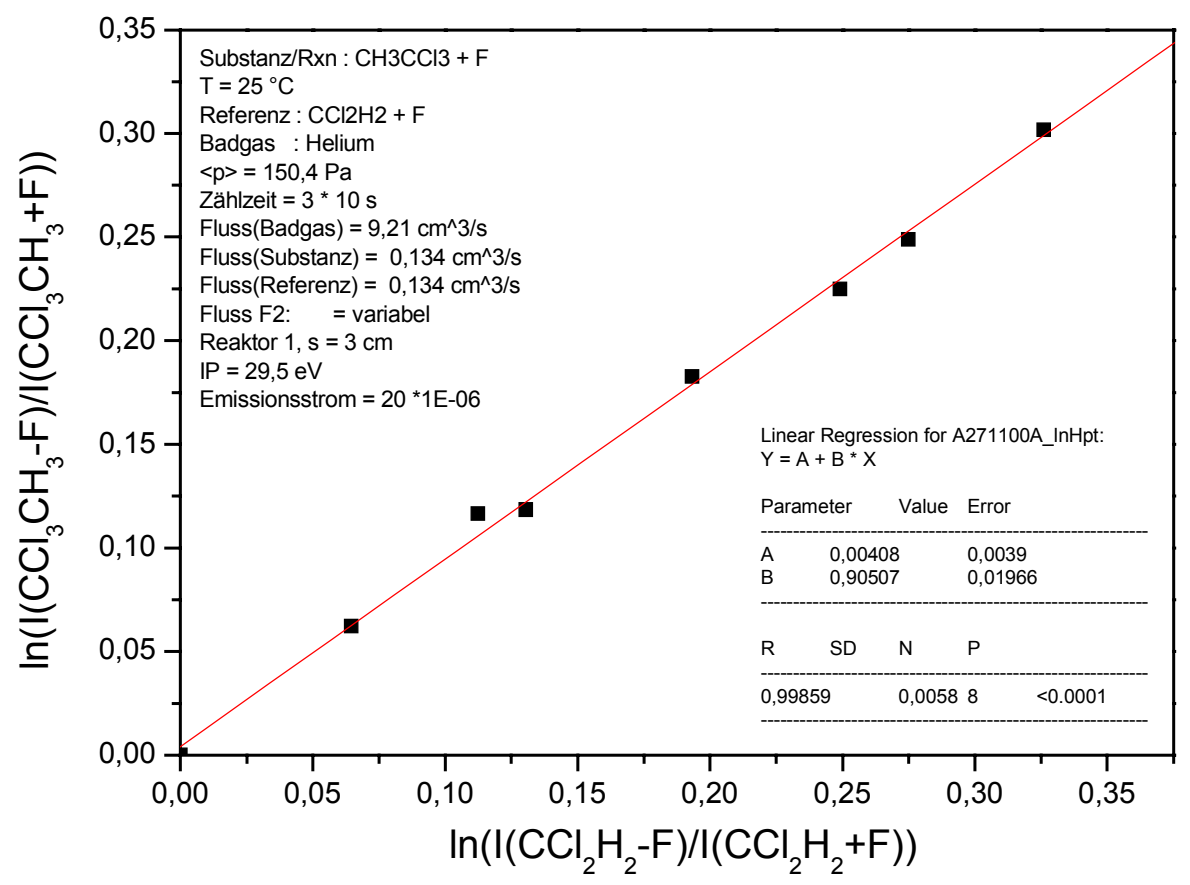

Abbildung 70: $\mathrm{CH}_{3} \mathrm{CCl}_{3}+\mathrm{F}$ relativ zu $\mathrm{CH}_{2} \mathrm{Cl}_{2}+\mathrm{F}, \mathrm{T}=25^{\circ} \mathrm{C}$

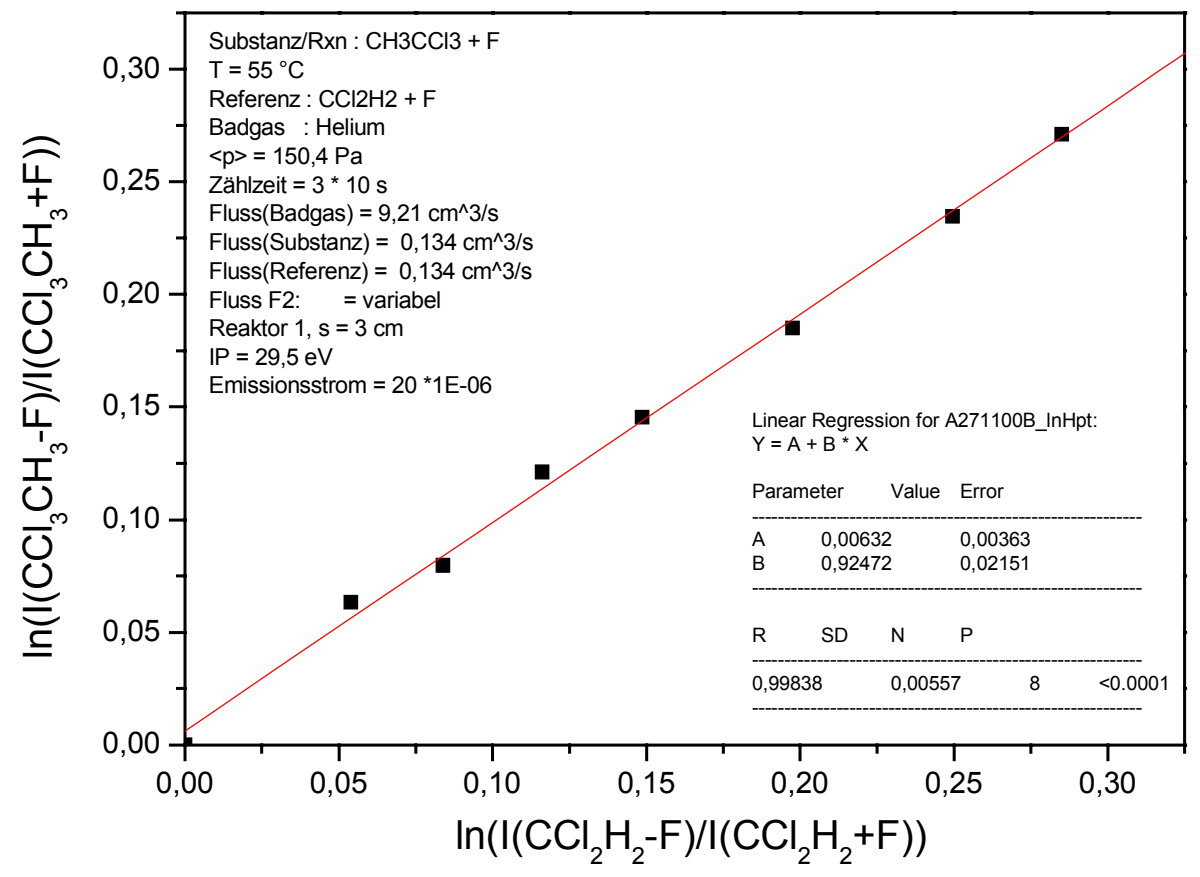

Abbildung 71: $\mathrm{CH}_{3} \mathrm{CCl}_{3}+\mathrm{F}$ relativ zu $\mathrm{CH}_{2} \mathrm{Cl}_{2}+\mathrm{F}, \mathrm{T}=55^{\circ} \mathrm{C}$ 


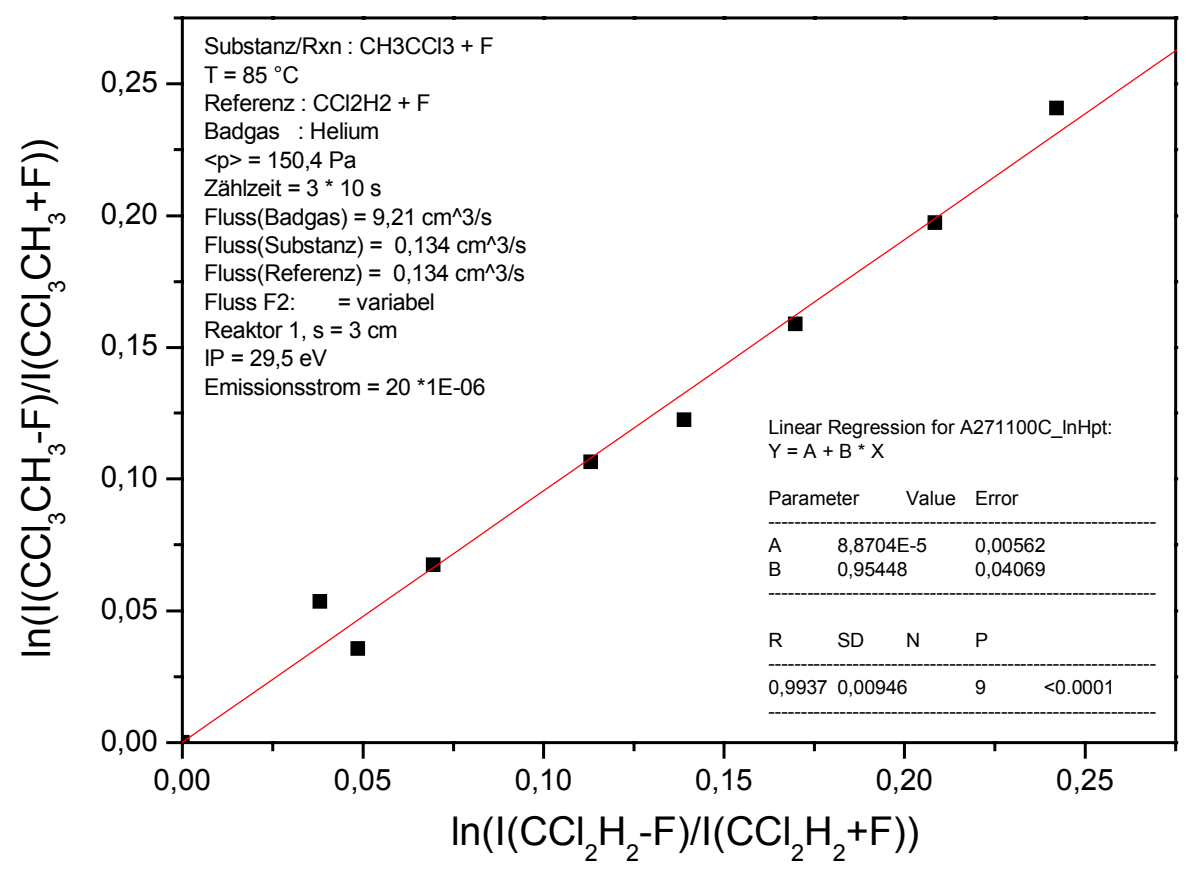

Abbildung 72: $\mathrm{CH}_{3} \mathrm{CCl}_{3}+\mathrm{F}$ relativ zu $\mathrm{CH}_{2} \mathrm{Cl}_{2}+\mathrm{F}, \mathrm{T}=85^{\circ} \mathrm{C}$

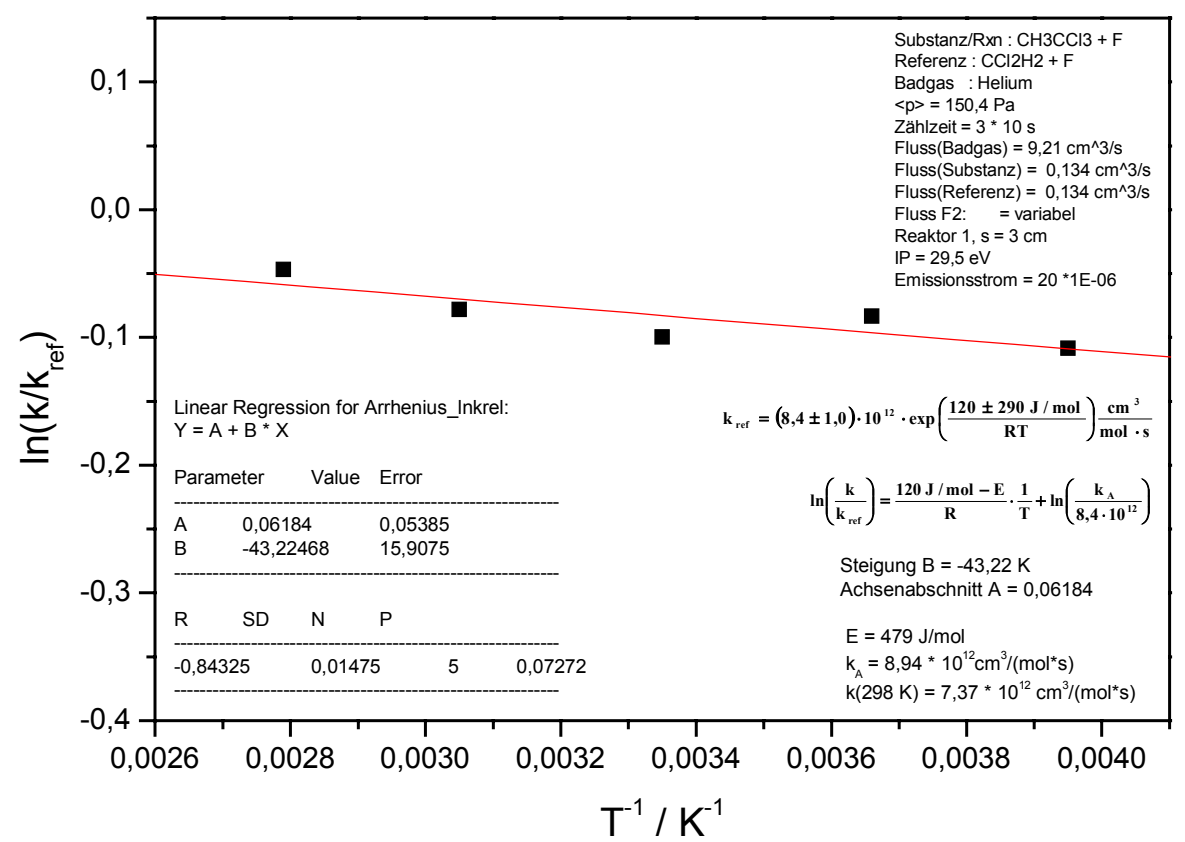

\section{Abbildung 73: $\mathrm{CH}_{3} \mathrm{CCl}_{3}+\mathrm{F}$ relativ zu $\mathrm{CH}_{2} \mathrm{Cl}_{2}+\mathrm{F}$, Arrheniusauftragung}

Die Daten für die Referenzreaktion $\mathrm{CH}_{2} \mathrm{Cl}_{2}+\mathrm{F}$ entstammen [HOL97]. Für die Reaktion $\mathrm{CH}_{3} \mathrm{CCl}_{3}+\mathrm{F}$ ergibt sich damit:

$$
\begin{gathered}
\mathrm{k}=(8,94 \pm 0,49) \cdot 10^{12} \exp \left\{-479 \pm 132 /(\mathrm{RT}) \mathrm{J} \cdot \mathrm{mol}^{-1}\right\} \mathrm{cm}^{3} \cdot \mathrm{mol}^{-1} \cdot \mathrm{s}^{-1} \\
\mathrm{k}^{298}=(7,37 \pm 0,76) \cdot 10^{12} \mathrm{~cm}^{3} \cdot \mathrm{mol}^{-1} \cdot \mathrm{s}^{-1}
\end{gathered}
$$

Die angegebenen Fehlerwerte resultieren aus der Standardabweichung der Regressionsgeraden. 
Diskussion: Die Geschwindigkeit der Reaktion $\mathrm{CH}_{3} \mathrm{CCl}_{3}+\mathrm{F}$ ist von Platz, Nielson, Sehested und Wallington bei Zimmertemperatur und einem Druck von 760 Torr zu k $=4,09 \cdot 10^{12} \mathrm{~cm}^{3} \cdot \mathrm{mol}^{-1} \cdot \mathrm{s}^{-1}$ bestimmt worden [PLA95]. Im Reaktionsraum befand sich ein $\mathrm{CH}_{3} \mathrm{CCl}_{3} / \mathrm{SF}_{6} / \mathrm{O}_{2}-\mathrm{Gemisch}$. Die F-Atome wurden durch Radiolyse von $\mathrm{SF}_{6}$ erzeugt, der Nachweis erfolgte mit UV-Absorptionsspektroskopie des entstehenden $\mathrm{CCl}_{3} \mathrm{CH}_{2} \mathrm{O}_{2}$ bei $\lambda=250 \mathrm{~nm}$. Es wurde nur der Abstraktionskanal (a) bei der Aufklärung der Kinetik berücksichtigt. Das kann ein Grund dafür sein, dass der ermittelte Wert geringer ausfällt als in dieser Arbeit.

\subsubsection{Isobutylchlorid $+F$}

Isobutylchlorid wird bei synthetischen Reaktionen zur Einführung der Isobutylgruppe und als Lösungsmittel eingesetzt [FAL91].

Messergebnisse: Die Reaktion des Isobutylchlorids (1-Chlor-2-Methyl-Propan) mit F-Atomen wurde mit Versuchsanordnung A (REMPI) untersucht. Die folgende Abbildung zeigt ein Flugzeitmassenspektrum bei $\lambda=440 \mathrm{~nm}$. Ein Untergrund durch das Edukt Isobutylchlorid war nicht zu verzeichnen.

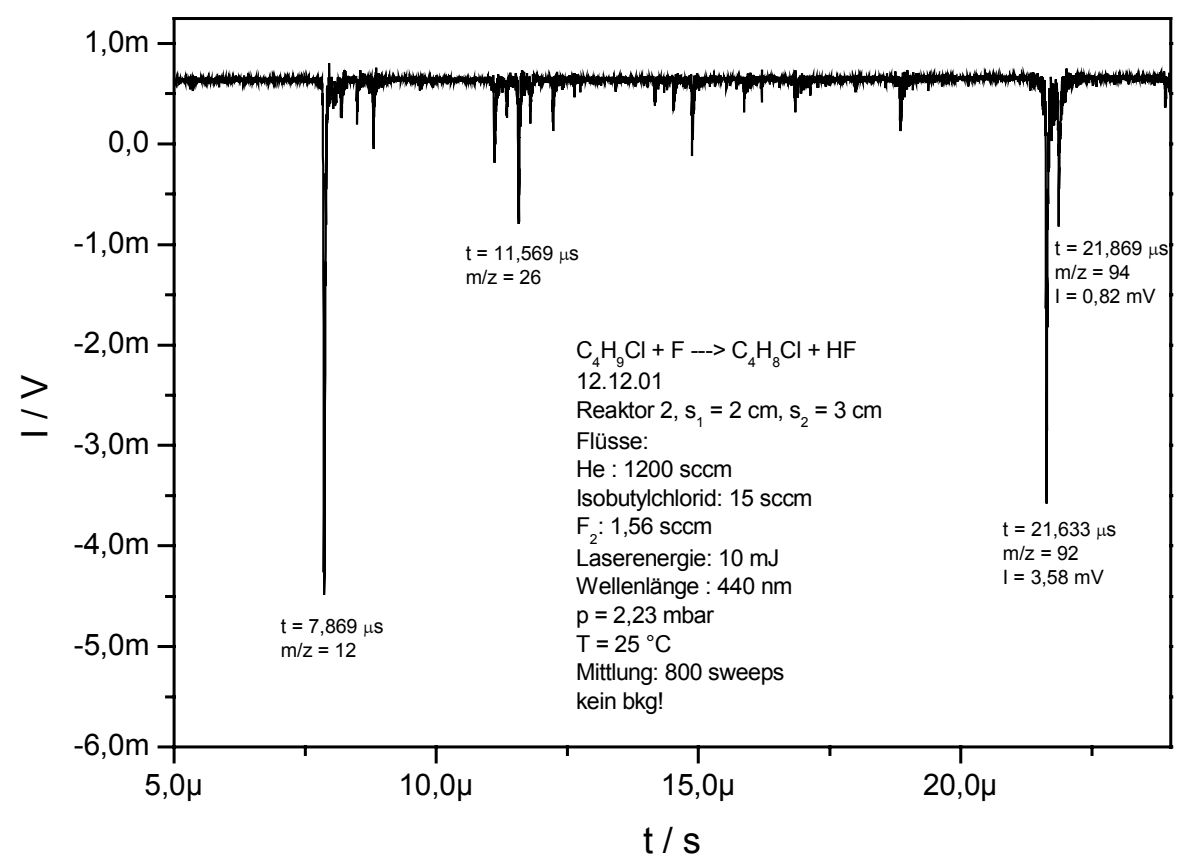

Abbildung 74: Flugzeitmassenspektrum Isobutylchlorid $+\mathrm{F}, \lambda=440 \mathrm{~nm}$

Interpretation: Wie bei der Reaktion des t-Butylchlorids mit F-Atomen (vgl. Abschnitt 3.1.11) zeigen sich intensive Peaks auf den Massen $\mathrm{m} / \mathrm{z}=92$ und 94, welche auf eine Radikalbildungsreaktion durch H-Atomabstraktion schließen lassen. Beachtliche Signale sind auch bei $\mathrm{m} / \mathrm{z}=26,24$ und 12 
festzustellen. Ein in analoger Weise durchgeführtes Experiment bei $\lambda=500 \mathrm{~nm}$ zeigt nur noch geringe Signale.

Die Reaktion des Isobutylchlorids mit F-Atomen unter Bildung von Fluorwasserstoff (H-Atomabstraktion) kann in drei isomere Radikale münden:<smiles>CCC(C)(C)CCl</smiles><smiles>CC(C)CCl</smiles><smiles>CC(C)C(Cl)Cl</smiles><smiles>CCCC(C)CCl</smiles>

Da mit den vorhandenen Mitteln nicht geklärt werden kann, welche der entstehenden Radikale zu den gefundenen Signalen bei $\mathrm{m} / \mathrm{z}=92$ und 94 beitragen, wird diese Reaktion nicht weiter betrachtet.

\subsubsection{Di-tertiär-Butyl-Peroxid + F}

Diskussion: Die Reaktion des Di-tertiär-Butyl-Peroxids (DtBP) mit F-Atomen wurde vom Autor bereits im Rahmen einer Staatsexamensarbeit umfassend mit Hilfe der Versuchsanordnung B (EI-MS) untersucht [WEH97]. Aufgrund der Messergebnisse wurde folgender Mechanismus vorgeschlagen:<smiles>CC(C)(C)OOC(C)(C)[In]F</smiles> 


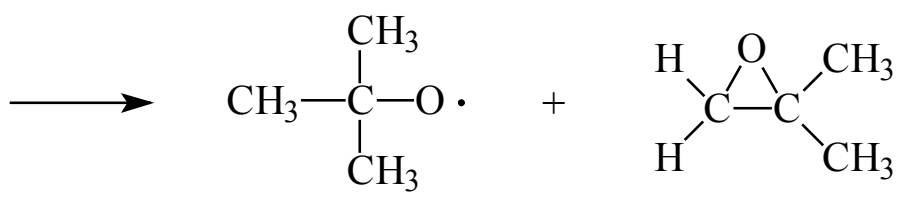

Die Geschwindigkeit dieser Reaktion wurde ermittelt zu k $\mathrm{k}^{298 \mathrm{~K}}=(1,5 \pm 0,05) \cdot 10^{14} \mathrm{~cm}^{3} /(\mathrm{mol} \cdot \mathrm{s})$. Das Epoxid konnte massenspektrometrisch nachgewiesen werden. Das t-Butoxyradikal konnte nicht direkt beobachtet werden.

Messergebnisse: Im Rahmen dieser Arbeit wurde das Thema durch Messungen mit Versuchsanordnung A (REMPI) neu aufgenommen. Im UV- bzw. kurzwelligen Vis-Bereich zeigt DtBP auch ohne Reaktion mit F-Atomen bereits starke Fragmentsignale auf diversen Massen. Deshalb konzentrierten sich die Untersuchungen auf den grünen Wellenlängenbereich $(\lambda=500-560 \mathrm{~nm})$, wo sich nur noch ein schwaches Molekülsignal bei $\mathrm{m} / \mathrm{z}=57$ findet. Die folgende Abbildung zeigt jeweils ein Flugzeitmassenspektrum unter den Bedingungen DtBP+F und DtBP-F bei $\lambda=520 \mathrm{~nm}$ :

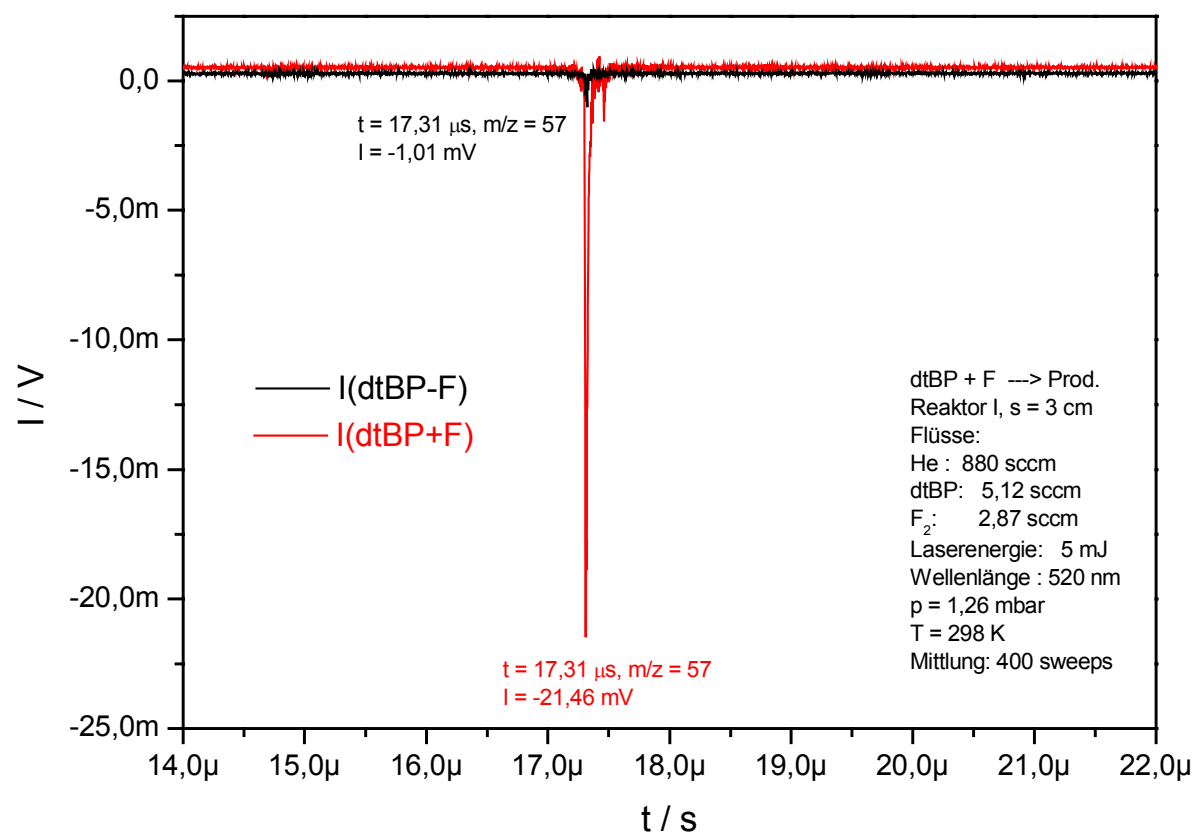

\section{Abbildung 75: Flugzeitmassenspektrum DtBP-F/DtBP+F, $\lambda=520 \mathrm{~nm}$}

Es zeigt sich ein starkes Ionensignal bei $\mathrm{m} / \mathrm{z}=57$. Dieses Signal ist nicht wellenlängenselektiv, es ist bei allen zugänglichen Wellenlängen unterhalb $570 \mathrm{~nm}$ zu beobachten. Der Untergrund durch das Molekül ohne Reaktion mit F-Atomen kann bei dieser Wellenlänge vernachlässigt werden. Ein Signal bei $\mathrm{m} / \mathrm{z}=73$, dem Mutterpeak des t-Butoxyradikals, kann nicht beobachtet werden. Es stellt sich die Frage, ob das beobachtete Signal nicht vom ebenfalls denkbaren Produkt t-Butylradikal 
herrühren könnte. Deshalb wurde das Verhalten des Signals bei zusätzlicher Zugabe von O-Atomen beobachtet und ein Vergleich zum t-Butylradikal, welches über die Reaktion Isobutan + F erzeugt wurde (vgl. Abschnitt 3.1.5, eindeutiger Nachweis des t-Butylradikals bei $\lambda>490 \mathrm{~nm}$ ), unternommen. Die folgende Darstellung zeigt Flugzeitmassenspektren von Isobutan $+\mathrm{F}-\mathrm{O}$ und Isobutan $+\mathrm{F}+\mathrm{O}$ bei $\lambda=520 \mathrm{~nm}$ :

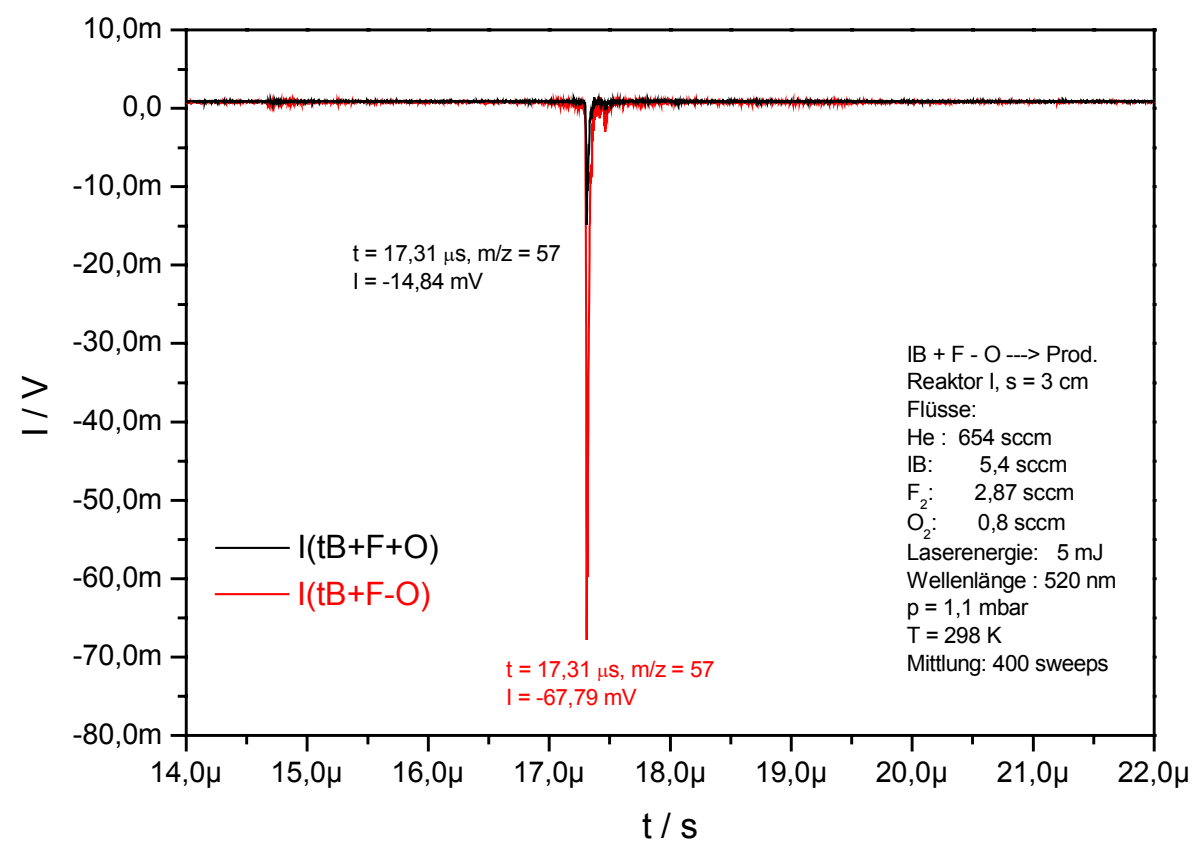

\section{Abbildung 76: Flugzeitmassenspektrum t-Butyl $+/-\mathrm{O}, \lambda=520 \mathrm{~nm}$}

Wie zu erwarten, zeigt sich infolge der Reaktion der t-Butylradikale mit O-Atomen eine deutliche Abnahme des Signals (vgl. Abschnitt 3.3.9). Die nächste Abbildung stellt Flugzeitmassenspektren von $\mathrm{DtBP}+\mathrm{F}-\mathrm{O}$ und $\mathrm{DtBP}+\mathrm{F}+\mathrm{O}$ bei derselben Wellenlänge dar: 


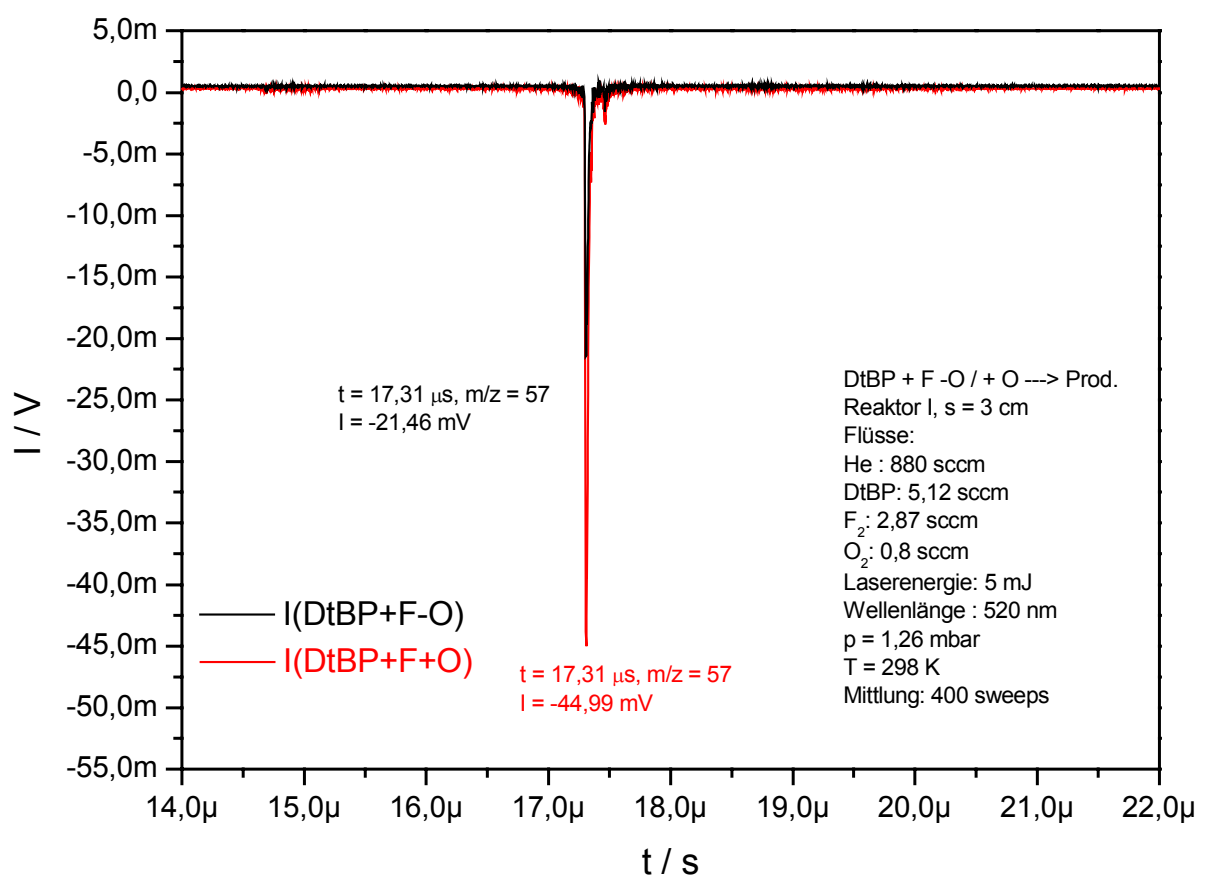

\section{Abbildung 77: Flugzeitmassenspektrum DtBP+F-O / DtBP+F+O, $\lambda=520 \mathrm{~nm}$}

Interpretation: Es zeigt sich ein völlig gegensätzliches Verhalten. Statt einer Abnahme von 78\%, wie es sich beim t-Butylradikal, erzeugt über Isobutan + F, findet, ist eine Zunahme um den Faktor 2 zu beobachten. Das beobachtete Ionensignal der Reaktion DtBP $+\mathrm{F}$ bei $\mathrm{m} / \mathrm{z}=57 \mathrm{kann}$ demnach nicht vom t-Butylradikal stammen. Es muss sich um ein anderes Teilchen handeln. Die Tatsache, dass sich das Signal auch im langwelligen Bereich so kräftig zeigt, legt einen radikalischen Ursprung nahe. Typischerweise sind Alkoxyradikale massenspektrometrisch inaktiv. Es kann ein Abspalten des O-Atoms in der Ionenquelle vermutet werden. Damit würde es beim Signal bei $\mathrm{m} / \mathrm{z}=57$ der Reaktion DtBP + F um ein Fragment des t-Butoxyradikals handeln. Wie ist dann die deutliche Zunahme bei Zugabe von O-Atomen zu erklären? Ausgehend von einer Anlagerung der O-Atome an die Radikalstelle des t- $\mathrm{C}_{4} \mathrm{H}_{9} \mathrm{O}$ bildet sich t- $\mathrm{C}_{4} \mathrm{H}_{9} \mathrm{OO}$. Dieses Peroxyradikal könnte nun durch die Wirkung des Laserlichts in der Ionenquelle unter Abspaltung von $\mathrm{O}_{2}$ bevorzugt auf $\mathrm{m} / \mathrm{z}=57$ fragmentieren. Allerdings kann auch ein Zerfall des Peroxyradikals zum t-Butylradikal und $\mathrm{O}_{2}$ im Reaktionsraum nicht ausgeschlossen werden, so dass das Ionensignal von $\mathrm{m} / \mathrm{z}=57$ unter der Bedingung $\mathrm{DtBP}+\mathrm{F}+\mathrm{O}$ eine Mischung aus dem fragmentierten t-Butoxy und einem Zerfallsprodukt t-Butylradikal darstellen würde. Eine Klärung dieser Frage erscheint nicht ohne weiteres möglich.

Eine Messung unter den Bedingungen DtBP-F+O ließ kein Ionensignal erscheinen, eine direkte Reaktion DtBP + O konnte bei den vorgegebenen Reaktionszeiten also nicht festgestellt werden. 
Zusammenfassend lässt sich sagen, dass aufgrund des chemischen Verhaltens das t-Butylradikal als Produkt der Reaktion DtBP + F ausgeschlossen werden muss. Die oben vorgeschlagene Reaktionsgleichung von DtBP + F zum 2,2-Di-Methyloxiran (Epoxid) und zum t-Butoxyradikal wird weiterhin aufrecht erhalten.

Ein Versuch eine spektrale Unterscheidung zwischen t-Butyl- und dem postulierten t-Butoxyradikal herbeizuführen, also eine Wellenlänge im langwelligen Bereich zu finden, bei der nur noch t-Butyl aus Isobutan + F oder das Produkt aus DtBP + F signalgebend ist, schlug fehl: Ab $560 \mathrm{~nm}$ streben die Signalintensitäten gegen null. Bis dahin ist das chemische Verhalten unter der Bedingung $+\mathrm{O}$ aber wie oben beschrieben.

\subsection{4 tertiär-Butyl-Alkohol + F}

Diskussion: Die Untersuchung der Reaktion t-Butylalkohol (tBAl) $+\mathrm{F}$ wurde anhand des an der Hydroxylgruppe deuterierten Kohlenwasserstoffs t- $\mathrm{C}_{4} \mathrm{H}_{9} \mathrm{OD}$ mit Hilfe der Versuchsanordnung A (REMPI) durchgeführt. Die Untersuchungen stehen in einem Interpretationszusammenhang zu der im vorigen Abschnitt untersuchten Reaktion DtBP + F. Es sind zwei Reaktionskanäle denkbar:

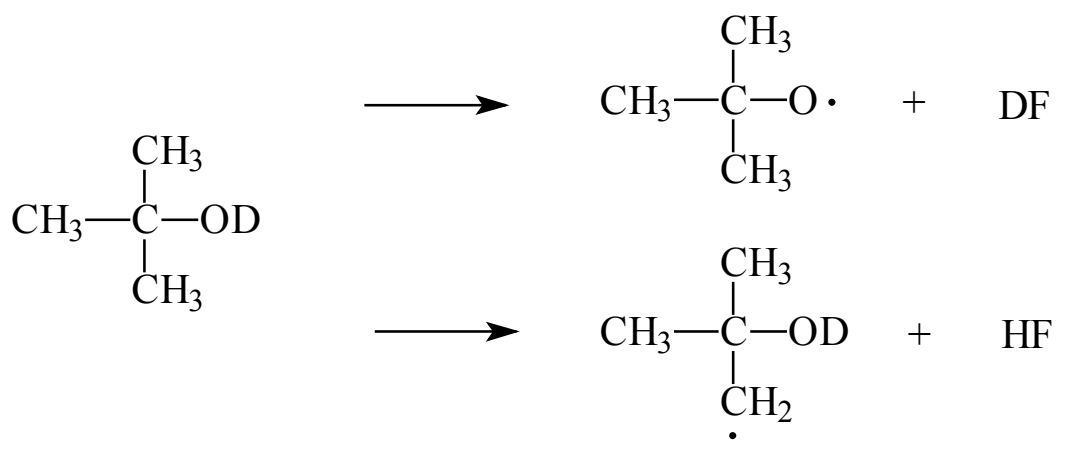

Für den Reaktionskanal (a) kann auf Basis des nicht-deuterierten Alkohols eine Reaktionsenthalpie von $\Delta_{\mathrm{R}} \mathrm{H}^{0}{ }_{298 \mathrm{~K}}=-129,8 \mathrm{~kJ} / \mathrm{mol}$ angegeben werden. Für Kanal (b) ist dieses mangels Daten für das entstehende Radikal nicht möglich. Ein Geschwindigkeitskoeffizient für diese Reaktion ist nicht bekannt. Jedoch findet sich in der Literatur für die Reaktion t- $\mathrm{C}_{4} \mathrm{H}_{9} \mathrm{OH}+\mathrm{Cl}$ bei 760 Torr ein Wert von $\mathrm{k}^{298}=1,96 \cdot 10^{13} \mathrm{~cm}^{3} /(\mathrm{mol} \cdot \mathrm{s})$ [WAL88]. Diese Angabe in Verbindung mit den Werten von Reaktionen vergleichbarer Kohlenwasserstoffe mit F-Atomen lässt vermuten, dass eine H-Abstraktionsreaktion unter den üblichen Bedingungen in Versuchsanordnung A stattfindet.

Messergebnisse: Es wurden Flugzeitmassenspektren bei verschiedenen Ionisierungswellenlängen aufgenommen. Bei einigen Messungen wurden zusätzlich O-Atome hinzugegeben. Die folgende Abbildung zeigt ein Flugzeitmassenspektrum unter den Bedingungen $\mathrm{KW}+\mathrm{F}-\mathrm{O} /+\mathrm{O}$ bei 
$\lambda=520 \mathrm{~nm}$. Das Spektrum ist untergrundfrei, d.h., t-Butylalkohol ist bei dieser Wellenlänge nicht signalgebend.

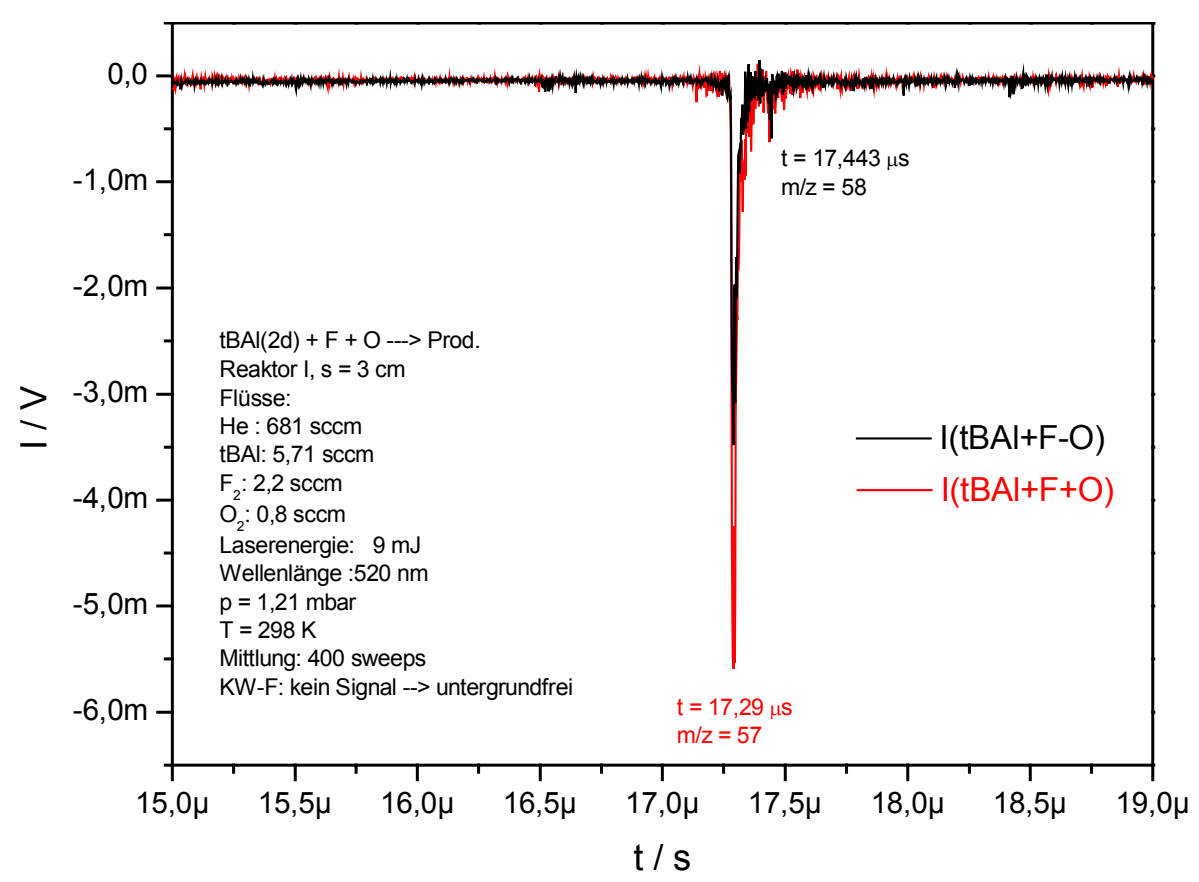

Abbildung 78: Flugzeitmassenspektrum t- $\mathrm{C}_{4} \mathrm{H}_{9} \mathrm{OD}+\mathrm{F}+\mathrm{O}, \lambda=520 \mathrm{~nm}$

Unter der Bedingung KW + F-O ist ein Ionensignal bei m/z = $57 \mathrm{zu}$ beobachten (schwarze Linie), welches bei zusätzlicher Zugabe von O-Atomen eine beachtliche Verstärkung findet (rote Linie).

Interpretation: Dieses Signal kann im Sinne der Überlegungen des Abschnitts Di-tertiär-Butyl-Peroxid + F (vgl. Abschnitt 3.2.3) als ein Fragment des t-Butoxyradikals interpretiert werden (Kanal (a)). Eine Auslegung unter Heranziehung des Kanals (b) erscheint dagegen nicht sinnvoll. Ein Fragmentierungsprozess des dort entstehenden $\mathrm{C}_{4} \mathrm{H}_{8} \mathrm{OD}$, der zu einem Signal von $\mathrm{m} / \mathrm{z}=57$ führt, ist nicht einleuchtend.

Das Ausmaß des Anwachsens unter der Zugabe von O-Atomen entspricht den Messungen des Systems DtBP + F -O/+O. Die Zunahme wurde dort durch eine $\mathrm{O}_{2}$-Abspaltung entweder als Folgereaktion oder als Ionenquellenprozess interpretiert. Es liegt der Schluss nahe, dass tBAl + F und DtBP + F zu demselben radikalischen Produkt, dem t-Butoxy, führen. Die Existenz des Kanals (b) der Reaktion $\mathrm{tBAl}+\mathrm{F}$, welcher zum $\mathrm{C}_{4} \mathrm{H}_{8} \mathrm{OD}$-Radikal führt, ist damit nicht ausgeschlossen. Es muss aber unterstellt werden, dass dieser Kanal bei der Wellenlänge $\lambda=520 \mathrm{~nm}$ zumindest nicht signalgebend ist. Die Messungen der Reaktion des deuterierten t-Butylalkohols mit F-Atomen 
ihrerseits bestärken wiederum die Argumentationslinie zur Erklärung der Signale der Reaktion $\mathrm{DtBP}+\mathrm{F}$.

Diskussion: Ein Vergleich mit der Literatur zeigt, dass bei Alkoholen die Abstraktion des an der Hydroxylgruppe positionierten H-Atoms (im Falle dieser Messungen des D-Atoms) begünstigt ist. Bei der Reaktion des Ethanols mit F-Atomen hat trotz eines statistischen Verhältnisses dieses H-Atoms zu den übrigen von 1:5 eine Abstraktion dieses Atoms gegenüber den anderen einen Kanalanteil von 40-48\% ([BAR82],[KHA83],[MEI85]). Im Analogieschluss scheint Kanal (a) der Reaktion $\mathrm{tBAl}+\mathrm{F}$ chemisch begünstigt zu sein.

Messergebnisse: Im folgenden ist ein Flugzeitmassenspektrum unter den Bedingungen KW -F/+F bei $\lambda=440 \mathrm{~nm}$ dargestellt:

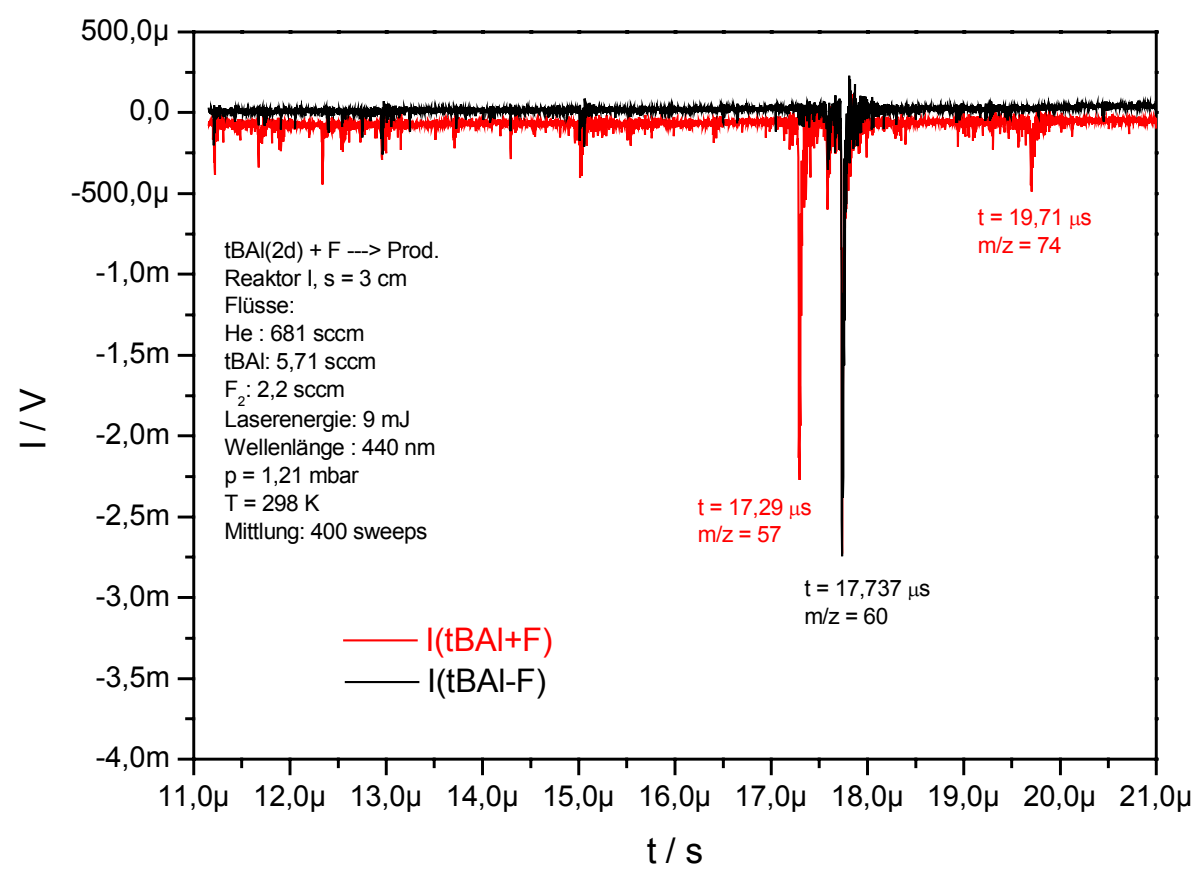

Abbildung 79: Flugzeitmassenspektrum t- $\mathrm{C}_{4} \mathrm{H}_{9} \mathrm{OD}+\mathrm{F}, \lambda=440 \mathrm{~nm}$

Interpretation: Erneut ist untergrundfrei ein Ionensignal bei $\mathrm{m} / \mathrm{z}=57$ unter der Bedingung $+\mathrm{F}$ zu beobachten, welches nach den vorhergehenden Überlegungen dem t-Butoxyradikal zuzuordnen ist. Des Weiteren ist ein schwaches Ionensignal bei $\mathrm{m} / \mathrm{z}=74$ unter der Bedingung $+\mathrm{F}$ zu detektieren. Das ist die Molekülmasse des $\mathrm{C}_{4} \mathrm{H}_{8} \mathrm{OD}$-Radikals aus Kanal (b). Zusätzlich existiert bei dieser Wellenlänge aber auch ein Molekülfragment bei $\mathrm{m} / \mathrm{z}=60$, welches unter der Bedingung -F bereits vorhanden ist. Es muss sich um das $\left(\left(\mathrm{CH}_{3}\right)_{2} \mathrm{COD}\right)^{+}$handeln, welches nach Abspaltung von $\mathrm{CH}_{3}$ in der Ionenquelle entsteht. Was in dieser Abbildung nicht unmittelbar zu sehen ist, durch Farbinversion aber visualisiert werden kann, ist die Tatsache, dass dieses Signal unter der Bedingung +F 
kaum abnimmt. Da der Umsatz des Alkohols mit F-Atomen aber auf ca. 15\% eingestellt ist, muss ein Produkt der Reaktion t- $\mathrm{C}_{4} \mathrm{H}_{9} \mathrm{OD}+\mathrm{F}$ auf dieser Masse ebenfalls fragmentieren. Hier wiederum ist eine Fragmentierung des $\mathrm{t}-\mathrm{C}_{4} \mathrm{H}_{9} \mathrm{O}$ aus Kanal (a) $\mathrm{zu} \mathrm{m} / \mathrm{z}=60$ nicht vorstellbar. Es muss sich also um ein Fragment des $\mathrm{C}_{4} \mathrm{H}_{8} \mathrm{OD}$-Radikals aus Kanal (b) handeln. Kanal (b) ist demnach ebenfalls relevant.

Messergebnisse: Aufschlussreich ist auch das Verhalten der Peaks bei zusätzlicher Zugabe von O-Atomen bei dieser Wellenlänge. Die folgenden beiden Abbildungen zeigen ein und dasselbe Flugzeitmassenspektrum jeweils unter der Bedingung $+\mathrm{F}-\mathrm{O} /+\mathrm{O}$, aber umgekehrt farblich dargestellt:
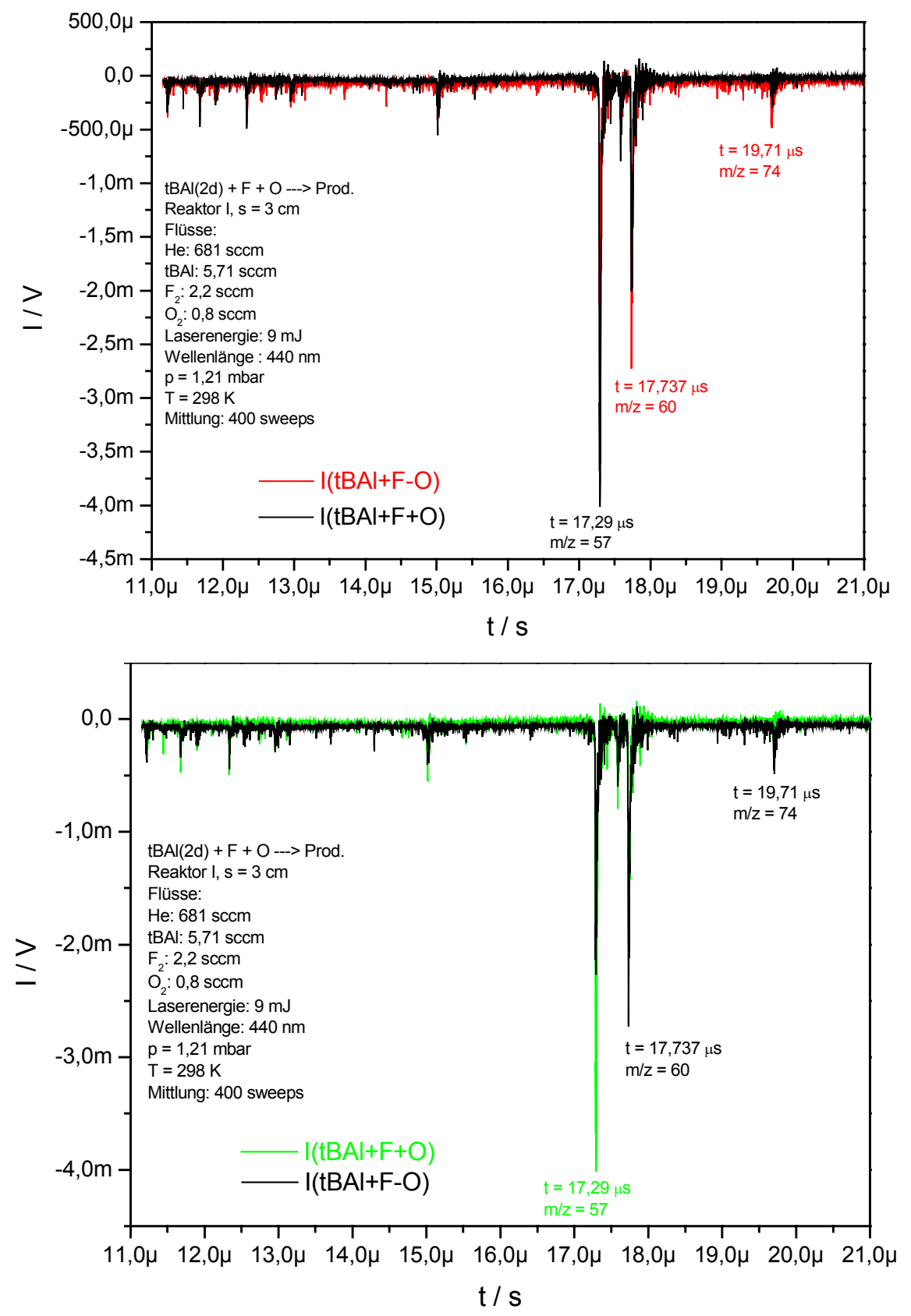

Abbildung 80: Flugzeitmassenspektrum t- $\mathrm{C}_{4} \mathrm{H}_{9} \mathrm{OD}+\mathrm{F}+\mathrm{O}, \lambda=440 \mathrm{~nm}$ 
Bei den Massen 74 und 60 sind bei Zugabe von O-Atomen Abnahmen zu verzeichnen, welche mit der Reaktion des aus Kanal (b) entstehenden Radikals $\mathrm{C}_{4} \mathrm{H}_{8} \mathrm{OD}$ mit O-Atomen zu erklären sind. Die Abnahme auf $\mathrm{m} / \mathrm{z}=60$ fällt anteilsmäßig geringer aus als die bei $\mathrm{m} / \mathrm{z}=74$. Das verwundert nicht, denn $\mathrm{m} / \mathrm{z}=60$ ist auch Fragment des Moleküls $\mathrm{C}_{4} \mathrm{H}_{9} \mathrm{OD}$. Bei $\mathrm{m} / \mathrm{z}=57$ ist wie schon bei $\lambda=520 \mathrm{~nm}$ unter der Bedingung $+\mathrm{O}$ eine beachtliche Zunahme zu registrieren, welche oben bereits interpretiert wurde.

Es wurden weitere Untersuchungen des Systems bei $\lambda=390 \mathrm{~nm}$ unternommen. Bei dieser kurzen Wellenlänge nimmt der Fragmentierungsanteil sowohl vom Edukt tBAl als auch von den Produkten der Reaktion mit F-Atomen insbesondere zu den Massen 12, 13, 14 und 15 deutlich zu. Bei den aussagekräftigen Massen 57, 60 und 74 ist dasselbe Verhalten wie bei $\lambda=440 \mathrm{~nm}$ zu beobachten. Unter der Bedingung KW $+F$ taucht bei $\mathrm{m} / \mathrm{z}=55$ ein zusätzliches schwaches Signal auf, welches untergrundfrei ist, d.h., bei KW-F nicht zu beobachten ist. Dieses Signal verhält sich bei Zugabe von O-Atomen genauso wie $\mathrm{m} / \mathrm{z}=74$ und ist deshalb dem $\mathrm{C}_{4} \mathrm{H}_{8} \mathrm{OD}$-Radikal aus Kanal (b) nach einer Abspaltung einer $\mathrm{CH}_{3}$-Gruppe und des D-Atoms oder zweier H-Atome in der Ionenquelle zuzuordnen. Da in diesem Vorgang aber kein neuer entscheidender Erkenntniswert enthalten ist, wird auf eine graphische Darstellung verzichtet.

\subsection{5 tertiär-Butyl-Peroxy-Pivalat + F}

Diskussion: Die Reaktion des t-Butyl-Peroxy-Pivalats (tBPP) mit F-Atomen wurde vom Autor bereits im Rahmen einer Staatsexamensarbeit umfassend mit Hilfe der Versuchsanordnung B (EI-MS) untersucht [WEH97]. Der Peroxy-Ester eröffnet aufgrund seines unsymmetrischen Molekülbaus zwei Möglichkeiten einer H-Atomabstraktion. Folgende Reaktionskanäle wurden auf Basis der Messergebnisse vorgeschlagen:<smiles>CC(C)(C)OOC(=O)C(C)(C)[In]C(F)F</smiles><smiles>CC(C)(C)[O+]C(=O)[OH2+]</smiles> 
<smiles>CC(C)(C)OOC(=O)C(C)(C)[In]C(F)F</smiles><smiles>C=C(C)CCCCCC(=O)OC(C)(C)C</smiles>

Die Geschwindigkeit dieser Reaktion wurde ermittelt $\mathrm{zu} \mathrm{k}^{298 \mathrm{~K}}=(3,2 \pm 0,3) \cdot 10^{14} \mathrm{~cm}^{3} /(\mathrm{mol} \cdot \mathrm{s})$. Wie bei DtBP + F wird ein Bruch der schwachen O-O Bindung unterstellt.22 Auch bei den Untersuchungen zur Reaktion tBPP + F konnten damals nur die nicht-radikalischen Produkte nachgewiesen werden.

Messergebnisse: Im Rahmen dieser Arbeit wurden die Untersuchungen mittels Versuchsanordnung A (REMPI) erneut aufgenommen. Wegen der nicht unerheblichen Fragmentierung des tBPP bei kurzen Wellenlängen konzentrierten sich die Messungen auf Wellenlängen größer als $\lambda=500 \mathrm{~nm}$. Die folgende Abbildung zeigt Flugzeitmassenspektren unter den Bedingungen tBPP-F und tBPP+F bei $\lambda=520 \mathrm{~nm}$ :

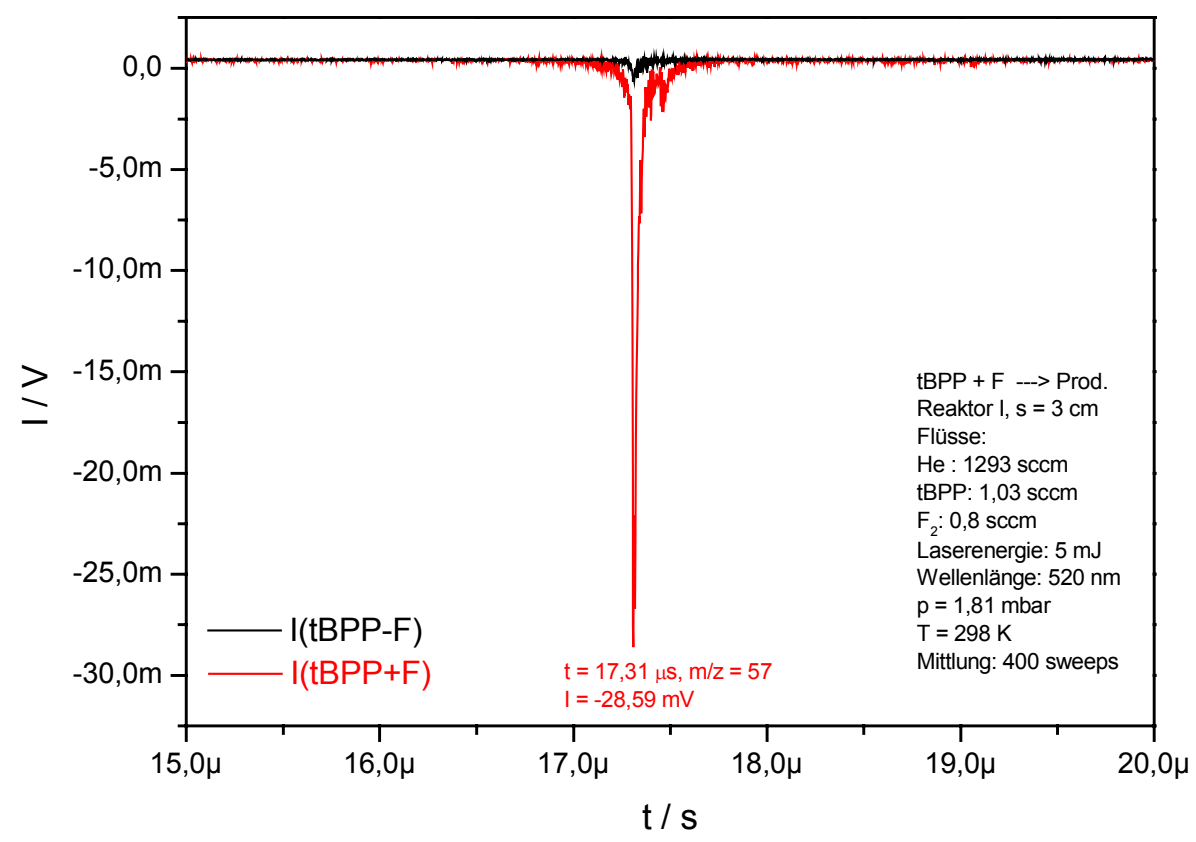

Abbildung 81: Flugzeitmassenspektrum tBPP - F/+ F, $\lambda=520 \mathrm{~nm}$

22 Die Bindungsdissoziationsenergie dieser Bindung wurde nach Werten von [BEN76] und [PED77] zu 166,5 kJ/mol errechnet. 
Es ist ein nahezu untergrundfreies intensives Ionensignal bei $\mathrm{m} / \mathrm{z}=57$ erkennbar. Unter Berücksichtigung der Ergebnisse der Reaktion Isobutan + F (vgl. Abschnitt 3.1.5, eindeutiger Nachweis des t-Butylradikals) sowie der Reaktionen DtBP + F und t-Butylalkohol + F (vgl. Abschnitte 3.2.3 und 3.2.4) könnten sowohl das t-Butylradikal aus Reaktionskanal (a) als auch das t-Butoxyradikal aus Reaktionskanal (b) signalgebend sein. Im folgenden wird der Versuch unternommen, aufgrund des chemischen Verhaltens bei Zugabe von O-Atomen zu weiteren Erkenntnissen zu kommen. Die folgende Abbildung zeigt ein Flugzeitmassenspektrum unter den Bedingungen tBPP $+\mathrm{F}-\mathrm{O} /+\mathrm{O}$ bei $\lambda=520 \mathrm{~nm}:$

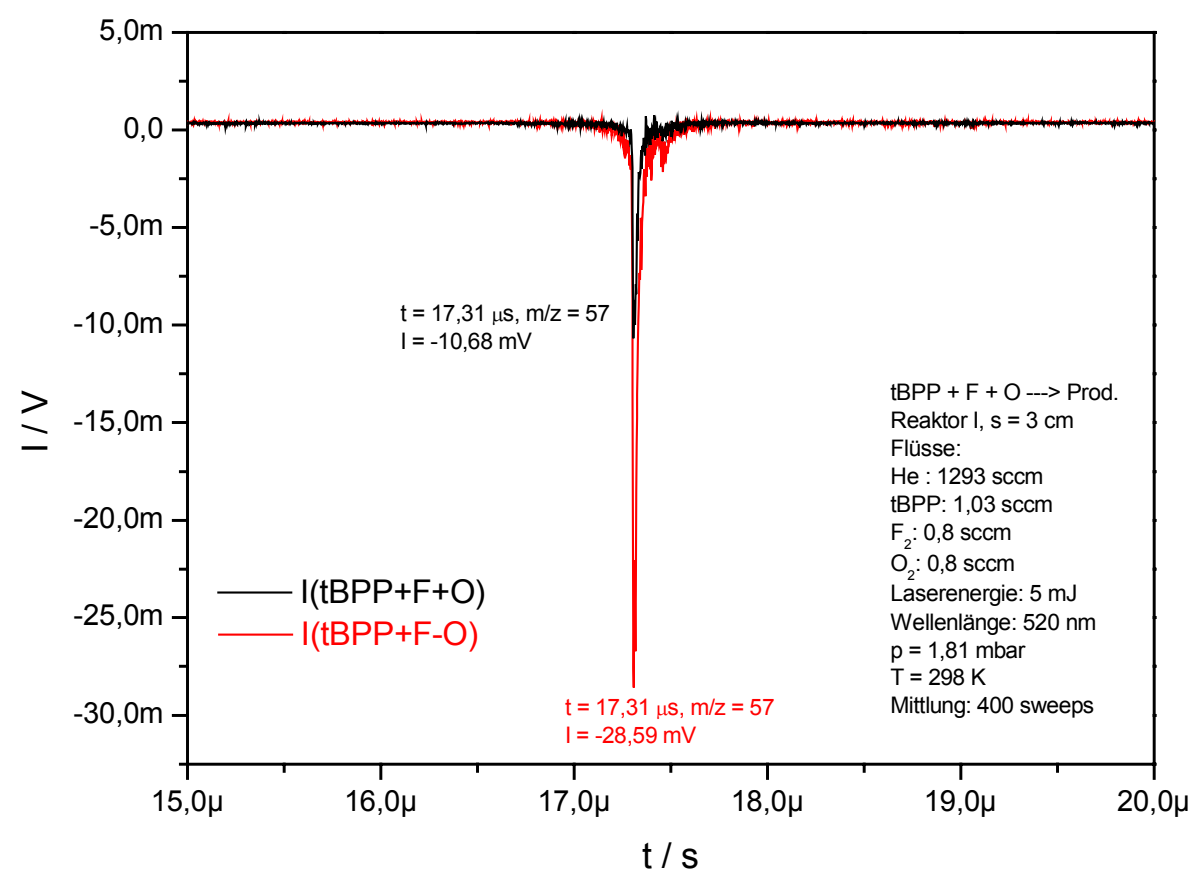

\section{Abbildung 82: Flugzeitmassenspektrum tBPP+F-O $/+F+O, \lambda=520 \mathrm{~nm}$}

Interpretation: Die Konzentration der O-Atome im Reaktor ist gleich derjenigen bei Isobutan+F+O und $\mathrm{DtBP}+\mathrm{F}+\mathrm{O}$. Die Konzentration des tBPP musste aufgrund des extrem niedrigen Dampfdruckes dieser Substanz ${ }^{23}$ weitaus geringer gehalten werden als die von DtBP und Isobutan. Entsprechend wurde die Konzentration der F-Atome gesenkt. Es ist eine Signalabnahme unter der Bedingung $+\mathrm{O}$ von $62 \%$ festzustellen. Bei der Reaktion t-Butyl $+\mathrm{O}$ (t-Butyl erzeugt durch Isobutan + F) war bei gleicher O-Atom-Konzentration und höherer Radikalkonzentration eine Abnahme von 78\% zu verzeichnen (vgl. Abschnitt 3.2.3). Das ist eine Hinweis, wenn auch nur ein schwacher, dass das Signal bei $\mathrm{m} / \mathrm{z}=57$ nicht nur aus dem t-Butylradikal aus Kanal (a), sondern möglicherweise aus dem t-Butoxyradikal aus Kanal (b) resultiert. Aufgrund des Verhaltens des Signals bei DtBP $+\mathrm{F}+\mathrm{O}$ (Zunahme um den Faktor 2) erscheint aber der Kanal (a) der Reaktion

${ }^{23} \mathrm{p}(\mathrm{tBPP}) \approx 25$ Torr, errechnet nach [STE94]. 
tBPP + F bevorzugt. Eine Quantifizierung erscheint wegen des teilweise hypothetischen Charakters der Argumentation und unbekannter spektroskopischer Empfindlichkeiten in der Ionenquelle nicht sinnvoll.

\subsubsection{Dimethoxymethan + F}

Produkte:

Diskussion: Dimethoxymethan (DMM) wird in großen Mengen als Lösungsmittel, zum Entparaffinieren von Mineral- und Schmieröl sowie als Hilfsstoff bei der Herstellung von Kunstharzen, Klebstoffen und dergleichen eingesetzt [FAL91]. Ferner wird DMM als Dieselersatzkraftstoff in Betracht gezogen, da es ähnlich aufgebaut ist wie Dimethylether, welcher gute Kraftstoffeigenschaften mit niedrigen Emissionswerten verbindet. Darüber hinaus ist DMM bei Zimmertemperatur und Normaldruck flüssig und somit leichter zu handhaben ist als Dimethylether [WAL97].

Es sind folgende Kanäle der Reaktion von $\mathrm{CH}_{3} \mathrm{OCH}_{2} \mathrm{OCH}_{3}$ mit Fluoratomen denkbar:<smiles>COCOC</smiles><smiles>CCCCCOC</smiles><smiles>CCCOCCOC</smiles>

Angaben über Existenz, Geschwindigkeit und Kanalverzweigungsverhältnis der Reaktionskanäle in der Literatur liegen nicht vor. Da alle H-Atome primär sind, sind die Geschwindigkeitskoeffizienten von (a) und (b) in gleicher Größenordnung zu erwarten. Für Kanal (a) kann eine Reaktionsenthalpie von $\Delta_{\mathrm{R}} \mathrm{H}^{0} 298 \mathrm{~K}=-166,8 \mathrm{~kJ} / \mathrm{mol}$ abgeschätzt werden. Für Kanal (b) ist eine ähnliche Größe anzunehmen.

Messergebnisse: Es wurde mit Hilfe von Versuchsanordnung A (REMPI) und B (EI-MS) untersucht, ob eines der aus (a) oder (b) entstehenden Radikale spezifisch nachgewiesen werden kann. Die folgende Abbildung zeigt ein Flugzeitmassenspektrum bei $\lambda=520 \mathrm{~nm}$ : 


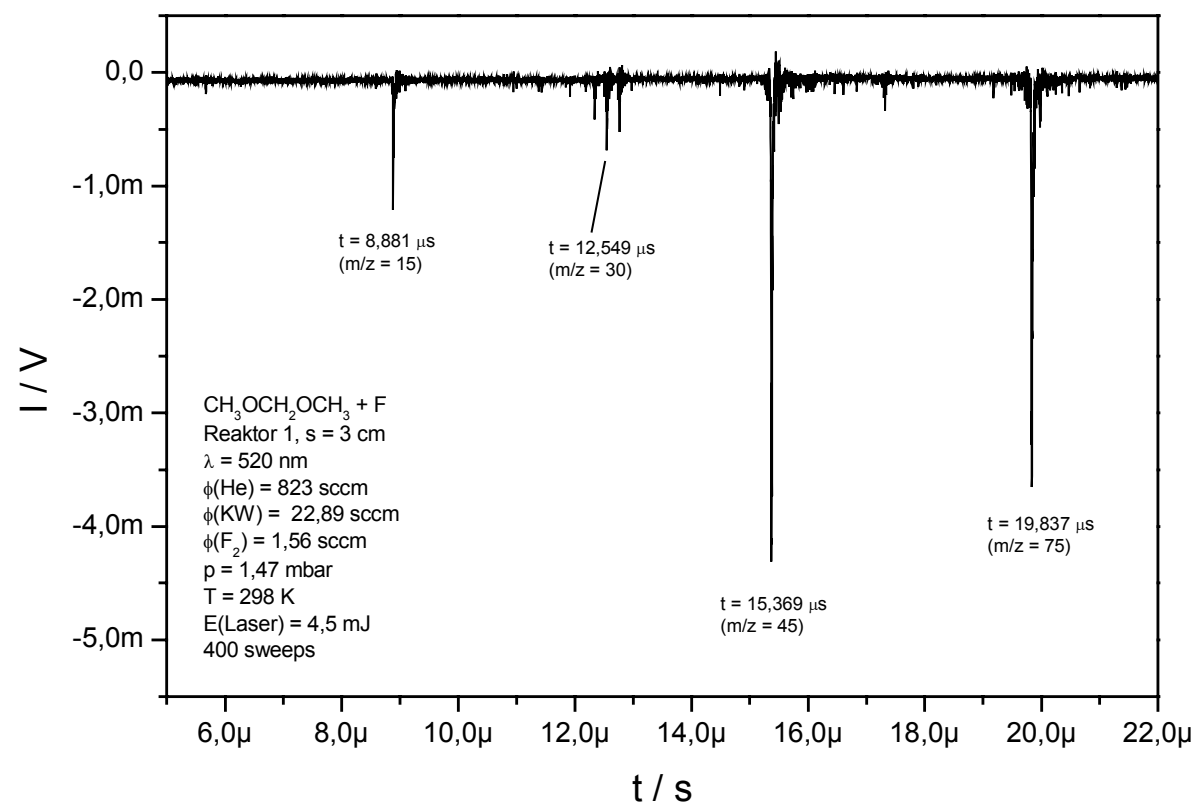

\section{Abbildung 83: Flugzeitmassenspektrum DMM + F, $\lambda=520 \mathrm{~nm}$}

Ein Untergrund, d.h. ein Signal von DMM - F, ist bei dieser Wellenlänge kaum vorhanden. Neben der Radikalmuttermasse $\mathrm{m} / \mathrm{z}=75$ sind bedeutende Fragmente bei $\mathrm{m} / \mathrm{z}=45$ und 15, kleinere Fragmente bei $\mathrm{m} / \mathrm{z}=29,30$ und $31 \mathrm{zu}$ erkennen. Beim Übergang zu kürzeren Wellenlängen sind zwei Tendenzen auszumachen: Erstens nimmt der Verhältnis des Signals von $\mathrm{m} / \mathrm{z}=45 \mathrm{zu}$ $\mathrm{m} / \mathrm{z}=75$ deutlich zu. Und zweitens steigt der Untergrund an. Das Fragmentierungsmuster des molekularen DMM gleicht dem der Radikale. Bei $\lambda=370 \mathrm{~nm}$ ist ein Unterschied zwischen DMM - F und DMM + F nicht mehr zu erkennen.

Interpretation: Das bei Wellenlängen $\mathrm{ab} \lambda=500 \mathrm{~nm}$ untergrundfreie Signal bei $\mathrm{m} / \mathrm{z}=75$ ist zunächst nicht einem der aus (a) und (b) entstehenden Radikale spezifisch zuweisbar. Dennoch gibt es einige Aspekte die bedenkenswert sind:

Mit Versuchsanordnung (B) (EI-MS) wurde nach dem Kombinationsprodukt des aus Kanal (b) entstehenden $\mathrm{CH}_{3} \mathrm{OCHOCH}_{3}$ gesucht. Dabei zeigte sich, dass mit wachsendem Umsatz der Reaktion DMM + F zunehmend Signaltätigkeit auf den Massen m/z $=73$ und 119 zu beobachten war (Messtabelle 1). Ein Vergleich mit dem Literaturmassenspektrum zeigt: Die Zunahme auf den untergrundfreien Massen ist ein Hinweis auf die Entstehung von 1,1,2,2-Tetra-Methoxy-Ethan $\left(\mathrm{C}_{6} \mathrm{O}_{4} \mathrm{H}_{14}\right)$, dem Kombinationsprodukt des $\mathrm{CH}_{3} \mathrm{OCHOCH}_{3}$. Das ist ein indirekter Nachweis für die Entstehung dieses Radikals.

Beim mittelständig radikalisierten $\mathrm{CH}_{3} \mathrm{OCHOCH}_{3}$ ist es nicht sinnvoll vorstellbar, dass es auf $\mathrm{m} / \mathrm{z}=45$ fragmentieren könnte. Ein Bruch der schwachen O-C Bindung in der Ionenquelle würde 
zu $\mathrm{CH}_{3}$ und $\mathrm{CH}_{3} \mathrm{OCHO}$ führen. Letzteres hat aber eine Masse von $\mathrm{m} / \mathrm{z}=44$. Die Masse $\mathrm{m} / \mathrm{z}=45$ muss demnach aus dem endständig radikalisierten $\mathrm{CH}_{3} \mathrm{OCH}_{2} \mathrm{OCH}_{2}$ resultieren. Damit stellt sich aber eine neue Frage: Ist $\mathrm{m} / \mathrm{z}=45$ ein Fragment dieses Radikals oder kommt es im Sinne der folgenden Reaktionsgleichung bereits im Reaktionsraum zu einem unimolekularen Zerfall mit dem Methoxymethylradikal und dem stabilen Formaldehyd als Zerfallsprodukte?<smiles>COC</smiles>

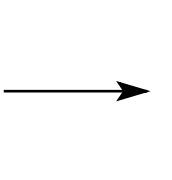<smiles>CO</smiles><smiles>C=O</smiles>

Zwar ist die Dissoziation mit $\Delta_{\mathrm{R}} \mathrm{H}^{0}{ }_{298 \mathrm{~K}}=42,5 \mathrm{~kJ} / \mathrm{mol}$ endotherm, jedoch wurde die Standardbildungsenthalpie für das $\mathrm{CH}_{3} \mathrm{OCH}_{2} \mathrm{OCH}_{2}$ mit Hilfe von [STE94] nur abgeschätzt und dürfte dementsprechend mit einem hohen Fehler behaftet sein. Es gibt einige Anzeichen für einen solchen Zerfall, so wurde z.B. mit Versuchsanordnung B (EI-MS) unter der Bedingung DMM+F im Vergleich zu DMM-F ein Zuwachs bei $\mathrm{m} / \mathrm{z}=30$ und 29 beobachtet, was für die Entstehung von Formaldehyd spricht. Ebenso ist ein Anwachsen bei m/z $=90$ festzustellen, der Molekülmasse des Kombinationsprodukts 1,2-Dimethoxyethan des Methoxymethylradikals. Auch ein Wellenlängenscan von $\mathrm{m} / \mathrm{z}=45$ mit Versuchsanordnung A (REMPI) ähnelt dem des Methoxymethylradikal (aus Dimethylether + F) sehr stark. Die in der nächsten Abbildung dargestellten zwei Wellenlängenscans wurden in unabhängigen Messreihen aufgenommen.

\section{Messergebnisse:}

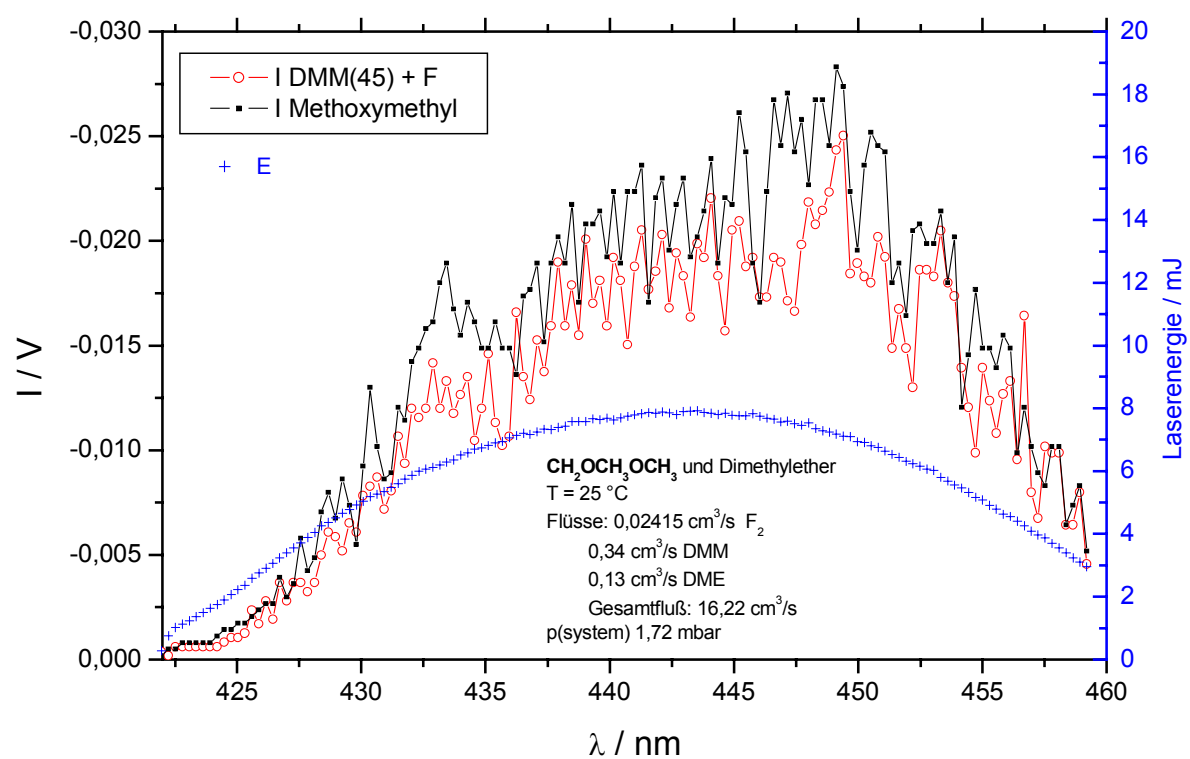

Abbildung 84: Vergleich der Wellenlängenabhängigkeit von $\mathrm{DMM}+\mathrm{F}(\mathrm{m} / \mathrm{z}=45)$ und $M M$ 
Diskussion: In früheren Arbeiten wurde von Nacke die Reaktion des Methoxymethylradikals mit H-Atomen mit Hilfe von Versuchsanordnung B (EI-MS) untersucht [NAC94]. Als einziger relevanter Reaktionskanal wurde der Zerfall zu $\mathrm{CH}_{3} \mathrm{O}$ und $\mathrm{CH}_{3}$ gefunden. Durch Messungen zur vorliegenden Arbeit mit Hilfe von Versuchsanordnung A (REMPI) kann dieser Kanal bestätigt werden. Das Methoxymethylradikal wurde sowohl mit H-Atomen als auch mit D-Atomen bei $\lambda=450,8 \mathrm{~nm}$ im Strömungsreaktor 2 umgesetzt $^{24}$. Neben einer Abnahme auf der Radikalmuttermasse $\mathrm{m} / \mathrm{z}=45$ war eine deutliche Zunahme auf der Masse $\mathrm{m} / \mathrm{z}=15(+\mathrm{H})$, welche durch ein Radikalfragment bereits belegt ist, bzw. das Auftauchen eines untergrundfreien Signals bei $\mathrm{m} / \mathrm{z}=16(+\mathrm{D}) \mathrm{zu}$ verzeichnen. Dieselbe Erscheinung ist bei der Reaktion DMM + F bei Zugabe von $\mathrm{H}$ - bzw. D-Atomen zu beobachten, was als Hinweis zur Entstehung von Methoxymethyl gedeutet werden kann.

Die diskutierten Beobachtungen sind jedoch nur Indizien für den oben vorgeschlagenen Zerfall des $\mathrm{CH}_{3} \mathrm{OCH}_{2} \mathrm{OCH}_{2}$. Eine in der Ionenquelle verursachte Fragmentierung könnte ähnliche Effekte hervorrufen. Das Entstehen von Methylradikalen bei der Umsetzung mit H-Atomen könnte auch durch direkte Reaktion nicht dissoziierter Dimethoxymethylradikale mit H erklärt werden. Eine Mischung zwischen Fragmentierung in der Ionenquelle und unimolekularem Zerfall im Reaktionsraum wäre ebenfalls denkbar. Wegen der fehlenden Eindeutigkeit des Nachweises der beiden Dimethoxymethylradikale wurde eine Untersuchung der Reaktionen der Radikale mit anderen Substanzen nicht unternommen.

\section{Reaktionsgeschwindigkeit:}

Messergebnisse: Mit Hilfe von Versuchsanordnung B (EI-MS) wurde für die Gesamtreaktion $\mathrm{DMM}+\mathrm{F}$ die Geschwindigkeitskonstante relativ zur Reaktion $\mathrm{CH}_{4}+\mathrm{F}$ im Temperaturbereich $-20{ }^{\circ} \mathrm{C}$ bis $90{ }^{\circ} \mathrm{C}$ bestimmt. Die folgenden Abbildungen stellen die Messungen in graphischer Auswertung (vgl. Abschnitt 2.4.2 Relativmessung) dar:

\footnotetext{
${ }^{24}$ Wie im experimentellen Teil erklärt, vermeidet dieser Reaktor die schnelle Reaktion von H-Atomen mit F-Atomen.
} 


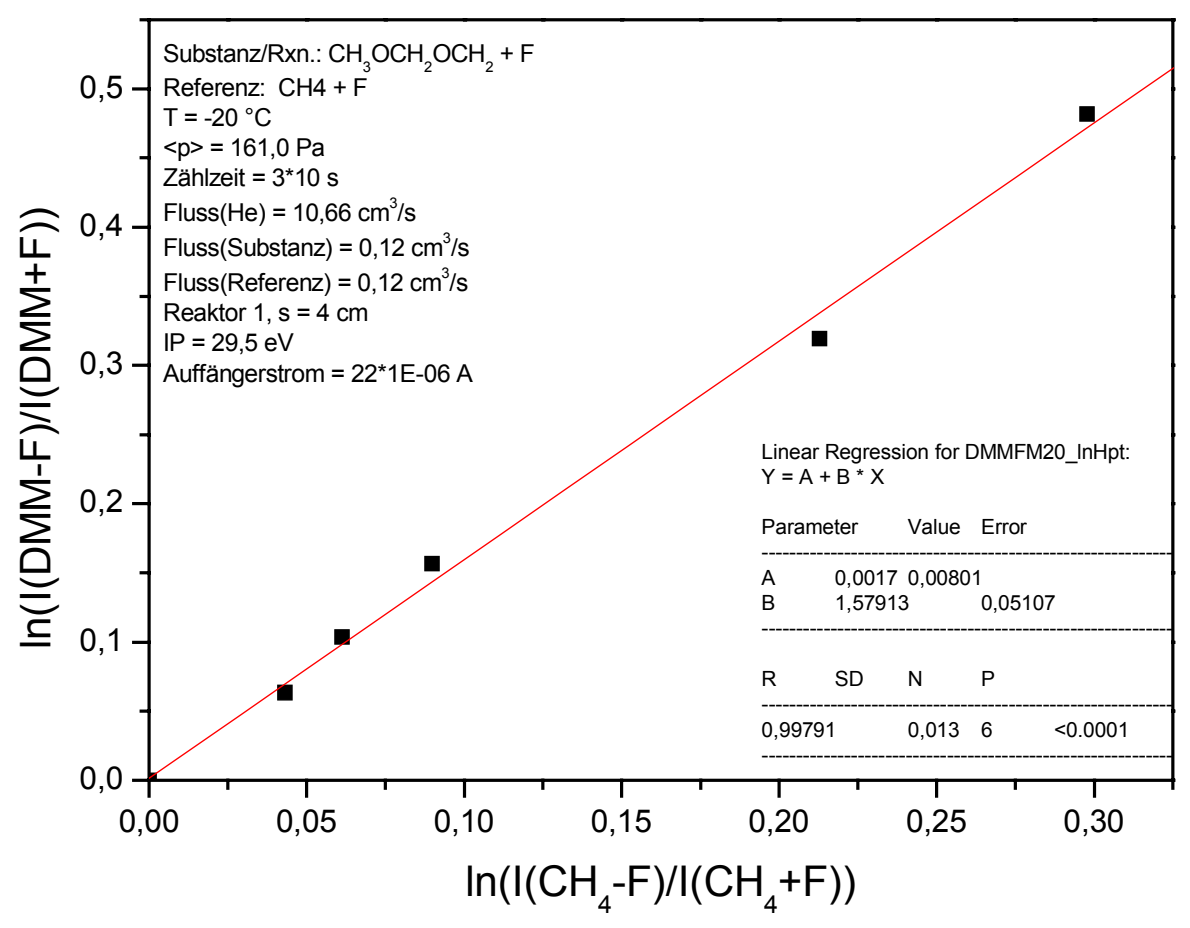

Abbildung 85: DMM $+\mathrm{F}$ relativ zu $\mathrm{CH}_{4}+\mathrm{F}, \mathrm{T}=-20^{\circ} \mathrm{C}$

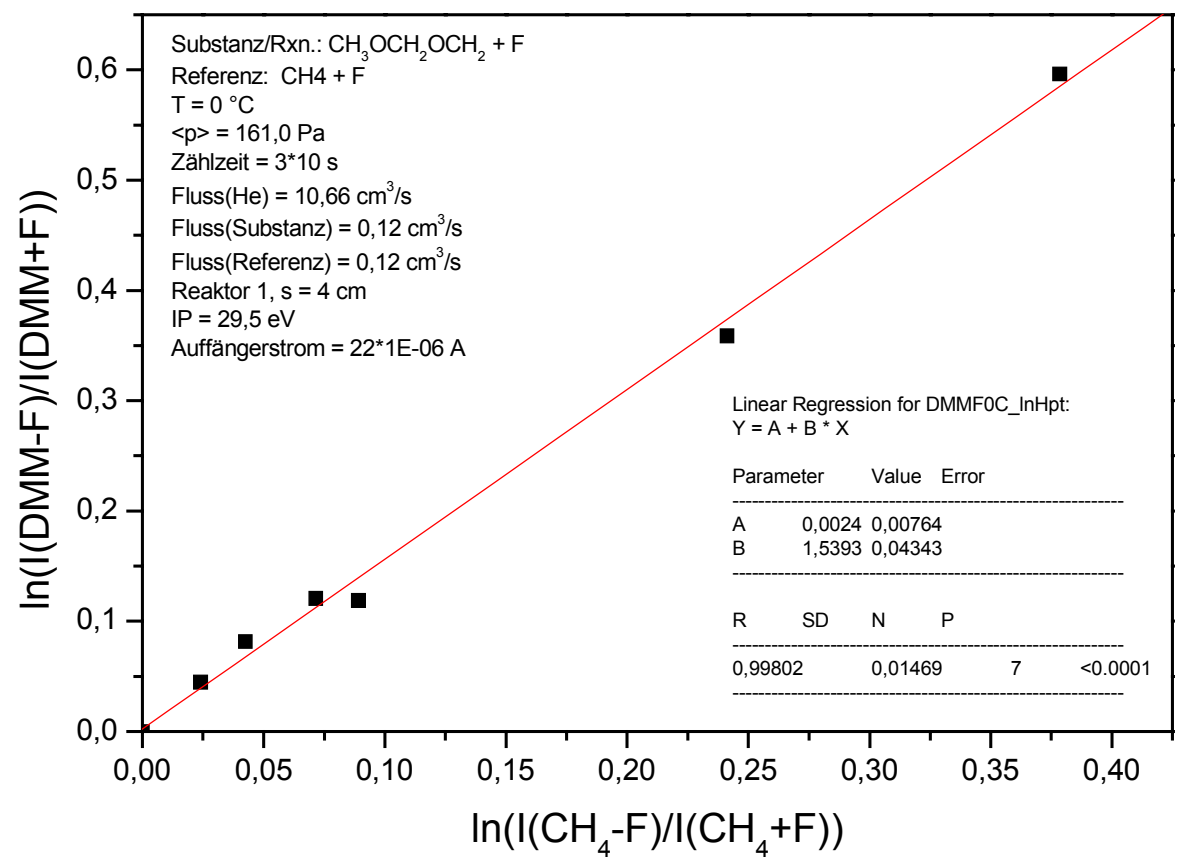

Abbildung 86: $\mathrm{DMM}+\mathrm{F}$ relativ $\mathrm{zu} \mathrm{CH}_{4}+\mathrm{F}, \mathrm{T}=0{ }^{\circ} \mathrm{C}$ 


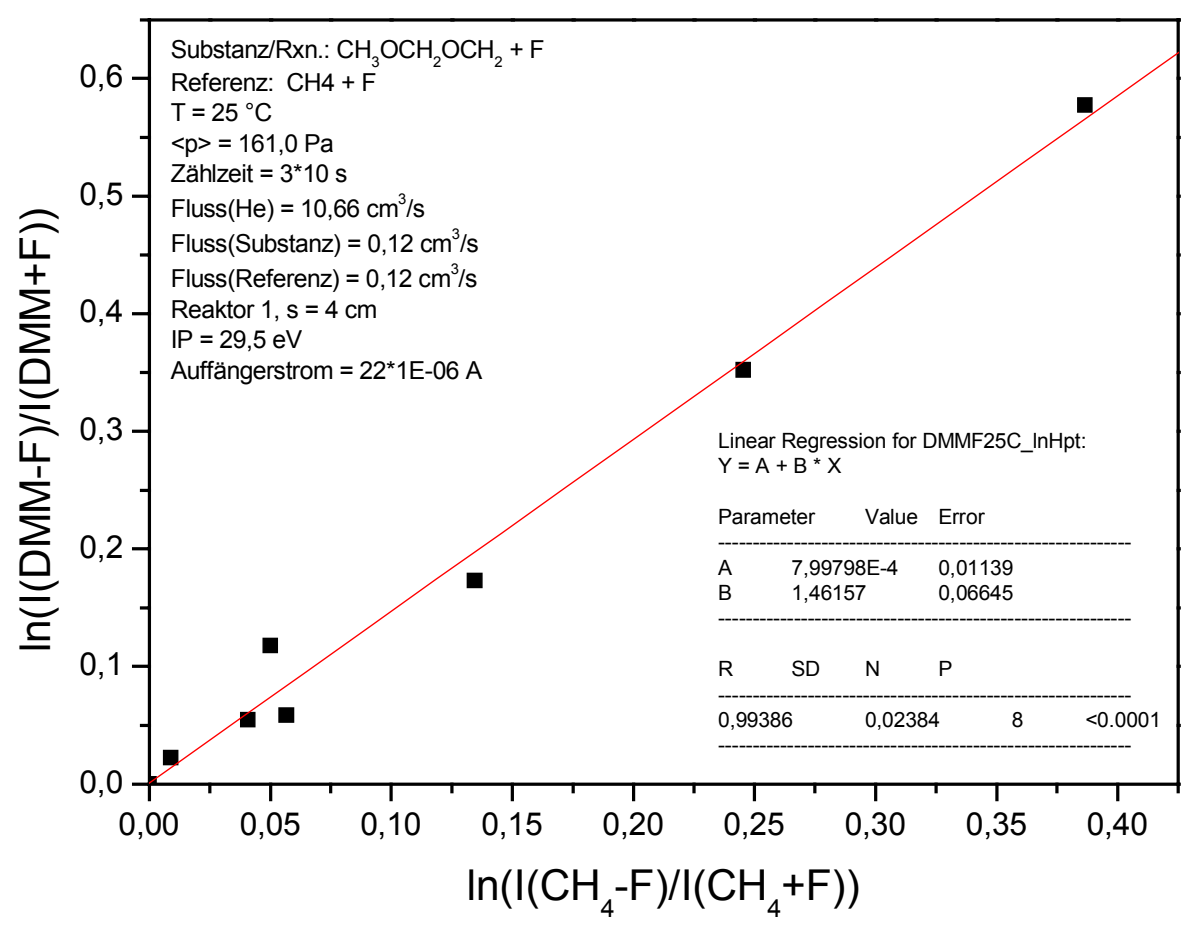

Abbildung 87: DMM $+\mathrm{F}$ relativ zu $\mathrm{CH}_{4}+\mathrm{F}, \mathrm{T}=25^{\circ} \mathrm{C}$

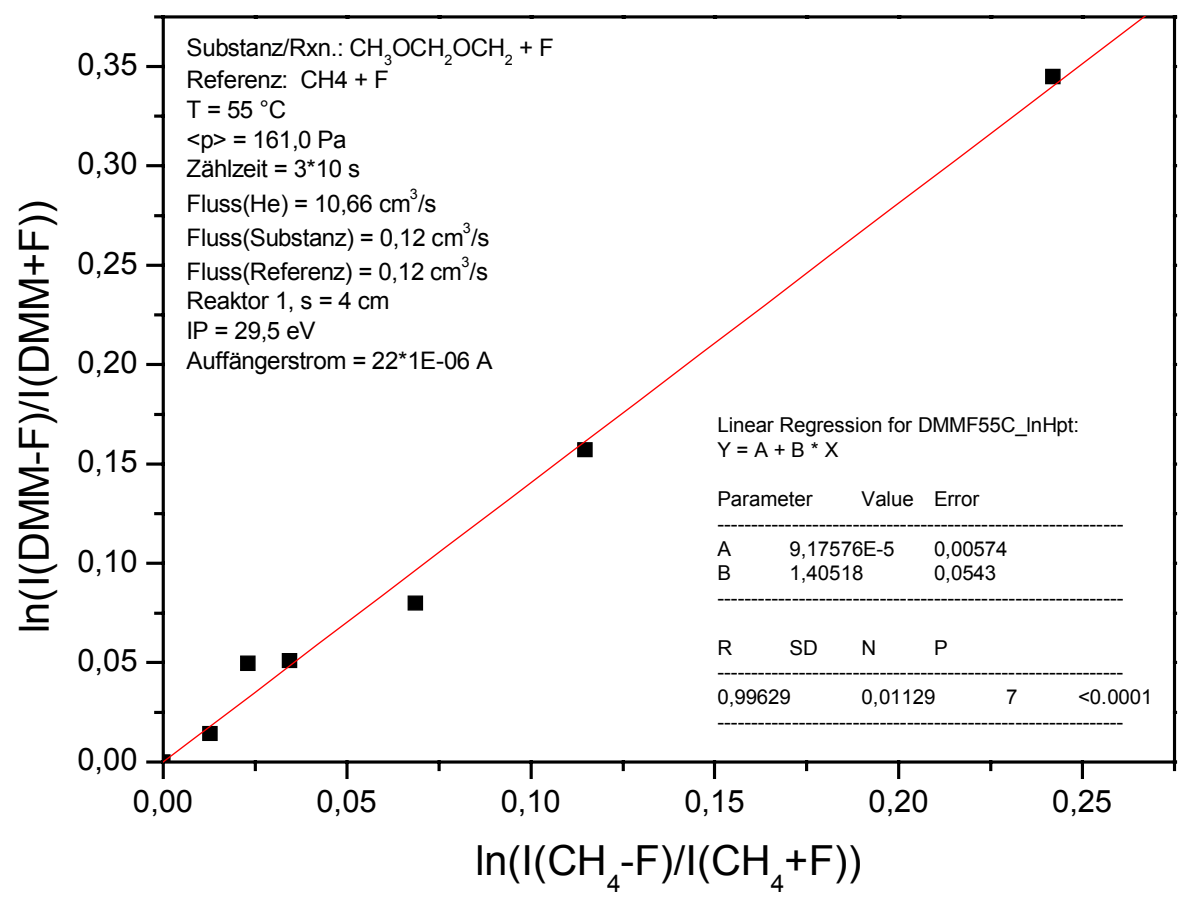

Abbildung 88: $\mathrm{DMM}+\mathrm{F}$ relativ $\mathrm{zu} \mathrm{CH}_{4}+\mathrm{F}, \mathrm{T}=55^{\circ} \mathrm{C}$ 


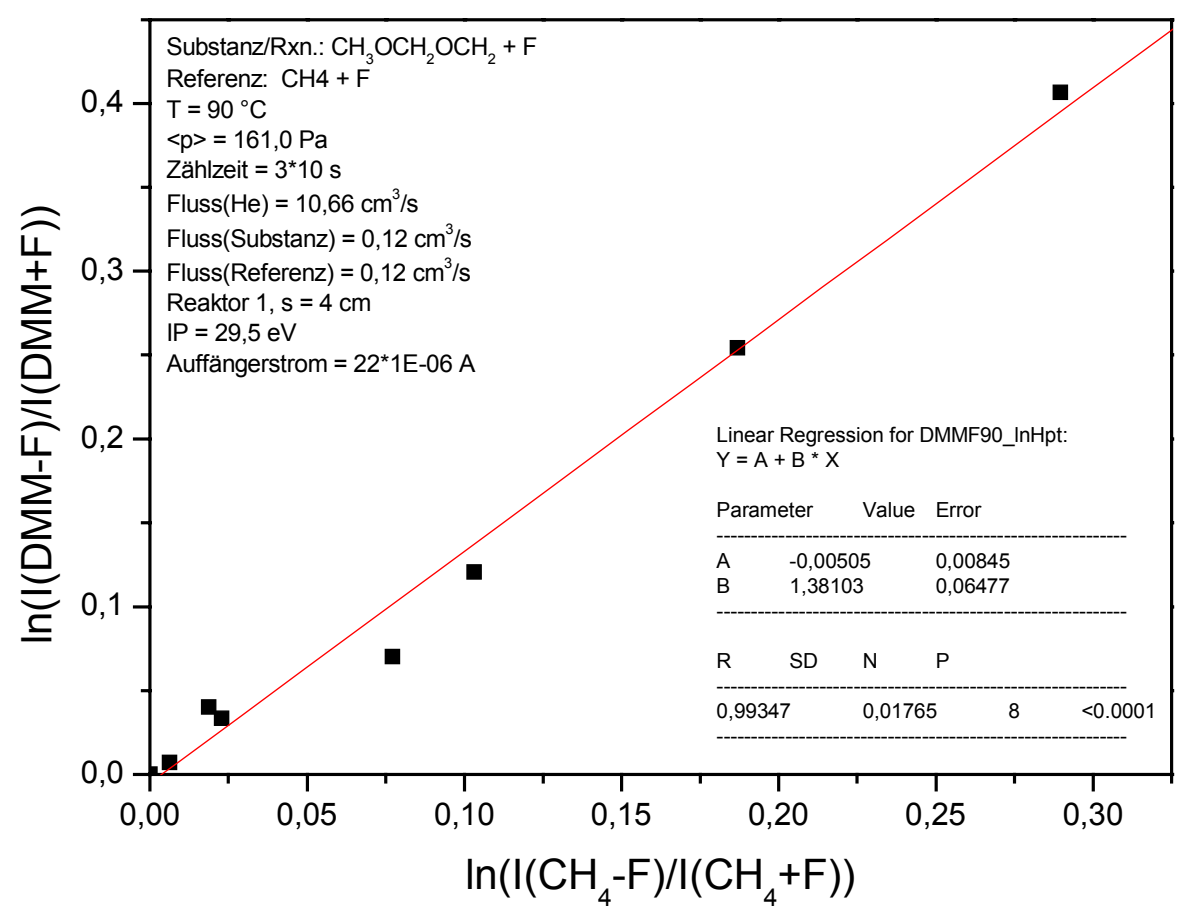

Abbildung 89: $\mathrm{DMM}+\mathrm{F}$ relativ $\mathrm{zu} \mathrm{CH}_{4}+\mathrm{F}, \mathrm{T}=90^{\circ} \mathrm{C}$

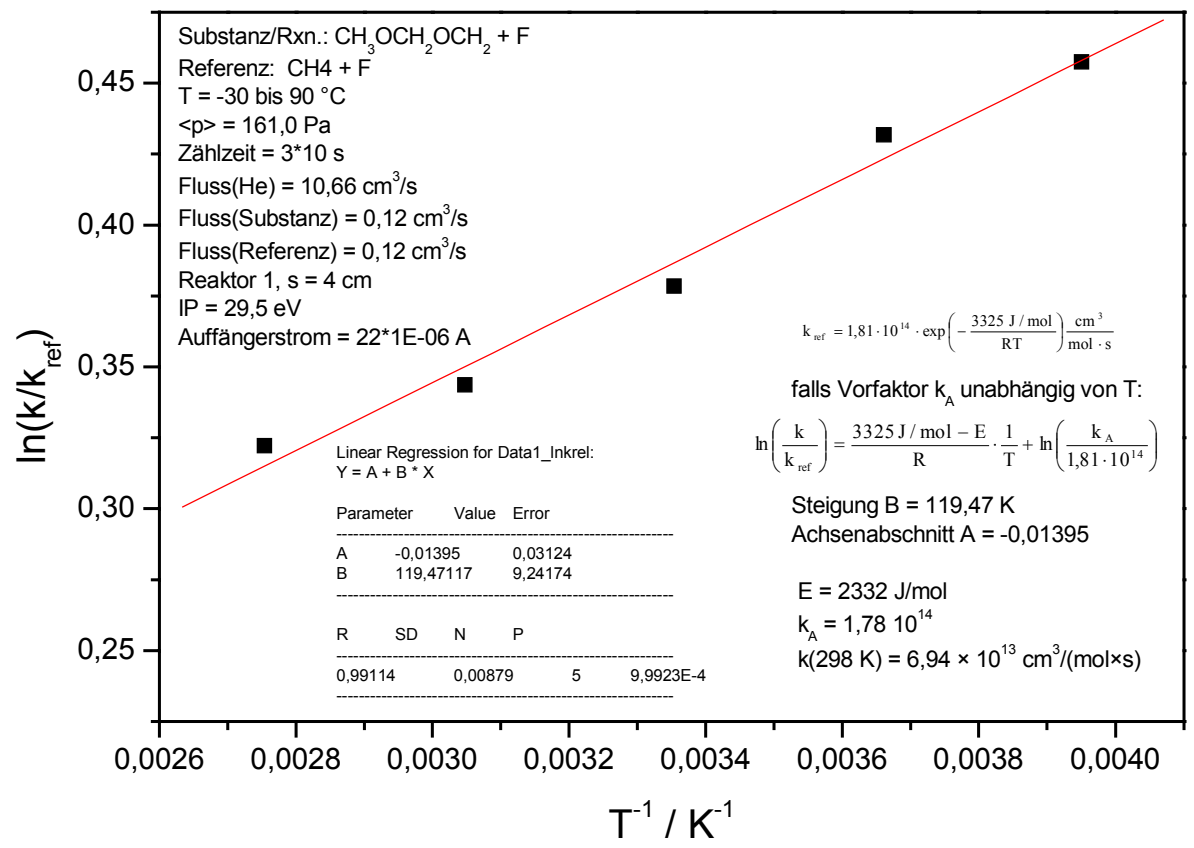

\section{Abbildung 90: DMM + F relativ zu $\mathrm{CH}_{4}+\mathrm{F}$, Arrheniusauftragung}

Die Daten für die ausgiebig untersuchte Referenzreaktion $\mathrm{CH}_{4}+\mathrm{F}$ entstammen [ATK97]. Für die Reaktion DMM + F ergibt sich damit:

$$
\begin{gathered}
\mathrm{k}=(1,78 \pm 0,05) \cdot 10^{14} \exp \{-2332 \pm 77 /(\mathrm{RT}) \mathrm{J} \mathrm{mol}-1\} \mathrm{cm}^{3} \cdot \mathrm{mol}^{-1} \cdot \mathrm{s}^{-1} \\
\mathrm{k}^{298}=(6,95 \pm 0,65) \cdot 10^{13} \mathrm{~cm}^{3} \cdot \mathrm{mol}^{-1} \cdot \mathrm{s}^{-1}
\end{gathered}
$$

Die angegebenen Fehlerwerte resultieren aus der Standardabweichung der Regressionsgeraden. 
Diskussion: Literaturwerte für diese Reaktion liegen nicht vor. Für die analoge Reaktion von Dimethoxymethan mit Chloratomen wird eine Geschwindigkeitskonstante von $\mathrm{k}^{298}=8,13 \cdot 10^{13}$ $\mathrm{cm}^{3} /(\mathrm{mol} \cdot \mathrm{s})$ angegeben [WAL97], allerdings für einen Druck von $\mathrm{p}=700$ Torr.

\subsection{Reaktionen von Radikalen mit O-Atomen}

Reaktionen von Kohlenwasserstoffradikalen $\mathrm{R}$ mit O-Atomen verlaufen gemäß folgendem allgemeinem Reaktionsschema:

$$
\begin{aligned}
& \mathrm{R}+\mathrm{O} \rightarrow[\mathrm{R}-\mathrm{O}]^{*} \rightarrow \text { Produkte } \\
& \mathrm{R}+\mathrm{O} \rightarrow \text { Alken }+\mathrm{OH}
\end{aligned}
$$

Die nach (1) erzeugten hochangeregten Addukte zerfallen zu Produkten, die aus sauerstoffhaltigen Verbindungen (Aldehyde, Ketone) und kleineren Radikalen bestehen können ([HOY79a],[SLA88]). Dieser Zerfall läuft mechanistisch häufig über einen Bruch der C-H-Bindung oder der C-C-Bindung an dem das addierte Sauerstoffatom tragenden C-Atom. Daneben wird gemäß (2) bei einigen Reaktionen von Kohlenwasserstoffradikalen mit O-Atomen auch eine H-Abstraktion unter Bildung von OH-Radikalen und entsprechendem Alken beobachtet, z.B. wird bei der Reaktion von Ethyl mit O-Atomen auch der Kanal unter Ausbildung von OH-Radikalen und Ethen beobachtet [HAC02]. Es gibt deutliche Hinweise dafür, dass in diesem Fall die Abstraktion direkt geschieht und nicht über das angeregte Addukt verläuft (zur Argumentation vgl. Abschnitt 3.3.8).

Im folgenden werden die Untersuchungen zu den Reaktionen der Radikale Ethyl, Methoxymethyl, c-Pentyl, Methyl, 2-Propyl, 1-Propyl, 2-Butyl, 1-Butyl, t-Butyl, Allyl (im Rahmen einer Vergleichskinetik), Isobutyl, c-Heptyl, c-Oktyl, c-Pentadienyl, Trichlorethyl und 2-Chlor-2-Methyl-Propyl mit O-Atomen vorgestellt. Die Reaktionen von Radikalen mit O-Atomen sind typischerweise wenig druck- und temperaturabhängig. Trotzdem wurde bei einigen Reaktionen die Temperaturabhängigkeit im Bereich $\mathrm{T}=-30 /+5{ }^{\circ} \mathrm{C}$ bis $+90^{\circ} \mathrm{C}$ untersucht.

\subsubsection{Ethyl + O}

\section{Produkte:}

Messergebnisse: Die Produktbildung der Reaktion Ethyl $+\mathrm{O}$ wurde mit Versuchsanordnung A (REMPI) bei $\lambda=340,8 \mathrm{~nm}$ untersucht. Dabei wurden zum einen $\mathrm{C}_{2} \mathrm{H}_{5}$-Radikale, zum anderen $\mathrm{C}_{2} \mathrm{D}_{5}$-Radikale über die Reaktion des entsprechenden Ethans mit F-Atomen erzeugt und gemäß Abschnitt 3.1.2 nachgewiesen. Die Sauerstoffatome wurden gemäß Abschnitt 2.6 dargestellt und den Radikalen in der Reaktionszone zugeführt. Die folgenden Abbildungen zeigen als 
Messergebnisse Flugzeitmassenspektren unter den Bedingungen Ethyl -O/+O, wobei die zweite und die dritte Abbildung dieselbe Messung, aber in Farbinversion darstellen.

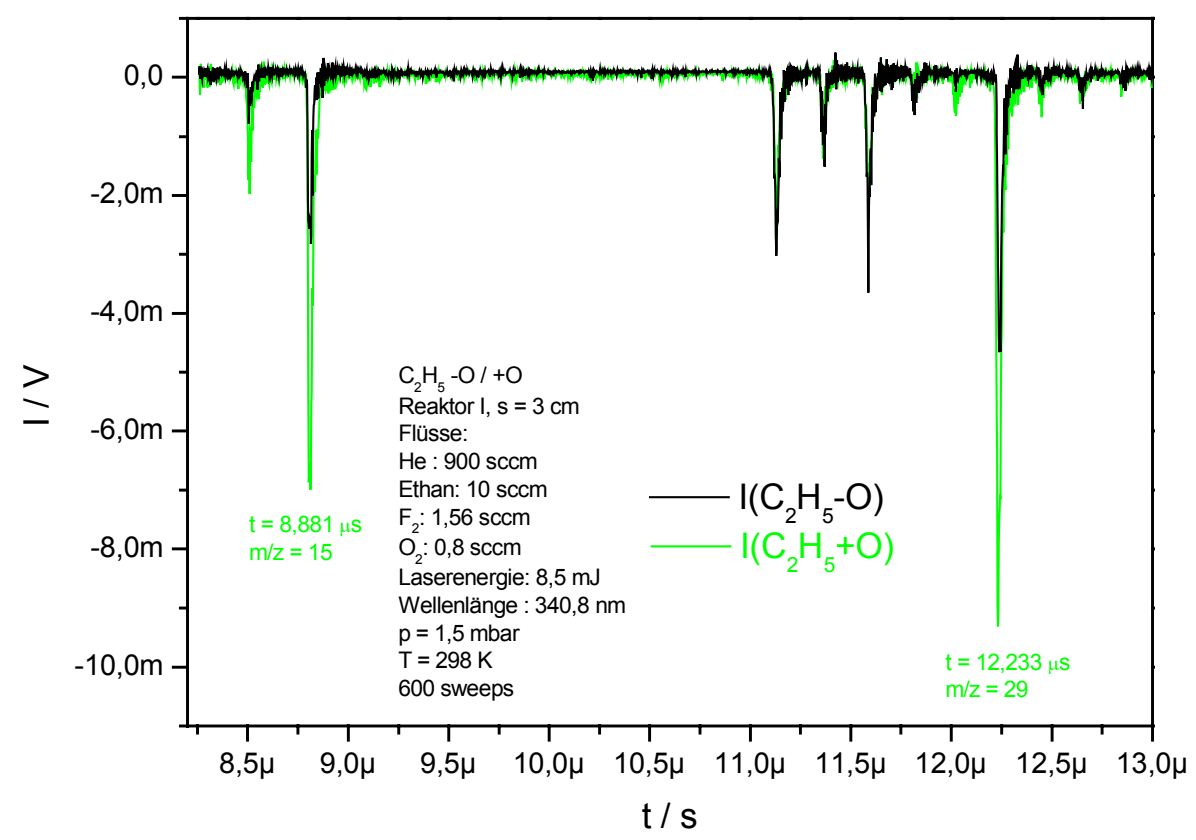

Abbildung 91: Flugzeitmassenspektrum $\mathrm{C}_{2} \mathrm{H}_{5}-\mathrm{O} /+\mathrm{O}, \lambda=340,8 \mathrm{~nm}$

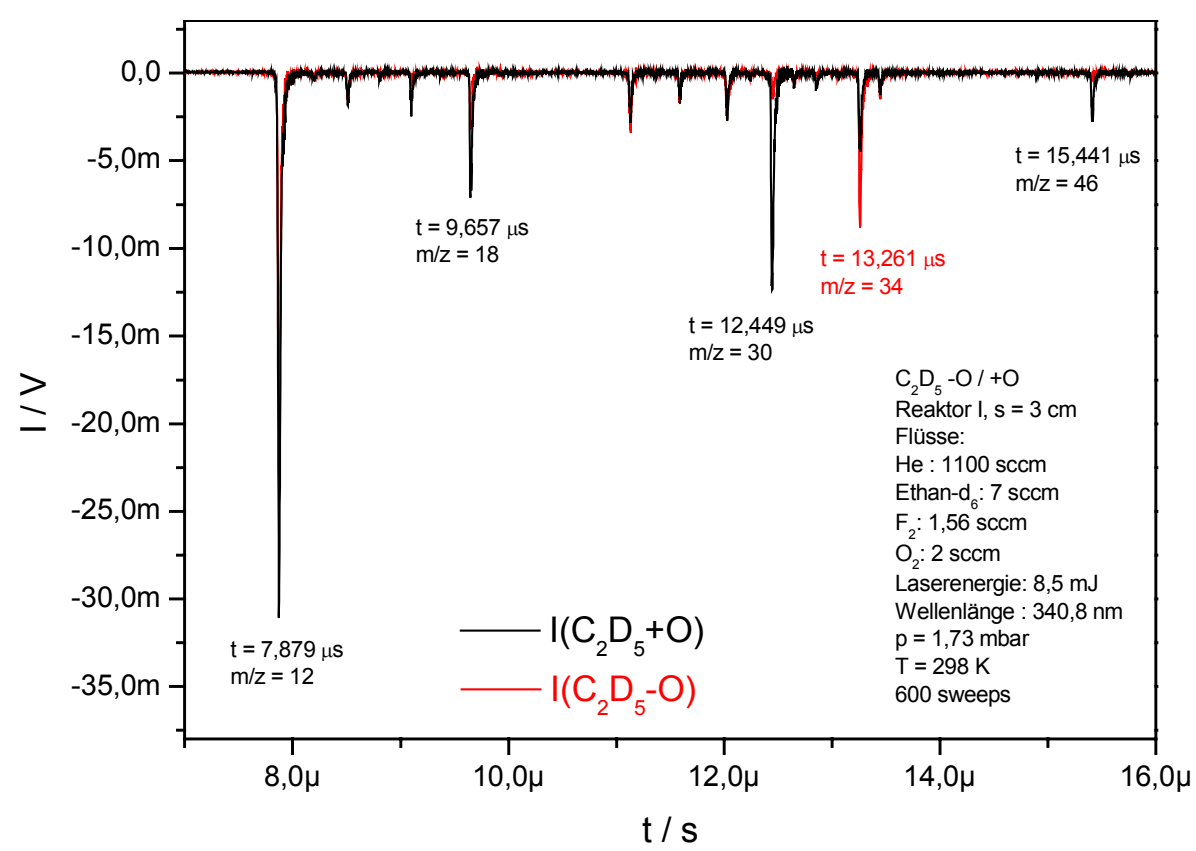

Abbildung 92: Flugzeitmassenspektrum $\mathrm{C}_{2} \mathrm{D}_{5}-\mathrm{O} /+\mathrm{O}, \lambda=340,8 \mathrm{~nm}$, Darst. 1 


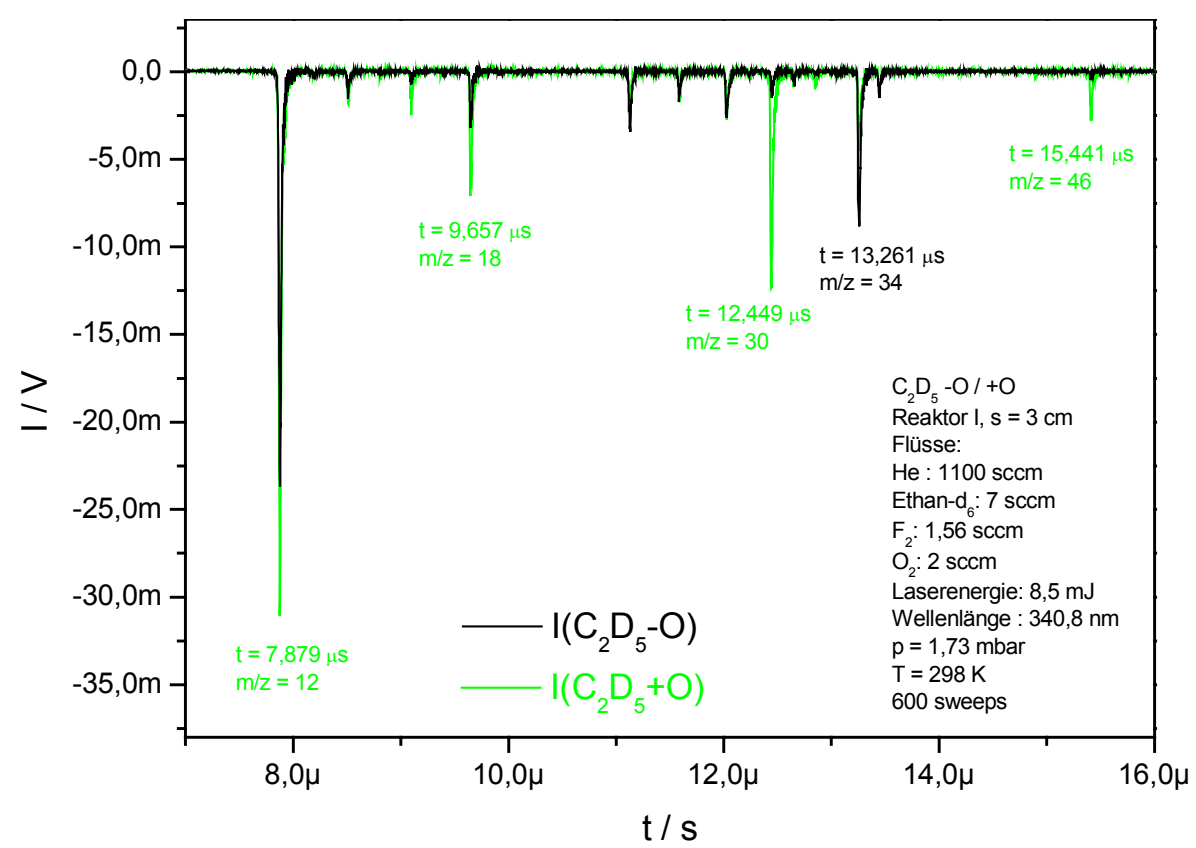

Abbildung 93: Flugzeitmassenspektrum $\mathrm{C}_{2} \mathrm{D}_{5}-\mathrm{O} /+\mathrm{O}, \lambda=340,8$ nm, Darst. 2

In Abbildung 91 sind bei Zugabe von O-Atomen deutliche Zunahmen auf den Massen $\mathrm{m} / \mathrm{z}=29$, 15 und 12 sowie eine leichte Zunahme bei $\mathrm{m} / \mathrm{z}=30$ und $28 \mathrm{zu}$ verzeichnen. In Abbildung 92 ist bei Anwesenheit von O-Atomen bei $\mathrm{m} / \mathrm{z}=34$ eine markante Abnahme festzustellen. In Abbildung 93 ist unter der Bedingung $+\mathrm{O}$ eine Zunahme bei $\mathrm{m} / \mathrm{z}=46,30,18,16$ und 12 und eine kleine Zunahme bei $\mathrm{m} / \mathrm{z}=32$ zu beobachten.

Interpretation: Für die Reaktion von Ethylradikalen mit O-Atomen sind drei Reaktionskanäle bekannt:

$$
\begin{array}{llll} 
& \rightarrow \mathrm{HCHO}+\mathrm{CH}_{3} & \text { (I) } & \Delta_{\mathrm{R}} \mathrm{H}^{0}{ }_{298 \mathrm{~K}}=-329,8 \mathrm{~kJ} / \mathrm{mol} \\
\mathrm{C}_{2} \mathrm{H}_{5}+\mathrm{O} & \rightarrow \mathrm{CH}_{3} \mathrm{CHO}+\mathrm{H} & \text { (II) } & \Delta_{\mathrm{R}} \mathrm{H}^{0}{ }_{298 \mathrm{~K}}=-324,3 \mathrm{~kJ} / \mathrm{mol} \\
& \rightarrow \mathrm{CH}_{2}=\mathrm{CH}_{2}+\mathrm{OH} & \text { (III) } & \Delta_{\mathrm{R}} \mathrm{H}^{0}{ }_{298 \mathrm{~K}}=-267,0 \mathrm{~kJ} / \mathrm{mol}
\end{array}
$$

Durch die Abnahme bei $\mathrm{m} / \mathrm{z}=34$ in Abbildung 92 ist ein Reaktionseffekt von Ethylradikalen mit O-Atomen festzustellen. Die Zunahme bei $\mathrm{m} / \mathrm{z}=29$ in Abbildung 91 sowie die Zunahme bei $\mathrm{m} / \mathrm{z}=30$ in Abbildung 93 ist der Entstehung von Formaldehyd nach (I) zuzuordnen, welches in massenspektrometrischen Ionenquellen typischerweise ein H- bzw. D-Atom verliert (vgl. Anhang 6.3, S. 260). Allerdings kann auch nicht ausgeschlossen werden, dass diese Zunahmen Fragmenten des Acetaldehyds aus Kanal (II) zuzurechnen sind. Kanal (I) wird aber auch noch durch die Zunahme von $\mathrm{m} / \mathrm{z}=15$ in Abbildung 91 und durch die Zunahme bei $\mathrm{m} / \mathrm{z}=18$ in Abbildung 93 bestätigt, die der Entstehung von $\mathrm{CH}_{3}$ bzw. $\mathrm{CD}_{3}$ zugewiesen werden. 
Die Zunahme bei $\mathrm{m} / \mathrm{z}=46$ in Abbildung 93 ist durch die Entstehung von Acetaldehyd nach Kanal (II) zu erklären, welches in der Ionenquelle ein D-Atom verliert.

$\mathrm{Ob}$ die kleinen Signale bei $\mathrm{m} / \mathrm{z}=28$ (Abbildung 91) und $\mathrm{m} / \mathrm{z}=32$ (Abbildung 93) bei O-Atomzugabe der Entstehung von Ethen aus Kanal (III), der von Formaldehyd aus Kanal (I) oder der von Acetaldehyd aus Kanal (II) zuzuordnen ist, bleibt spekulativ.

Eine Bestimmung des Kanalverzweigungsverhältnisses ist aufgrund der erwähnten Überlagerungen auf einigen Massen und aufgrund unbekannter spektroskopischer Empfindlichkeiten in der Mehrphotonenionenquelle nicht möglich.

Diskussion: Gutman et al. haben die Reaktion Ethyl + O durch Photolyse einer $\left(\mathrm{C}_{2} \mathrm{H}_{5}\right)_{2} \mathrm{CO} / \mathrm{SO}_{2}$ Mischung mit massenspektrometrischem Nachweis untersucht. Es wurde folgender Mechanismus vorgeschlagen [SLA88]:

$\begin{array}{lll}\mathrm{C}_{2} \mathrm{H}_{5}+\mathrm{O} & \rightarrow & \mathrm{C}_{2} \mathrm{H}_{5} \mathrm{O}^{*} \\ \mathrm{C}_{2} \mathrm{H}_{5} \mathrm{O}^{*} & \rightarrow & \mathrm{HCHO}+\mathrm{CH}_{3} \\ & \rightarrow & \mathrm{CH}_{3} \mathrm{CHO}+\mathrm{H} \\ \mathrm{C}_{2} \mathrm{H}_{5}+\mathrm{O} & \rightarrow & \mathrm{OH}+\mathrm{C}_{2} \mathrm{H}_{4}\end{array}$

Dabei wurden den einzelnen Kanälen folgende Anteile zugewiesen: 32\% (a $a_{1}$, 40\% (a $)$ und $23 \%$ (b). Es wurde postuliert, dass Kanal (b) eine direkte Abstraktionsreaktion darstellt, da der gemessene Kanalanteil von 23\% aus einer Isomerisierung des angeregten Addukts theoretisch nicht reproduziert werden konnte. Die zugehörige Rechnung hatte dem über ein Addukt verlaufenden Kanal (b) nur 2\% Anteil zugeteilt. Auch Leone et al. befürworten die direkte Abstraktion [LIN98]. Bei ihren zeitaufgelösten IR-Chemilumineszenzuntersuchungen der Schwingungszustände der entstehenden OH-Radikale ergab sich eine nicht-thermische Besetzung der Schwingungszustände. Untersuchungen innerhalb der eigenen Arbeitsgruppe durch Hack, Hoyermann und Zeuch mit Versuchsanordnung C (FTIR) und Diethylketon, Ethyljodid und Ethan $\left(+\mathrm{CFCl}_{3}\right)$ als Radikalvorläufer kommen zu einem ähnlichen Kanalverzweigungsverhältnis wie Gutman et al.: 32\% (a 1 ), 44\% (a $\mathrm{a}_{2}$ und 24\% (b) [HAC02], [ZEU02]. Ein Vergleich der Besetzungsverhältnisse der Schwingungszustände von $\mathrm{OH}$ aus einer LIF-Messung einerseits und einer Rechnung zur OH-Bildung aus einem Ethyl-O Addukt andererseits spricht auch hier für einen direkten Abstraktionsprozess (zur Argumentation vgl. Abschnitt 3.3.8). 


\section{Reaktionsgeschwindigkeit:}

Die Reaktionsgeschwindigkeit der Reaktion Ethyl $+\mathrm{O}$ wurde in dieser Arbeit nicht bestimmt. Jedoch wurde sie auf einen möglichen Isotopeneffekt hin untersucht, indem die Geschwindigkeit von $\mathrm{C}_{2} \mathrm{D}_{5}+\mathrm{O}$ relativ zu der von $\mathrm{C}_{2} \mathrm{H}_{5}+\mathrm{O}$ ermittelt wurde. Die Geschwindigkeit von $\mathrm{C}_{2} \mathrm{H}_{5}+\mathrm{O}$ bildet das Fundament der in den folgenden Abschnitten dargestellten Relativmessungen. Im Rahmen dieser Messungen wurden immer wieder Vergleichsmessungen durchgeführt, um die Ergebnisse auf Konsistenz zu prüfen. Soweit die Konsistenz innerhalb eines Vergleichszyklus gegeben ist und auch Teile des Zyklus in Einklang mit dem Großteil der anerkannten Literatur stehen, kann das durchaus als eine Bestätigung der verwendeten Basisgeschwindigkeitskonstanten der Reaktion Ethyl $+\mathrm{O}$ angesehen werden.

Diskussion: Trotz ihrer enormen Bedeutung in vielen Kohlenwasserstoffverbrennungssystemen lagen in der Literatur bis vor kurzem nur zwei gemessene Werte zur Geschwindigkeit der Reaktion Ethyl $+\mathrm{O}$ vor. Gutman et al.: $\mathrm{k}^{298 \mathrm{~K}}=1,32 \cdot 10^{14} \mathrm{~cm}^{3} /(\mathrm{mol} \cdot \mathrm{s})$ [SLA88], Washida et al.: $\mathrm{k}^{298 \mathrm{~K}}=9,03 \cdot 10^{13} \mathrm{~cm}^{3} /(\mathrm{mol} \cdot \mathrm{s})$ [WAS00]. Daneben existieren in Reviews vorgeschlagene Werte, die bis zum Faktor 2 von den gemessenen Werten abweichen: $\mathrm{k}^{298 \mathrm{~K}}=6,6 \cdot 10^{13} \mathrm{~cm}^{3} /(\mathrm{mol} \cdot \mathrm{s})$ [BAU92], $\mathrm{k}^{298 \mathrm{~K}}=8 \cdot 10^{13} \mathrm{~cm}^{3} /(\mathrm{mol} \cdot \mathrm{s})$ [TSA86], $\mathrm{k}^{298 \mathrm{~K}}=1,3 \cdot 10^{14} \mathrm{~cm}^{3} /(\mathrm{mol} \cdot \mathrm{s})$ [HER88]. In einer Literaturstelle ist die Abweichung des Werts dagegen sehr viel größer: $\mathrm{k}^{298 \mathrm{~K}}=1,75 \cdot 10^{13} \mathrm{~cm}^{3} /(\mathrm{mol} \cdot \mathrm{s})$ [BEN98]. Wegen der nicht unbeachtlichen Unterschiede wurden kürzlich in der eigenen Arbeitsgruppe Anstrengungen zu einer erneuten Bestimmung des Geschwindigkeitskoeffizienten der Reaktion Ethyl + O unternommen. Mittels einer LIF-Analyse des zeitlichen Anstiegprofils der entstehenden OH-Radikale wurde der Koeffizient in pseudoerster Ordnung (O-Atom Überschuss) absolut von Hack, Hoyermann und Zeuch gemessen ([HAC02], [ZEU02]). Das Ergebnis: $\mathbf{k}^{\mathbf{2 9 8 K}}\left(\mathbf{C}_{2} \mathbf{H}_{5}+\mathbf{O}\right)=$

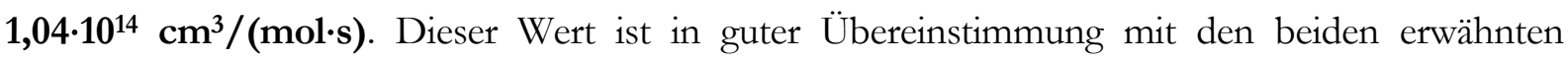
gemessenen Konstanten von Gutman und Washida. Er wird als Basisgeschwindigkeitskonstante für die aus den Relativmessungen der nächsten Abschnitte abgeleiteten Geschwindigkeitskoeffizienten der Reaktionen anderer Radikale mit O-Atomen verwendet.

\section{Isotopeneffekt:}

Messergebnisse: Mit Versuchsanordnung A (REMPI) wurde die Geschwindigkeit der Reaktion $\mathrm{C}_{2} \mathrm{D}_{5}+\mathrm{O}$ relativ zu $\mathrm{C}_{2} \mathrm{H}_{5}+\mathrm{O}$ bei Zimmertemperatur bestimmt. Die Radikale wurden gemäß Abschnitt 3.1.2 erzeugt und auf ihrer Muttermasse nachgewiesen. Zur Bestimmung des Geschwindigkeitskoeffizienten wurden die Signalintensitäten über Peakintegration (vgl. Abschnitt 2.1.6) bei An- und Abwesenheit von Sauerstoffatomen gemessen. Durch Variation der Sauerstoffkonzentration wurden verschiedene Umsätze erzielt. Die folgende Abbildung zeigt in doppelt 
logarithmischer Auftragung (vgl. Abschnitt 2.4.2) die Messpunkte. Die zugehörigen Werte finden sich in Abschnitt 5 (Messwerte). Die gewählten Versuchsbedingungen sind dem Text in der Abbildung oder bei den Messtabellen zu entnehmen. Wie bei allen Messungen wurde sorgfältig geprüft, ob es zu irgendwelchen Überlagerungen durch Edukte oder Produkte bei den verwendeten Massensignalen kommt. Das wäre z.B. bei $\lambda=340,8 \mathrm{~nm}$ durchaus der Fall (vgl. Produkte), nicht aber bei der für diese Messung eingestellten Wellenlänge von $\lambda=435 \mathrm{~nm}$.

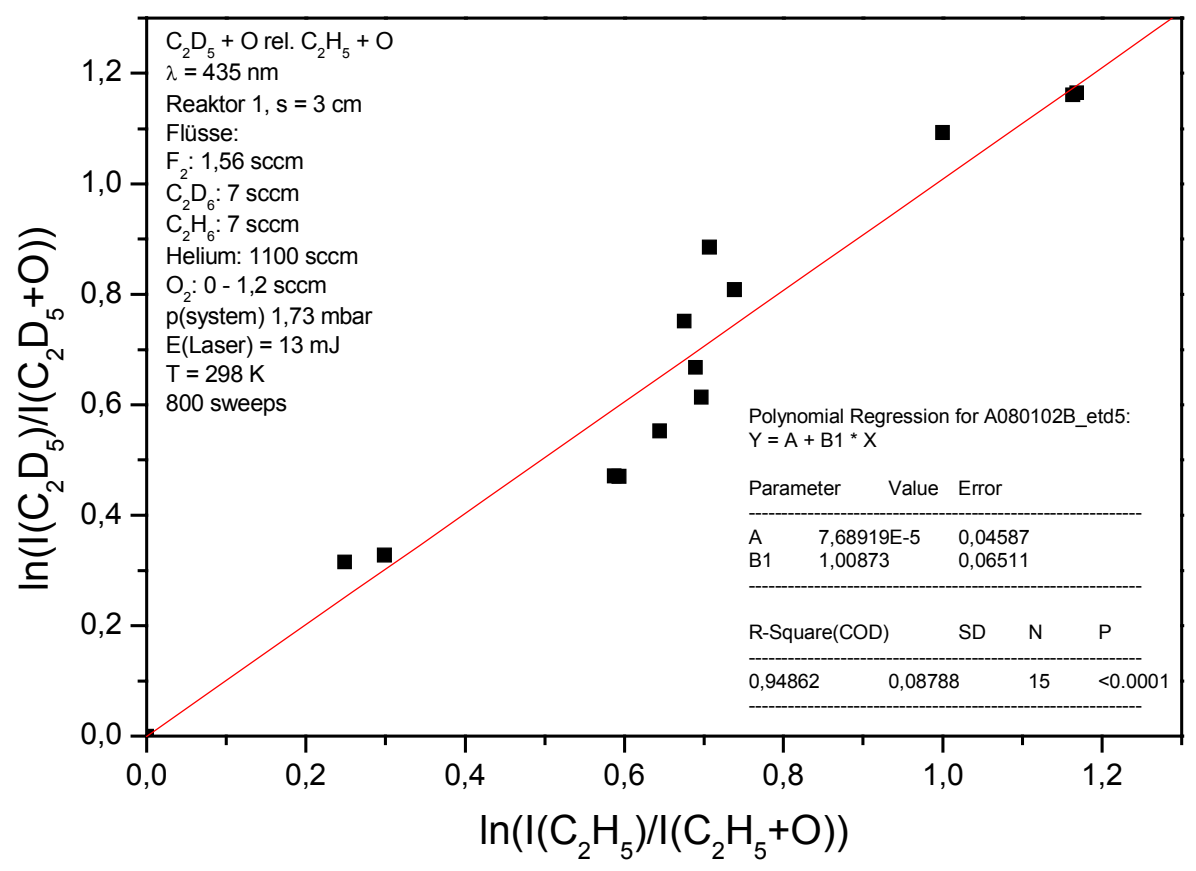

Abbildung 94: $\mathrm{C}_{2} \mathrm{D}_{5}+\mathrm{O}$ relativ zu $\mathrm{C}_{2} \mathrm{H}_{5}+\mathrm{O}, \mathrm{T}=25^{\circ} \mathrm{C}$

Aus der linearen Regression der aufgetragenen Messwerte ergibt sich ein relativer Geschwindigkeitskoeffizient von

$$
\mathrm{k}_{\mathrm{rel}}=\mathrm{k}\left(\mathrm{C}_{2} \mathrm{D}_{5}+\mathrm{O}\right) / \mathrm{k}\left(\mathrm{C}_{2} \mathrm{H}_{5}+\mathrm{O}\right)=1,01 \pm 0,07
$$

Der angegebene Fehler resultiert aus der Standardabweichung der Regressionsgeraden.

Aus der Messung ist ein merklicher Isotopeneffekt nicht abzuleiten. 


\subsubsection{Methoxymethyl + O}

\section{Produkte:}

Diskussion: Die Produkte der Reaktion von $\mathrm{CH}_{3} \mathrm{OCH}_{2}$ mit atomarem Sauerstoff wurden in der eigenen Arbeitsgruppe von Hoyermann und Nacke mit Hilfe von Versuchsanordnung B (EI-MS) durchgeführt ([HOY96], [NAC94]). Folgende Reaktionskanäle sind diskutiert worden:

$$
\begin{array}{lll}
\mathrm{CH}_{3} \mathrm{OCH}_{2}+\mathrm{O} & \rightarrow & \mathrm{CH}_{3} \mathrm{OCHO}+\mathrm{H} \\
& \rightarrow & \mathrm{CH}_{3} \mathrm{O}+\mathrm{CH}_{2} \mathrm{O} \\
& \left.\rightarrow \mathrm{CH}_{2}\right)_{2} \mathrm{O}+\mathrm{OH}
\end{array}
$$

Alle drei Kanäle sind stark exotherm: $\Delta_{\mathrm{R}} \mathrm{H}^{0}{ }_{298 \mathrm{~K}} /(\mathrm{kJ} / \mathrm{mol})$ : $-450,1$ (a), -338,4 (b), -249,6 (c). Für Kanal (c) wurden keine Hinweise gefunden. Das Verzweigungsverhältnis des C-H-Bindungsbruchkanals (a) zum C-C-Bindungsbruch-Kanals wird angegeben mit $(95 \pm 18) \%:(5 \pm 1) \%$.

\section{Reaktionsgeschwindigkeit:}

Messergebnisse: Die Geschwindigkeit der Reaktion $\mathrm{CH}_{3} \mathrm{OCH}_{2}+\mathrm{O}$ wurde relativ zur Reaktion Ethyl $+\mathrm{O}$ bei Zimmertemperatur mit Versuchsanordnung A (REMPI) bei $\lambda=433 \mathrm{~nm}$ bestimmt. Die Radikale wurden gemäß Abschnitt 3.1.2 und 3.1.14 erzeugt und auf ihrer Muttermasse nachgewiesen. Zur Bestimmung des Geschwindigkeitskoeffizienten wurden die Signalintensitäten bei An- und Abwesenheit von Sauerstoffatomen gemessen. Durch Variation der Sauerstoffkonzentration wurden verschiedene Umsätze erzielt. Die folgende Abbildung zeigt in doppeltlogarithmischer Auftragung die Messpunkte. Die zugehörigen Werte finden sich in Abschnitt 5 (Messtabellen). Die gewählten Versuchsbedingungen sind dem Text in der Abbildung oder bei den Messtabellen zu entnehmen. 


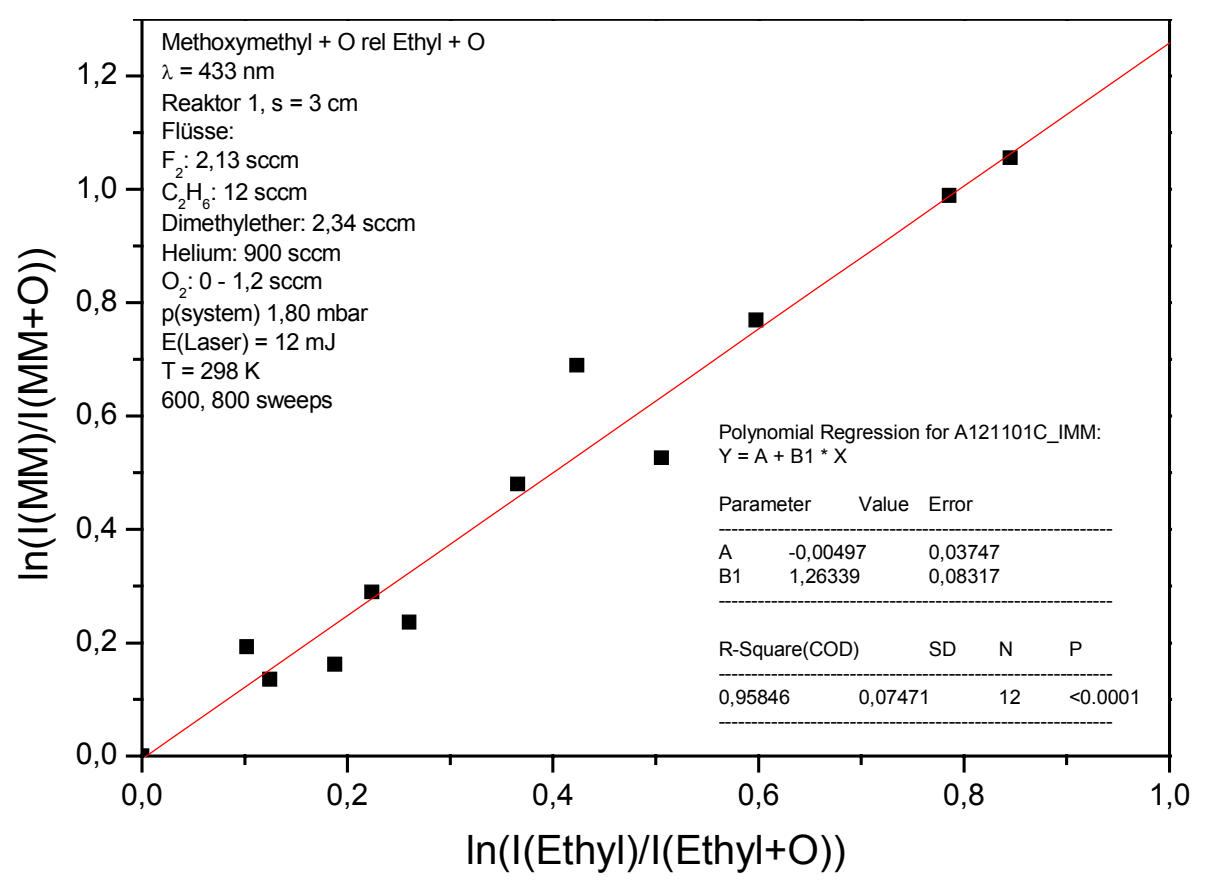

Abbildung 95: Methoxymethyl $+\mathrm{O}$ relativ zu Ethyl $+\mathrm{O}, \mathrm{T}=25^{\circ} \mathrm{C}$

Aus der Auftragung ergibt sich:

$$
\mathrm{k}_{\mathrm{rel}}=\mathrm{k}\left(\mathrm{CH}_{3} \mathrm{OCH}_{2}+\mathrm{O}\right) / \mathrm{k}\left(\mathrm{C}_{2} \mathrm{H}_{5}+\mathrm{O}\right)=1,26 \pm 0,08 \text {. }
$$

Mit der in Abschnitt 3.3.1 erläuterten Geschwindigkeitskonstante $k_{\text {ref }}=1,04 \cdot 10^{14} \mathrm{~cm}^{3} /(\mathrm{mol} \cdot \mathrm{s})$ für die Referenzreaktion Ethyl + O erhält man:

$$
\mathrm{k}^{298 \mathrm{~K}}\left(\mathrm{CH}_{3} \mathrm{OCH}_{2}+\mathrm{O}\right)=(1,31 \pm 0,08) \cdot 10^{14} \mathrm{~cm}^{3} /(\mathrm{mol} \cdot \mathrm{s})
$$

Diskussion: Die Geschwindigkeit der Reaktion $\mathrm{CH}_{3} \mathrm{OCH}_{2}+\mathrm{O}$ wurde innerhalb der eigenen Arbeitsgruppe bereits von Hoyermann und Nacke mit Versuchsanordnung B (EI-MS) relativ zur Reaktion Ethyl + O bestimmt ([HOY96], [NAC94]). Die zugehörige Geschwindigkeitskonstante wird mit $\mathrm{k}^{298 \mathrm{~K}}=(1,54 \pm 0,03) \cdot 10^{14} \mathrm{~cm}^{3} /(\mathrm{mol} \cdot \mathrm{s})$ angegeben. Als Referenzgeschwindigkeitskoeffizient wurde der von Gutman et al. angegebene Wert $\mathrm{k}\left(\right.$ Ethyl+O) $=1,33 \cdot 10^{14} \mathrm{~cm}^{3} /(\mathrm{mol} \cdot \mathrm{s})$ [SLA88] verwendet. Legt man den in dieser Arbeit verwendeten Wert $k_{\text {ref }}=1,04 \cdot 10^{14} \mathrm{~cm}^{3} /(\mathrm{mol} \cdot \mathrm{s})$ ([HAC02], [ZEU02]) zugrunde, so ergibt sich aus dem relativen Geschwindigkeitskoeffizient $\mathrm{k}_{\text {rel }}=1,16$ ein Wert von $\mathrm{k}\left(\mathrm{CH}_{3} \mathrm{OCH}_{2}+\mathrm{O}\right)=1,20 \cdot 10^{14} \mathrm{~cm}^{3} /(\mathrm{mol} \cdot \mathrm{s})$, was in sehr guter Übereinstimmung mit dem in dieser Arbeit ermittelten Wert ist. Die Geschwindigkeit der Reaktion Methoxymethyl $+\mathrm{O}$ soll deshalb als so gesichert angesehen werden, dass sie als zuverlässige Referenz für andere Reaktionen tauglich ist. 


\subsubsection{Cyclopentyl + O}

\section{Produkte:}

Es wurden keine eigenen Produktuntersuchungen vorgenommen. Folgende Reaktionskanäle erscheinen mechanistisch möglich:

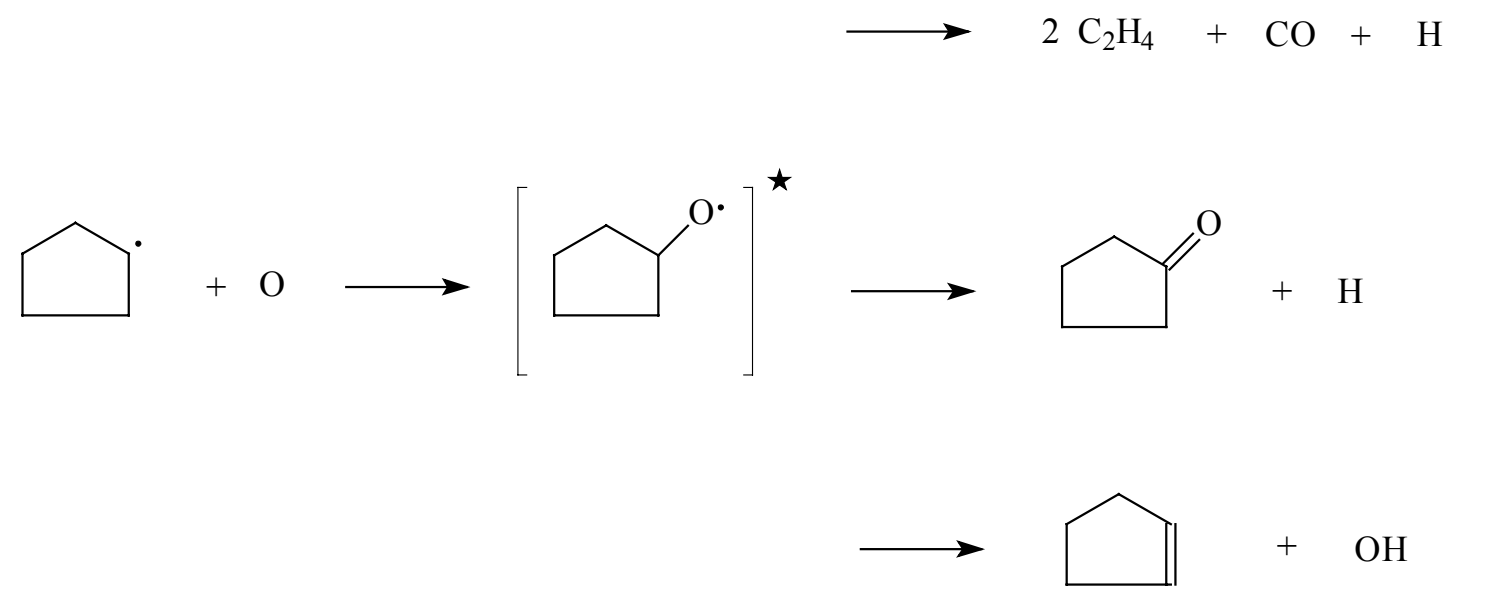

Kanal (a) (Zerfall) entspricht dem C-C-Bindungsbruch $\left(\Delta_{\mathrm{R}} \mathrm{H}^{0}{ }_{298 \mathrm{~K}}=-139,0 \mathrm{~kJ} / \mathrm{mol}\right)$, Kanal (b) (Substitution) dem C-H-Bindungsbruch $\left(\Delta_{\mathrm{R}} \mathrm{H}^{0}{ }_{298 \mathrm{~K}}=-325,6 \mathrm{~kJ} / \mathrm{mol}\right)$. Kanal (c) wird als Abstraktion bezeichnet $\left(\Delta_{\mathrm{R}} \mathrm{H}^{0} 298 \mathrm{~K}=-276,2 \mathrm{~kJ} / \mathrm{mol}\right)$, wobei offen bleiben soll, ob dieser Weg über den angeregten Adduktkomplex verläuft oder nicht.

Diskussion: Teile des aufgeführten Reaktionsmechanismus sind schon von Washida et al. erwähnt worden [TAK81]. Diese untersuchten die Photooxidation von Cyclopentan in einem $\mathrm{NO}_{-} \mathrm{H}_{2} \mathrm{O}-$ Luft-System. Es wurde Cyclopentanon nachgewiesen. Durch die Vielzahl der zur Verfügung stehenden Reaktionspartner ( $\mathrm{NO}, \mathrm{O}_{2}$, als Folgeprodukte: $\mathrm{O}, \mathrm{OH}$ ) und damit auch der möglichen Reaktionen kann jedoch nicht von einer spezifischen Untersuchung der Reaktion c-Pentyl $+\mathrm{O}$ gesprochen werden. Innerhalb der eigenen Arbeitsgruppe wurden von Nacke mit Versuchsanordnung B (EI-MS) Produktuntersuchungen angestrengt, die aber nicht zum Nachweis bestimmter Produkte geführt haben [NAC98]. Kanal (c) wurde ausgeschlossen, da kein Hinweis auf c-Penten $(\mathrm{m} / \mathrm{z}=68)$ gefunden wurde. Es wurden weiterhin Kanäle zur Bildung von 4-Pentenal und zu 1-Buten, die über einen H-Shift verlaufen würden (nicht im obigen Mechanismus erwähnt), ausgeschlossen. Es wurden keine Hinweise zu Kanal (b) gefunden.

Die Messungen von Nacke stehen im Widerspruch zu einer früheren Untersuchung von Rohde ([HEI90], [ROH88]), die mit Versuchsanordnung B (EI-MS) Anhaltspunkte für eine Entstehung von Cyclopentanon (Kanal (b)) und Cyclopenten (Kanal (c)) gefunden hat. Die Vielzahl von Überlagerungen im Massenspektrum, die sich aus den ähnlichen Fragmentierungsmustern der 
möglichen Produkte ergibt, lässt genauere Aussagen jedoch nicht zu. Eine weitere Untersuchung von Rohde mit Versuchsanordnung A (REMPI) schien Kanal (c) durch den vermeintlichen Nachweis von $\mathrm{OH}$ zu bestärken [ROH91]. Da der REMPI-Nachweis des OH-Radikals aber fehlerhaft war und später auch zurückgenommen wurde, muss diese Untersuchung verworfen werden.

Neuere Untersuchungen in der eigenen Arbeitsgruppe mit Versuchsanordnung C (FTIR) von Nothdurft scheinen dagegen erfolgreicher zu verlaufen. Es wird in eindeutiger Weise gezeigt, dass Cyclopentanon entsteht [NOT02]. Kanal (a) wird anhand des entstehenden Ethens und Kohlenmonoxids ebenfalls nachgewiesen. Kanal (c) wird in Übereinstimmung mit den Untersuchungen von Nacke ausgeschlossen. Eine Bestimmung des Kanalverzweigungsverhältnisses steht kurz bevor.

$\underline{\text { Reaktionsgeschwindigkeit: }}$

Messergebnisse: Die Geschwindigkeit der Reaktion c-Pentyl $+\mathrm{O}$ wurde relativ zur Reaktion Ethyl + O bei Zimmertemperatur mit Versuchsanordnung A (REMPI) bei $\lambda=433 \mathrm{~nm}$ bestimmt. Die Radikale wurden gemäß Abschnitt 3.1.2 und 3.1.6 erzeugt und auf ihrer Muttermasse nachgewiesen. Zur Bestimmung des Geschwindigkeitskoeffizienten wurden die Signalintensitäten bei An- und Abwesenheit von Sauerstoffatomen gemessen. Durch Variation der Sauerstoffkonzentration wurden verschiedene Umsätze erzielt. Die folgende Abbildung zeigt in doppeltlogarithmischer Auftragung die Messpunkte. Die zugehörigen Werte finden sich in Abschnitt 5 (Messtabellen). Die gewählten Versuchsbedingungen sind dem Text in der Abbildung oder bei den Messtabellen zu entnehmen.

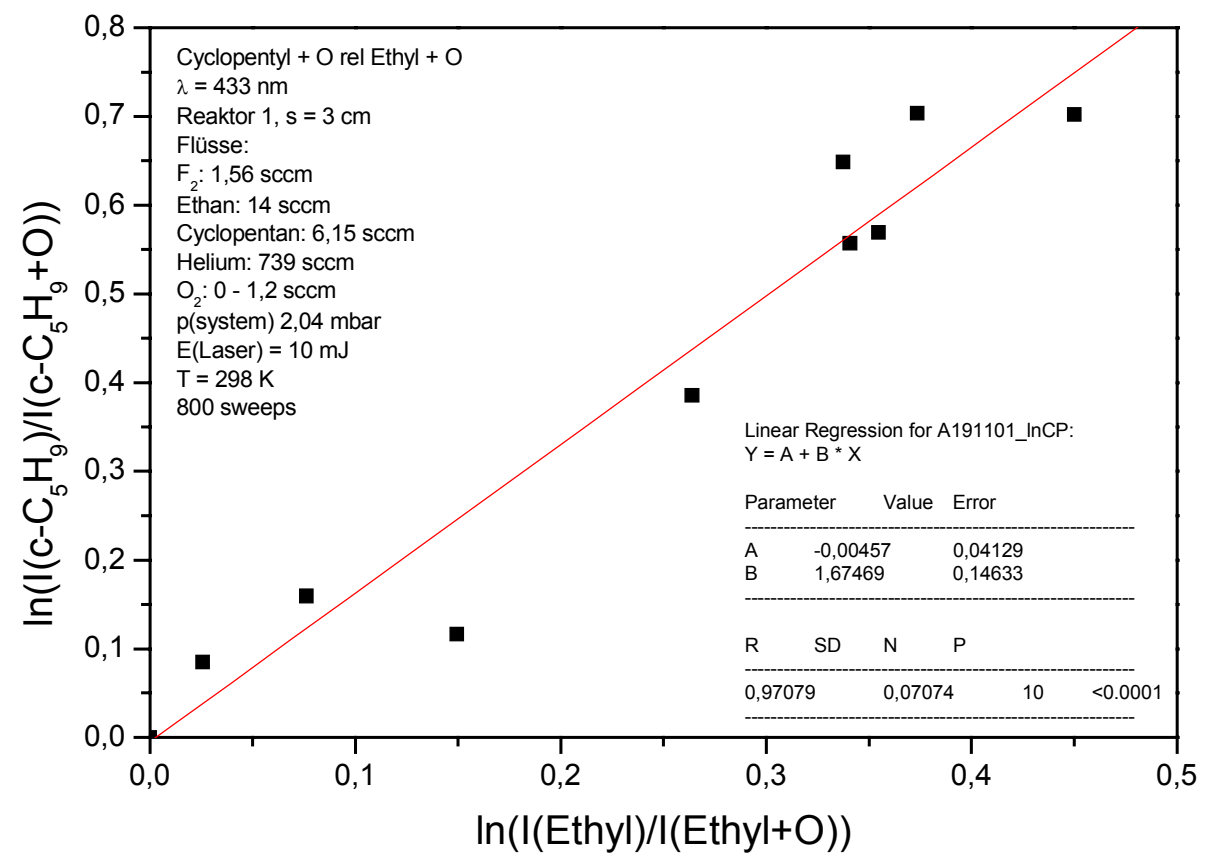

Abbildung 96: Cyclopentyl $+\mathrm{O}$ relativ zu Ethyl $+\mathrm{O}, \mathrm{T}=25^{\circ} \mathrm{C}$ 
Aus der Auftragung ergibt sich:

$$
\mathrm{k}_{\mathrm{rel}}=\mathrm{k}(\mathrm{c}-\mathrm{Pentyl}+\mathrm{O}) / \mathrm{k}\left(\mathrm{C}_{2} \mathrm{H}_{5}+\mathrm{O}\right)=1,67 \pm 0,15
$$

Mit der in Abschnitt 3.3.1 erläuterten Geschwindigkeitskonstante $\mathrm{k}_{\mathrm{ref}}=1,04 \cdot 10^{14} \mathrm{~cm}^{3} /(\mathrm{mol} \cdot \mathrm{s})$ für die Referenzreaktion Ethyl + O erhält man:

$$
\mathrm{k}^{298 \mathrm{~K}}(\mathrm{c}-\text { Pentyl+O})=(1,73 \pm 0,16) \cdot 10^{14} \mathrm{~cm}^{3} /(\mathrm{mol} \cdot \mathrm{s})
$$

\section{Vergleichskinetik:}

Die Geschwindigkeit der Reaktion von c-Pentyl $+\mathrm{O}$ wurde zur Kontrolle auch noch relativ zur Reaktion von Methoxymethyl + O bei Zimmertemperatur mit Versuchsanordnung A (REMPI) bei $\lambda=433 \mathrm{~nm}$ bestimmt. Die Radikale wurden gemäß Abschnitt 3.1.14 und 3.1.6 erzeugt und auf ihrer Muttermasse nachgewiesen. Die folgende Abbildung zeigt die zugehörige Auftragung:

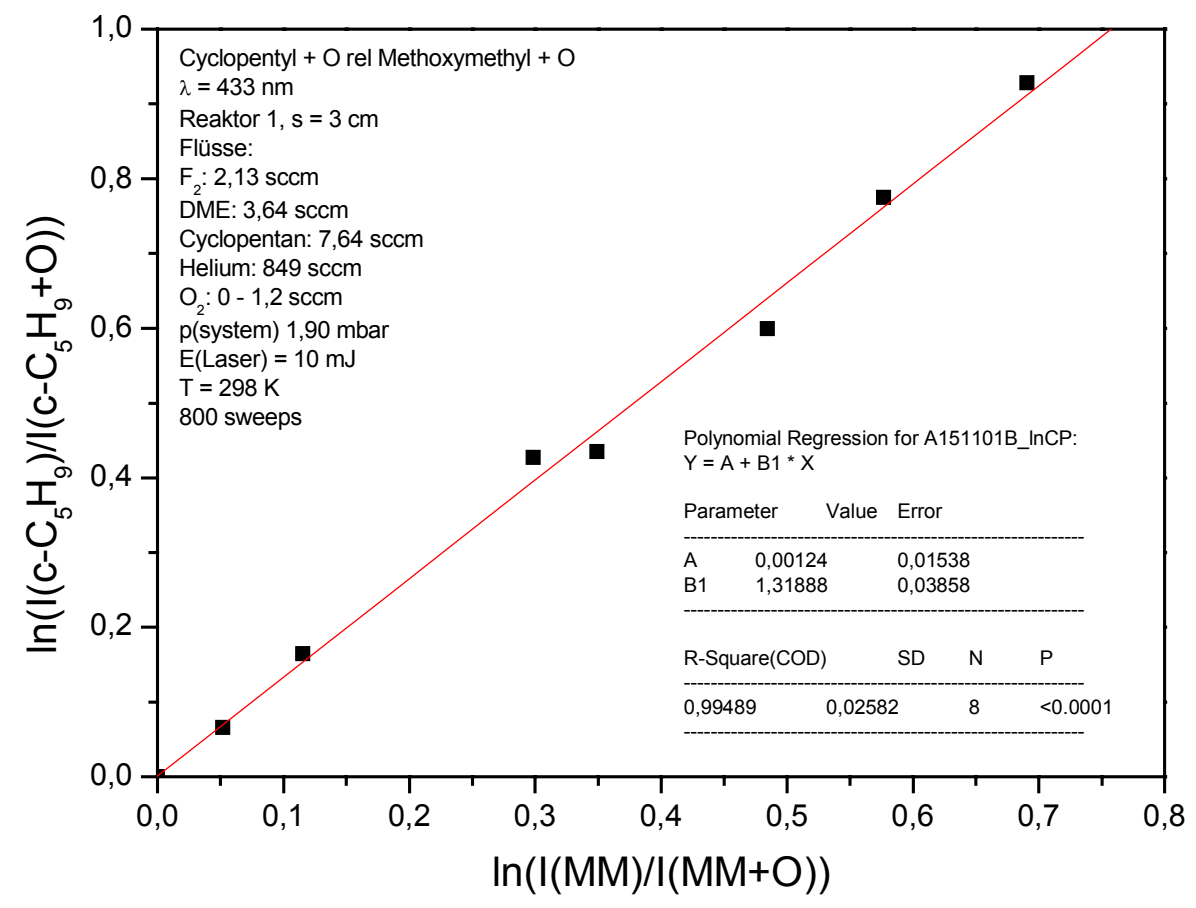

Abbildung 97: Cyclopentyl $+\mathrm{O}$ relativ zu Methoxymethyl $+\mathrm{O}, \mathrm{T}=25^{\circ} \mathrm{C}$

Aus der Auftragung ergibt sich:

$$
\mathrm{k}_{\mathrm{rel}}=\mathrm{k}(\mathrm{c}-\mathrm{Pentyl}+\mathrm{O}) / \mathrm{k}\left(\mathrm{CH}_{3} \mathrm{OCH}_{2}+\mathrm{O}\right)=1,32 \pm 0,04
$$

Mit der in Abschnitt 3.3.2 erläuterten Geschwindigkeitskonstante $k_{\text {ref }}=1,31 \cdot 10^{14} \mathrm{~cm}^{3} /(\mathrm{mol} \cdot \mathrm{s})$ für die Referenzreaktion Methoxymethyl + O erhält man: 


\section{$\mathrm{k}^{298 \mathrm{~K}}(\mathrm{c}-$ Pentyl+O$)=(1,73 \pm 0,05) \cdot 10^{14} \mathrm{~cm}^{3} /(\mathrm{mol} \cdot \mathrm{s})$}

Es bleibt festzuhalten, dass beide Messreihen zu dem selben Wert kommen.

Diskussion: Die Geschwindigkeit der Reaktion c-Pentyl + O wurde bereits mehrfach innerhalb der eigenen Arbeitsgruppe untersucht. Durch Rohde ist das mit Versuchsanordnung A (REMPI) relativ zur Reaktion Ethyl $+\mathrm{O}$ geschehen [ROH91]. Die zugehörige Konstante wird mit $\mathrm{k}^{298 \mathrm{~K}}=(2,00 \pm 0,6) \cdot 10^{14} \mathrm{~cm}^{3} /(\mathrm{mol} \cdot \mathrm{s})$ angegeben. Als Referenzgeschwindigkeitskoeffizient wurde der von Gutman et al. angegebene Wert $\mathrm{k}\left(\right.$ Ethyl+O) $=1,33 \cdot 10^{14} \mathrm{~cm}^{3} /(\mathrm{mol} \cdot \mathrm{s})$ [SLA88] verwendet. Legt man den in dieser Arbeit verwendeten Wert $\mathrm{k}_{\text {ref }}=1,04 \cdot 10^{14} \mathrm{~cm}^{3} /(\mathrm{mol} \cdot \mathrm{s})([\mathrm{HAC02}]$, [ZEU02]) zugrunde, so ergibt sich aus der von Rohde ermittelten relativen Geschwindigkeitskonstanten $k_{\text {rel }}=$ 1,52 ein Wert von $\mathrm{k}\left(\mathrm{c}-\right.$ Pentyl+O) $=1,58 \cdot 10^{14} \mathrm{~cm}^{3} /(\mathrm{mol} \cdot \mathrm{s})$, welcher in sehr guter Übereinstimmung mit dem in dieser Arbeit ermitteltem Wert ist. Als Ergebnis einer zweiten Messung mit einem Lavaldüsenreaktor und massenspektrometrischem Nachweis wird von Rohde der Wert k(cPentyl $+\mathrm{O})=(1,7 \pm 0,1) \cdot 10^{14} \mathrm{~cm}^{3} /(\mathrm{mol} \cdot \mathrm{s})$ angegeben [HEI90]. Für die Referenzreaktion $\mathrm{CH}_{3}+\mathrm{O}$ wurde der Geschwindigkeitskoeffizient $\mathrm{k}_{\mathrm{ref}}=8,4 \cdot 10^{13} \mathrm{~cm}^{3} /(\mathrm{mol} \cdot \mathrm{s})$ [SLA87] verwendet. Von Nacke ist mit Versuchsanordnung A (REMPI) für die Reaktion c-Pentyl + O ein Wert von $\mathrm{k}^{298 \mathrm{~K}}=(1,8 \pm$ 0,5) $10^{14} \mathrm{~cm}^{3} /(\mathrm{mol} \cdot \mathrm{s})$ ermittelt worden. Dabei wurde als Referenzreaktion $\mathrm{CH}_{3} \mathrm{OCH}_{2}+\mathrm{O}$ verwendet worden. Korrigiert man diese Messung gemäß der Diskussion aus Abschnitt 3.3.2, so erhält man $\mathrm{k}^{298 \mathrm{~K}}(\mathrm{c}-\mathrm{Pentyl}+\mathrm{O})=1,53 \cdot 10^{14} \mathrm{~cm}^{3} /(\mathrm{mol} \cdot \mathrm{s})$. Im Vergleich zu den bisher gemachten Messungen liegen die eigenen Werte für die Geschwindigkeitskonstante der Reaktion c-Pentyl + O zwar leicht höher, aber im Rahmen der zu erreichenden Messgenauigkeit kann man nicht von einer gravierenden Abweichung sprechen. Die Geschwindigkeit der Reaktion c-Pentyl + O soll deshalb als so gesichert angesehen werden, dass sie als zuverlässige Referenz für andere Reaktionen tauglich ist.

\subsubsection{Methyl + O}

\section{Produkte:}

Messergebnisse: Die Produktbildung der Reaktion Methyl + O wurde mit Versuchsanordnung A (REMPI) bei $\lambda=340,8 \mathrm{~nm}$ untersucht. Dabei wurden zum einen $\mathrm{CH}_{3}$-Radikale, zum anderen $\mathrm{CD}_{3}$ Radikale über die Reaktion des entsprechenden Methans mit F-Atomen erzeugt und gemäß Abschnitt 3.1.1 nachgewiesen. Die Sauerstoffatome wurde gemäß Abschnitt 2.6 dargestellt und den Radikalen in der Reaktionszone zugeführt. Die folgenden Abbildungen zeigen als Messergebnisse Flugzeitmassenspektren unter den Bedingungen Methyl -O/+O, wobei die zweite und die dritte Abbildung dieselbe Messung, aber in Farbinversion darstellen. 


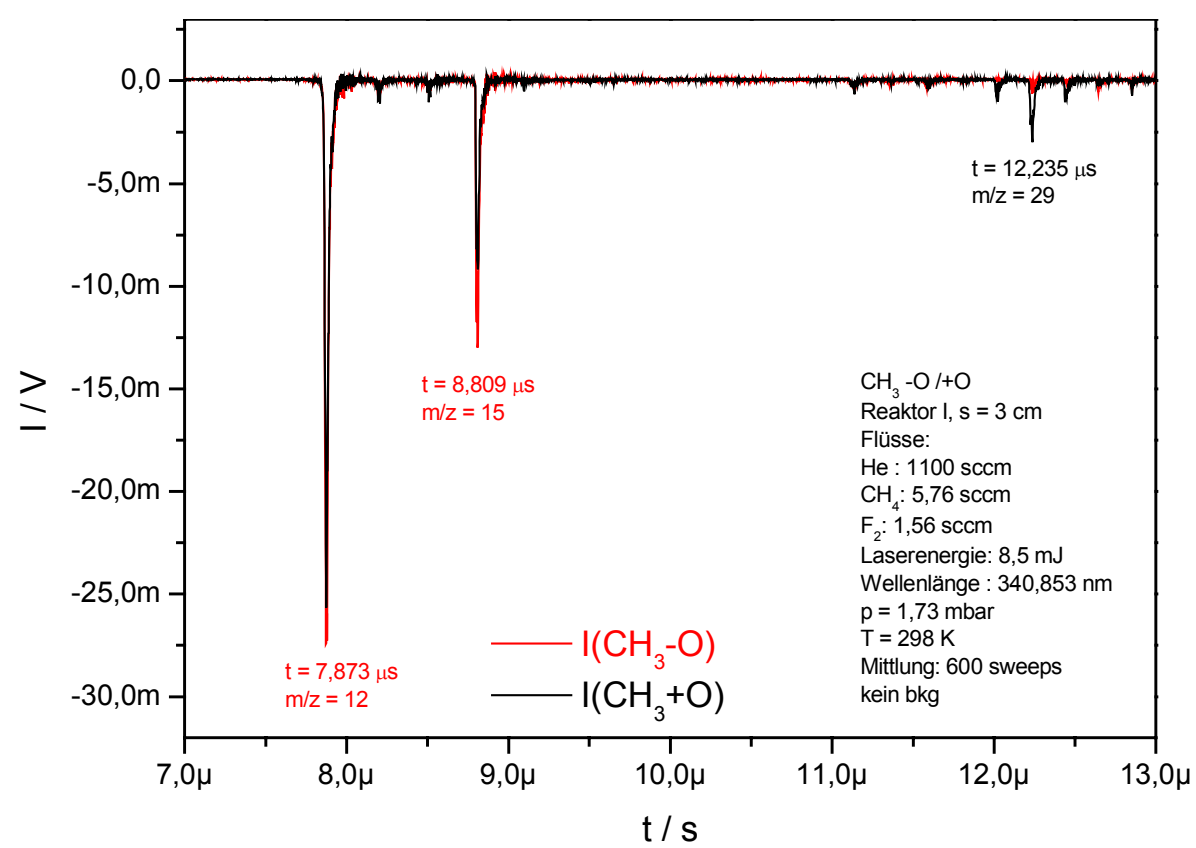

Abbildung 98: Flugzeitmassenspektrum $\mathrm{CH}_{3}-\mathrm{O} /+\mathrm{O}, \lambda=340,8 \mathrm{~nm}$

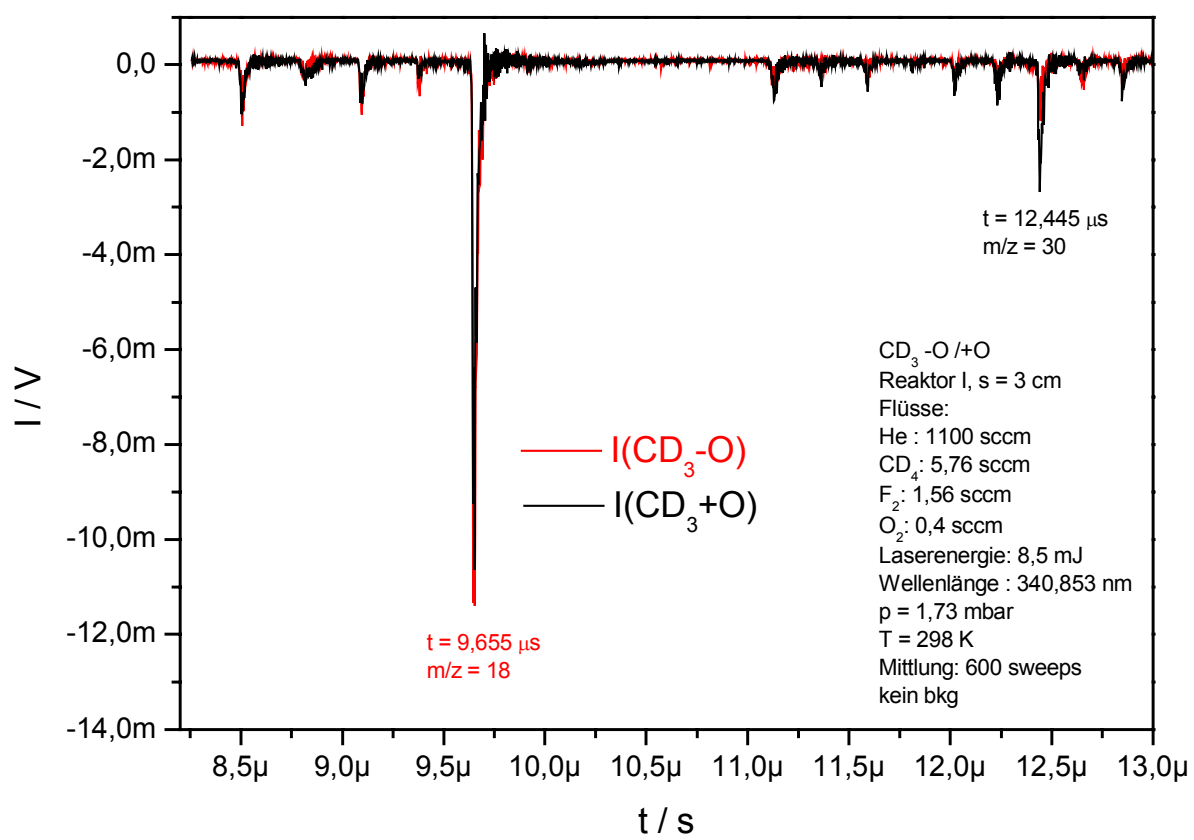

Abbildung 99: Flugzeitmassenspektrum $\mathrm{CD}_{3}-\mathrm{O} /+\mathrm{O}, \lambda=340,8 \mathrm{~nm}$, Darst. 1 


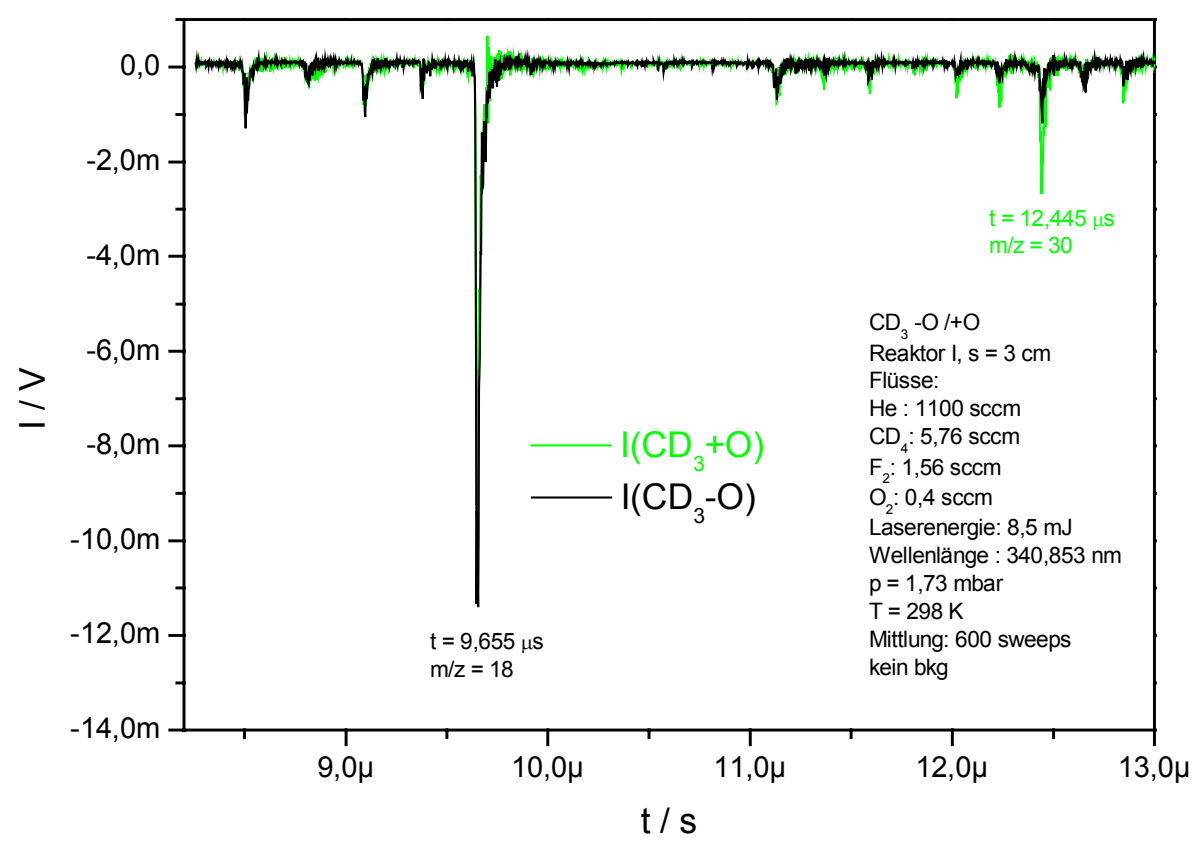

Abbildung 100: Flugzeitmassenspektrum $\mathrm{CD}_{3}-\mathrm{O} /+\mathrm{O}, \lambda=340,8 \mathrm{~nm}$, Darst. 2

Interpretation: Für die Reaktion von Methylradikalen mit O-Atomen sind zwei Reaktionskanäle bekannt:

$$
\rightarrow \mathrm{HCHO}+\mathrm{H}
$$

$$
\mathrm{CH}_{3}+\mathrm{O}
$$

(a) $\Delta_{\mathrm{R}} \mathrm{H}^{0}{ }_{298 \mathrm{~K}}=-286 \mathrm{~kJ} / \mathrm{mol}$

(b) $\Delta_{\mathrm{R}} \mathrm{H}^{0}{ }_{298 \mathrm{~K}}=-287 \mathrm{~kJ} / \mathrm{mol}$

Durch die Abnahme bei $\mathrm{m} / \mathrm{z}=15$ in Abbildung 98 ist ein Reaktionseffekt von Methylradikalen mit O-Atomen festzustellen. Die Zunahme bei $\mathrm{m} / \mathrm{z}=29$ in Abbildung 98 sowie die Zunahme bei $\mathrm{m} / \mathrm{z}=30$ in Abbildung 100 ist der Entstehung von Formaldehyd nach (a) zuzuordnen, welches in massenspektrometrischen Ionenquellen typischerweise ein H- bzw. D-Atom verliert (vgl. Anhang 6.3, S. 260). Eine kleine Zunahme ist auch bei $\mathrm{m} / \mathrm{z}=28 \mathrm{zu}$ erkennen. Ob diese Zunahme aber dem nach (b) entstehenden CO zuzuordnen ist oder ein Fragment des Formaldehyds nach (a) ist, kann nicht sicher beurteilt werden. Über einen REMPI-Nachweis des Kohlenmonoxids bei der verwendeten Wellenlänge nichts bekannt ist. $\mathrm{Da}$ aber auch bei $\mathrm{m} / \mathrm{z}=29$ in Abbildung 100 eine kleine Zunahme zu verzeichnen ist, ist zu vermuten, dass diese nur kleinen Intensitätssteigerung bei $\mathrm{m} / \mathrm{z}=28$ auf nicht-deuteriertes Formaldehyd zurückzuführen ist, welches infolge einer nur 98\%igen D-Atom Reinheit des verwendeten deuterierten Methans gebildet wird.

Diskussion: Kanal (a) der Reaktion $\mathrm{CH}_{3}+\mathrm{O}$ zur Bildung von Formaldehyd und H-Atomen wurde lange Zeit als der einzig relevante Reaktionskanal angesehen (vgl. [SLA87]). Von Seakins und Leone wurde eine abweichende Meinung vertreten. Mittels FTIR-Messungen des Primärproduktes CO 
wurde für Kanal (b) ein Anteil von (40 \pm 10)\% ermittelt [SEA92]. Neuere Untersuchungen in der eigenen Arbeitsgruppe bestätigen das. So wurde von Hold mit Versuchsanordnung B (EI-MS) durch Bestimmung der massenspektrometrischen Nachweisempfindlichkeit des Kohlenmonoxids in Verbindung mit einer Titration des Verbrauchs an Methyl ein Kanalanteil für (b) von (46 \pm 6$) \%$ bestimmt [HOL01]. In einer davon unabhängigen Messung von Zeuch mit Versuchsanordnung C (FTIR) wurde über eine Massenbilanz ein CO-Kanalanteil von $(45 \pm 2) \%$ ermittelt [ZEU02], was in sehr guter Übereinstimmung mit den Ergebnissen von Seakins und Leone und von Hold ist.

Muckerman et al. haben jüngst die Reaktion $\mathrm{CH}_{3}+\mathrm{O}$ bei Zimmertemperatur und einem Druck von $\mathrm{p}=1$ Torr in einem Strömungsreaktor mit Time-of-Flight-Massenspektrometer (TOF-MS) und Photoionisation durch eine Ar- oder Ne-Hohlkathodenlampe im VUV-Bereich $(\lambda=104,6-106,5 \mathrm{~nm}, \lambda=73,4-74,2 \mathrm{~nm})$ untersucht [FOC99]. Die Erzeugung der Sauerstoffatome geschah photolytisch aus $\mathrm{SO}_{2}$ bei einer Wellenlänge von $\lambda=193 \mathrm{~nm}$. Gleichzeitig wurden Methylradikale kophotolytisch aus Aceton erzeugt. Formaldehyd aus dem Reaktionskanal (a) konnte klar nachgewiesen werden. Kohlenmonoxid aus Kanal (b) wurde ein Kanalanteil von 0,17 \pm 0,11 zugewiesen. Einschränkend lässt sich anführen, dass das Signal bei $\mathrm{m} / \mathrm{z}=28$ nicht nur durch CO aus Kanal (b), sondern auch durch $\mathrm{CO}$ aus einem Photolysekanal und durch $\mathrm{N}_{2}$ (bkg) sowie Fragmente von Aceton (Vorläufer) und Formaldehyd (Kanal (a)) belegt war. Zwar wurden diese Beiträge durch umfangreiche und detaillierte Kalibrierungen herausgerechnet, aber die daraus ergebende Unsicherheit in Verbindung mit der beachtlichen Streuung der Signale, die auch nach einer Durchschnittsbildung von 100000-200000 Messpunkte erhalten blieb, lassen die Ergebnisse nur bedingt zuverlässig erscheinen. Der Einsatz von $\mathrm{CH}_{3} \mathrm{Br}$ als Radikalvorläufer erwies sich aufgrund eines geringen Photolysegrads von 1,5\% als ebenfalls problematisch.

\section{$\underline{\text { Reaktionsgeschwindigkeit: }}$}

Messergebnisse: Die Geschwindigkeit der Reaktion $\mathrm{CH}_{3}+\mathrm{O}$ wurde relativ zur Reaktion $\mathrm{C}_{2} \mathrm{D}_{5}+\mathrm{O}$ bei Zimmertemperatur mit Versuchsanordnung A (REMPI) bei $\lambda=340,8 \mathrm{~nm}$ bestimmt. Die Radikale wurden gemäß Abschnitt 3.1.1 und 3.1.2 erzeugt und auf ihren Muttermassen $\mathrm{m} / \mathrm{z}=15$ und $\mathrm{m} / \mathrm{z}=34$ nachgewiesen. Es wurde mit dem deuterierten Ethyl $\mathrm{C}_{2} \mathrm{D}_{5}$ gearbeitet, um Überlagerungen auf den Massen $\mathrm{m} / \mathrm{z}=15$ (Muttermasse des $\mathrm{CH}_{3}$, Fragment des $\mathrm{C}_{2} \mathrm{H}_{5}$ ) und $\mathrm{m} / \mathrm{z}=29$ (Muttermasse des $\mathrm{C}_{2} \mathrm{H}_{5}$, Fragment des bei beiden Reaktionen entstehenden Formaldehyds) zu vermeiden. Wie in Abschnitt 3.3.1 erläutert, konnte bei der Reaktion Ethyl + O kein Isotopeneffekt festgestellt werden. Zur Bestimmung des Geschwindigkeitskoeffizienten wurden die Signalintensitäten bei An- und Abwesenheit von Sauerstoffatomen gemessen. Durch Variation der Sauerstoffkonzentration wurden verschiedene Umsätze erzielt. Die folgende Abbildung zeigt in 
doppeltlogarithmischer Auftragung die Messpunkte. Die zugehörigen Werte finden sich in Abschnitt 5 (Messtabellen). Die gewählten Versuchsbedingungen sind dem Text in der Abbildung oder bei den Messtabellen zu entnehmen.

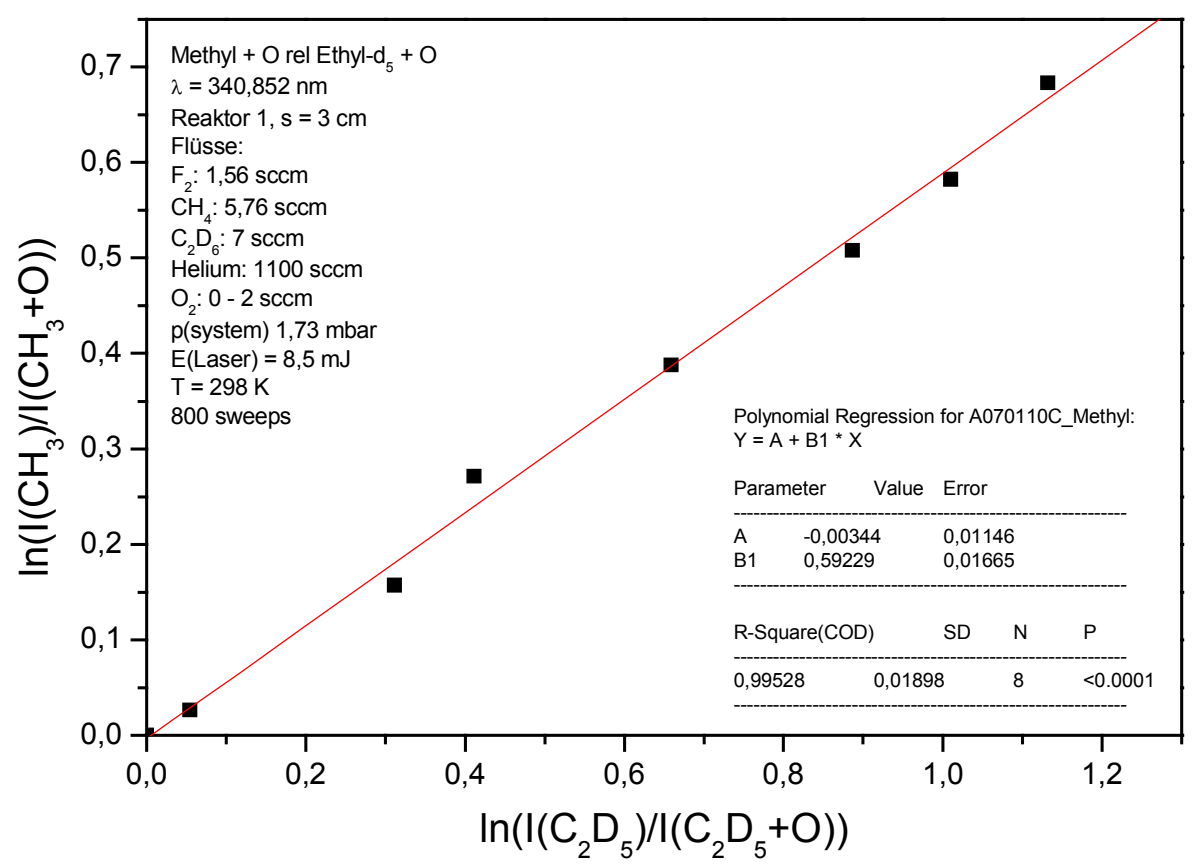

Abbildung 101: $\mathrm{CH}_{3}+\mathrm{O}$ relativ zu $\mathrm{C}_{2} \mathrm{D}_{5}+\mathrm{O}, \mathrm{T}=25^{\circ} \mathrm{C}$

Aus der Auftragung ergibt sich:

$$
\mathrm{k}_{\mathrm{rel}}=\mathrm{k}\left(\mathrm{CH}_{3}+\mathrm{O}\right) / \mathrm{k}\left(\mathrm{C}_{2} \mathrm{D}_{5}+\mathrm{O}\right)=0,59 \pm 0,02
$$

Mit der in Abschnitt 3.3.1 erläuterten Geschwindigkeitskonstante $k_{\text {ref }}=1,04 \cdot 10^{14} \mathrm{~cm}^{3} /(\mathrm{mol} \cdot \mathrm{s})$ für die Referenzreaktion Ethyl + O erhält man:

$$
\mathrm{k}^{298 \mathrm{~K}}\left(\mathrm{CH}_{3}+\mathrm{O}\right)=(6,14 \pm 0,2) \cdot 10^{13} \mathrm{~cm}^{3} /(\mathrm{mol} \cdot \mathrm{s}) .
$$

Vergleichskinetik:

Die Geschwindigkeit der Reaktion von $\mathrm{CH}_{3}+\mathrm{O}$ wurde zur Kontrolle auch relativ zur Reaktion Methoxymethyl + O bei Zimmertemperatur mit Versuchsanordnung A (REMPI) bei $\lambda=450,7 \mathrm{~nm}$ bestimmt. Die Radikale wurden gemäß Abschnitt 3.1.1 und 3.1.14 erzeugt und auf ihrer Muttermasse nachgewiesen. Die folgende Abbildung zeigt die zugehörige Auftragung: 


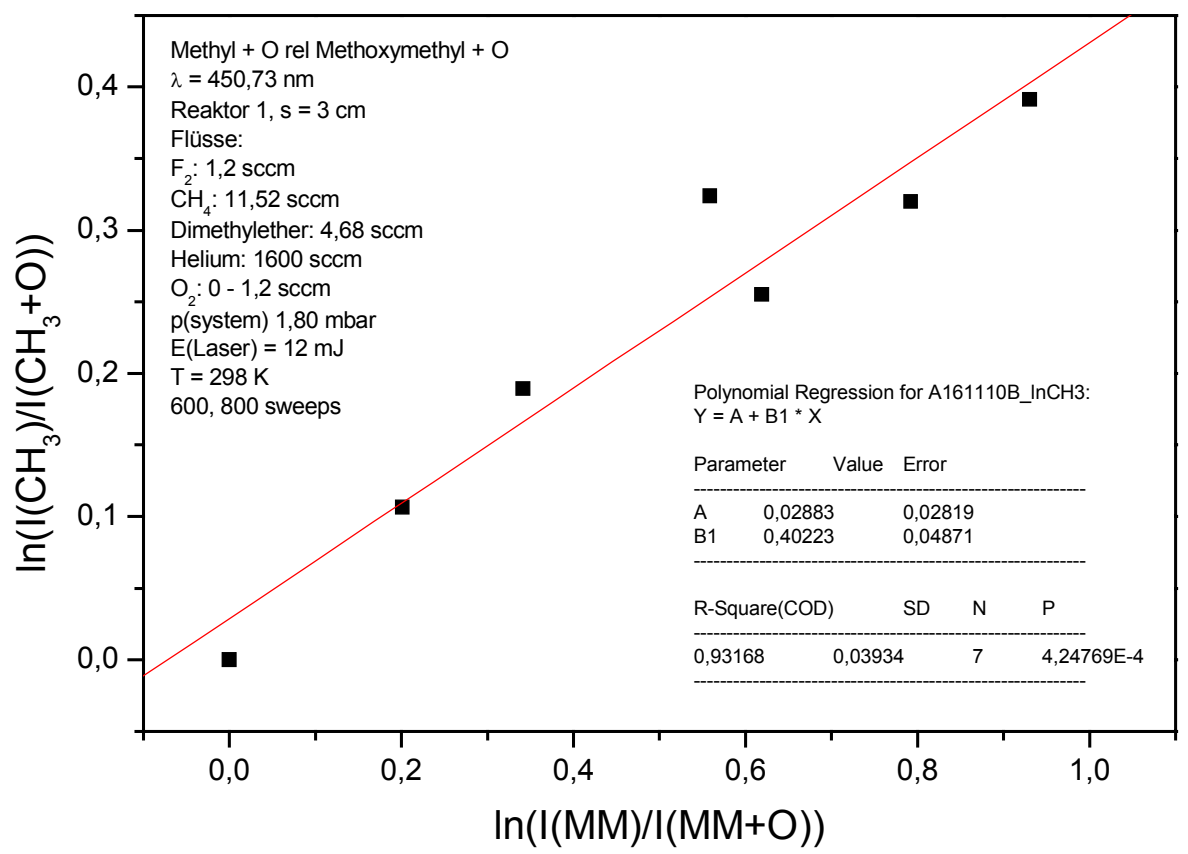

Abbildung 102: Methyl $+\mathrm{O}$ relativ zu Methoxymethyl $+\mathrm{O}, \mathrm{T}=25^{\circ} \mathrm{C}$

Aus der Auftragung ergibt sich:

$$
\mathrm{k}_{\mathrm{rel}}=\mathrm{k}\left(\mathrm{CH}_{3}+\mathrm{O}\right) / \mathrm{k}\left(\mathrm{CH}_{3} \mathrm{OCH}_{2}+\mathrm{O}\right)=0,40 \pm 0,05 \text {. }
$$

Mit der in Abschnitt 3.3.2 erläuterten Geschwindigkeitskonstante $k_{\text {ref }}=1,31 \cdot 10^{14} \mathrm{~cm}^{3} /(\mathrm{mol} \cdot \mathrm{s})$ für die Referenzreaktion Methoxymethyl + O erhält man:

$$
\mathrm{k}^{298 \mathrm{~K}}\left(\mathrm{CH}_{3}+\mathrm{O}\right)=(5,24 \pm 0,7) \cdot 10^{13} \mathrm{~cm}^{3} /(\mathrm{mol} \cdot \mathrm{s}) .
$$

Die Geschwindigkeit der Reaktion von $\mathrm{CH}_{3}+\mathrm{O}$ wurde des Weiteren auch relativ zur Reaktion c-Pentyl $+O$ bei Zimmertemperatur mit Versuchsanordnung A (REMPI) bei $\lambda=450,7 \mathrm{~nm}$ bestimmt. Die Radikale wurden gemäß Abschnitt 3.1.1 und 3.1.6 erzeugt und auf ihrer Muttermasse nachgewiesen. Die folgende Abbildung zeigt die zugehörige Auftragung: 


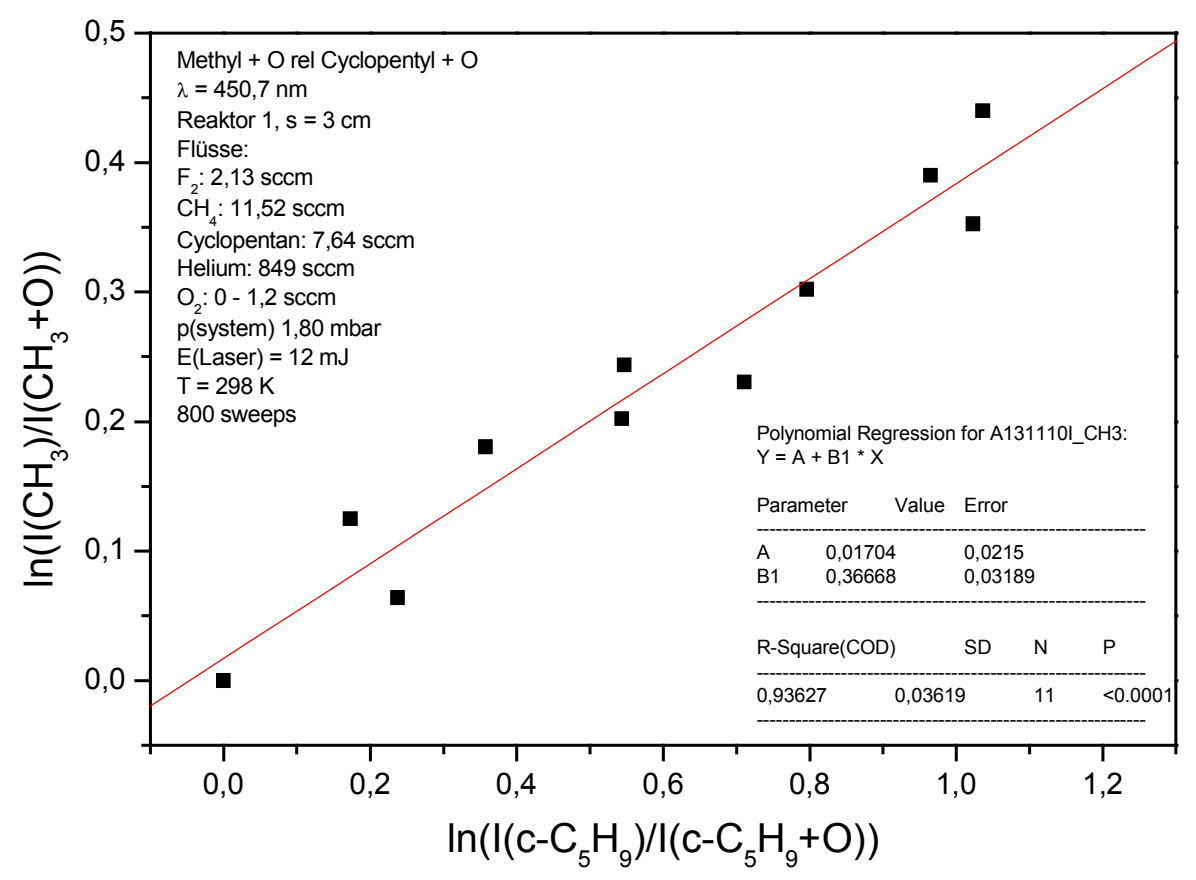

Abbildung 103: Methyl $+\mathrm{O}$ relativ zu c-Pentyl $+\mathrm{O}, \mathrm{T}=25^{\circ} \mathrm{C}$

Aus der Auftragung ergibt sich:

$$
\mathrm{k}_{\mathrm{rel}}=\mathrm{k}\left(\mathrm{CH}_{3}+\mathrm{O}\right) / \mathrm{k}(\mathrm{c}-\text { Pentyl }+\mathrm{O})=0,37 \pm 0,03 \text {. }
$$

Mit der in Abschnitt 3.3.3 erläuterten Geschwindigkeitskonstante $k_{\text {ref }}=1,73 \cdot 10^{14} \mathrm{~cm}^{3} /(\mathrm{mol} \cdot \mathrm{s})$ für die Referenzreaktion c-Pentyl + O erhält man:

$$
\mathrm{k}^{298 \mathrm{~K}}\left(\mathrm{CH}_{3}+\mathrm{O}\right)=(6,40 \pm 0,5) \cdot 10^{13} \mathrm{~cm}^{3} /(\mathrm{mol} \cdot \mathrm{s}) .
$$

Interpretation: Die aus den Vergleichsmessungen abgeleiteten Geschwindigkeitskoeffizienten der Reaktion Methyl + O stimmen innerhalb der Fehlergrenzen mit dem relativ zur Reaktion $\mathrm{C}_{2} \mathrm{D}_{5}+\mathrm{O}$ gemessenen Wert überein. Gleichzeitig wird damit die Konsistenz innerhalb des Systems der kinetischen Messungen zu $\mathrm{C}_{2} \mathrm{H}_{5}+\mathrm{O}, \mathrm{C}_{2} \mathrm{D}_{5}+\mathrm{O}, \mathrm{CH}_{3} \mathrm{OCH}_{2}+\mathrm{O}$, c-Pentyl $+\mathrm{O}$ bestärkt.

Diskussion: Die Geschwindigkeitskonstante der Reaktion $\mathrm{CH}_{3}+\mathrm{O}$ ist schon mehrfach experimentell bestimmt worden. Die zugehörigen Werte werden in sehr vielen zusammenfassenden und evaluierenden Publikationen und Datenbanken zitiert, die eine Basis für Modellierungen in der Atmosphärenchemie und bei Verbrennungsprozessen bilden ([ATK97], [DEM97]). Das ist damit zu erklären, dass die Reaktion von Methylradikalen mit atomarem Sauerstoff als eine der Schlüsselreaktion bei der Oxidation von Kohlenwasserstoffen angesehen wird. Eine Temperaturabhängigkeit der Reaktion kann im Bereich 300-2000 K nicht festgestellt werden [HER88]. Eine der am häufigsten zitierten gemessenen Geschwindigkeitskonstanten stammt von 
Gutman et al.: $\mathrm{k}^{298 \mathrm{~K}}=8,43 \cdot 10^{14} \mathrm{~cm}^{3} /(\mathrm{mol} \cdot \mathrm{s})$ [SLA87]. Die Reaktion erweist sich zwischen 1 und 10 mbar als druckunabhängig. Die Messungen wurden in einem Rohrreaktor bei niedrigem Fluss ausgeführt. Die Radikalerzeugung geschah photolytisch, der Nachweis erfolgte mit einem Massenspektrometer mit Photoionisation. Zwar gehen Gutman et al. nur von einem Reaktionskanal zur Bildung von Formaldehyd und $\mathrm{H}$ aus (vgl. oben, Produkte), das berührt die Bestimmung des Geschwindigkeitskoeffizienten aber nicht, weil die Grundlage der kinetischen Messung das Abnahmeprofil von $\mathrm{CH}_{3}$ war. Der ermittelte Koeffizient gilt somit für die Gesamtreaktion. Ähnliche Überlegungen gelten für eine Untersuchung von Oser et al., die für die Reaktion $\mathrm{CH}_{3}+\mathrm{O}$ in einem Strömungssystem mit massenspektrometrischem Nachweis einen Geschwindigkeitskoeffizienten von $\mathrm{k}^{298 \mathrm{~K}}=7,82 \cdot 10^{13} \mathrm{~cm}^{3} /(\mathrm{mol} \cdot \mathrm{s})$ angegeben [OSE91]. Im Vergleich zu den beiden diskutierten Veröffentlichungen ist der in dieser Arbeit vorgestellte Koeffizient um ca. 25\% niedriger.

Wie im obigen Abschnitt Produkte erläutert, wurde das System $\mathrm{CH}_{3}+\mathrm{O}$ auch von Seakins und Leone untersucht [SEA92]. Die Kinetik wurde dabei anhand des Anstiegprofils der entstehenden Produkte Formaldehyd einerseits und Kohlenmonoxid andererseits bestimmt. Leider geht aus der Publikation nicht eindeutig hervor, ob ein Geschwindigkeitskoeffizient für jeden der beiden Kanäle einzeln bestimmt wurde (Fall1) oder ob gemäß einer Auswertung, wie sie bei [HAC02] erläutert wird, der Gesamtgeschwindigkeitskoeffizient anhand des jeweiligen Produktsignals ermittelt wurde (Fall 2). Ausgehend von Fall 1 wird für den Kanal (a) zum Formaldehyd ein Wert von $\mathrm{k}_{\mathrm{a}}=(5,66 \pm 1,81) \cdot 10^{13} \mathrm{~cm}^{3} /(\mathrm{mol} \cdot \mathrm{s})$ angegeben ${ }^{25}$, für den Kanal (b) zum CO der Wert $\mathrm{k}_{\mathrm{b}}=(3,61 \pm 2,17) \cdot 10^{13} \mathrm{~cm}^{3} /(\mathrm{mol} \cdot \mathrm{s})$. Nach den Gesetzen der chemischen Kinetik der Parallelreaktionen setzt sich der Gesamtgeschwindigkeitskoeffizient additiv aus beiden Werten zusammen [WED87], so dass sich $\mathrm{k}^{298 \mathrm{~K}}=9,23 \cdot 10^{13} \mathrm{~cm}^{3} /(\mathrm{mol} \cdot \mathrm{s})$ ergibt. Im Vergleich dazu ist der in dieser Arbeit bestimmte Wert niedriger. Wird von Fall 2 ausgegangen, so liegt der Gesamtgeschwindigkeitskoeffizient, die fehlerärmere Bestimmung über das Produkt Formaldehyd zu Grunde legend, sehr nahe bei dem in dieser Arbeit bestimmten Wert.

Washida et al. haben vor kurzem die Geschwindigkeit der Reaktion $\mathrm{CH}_{3}+\mathrm{O}$ in einem Strömungsreaktor mit photolytischer Radikal- und Sauerstoffatomerzeugung und anschließendem Photoionisationsmassenspektrometer untersucht [WAS00]. Die angegebene Konstante beträgt

\footnotetext{
${ }^{25}$ Den Fall 1 vorausgesetzt: Seakins und Leone vergleichen in ihrer Publikation den Geschwindigkeitskoeffizienten von Kanal (a) zur Bildung von Formaldehyd unmittelbar mit den bis dato in der Literatur angegebenen. Bei diesen in der Literatur angegebenen Konstanten wird zwar nur von der überwiegenden Wirksamkeit dieses Kanals ausgegangen (Anteil Kanal (a) $\geq 90 \%$ ), aber in diesen Messungen wurde, soweit das übersehen werden kann, die Kinetik aufgrund der Abnahme des Edukts $\mathrm{CH}_{3}$ bestimmt. Damit wurde der Gesamtgeschwindigkeitskoeffizient ermittelt. Die Vergleichbarkeit wäre dann nicht gegeben.
} 
$\mathrm{k}=1,02 \cdot 10^{14} \mathrm{~cm}^{3} /(\mathrm{mol} \cdot \mathrm{s})$ und liegt um den Faktor 1,7 höher als die in dieser Arbeit gemessene Geschwindigkeitskonstante. Nach den Messungen von Washida et al. ist die Reaktion $\mathrm{CH}_{3}+\mathrm{O}$ sogar schneller als die Reaktion $\mathrm{C}_{2} \mathrm{H}_{5}+\mathrm{O}$, was in Anbetracht der eigenen Ergebnisse abgelehnt werden muss.

Der eigene Wert liegt dagegen deutlich höher als der Geschwindigkeitskoeffizient von Benson und Dobis $\left(\mathrm{k}^{298 \mathrm{~K}}=2,31 \cdot 10^{13} \mathrm{~cm}^{3} /(\mathrm{mol} \cdot \mathrm{s})\right.$, [BEN98]), errechnet aus einem Vergleich der Stoßparameter des gemessenen Werts von Gutman et al. und eines modifizierten Gorinmodells.

Die Geschwindigkeit der Reaktion $\mathrm{CH}_{3}+\mathrm{O}$ ist vor kurzem auch von Muckerman et al. bei Zimmertemperatur und einem Druck von $\mathrm{p}=1$ Torr in einem Strömungsreaktor mit Time-ofFlight-Massenspektrometer (TOF-MS) und Photoionisation durch eine Ar- oder NeHohlkathodenlampe im VUV-Bereich $(\lambda=104,6-106,5 \mathrm{~nm}, \lambda=73,4-74,2 \mathrm{~nm})$ bestimmt worden: $\mathrm{k}^{298 \mathrm{~K}}=(1,02 \pm 0,18) \cdot 10^{14} \mathrm{~cm}^{3} /(\mathrm{mol} \cdot \mathrm{s})$ [FOC99]. Die Erzeugung der Sauerstoffatome geschah photolytisch aus $\mathrm{SO}_{2}$ bei einer Wellenlänge von $\lambda=193 \mathrm{~nm}$. Gleichzeitig wurden Methylradikale kophotolytisch aus Aceton oder aus $\mathrm{CH}_{3} \mathrm{Br}$ erzeugt. Die Kinetik wurde auf Basis des zeitlichen Absinkprofils der Methylradikalsignale und des Anstiegprofils des nach Reaktionskanal (a) entstehenden Formaldehyds bei verschiedenen O-Konzentrationen bestimmt. Die Verwendung von $\mathrm{CH}_{3} \mathrm{Br}$ als Radikalvorläufer resultierte in starken Streuungen, was auf Wandreaktionen verursacht durch Wandbelegung von Brom oder Brommethan zurückgeführt wurde. Aus diesem Grund wurden diese Daten nicht verwendet. Um die Streuung bei der Benutzung von Aceton als Radikalquelle zu minimieren, wurde eine Mittelwertbildung über 100000-200000 Photolyselaserpulse durchgeführt. Kritisch ist anzumerken, dass die Probennahme am Rande des zylindrischen Strömungsreaktors Fragestellungen aufwirft, inwieweit die genommene Probe unter strömungsmechanischen Gesichtspunkten das reale Reaktionsgemisch auch korrekt widerspiegelt. Radiale Diffusion und Wandreaktionen der Methylradikale könnten die Ergebnisse verfälscht haben. Außerdem ist die Signalintensität der Methylradikale und des Formaldehyds eher klein gewesen. Die massenspektrometrische Auflösung ist trotz der Verwendung eines Reflektron-TOFMassenspektrometers und der hohen Zahl an Mittlungen nicht überzeugend (vgl. Figure 2, [FOC99]).

\section{Isotopeneffekt:}

Messergebnisse: Es wurde die Geschwindigkeit der Reaktion $\mathrm{CD}_{3}+\mathrm{O}$ mit Versuchsanordnung A (REMPI) bei $\lambda=345 \mathrm{~nm}$ relativ zur Reaktion $\mathrm{CH}_{3}+\mathrm{O}$ bei Zimmertemperatur bestimmt. Die Radikale wurden gemäß Abschnitt 3.1.1 erzeugt und auf ihrer Muttermasse nachgewiesen. Zur 
Bestimmung des Geschwindigkeitskoeffizienten wurden die Signalintensitäten bei An- und Abwesenheit von Sauerstoffatomen gemessen. Durch Variation der Sauerstoffkonzentration wurden verschiedene Umsätze erzielt. Die folgende Abbildung zeigt in doppeltlogarithmischer Auftragung die Messpunkte. Die zugehörigen Werte finden sich in Abschnitt 5 (Messtabellen). Die gewählten Versuchsbedingungen sind dem Text in der Abbildung oder bei den Messtabellen zu entnehmen.

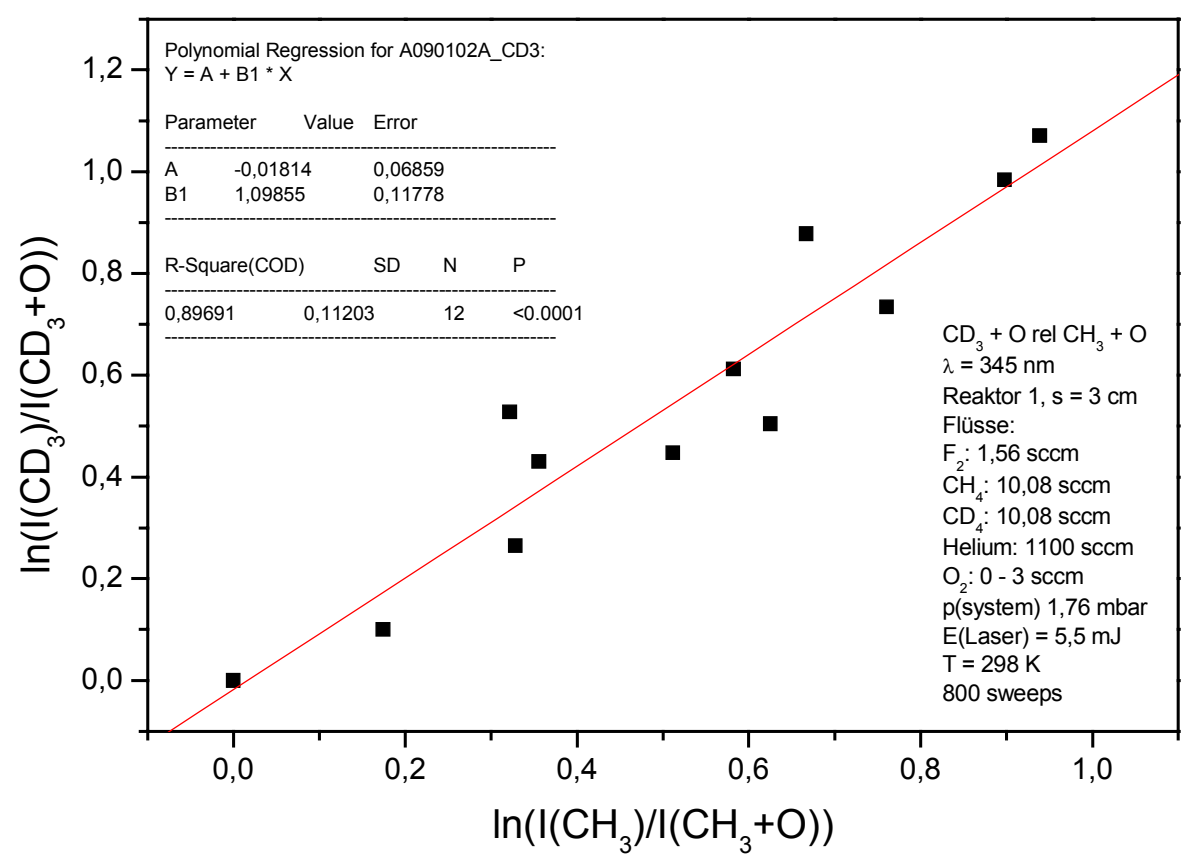

\section{Abbildung 104: $\mathrm{CD}_{3}+\mathrm{O}$ relativ zu $\mathrm{CH}_{3}+\mathrm{O}, \mathrm{T}=25^{\circ} \mathrm{C}$}

Aus der linearen Regression der aufgetragenen Messwerte ergibt sich ein relativer Geschwindigkeitskoeffizient von

$$
\mathrm{k}_{\mathrm{rel}}=\mathrm{k}\left(\mathrm{CD}_{3}+\mathrm{O}\right) / \mathrm{k}\left(\mathrm{CH}_{3}+\mathrm{O}\right)=1,01 \pm 0,12 \text {. }
$$

Aus der Messung ist ein merklicher Isotopeneffekt nicht abzuleiten.

Diskussion: Literatur über eine Messung des Isotopeneffekts zur Reaktion Methyl + O liegt nicht vor. Von Washida wurde nach einer persönlichen Mitteilung ${ }^{26}$ als relative Geschwindigkeitskonstante $\mathrm{k}_{\text {rel }}=\mathrm{k}\left(\mathrm{CH}_{3}+\mathrm{O}\right) / \mathrm{k}\left(\mathrm{CD}_{3}+\mathrm{O}\right)=1,07 \pm 0,05$ gemessen. Nach einer Abschätzung von Hoyermann ${ }^{27}$ über den Einfluss der Änderung der reduzierten Masse auf die Relativgeschwindigkeit der Teilchen ergibt sich $\mathrm{k}_{\mathrm{rel}}=\mathrm{k}\left(\mathrm{CH}_{3}+\mathrm{O}\right) / \mathrm{k}\left(\mathrm{CD}_{3}+\mathrm{O}\right)=1,05$, wonach in Übereinstimmung mit dem gemessenen Wert von Washida die Reaktion $\mathrm{CH}_{3}+\mathrm{O}$ leicht schneller sein sollte als $\mathrm{CD}_{3}+\mathrm{O}$. Der

\footnotetext{
${ }^{26}$ Von Washida an Hoyermann im Rahmen des 16th Int. Symp. on Gas Kinetics, Cambridge (GB), P[C6], (2000), vgl. auch [WAS00].

27 Persönliche Mitteilung
} 
in dieser Arbeit angegebene relative Geschwindigkeitskoeffizient stimmt im Rahmen der Fehlergrenzen mit den beiden diskutierten Werten überein.

\subsubsection{2-Propyl + O}

\section{Produkte:}

Diskussion: Für die Reaktion von 2-Propylradikalen mit O-Atomen erscheinen drei Reaktionskanäle, die alle stark exotherm sind, mechanistisch möglich:

$$
\begin{array}{rll} 
& \rightarrow \mathrm{CH}_{3} \mathrm{CHO}+\mathrm{CH}_{3} \quad \text { (a) } \Delta_{\mathrm{R}} \mathrm{H}^{0}{ }_{298 \mathrm{~K}}=-369,9 \mathrm{~kJ} / \mathrm{mol} \\
\text { iso- } \mathrm{C}_{3} \mathrm{H}_{7}+\mathrm{O} & \rightarrow \mathrm{CH}_{3} \mathrm{COCH}_{3}+\mathrm{H} & \text { (b) } \Delta_{\mathrm{R}} \mathrm{H}^{0}{ }_{298 \mathrm{~K}}=-348,9 \mathrm{~kJ} / \mathrm{mol} \\
& \rightarrow \mathrm{CH}_{2}=\mathrm{CHCH}_{3}+\mathrm{OH} & \text { (c) } \Delta_{\mathrm{R}} \mathrm{H}^{0}{ }_{298 \mathrm{~K}}=-290,8 \mathrm{~kJ} / \mathrm{mol}
\end{array}
$$

Kanal (a) zur Bildung von Acetaldehyd verläuft über einen C-C-Bindungsbruch, Kanal (b) über einen C-H-Bindungsbruch am 2-C-Atom, welches im angeregten Komplex das addierte Sauerstoffatom trägt. Inwiefern Kanal (c) über den angeregten Komplex verläuft oder eine direkte Abstraktion darstellt, muss offen gelassen werden. Die direkte Abstraktion ist in Anbetracht der Ergebnisse zur Reaktion Ethyl $+\mathrm{O}$ wahrscheinlicher. Produktuntersuchungen zur Reaktion des 2-Propylradikals mit atomarem Sauerstoff sind selten. Hoyermann und Sievert geben als Produktkanal Aceton $+\mathrm{H}$ an [HOY79a]. Das Kanalverzweigungsverhältnis wurde innerhalb der eigenen Arbeitsgruppe von Zeuch mit Versuchsanordnung C (FTIR) bei einem Druck von 4 mbar ermittelt [ZEU02]. Als Radikalvorläufer in der Photolysezelle diente Di-iso-Propyl-Keton. Auf Kanal (a) entfallen danach 39\%, auf (b) $37 \%$ und auf (c) $24 \%$.

$\underline{\text { Reaktionsgeschwindigkeit: }}$

Messergebnisse: Die Geschwindigkeit der Reaktion 2-Propyl + O wurde relativ zur Reaktion Methoxymethyl + O bei Zimmertemperatur mit Versuchsanordnung A (REMPI) bei $\lambda=465 \mathrm{~nm}$ bestimmt. Die Radikale wurden gemäß Abschnitt 3.1.3 und 3.1.14 erzeugt und auf ihren Muttermassen $\mathrm{m} / \mathrm{z}=43$ und $\mathrm{m} / \mathrm{z}=45$ in eindeutiger Weise nachgewiesen. Zur Bestimmung des Geschwindigkeitskoeffizienten wurden die Signalintensitäten bei An- und Abwesenheit von Sauerstoffatomen gemessen. Durch Variation der Sauerstoffkonzentration wurden verschiedene Umsätze erzielt. Die folgende Abbildung zeigt in doppeltlogarithmischer Auftragung die Messpunkte. Die zugehörigen Werte finden sich in Abschnitt 5 (Messtabellen). Die gewählten Versuchsbedingungen sind dem Text in der Abbildung oder bei den Messtabellen zu entnehmen. 


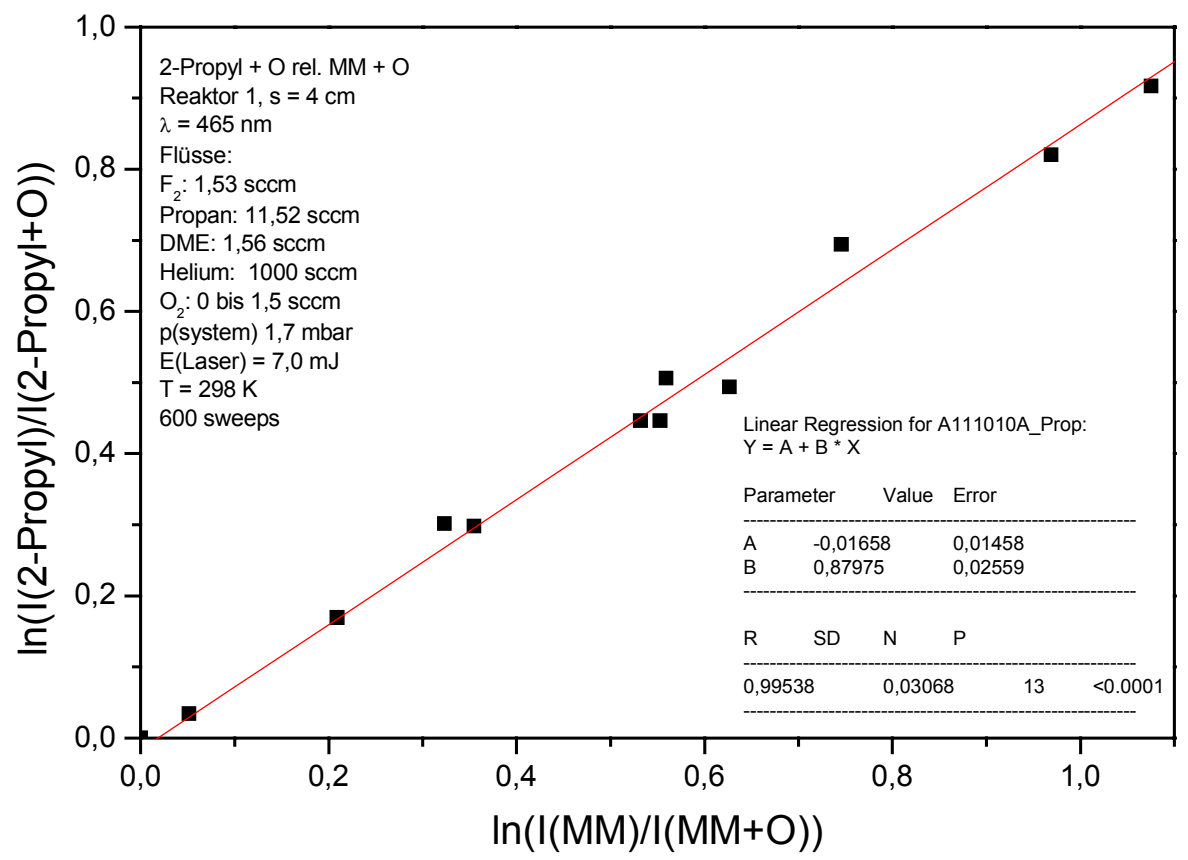

Abbildung 105: 2-Propyl $+\mathrm{O}$ relativ zu Methoxymethyl $+\mathrm{O}, \mathrm{T}=25^{\circ} \mathrm{C}$

Aus der Auftragung ergibt sich:

$$
\mathrm{k}_{\text {rel }}=\mathrm{k}\left(\mathrm{iso}_{3} \mathrm{C}_{3} \mathrm{H}_{7}+\mathrm{O}\right) / \mathrm{k}\left(\mathrm{CH}_{3} \mathrm{OCH}_{2}+\mathrm{O}\right)=0,87 \pm 0,03 \text {. }
$$

Mit der in Abschnitt 3.3.2 erläuterten Geschwindigkeitskonstante $k_{\text {ref }}=1,31 \cdot 10^{14} \mathrm{~cm}^{3} /(\mathrm{mol} \cdot \mathrm{s})$ für die Referenzreaktion Methoxymethyl + O erhält man:

$$
\mathrm{k}^{298 \mathrm{~K}}\left(\mathrm{iso}-\mathrm{C}_{3} \mathrm{H}_{7}+\mathrm{O}\right)=(1,14 \pm 0,04) \cdot 10^{14} \mathrm{~cm}^{3} /(\mathrm{mol} \cdot \mathrm{s}) .
$$

Diskussion: Zwar werden in einigen Publikationen Geschwindigkeitskonstanten angegeben ([HER88], [TSA88]), diese beziehen sich jedoch teilweise nur auf einzelne Reaktionskanäle und beruhen nicht auf Messungen. Sie sollen hier nicht weiter diskutiert werden. Von Kersten wurde mittels einer LIF-Analyse des zeitlichen Anstiegprofils der entstehenden OH-Radikale der Geschwindigkeitskoeffizient in pseudoerster Ordnung (O-Atom-Überschuss) gemessen [KER99]. Es wurde mit zwei Auswertungsmethoden gearbeitet. Das Ergebnis nach Methode 1 lautet $\mathrm{k}^{298 \mathrm{~K}}\left(\right.$ iso- $\left.\mathrm{C}_{3} \mathrm{H}_{7}+\mathrm{O}\right)=5,87 \cdot 10^{14} \mathrm{~cm}^{3} /(\mathrm{mol} \cdot \mathrm{s})$, nach Methode $2 \mathrm{k}^{298 \mathrm{~K}}\left(\right.$ iso- $\left.\mathrm{C}_{3} \mathrm{H}_{7}+\mathrm{O}\right)=2,66 \cdot 10^{14}$ $\mathrm{cm}^{3} /(\mathrm{mol} \cdot \mathrm{s})$. Beide Werte liegen deutlich höher als der in dieser Arbeit gemessene Geschwindigkeitskoeffizient. Vor Kurzem von Zeuch angestellte Überlegungen ergeben, dass die sehr starken Einfluss nehmende Nebenreaktion von O-Atomen mit dem Radikalvorläufer 2-Jodpropan zur Bildung von IO und 2-Propyl bei der Auswertung nicht berücksichtigt wurde. Eine erneute LIFAnalyse des zeitlichen Anstiegprofils der entstehenden OH-Radikale von Zeuch, dieses Mal mit Diiso-Propyl-Keton als Radikalvorläufer, ergibt $\mathrm{k}^{298 \mathrm{~K}}\left(\right.$ iso- $\left._{3} \mathrm{H}_{7}+\mathrm{O}\right)=(1,14 \pm 0,1) \cdot 10^{14} \mathrm{~cm}^{3} /(\mathrm{mol} \cdot \mathrm{s})$, was in exakter Übereinstimmung mit dem in dieser Arbeit ermittelten Wert ist [ZEU02]. 
Auch Washida et al. haben vor kurzem die Geschwindigkeit der Reaktion iso- $\mathrm{C}_{3} \mathrm{H}_{7}+\mathrm{O}$ in einem Strömungsreaktor mit photolytischer Radikal- und Sauerstoffatomerzeugung und anschließendem Photoionisationsmassenspektrometer untersucht [WAS00]. Der angegebene Geschwindigkeitskoeffizient beträgt $\mathrm{k}=1,32 \cdot 10^{14} \mathrm{~cm}^{3} /(\mathrm{mol} \cdot \mathrm{s})$ und liegt damit nahe bei dem durch diese Arbeit bestimmten Geschwindigkeitskoeffizienten.

\subsubsection{1-Propyl + O}

\section{Produkte:}

Diskussion: Für die Reaktion von 1-Propylradikalen mit O-Atomen erscheinen drei Reaktionskanäle, die alle stark exotherm sind, mechanistisch möglich:

$$
\begin{array}{llll}
\mathrm{n}-\mathrm{C}_{3} \mathrm{H}_{7}+\mathrm{O} & \rightarrow & \mathrm{n}_{-} \mathrm{C}_{3} \mathrm{H}_{7} \mathrm{O}^{*} & \\
\mathrm{n}-\mathrm{C}_{3} \mathrm{H}_{7} \mathrm{O}^{*} & \rightarrow & \mathrm{HCHO}+\mathrm{C}_{2} \mathrm{H}_{5} & \Delta_{\mathrm{R}} \mathrm{H}_{298 \mathrm{~K}}=-341,4 \mathrm{~kJ} / \mathrm{mol} \\
& \rightarrow & \mathrm{CH}_{3} \mathrm{CH}_{2} \mathrm{CHO}+\mathrm{H} & \Delta_{\mathrm{R}} \mathrm{H}^{0}{ }_{298 \mathrm{~K}}=-319,2 \mathrm{~kJ} / \mathrm{mol} \\
& \left(\mathrm{a}_{2}\right) \\
\mathrm{n}-\mathrm{C}_{3} \mathrm{H}_{7}+\mathrm{O} & \rightarrow & \mathrm{OH}+\mathrm{CH}_{2} \mathrm{CH}=\mathrm{CH}_{2} & \Delta_{\mathrm{R}} \mathrm{H}^{0}{ }_{298 \mathrm{~K}}=-290,8 \mathrm{~kJ} / \mathrm{mol}
\end{array}
$$

Im Rahmen einer Vergleichsstudie zur IR-Chemielumineszenz der entstehenden OH-Radikale mit $\mathrm{C}_{2} \mathrm{H}_{5} \mathrm{I}$ und 1- $\mathrm{C}_{3} \mathrm{H}_{7} \mathrm{I}$ als Propylradikalvorläufer wurde dieser Mechanismus in Analogie zur Reaktion Ethyl + O von Leone et al. vorgeschlagen [LIN98]. Genauere Untersuchungen wurden innerhalb der eigenen Arbeitsgruppe kürzlich von Hack, Hoyermann und Zeuch mit Versuchsanordnung C (FTIR) durchgeführt ([HAC02], [ZEU02]). Als Radikalvorläufer wurde Dipropylketon eingesetzt, Sauerstoffatome wurden in Kophotolyse von $\mathrm{SO}_{2}$ erzeugt. Der Beitrag der einzelnen Kanäle ergibt sich wie folgt: $(44,2 \pm 2) \%\left(a_{1}\right),(31,8 \pm 2) \%\left(a_{2}\right)$ und $(24,0 \pm 1) \%$ (b). Ein Vergleich der Besetzungsverhältnisse der Schwingungszustände von $\mathrm{OH}$ aus einer LIF-Messung einerseits und einer Rechnung zur OH-Bildung aus einem Propyl-O-Addukt andererseits spricht für einen direkten Abstraktionsprozess im Reaktionskanal (b) (zur Argumentation vgl. Abschnitt 3.3.8).

\section{Reaktionsgeschwindigkeit:}

Messergebnisse: Die Geschwindigkeit der Reaktion 1-Propyl + O wurde relativ zur Reaktion 2-Propyl + O bei Zimmertemperatur mit Versuchsanordnung A (REMPI) bei $\lambda=430 \mathrm{~nm}$ und $\lambda=450 \mathrm{~nm}$ (Messpunkte in nachfolgender Abbildung in rot) bestimmt. Beide Radikale wurden aus der Reaktion von $\mathrm{CH}_{3} \mathrm{CD}_{2} \mathrm{CH}_{3}$ mit F-Atomen erzeugt und konnten aufgrund ihrer unterschiedlichen Masse (1-Propyl: $\mathrm{m} / \mathrm{z}=45$, 2-Propyl: $\mathrm{m} / \mathrm{z}=44$ ) gemäß Abschnitt 3.1.3 nachgewiesen werden. Zur Bestimmung des Geschwindigkeitskoeffizienten wurden die Signalintensitäten bei Anund Abwesenheit von Sauerstoffatomen gemessen. Durch Variation der Sauerstoffkonzentration wurden verschiedene Umsätze erzielt. Die folgende Abbildung zeigt in doppeltlogarithmischer 
Auftragung die Messpunkte. Die zugehörigen Werte finden sich in Abschnitt 5 (Messtabellen). Die gewählten Versuchsbedingungen sind dem Text in der Abbildung oder bei den Messtabellen zu entnehmen.

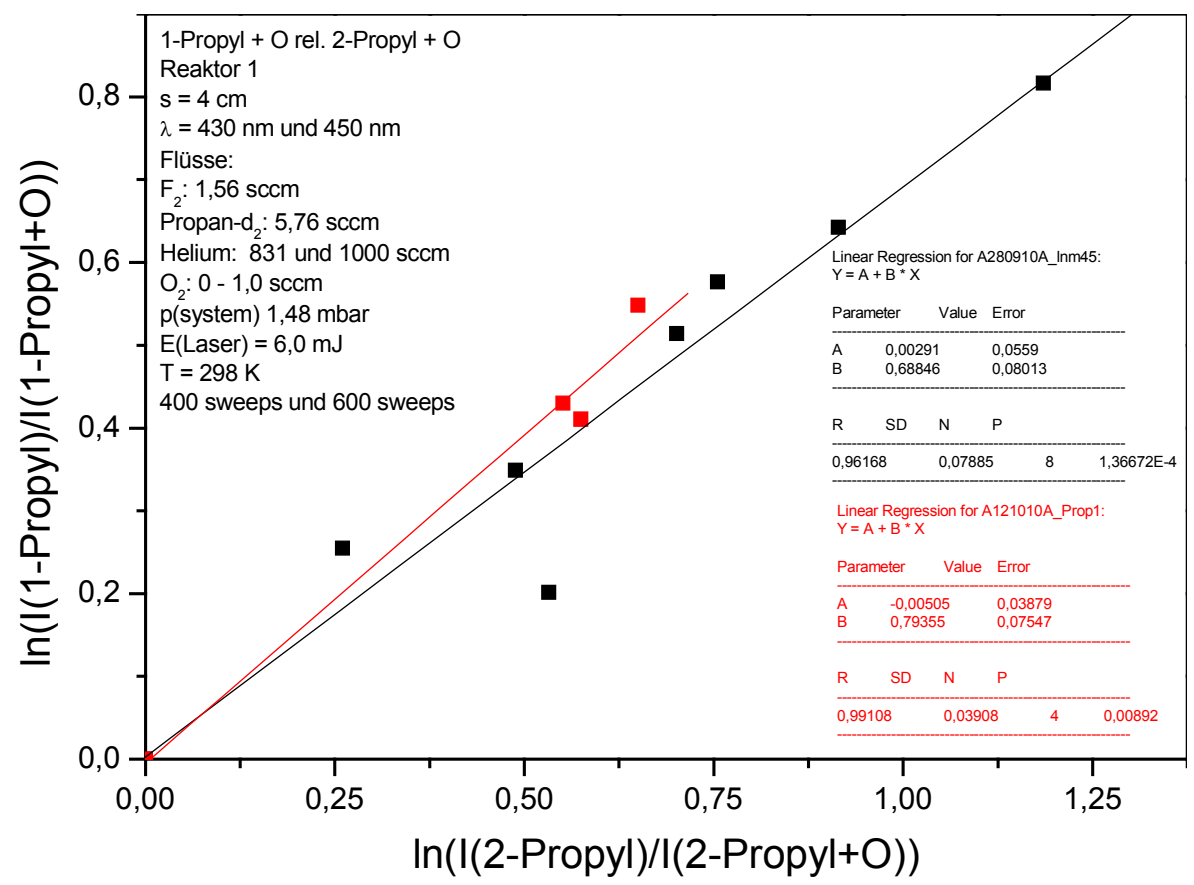

Abbildung 106: 1-Propyl $+\mathrm{O}$ relativ zu 2-Propyl $+\mathrm{O}, \mathrm{T}=25^{\circ} \mathrm{C}$

Als Mittelwert aus den Auftragungen ergibt sich:

$$
\mathrm{k}_{\mathrm{rel}}=\mathrm{k}\left(\mathrm{n}-\mathrm{C}_{3} \mathrm{H}_{7}+\mathrm{O}\right) / \mathrm{k}\left(\text { iso- } \mathrm{C}_{3} \mathrm{H}_{7}+\mathrm{O}\right)=0,74 \pm 0,08 \text {. }
$$

Mit der in Abschnitt 3.3.5 erläuterten Geschwindigkeitskonstante $k_{\text {ref }}=1,14 \cdot 10^{14} \mathrm{~cm}^{3} /(\mathrm{mol} \cdot \mathrm{s})$ für die Referenzreaktion 2-Propyl + O erhält man:

$$
\mathrm{k}^{298 \mathrm{~K}}\left(\mathrm{n}-\mathrm{C}_{3} \mathrm{H}_{7}+\mathrm{O}\right)=(8,43 \pm 0,09) \cdot 10^{13} \mathrm{~cm}^{3} /(\mathrm{mol} \cdot \mathrm{s}) .
$$

Diskussion: In Datensammlungen werden Geschwindigkeitskoeffizienten angegeben, die aber nicht experimentell verifiziert wurden: $\mathrm{k}^{298 \mathrm{~K}}\left(\mathrm{n}-\mathrm{C}_{3} \mathrm{H}_{7}+\mathrm{O}\right)=2 \cdot 10^{14} \mathrm{~cm}^{3} /(\mathrm{mol} \cdot \mathrm{s}) \quad$ [HER88], $\mathrm{k}^{298 \mathrm{~K}}\left(\mathrm{n}-\mathrm{C}_{3} \mathrm{H}_{7}+\mathrm{O}\right)=9,6 \cdot 10^{13} \mathrm{~cm}^{3} /(\mathrm{mol} \cdot \mathrm{s})$ [TSA88]. Mittels einer LIF-Analyse des zeitlichen Anstiegprofils der entstehenden OH-Radikale wurde der Geschwindigkeitskoeffizient in pseudoerster Ordnung (O-Atom Überschuss) von Hack, Hoyermann und Zeuch bestimmt ([HAC02], [ZEU02]). Das Ergebnis $\mathrm{k}^{298 \mathrm{~K}}\left(\mathrm{n}-\mathrm{C}_{3} \mathrm{H}_{7}+\mathrm{O}\right)=(8,2 \pm 1) \cdot 10^{14} \mathrm{~cm}^{3} /(\mathrm{mol} \cdot \mathrm{s})$ ist in enger Übereinstimmung mit dem in dieser Arbeit ermittelten Geschwindigkeitskoeffizienten. 


\subsubsection{2-Butyl + O}

\section{Produkte:}

Diskussion: Für die Reaktion von 2-Butylradikalen mit O-Atomen erscheinen die folgenden Reaktionskanäle, die alle stark exotherm sind, mechanistisch möglich:

$$
\begin{array}{rlll} 
& \rightarrow & \mathrm{C}_{2} \mathrm{H}_{5} \mathrm{CHO}+\mathrm{CH}_{3} & \Delta_{\mathrm{R}} \mathrm{H}^{0}{ }_{298 \mathrm{~K}}=-362,3 \mathrm{~kJ} / \mathrm{mol} \text { (a) } \\
& \rightarrow & \mathrm{CH}_{3} \mathrm{CHO}+\mathrm{C}_{2} \mathrm{H}_{5} & \Delta_{\mathrm{R}} \mathrm{H}^{0}{ }_{298 \mathrm{~K}}=-369,0 \mathrm{~kJ} / \mathrm{mol} \text { (b) } \\
2-\mathrm{C}_{4} \mathrm{H}_{9}+\mathrm{O} & \rightarrow & \text { Butanon }+\mathrm{H} & \Delta_{\mathrm{R}} \mathrm{H}^{0}{ }_{298 \mathrm{~K}}=-343,1 \mathrm{~kJ} / \mathrm{mol}(\mathrm{c}) \\
& \rightarrow & \mathrm{CH}_{2}=\mathrm{CHCH}_{2} \mathrm{CH}_{3}+\mathrm{OH} & \Delta_{\mathrm{R}} \mathrm{H}^{0}{ }_{298 \mathrm{~K}}=-282,0 \mathrm{~kJ} / \mathrm{mol}(\mathrm{d}) \\
& \rightarrow & \mathrm{CH}_{3} \mathrm{CH}=\mathrm{CHCH}_{3}+\mathrm{OH} & \Delta_{\mathrm{R}} \mathrm{H}^{0}{ }_{298 \mathrm{~K}}=-289,5 \mathrm{~kJ} / \mathrm{mol}(\mathrm{e})
\end{array}
$$

Externe Literatur zur Reaktion 2-Butyl + O liegt nicht vor. In der eigenen Arbeitsgruppe wurden Produktuntersuchungen von Seeba mit Versuchsanordnung B (EI-MS) durchgeführt [SEE94]. Als Produkt konnte Acetaldehyd nach Kanal (b) nachgewiesen werden. Auch für die Alkenbildung nach (d) und (e) gibt es Hinweise. Eine kritische Betrachtung der Versuchsbedingungen lässt Produktuntersuchungen dieser Art allerdings von geringem Wert erscheinen: Die 2-Butylradikale wurden über die Reaktion n-Butan + F erzeugt. Da gleichzeitig das 1-Butylradikal gebildet wird (vgl. Abschnitt 3.1.4), kann nicht von einer spezifischen Radikalerzeugung gesprochen werden, es fand somit immer die Reaktion 1-Butyl + O gleichzeitig statt. Die Vielzahl paralleler Reaktionskanäle und Überlagerungen auf den entscheidenden Massen machen eine sinnvolle Interpretation aussichtslos. Die Aussagekraft könnte durch den Einsatz teildeuterierten Butans als Radikalquelle bei einer solchen Versuchsanordnung erhöht werden. Andere Methoden sind aber weitaus vielversprechender. Produktuntersuchungen von Kersten mit Versuchsanordnung C (FTIR) und 2-Jodbutan als photolytische Radikalquelle zeigen die Existenz von (b), (d) und (e). Diesen Reaktionskanälen werden folgenden Anteile zugewiesen: 18,6\% (b), 60,0\% (d) und 21,4\% (e). Der Einsatz von Alkyljodiden als Radikalquelle in Photolysezellen zur Untersuchung von Reaktionen Alkyl $+\mathrm{O}$ ist nicht unproblematisch. Alkyljodide reagieren in schneller Reaktion mit O-Atomen zur Bildung von IO und den entsprechenden Alkylradikalen [GIL96], was die Ergebnisse verfälschen kann. Die nachgebildeten Alkylradikale reagieren mit $\mathrm{OH}$ zu Alken, wodurch der zugehörige Kanalanteil zu hoch bewertet wird. Weitere Untersuchungen zur Reaktion 2-Butyl + O mit anderen Radikalquellen sind in der eigenen Arbeitsgruppe geplant.

$\underline{\text { Reaktionsgeschwindigkeit: }}$

Messergebnisse: Die Geschwindigkeit der Reaktion 2-Butyl + O wurde relativ zur Reaktion c-Pentyl + O bei Zimmertemperatur mit Versuchsanordnung A (REMPI) bei $\lambda=470 \mathrm{~nm}$ bestimmt. Die Radikale wurden gemäß Abschnitt 3.1.4 und 3.1.6 erzeugt und auf ihren 
Muttermassen $\mathrm{m} / \mathrm{z}=57$ und $\mathrm{m} / \mathrm{z}=69$ in eindeutiger Weise nachgewiesen. Zur Bestimmung des Geschwindigkeitskoeffizienten wurden die Signalintensitäten bei An- und Abwesenheit von Sauerstoffatomen gemessen. Durch Variation der Sauerstoffkonzentration wurden verschiedene Umsätze erzielt. Die folgende Abbildung zeigt in doppeltlogarithmischer Auftragung die Messpunkte. Die zugehörigen Werte finden sich in Abschnitt 5 (Messtabellen). Die gewählten Versuchsbedingungen sind dem Text in der Abbildung oder bei den Messtabellen zu entnehmen.

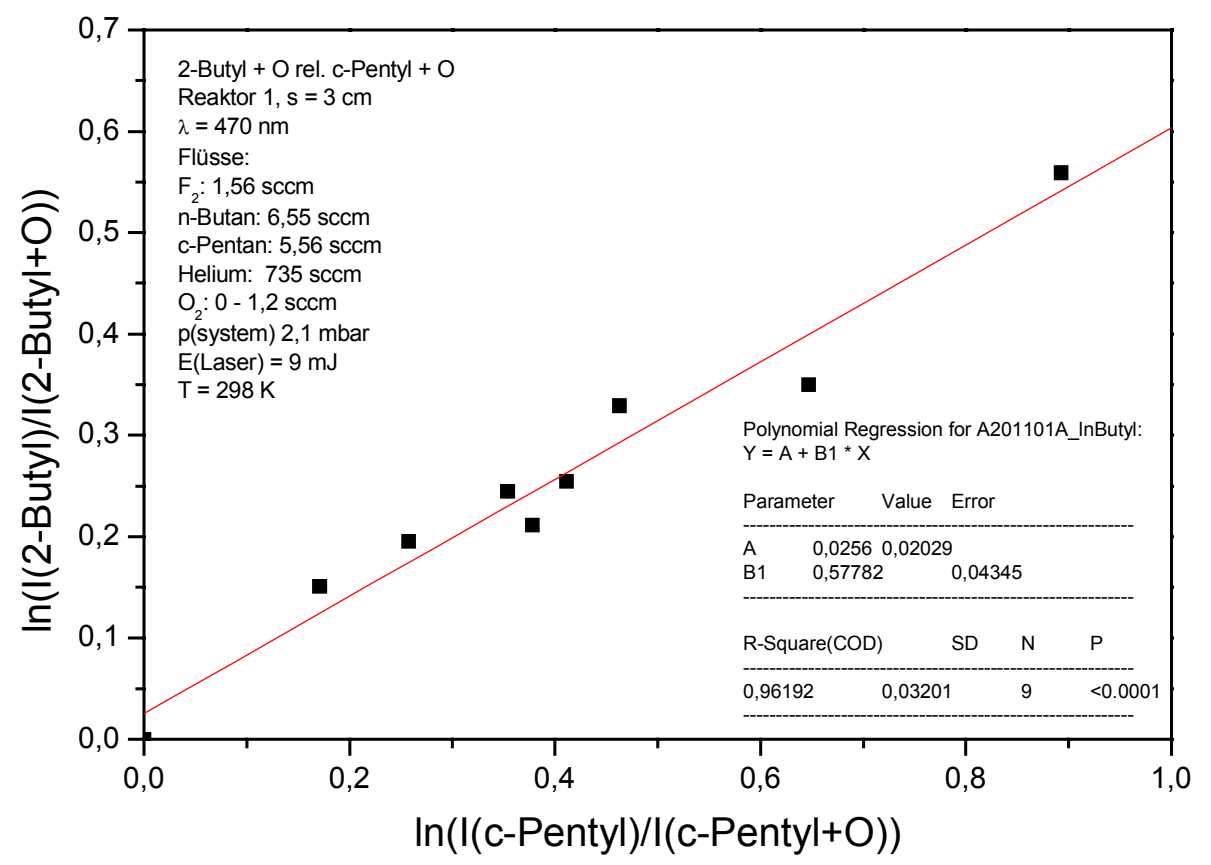

Abbildung 107: 2-Butyl $+\mathrm{O}$ relativ zu Cyclopentyl $+\mathrm{O}, \mathrm{T}=25^{\circ} \mathrm{C}$

Aus der Auftragung erhält man:

$$
\mathrm{k}_{\mathrm{rel}}=\mathrm{k}\left(2-\mathrm{C}_{4} \mathrm{H}_{9}\right) / \mathrm{k}(\mathrm{cPentyl}+\mathrm{O})=0,58 \pm 0,04
$$

Mit der in Abschnitt 3.3.3 erläuterten Geschwindigkeitskonstante $\mathrm{k}_{\mathrm{ref}}=1,67 \cdot 10^{14} \mathrm{~cm}^{3} /(\mathrm{mol} \cdot \mathrm{s})$ für die Referenzreaktion c-Pentyl + O ergibt sich:

$$
\mathrm{k}^{298 \mathrm{~K}}(2-\mathrm{Butyl}+\mathrm{O})=(9,69 \pm 0,07) \cdot 10^{13} \mathrm{~cm}^{3} /(\mathrm{mol} \cdot \mathrm{s}) \text {. }
$$

Diskussion: Der Geschwindigkeitskoeffizient der Reaktion 2-Butyl + O wurde in der eigenen Arbeitsgruppe von Seeba mit Versuchsanordnung A (REMPI) relativ zur Reaktion Methyl + O gemessen: $\mathrm{k}^{298 \mathrm{~K}}=(2,2 \pm 0,7) \cdot 10^{14} \mathrm{~cm}^{3} /(\mathrm{mol} \cdot \mathrm{s})$ [SEE94]. Als Wert für die Referenzreaktion $\mathrm{CH}_{3}+\mathrm{O}$ wurde $\mathrm{k}=8,43 \cdot 10^{14} \mathrm{~cm}^{3} /(\mathrm{mol} \cdot \mathrm{s})$ nach Gutman et al. verwendet [SLA87]. Korrigiert man auf den in dieser Arbeit verwendeten Geschwindigkeitskoeffizienten von Methyl $+\mathrm{O}$ $\left(\mathrm{k}=6,14 \cdot 10^{14} \mathrm{~cm}^{3} /(\mathrm{mol} \cdot \mathrm{s})\right)$, so ergibt sich $\mathrm{k}(2-$ Butyl $+\mathrm{O})=1,60 \cdot 10^{14} \mathrm{~cm}^{3} /(\mathrm{mol} \cdot \mathrm{s})$. Die Radikalerzeugung geschah wie in dieser Arbeit über die Reaktion von n-Butan mit F-Atomen, allerdings 
wurde von Seeba das Signal bei $\mathrm{m} / \mathrm{z}=57$ bei einer Ionisierungswellenlänge von $\lambda=450,8 \mathrm{~nm}$ verarbeitet. Bei dieser Wellenlänge ist, wie in Abschnitt 3.1.4 ausführlich erläutert, auch das 1-Butylradikal noch stark signalgebend. Von Seeba wurde demnach fälschlicherweise ein Mischsignal registriert und ausgewertet. Nun ist, wie im folgenden Abschnitt dargelegt wird, die Geschwindigkeit von 1-Butyl $+\mathrm{O}$ nicht wesentlich anders als die von 2-Butyl $+\mathrm{O}$, insofern ist ein Vergleich statthaft. Die von Seeba bestimmte Geschwindigkeitskonstante liegt um den Faktor 1,6 höher als in dieser Arbeit gemessen.

Von Kersten wurde mittels einer LIF-Analyse des zeitlichen Anstiegprofils der entstehenden OHRadikale der Koeffizient in pseudoerster Ordnung (O-Atom Überschuss) gemessen [KER99]. Es wurde mit zwei Auswertungsmethoden gearbeitet. Das Ergebnis nach Methode 1 lautet $\mathrm{k}^{298 \mathrm{~K}}\left(2-\mathrm{C}_{4} \mathrm{H}_{9}+\mathrm{O}\right)=3,21 \cdot 10^{14} \mathrm{~cm}^{3} /(\mathrm{mol} \cdot \mathrm{s})$, das nach Methode $2 \mathrm{k}^{298 \mathrm{~K}}\left(2-\mathrm{C}_{4} \mathrm{H}_{9}+\mathrm{O}\right)=1,09 \cdot 10^{14}$ $\mathrm{cm}^{3} /$ (mol·s). Ein Vergleich mit anderen von Kersten durchgeführten kinetischen Messungen zeigt, dass Methode 1 unverhältnismäßig hohe Werte liefert. Der mit Methode 2 ermittelte Wert stimmt dagegen sehr gut mit dem in dieser Arbeit bestimmten Wert überein. Weitere Literatur über die Reaktion 2-Butyl + O liegt nicht vor.

\subsubsection{1-Butyl + O}

\section{Produkte:}

Diskussion: Für die Reaktion von 1-Butylradikalen mit O-Atomen erscheinen die folgenden Reaktionskanäle, die alle stark exotherm sind, mechanistisch möglich:

$$
\begin{array}{rlll} 
& \rightarrow & \mathrm{HCHO}+\mathrm{n}_{-} \mathrm{C}_{3} \mathrm{H}_{7} & \Delta_{\mathrm{R}} \mathrm{H}^{0}{ }_{298 \mathrm{~K}}=-333,1 \mathrm{~kJ} / \mathrm{mol}(\mathrm{a}) \\
1-\mathrm{C}_{4} \mathrm{H}_{9}+\mathrm{O} & \rightarrow & \mathrm{CH}_{3} \mathrm{CH}_{2} \mathrm{CH}_{2} \mathrm{CHO}+\mathrm{H} & \Delta_{\mathrm{R}} \mathrm{H}^{0}{ }_{298 \mathrm{~K}}=-314,2 \mathrm{~kJ} / \mathrm{mol} \text { (b) } \\
& \rightarrow & \mathrm{CH}_{3} \mathrm{CH}_{2} \mathrm{CH}=\mathrm{CH}_{2}+\mathrm{OH} & \Delta_{\mathrm{R}} \mathrm{H}^{0}{ }_{298 \mathrm{~K}}=-286,2 \mathrm{~kJ} / \mathrm{mol}(\mathrm{c})
\end{array}
$$

Die Reaktionskanäle (a) und (b) stellen jeweils den C-C und den C-H Bindungsbruch am radikalischen 1-C-Atom dar. Kanal (c) ist der Abstraktionskanal, wobei zunächst nicht unterschieden werden soll zwischen einer Eliminierung aus dem aktivierten Additionskomplex einerseits und einer direkten Abstraktion andererseits. Neben den hier vorgestellten Reaktionskanälen sind noch 1-2, 1-3, 1-4 und 1-5 Isomerisierungen des durch Sauerstoffaddition entstehenden Butoxyradikals denkbar. Für diese wurden aber in den folgenden diskutierten Literaturstellen keine Hinweise gefunden.

In der eigenen Arbeitsgruppe wurden Produktuntersuchungen von Seeba mit Versuchsanordnung B (EI-MS) durchgeführt [SEE94]. Als stabile Produkte konnten Formaldehyd nach Kanal (a), ein 
O-haltiges Produkt bei $\mathrm{m} / \mathrm{z}=72\left(\mathrm{C}_{4} \mathrm{H}_{8} \mathrm{O}\right)$ und ein ungesättigter Kohlenwasserstoff bei $\mathrm{m} / \mathrm{z}=56$ $\left(\mathrm{C}_{4} \mathrm{H}_{8}\right)$ nachgewiesen werden. Es muss aber berücksichtigt werden, dass neben dem 1-Butylradikal das 2-Butylradikal in gleicher Größenordnung erzeugt wurde, wie in den Abschnitten 3.1.4 und 3.3.7 bereits diskutiert, und die Messungen deshalb nur bedingt aussagekräftig sind.

Produktuntersuchungen von Kersten mit Versuchsanordnung C (FTIR) und 1-Jodbutan als photolytische Radikalquelle zeigen die Existenz von (a), (b) und (c). Diesen Reaktionskanälen werden folgenden Ausbeuten zugewiesen: 39,7\% (a), 5,3\% (b) und 55,0\% (c) ([KER99], [HAC01]).

Der OH-Produktnachweis wurde ferner über laserinduzierte Fluoreszenz geführt. Das OH-Radikal wurde in den Schwingungszuständen $v=0,1,2$ in nicht-thermischer Besetzung nachgewiesen. Würde dagegen die $\mathrm{OH}$-Bildung über einen Additionskomplex mit einer gewissen Lebensdauer verlaufen, so käme es zu einer intramolekularen Energieverteilung. Eine zugehörige Abschätzung der Besetzung nach dem Phasenraummodell in der statistischen Grenze ${ }^{28}$ liefert eine relative Besetzung von $1(\mathrm{v}=0): 5,5 \cdot 10^{-2}(\mathrm{v}=1): 2,5 \cdot 10^{-3}(\mathrm{v}=2): 9,5 \cdot 10^{-5}(\mathrm{v}=3)$, welche nicht der gemessenen Verteilung entspricht. Aus diesem Grund wird Kanal (c) als eine direkte Abstraktion formuliert [HAC01].

Wie bereits erwähnt, ist der Einsatz von Alkyljodiden als Radikalquelle in Photolysezellen zur Untersuchung der Reaktion Alkyl $+\mathrm{O}$ problematisch (vgl. Abschnitt 3.3.7, Produkte). Weitere Untersuchungen zur Reaktion 1-Butyl $+\mathrm{O}$ mit anderen Radikalquellen sind in der eigenen Arbeitsgruppe geplant.

\section{$\underline{\text { Reaktionsgeschwindigkeit: }}$}

Messergebnisse: Die Geschwindigkeit der Reaktion 1-Butyl $+\mathrm{O}$ wurde relativ zur Reaktion 2-Butyl + O bei Zimmertemperatur mit Versuchsanordnung A (REMPI) bei $\lambda=430 \mathrm{~nm}$ bestimmt. Beide Radikale wurden aus der Reaktion von $\mathrm{CD}_{3} \mathrm{CH}_{2} \mathrm{CH}_{2} \mathrm{CD}_{3}$ mit F-Atomen erzeugt und konnten aufgrund ihrer unterschiedlichen Masse (1-Butyl: $\mathrm{m} / \mathrm{z}=62,2$-Butyl: $\mathrm{m} / \mathrm{z}=63$ ) gemäß Abschnitt 3.1.4 nachgewiesen werden. Zur Bestimmung des Geschwindigkeitskoeffizienten wurden die Signalintensitäten bei An- und Abwesenheit von Sauerstoffatomen gemessen. Durch Variation der Sauerstoffkonzentration wurden verschiedene Umsätze erzielt. Die folgende Abbildung zeigt in doppeltlogarithmischer Auftragung die Messpunkte. Die zugehörigen Werte finden sich in Abschnitt 5 (Messtabellen). Die gewählten Versuchsbedingungen sind dem Text in der Abbildung oder bei den Messtabellen zu entnehmen. 


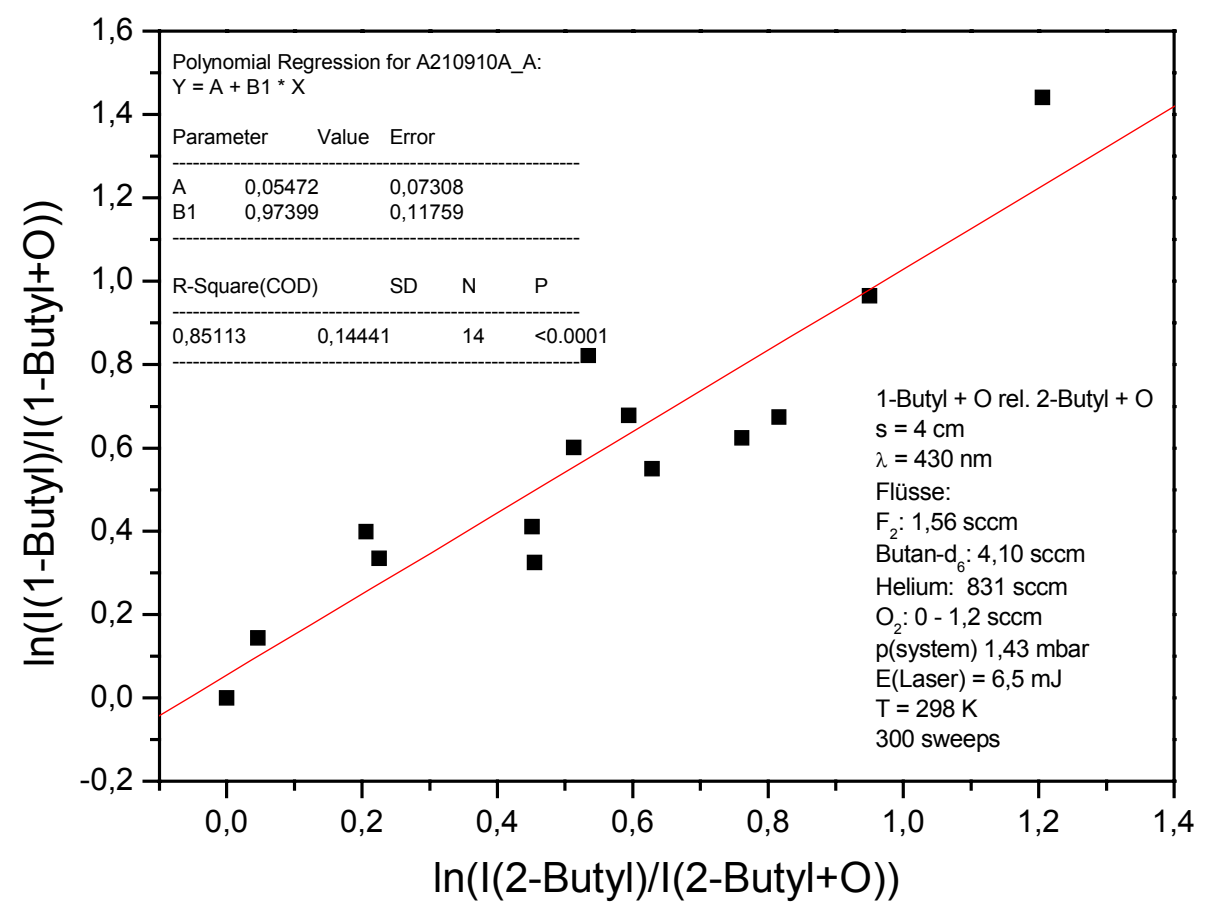

Abbildung 108: 1-Butyl + O relativ zu 2-Butyl $+\mathrm{O}, \mathrm{T}=25^{\circ} \mathrm{C}$

Aus der Auftragung ergibt sich:

$$
k_{\text {rel }}=k\left(1-C_{4} H_{9}\right) / k\left(2-C_{4} H_{9}\right)=0,97 \pm 0,12
$$

Mit der in Abschnitt 3.3.3 erläuterten Geschwindigkeitskonstante $k_{\text {ref }}=9,69 \cdot 10^{13} \mathrm{~cm}^{3} /(\mathrm{mol} \cdot \mathrm{s})$ für die Referenzreaktion 2-Butyl + O erhält man:

$$
\mathrm{k}^{298 \mathrm{~K}}(1-\mathrm{Butyl}+\mathrm{O})=(9,40 \pm 0,12) \cdot 10^{13} \mathrm{~cm}^{3} /(\mathrm{mol} \cdot \mathrm{s})
$$

Diskussion: Der Geschwindigkeitskoeffizient der Reaktion 1-Butyl + O wurde in der eigenen Arbeitsgruppe von Kersten mittels einer LIF-Analyse des zeitlichen Anstiegprofils der entstehenden OH-Radikale in pseudoerster Ordnung (O-Atom Überschuss) gemessen [KER99]. Es wurde mit zwei Auswertungsmethoden gearbeitet. Das Ergebnis nach Methode 1 lautet $\mathrm{k}^{298 \mathrm{~K}}\left(1-\mathrm{C}_{4} \mathrm{H}_{9}+\mathrm{O}\right)=$ $3,12 \cdot 10^{14} \mathrm{~cm}^{3} /(\mathrm{mol} \cdot \mathrm{s})$, das nach Methode $2 \mathrm{k}^{298 \mathrm{~K}}\left(1-\mathrm{C}_{4} \mathrm{H}_{9}+\mathrm{O}\right)=1,39 \cdot 10^{14} \mathrm{~cm}^{3} /(\mathrm{mol} \cdot \mathrm{s})$. Ein Vergleich mit anderen von Kersten durchgeführten kinetischen Messungen zeigt, dass Methode 1 unverhältnismäßig hohe Werte liefert. Der mit Methode 2 ermittelte Wert liegt um den Faktor 1,4 höher als der in dieser Arbeit vorgestellte Geschwindigkeitskoeffizient. Weitere Literatur zur Geschwindigkeit der Reaktion 1-Butyl + O liegt nicht vor.

28 Die vorhergesagte Lebensdauer von hochangeregten n-Alkoxyradikalen liegt im Pikosekundenbereich. Eine solch kurze Lebensdauer liegt am Limit der Anwendbarkeit der statistischen Theorie. Eine Stoßstabilisierung erfordert dagegen Drücke von mehr als 100 bar [HAC02]. 


\subsection{9 t-Butyl + O}

\section{Produkte:}

Messergebnisse: Die Produkte der Reaktion t-Butyl + O wurden mit Versuchsanordnung C (FTIR) bei Zimmertemperatur untersucht. Als Radikalvorläufer wurde dabei Di-tertiär-Butyl-Keton (DtBK, IUPAC-Name: 2,2,4,4-Tetramethylpentan-3-on) eingesetzt. Die folgende Abbildung zeigt ein IRAbsorbanzspektrum des DtBK sowie die Kennzeichnung von einigen Wellenzahlbereichen zu IRaktiven Gruppen (Gruppenfrequenzen). Die gewählten Messbedingungen sind dem Text in der Abbildung zu entnehmen.

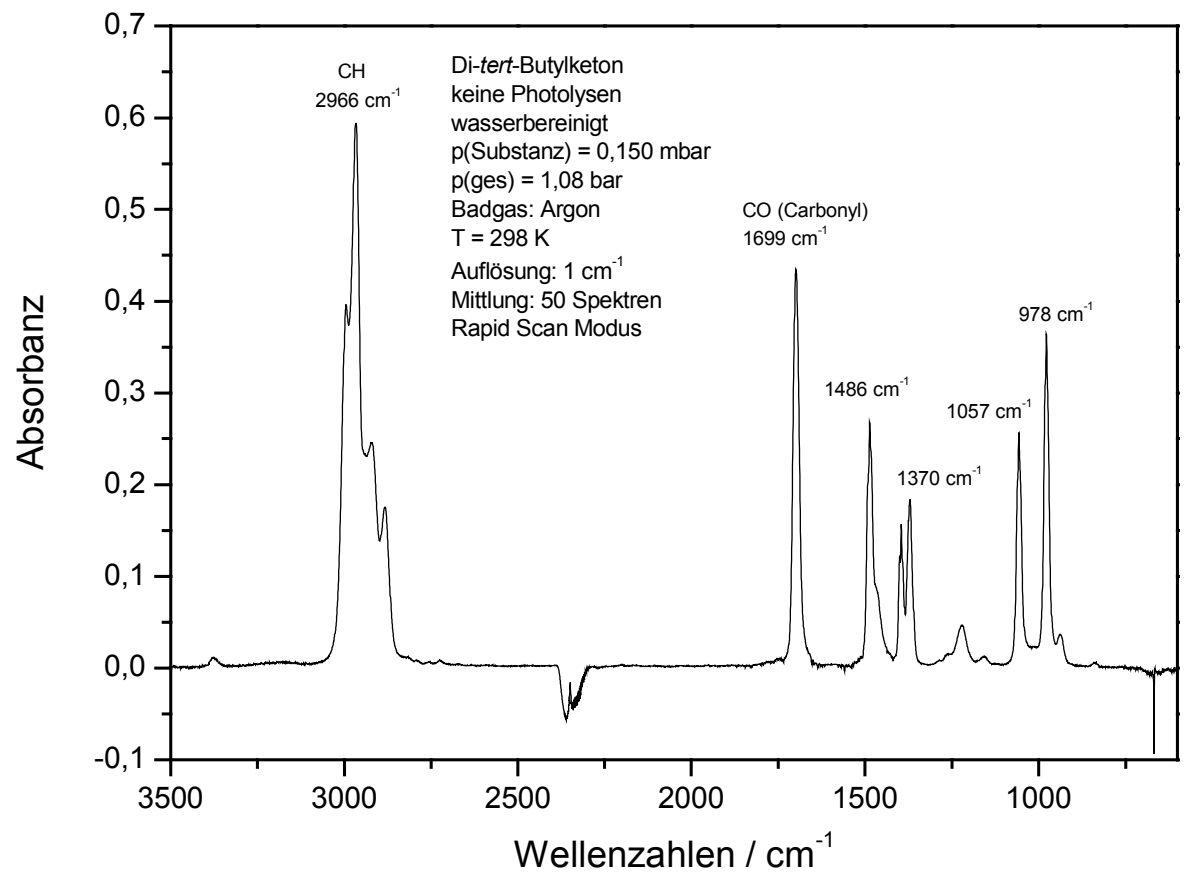

\section{Abbildung 109: FTIR-Spektrum des DtBK}

DtBK wurde anschließend bei $\lambda=193 \mathrm{~nm}$ photolysiert mit dem Ziel, t-Butylradikale zu erzeugen. Da aber auch andere Photolyseprodukte möglich sind, wurden Untersuchungen angestellt, um den Photolyseprozess aufzuklären. Die folgende Abbildung zeigt ein Absorbanzspektrum der Photolyseprodukte nach 250 Laserpulsen. Das Spektrum des DtBK geht in die Berechnung der Absorbanz als $\mathrm{I}_{0}$-Wert ein. Das Spektrum wurde wasserbereinigt. 


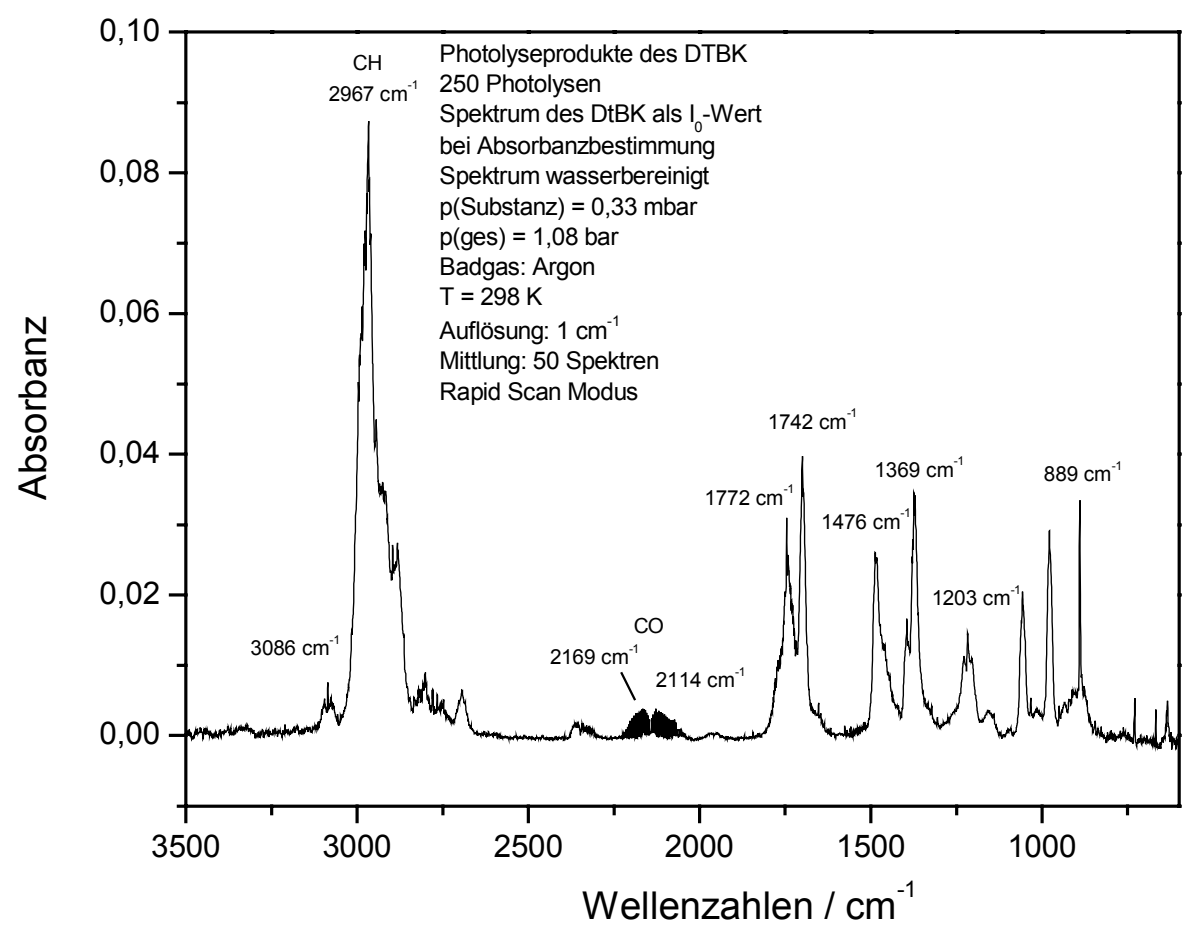

\section{Abbildung 110: Photolyseprodukte des DtBK}

Interpretation: Die Entstehung von CO ist an der typischen Form des Absorbanzbereichs bei $2169 \mathrm{~cm}^{-1}$ und $2114 \mathrm{~cm}^{-1}$ zu erkennen. Somit findet der erwünschte Photolysekanal zur Produktion des t-Butylradikals gemäß folgender Reaktionsgleichung statt:

$$
\mathrm{t}-\mathrm{C}_{4} \mathrm{H}_{9}-\mathrm{CO}-\mathrm{t}-\mathrm{C}_{4} \mathrm{H}_{9} \rightarrow 2 \mathrm{t}-\mathrm{C}_{4} \mathrm{H}_{9}+\mathrm{CO} .
$$

Die zu beobachtenden Absorbanzen bei 2979, 1476, 1369 und $1203 \mathrm{~cm}^{-1}$ sind zumindest teilweise auf die Entstehung des Kombinationsprodukts 2,2,4,4-Tetramethylbutan der t-Butylradikale gemäß der folgenden Reaktionsgleichung zurückzuführen. Ein entsprechendes Vergleichsspektrum des 2,2,4,4-Tetramethylbutan ist im Internet unter der Adresse http://webbook.nist.gov/chemistry erhältlich.

2<smiles>CC(C)(C)C</smiles>

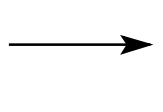<smiles>CC(C)(C)C(C)(C)C</smiles>

Dieser Reaktion wird ein Geschwindigkeitskoeffizient von $\mathrm{k}^{298 \mathrm{~K}}=2,41 \cdot 10^{12} \mathrm{~cm}^{3} /(\mathrm{mol} \cdot \mathrm{s})$ zugewiesen [TSA90]. Bei Anwesenheit von O-Atomen wird die Kombinationsreaktion jedoch zurückgedrängt. 
Am typischen Verlauf der Absorbanzen bei 1772 und $1742 \mathrm{~cm}^{-1}$ ist die Entstehung von Pivaldehyd (2,2-Dimethylpropanal) zu erkennen. Ein entsprechendes Eichspektrum ist bei [KER99] im Anhang zu finden. Damit verbunden ist auch die Entstehung von Isobuten direkt aus der Photolyse. Das ist auch an der Absorbanz bei $890 \mathrm{~cm}^{-1}$ eindeutig zu erkennen. Auch die Absorbanzen bei 2979, 1476, 1369 und $1203 \mathrm{~cm}^{-1}$ sind teilweise auf die Entstehung von Isobuten zurückzuführen. Ein entsprechendes Vergleichsspektrum ist im Internet unter der Adresse http://webbook.nist.gov/chemistry erhältlich. Der geschilderte Photolysekanal stellt sich wie folgt dar:<smiles>CC(C)(C)C(=O)C(C)(C)C</smiles><smiles>CC(C)(C)C=O</smiles><smiles>C=C(C)C</smiles>

Isobuten kann aber auch aus einer Disproportionierungsreaktion der t-Butylradikale entstehen:

$$
2 \mathrm{t}^{-} \mathrm{C}_{4} \mathrm{H}_{9} \rightarrow \text { Isobutan }+ \text { Isobuten. }
$$

Der Geschwindigkeitskoeffizient dieser Reaktion wird angegeben mit $\mathrm{k}^{298 \mathrm{~K}}=2,5 \cdot 10^{12} \mathrm{~cm}^{3} /(\mathrm{mol} \cdot \mathrm{s})$ [WAR84].

Kalibrierungsmessungen von Kohlenmonoxid und Isobuten haben für den hier erläuterten Photolyseprozess ergeben: $\mathrm{c}(\mathrm{CO}): \mathrm{c}($ Isobuten $)=0,7: 0,6$. Zum Zeitpunkt unmittelbar nach der Photolyse ist also $\mathrm{c}(\mathrm{t}-\mathrm{Butyl}) / \mathrm{c}($ Isobuten aus Photolyse) $\geq 14 / 6$. Das Verhältnis des erwünschten Photolysekanals zum unerwünschten entspricht also mindestens diesem Quotienten. Eine genauere Quantifizierung scheint nur mit weiteren Kalibrierungsmessungen unter Berücksichtigung des Pivaldehyds möglich.

Messergebnisse: Für die Untersuchung der Produkte der Reaktion t-Butyl $+\mathrm{O}$ wurden die Sauerstoffatome durch Kophotolyse von $\mathrm{SO}_{2}$ erzeugt. Das Spektrum der Reaktionsmischung vor der Photolyse geht in die Berechnung der Absorbanz als $\mathrm{I}_{0}$-Wert ein. Die folgende Abbildung zeigt die Produktbildung der Reaktion t-Butyl + O. Das oben beschriebene Spektrum der Photolyseprodukte wurde, mit einem entsprechenden Skalierungsfaktor versehen, von der Absorbanz subtrahiert, so dass nur die Reaktionsprodukte von t-Butyl $+\mathrm{O}$ signalgebend sein sollten: 


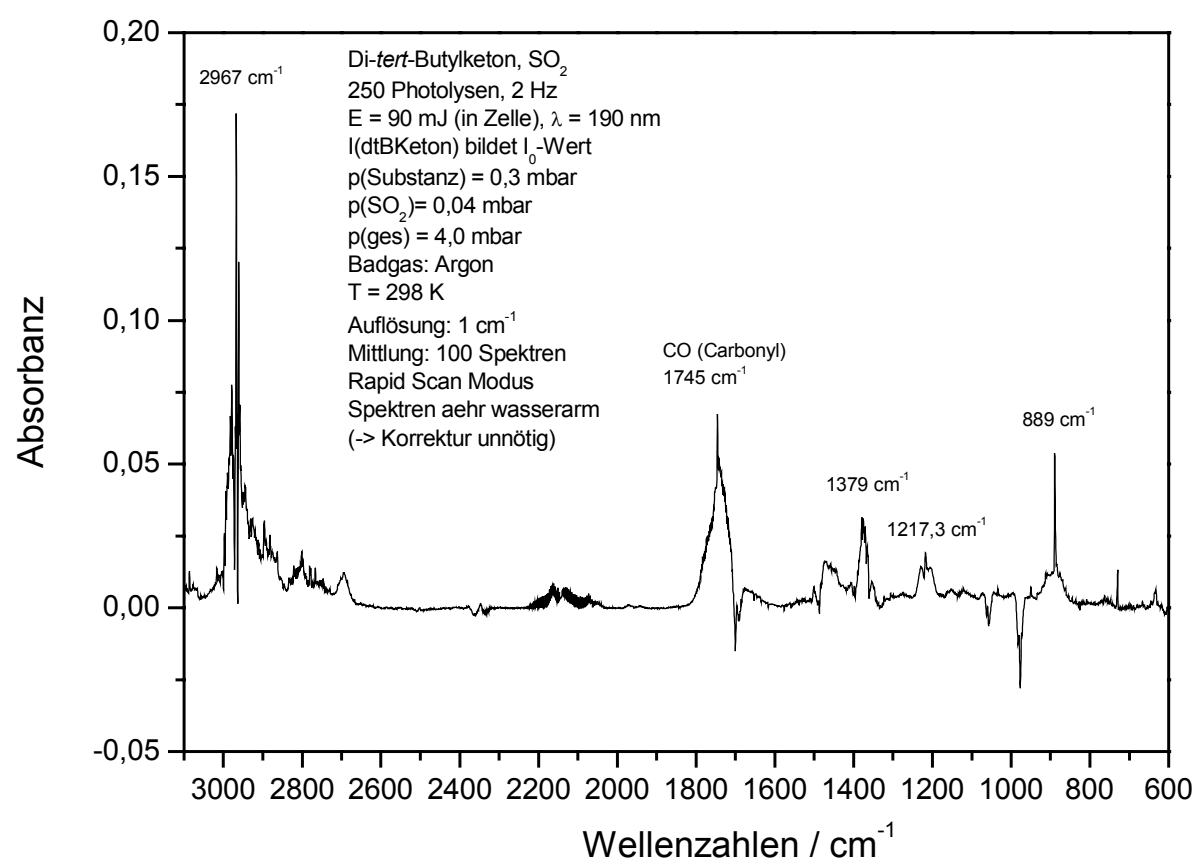

Abbildung 111: Produktanalyse t-Butyl + O

Interpretation: Zur Reaktion t-Butyl + O wird folgender Mechanismus diskutiert:

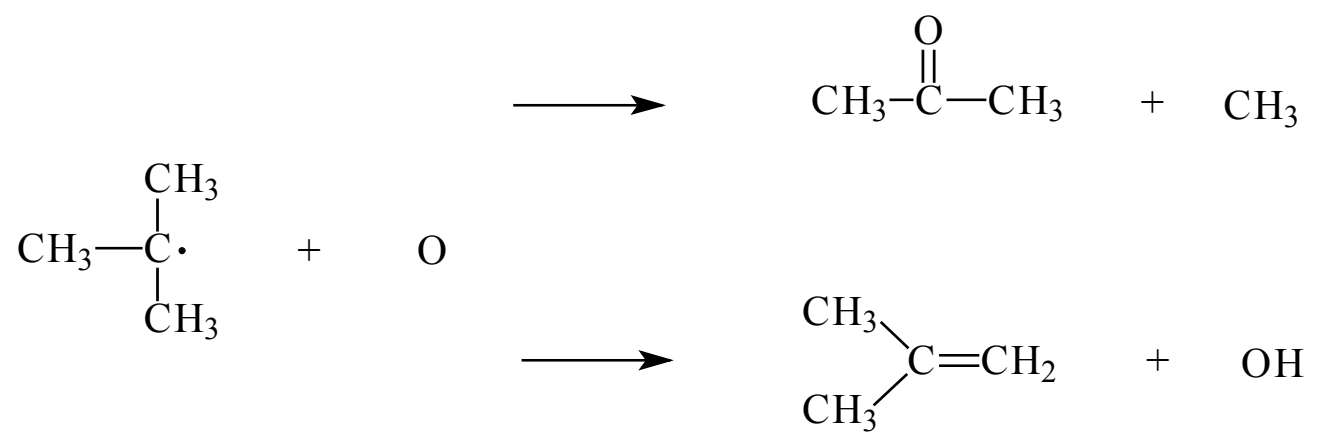

Beide Reaktionskanäle sind stark exotherm: $\Delta_{\mathrm{R}} \mathrm{H}^{0}{ }_{298 \mathrm{~K}}(\mathrm{a})=-366,9 \mathrm{~kJ} / \mathrm{mol}, \Delta_{\mathrm{R}} \mathrm{H}^{0}{ }_{298 \mathrm{~K}}(\mathrm{~b})=-273,2$ $\mathrm{kJ} / \mathrm{mol}$. An den Absorbanzen bei 2967, 1745, 1379 und $1217 \mathrm{~cm}^{-1}$ ist die Entstehung von Aceton eindeutig zu erkennen. Das Signal bei $889 \mathrm{~cm}^{-1}$ weist Isobuten als Produkt nach. Entsprechende Vergleichsspektren sind im Internet unter http://webbook.nist.gov/chemistry erhältlich.

Eine kritische Betrachtung lässt eine darüber hinausgehende Interpretation als problematisch erscheinen: Isobuten entsteht im betrachteten System aus drei Quellen: Direkt aus der Photolyse, aus einer Disproportionierungsreaktion von $\mathrm{t}_{-} \mathrm{C}_{4} \mathrm{H}_{9}$ und aus der Reaktion $\mathrm{t}-\mathrm{C}_{4} \mathrm{H}_{9}+\mathrm{O}$. Eine Bestimmung des Kanalverzweigungsverhältnisses ist auf diesem Wege somit fragwürdig. Berücksichtigt man mögliche Folgereaktionen, wird die Lage sogar noch komplizierter: Es ist zu erwarten, dass Isobuten durch eine schnelle Reaktion mit O-Atomen $\left(\mathrm{k}^{298 \mathrm{~K}}=1,06 \cdot 10^{13} \mathrm{~cm}^{3} /(\mathrm{mol} \cdot \mathrm{s})\right.$, [BIE95]) auch wieder abgebaut wird. Es ergibt sich ein Dilemma: Wird in O-Atom-Unterschuss gearbeitet, wie bei den Untersuchungen hier, ist die Disproportionierung des $\mathrm{t}-\mathrm{C}_{4} \mathrm{H}_{9}$ zur Bildung 
von Isobuten relevant. Wird dagegen im O-Atom-Überschuss gearbeitet, wird Isobuten durch Reaktion mit O-Atomen schnell abgebaut. Zusammenfassend lässt sich sagen, dass für eine Quantifizierung der Produktbildung der Reaktion t-Butyl + O bei der verwendeten Untersuchungsmethode zu viele schnelle Neben- und Folgereaktionen Einfluss nehmen. Alternative Aufklärungsmethoden werden in der sich anschließenden Diskussion erläutert.

Diskussion: Produktuntersuchungen in der eigenen Arbeitsgruppe von Kersten mit Versuchsanordnung C (FTIR) und t-Butyljodid als photolytische Radikalquelle zeigen ebenfalls die Existenz von Reaktionskanal (a) und (b). Diesen Reaktionskanälen werden folgende Anteile zugewiesen: 49\% (a) und 51\% (b) [KER99]. Die erwünschte Photolysereaktion zur Erzeugung des t- $\mathrm{C}_{4} \mathrm{H}_{9}$ lautet wie folgt:

$$
\mathrm{t}-\mathrm{C}_{4} \mathrm{H}_{9} \mathrm{I} \quad \rightarrow \quad \mathrm{t}-\mathrm{C}_{4} \mathrm{H}_{9}+\mathrm{I} .
$$

Es wurde aber nicht untersucht, ob auch folgender Photolysekanal eine Rolle spielt:

$$
\mathrm{t}-\mathrm{C}_{4} \mathrm{H}_{9} \mathrm{I} \quad \rightarrow \quad \text { iso- } \mathrm{C}_{4} \mathrm{H}_{8}+\mathrm{HI} \text {. }
$$

Falls diese zweite unerwünschte Photolysereaktion ebenfalls abläuft, muss ihr Anteil unbedingt berücksichtigt werden.

Des Weiteren nimmt sicherlich auch hier die Reaktion der Alkyljodide mit Sauerstoffatomen zur Bildung von IO und t-Butylradikalen Einfluss auf das Reaktionsgeschehen, wie schon in Abschnitt 3.3.7 (Produkte) erläutert wurde. Es ist davon auszugehen, dass die nachgebildeten Radikale Folgereaktionen mit den Primärprodukten eingehen.

Eine alternative Quelle zur Darstellung des t-Butylradikals in Photolysezellen könnte in folgendem Mechanismus bestehen:

i) Neopentan $+\mathrm{Cl}$ (aus Photolyse von $\left.\mathrm{CFCl}_{3}\right) \rightarrow$ Neopentyl $+\mathrm{HCl}$

ii) Neopentyl $+\mathrm{O}$ (aus Kophotolyse von $\left.\mathrm{SO}_{2}\right) \rightarrow$ t- $\mathrm{C}_{4} \mathrm{H}_{9}+$ Formaldehyd

Die so erzeugten t-Butylradikale stehen nun für eine Reaktion mit O-Atomen zur Verfügung. Vorteil dieser Methode ist, dass weder Aceton noch Isobuten aus dem Photolyseprozess hervorgehen, was eine Bestimmung des Verzweigungsverhältnisses der beiden Kanäle der Reaktion t-Butyl + O ermöglichen könnte. Es sollte dabei ein leichter O-Atom-Überschuss hergestellt werden: Eine Disproportionierung von $\mathrm{t}-\mathrm{C}_{4} \mathrm{H}_{9}$ wird damit zurückgedrängt, andererseits führt der nur leichte O-Atom-Überschuss dazu, dass wenig Isobuten durch Reaktion mit O gleich wieder 
verbraucht wird. Ein Teil der O-Atome wird nach einer gewissen Zeit auch durch das als Primärprodukt aus Reaktionskanal (a) entstehende $\mathrm{CH}_{3}$ reaktiv aufgefangen.

\section{$\underline{\text { Reaktionsgeschwindigkeit: }}$}

Messergebnisse: Die Geschwindigkeit der Reaktion t-Butyl + O wurde bei Zimmertemperatur relativ zur Reaktion Methoxymethyl $+\mathrm{O}$ mit Versuchsanordnung A (REMPI) bei $\lambda=510 \mathrm{~nm}$ bestimmt. Die Radikale wurden gemäß Abschnitt 3.1.5 und 3.1.14 erzeugt und auf ihren Muttermassen $\mathrm{m} / \mathrm{z}=57$ und $\mathrm{m} / \mathrm{z}=45$ in eindeutiger Weise nachgewiesen. Zur Bestimmung des Geschwindigkeitskoeffizienten wurden die Signalintensitäten bei An- und Abwesenheit von Sauerstoffatomen gemessen. Durch Variation der Sauerstoffkonzentration wurden verschiedene Umsätze erzielt. Die folgende Abbildung zeigt in doppeltlogarithmischer Auftragung die Messpunkte. Die zugehörigen Werte finden sich in Abschnitt 5 (Messtabellen). Die gewählten Versuchsbedingungen sind dem Text in der Abbildung oder bei den Messtabellen zu entnehmen.

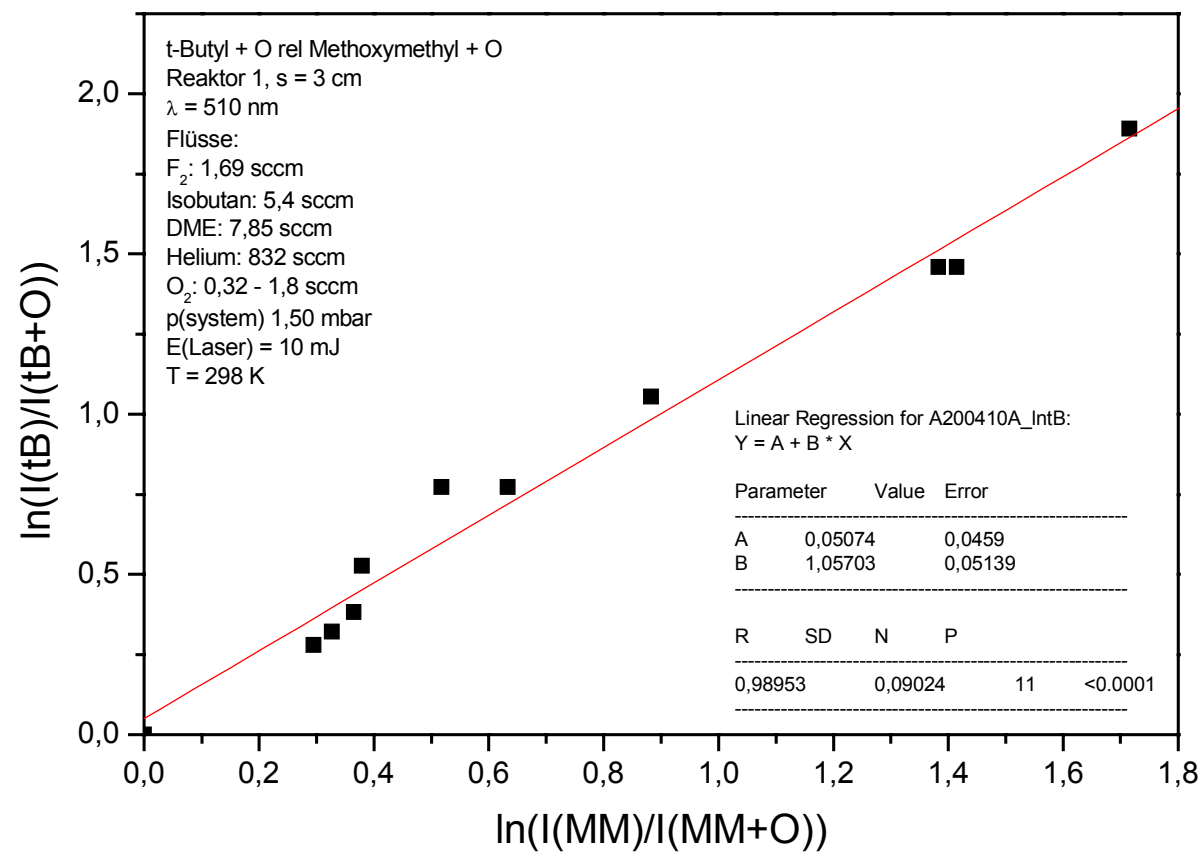

Abbildung 112: t-Butyl $+\mathrm{O}$ relativ zu Methoxymethyl $+\mathrm{O}, \mathrm{T}=25^{\circ} \mathrm{C}$

Aus der Auftragung ergibt sich:

$$
\mathrm{k}_{\mathrm{rel}}=\mathrm{k}\left(\mathrm{t}-\mathrm{C}_{4} \mathrm{H}_{9}+\mathrm{O}\right) / \mathrm{k}\left(\mathrm{CH}_{3} \mathrm{OCH}_{2}+\mathrm{O}\right)=1,06 \pm 0,06 \text {. }
$$

Mit der in Abschnitt 3.3.2 erläuterten Geschwindigkeitskonstante $k_{\text {ref }}=1,31 \cdot 10^{14} \mathrm{~cm}^{3} /(\mathrm{mol} \cdot \mathrm{s})$ für die Referenzreaktion Methoxymethyl + O erhält man:

$$
\mathrm{k}^{298 \mathrm{~K}}\left(\mathrm{t}-\mathrm{C}_{4} \mathrm{H}_{9}+\mathrm{O}\right)=(1,38 \pm 0,08) \cdot 10^{14} \mathrm{~cm}^{3} /(\mathrm{mol} \cdot \mathrm{s})
$$


Diskussion: Washida et al. haben vor kurzem die Geschwindigkeit der Reaktion t-Butyl + O in einem Strömungsreaktor mit photolytischer Radikal- und Sauerstoffatomerzeugung und massenspektrometrischem Nachweis untersucht [WAS00]. Der angegebene Geschwindigkeitskoeffizient beträgt $\mathrm{k}^{298 \mathrm{~K}}=1,32 \cdot 10^{14} \mathrm{~cm}^{3} /(\mathrm{mol} \cdot \mathrm{s})$. Er stimmt mit dem in dieser Arbeit gemessenen Geschwindigkeitskoeffizienten überein. Die einige Jahre zuvor angegebene Geschwindigkeitskonstante $\mathrm{k}^{298 \mathrm{~K}}=5,24 \cdot 10^{14} \mathrm{~cm}^{3} \cdot \mathrm{mol}^{-1} \cdot \mathrm{s}^{-1}$ [WAS80] wurde bei der Vorstellung des neuen Werts von Washida selbst als unrealistisch hoch bezeichnet [WAS00].

Der Geschwindigkeitskoeffizient der Reaktion t-Butyl + O wurde in der eigenen Arbeitsgruppe von Kersten mittels einer LIF-Analyse des zeitlichen Anstiegprofils der entstehenden OH-Radikale in pseudoerster Ordnung (O-Atom-Überschuss) gemessen [KER99]. Es wurde mit zwei Auswertungsmethoden gearbeitet. Das Ergebnis nach Methode 1 lautet $\mathrm{k}^{298 \mathrm{~K}}\left(\mathrm{t}-\mathrm{C}_{4} \mathrm{H}_{9}+\mathrm{O}\right)=$ $7,63 \cdot 10^{14} \mathrm{~cm}^{3} /(\mathrm{mol} \cdot \mathrm{s})$, das nach Methode $2 \mathrm{k}^{298 \mathrm{~K}}\left(\mathrm{t}-\mathrm{C}_{4} \mathrm{H}_{9}+\mathrm{O}\right)=1,39 \cdot 10^{14} \mathrm{~cm}^{3} /(\mathrm{mol} \cdot \mathrm{s})$. Ein Vergleich mit anderen von Kersten durchgeführten kinetischen Messungen zeigt, dass Methode 1 unverhältnismäßig hohe Werte liefert, was sich hier bestätigt. Der mit Methode 2 ermittelte Wert stimmt mit dem in dieser Arbeit vorgestellten Geschwindigkeitskoeffizienten überein.

\section{Temperaturabhängigkeit:}

Messergebnisse: Die Temperaturabhängigkeit der Reaktion t-Butyl $+\mathrm{O}$ wurde im Intervall $-30{ }^{\circ} \mathrm{C}$ bis $+90{ }^{\circ} \mathrm{C}$ nach dem selben Verfahren untersucht. Die Temperierung erfolgte mit der in Abschnitt 2.1.2 beschriebenen Methode für Reaktor 1. Die nachfolgenden Abbildungen zeigen die Ergebnisse. Die zugehörigen Werte finden sich in Abschnitt 5 (Messtabellen). Die gewählten Versuchsbedingungen sind dem Text in der Abbildung oder bei den Messtabellen zu entnehmen. 


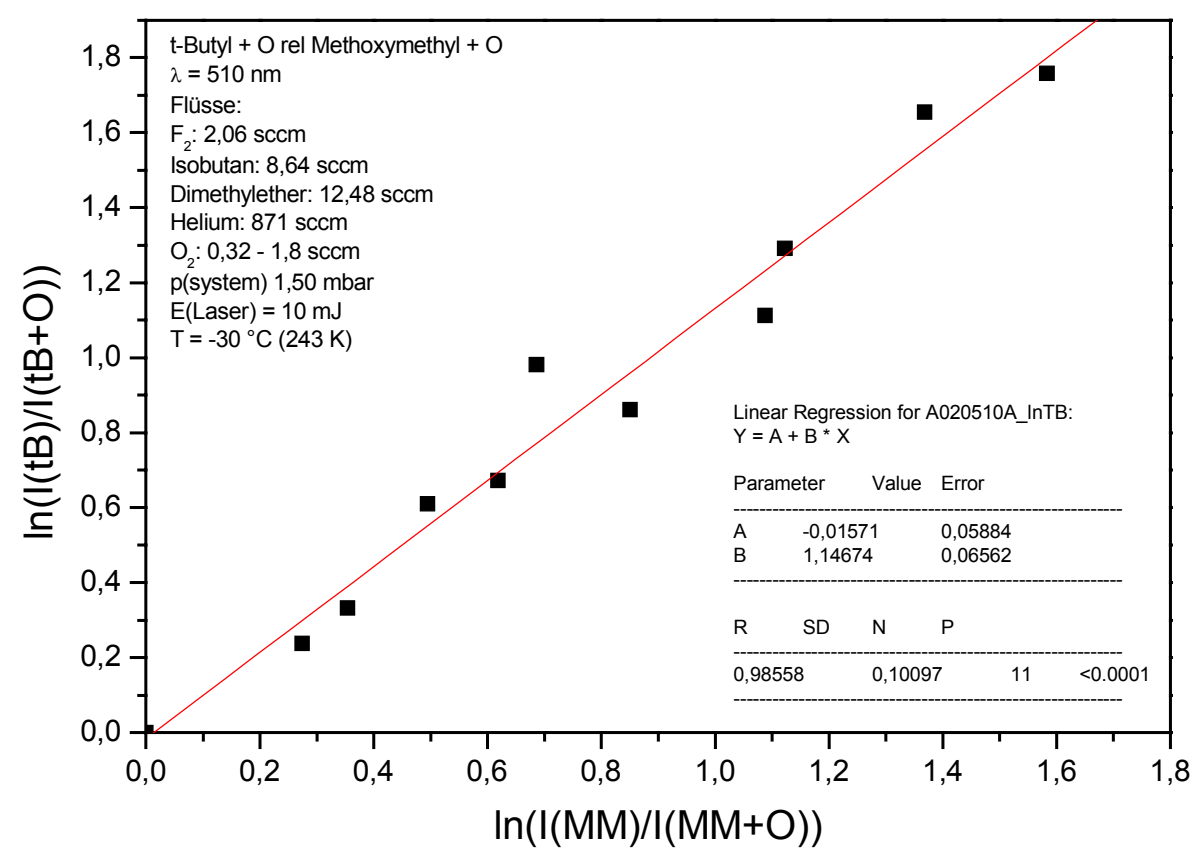

Abbildung 113: t-Butyl $+\mathrm{O}$ relativ zu Methoxymethyl $+\mathrm{O}, \mathrm{T}=-30^{\circ} \mathrm{C}$

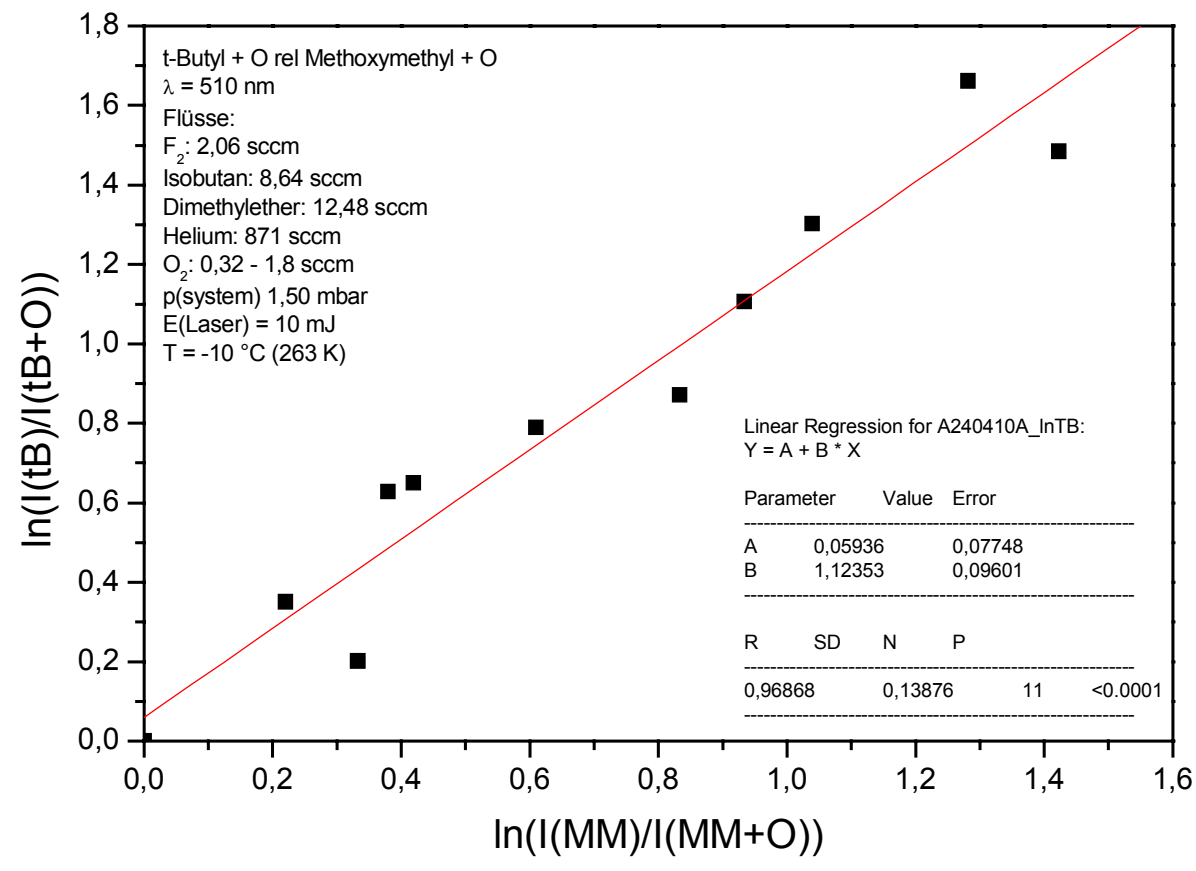

Abbildung 114: t-Butyl $+\mathrm{O}$ relativ zu Methoxymethyl $+\mathrm{O}, \mathrm{T}=-10^{\circ} \mathrm{C}$ 


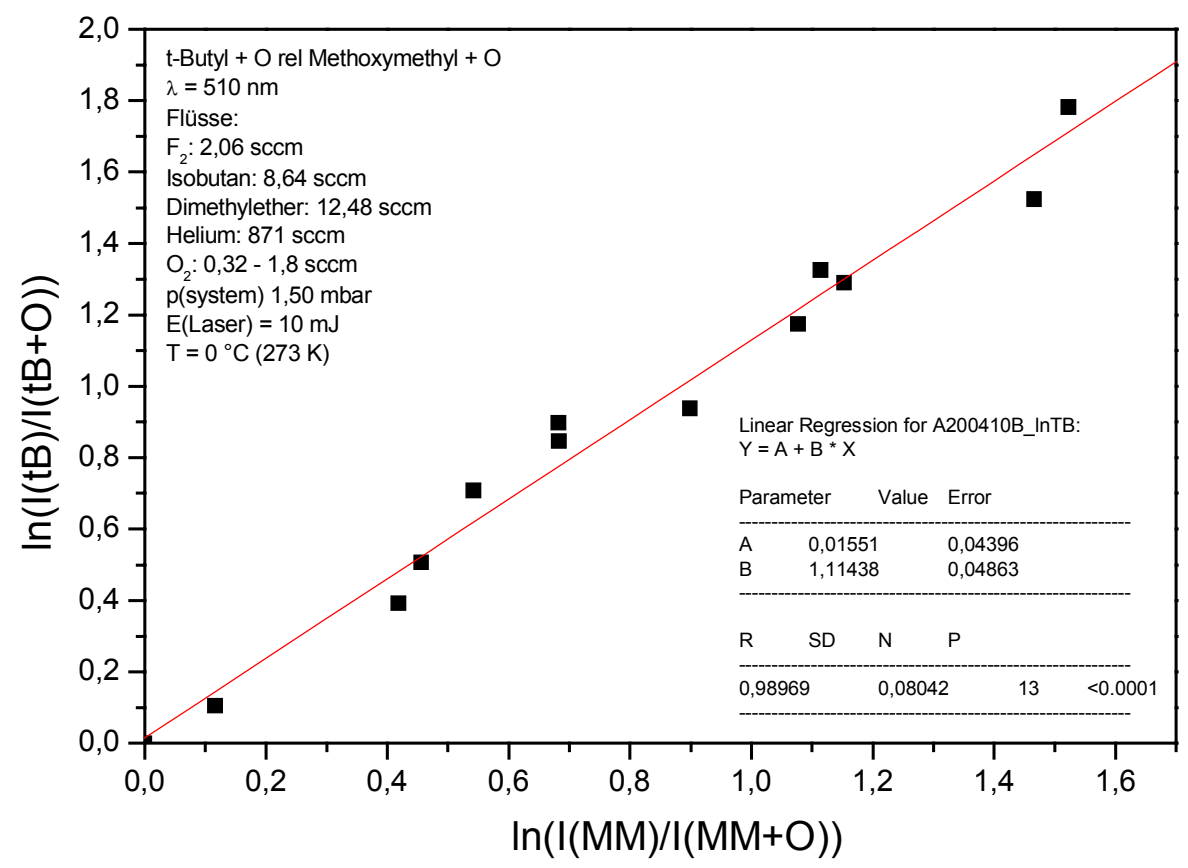

Abbildung 115: t-Butyl $+\mathrm{O}$ relativ zu Methoxymethyl $+\mathrm{O}, \mathrm{T}=0^{\circ} \mathrm{C}$

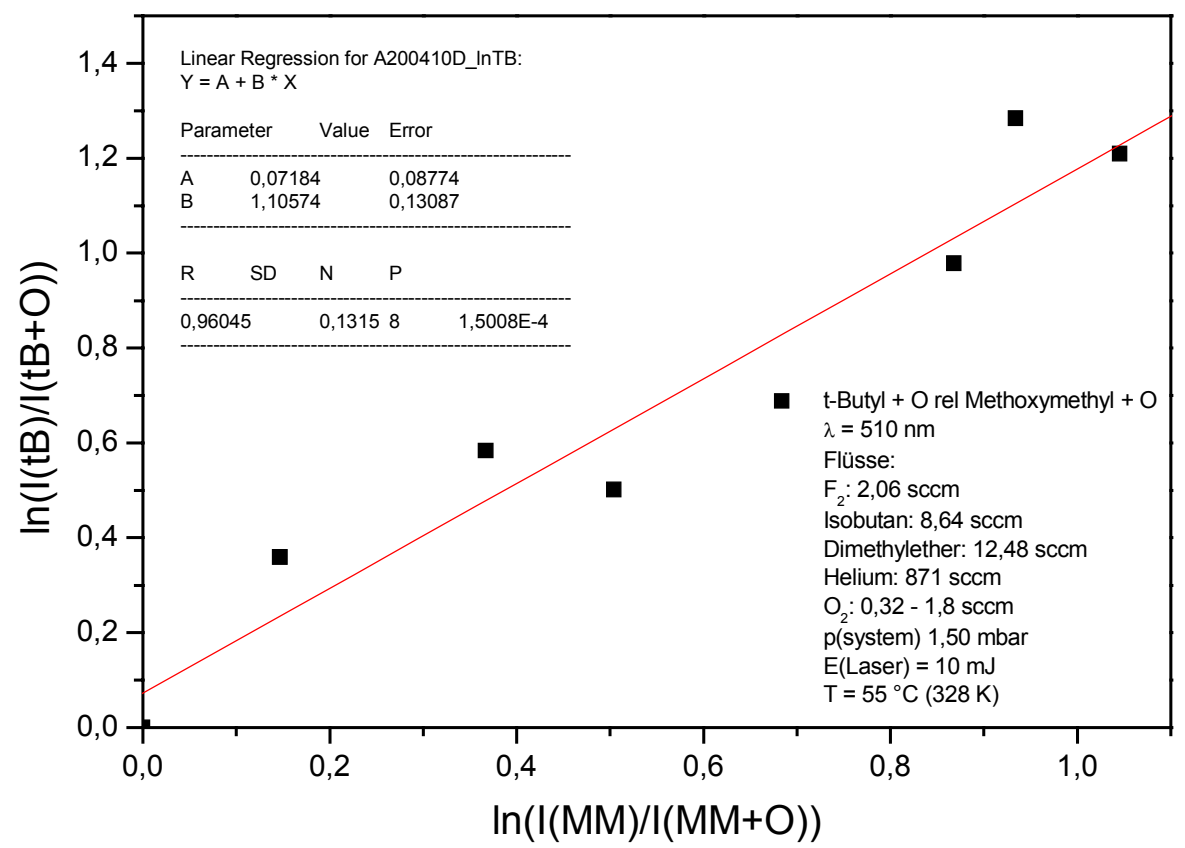

Abbildung 116: t-Butyl $+\mathrm{O}$ relativ zu Methoxymethyl $+\mathrm{O}, \mathrm{T}=55^{\circ} \mathrm{C}$ 


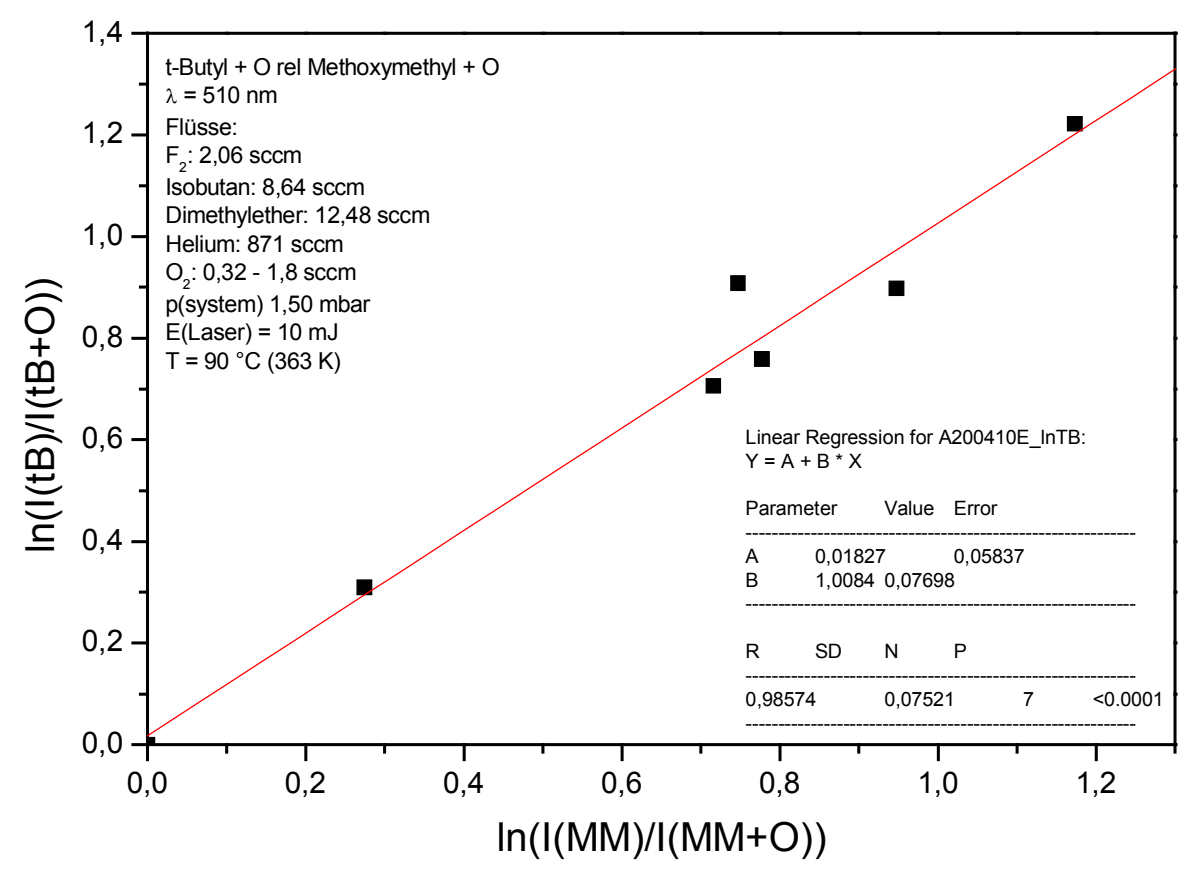

Abbildung 117: t-Butyl $+\mathrm{O}$ relativ zu Methoxymethyl $+\mathrm{O}, \mathrm{T}=90^{\circ} \mathrm{C}$

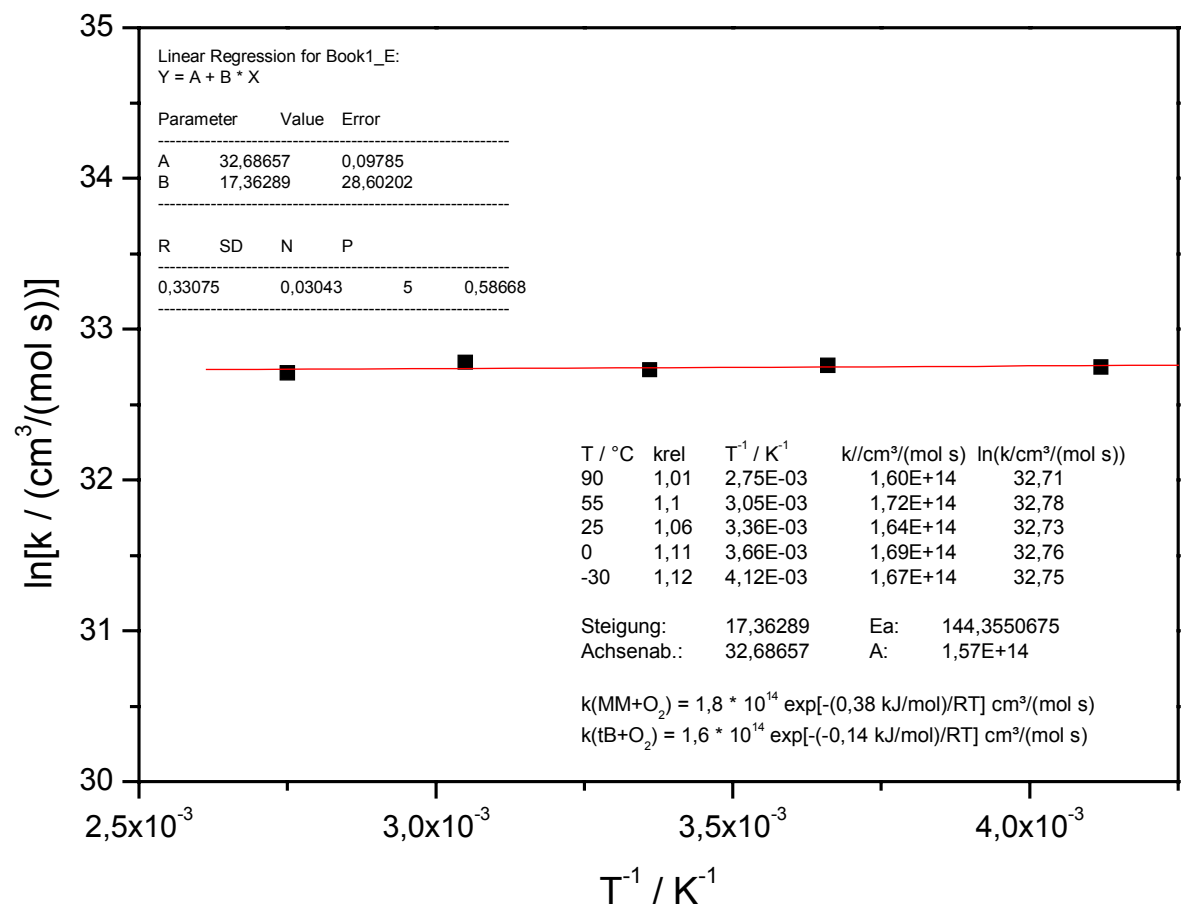

\section{Abbildung 118: t-Butyl + O relativ zu Methoxymethyl + O, Arrheniusauftragung}

Interpretation: Die Temperaturabhängigkeit der Referenzreaktion $\mathrm{CH}_{3} \mathrm{OCH}_{2}+\mathrm{O}$ wurde in der eigenen Arbeitsgruppe von Nacke mit Hilfe von Versuchsanordnung B (EI-MS) relativ zur temperaturunabhängigen Reaktion Ethyl + O untersucht ([NAC94], [HOY96]). Es wurde folgende Temperaturabhängigkeit festgestellt: $\mathrm{k}\left(\mathrm{CH}_{3} \mathrm{OCH}_{2}+\mathrm{O}\right)=1,54 \cdot 10^{14} \cdot(\mathrm{T} / 298 \mathrm{~K})^{0,15} \mathrm{~cm}^{3} /(\mathrm{mol} \cdot \mathrm{s})$ (243 $\leq \mathrm{T} / \mathrm{K} \leq 363)$. Der Geschwindigkeitskoeffizient dieser Reaktion bei Zimmertemperatur wurde 
auf Basis der eigenen Messungen und aufgrund einer leicht veränderten Geschwindigkeit der Reaktion Ethyl $+\mathrm{O}$ in dieser Arbeit ein wenig nach unten korrigiert (vgl. Abschnitt 3.3.2), was in obige Arrheniusauftragung eingearbeitet wurde. Unter Berücksichtigung der geringen Temperaturabhängigkeit der Reaktion $\mathrm{CH}_{3} \mathrm{OCH}_{2}+\mathrm{O}$ wird im Rahmen der Messgenauigkeit für die Reaktion t-Butyl + O Temperaturunabhängigkeit im untersuchten Temperaturintervall festgestellt.

\subsubsection{Eine Vergleichskinetik}

Messergebnisse: Um die Ergebnisse des kinetischen Messverfahrens auf Konsistenz zu prüfen, wurde ein Vergleich der relativen Geschwindigkeitskoeffizienten von drei Reaktionen durchgeführt. Es wurden die Geschwindigkeiten der Reaktionen des t-Butylradikals, des Methoxymethylradikals und des Allylradikals mit O-Atomen relativ zueinander gemessen (zur Methode vgl. Abschnitt 2.4.2). Die folgende Abbildung zeigt die Messung der Geschwindigkeiten von t-Butyl $+\mathrm{O}$ relativ zu Allyl + O. Die zugehörigen Werte finden sich in Abschnitt 5 (Messtabellen). Die gewählten Versuchsbedingungen sind dem Text in der Abbildung oder bei den Messtabellen zu entnehmen.

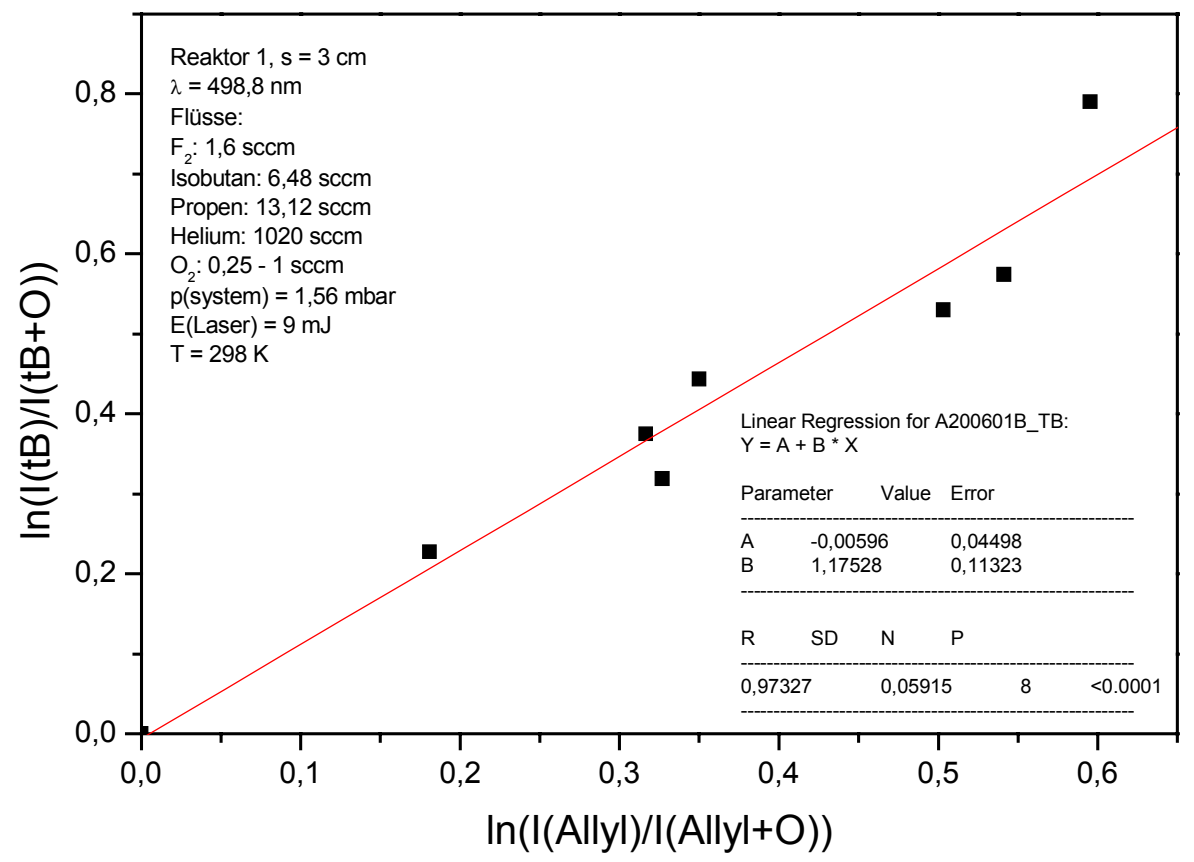

Abbildung 119: t-Butyl $+\mathrm{O}$ relativ zu Allyl $+\mathrm{O}, \mathrm{T}=25^{\circ} \mathrm{C}$

Aus der Auftragung ergibt sich:

$$
\mathrm{k}_{\mathrm{rel}}=\mathrm{k}\left(\mathrm{t}-\mathrm{C}_{4} \mathrm{H}_{9}+\mathrm{O}\right) / \mathrm{k}(\mathrm{Allyl}+\mathrm{O})=1,18 \pm 0,12
$$

Aus den Ergebnissen der Messungen t-Butyl $+\mathrm{O}$ relativ zu $\mathrm{CH}_{3} \mathrm{OCH}_{2}+\mathrm{O}$ und t-Butyl $+\mathrm{O}$ relativ zu Allyl + O ergibt sich rechnerisch dann folgender Geschwindigkeitskoeffizient für die Reaktion $\mathrm{CH}_{3} \mathrm{OCH}_{2}+\mathrm{O}$ relativ zu Allyl $+\mathrm{O}$ : 


$$
\frac{\mathrm{k}_{\mathrm{MM}+\mathrm{O}}}{\mathrm{k}_{\mathrm{Allyl}+\mathrm{O}}}=\frac{\frac{\mathrm{k}_{\mathrm{tB}+\mathrm{O}}}{\mathrm{k}_{\mathrm{Allyl}+\mathrm{O}}}}{\frac{\mathrm{k}_{\mathrm{tB}+\mathrm{O}}}{\mathrm{k}_{\mathrm{MM}+\mathrm{O}}}}=\frac{1,18}{1,06}=1,11 .
$$

Die folgende Abbildung zeigt die Messung zur Reaktion des $\mathrm{CH}_{3} \mathrm{OCH}_{2}+\mathrm{O}$ relativ zu Allyl $+\mathrm{O}$. Die zugehörigen Werte finden sich in Abschnitt 5 (Messtabellen). Die gewählten Versuchsbedingungen sind dem Text in der Abbildung oder bei den Messtabellen zu entnehmen.

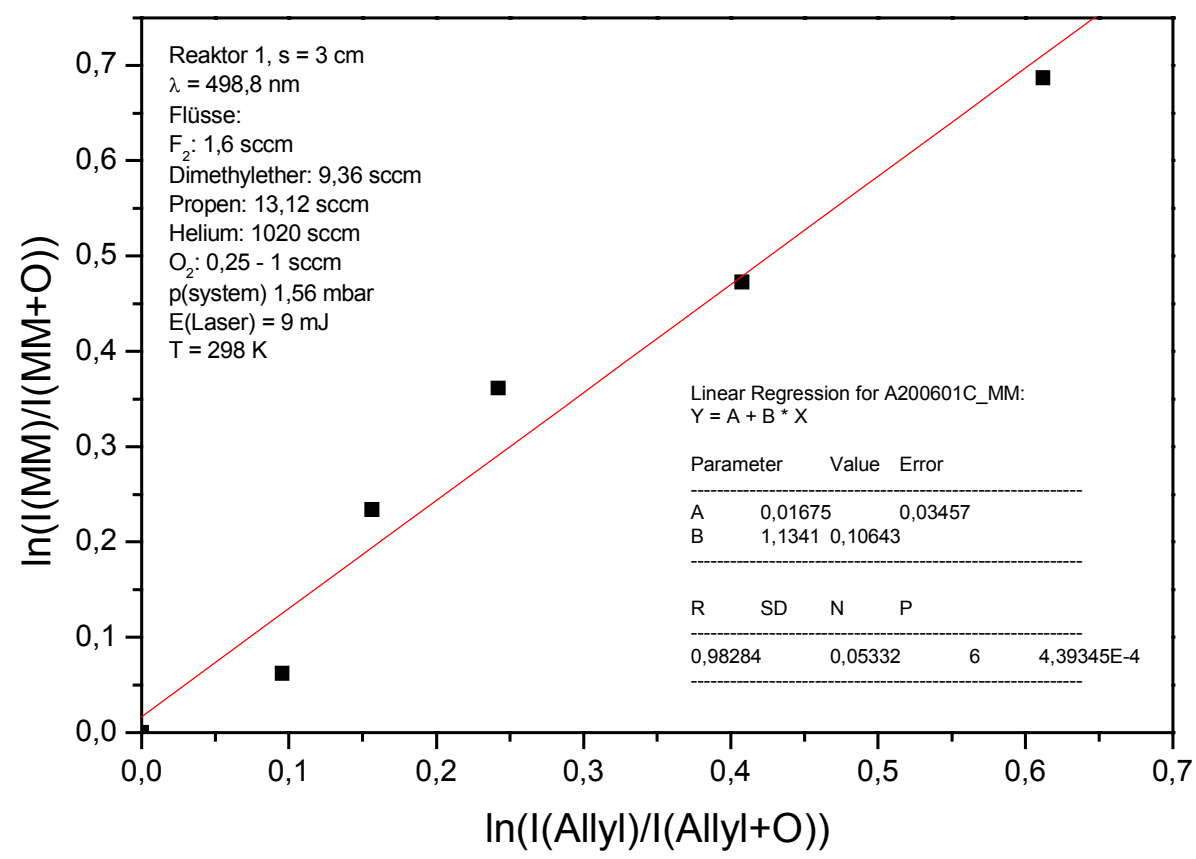

\section{Abbildung 120: Methoxymethyl $+\mathrm{O}$ relativ zu Allyl $+\mathrm{O}, \mathrm{T}=25^{\circ} \mathrm{C}$}

Aus der Auftragung ergibt sich:

$$
\mathrm{k}_{\text {rel }}=\mathrm{k}\left(\mathrm{CH}_{3} \mathrm{OCH}_{2}+\mathrm{O}\right) / \mathrm{k}(\mathrm{Allyl}+\mathrm{O})=1,18 \pm 0,12 \text {. }
$$

Der Vergleich zwischen dem rechnerischen und dem gemessenen relativen Geschwindigkeitskoeffizienten bestätigt die Konsistenz der Messungen zum System der relativen Geschwindigkeitskoeffizienten der genannten drei Reaktionen.

Mit der in Abschnitt 3.3.2 erläuterten Geschwindigkeitskonstanten $k_{\text {ref }}=1,31 \cdot 10^{14} \mathrm{~cm}^{3} /(\mathrm{mol} \cdot \mathrm{s})$ für die Referenzreaktion Methoxymethyl + O ergibt sich für die Reaktion Allyl + O:

$$
\mathrm{k}^{298 \mathrm{~K}}(\mathrm{Ally}+\mathrm{O})=(1,11 \pm 0,2) \cdot 10^{14} \mathrm{~cm}^{3} /(\mathrm{mol} \cdot \mathrm{s})
$$


Diskussion: Nacke hat die relative Geschwindigkeitskonstante $\mathrm{k}_{\mathrm{rel}}=\mathrm{k}\left(\mathrm{CH}_{3} \mathrm{OCH}_{2}+\mathrm{O}\right) / \mathrm{k}(\mathrm{Allyl}+\mathrm{O})$ mit Versuchsanordnung A (REMPI) zu 1,058 bestimmt [NAC98], was in sehr guter Übereinstimmung mit der hier vorgestellten Messung ist.

Die in der Literatur gefundenen Werte für die Geschwindigkeitskoeffizienten der betrachteten Reaktionen sind zusammen mit den eigenen Werten in folgender Tabelle zusammengefasst:

\begin{tabular}{|c|c|c|}
\hline Reaktion & $\mathrm{k} /\left(\mathrm{cm}^{3} \cdot \mathrm{mol}^{-1} \cdot \mathrm{s}^{-1}\right)$ & Autor \\
\hline \multirow{3}{*}{ t-Butyl $+\mathrm{O}$} & $5,24 \cdot 10^{14}$ & Washida et al. [WAS80] \\
\hline & $1,39 \cdot 10^{14}$ & Washida [WAS00] \\
\hline & $1,38 \cdot 10^{14}$ & diese Arbeit \\
\hline \multirow{3}{*}{ Allyl + O } & $6,03 \cdot 10^{13}$ & Tsang [TSA91] \\
\hline & $1,81 \cdot 10^{14}$ & Slagle et al [SLA90] \\
\hline & $1,11 \cdot 10^{14}$ & diese Arbeit \\
\hline \multirow{2}{*}{ Methoxymethyl $+\mathrm{O}$} & $1,20 \cdot 10^{14}$ (korrigiert) & Hoyermann, Nacke [HOY96] \\
\hline & $1,31 \cdot 10^{14}$ & diese Arbeit \\
\hline
\end{tabular}

Tabelle 4: Geschwindigkeitskonstanten ausgewählter Radikale mit O-Atomen

Die Geschwindigkeitskoeffizienten der Reaktionen $\mathrm{CH}_{3} \mathrm{OCH}_{2}+\mathrm{O}$ und t-Butyl $+\mathrm{O}$ wurden in den zugehörigen Abschnitten bereits diskutiert. Für die Reaktion Allyl + O erzielt ein Mittelwert aus den beiden in der Tabelle angegebenen Geschwindigkeitskoeffizienten die beste Übereinstimmung mit dem hier vorgestellten Messzyklus.

\subsubsection{Isobutyl + O}

Produkte:

Diskussion: Für die Reaktion von Isobutylradikalen mit O-Atomen erscheinen die folgenden Reaktionskanäle, die alle stark exotherm sind, mechanistisch möglich:
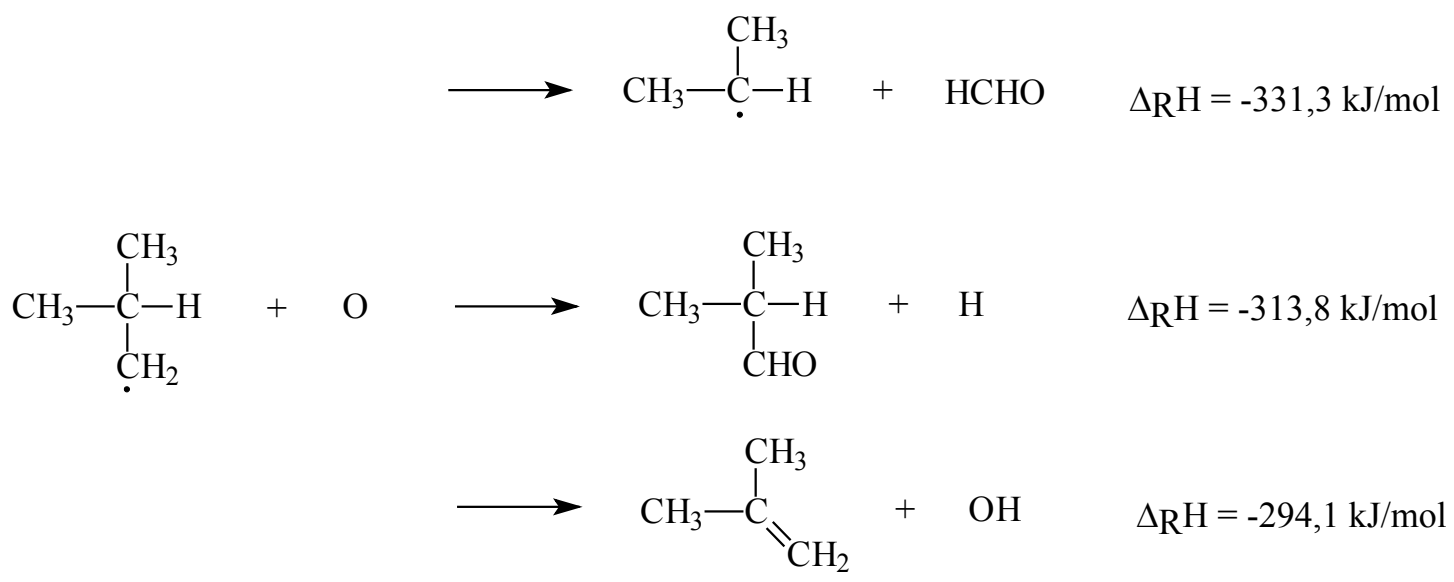
Produktuntersuchungen in der eigenen Arbeitsgruppe von Kersten mit Versuchsanordnung C (FTIR) und 1-Jod-2-Methylpropan als photolytische Radikalquelle zeigen die Existenz von (a), (b) und (c). Diesen Reaktionskanälen werden folgende Anteile zugewiesen: 9,2\% (a), 22,0\% (b) und $88,8 \%$ (c) [KER99].

\section{$\underline{\text { Reaktionsgeschwindigkeit: }}$}

Messergebnisse: Die Geschwindigkeit der Reaktion Isobutyl + O wurde relativ zur Reaktion t-Butyl + O bei Zimmertemperatur mit Versuchsanordnung A (REMPI) bei $\lambda=430 \mathrm{~nm}$ bestimmt. Beide Radikale wurden aus der Reaktion von 2-Methylpropan-2-d mit F-Atomen erzeugt und konnten aufgrund ihrer unterschiedlichen Masse (t-Butyl: $\mathrm{m} / \mathrm{z}=57$, Isobutyl: $\mathrm{m} / \mathrm{z}=58$ ) gemäß Abschnitt 3.1.5 nachgewiesen werden. Zur Bestimmung des Geschwindigkeitskoeffizienten wurden die Signalintensitäten bei An- und Abwesenheit von Sauerstoffatomen gemessen. Durch Variation der Sauerstoffkonzentration wurden verschiedene Umsätze erzielt. Die folgende Abbildung zeigt in doppeltlogarithmischer Auftragung die Messpunkte. Die zugehörigen Werte finden sich in Abschnitt 5 (Messtabellen). Die gewählten Versuchsbedingungen sind dem Text in der Abbildung oder bei den Messtabellen zu entnehmen.

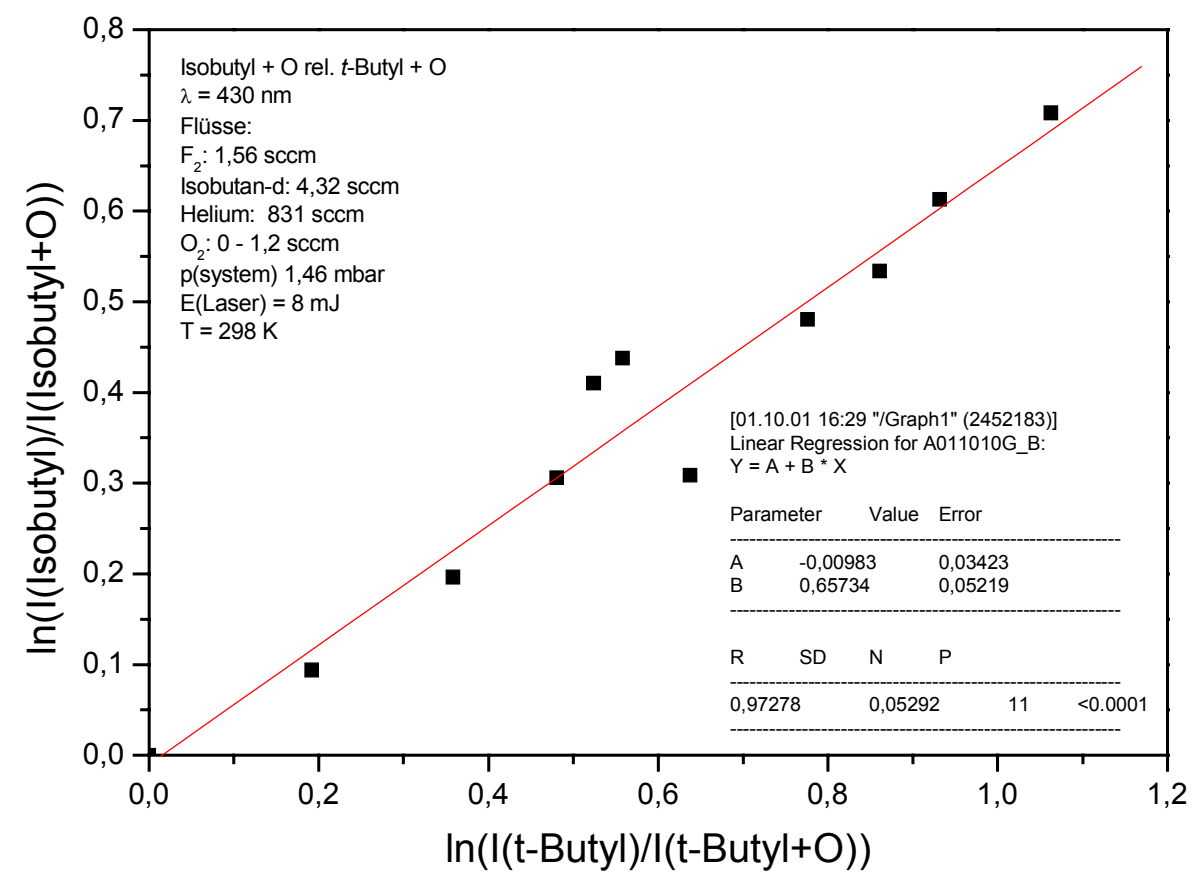

Abbildung 121: Isobutyl $+\mathrm{O}$ relativ zu t-Butyl $+\mathrm{O}, \mathrm{T}=25^{\circ} \mathrm{C}$

Aus der Auftragung ergibt sich:

$$
\mathrm{k}_{\mathrm{rel}}=\mathrm{k}\left(\text { iso- } \mathrm{C}_{4} \mathrm{H}_{9}+\mathrm{O}\right) / \mathrm{k}\left(\mathrm{t}-\mathrm{C}_{4} \mathrm{H}_{9}+\mathrm{O}\right)=0,66 \pm 0,06
$$


Mit der in Abschnitt 3.3.2 erläuterten Geschwindigkeitskonstante $k_{\text {ref }}=1,38 \cdot 10^{14} \mathrm{~cm}^{3} /(\mathrm{mol} \cdot \mathrm{s})$ für die Referenzreaktion t-Butyl + O erhält man:

$$
\mathrm{k}^{298 \mathrm{~K}}\left(\mathrm{iso}-\mathrm{C}_{4} \mathrm{H}_{9}+\mathrm{O}\right)=(9,11 \pm 0,09) \cdot 10^{13} \mathrm{~cm}^{3} /(\mathrm{mol} \cdot \mathrm{s})
$$

Diskussion: Der Geschwindigkeitskoeffizient der Reaktion Isobutyl + O wurde in der eigenen Arbeitsgruppe von Kersten mittels einer LIF-Analyse des zeitlichen Anstiegprofils der entstehenden OH-Radikale in pseudoerster Ordnung (O-Atom-Überschuss) gemessen [KER99]. Es wurde mit zwei Auswertungsverfahren gearbeitet. Das Ergebnis nach Verfahren 1 lautet $\mathrm{k}^{298 \mathrm{~K}}\left(\right.$ iso- $\left._{4} \mathrm{H}_{9}+\mathrm{O}\right)=$ $3,86 \cdot 10^{14} \mathrm{~cm}^{3} /(\mathrm{mol} \cdot \mathrm{s})$, das nach Verfahren 2 lautet $\mathrm{k}^{298 \mathrm{~K}}\left(\right.$ iso- $\left.\mathrm{C}_{4} \mathrm{H}_{9}+\mathrm{O}\right)=1,33 \cdot 10^{14} \mathrm{~cm}^{3} /(\mathrm{mol} \cdot \mathrm{s})$. Ein Vergleich mit anderen von Kersten durchgeführten kinetischen Messungen zeigt, dass Verfahren 1 unverhältnismäßig hohe Werte liefert. Der mit Verfahren 2 ermittelte Wert liegt um den Faktor 1,5 höher als der in dieser Arbeit vorgestellte Geschwindigkeitskoeffizient. Weitere Literatur zur Geschwindigkeit der Reaktion Isobutyl + O liegt nicht vor.

\subsubsection{Cycloheptyl + O}

\section{Produkte:}

Diskussion: Es wurden keine Produktuntersuchungen vorgenommen. Folgende Reaktionskanäle erscheinen mechanistisch möglich:
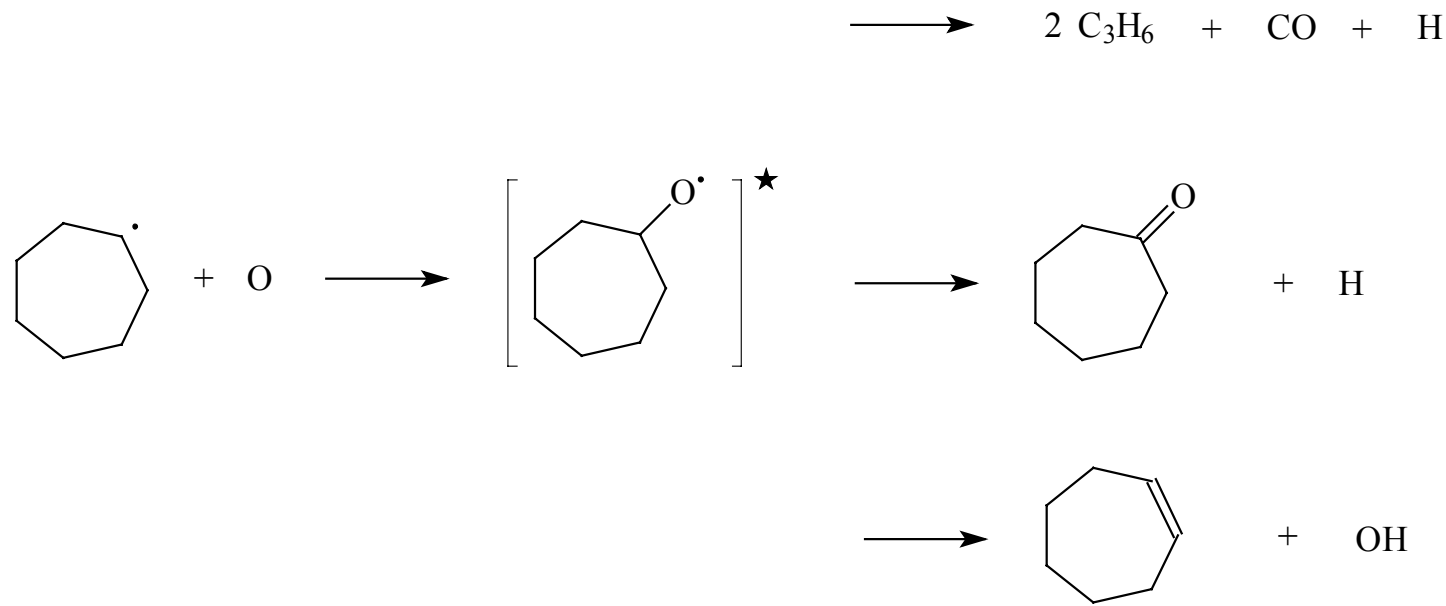

Alle drei Kanäle sind exotherm: $\Delta_{\mathrm{R}} \mathrm{H}^{0}{ }_{298 \mathrm{~K}} /(\mathrm{kJ} / \mathrm{mol}):-176,1$ (a), $-353,1$ (b), $-294,1$ (c). Der Ringzerfall nach (a) ist auch noch in anderen Varianten denkbar.

Es lag keine Literatur über Produktuntersuchungen der Reaktion c-Heptyl + O vor. 


\section{Reaktionsgeschwindigkeit:}

Messergebnisse: Die Geschwindigkeit der Reaktion c-Heptyl + O wurde bei Zimmertemperatur relativ zur Reaktion Methoxymethyl $+\mathrm{O}$ mit Versuchsanordnung A (REMPI) bei $\lambda=440 \mathrm{~nm}$ bestimmt. Die Radikale wurden gemäß Abschnitt 3.1.8 und 3.1.14 erzeugt und auf ihren Muttermassen $\mathrm{m} / \mathrm{z}=97$ und $\mathrm{m} / \mathrm{z}=45$ nachgewiesen. Zur Bestimmung des Geschwindigkeitskoeffizienten wurden die Signalintensitäten bei An- und Abwesenheit von Sauerstoffatomen gemessen. Durch Variation der Sauerstoffkonzentration wurden verschiedene Umsätze erzielt. Die folgende Abbildung zeigt in doppeltlogarithmischer Auftragung die Messpunkte. Die zugehörigen Werte finden sich in Abschnitt 5 (Messtabellen). Die gewählten Versuchsbedingungen sind dem Text in der Abbildung oder bei den Messtabellen zu entnehmen.

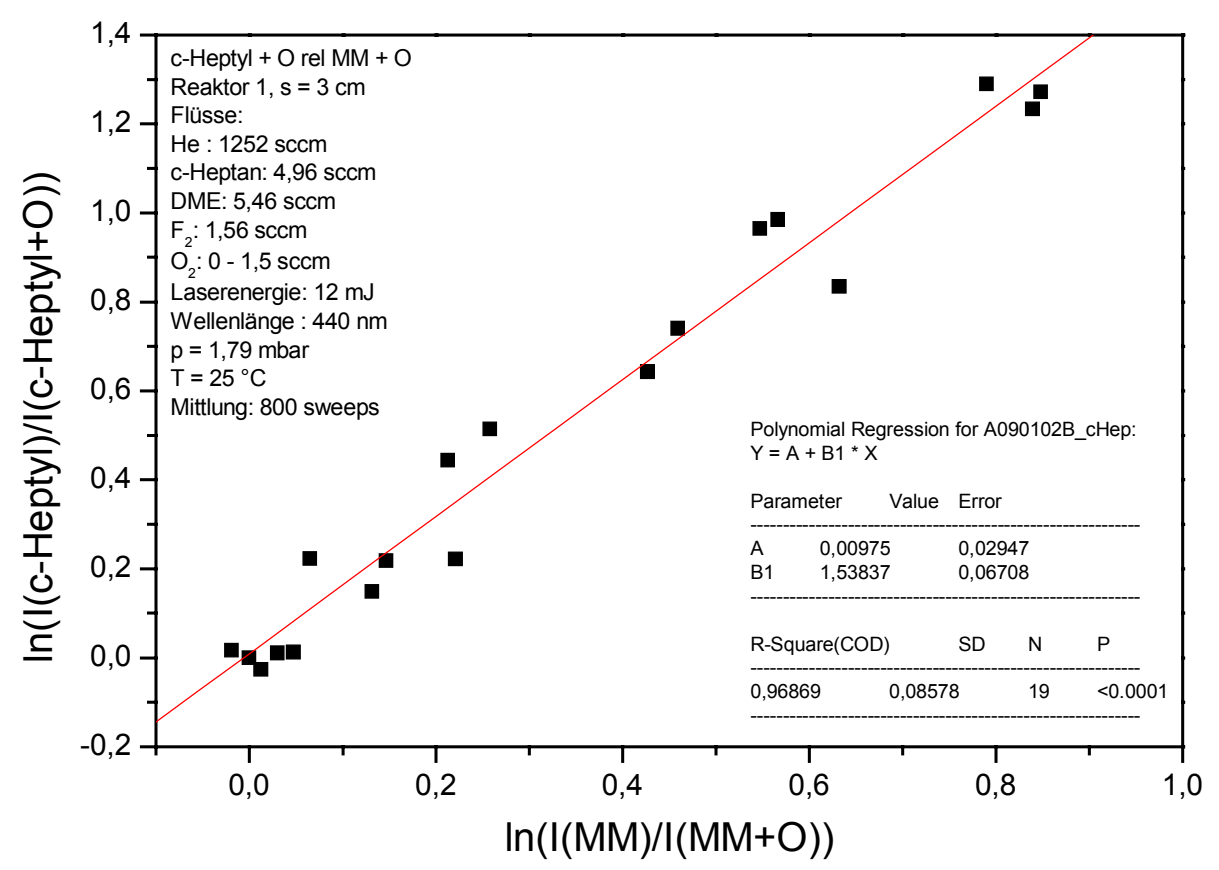

Abbildung 122: c-Heptyl $+\mathrm{O}$ relativ zu Methoxymethyl $+\mathrm{O}, \mathrm{T}=25^{\circ} \mathrm{C}$

Aus der Auftragung ergibt sich:

$$
\mathrm{k}_{\mathrm{rel}}=\mathrm{k}\left(\mathrm{c}-\mathrm{C}_{7} \mathrm{H}_{13}+\mathrm{O}\right) / \mathrm{k}\left(\mathrm{CH}_{3} \mathrm{OCH}_{2}+\mathrm{O}\right)=1,54 \pm 0,07 \text {. }
$$

Mit der in Abschnitt 3.3.2 erläuterten Geschwindigkeitskonstante $k_{\text {ref }}=1,31 \cdot 10^{14} \mathrm{~cm}^{3} /(\mathrm{mol} \cdot \mathrm{s})$ für die Referenzreaktion Methoxymethyl + O erhält man:

$$
\mathrm{k}^{298 \mathrm{~K}}\left(\mathrm{c}-\mathrm{C}_{7} \mathrm{H}_{13}+\mathrm{O}\right)=(2,01 \pm 0,10) \cdot 10^{14} \mathrm{~cm}^{3} /(\mathrm{mol} \cdot \mathrm{s})
$$

Es lag keine Literatur über die Geschwindigkeit der Reaktion c-Heptyl + O vor. 


\subsubsection{Cyclooktyl + O}

\section{Produkte:}

Diskussion: Es wurden keine Produktuntersuchungen vorgenommen. Folgende Reaktionskanäle erscheinen mechanistisch möglich:
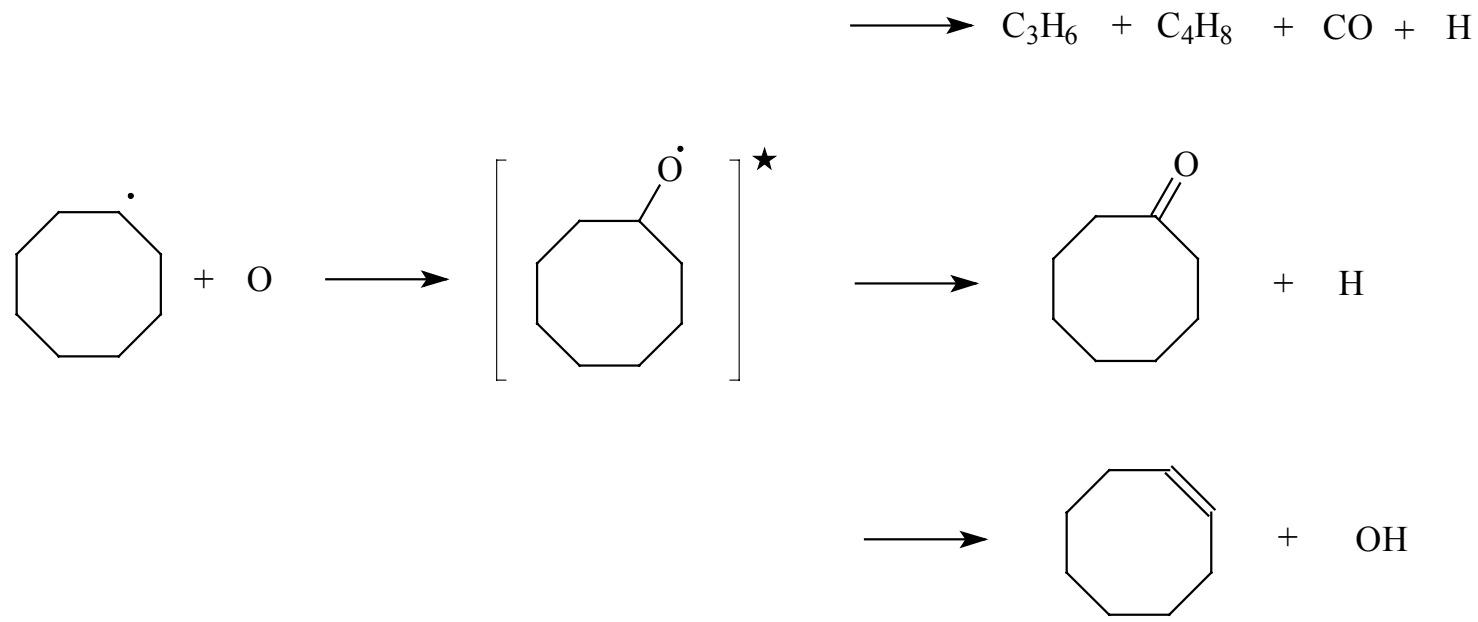

(b)

Alle drei Kanäle sind exotherm: $\Delta_{\mathrm{R}} \mathrm{H}^{0}{ }_{298 \mathrm{~K}} /(\mathrm{kJ} / \mathrm{mol})$ : $-190,7$ (a), $-371,9$ (b), $-306,2$ (c). Der Ringzerfall nach (a) ist auch noch in anderen Varianten denkbar.

Es lag keine Literatur über Produktuntersuchungen der Reaktion c-Oktyl + O vor.

\section{Reaktionsgeschwindigkeit:}

Messergebnisse: Die Geschwindigkeit der Reaktion c-Oktyl + O wurde bei Zimmertemperatur relativ zur Reaktion Methoxymethyl $+\mathrm{O}$ mit Versuchsanordnung A (REMPI) bei $\lambda=456 \mathrm{~nm}$ bestimmt. Die Radikale wurden gemäß Abschnitt 3.1.9 und 3.1.14 erzeugt und auf ihren Muttermassen $\mathrm{m} / \mathrm{z}=111$ und $\mathrm{m} / \mathrm{z}=45$ nachgewiesen. Zur Bestimmung des Geschwindigkeitskoeffizienten wurden die Signalintensitäten bei An- und Abwesenheit von Sauerstoffatomen gemessen. Durch Variation der Sauerstoffkonzentration wurden verschiedene Umsätze erzielt. Die folgende Abbildung zeigt in doppeltlogarithmischer Auftragung die Messpunkte. Die zugehörigen Werte finden sich in Abschnitt 5 (Messtabellen). Die gewählten Versuchsbedingungen sind dem Text in der Abbildung oder bei den Messtabellen zu entnehmen. 


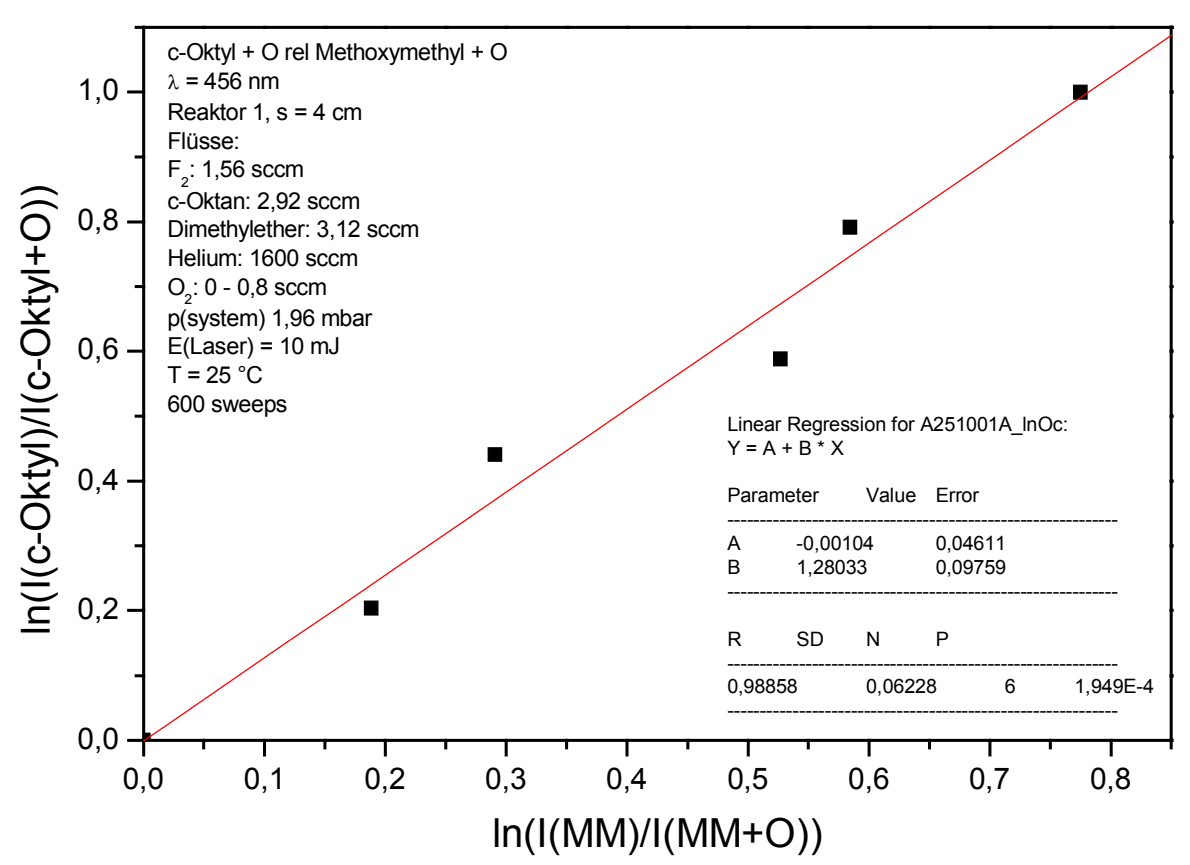

Abbildung 123: c-Oktyl $+\mathrm{O}$ relativ zu Methoxymethyl $+\mathrm{O}, \mathrm{T}=25^{\circ} \mathrm{C}$

Aus der Auftragung ergibt sich:

$$
\mathrm{k}_{\mathrm{rel}}=\mathrm{k}\left(\mathrm{c}-\mathrm{C}_{8} \mathrm{H}_{15}+\mathrm{O}\right) / \mathrm{k}\left(\mathrm{CH}_{3} \mathrm{OCH}_{2}+\mathrm{O}\right)=1,28 \pm 0,10 \text {. }
$$

Mit der in Abschnitt 3.3.2 erläuterten Geschwindigkeitskonstante $k_{\text {ref }}=1,31 \cdot 10^{14} \mathrm{~cm}^{3} /(\mathrm{mol} \cdot \mathrm{s})$ für die Referenzreaktion Methoxymethyl + O erhält man:

$$
\mathrm{k}^{298 \mathrm{~K}}\left(\mathrm{c}-\mathrm{C}_{8} \mathrm{H}_{15}+\mathrm{O}\right)=(1,68 \pm 0,14) \cdot 10^{14} \mathrm{~cm}^{3} /(\mathrm{mol} \cdot \mathrm{s})
$$

Es lag keine Literatur über die Geschwindigkeit der Reaktion c-Oktyl + O vor.

Temperaturabhängigkeit:

Messergebnisse: Die Temperaturabhängigkeit der Reaktion c-Oktyl $+\mathrm{O}$ wurde im Intervall $+5{ }^{\circ} \mathrm{C}$ bis $+90{ }^{\circ} \mathrm{C}$ mit dem selben Verfahren untersucht. Die Temperierung erfolgte mit der in Abschnitt 2.1.2 beschriebenen Methode für Reaktor 1. Die nachfolgenden Abbildungen zeigen die Ergebnisse. Die zugehörigen Werte finden sich in Abschnitt 5 (Messtabellen). Die gewählten Versuchsbedingungen sind dem Text in der Abbildung oder bei den Messtabellen zu entnehmen. 


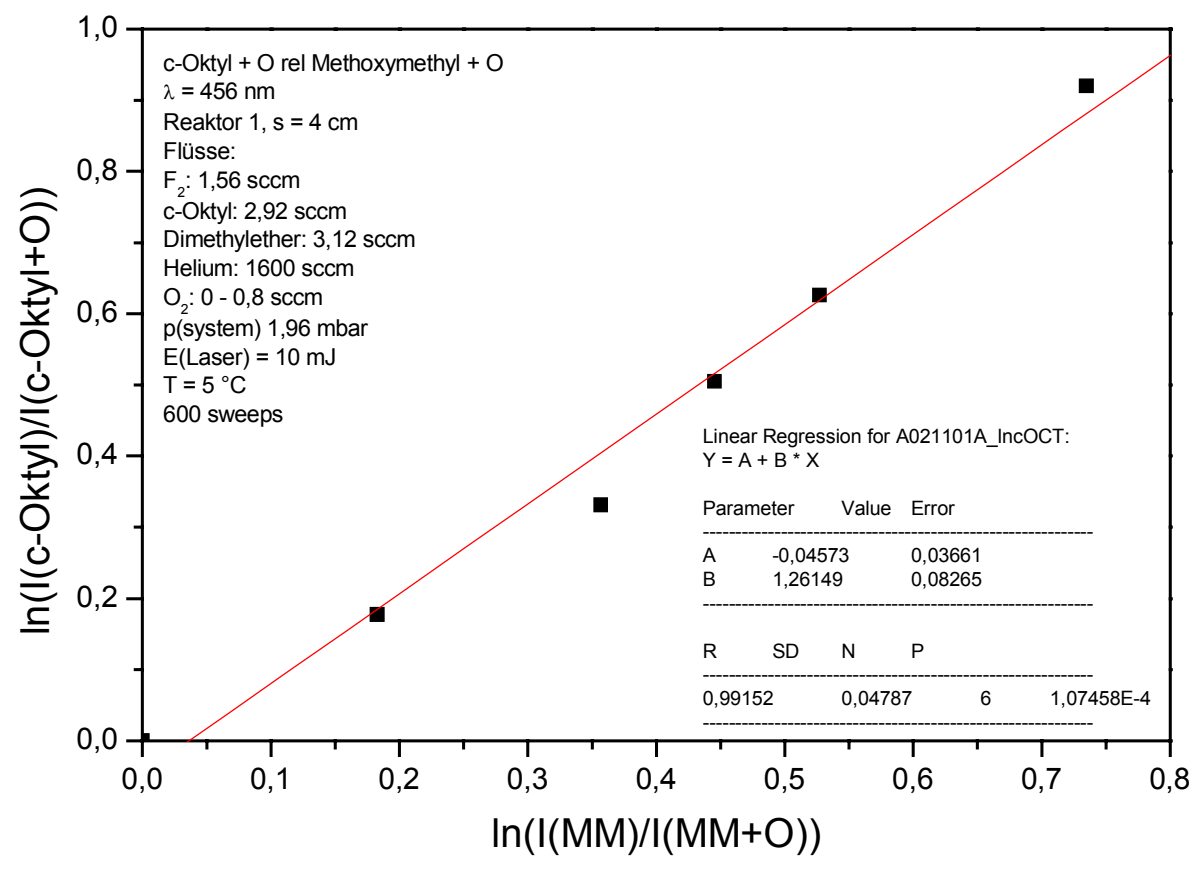

Abbildung 124: c-Oktyl $+\mathrm{O}$ relativ zu Methoxymethyl $+\mathrm{O}, \mathrm{T}=5^{\circ} \mathrm{C}$

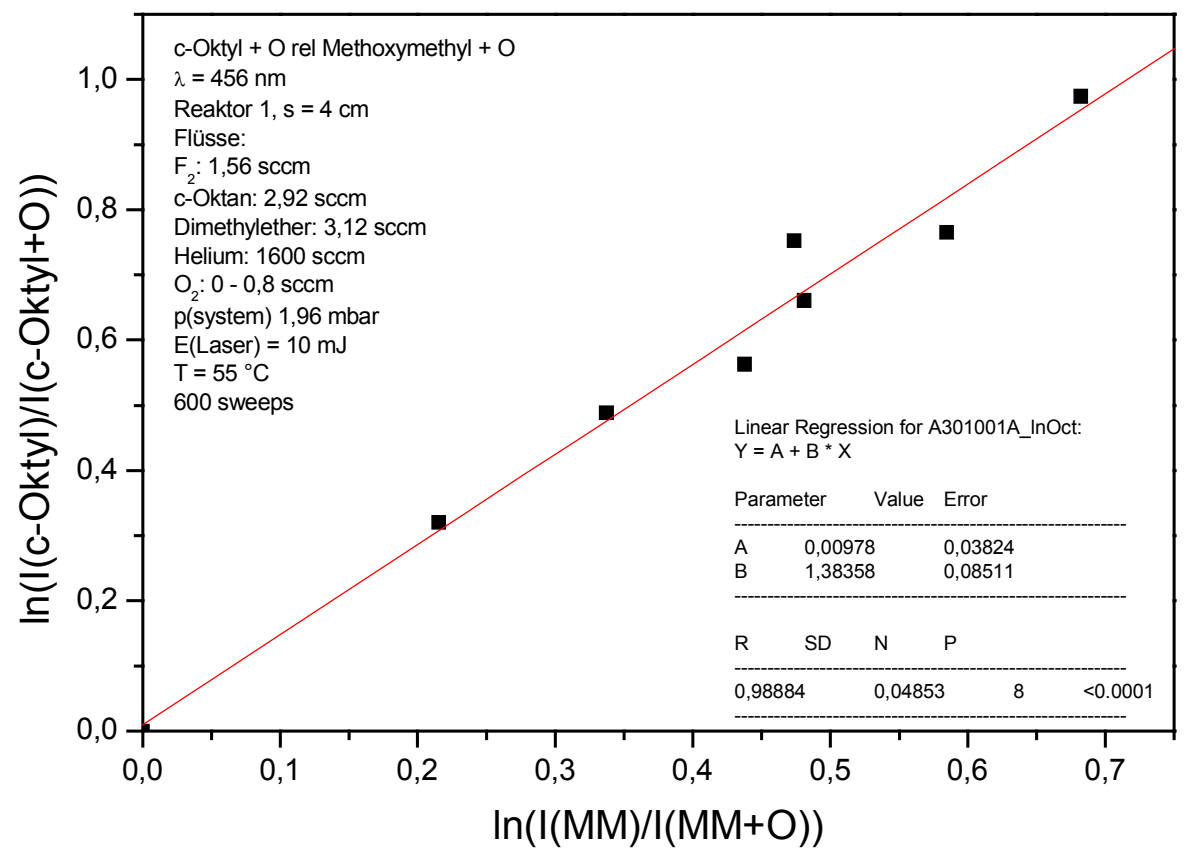

Abbildung 125: c-Oktyl $+\mathrm{O}$ relativ zu Methoxymethyl $+\mathrm{O}, \mathrm{T}=55^{\circ} \mathrm{C}$ 


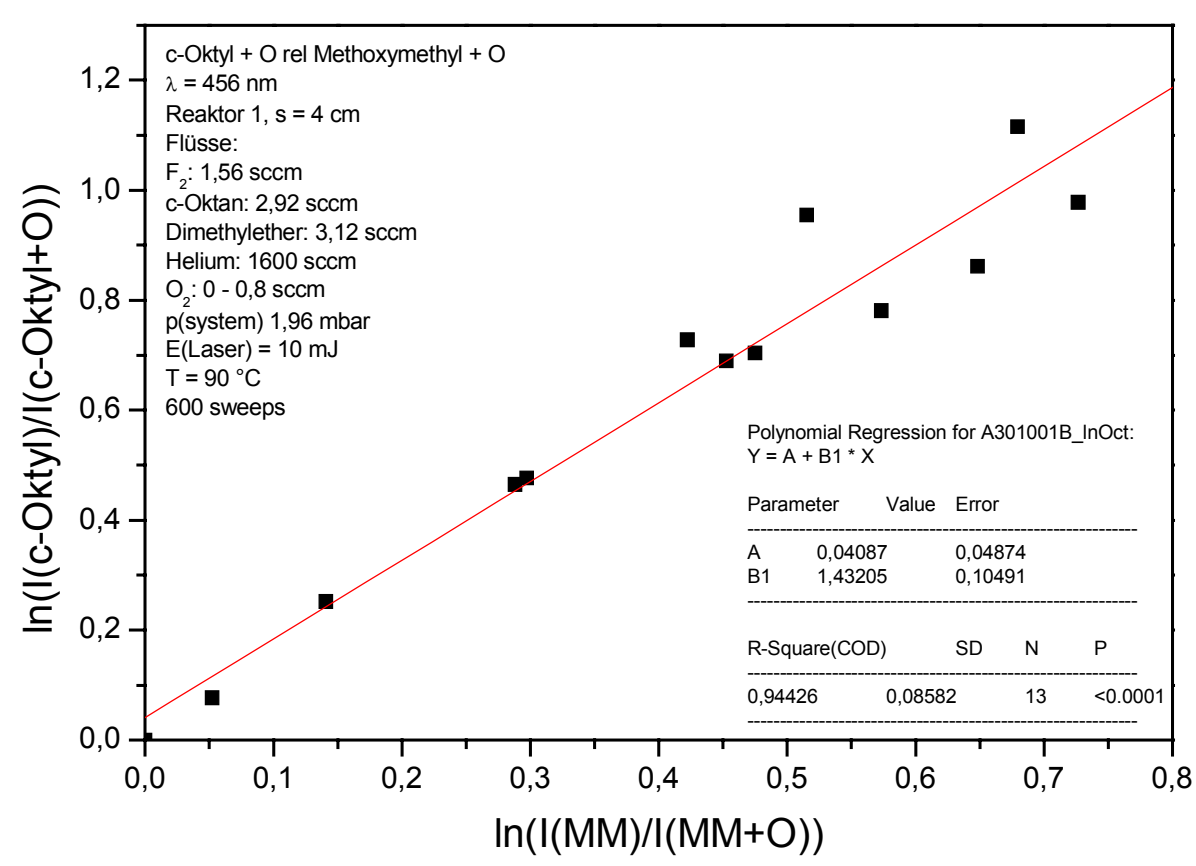

Abbildung 126: c-Oktyl $+\mathrm{O}$ relativ zu Methoxymethyl $+\mathrm{O}, \mathrm{T}=90^{\circ} \mathrm{C}$

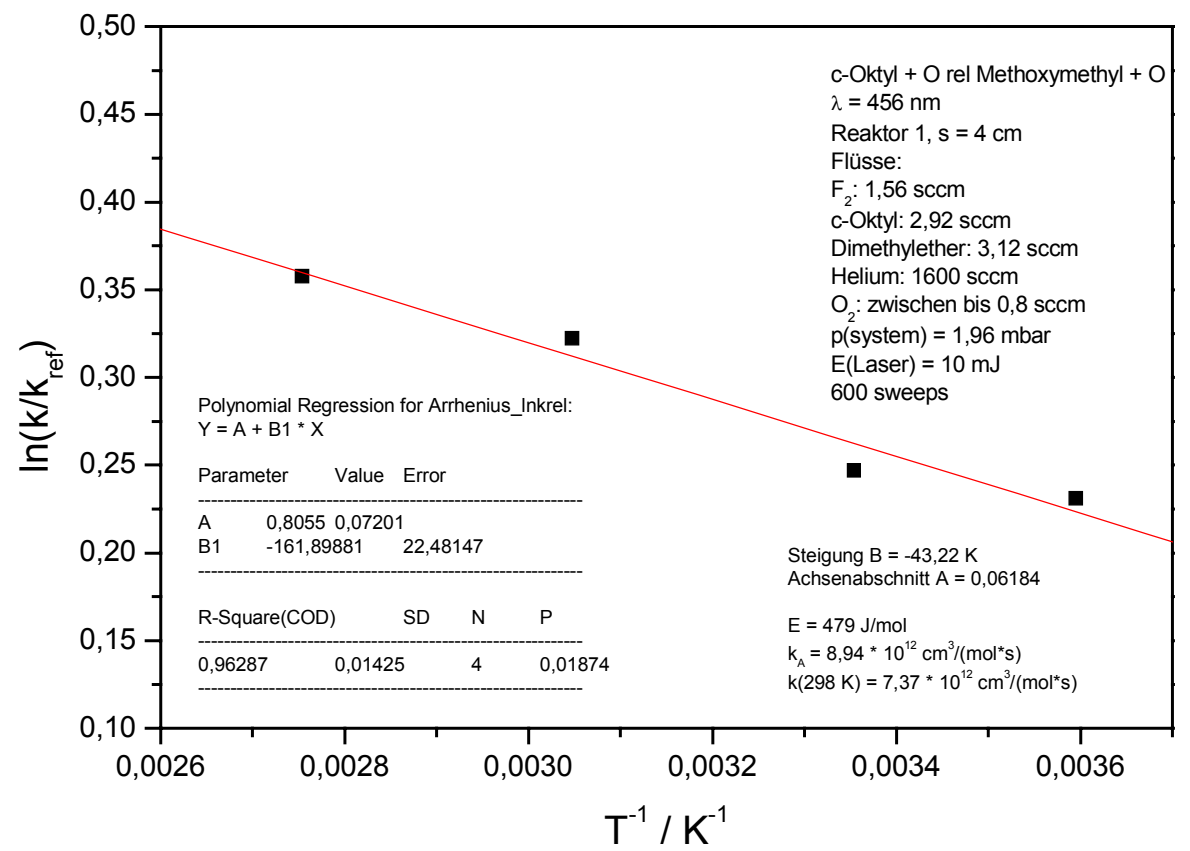

Abbildung 127: c-Oktyl + O relativ zu Methoxymethyl + O, Arrheniusauftragung

Aus der Arrheniusauftragung ergibt sich:

$$
\begin{gathered}
\mathrm{k}=(3,42 \pm 0,30) \cdot 10^{14} \exp \left\{(-1726 \pm 240) /(\mathrm{RT}) \mathrm{J} \cdot \mathrm{mol}^{-1}\right\} \mathrm{cm}^{3} \cdot \mathrm{mol}^{-1} \cdot \mathrm{s}^{-1} \\
\mathrm{k}^{298}=(1,70 \pm 0,4) \cdot 10^{14} \mathrm{~cm}^{3} \cdot \mathrm{mol}^{-1} \cdot \mathrm{s}^{-1}
\end{gathered}
$$


Die in diese Werte eingegangene Geschwindigkeitskonstante der Referenzreaktion wurde entsprechend der Ausführungen in den Abschnitten 3.3.2 und 3.3.9 um den Faktor 0,85 nach unten korrigiert.

In der Literatur finden sich keine Angaben über die Geschwindigkeit der Reaktion c-Oktyl + O.

\subsubsection{Cyclopentadienyl + O}

Produkte:

Diskussion: Im Rahmen der vorliegenden Arbeit wurden keine Produktuntersuchungen vorgenommen. In der eigenen Arbeitsgruppe wurde die Reaktion Cyclopentadienyl + O von Buth mit Versuchsanordnung B (EI-MS) untersucht [BUT93]. Es wurde folgender Mechanismus vorgeschlagen:
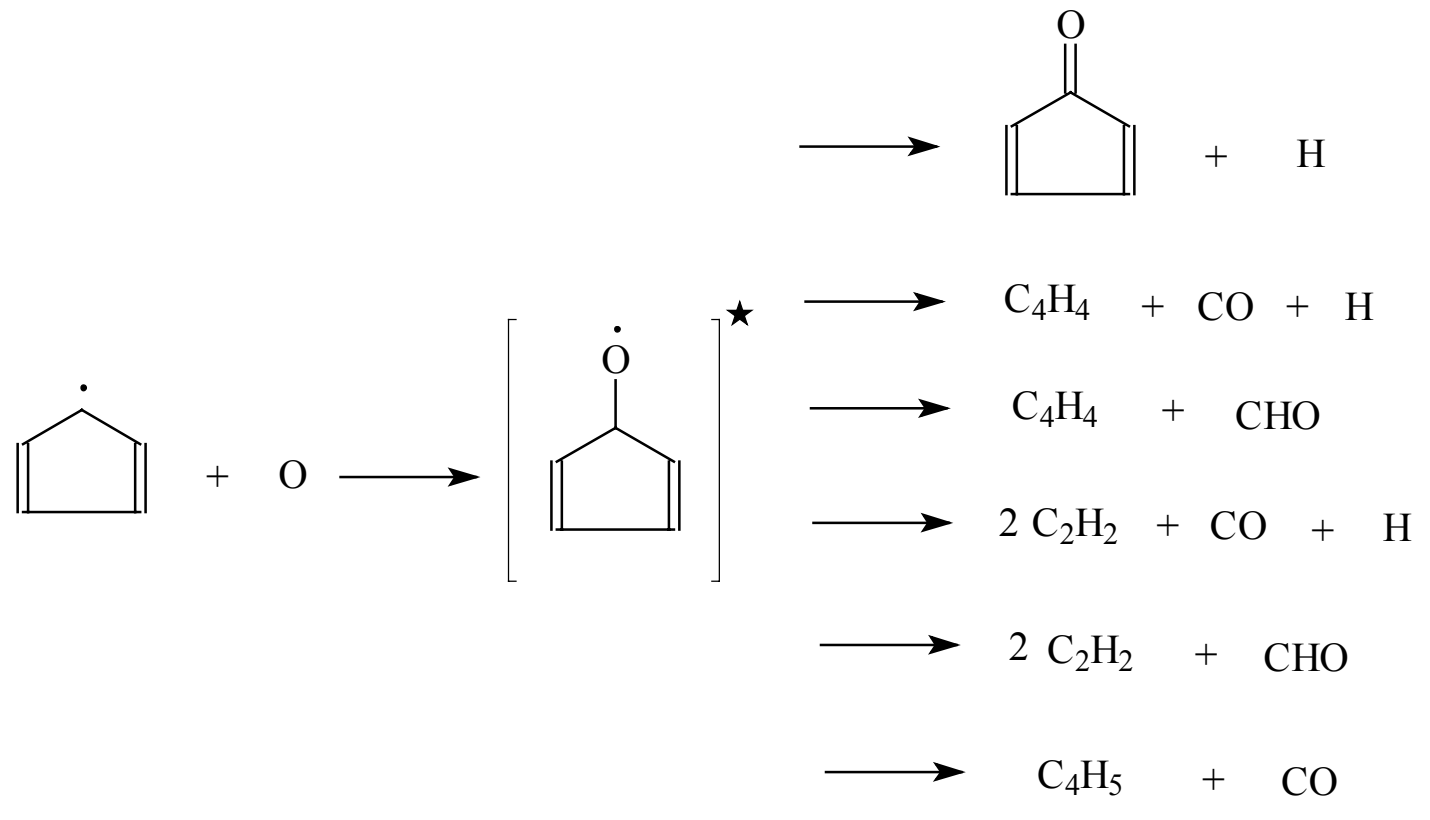

Die Reaktionsenthalpien werden wie folgt angegeben: $\Delta_{\mathrm{R}} \mathrm{H}^{0}{ }_{298 \mathrm{~K}} /(\mathrm{kJ} / \mathrm{mol})$ : -321 (a), -95 (b), -154 (c), 54 (d), -6 (e), -318 (f). Die Zuordnung der Massensignale zu den Produkten ist aufgrund beträchtlicher Überlagerungen und reichhaltiger Fragmentierungsmuster nicht eindeutig. Neben der Reaktion Cyclopentadienyl + O existieren noch zwei weitere konkurrierende Radikal-O-AtomReaktionen, nämlich $\mathrm{C}_{5} \mathrm{H}_{5} \mathrm{~F}+\mathrm{O}$ und $\mathrm{C}_{5} \mathrm{H}_{6} \mathrm{~F}+\mathrm{O}$, welche sich aus der Radikalerzeugungsreaktion Cyclopentadien + F ergeben (vgl. Abschnitt 3.1.13). Auch der Radikalvorläufer Cyclopentadien reagiert schnell mit O-Atomen: $\mathrm{k}^{298 \mathrm{~K}}=2,9 \cdot 10^{11} \mathrm{~cm}^{3} \cdot \mathrm{mol}^{-1} \cdot \mathrm{s}^{-1}$ [BUT93], was irrtümlich Produkte der Reaktion Cyclopentadienyl $+\mathrm{O}$ anzeigen könnte. Es wurden Anzeichen für $\mathrm{C}_{2} \mathrm{H}_{2}(\mathrm{~m} / \mathrm{z}=26)$ nach (d) oder (e), $\mathrm{C}_{4} \mathrm{H}_{4}(\mathrm{~m} / \mathrm{z}=54)$ nach (b) oder (c) und in geringem Umfang für c-Pentadienon 
$(\mathrm{m} / \mathrm{z}=80)$ nach (a) gefunden. Hochauflösende Messungen bei $\mathrm{m} / \mathrm{z}=28$ ergaben als Produkt CO, jedoch nicht $\mathrm{C}_{2} \mathrm{H}_{4}$.

\section{$\underline{\text { Reaktionsgeschwindigkeit: }}$}

Messergebnisse: Die Geschwindigkeit der Reaktion c-Pentadienyl + O wurde bei Zimmertemperatur relativ zur Reaktion Methoxymethyl + O mit Versuchsanordnung A (REMPI) bei $\lambda=424,87 \mathrm{~nm}$ bestimmt. Die Radikale wurden gemäß Abschnitt 3.1 .13 und 3.1 .14 erzeugt und auf ihren Muttermassen $\mathrm{m} / \mathrm{z}=65$ und $\mathrm{m} / \mathrm{z}=45$ nachgewiesen. Zur Bestimmung des Geschwindigkeitskoeffizienten wurden die Signalintensitäten bei An- und Abwesenheit von Sauerstoffatomen gemessen. Durch Variation der Sauerstoffkonzentration wurden verschiedene Umsätze erzielt. Die folgende Abbildung zeigt in doppeltlogarithmischer Auftragung die Messpunkte. Die zugehörigen Werte finden sich in Abschnitt 5 (Messtabellen). Die gewählten Versuchsbedingungen sind dem Text in der Abbildung oder bei den Messtabellen zu entnehmen.

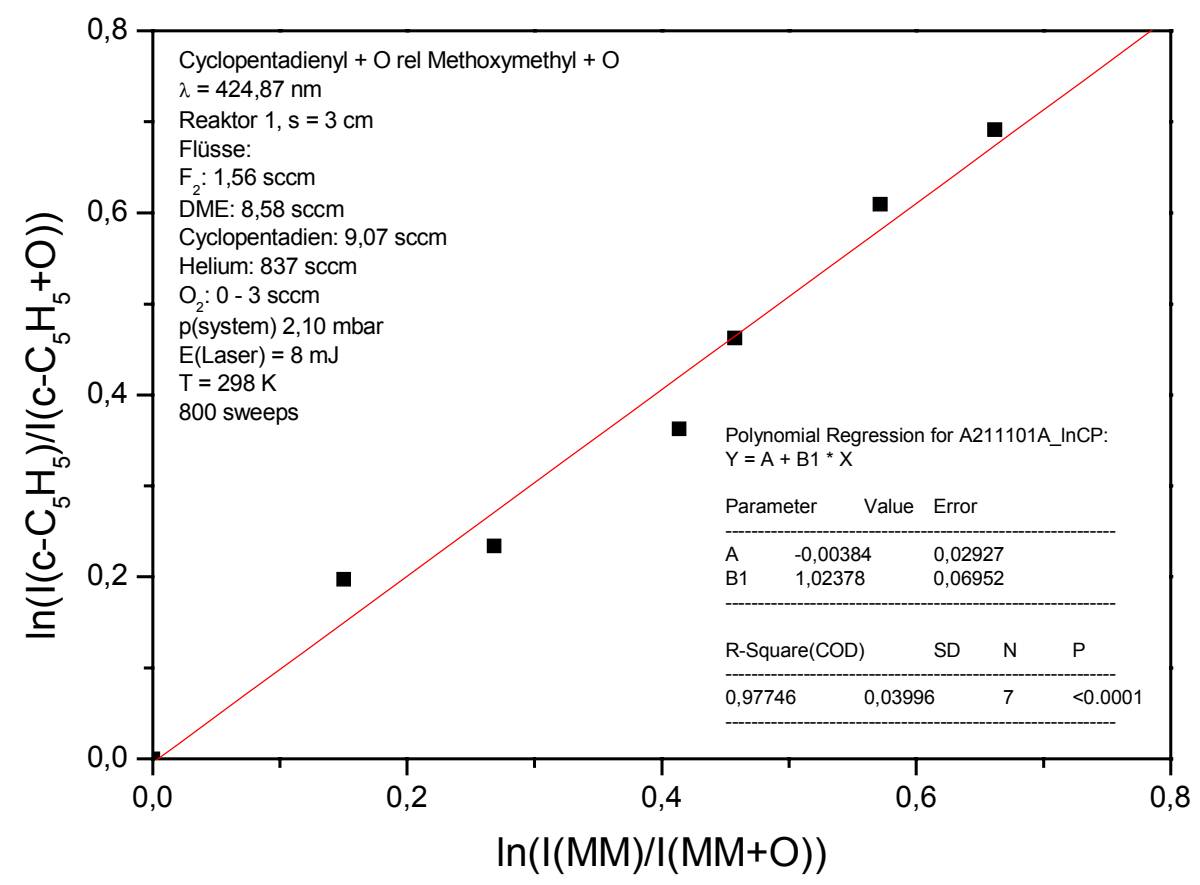

Abbildung 128: Cyclopentadienyl $+\mathrm{O}$ relativ zu Methoxymethyl $+\mathrm{O}, \mathrm{T}=25^{\circ} \mathrm{C}$

Aus der Auftragung ergibt sich:

$$
\mathrm{k}_{\text {rel }}=\mathrm{k}\left(\mathrm{c}-\mathrm{C}_{5} \mathrm{H}_{5}+\mathrm{O}\right) / \mathrm{k}\left(\mathrm{CH}_{3} \mathrm{OCH}_{2}+\mathrm{O}\right)=1,02 \pm 0,07
$$

Mit der in Abschnitt 3.3.2 erläuterten Geschwindigkeitskonstante $k_{\text {ref }}=1,31 \cdot 10^{14} \mathrm{~cm}^{3} /(\mathrm{mol} \cdot \mathrm{s})$ für die Referenzreaktion Methoxymethyl + O erhält man:

$$
\mathrm{k}^{298 \mathrm{~K}}\left(\mathrm{c}-\mathrm{C}_{5} \mathrm{H}_{5}+\mathrm{O}\right)=(1,34 \pm 0,09) \cdot 10^{14} \mathrm{~cm}^{3} /(\mathrm{mol} \cdot \mathrm{s}) \text {. }
$$


Diskussion: Die Geschwindigkeit der Reaktion c-Pentadienyl + O wurde bereits innerhalb der eigenen Arbeitsgruppe mit Versuchsanordnung B (EI-MS) von Buth relativ zur Reaktion $\mathrm{CH}_{3}+\mathrm{O}$ bestimmt [BUT93]. Die zugehörige Konstante wird mit $\mathrm{k}^{298 \mathrm{~K}}=\left(1,4 \cdot 10^{14} \pm 0,2\right) \mathrm{cm}^{3} /(\mathrm{mol} \cdot \mathrm{s})$ angegeben. Als Referenzgeschwindigkeitskoeffizient wurde der von Gutman et al. ermittelte Wert $\mathrm{k}\left(\mathrm{CH}_{3}+\mathrm{O}\right)=8,43 \cdot 10^{13} \mathrm{~cm}^{3} /(\mathrm{mol} \cdot \mathrm{s})$ [SLA87] verwendet. Legt man den in dieser Arbeit ermittelten Wert von $\mathrm{k}_{\text {ref }}=6,14 \cdot 10^{13} \mathrm{~cm}^{3} /(\mathrm{mol} \cdot \mathrm{s})$ zugrunde, so ergibt sich ein Wert von $\mathrm{k}\left(\mathrm{c}-\mathrm{C}_{5} \mathrm{H}_{5}+\mathrm{O}\right)=$ $1,02 \cdot 10^{14} \mathrm{~cm}^{3} /(\mathrm{mol} \cdot \mathrm{s})$, welcher um den Faktor 1,3 niedriger als der hier ermittelte Wert ist.

Bei Untersuchungen von Frank, Herzler, Just und Wahl zur Phenyloxidation mit einer Stoßwellenanordnung $(\mathrm{T}=900-1800 \mathrm{~K}, \mathrm{p}=1,3-1,5$ bar) und Atom-Resonanz-AbsorptionsSpektroskopie $(A R A S)$ als Nachweis gehen die Autoren nur von der Existenz des Reaktionskanals (a) aus [FRA94]. Die Untersuchungen von Buth zur Reaktion Cyclopentadienyl + O scheinen den Autoren nicht bekannt gewesen zu sein. Es wurde ein Reaktionssystem betrachtet, in dem die genannte Reaktion als Folgereaktion vorkommt. Dabei wurden die Signale von O-, H- und I-Atomen und von CO (mittels MRAS) im Wellenlängenbereich 121-165 nm verfolgt und die kinetischen Daten des angenommenen Reaktionssystems per Simulation angepasst. Die beste Übereinstimmung wurde für die Reaktion c-Pentadienyl $+\mathrm{O}$ erzielt, indem der Geschwindigkeitskoeffizient $\mathrm{k}^{298 \mathrm{~K}}\left(\mathrm{c}-\mathrm{C}_{5} \mathrm{H}_{5}+\mathrm{O}\right)=7 \cdot 10^{13} \mathrm{~cm}^{3} \cdot \mathrm{mol}^{-1} \cdot \mathrm{s}^{-1}$ gesetzt wurde. Obwohl die Reaktionen von Kohlenwasserstoffradikalen mit O-Atomen über einen weiten Bereich druck- und temperaturunabhängig sind, bleibt es dennoch fraglich, ob die eingestellten experimentellen Bedingungen vergleichbar mit den hier gewählten sind. Außerdem ist die verwendete Bestimmungsmethode wenig direkt. Die Komplexität des Reaktionssystems trägt ebenfalls zur Unsicherheit des Verfahrens bei.

\subsubsection{5 $\mathrm{CH}_{2} \mathrm{CCl}_{3}+\mathrm{O}$}

\section{Produkte:}

Messergebnisse: Die Reaktion $\mathrm{CH}_{2} \mathrm{CCl}_{3}+\mathrm{O}$ wurde mit Versuchsanordnung B (EI-MS) nach Produkten untersucht. Die Radikale wurden über die Reaktion $\mathrm{CH}_{3} \mathrm{CCl}_{3}+\mathrm{F}$ erzeugt. Bei der Interpretation der Daten ist zu berücksichtigen, dass diese Radikalbildungsreaktion weitere Reaktionskanäle aufweist (vgl. Abschnitt 3.2.1). Folgende Reaktionskanäle der Reaktion $\mathrm{CH}_{2} \mathrm{CCl}_{3}+\mathrm{O}$ sind denkbar: 


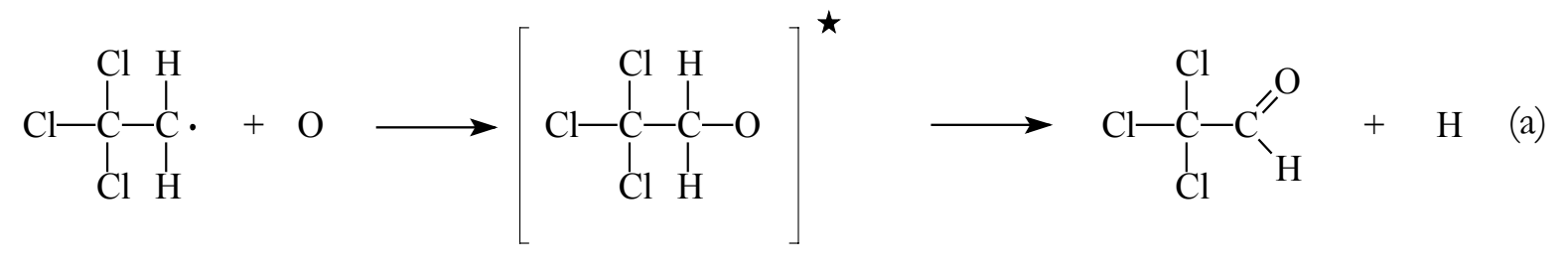

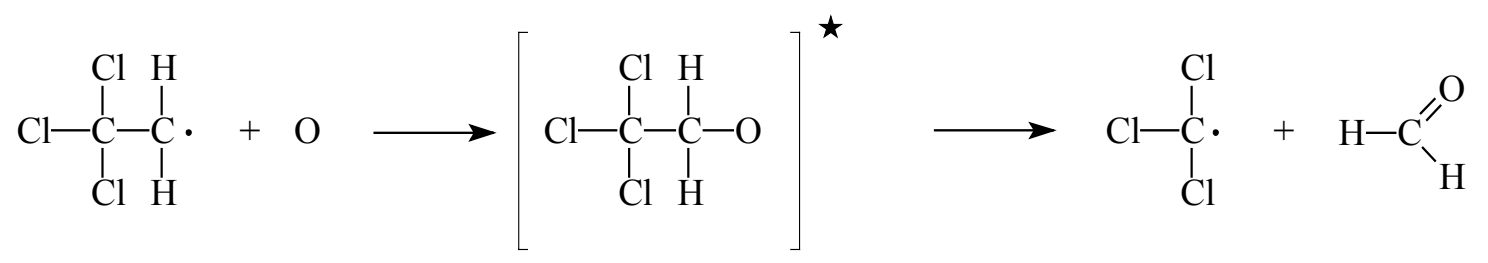<smiles>CC(C)C(Cl)(Cl)C(O)C1(C)CCCCC1C</smiles>

Die Gesamtreaktionsenthalpie des Kanals (a) beträgt -272,9 kJ/mol, die des Kanals (b) $-323,5 \mathrm{~kJ} / \mathrm{mol}$ und die des Kanals (c) -190,1 kJ/mol. Kanal (c) soll neben den hier skizzierten Verlauf über ein Addukt auch die direkte Abstraktion beinhalten. Die Ergebnisse der Produktuntersuchung sind in Messtabelle 2 abgelegt (vgl. Abschnitt 5). Die Messungen wurden, nachdem Übersichtsspektren in Form von Magnetstromscans aufgenommen waren, im Modus Einzelmassenzählung bei Ionisierungsenergie von IE $=29,5 \mathrm{eV}$ aufgenommen. Die Bedingungen wurden so eingestellt, dass sich, gemessen auf der Radikalmuttermasse $\mathrm{m} / \mathrm{z}=131$, ein Umsatz der Reaktion $\mathrm{CH}_{2} \mathrm{CCl}_{3}+\mathrm{O}$ von $65 \%$ ergab. Die Massen, die Radikalfragmenten zuzuordnen sind, zeigten ebenfalls Abnahmen in ähnlicher Größenordnung. Die folgende Tabelle gibt die Massen an, bei denen eine Zunahme der Intensität unter der Bedingung $+\mathrm{O} \mathrm{zu}$ beobachten war. Die angegebenen Anstiegsfaktoren sind abhängig von der Überlagerung der Massen durch verschiedene Substanzen und deren massenspektrometrischer Empfindlichkeit. Sie dienen lediglich der groben Orientierung. 


\begin{tabular}{|c|c|c|c|l|}
\hline $\mathrm{m} / \mathrm{z}$ & $\begin{array}{c}\mathrm{I}(\mathrm{KW}+\mathrm{F}-\mathrm{O}) \\
\text { /counts }\end{array}$ & $\begin{array}{c}\mathrm{I}(\mathrm{KW}+\mathrm{F}+\mathrm{O}) \\
\text { /counts }\end{array}$ & $\begin{array}{c}\text { Anstieg } \\
\text { Faktor } \\
\text { Fakt }\end{array}$ & Anmerkungen \\
\hline 150 & 371 & 387 & 1,04 & \\
\hline 148 & 346 & 1153 & 3,33 & \\
\hline 146 & 180 & 1143 & 6,35 & \\
\hline 120 & 1710 & 2290 & 1,34 & \\
\hline 111 & 819 & 3713 & 4,53 & \\
\hline 110 & 140 & 932 & 6,66 & \\
\hline 85 & 3613 & 4483 & 1,24 & \\
\hline 84 & 5910 & 12270 & 2,08 & \\
\hline 83 & 2460 & 4410 & 1,79 & \\
\hline 82 & 7933 & 17000 & 2,14 & \\
\hline 76 & 364 & 830 & 2,28 & \\
\hline 65 & 459 & 1917 & 4,18 & \\
\hline 47 & 2673 & 4670 & 1,74 & \\
\hline 44 & 3793 & 11830 & 3,12 & \\
\hline 42 & 983 & 1213 & 1,23 & \\
\hline 41 & 2570 & 2917 & 1,14 & \\
\hline 38 & 12600 & 18500 & 1,47 & \\
\hline 37 & 8430 & 20000 & 2,37 & \\
\hline 36 & 37830 & 56700 & 1,50 & \\
\hline 35 & 26200 & 62900 & 2,40 & \\
\hline 32 & 1730 & 6000 & 3,47 & \\
\hline 30 & 2593 & 4660 & 1,80 & \\
\hline 29 & 1767 & 6000 & 3,40 & \\
\hline 28 & 54530 & 77370 & 1,42 & stark überlagert durch $\mathrm{N}_{2}$-Untergrund \\
\hline 20 & 11170 & 13600 & 1,28 & \\
\hline 16 & 781 & 95070 & 121 & \\
\hline
\end{tabular}

Tabelle 5: Produktanalyse der Reaktion $\mathrm{CH}_{2} \mathrm{CCl}_{3}+\mathrm{O}$

Interpretation: Im folgenden sind Auszüge des Literaturmassenspektrums des als Produkt vermuteten Trichlorethanals gegeben ([STE90], IE $=70 \mathrm{eV}$ ). Die nicht aufgeführten Massen sind entweder überlagert oder wurden nicht in Einzelmassenzählung erfasst, meist mangels Signalstärke. Das vollständig Literaturmassenspektrum findet sich im Anhang. Die Intensitäten sind auf das Maximum (99,9\%) standardisiert.

$\mathrm{CHOCCl}_{3}, \mathrm{M}=146 \mathrm{~g} / \mathrm{mol}$

\begin{tabular}{|l|c|c|c|c|c|c|c|c|c|c|c|c|c|}
\hline $\mathbf{m} / \mathbf{z}$ & 150 & 148 & 146 & 122 & 121 & 120 & 111 & 110 & 85 & 84 & 83 & 82 & 47 \\
\hline $\mathbf{I} / \mathbf{~} \mathbf{0}$ & 1,5 & 4,4 & 4,6 & 4,5 & 4,3 & 14,0 & 35,3 & 9,8 & 20,6 & 66,4 & 31,9 & 99,9 & 18,3 \\
\hline
\end{tabular}

\begin{tabular}{|l|c|c|c|c|c|}
\hline $\mathbf{m} / \mathbf{z}$ & 37 & 36 & 35 & 29 & 28 \\
\hline $\mathbf{I} / \mathbf{~} \mathbf{0}$ & 1,9 & 1,8 & 5,5 & 31,0 & 3,1 \\
\hline
\end{tabular}


Der Vergleich zwischen den Messwerten und dem Literaturmassenspektrum ergibt, dass Trichlorethanal als Produkt eindeutig nachgewiesen wird. Dies wird besonders deutlich beim Anstieg auf der Molekülmasse des $\mathrm{CHOCCl}_{3}, \mathrm{~m} / \mathrm{z}=146$, und auf den intensivsten Massen 84 und 82. Der vermutete Reaktionskanal (a) ist somit bewiesen.

Nicht mit Kanal (a) erklärt werden kann der Anstieg der Signalstärke bei m/z = 76, 65, 44, 42, 41, 38,30 und 20 .

Der Anstieg bei m/z = 30 kann mit der Entstehung von Formaldehyd begründet werden, welches sein Hauptfragment bei nicht zu niedrigen Ionisierungsenergien bei $\mathrm{m} / \mathrm{z}=29$ hat. Der Anstieg bei $\mathrm{m} / \mathrm{z}=29$ rührt demnach also nicht nur von Kanal (a) her. Kanal (b) muss somit ebenfalls als relevanter Reaktionskanal angenommen werden.

Für die Existenz der Elimination (Kanal (c)) wurden keine Anzeichen gefunden. Literatur zu Produktuntersuchungen der Reaktion $\mathrm{CH}_{2} \mathrm{CCl}_{3}+\mathrm{O}$ liegt nicht vor.

\section{$\underline{\text { Reaktionsgeschwindigkeit: }}$}

Messergebnisse: Die Geschwindigkeit der Reaktion $\mathrm{CH}_{2} \mathrm{CCl}_{3}+\mathrm{O}$ wurde bei Zimmertemperatur relativ zur Reaktion $\mathrm{CHCl}_{2}+\mathrm{O}$ mit Versuchsanordnung B (EI-MS) bei einer Ionisierungsenergie von IE $=29,5 \mathrm{eV}$ bestimmt. Das Trichlorethylradikal wurden gemäß Abschnitt 3.1.10 erzeugt und auf seiner Muttermasse $\mathrm{m} / \mathrm{z}=131$ nachgewiesen. Das Dichlormethylradikal wurde wie bei [HOL97] beschrieben erzeugt und nachgewiesen. Zur Bestimmung des Geschwindigkeitskoeffizienten wurden die Signalintensitäten bei An- und Abwesenheit von Sauerstoffatomen gemessen. Durch Variation der Sauerstoffkonzentration wurden verschiedene Umsätze erzielt. Die folgende Abbildung zeigt in doppeltlogarithmischer Auftragung die Messpunkte. Die zugehörigen Werte finden sich in Abschnitt 5 (Messtabellen). Die gewählten Versuchsbedingungen sind dem Text in der Abbildung oder bei den Messtabellen zu entnehmen. 


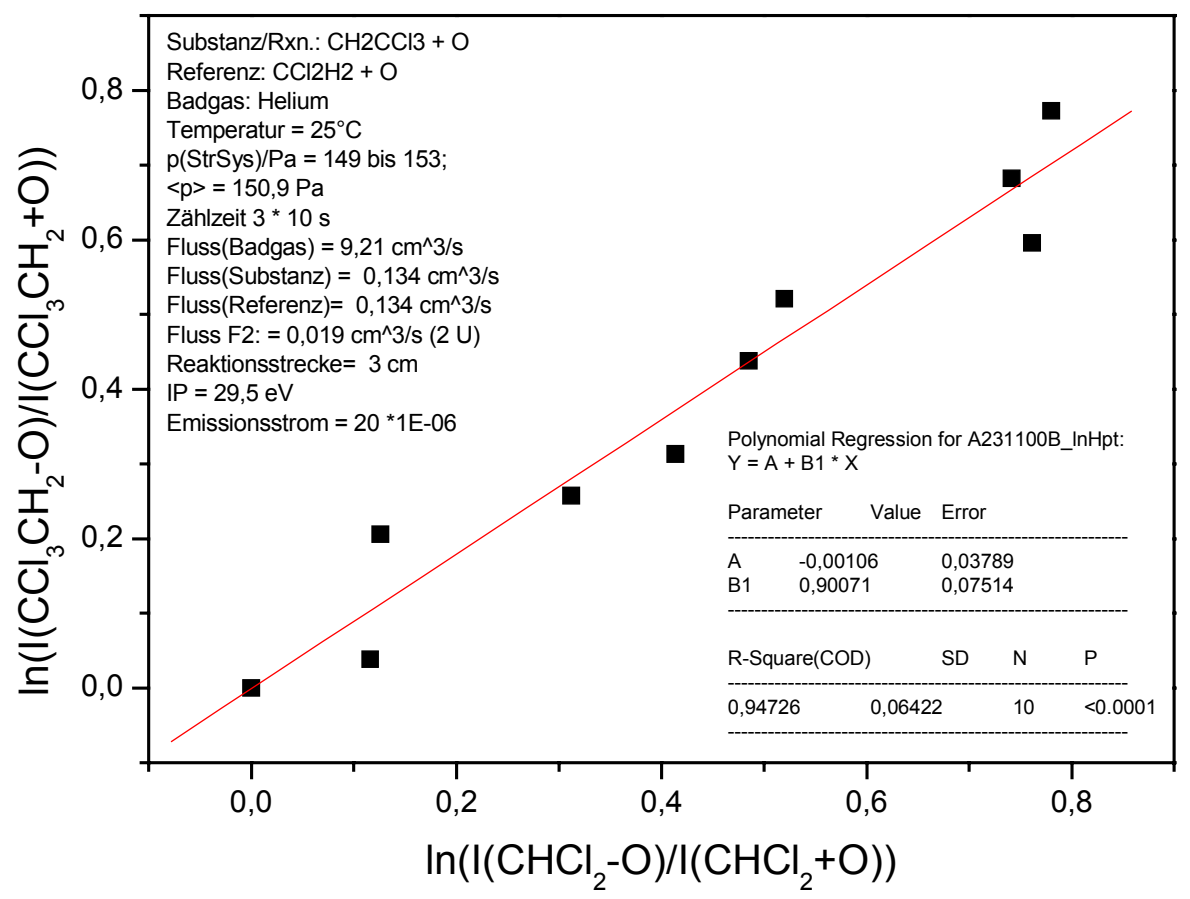

Abbildung 129: $\mathrm{CH}_{2} \mathrm{CCl}_{3}+\mathrm{O}$ relativ zu $\mathrm{CHCl}_{2}+\mathrm{O}, \mathrm{T}=25^{\circ} \mathrm{C}$

Aus der Auftragung ergibt sich:

$$
\mathrm{k}_{\mathrm{rel}}=\mathrm{k}\left(\mathrm{CH}_{2} \mathrm{CCl}_{3}+\mathrm{O}\right) / \mathrm{k}\left(\mathrm{CHCl}_{2}+\mathrm{O}\right)=0,90 \pm 0,08
$$

Bei [HOL97] ist für die Referenzreaktion $\mathrm{CHCl}_{2}+\mathrm{O}$ eine Geschwindigkeitskonstante von $\mathrm{k}^{298 \mathrm{~K}}=4,3 \cdot 10^{13} \mathrm{~cm}^{3} /(\mathrm{mol} \cdot \mathrm{s})$ angegeben. Dieser Wert wurde aus einer Relativmessung $\mathrm{zu} \mathrm{CH}_{3}+\mathrm{O}$ gewonnen, als Referenzgeschwindigkeitskoeffizient wurde der von Gutman et al. angegebene Wert $\mathrm{k}\left(\right.$ Methyl+O) $=8,43 \cdot 10^{13} \mathrm{~cm}^{3} /(\mathrm{mol} \cdot \mathrm{s})$ [SLA87] verwendet. Legt man den in dieser Arbeit ermittelten Wert von $\mathrm{k}\left(\mathrm{CH}_{3}+\mathrm{O}\right)=6,14 \cdot 10^{13} \mathrm{~cm}^{3} /(\mathrm{mol} \cdot \mathrm{s})$ zugrunde, so ergibt sich der Wert $\mathrm{k}\left(\mathrm{CHCl}_{2}+\mathrm{O}\right)=3,13 \cdot 10^{13} \mathrm{~cm}^{3} /(\mathrm{mol} \cdot \mathrm{s})$ und damit für die betrachtete Reaktion:

$$
\mathrm{k}^{298 \mathrm{~K}}\left(\mathrm{CH}_{2} \mathrm{CCl}_{3}+\mathrm{O}\right)=(2,82 \pm 0,3) \cdot 10^{13} \mathrm{~cm}^{3} /(\mathrm{mol} \cdot \mathrm{s})
$$

\section{Temperaturabhängigkeit:}

Messergebnisse: Die Temperaturabhängigkeit der Reaktion $\mathrm{CH}_{2} \mathrm{CCl}_{3}+\mathrm{O}$ wurde im Intervall $-20{ }^{\circ} \mathrm{C}$ bis $+85^{\circ} \mathrm{C}$ nach dem selben Verfahren untersucht. Die Temperierung erfolgte mit der in Abschnitt 2.1.2 beschriebenen Methode für Reaktor 1. Die nachfolgenden Abbildungen zeigen die Ergebnisse. Die zugehörigen Werte finden sich in Abschnitt 5 (Messtabellen). Die gewählten Versuchsbedingungen sind dem Text in der Abbildung oder bei den Messtabellen zu entnehmen. 


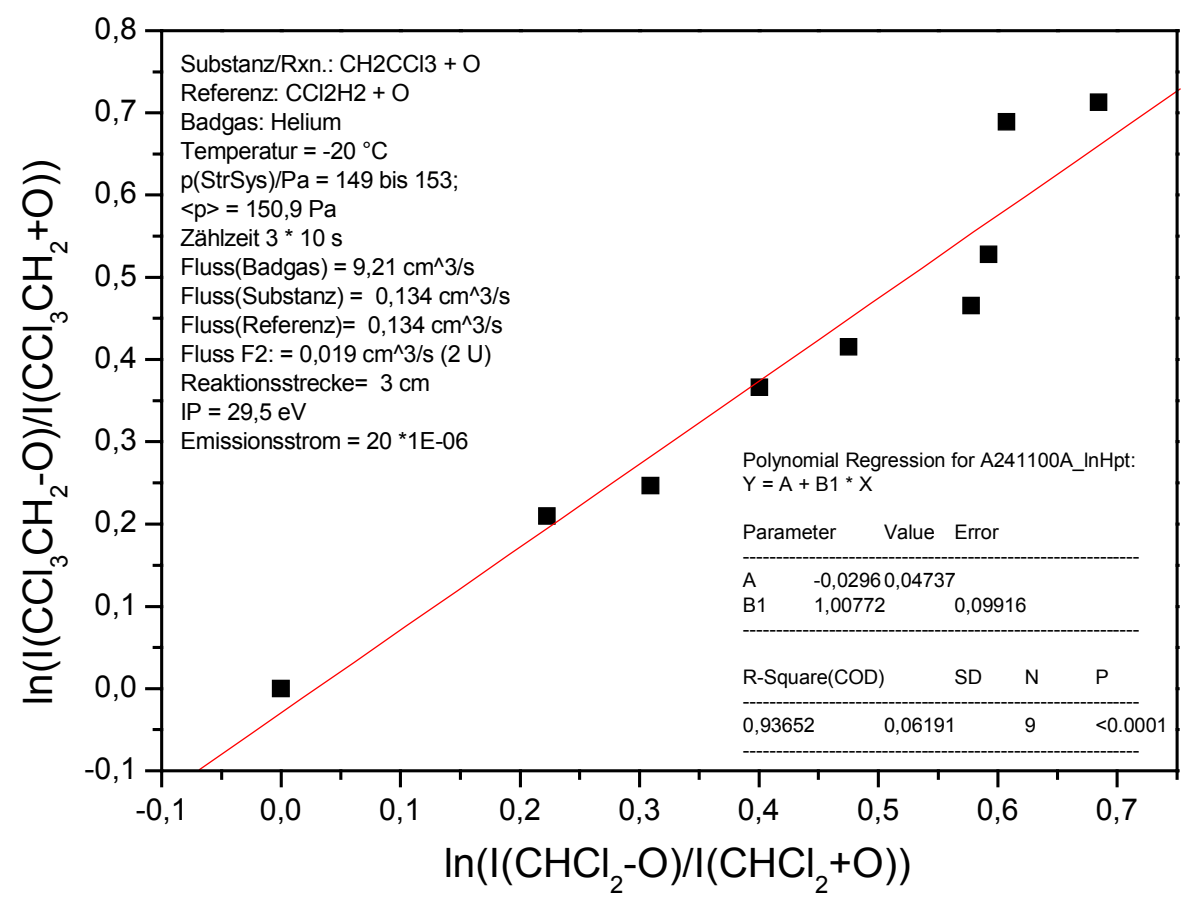

Abbildung 130: $\mathrm{CH}_{2} \mathrm{CCl}_{3}+\mathrm{O}$ relativ zu $\mathrm{CHCl}_{2}+\mathrm{O}, \mathrm{T}=-20^{\circ} \mathrm{C}$

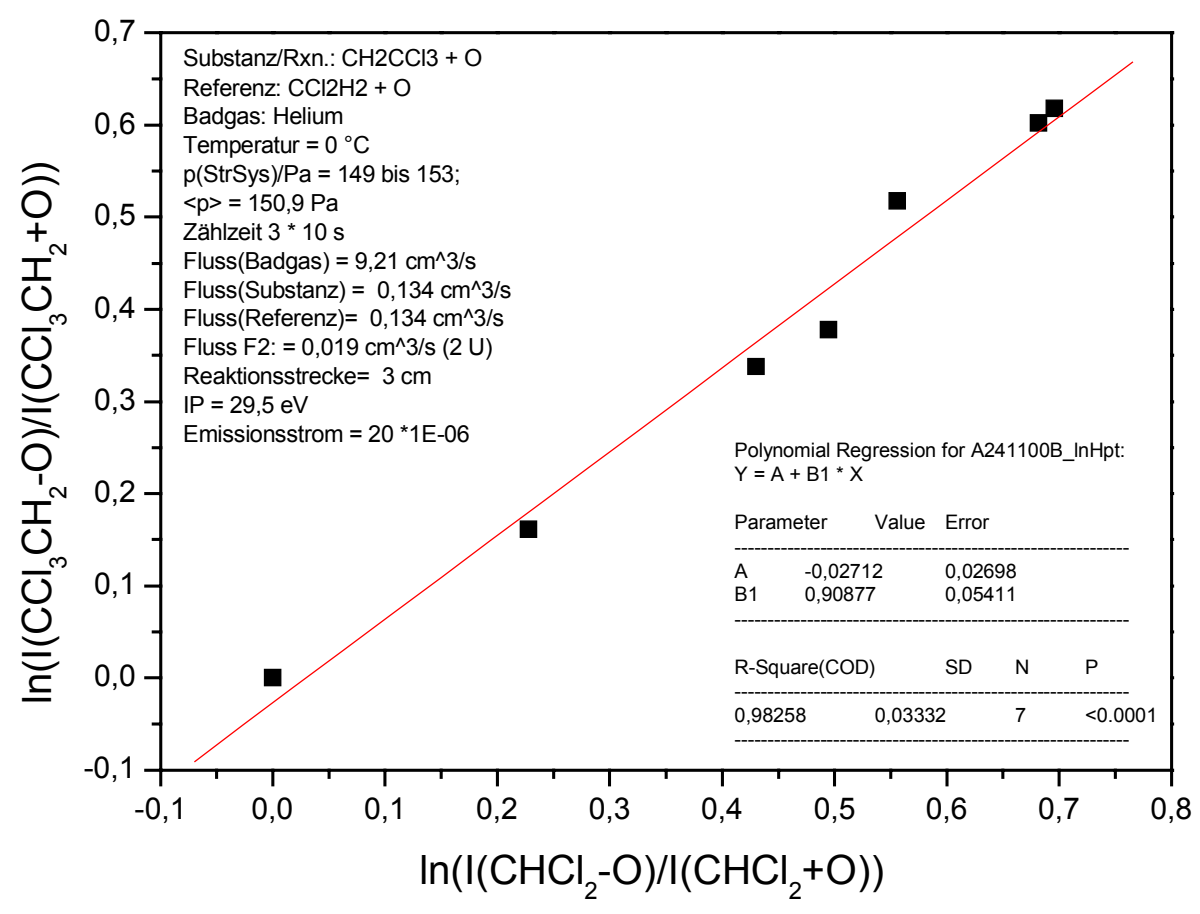

Abbildung 131: $\mathrm{CH}_{2} \mathrm{CCl}_{3}+\mathrm{O}$ relativ zu $\mathrm{CHCl}_{2}+\mathrm{O}, \mathrm{T}=0{ }^{\circ} \mathrm{C}$ 


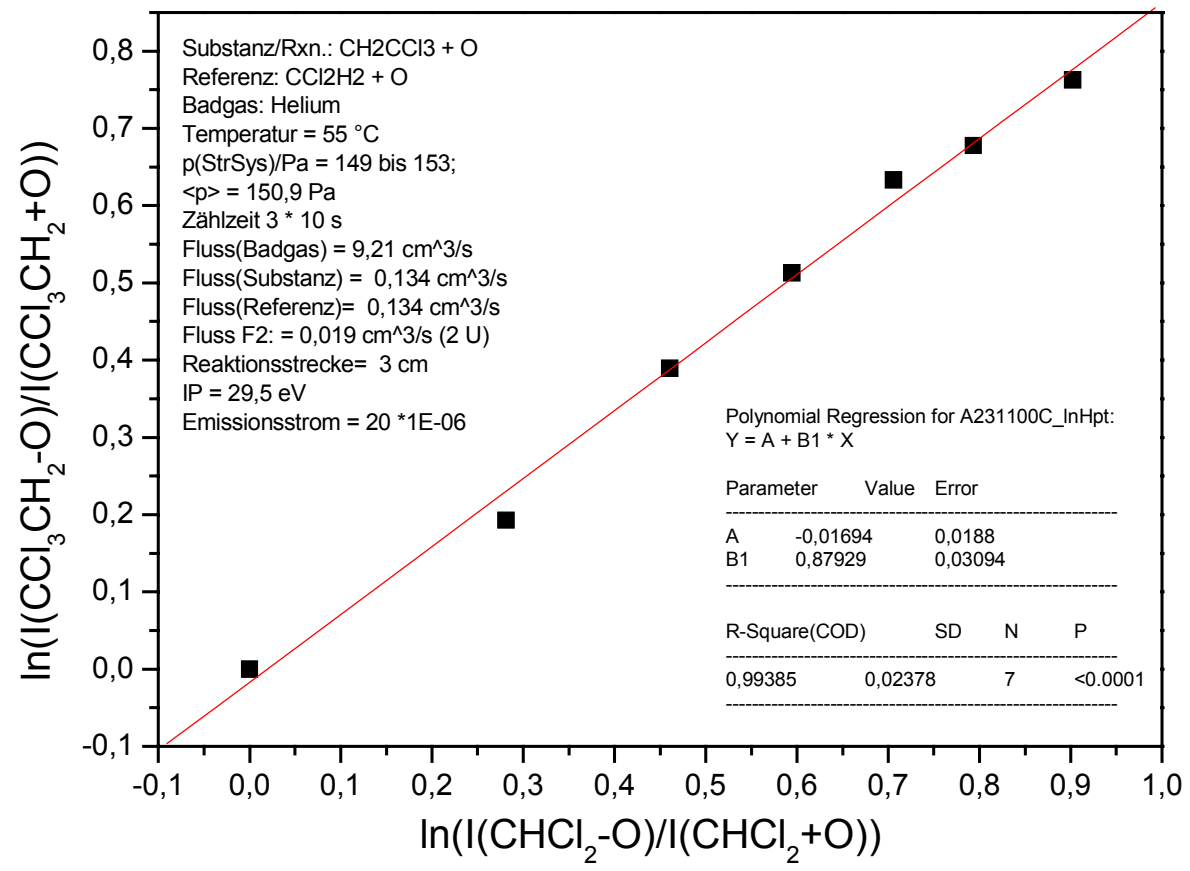

Abbildung 132: $\mathrm{CH}_{2} \mathrm{CCl}_{3}+\mathrm{O}$ relativ zu $\mathrm{CHCl}_{2}+\mathrm{O}, \mathrm{T}=55^{\circ} \mathrm{C}$

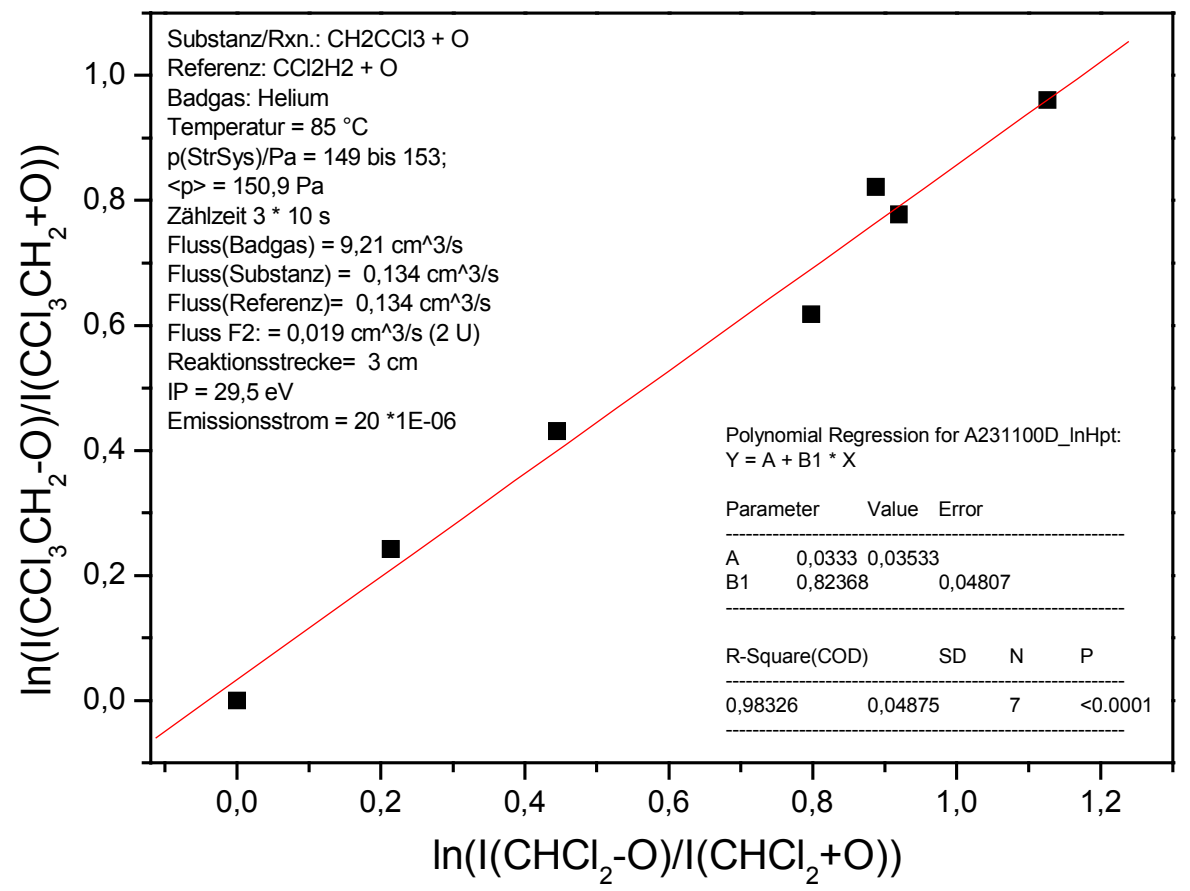

Abbildung 133: $\mathrm{CH}_{2} \mathrm{CCl}_{3}+\mathrm{O}$ relativ zu $\mathrm{CHCl}_{2}+\mathrm{O}, \mathrm{T}=85^{\circ} \mathrm{C}$ 


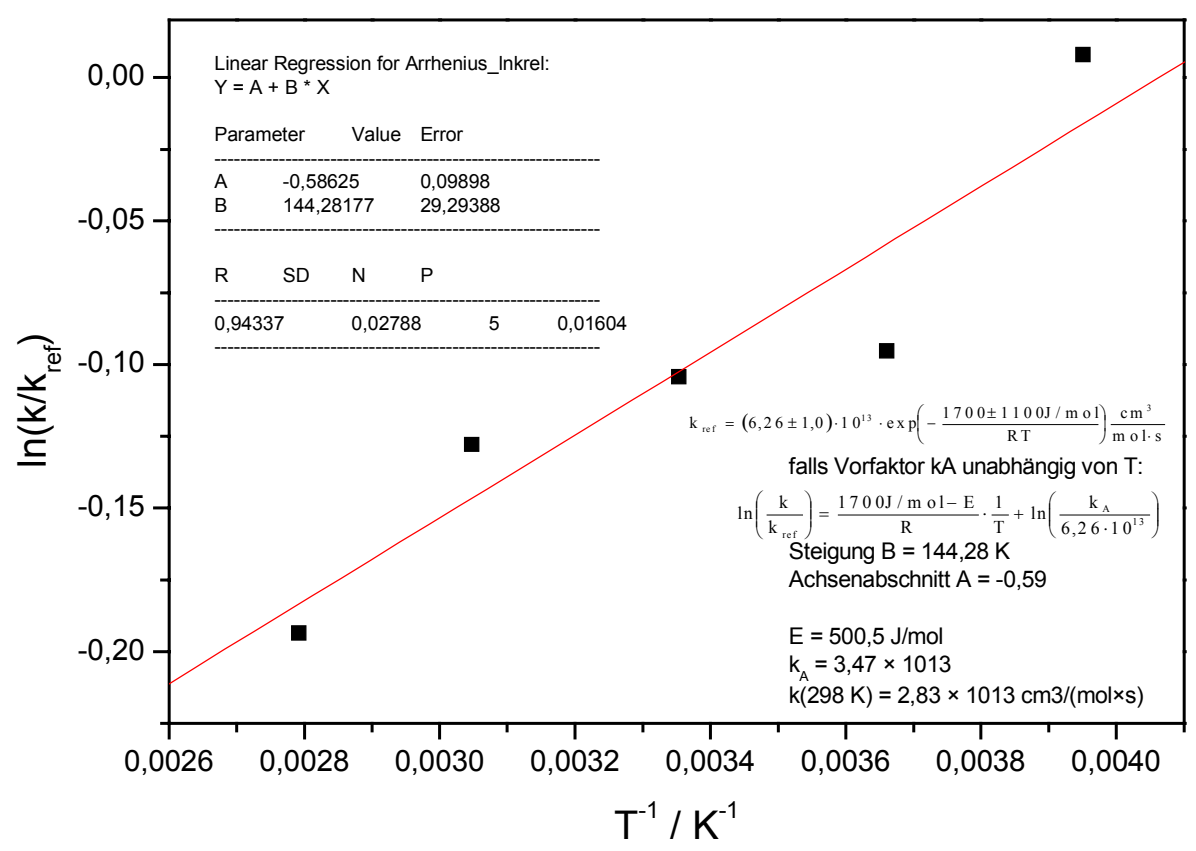

\section{Abbildung 134: $\mathrm{CH}_{2} \mathrm{CCl}_{3}+\mathrm{O}$ relativ zu $\mathrm{CHCl}_{2}+\mathrm{O}$, Arrheniusauftragung}

Aus der Arrheniusauftragung ergibt sich:

$$
\begin{gathered}
\mathrm{k}=(3,47 \pm 0,30) \cdot 10^{13} \exp \left\{(-501 \pm 246) /(\mathrm{RT}) \mathrm{J} \cdot \mathrm{mol}^{-1}\right\} \mathrm{cm}^{3} \cdot \mathrm{mol}^{-1} \cdot \mathrm{s}^{-1} \\
\mathrm{k}^{298}=(2,83 \pm 0,5) \cdot 10^{13} \mathrm{~cm}^{3} \cdot \mathrm{mol}^{-1} \cdot \mathrm{s}^{-1}
\end{gathered}
$$

Die in diesen Werten eingegangene Geschwindigkeitskonstante der Referenzreaktion wurde entsprechend den Ausführungen oben und im Abschnitt 3.3.4 nach unten korrigiert.

Diskussion: In der Literatur finden sich keine Angaben über die Geschwindigkeit der Reaktion $\mathrm{CH}_{2} \mathrm{CCl}_{3}+\mathrm{O}$. Auffällig ist die im Vergleich zu anderen Reaktionen von Kohlenwasserstoffradikalen mit O-Atomen kleine Geschwindigkeitskonstante - im Vergleich zu Ethyl + O ist sie um den Faktor 5 kleiner. Ein solches Verhalten ist für stark chlorsubstituierte Radikale aber nicht ungewöhnlich. Auch der Geschwindigkeitskoeffizient der Referenzreaktion $\mathrm{CHCl}_{3}+\mathrm{O}$ ist im Vergleich zu Methyl + O um den Faktor 2 kleiner. Ein möglicher Wegfall der direkten Abstraktion, also des oben erläuterten nicht über ein Addukt verlaufenden Kanals (c), könnte eine der Ursachen für die vergleichsweise kleine Geschwindigkeitskonstante der Reaktion $\mathrm{CH}_{2} \mathrm{CCl}_{3}+\mathrm{O}$ sein. Es sollte auch nicht vergessen werden, dass die mehrfache Substitution mit Chloratomen für eine ganz andere Elektronendichteverteilung im Molekül sorgt, was sich entscheidend für die Kinetik der Adduktbildung auswirken kann. 


\subsubsection{2-Chlor-2-Methyl-Propyl + O}

\section{Produkte:}

Diskussion: Es wurden keine Produktuntersuchungen vorgenommen. Folgende Reaktionskanäle erscheinen mechanistisch möglich:

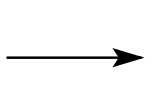

$\mathrm{CH}_{3}-\underset{\stackrel{\mathrm{C}}{\mathrm{C}}-\stackrel{\mathrm{CH}}{\mathrm{C}}-\mathrm{Cl}}{\mathrm{C}}+\mathrm{O}$<smiles>C=C(C)CCCCCCCC(C)(Cl)C=O</smiles><smiles>CC(C)(Cl)C=O</smiles>

Es lag keine Literatur über Produktuntersuchungen der Reaktion iso- $\mathrm{C}_{4} \mathrm{H}_{8} \mathrm{Cl}+\mathrm{O}$ vor.

\section{$\underline{\text { Reaktionsgeschwindigkeit: }}$}

Messergebnisse: Die Geschwindigkeit der Reaktion iso- $\mathrm{C}_{4} \mathrm{H}_{8} \mathrm{Cl}+\mathrm{O}$ wurde bei Zimmertemperatur relativ zur Reaktion Methoxymethyl $+\mathrm{O}$ mit Versuchsanordnung A (REMPI) bei $\lambda=440 \mathrm{~nm}$ bestimmt. Die Radikale wurden gemäß Abschnitt 3.1.11 und 3.1.14 erzeugt und auf ihren Muttermassen $\mathrm{m} / \mathrm{z}=91$ und $\mathrm{m} / \mathrm{z}=45$ nachgewiesen. Zur Bestimmung des Geschwindigkeitskoeffizienten wurden die Signalintensitäten bei An- und Abwesenheit von Sauerstoffatomen gemessen. Durch Variation der Sauerstoffkonzentration wurden verschiedene Umsätze erzielt. Die folgende Abbildung zeigt in doppeltlogarithmischer Auftragung die Messpunkte. Die zugehörigen Werte finden sich in Abschnitt 5 (Messtabellen). Die gewählten Versuchsbedingungen sind dem Text in der Abbildung oder bei den Messtabellen zu entnehmen. 


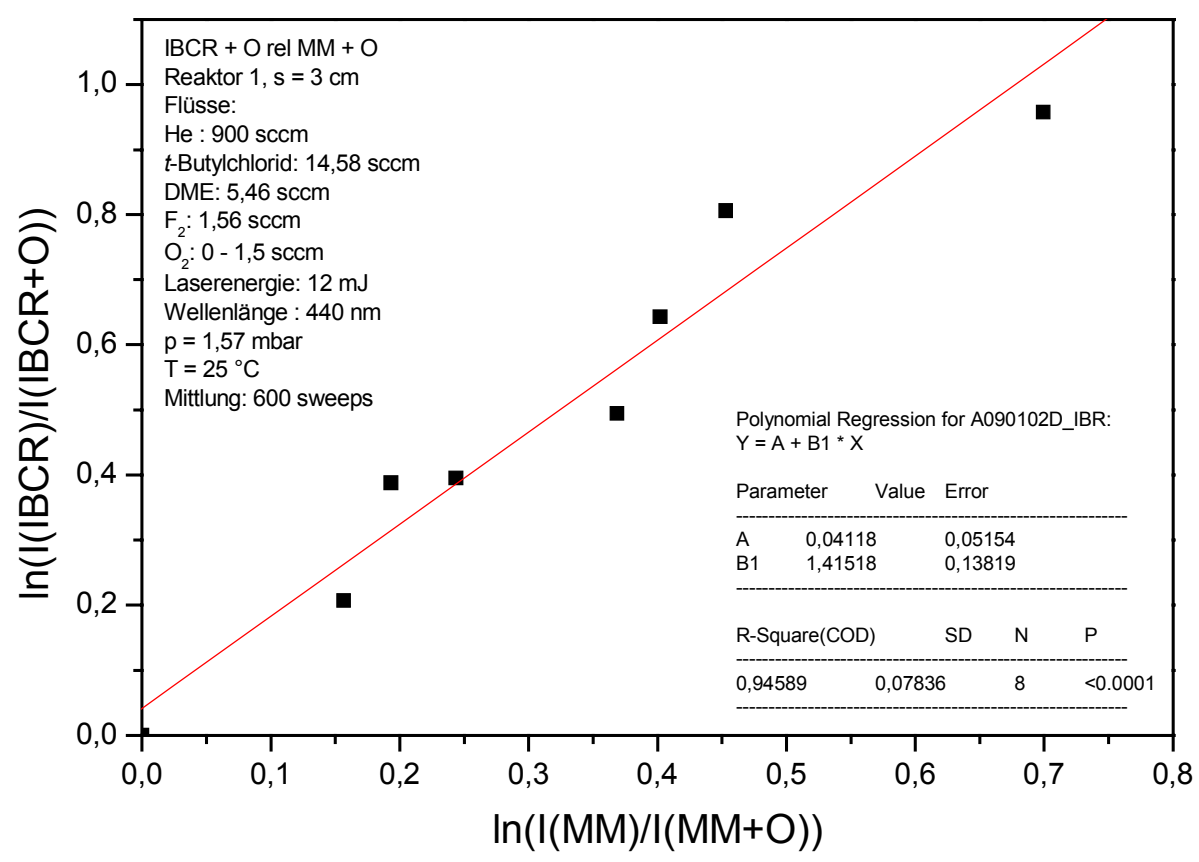

Abbildung 135: iso- $\mathrm{C}_{4} \mathrm{H}_{8} \mathrm{Cl}+\mathrm{O}$ relativ zu Methoxymethyl $+\mathrm{O}, \mathrm{T}=25^{\circ} \mathrm{C}$

Aus der Auftragung ergibt sich:

$$
\mathrm{k}_{\mathrm{rel}}=\mathrm{k}\left(\text { iso- } \mathrm{C}_{4} \mathrm{H}_{8} \mathrm{Cl}+\mathrm{O}\right) / \mathrm{k}\left(\mathrm{CH}_{3} \mathrm{OCH}_{2}+\mathrm{O}\right)=1,42 \pm 0,14 \text {. }
$$

Mit der in Abschnitt 3.3.2 erläuterten Geschwindigkeitskonstante $k_{\text {ref }}=1,31 \cdot 10^{14} \mathrm{~cm}^{3} /(\mathrm{mol} \cdot \mathrm{s})$ für die Referenzreaktion Methoxymethyl + O erhält man:

$$
\mathrm{k}^{298 \mathrm{~K}}\left(\text { iso- } \mathrm{C}_{4} \mathrm{H}_{8} \mathrm{Cl}+\mathrm{O}\right)=(1,86 \pm 0,19) \cdot 10^{14} \mathrm{~cm}^{3} /(\mathrm{mol} \cdot \mathrm{s}) \text {. }
$$

Es lag keine Literatur über die Geschwindigkeit der Reaktion iso- $\mathrm{C}_{4} \mathrm{H}_{8} \mathrm{Cl}+\mathrm{O}$ vor.

\subsubsection{Vergleich der Geschwindigkeiten der Reaktionen Radikal + O}

Die einzelnen gemessenen Geschwindigkeitskonstanten der Reaktionen von Kohlenwasserstoffradikalen mit Sauerstoffatomen sind in den zugehörigen Abschnitten bereits diskutiert worden. In diesem Abschnitt soll versucht werden, einen Gesamtzusammenhang zwischen den hier bestimmten Werten herzustellen. In erster Näherung lassen sich Geschwindigkeitskoeffizienten der Reaktionen Radikal + O nach der einfachen Stoßtheorie aus dem Produkt des Reaktionsquerschnitts und der Relativgeschwindigkeit bestimmen:

$$
\mathrm{k}=\langle\mathrm{v}\rangle \cdot \sigma \operatorname{mit}\langle\mathrm{v}\rangle=\sqrt{\frac{8 \mathrm{k}_{\mathrm{B}} \mathrm{T}}{\pi \mu}},
$$

$\mathrm{k}=$ Geschwindigkeitskoeffizient, $\langle\mathrm{v}\rangle=$ Relativgeschwindigkeit, $\sigma=$ Reaktionsquerschnitt, $\mathrm{k}_{\mathrm{B}}=$ Boltzmannkonstante, $\mathrm{T}=$ absolute Temperatur, $\mu=$ reduzierte Masse. 
Diese Formel ist bei der Beurteilung der Geschwindigkeit der Reaktion $\mathrm{CD}_{3}+\mathrm{O}$ relativ zu $\mathrm{CH}_{3}+\mathrm{O}$ unter der Annahme gleicher Reaktionsquerschnitte im Abschnitt 3.3.4 mit gutem Ergebnis angewendet worden.

Die Reaktionen von Kohlenwasserstoffradikalen mit O-Atomen sind sehr schnell und besitzen im untersuchten Bereich nur eine sehr kleine Temperaturabhängigkeit, was eine geringe Aktivierungsenergie bedeutet. Für solche Reaktionen wurde von Paltenghi, Ogryzlo und Bayes eine Korrelation vorgeschlagen, nach welcher der Logarithmus der Geschwindigkeitskoeffizienten umgekehrt proportional zu den Ionisierungspotenzialen der Radikale ist [PAL84]. In Anlehnung an das Harpunenmodell wird mit abnehmendem Ionisierungspotenzial von einer verstärkten Wechselwirkung zwischen dem Radikal und dem Reaktionspartner ausgegangen. Am Beispiel der Reaktionen von Radikalen mit $\mathrm{O}_{3}$ haben die genannten Autoren eine solche Korrelation feststellen können. Schon mehrfach wurde ein solcher funktionaler Zusammenhang auch für die Reaktionen von Radikalen mit O-Atomen aufgestellt ([SEE94], [BEI91], [NAC98]). Auffällig war, dass das tButylradikal auf Grund einer sehr hoch bestimmten Geschwindigkeitskonstante nicht in ein solches Schema passte. Wie in Abschnitt 3.3.9 erläutert, ist der Geschwindigkeitskoeffizient der Reaktion tButyl $+\mathrm{O}-$ auch aufgrund der in dieser Arbeit vorgestellten Messungen - viermal kleiner anzunehmen als bisher. Die folgende Tabelle stellt die in dieser Arbeit bestimmten Geschwindigkeitskoeffizienten bei Zimmertemperatur zusammen. Ferner sind die Ionisierungspotenziale nach [STE94] und die dekadischen Logarithmen der Geschwindigkeitskoeffizienten aufgeführt. Die von Zeuch bestimmte Geschwindigkeitskonstante der Reaktion Ethyl + O (vgl. Abschnitt 3.3.1) ist ebenfalls erwähnt.

\begin{tabular}{|c|c|c|c|}
\hline Radikal & $\mathrm{k}^{298 \mathrm{~K}} /\left(\mathrm{cm}^{3} \cdot \mathrm{mol}^{-1} \cdot \mathrm{s}^{-1}\right)$ & $\lg \left\{\mathrm{k} /\left(\mathrm{cm}^{3} \cdot \mathrm{mol}^{-1} \cdot \mathrm{s}^{-1}\right)\right\}$ & $\mathrm{IE} / \mathrm{eV}$ \\
\hline \hline $\mathrm{CH}_{3}$ & $6,14 \cdot 10^{13}$ & 13,78817 & 9,84 \\
\hline $\mathrm{C}_{2} \mathrm{H}_{5}$ & $1,04 \cdot 10^{14}$ & 14,01703 & 8,12 \\
\hline $2-\mathrm{C}_{3} \mathrm{H}_{7}$ & $1,14 \cdot 10^{14}$ & 14,0569 & 7,37 \\
\hline $1-\mathrm{C}_{3} \mathrm{H}_{7}$ & $8,43 \cdot 10^{13}$ & 13,92583 & 8,09 \\
\hline $\mathrm{CH}_{3} \mathrm{OCH}_{2}$ & $1,31 \cdot 10^{14}$ & 14,11727 & 6,94 \\
\hline $\mathrm{c}^{-} \mathrm{C}_{5} \mathrm{H}_{9}$ & $1,73 \cdot 10^{14}$ & 14,23805 & 7,21 \\
\hline $2-\mathrm{C}_{4} \mathrm{H}_{9}$ & $9,69 \cdot 10^{13}$ & 13,98632 & 7,25 \\
\hline $1-\mathrm{C}_{4} \mathrm{H}_{9}$ & $9,4 \cdot 10^{13}$ & 13,97313 & 8,02 \\
\hline $\mathrm{t}-\mathrm{C}_{4} \mathrm{H}_{9}$ & $1,38 \cdot 10^{14}$ & 14,13988 & 6,7 \\
\hline iso- $\mathrm{C}_{4} \mathrm{H}_{9}$ & $9,11 \cdot 10^{13}$ & 13,95952 & 7,93 \\
\hline $\mathrm{Alllyl}$ & $1,11 \cdot 10^{14}$ & 14,04532 & 8,13 \\
\hline $\mathrm{c}-\mathrm{C}_{7} \mathrm{H}_{13}$ & $2,01 \cdot 10^{14}$ & -- & -- \\
\hline $\mathrm{c}-\mathrm{C}_{8} \mathrm{H}_{15}$ & $1,68 \cdot 10^{14}$ & -- & -- \\
\hline $\mathrm{C}_{5} \mathrm{H}_{5}$ & $1,34 \cdot 10^{14}$ & -- & -- \\
\hline $\mathrm{CH}_{2} \mathrm{CCl}_{3}$ & $2,82 \cdot 10^{13}$ & -- & -- \\
\hline iso- $\mathrm{C}_{4} \mathrm{H}_{8} \mathrm{Cl}$ & $1,86 \cdot 10^{14}$ & -- & \\
\hline
\end{tabular}

Tabelle 6: Liste der Geschwindigkeitskoeffizienten Radikal + O 
Die Ionisierungsenergien der letzten fünf in der Tabelle aufgeführten Radikale waren nicht zu ermitteln.

Die folgende Abbildung zeigt die Auftragung der Logarithmen der in dieser Arbeit bestimmten Geschwindigkeitskoeffizienten gegen das Ionisierungspotenzial der zugehörigen Kohlenwasserstoffradikale. Der nach [HAC02] bestimmte Wert für die Reaktion Ethyl + O ist ebenfalls aufgetragen.

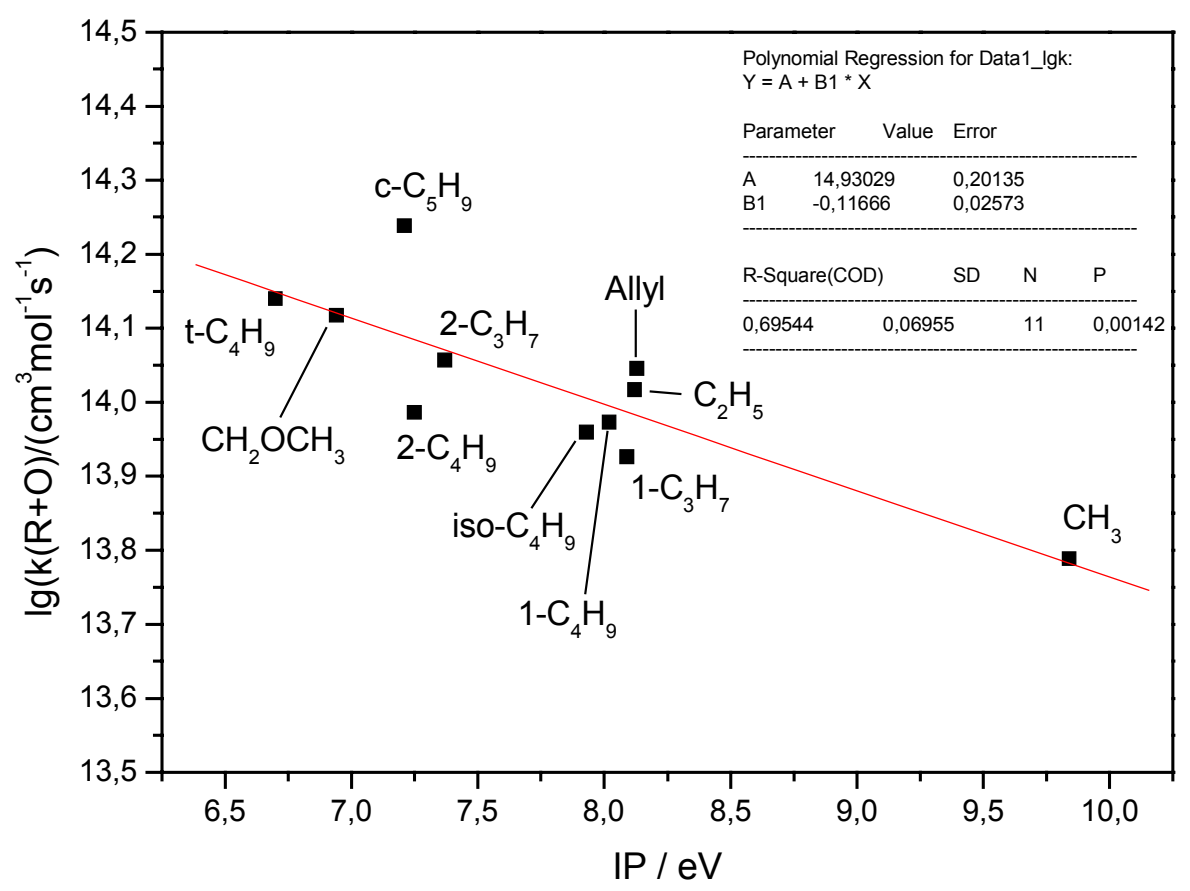

\section{Abbildung 136: $\lg \{k(R+O)\}$ gegen IP}

Die Auftragung zeigt, dass für die in dieser Arbeit ermittelten Werte eine gute Korrelation gegeben ist. Lediglich die Werte für c-Pentyl $+\mathrm{O}$ und 2-Butyl $+\mathrm{O}$ fallen nicht in unmittelbare Nähe zur Regressionsgeraden. Allerdings ist bei diesen beiden Werten die Abweichung zu einem passenden $\mathrm{k}$ auch nicht sehr groß, sie liegt bei 20-30\%. Ein vermuteter Zusammenhang zwischen den Geschwindigkeitskonstanten der Reaktionen Radikal + O und den Ionisierungspotenzialen der Radikale kann durch das im Rahmen dieser Arbeit ermittelte System von Geschwindigkeitskonstanten, welches durch viele Vergleichsmessungen auf innere Konsistenz überprüft wurde, im wesentlichen bestätigt werden. 


\subsection{Reaktionen von Radikalen mit $\mathbf{O}_{2}$}

Die Reaktionen von Kohlenwasserstoffradikalen mit Sauerstoffmolekülen sind von hoher Bedeutung für Verbrennungsprozesse und die Chemie der Atmosphäre. Sie gelten als Assoziationsreaktionen, die sich durch folgendes Reaktionsschema beschreiben lassen [NIE97]:

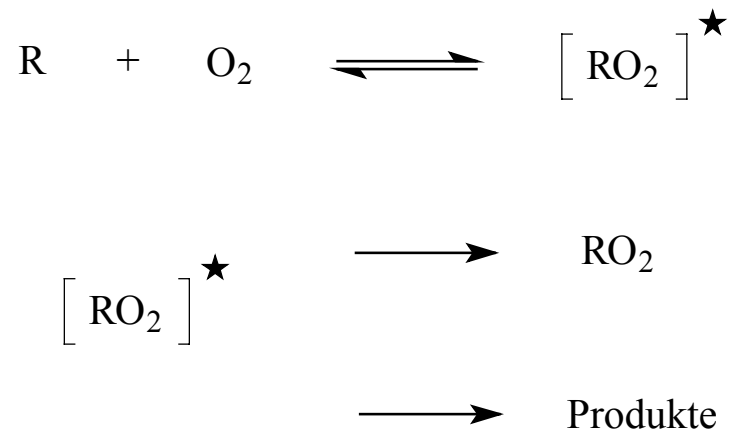

Die Peroxyradikalbildungsreaktion (1) hin ist eine schnelle Reaktion, doch schon bei mäßigen Temperaturen hat die Rückreaktion zum Kohlenwasserstoffradikal $\mathrm{R}$ und zu $\mathrm{O}_{2}$ aufgrund der geringen Stärke der $\mathrm{R}-\mathrm{O}_{2}$ Bindung eine hohe Bedeutung. Bei höheren Temperaturen steigt die Wahrscheinlichkeit für einen Zerfall des Additionskomplexes $\mathrm{RO}_{2} *$ über (1)rück an, weshalb sich in vielen Fällen eine negative Temperaturabhängigkeit der zugehörigen Geschwindigkeitskonstanten ergibt. Jedoch muss auch die Wirkung der Kanäle (2a) und (2b) beachtet werden.

Kanal (2a) ist die Stabilisierung, die im allgemeinen über Stoßpartner M erfolgt. Dadurch ergibt sich eine bedeutende Druckabhängigkeit des Gesamtgeschwindigkeitskoeffizienten bis zu einer Hochdruckgrenze, die bei den verschiedenen Radikalen bei ganz unterschiedlichen Drücken liegen kann. Größere $\mathrm{RO}_{2}$-Komplexe können sich aufgrund einer höheren Anzahl von Molekülfreiheitsgraden auch molekülintern stabilisieren. Die Hochdruckgrenze des Gesamtgeschwindigkeitskoeffizienten wird für große Radikale bei etwa 20 Torr erreicht [NIE97], für kleine Radikale kann sie jedoch sehr viel höher liegen. Typischerweise liegt der Wert für $\mathrm{k}$ (Hochdruck) im Bereich $6 \cdot 10^{11}-6 \cdot 10^{12}$ $\mathrm{cm}^{3} /(\mathrm{mol} \cdot \mathrm{s})$ [NIE97].

Unter Reaktion (2b) soll die unimolekulare Reaktion des Additionskomplexes verstanden werden. Das kann eine Isomerisierung, der Zerfall zum Alkoxyradikal und zu $\mathrm{OH}$ $\left(\mathrm{RO}_{2} \rightarrow \mathrm{R}^{\prime} \mathrm{OOH} \rightarrow \mathrm{R}^{\prime} \mathrm{O}+\mathrm{OH}\right)$ oder eine sonstige Dissoziation sein. Solche Reaktionen spielen bei hohen Temperaturen eine große Rolle [BEN86]. Des Weiteren kann (2b) die Reaktion zu Alken und $\mathrm{HO}_{2}$ sein. Letzteres ist bei Drücken kleiner als 10 Torr bedeutungsvoll [NIE97]. Als Beispiel sei die Reaktion $\mathrm{C}_{2} \mathrm{H}_{5}+\mathrm{O}_{2}$ bei Zimmertemperatur genannt: Die Bildung des $\mathrm{C}_{2} \mathrm{H}_{4}$ folgt einer $\mathrm{p}^{-0,8} 8^{ \pm 0,1}$ Druckabhängigkeit. Bei einem Druck von 10 Torr reagieren 2\% des Ethyls zum Ethen, bei 1 Torr 
sind es schon $12 \%$. Es ist nachgewiesen worden, dass die Alkenbildung nicht über eine direkte Abstraktion, sondern über den angeregten Ethylperoxy-Komplex verläuft [ING69].

In obigem Mechanismus ist nicht die Reaktion des Peroxyradikals $\mathrm{RO}_{2}$, angeregt oder stabilisiert, mit anderen Reaktionspartnern berücksichtigt. Solche Folgereaktionen hängen natürlich entscheidend von der chemischen Umgebung ab, können aber auch Einfluss auf das vorgelagerte Gleichgewicht (1) und damit auf die Gesamtgeschwindigkeitskonstante der Reaktion $\mathrm{R}+\mathrm{O}_{2}$ haben. Bei den Untersuchungen zur vorliegenden Arbeit wurde versucht, solche Folgereaktionen zu vermeiden.

Für die in den folgenden Abschnitten dargestellten Messungen zu den Reaktionen Radikal $+\mathrm{O}_{2}$ dient die intensiv untersuchte Reaktion $\mathrm{CH}_{2} \mathrm{OCH}_{3}+\mathrm{O}_{2}$ als Referenzreaktion. Die dazu in der Literatur angegeben, mit verschiedenen Methoden bestimmten Geschwindigkeitskonstanten stimmen sehr gut überein: $\mathrm{k}^{298 \mathrm{~K}}=5,97 \cdot 10^{12} \mathrm{~cm}^{3} /(\mathrm{mol} \cdot \mathrm{s}), \mathrm{p}=2$ Torr ([HOY96], [NAC94]); $\mathrm{k}^{298 \mathrm{~K}}=6,0 \cdot 10^{12} \mathrm{~cm}^{3} /(\mathrm{mol} \cdot \mathrm{s}), \mathrm{p}=2$ Torr $[\mathrm{NAC} 98] ; \mathrm{k}^{298 \mathrm{~K}}=6,62 \cdot 10^{12} \mathrm{~cm}^{3} /(\mathrm{mol} \cdot \mathrm{s}), \mathrm{p}=1$ Torr [MAR97]; $\mathrm{k}^{298 \mathrm{~K}}=6,87 \cdot 10^{12} \mathrm{~cm}^{3} /(\mathrm{mol} \cdot \mathrm{s}), \mathrm{p}=19$ Torr [SEH97]. Der in der eigenen Arbeitsgruppe ermittelte Wert von Hoyermann und Nacke ([HOY96], [NAC94]) soll als Basisgeschwindigkeitskoeffizient verwendet werden. Im folgenden werden die Untersuchungen zu den Reaktionen der Radikale t-Butyl, c-Heptyl, c-Oktyl, c-Pentadienyl und 2-Chlor-2-Methyl-Propyl mit $\mathrm{O}_{2}$ vorgestellt.

\subsection{1 t-Butyl $+\mathrm{O}_{2}$}

Produkte:

Messergebnisse: Die Produkte der Reaktion t-Butyl $+\mathrm{O}_{2}$ wurden bei Zimmertemperatur mit Versuchsanordnung C (FTIR) untersucht. Als Radikalvorläufer wurde Di-tertiär-Butyl-Keton (DtBK, IUPAC-Name: 2,2,4,4-Tetramethylpentan-3-on) eingesetzt. Die in Abschnitt 3.3.9 (Produktuntersuchungen t-Butyl + O) gemachten Äußerungen zum Radikalvorläufer und zum Photolyseprozess gelten auch hier. Das Spektrum der Reaktionsmischung vor der Photolyse geht in die Berechnung der Absorbanz als $\mathrm{I}_{0}$-Wert ein. Die folgende Abbildung zeigt die Produktbildung der Reaktion t-Butyl $+\mathrm{O}_{2}$ bei einem Druck von 4 mbar. Das in Abschnitt 3.3.9 beschriebene Spektrum der Photolyseprodukte wurde - soweit möglich ${ }^{29}$ - von der Absorbanz subtrahiert, so dass nur die Reaktionsprodukte von t-Butyl $+\mathrm{O}_{2}$ signalgebend sein sollten.

\footnotetext{
${ }^{29}$ Es erwies sich als problematisch die Zelle im Niederdruckbereich über den ganzen Messzeitraum hinweg völlig luftfrei zu halten. Das "reine" Photolysespektrum, welches subtrahiert wurde, enthält durch die in der Zelle vorhandenen Luftspuren demnach auch einen Teil der Produkte der Reaktion t-Butyl $+\mathrm{O}_{2}$.
} 


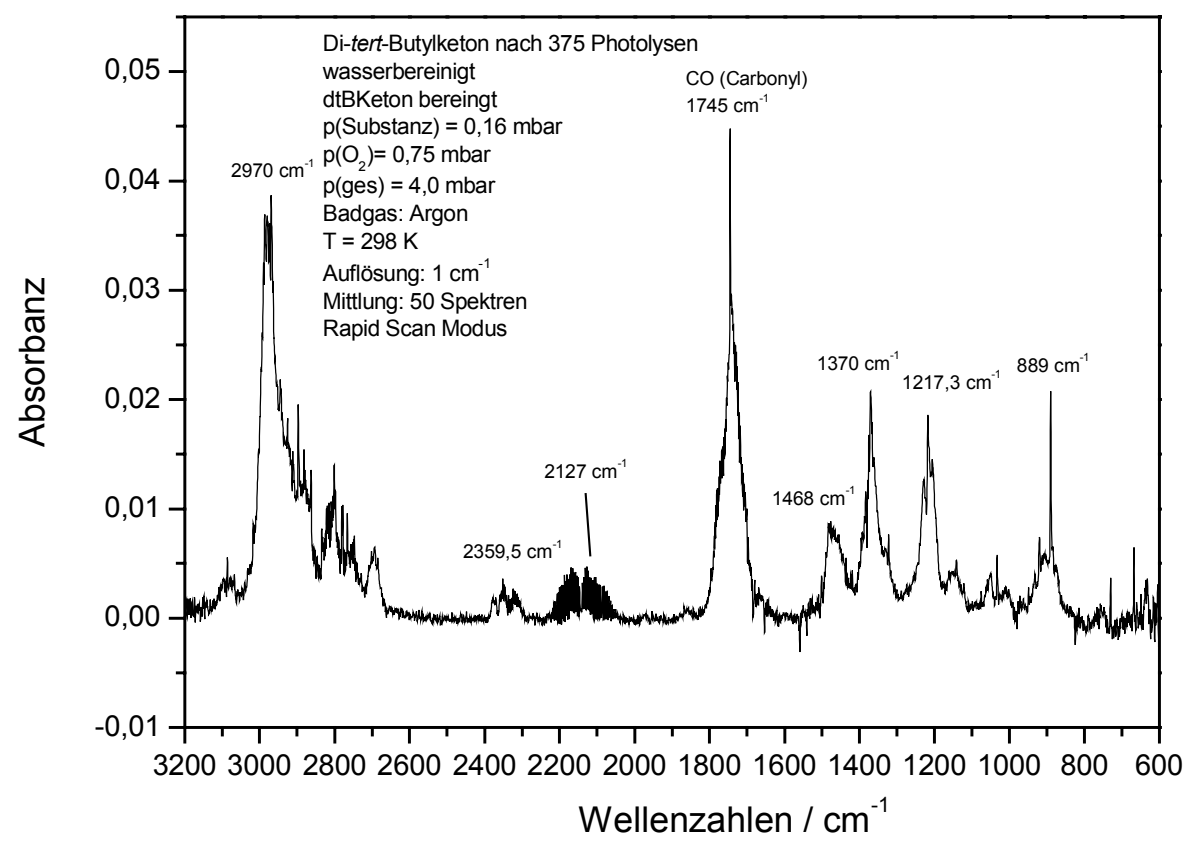

Abbildung 137: Produkte t-Butyl $+\mathrm{O}_{2}, \mathrm{p}=4$ mbar

Interpretation: Zur Reaktion t-Butyl $+\mathrm{O}_{2}$ wird folgender Mechanismus diskutiert:<smiles>C=CC(C)(C)OO</smiles>

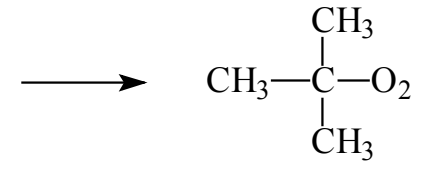

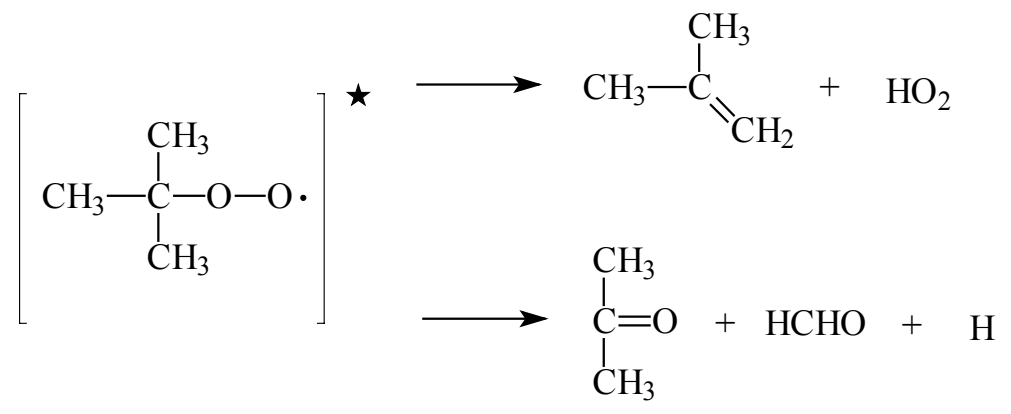

$$
\begin{aligned}
& \longrightarrow \mathrm{CH}_{3}-\stackrel{\stackrel{\mathrm{C}}{\mathrm{C}}-\mathrm{C}}{\mathrm{C} / \mathrm{C}_{2}}+\mathrm{OH}
\end{aligned}
$$


Alle genannten Reaktionskanäle sind (von den Edukten t-Butyl und $\mathrm{O}_{2}$ ausgehend) exotherm: $\Delta_{\mathrm{R}} \mathrm{H}^{0}{ }_{298 \mathrm{~K}}(2 \mathrm{a})=-126,3 \mathrm{~kJ} / \mathrm{mol}, \Delta_{\mathrm{R}} \mathrm{H}^{0}{ }_{298 \mathrm{~K}}(2 \mathrm{~b})=-52,2 \mathrm{~kJ} / \mathrm{mol}, \Delta_{\mathrm{R}} \mathrm{H}^{0}{ }_{298 \mathrm{~K}}(2 \mathrm{c})=-153,8 \mathrm{~kJ} / \mathrm{mol}$, $\Delta_{\mathrm{R}} \mathrm{H}^{0}{ }_{298 \mathrm{~K}}(2 \mathrm{~d})=-141 \mathrm{~kJ} / \mathrm{mol}$. An den Absorbanzen bei 2970, 1745, 1370 und $1217 \mathrm{~cm}^{-1}$ ist die Entstehung von Aceton eindeutig zu erkennen. Ein entsprechendes Vergleichsspektrum ist im Internet unter der Adresse http://webbook.nist.gov/chemistry erhältlich. Ein weiterer Hinweis für die Existenz des am stärksten exothermen Reaktionskanals (2c) ist die Entstehung von Formaldehyd. Zum Nachweis wurde das obige Produktspektrum von allen bekannten Produkten rechnerisch bereinigt. Die folgende Abbildung zeigt das Ergebnis im entscheidenden Wellenzahlbereich:

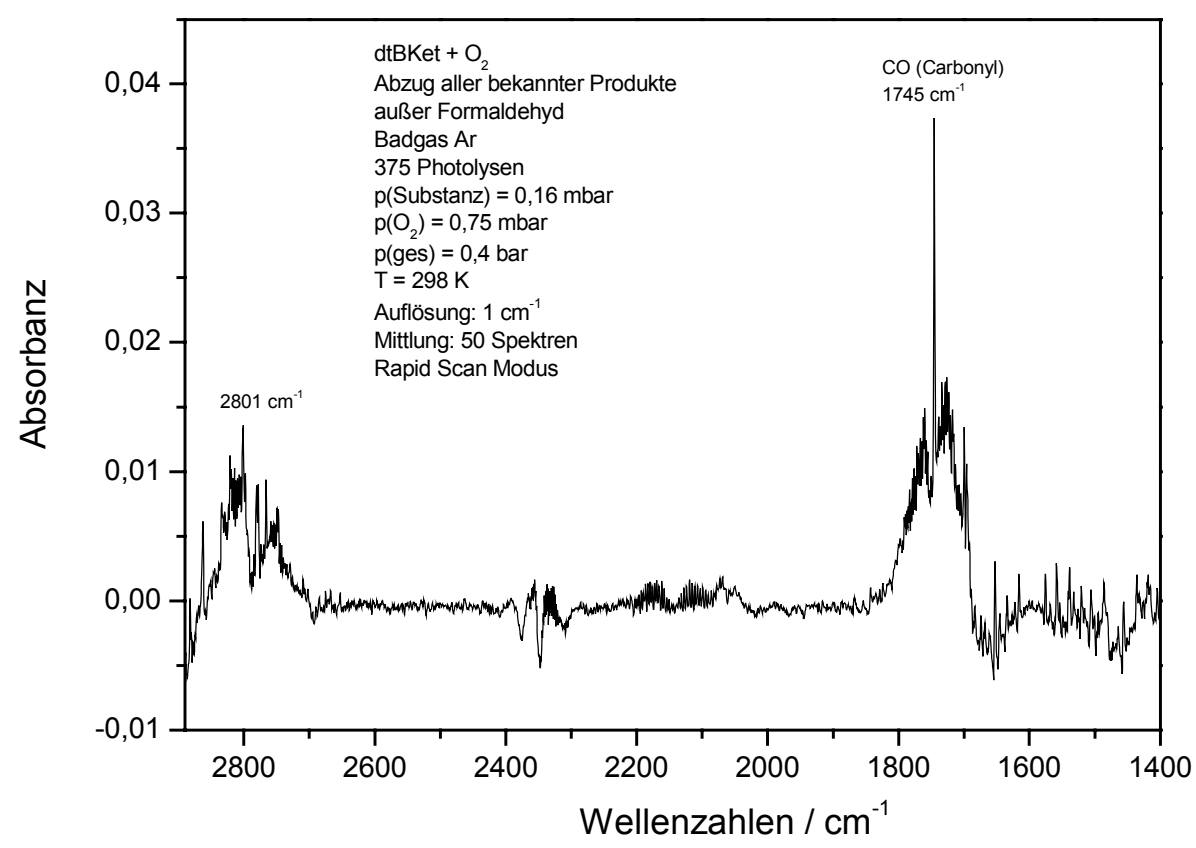

\section{Abbildung 138: Nachweis Formaldehyd als Produkt von t-Butyl $+\mathrm{O}_{2}$}

Es wurden keine Literaturspektren zum Formaldehyd gefunden, was dadurch zu erklären ist, dass Formaldehyd in der Gasphase nicht als Monomer vorliegt. Als Vergleichsspektrum wird eine Messung von Zeuch herangezogen [ZEU02]. Dazu wurde in Versuchsanordnung C die mittlerweile intensiv untersuchte Reaktion $\mathrm{CH}_{3}+\mathrm{O}$ ausgeführt. Es wurden sämtliche bekannten signalgebenden Produkte subtrahiert. Das Ergebnis kann als Kalibrierspektrum von Formaldehyd gewertet werden: 


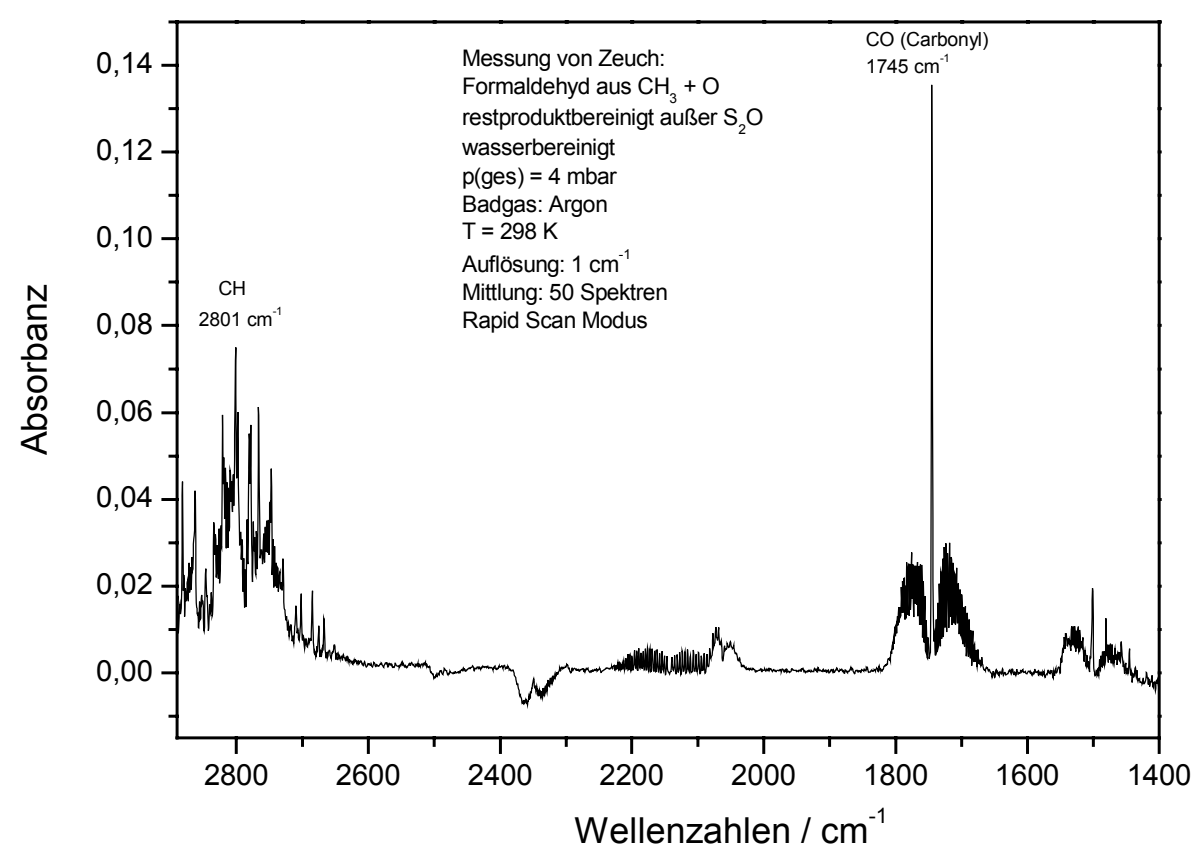

\section{Abbildung 139: Kalibrierspektrum Formaldehyd, [ZEU02]}

Kanal (2c) ist durch den Nachweis von Aceton und Formaldehyd sehr wahrscheinlich aktiv. In Anbetracht der Tatsache, dass es hier sich aufgrund der zur Verfügung stehenden langen Reaktionszeiten (im Sekundenbereich) um eine Endproduktanalyse handelt, kann aber nicht vollständig ausgeschlossen werden, dass Aceton und Formaldehyd aus einer Reihe eher langsamer Folgereaktionen entsteht. So könnte das nach Kanal (2a) stabilisierte t-Butylperoxyradikal kombinieren und anschließend zu $\mathrm{O}_{2}$ und zwei Butoxyradikalen zerfallen. Die Geschwindigkeit eines solchen Zerfalls wird angegeben mit $\mathrm{k}^{298 \mathrm{~K}}=1,21 \cdot 10^{7} \mathrm{~cm}^{3} /(\mathrm{mol} \cdot \mathrm{s})$ [WAL92], $\mathrm{k}^{298 \mathrm{~K}}=2,11 \cdot 10^{5}$ $\mathrm{cm}^{3} /(\mathrm{mol} \cdot \mathrm{s})$ [LIG90]. Die Butoxyradikale ihrerseits könnten dann zu Aceton und $\mathrm{CH}_{3}$ weiterzerfallen. Das Formaldehyd könnte dann aus einer Reaktion von Methylradikalen mit $\mathrm{O}_{2}$ gebildet werden $\left(\mathrm{k}^{298 \mathrm{~K}}=7,94 \cdot 10^{9} \mathrm{~cm}^{3} /(\mathrm{mol} \cdot \mathrm{s})[\mathrm{WAS} 76]\right)$.

Das Signal bei $889 \mathrm{~cm}^{-1}$ weist Isobuten als Produkt nach. Ein entsprechendes Vergleichsspektrum ist im Internet unter der Adresse http://webbook.nist.gov/chemistry erhältlich. Von Tsang wird für den Reaktionskanal (2b) zur Bildung von Isobuten und Hydroperoxyd ein Geschwindigkeitskoeffizient von $\mathrm{k}^{298 \mathrm{~K}}=5,48 \cdot 10^{11} \mathrm{~cm}^{3} /(\mathrm{mol} \cdot \mathrm{s})$ abgeschätzt [TSA90], eine Größenordnung, bei welcher der Kanal durchaus zur Produktbildung beitragen kann. Wie in den Vorbemerkungen erläutert, sollte Kanal (2b) vor allem bei niedrigen Drücken relevant sein. Ein in $\mathrm{O}_{2}$-Atomsphäre aufgenommenes Produktspektrum bei $\mathrm{p}=1$ bar zeigt kein Isobutensignal mehr, wie in der folgenden Abbildung zu sehen ist. Es muss deshalb angenommen werden, dass Kanal (2b) bei höheren Drücken ausbleibt. 


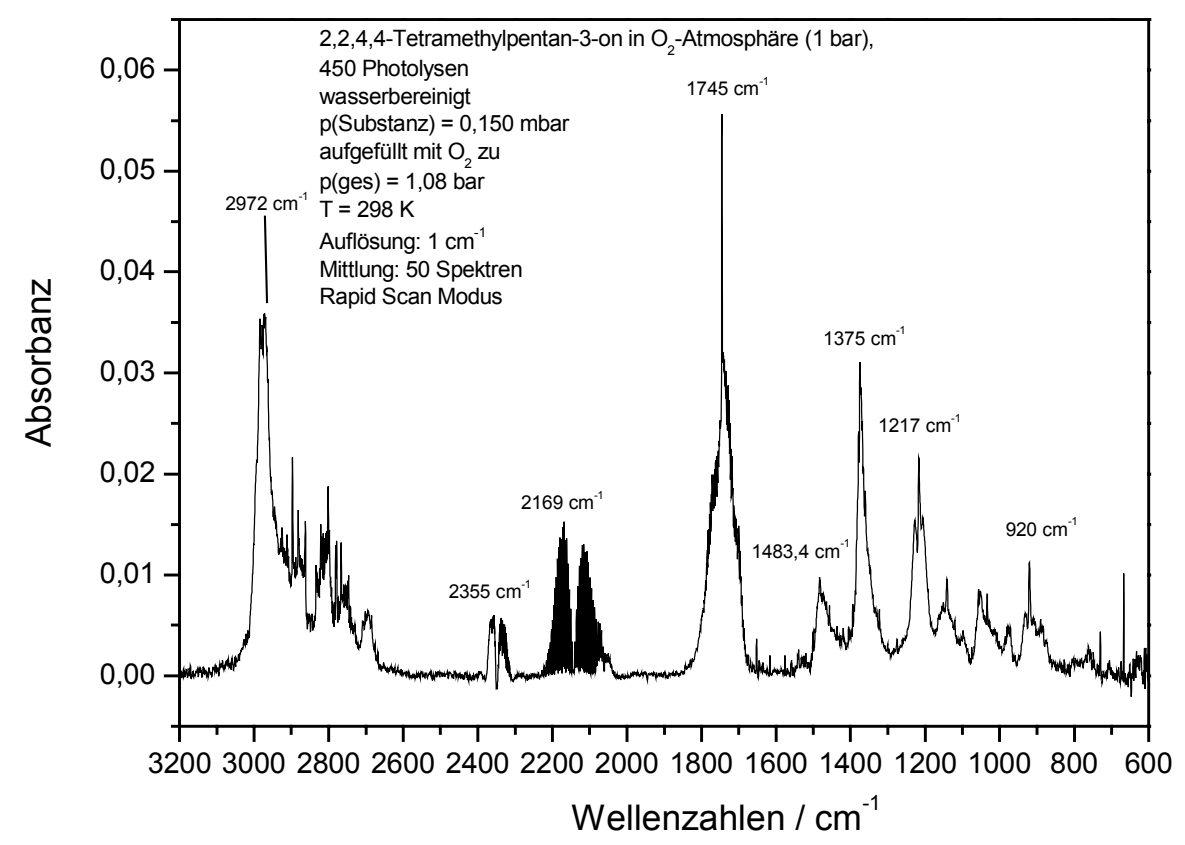

\section{Abbildung 140: Produkte t-Butyl $+\mathrm{O}_{2}, \mathrm{p}=1$ bar}

Für Kanal (2d) werden keine Hinweise gefunden.

\section{Reaktionsgeschwindigkeit:}

Messergebnisse: Die Geschwindigkeit der Reaktion t-Butyl $+\mathrm{O}_{2}$ wurde bei Zimmertemperatur relativ zur Reaktion Methoxymethyl $+\mathrm{O}_{2}$ mit Versuchsanordnung A (REMPI) bei $\lambda=510 \mathrm{~nm}$ bestimmt. Die Radikale wurden gemäß Abschnitt 3.1.5 und 3.1.14 erzeugt und auf ihren Muttermassen $\mathrm{m} / \mathrm{z}=57$ und $\mathrm{m} / \mathrm{z}=45$ in eindeutiger Weise nachgewiesen. Zur Bestimmung des Geschwindigkeitskoeffizienten wurden die Signalintensitäten bei An- und Abwesenheit von Sauerstoffmolekülen gemessen. Durch Variation der Sauerstoffkonzentration wurden verschiedene Umsätze erzielt. Die folgende Abbildung zeigt in doppeltlogarithmischer Auftragung die Messpunkte. Die zugehörigen Werte finden sich in Abschnitt 5 (Messtabellen). Die gewählten Versuchsbedingungen sind dem Text in der Abbildung oder bei den Messtabellen zu entnehmen. 


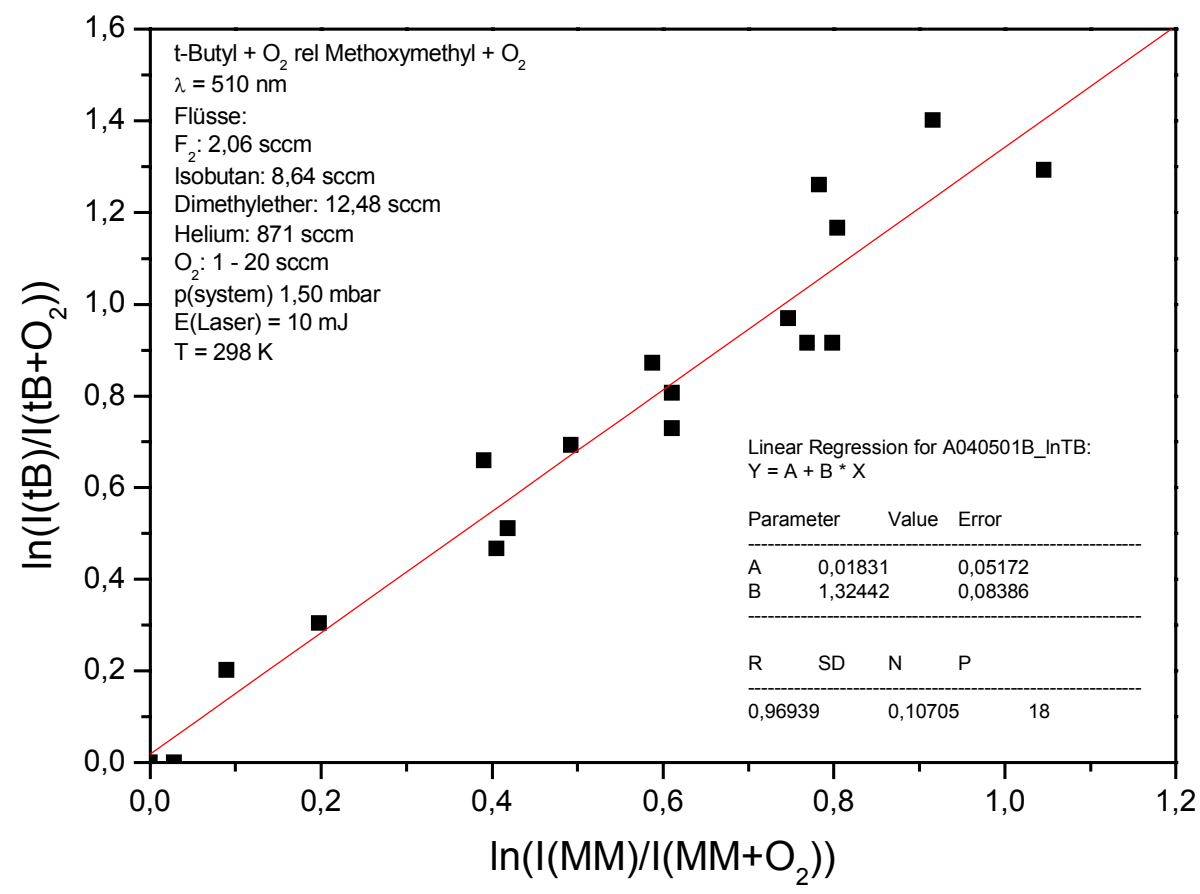

Abbildung 141: t-Butyl $+\mathrm{O}_{2}$ relativ zu Methoxymethyl $+\mathrm{O}_{2}, \mathrm{~T}=25^{\circ} \mathrm{C}$

Aus der Auftragung ergibt sich:

$$
\mathrm{k}_{\mathrm{rel}}=\mathrm{k}\left(\mathrm{t}-\mathrm{C}_{4} \mathrm{H}_{9}+\mathrm{O}_{2}\right) / \mathrm{k}\left(\mathrm{CH}_{3} \mathrm{OCH}_{2}+\mathrm{O}_{2}\right)=1,32 \pm 0,09 \text {. }
$$

Mit der in den Vorbemerkungen erläuterten Geschwindigkeitskonstante $\mathrm{k}_{\mathrm{ref}}=5,97 \cdot 10^{12}$ $\mathrm{cm}^{3} /(\mathrm{mol} \cdot \mathrm{s})$ für die Referenzreaktion Methoxymethyl $+\mathrm{O}_{2}$ erhält man:

$$
\mathrm{k}^{298 \mathrm{~K}}\left(\mathrm{t}-\mathrm{C}_{4} \mathrm{H}_{9}+\mathrm{O}_{2}\right)=(7,88 \pm 0,12) \cdot 10^{12} \mathrm{~cm}^{3} /(\mathrm{mol} \cdot \mathrm{s})
$$

Temperaturabhängigkeit:

Messergebnisse: Die Temperaturabhängigkeit der Reaktion t-Butyl $+\mathrm{O}_{2}$ wurde im Intervall $-30{ }^{\circ} \mathrm{C}$ bis $+90{ }^{\circ} \mathrm{C}$ nach dem selben Verfahren untersucht. Die Temperierung erfolgte mit der in Abschnitt 2.1.2 beschriebenen Methode für Reaktor 1. Die nachfolgenden Abbildungen zeigen die Ergebnisse. Die zugehörigen Werte finden sich in Abschnitt 5 (Messtabellen). Die gewählten Versuchsbedingungen sind dem Text in der Abbildung oder bei den Messtabellen zu entnehmen. 


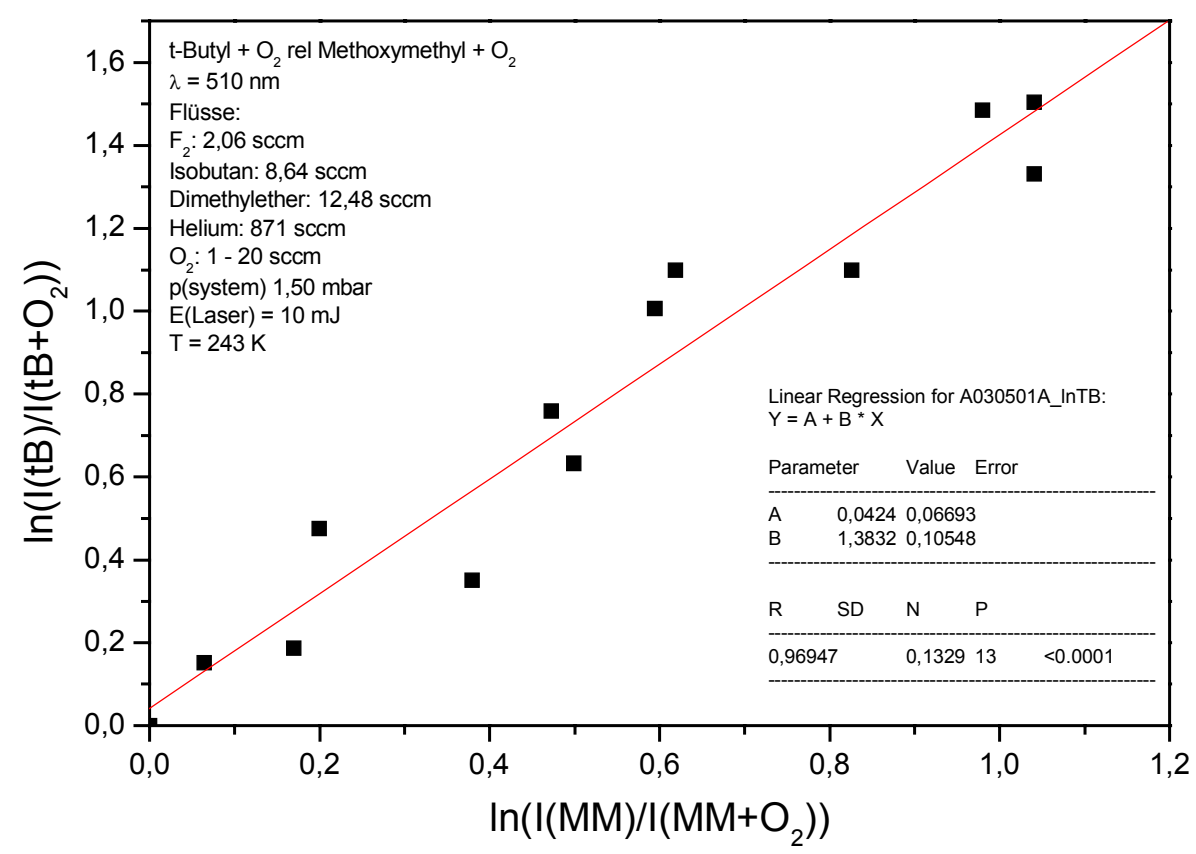

Abbildung 142: t-Butyl $+\mathrm{O}_{2}$ relativ zu Methoxymethyl $+\mathrm{O}_{2}, \mathrm{~T}=-30^{\circ} \mathrm{C}$

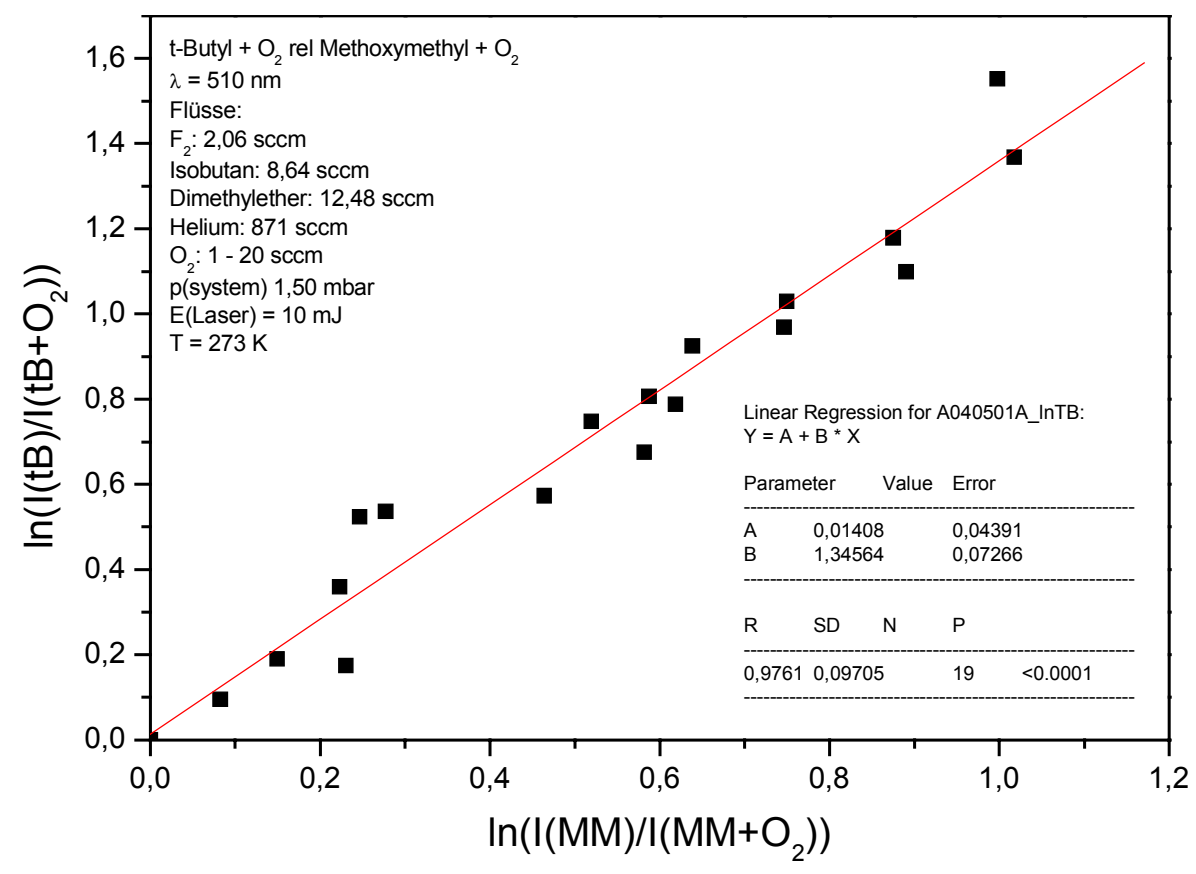

Abbildung 143: t-Butyl $+\mathrm{O}_{2}$ relativ zu Methoxymethyl $+\mathrm{O}_{2}, \mathrm{~T}=0^{\circ} \mathrm{C}$ 


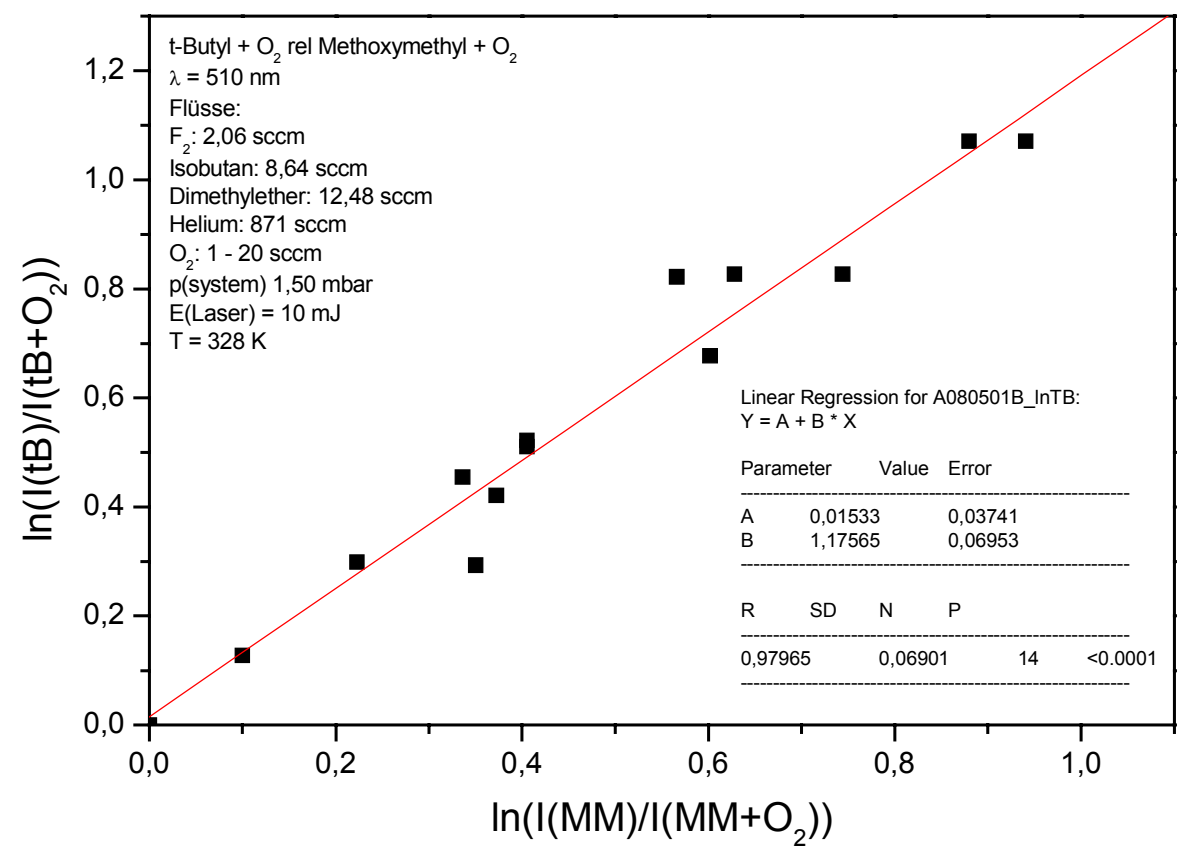

Abbildung 144: t-Butyl $+\mathrm{O}_{2}$ relativ zu Methoxymethyl $+\mathrm{O}_{2}, \mathrm{~T}=55^{\circ} \mathrm{C}$

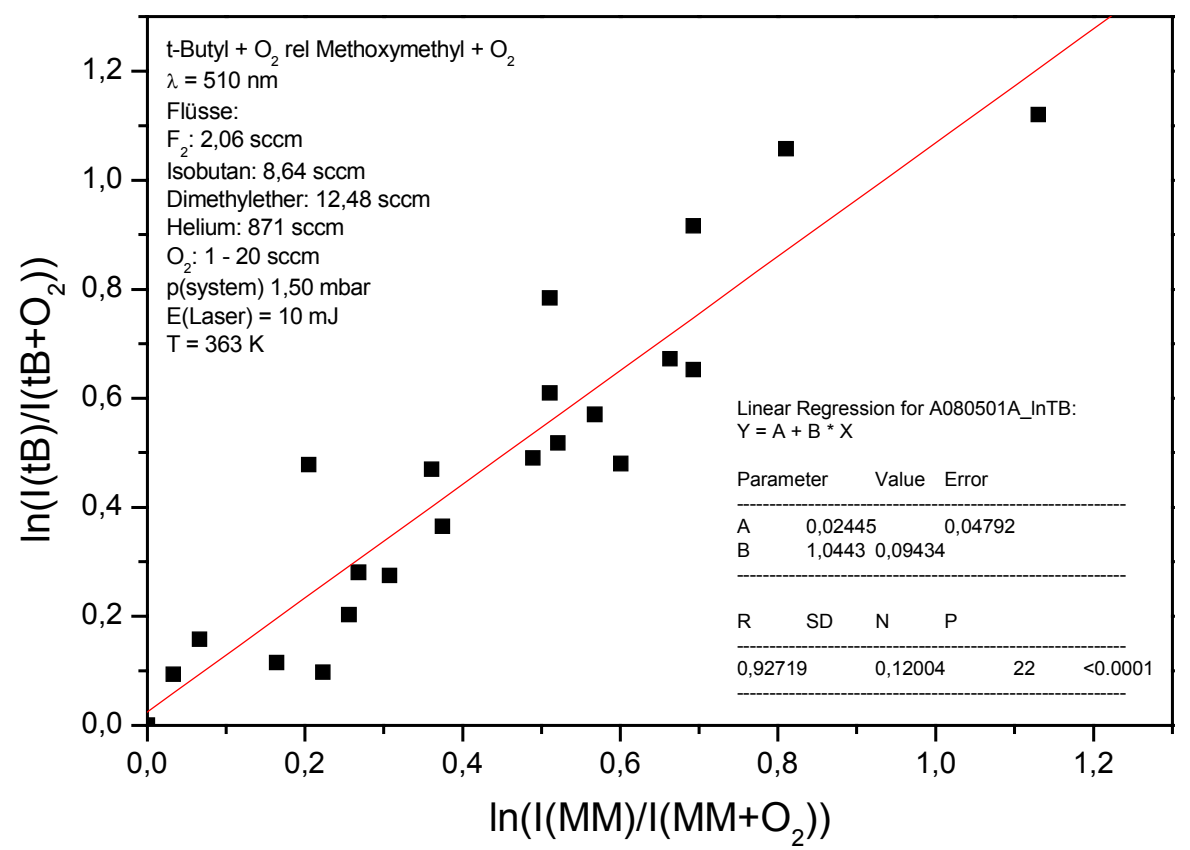

Abbildung 145: t-Butyl $+\mathrm{O}_{2}$ relativ zu Methoxymethyl $+\mathrm{O}_{2}, \mathrm{~T}=90^{\circ} \mathrm{C}$ 


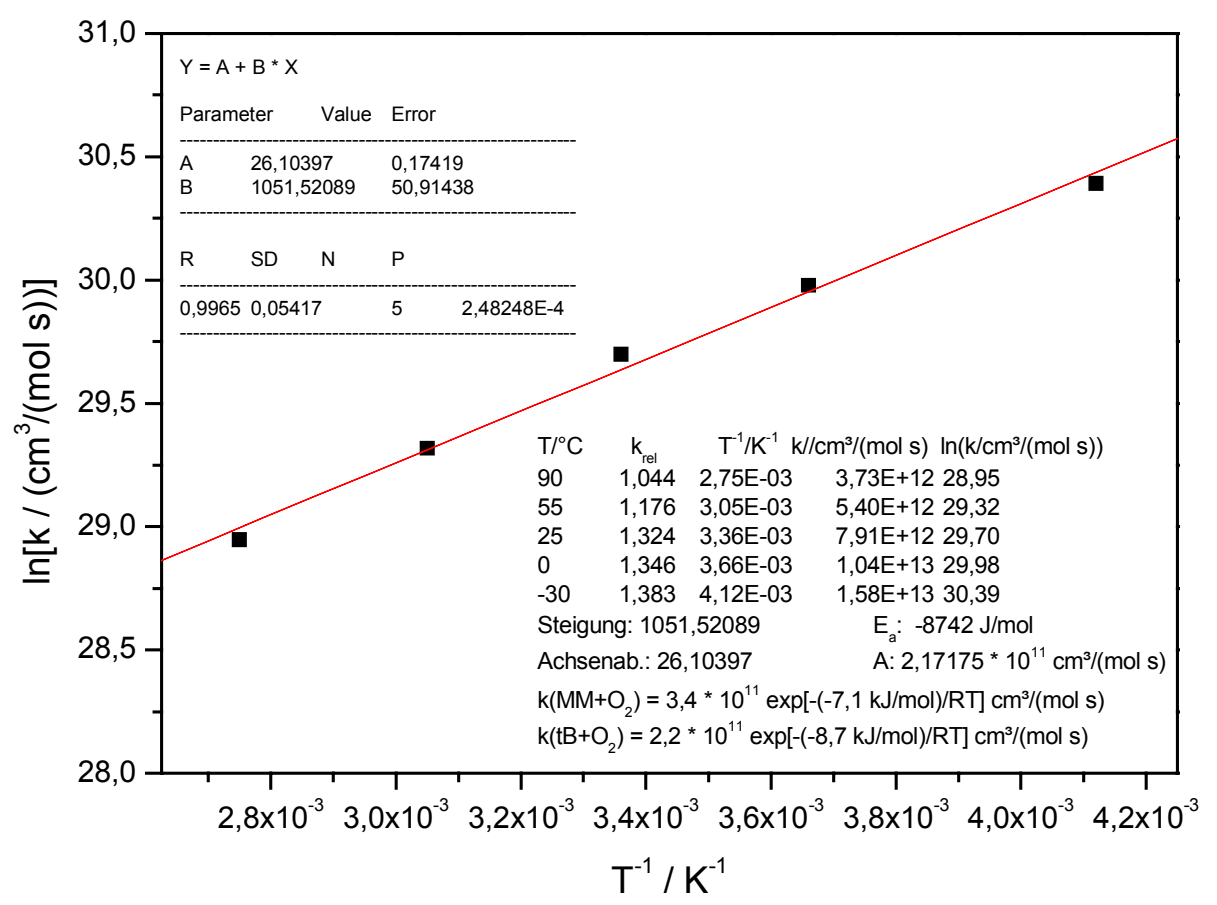

\section{Abbildung 146: t-Butyl $+\mathrm{O}_{2}$ relativ zu Methoxymethyl $+\mathrm{O}_{2}$, Arrheniusauftragung}

Aus der Arrheniusauftragung ergibt sich:

$$
\begin{gathered}
\mathrm{k}=(2,2 \pm 0,5) \cdot 10^{11} \exp \left\{-(-8,7 \pm 0,4) /(\mathrm{RT}) \mathrm{kJ} \cdot \mathrm{mol}^{-1}\right\} \mathrm{cm}^{3} \cdot \mathrm{mol}^{-1} \cdot \mathrm{s}^{-1} \\
\mathrm{k}^{298}=(7,4 \pm 2,6) \cdot 10^{12} \mathrm{~cm}^{3} \cdot \mathrm{mol}^{-1} \cdot \mathrm{s}^{-1}
\end{gathered}
$$

Diskussion: Warnatz gibt für die Reaktion $\mathrm{t}-\mathrm{Butyl}+\mathrm{O}_{2}$ den Arrheniusausdruck $\mathrm{k}=8 \cdot 10^{11} \exp \left\{-9 /(\mathrm{RT}) \mathrm{kJ} \cdot \mathrm{mol}^{-1}\right\} \mathrm{cm}^{3} \cdot \mathrm{mol}^{-1} \cdot \mathrm{s}^{-1}$ an [WAR84], bezieht sich dabei aber auf den wesentlich höheren Temperaturbereich 500-2000 K. Außerdem beruhen die Werte nicht auf eigene Messungen, sondern sind lediglich Abschätzungen. Ein gemessener Wert für die Gesamtgeschwindigkeitskonstante der Reaktion t-Butyl $+\mathrm{O}_{2}$ im Druckbereich $\mathrm{p}<10$ Torr existiert nur von Bayes et al.: $\mathrm{k}^{298}=(1,41 \pm 0,24) \cdot 10^{13} \mathrm{~cm}^{3} \cdot \mathrm{mol}^{-1} \cdot \mathrm{s}^{-1}$ [LEN80]. Dieser Wert ist um den Faktor 2 größer als der in dieser Arbeit bestimmte Geschwindigkeitskoeffizient. Eine Messung zur Temperaturabhängigkeit der Geschwindigkeitskonstanten wurde von Dilger et al. durchgeführt: $\mathrm{k}=(4,09 \pm 1,1) \cdot 10^{12} \exp \left\{-(-2,4 \pm 0,7) /(\mathrm{RT}) \mathrm{kJ} \cdot \mathrm{mol}^{-1}\right\} \mathrm{cm}^{3} \cdot \mathrm{mol}^{-1} \cdot \mathrm{s}^{-1}$ [DIL97], $241 \mathrm{~K} \leq \mathrm{T} \leq 462 \mathrm{~K}$ Zwar wurde die Reaktion bei 1140 Torr durchgeführt, da aber die Hochdruckgrenze der Geschwindigkeitskonstanten bei einem relativ großen Molekül wie dem t-Butylperoxyradikal bereits erreicht sein könnte, erscheint ein Vergleich nicht völlig abwegig. Für Zimmertemperatur ergibt sich ein Wert von $\mathrm{k}^{298}=1,08 \cdot 10^{13} \mathrm{~cm}^{3} \cdot \mathrm{mol}^{-1} \cdot \mathrm{s}^{-1}$, welcher innerhalb der Fehlergrenzen des Wertes dieser Arbeit liegt. Die Aktivierungsenergie ist ebenfalls negativ, vom Betrage her aber um den Faktor 3,5 kleiner. 


\subsubsection{Cycloheptyl $+\mathrm{O}_{2}$}

\section{Produkte:}

Es wurden keine Produktuntersuchungen durchgeführt. Literatur zu Produkten liegt nicht vor.

$\underline{\text { Reaktionsgeschwindigkeit: }}$

Messergebnisse: Die Geschwindigkeit der Reaktion c-Heptyl $+\mathrm{O}_{2}$ wurde bei Zimmertemperatur relativ zur Reaktion Methoxymethyl $+\mathrm{O}_{2}$ mit Versuchsanordnung A (REMPI) bei $\lambda=440 \mathrm{~nm}$ bestimmt. Die Radikale wurden gemäß Abschnitt 3.1.8 und 3.1.14 erzeugt und auf ihren Muttermassen $\mathrm{m} / \mathrm{z}=97$ und $\mathrm{m} / \mathrm{z}=45$ nachgewiesen. Zur Bestimmung des Geschwindigkeitskoeffizienten wurden die Signalintensitäten bei An- und Abwesenheit von Sauerstoffmolekülen gemessen. Durch Variation der Sauerstoffkonzentration wurden verschiedene Umsätze erzielt. Die folgende Abbildung zeigt in doppeltlogarithmischer Auftragung die Messpunkte. Die zugehörigen Werte finden sich in Abschnitt 5 (Messtabellen). Die gewählten Versuchsbedingungen sind dem Text in der Abbildung oder bei den Messtabellen zu entnehmen.

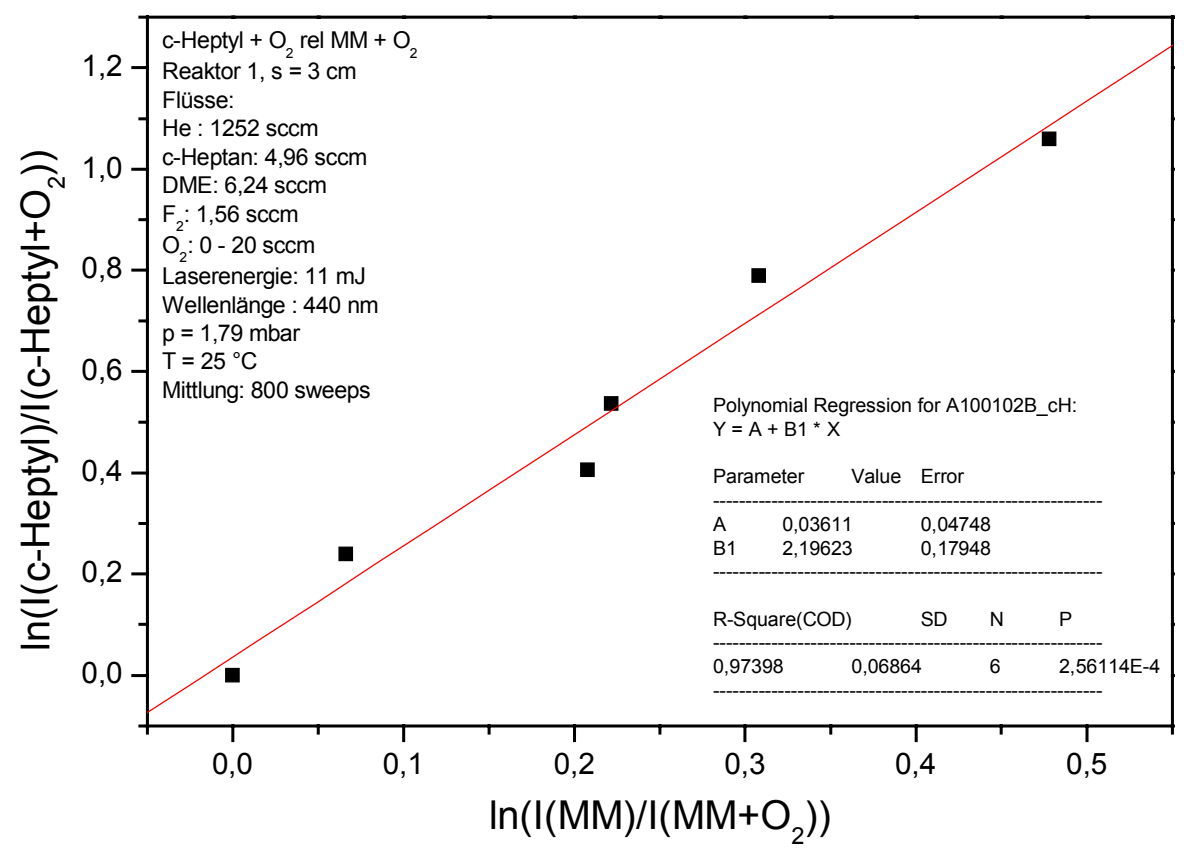

Abbildung 147: c-Heptyl $+\mathrm{O}_{2}$ relativ zu Methoxymethyl $+\mathrm{O}_{2}, \mathrm{~T}=25^{\circ} \mathrm{C}$

Aus der Auftragung ergibt sich:

$$
\mathrm{k}_{\text {rel }}=\mathrm{k}\left(\mathrm{c}-\mathrm{C}_{7} \mathrm{H}_{13}+\mathrm{O}_{2}\right) / \mathrm{k}\left(\mathrm{CH}_{3} \mathrm{OCH}_{2}+\mathrm{O}_{2}\right)=2,20 \pm 0,18 \text {. }
$$

Mit der in Abschnitt 3.4.1 erläuterten Geschwindigkeitskonstante $k_{\text {ref }}=5,97 \cdot 10^{12} \mathrm{~cm}^{3} /(\mathrm{mol} \cdot \mathrm{s})$ für die Referenzreaktion Methoxymethyl $+\mathrm{O}_{2}$ erhält man:

$$
\mathrm{k}^{298 \mathrm{~K}}\left(\mathrm{c}-\mathrm{C}_{7} \mathrm{H}_{13}+\mathrm{O}_{2}\right)=(1,31 \pm 0,12) \cdot 10^{13} \mathrm{~cm}^{3} /(\mathrm{mol} \cdot \mathrm{s}) .
$$

Es lag keine Literatur über die Geschwindigkeit der Reaktion c-Heptyl $+\mathrm{O}_{2}$ vor. 


\subsubsection{Cyclooktyl $+\mathrm{O}_{2}$}

\section{Produkte:}

Es wurden keine Produktuntersuchungen durchgeführt. Literatur zu Produkten liegt nicht vor.

$\underline{\text { Reaktionsgeschwindigkeit: }}$

Messergebnisse: Die Geschwindigkeit der Reaktion c-Oktyl $+\mathrm{O}_{2}$ wurde bei Zimmertemperatur relativ zur Reaktion Methoxymethyl $+\mathrm{O}_{2}$ mit Versuchsanordnung A (REMPI) bei $\lambda=456 \mathrm{~nm}$ bestimmt. Die Radikale wurden gemäß Abschnitt 3.1.9 und 3.1.14 erzeugt und auf ihren Muttermassen $\mathrm{m} / \mathrm{z}=$ 111 und $\mathrm{m} / \mathrm{z}=45$ nachgewiesen. Zur Bestimmung des Geschwindigkeitskoeffizienten wurden die Signalintensitäten bei An- und Abwesenheit von $\mathrm{O}_{2}$ gemessen. Durch Variation der Sauerstoffkonzentration wurden verschiedene Umsätze erzielt. Die folgende Abbildung zeigt in doppeltlogarithmischer Auftragung die Messpunkte. Die zugehörigen Werte finden sich in Abschnitt 5 (Messtabellen). Die gewählten Versuchsbedingungen sind dem Text in der Abbildung oder bei den Messtabellen zu entnehmen.

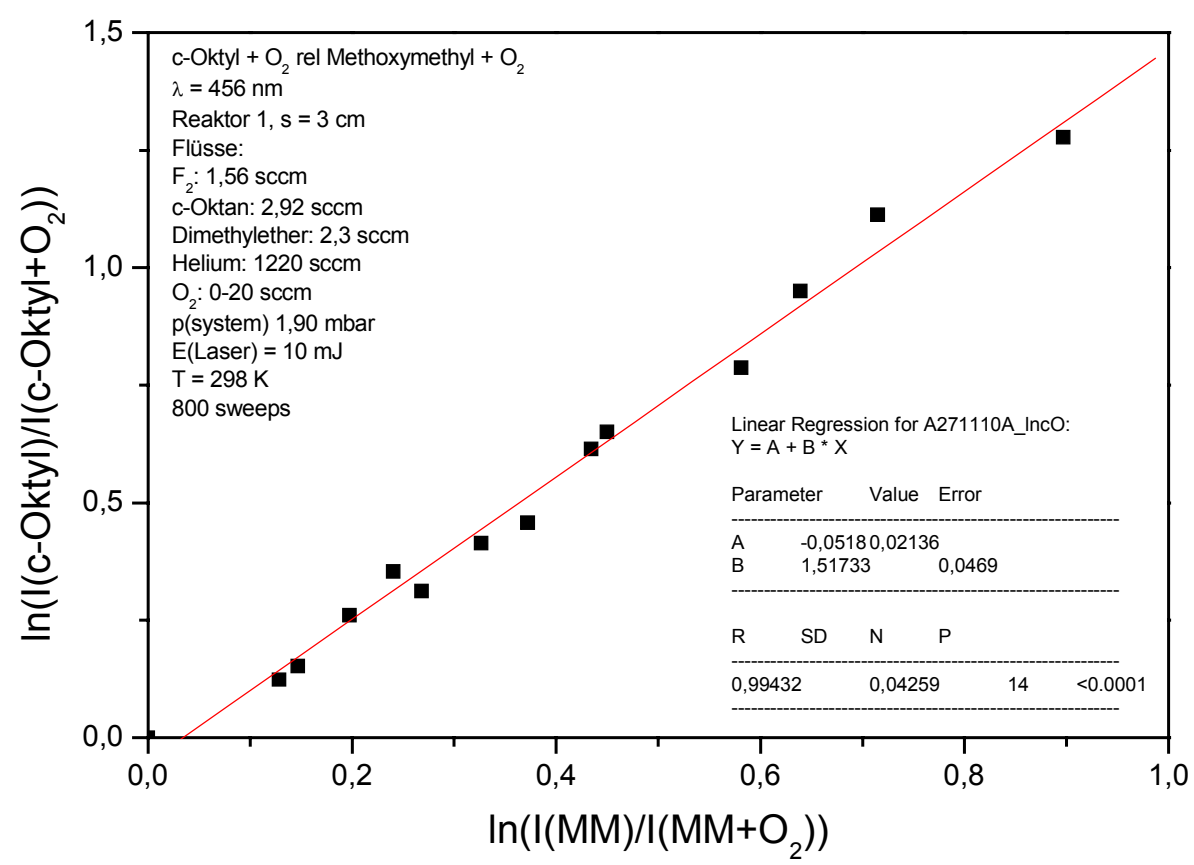

Abbildung 148: c-Oktyl $+\mathrm{O}_{2}$ relativ zu Methoxymethyl $+\mathrm{O}_{2}, \mathrm{~T}=25^{\circ} \mathrm{C}$

Aus der Auftragung ergibt sich:

$$
\mathrm{k}_{\mathrm{rel}}=\mathrm{k}\left(\mathrm{c}-\mathrm{C}_{8} \mathrm{H}_{15}+\mathrm{O}_{2}\right) / \mathrm{k}\left(\mathrm{CH}_{3} \mathrm{OCH}_{2}+\mathrm{O}_{2}\right)=1,52 \pm 0,05 \text {. }
$$

Mit der in Abschnitt 3.4.1 erläuterten Geschwindigkeitskonstante $k_{\text {ref }}=5,97 \cdot 10^{12} \mathrm{~cm}^{3} /(\mathrm{mol} \cdot \mathrm{s})$ für die Referenzreaktion Methoxymethyl $+\mathrm{O}_{2}$ erhält man:

$$
\mathrm{k}^{298 \mathrm{~K}}\left(\mathrm{c}-\mathrm{C}_{8} \mathrm{H}_{15}+\mathrm{O}_{2}\right)=(9,07 \pm 0,30) \cdot 10^{12} \mathrm{~cm}^{3} /(\mathrm{mol} \cdot \mathrm{s})
$$


Temperaturabhängigkeit:

Messergebnisse: Die Temperaturabhängigkeit der Reaktion c-Oktyl $+\mathrm{O}_{2}$ wurde im Intervall $+5{ }^{\circ} \mathrm{C}$ bis $+90{ }^{\circ} \mathrm{C}$ nach dem selben Verfahren untersucht. Die Temperierung erfolgte mit der in Abschnitt 2.1.2 beschriebenen Methode für Reaktor 1. Die nachfolgenden Abbildungen zeigen die Ergebnisse. Die zugehörigen Werte finden sich in Abschnitt 5 (Messtabellen). Die gewählten Versuchsbedingungen sind dem Text in der Abbildung oder bei den Messtabellen zu entnehmen.

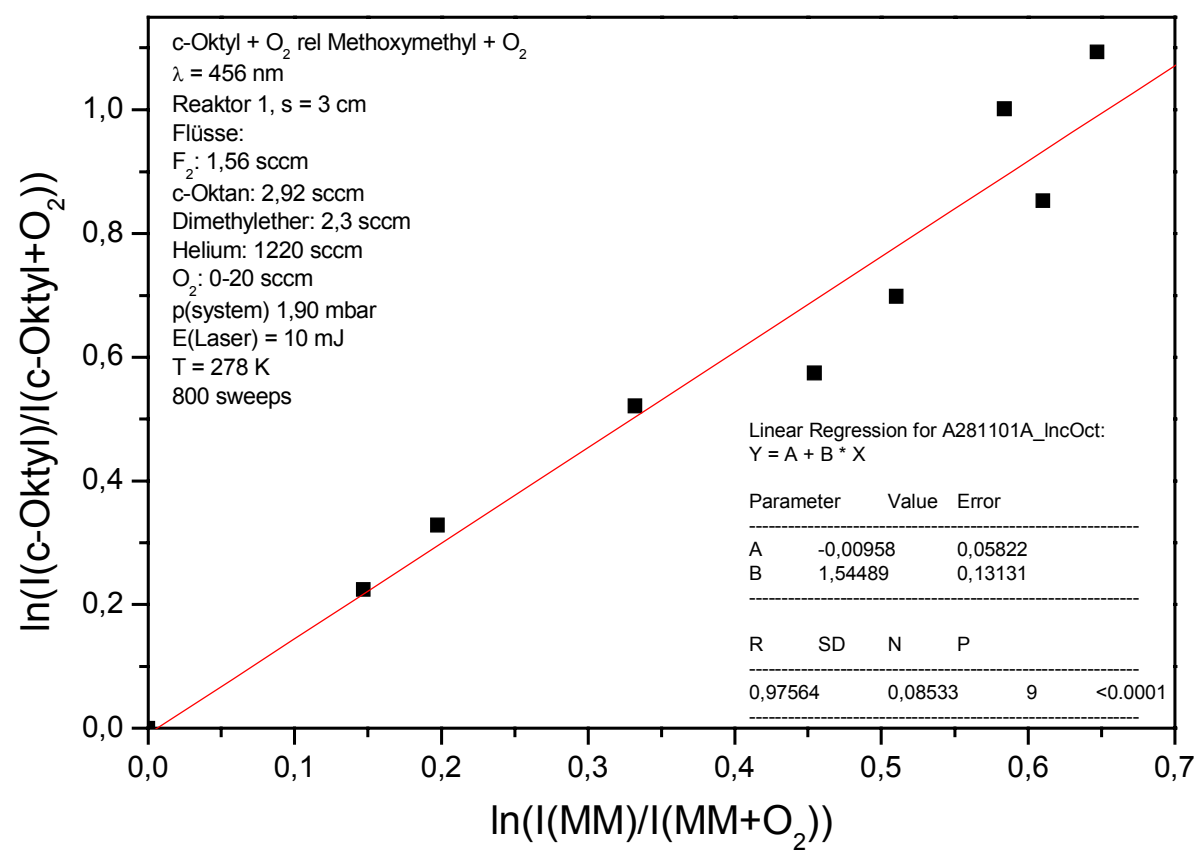

Abbildung 149: c-Oktyl $+\mathrm{O}_{2}$ relativ zu Methoxymethyl $+\mathrm{O}_{2}, \mathrm{~T}=5^{\circ} \mathrm{C}$

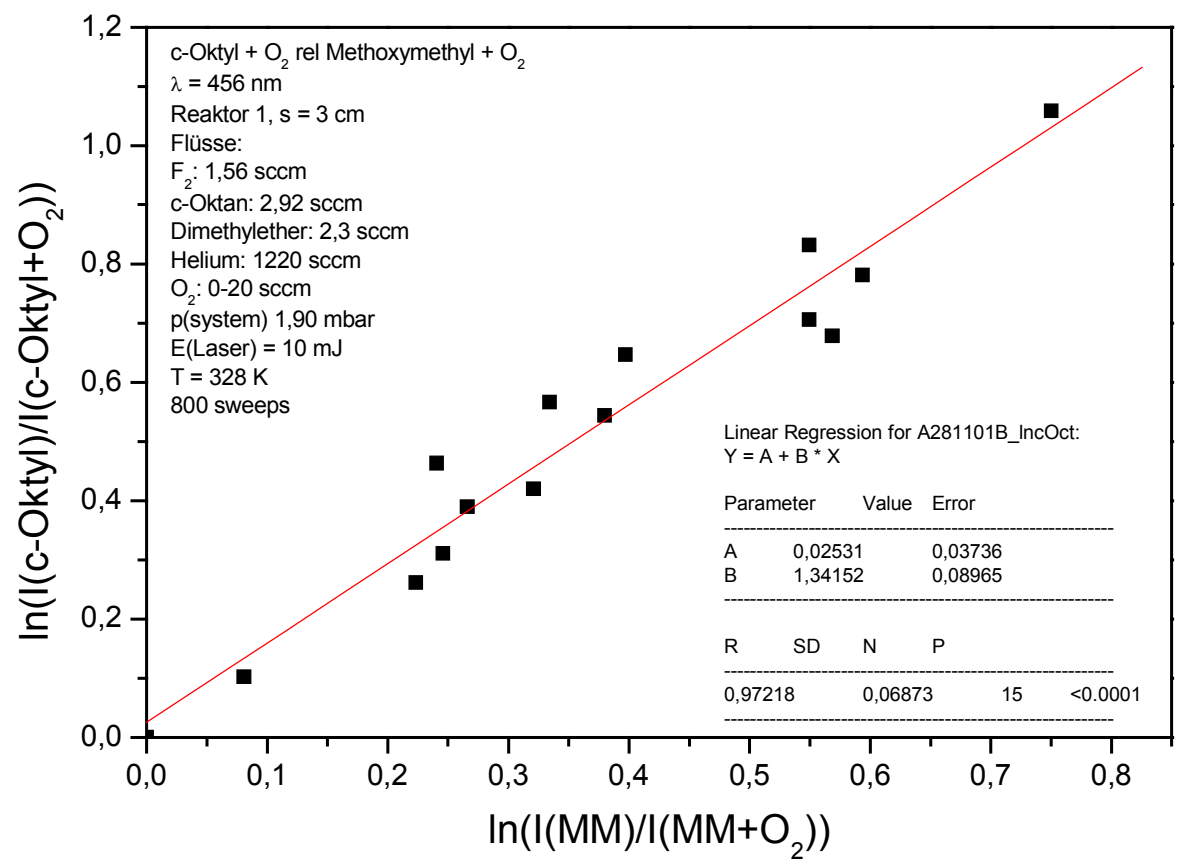

Abbildung 150: c-Oktyl $+\mathrm{O}_{2}$ relativ zu Methoxymethyl $+\mathrm{O}_{2}, \mathrm{~T}=55{ }^{\circ} \mathrm{C}$ 


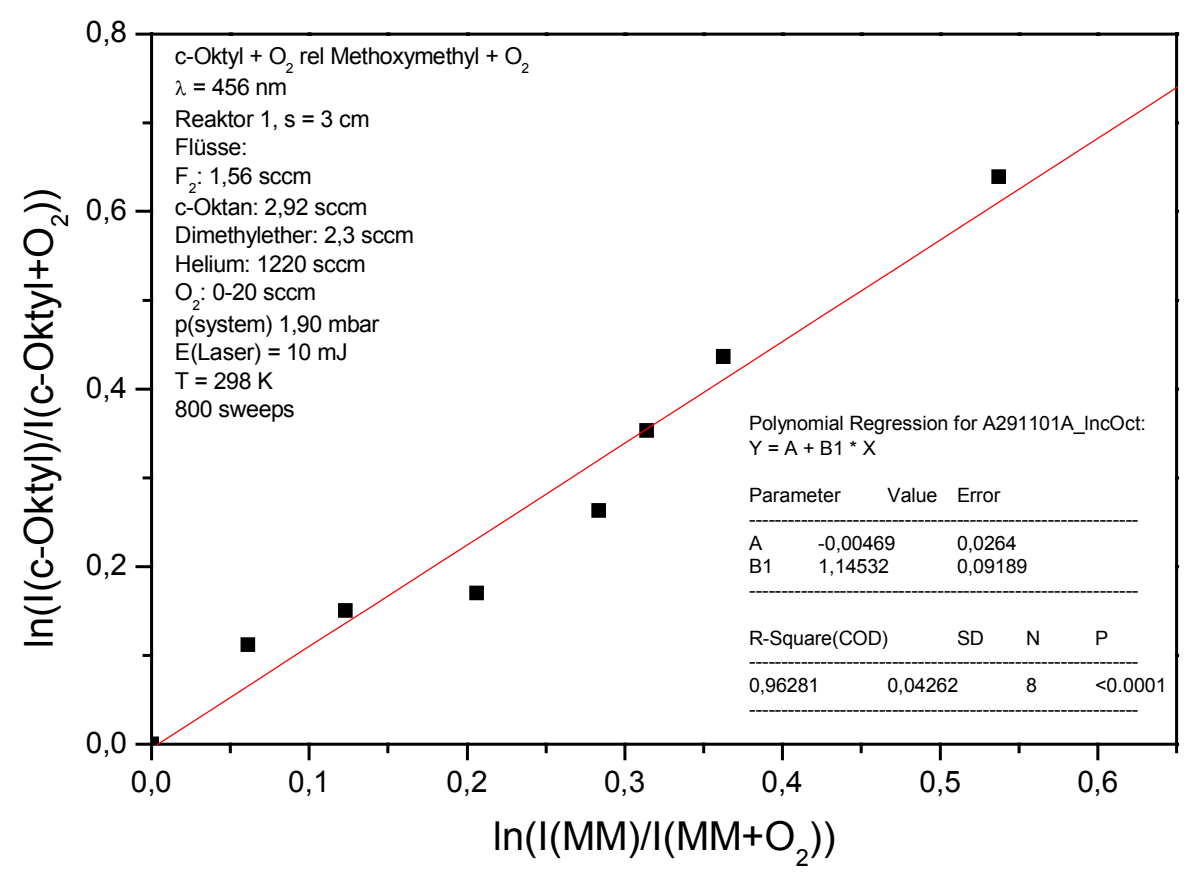

Abbildung 151: c-Oktyl $+\mathrm{O}_{2}$ relativ zu Methoxymethyl $+\mathrm{O}_{2}, \mathrm{~T}=90^{\circ} \mathrm{C}$

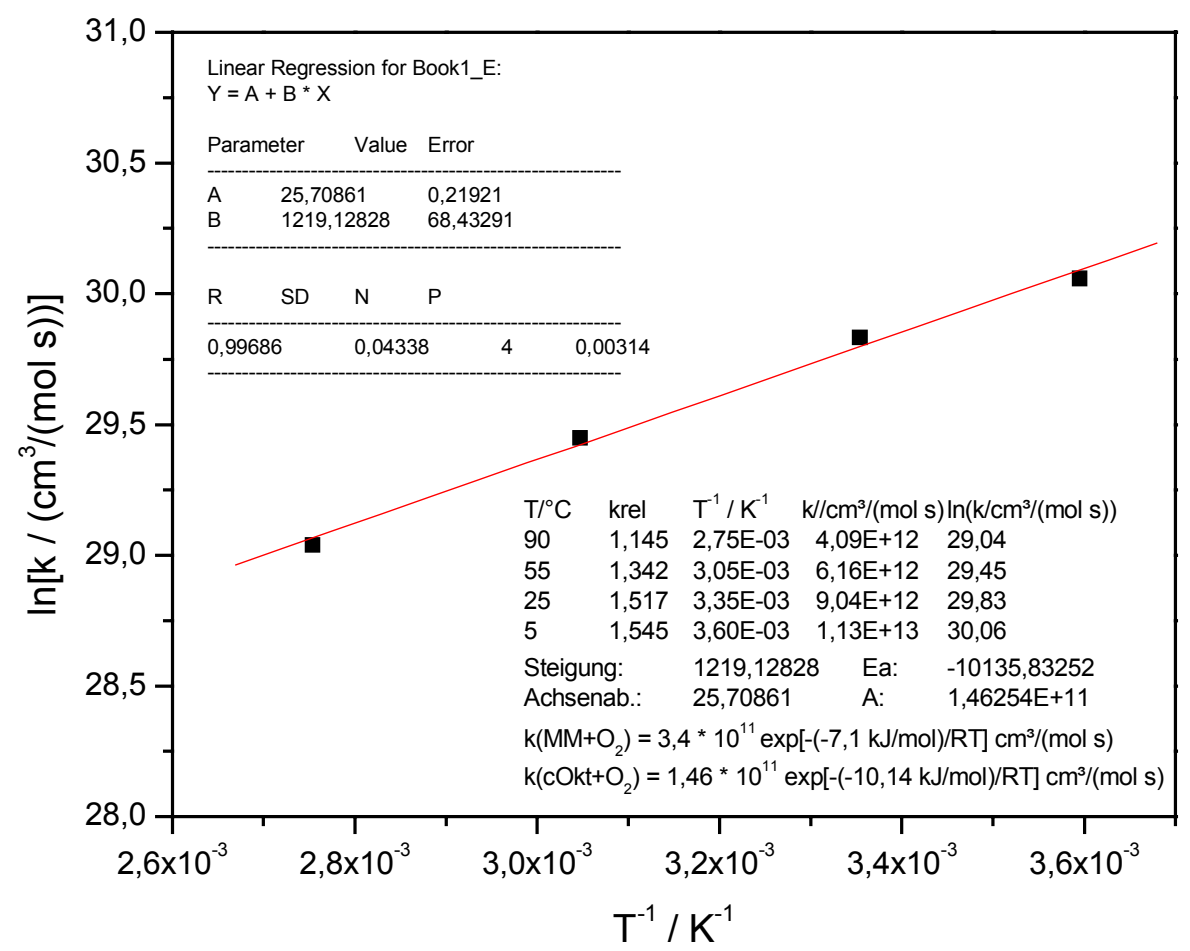

Abbildung 152: c-Oktyl $+\mathbf{O}_{2}$ relativ zu Methoxymethyl $+\mathbf{O}_{2}$, Arrheniusauftragung

Aus der Arrheniusauftragung ergibt sich:

$$
\begin{gathered}
\mathrm{k}=(1,46 \pm 0,36) \cdot 10^{11} \exp \left\{-(-10,14 \pm 0,57) /(\mathrm{RT}) \mathrm{kJ} \cdot \mathrm{mol}^{-1}\right\} \mathrm{cm}^{3} \cdot \mathrm{mol}^{-1} \cdot \mathrm{s}^{-1} \\
\mathrm{k}^{298}=(8,72 \pm 4,9) \cdot 10^{12} \mathrm{~cm}^{3} \cdot \mathrm{mol}^{-1} \cdot \mathrm{s}^{-1}
\end{gathered}
$$

Es liegt keine Literatur über die Geschwindigkeit der Reaktion c-Oktyl $+\mathrm{O}_{2}$ vor. 


\subsubsection{Cyclopentadienyl $+\mathrm{O}_{2}$}

\section{Produkte:}

Es wurden keine Produktuntersuchungen durchgeführt. Literatur zu Produkten liegt nicht vor.

Reaktionsgeschwindigkeit:

Messergebnisse: Die Geschwindigkeit der Reaktion c-Pentadienyl $+\mathrm{O}_{2}$ wurde bei Zimmertemperatur relativ zur Reaktion Ethyl $+\mathrm{O}_{2}$ mit Versuchsanordnung A (REMPI) bei $\lambda=424,87 \mathrm{~nm}$ bestimmt. Die Radikale wurden gemäß Abschnitt 3.1.13 und 3.1.2 erzeugt und auf ihren Muttermassen $\mathrm{m} / \mathrm{z}=65$ und $\mathrm{m} / \mathrm{z}=29$ nachgewiesen. Zur Bestimmung des Geschwindigkeitskoeffizienten wurden die Signalintensitäten bei An- und Abwesenheit von Sauerstoffatomen gemessen. Durch Variation der Sauerstoffkonzentration wurden verschiedene Umsätze erzielt. Die folgende Abbildung zeigt in doppeltlogarithmischer Auftragung die Messpunkte. Die zugehörigen Werte finden sich in Abschnitt 5 (Messtabellen). Die gewählten Versuchsbedingungen sind dem Text in der Abbildung oder bei den Messtabellen zu entnehmen.

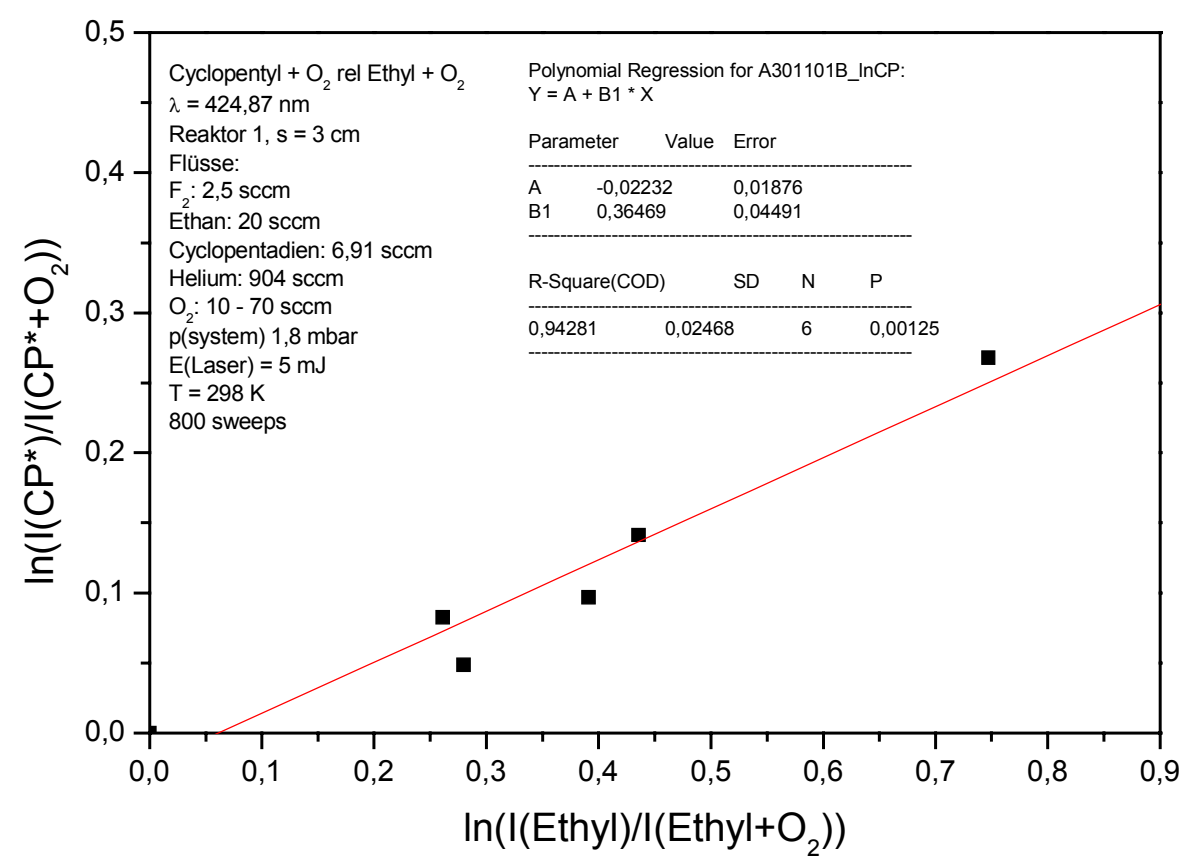

Abbildung 153: c-Pentadienyl $+\mathrm{O}_{2}$ relativ zu Ethyl $+\mathrm{O}_{2}, \mathrm{~T}=25^{\circ} \mathrm{C}$

Aus der Auftragung ergibt sich:

$$
\mathrm{k}_{\text {rel }}=\mathrm{k}\left(\mathrm{c}-\mathrm{C}_{5} \mathrm{H}_{5}+\mathrm{O}_{2}\right) / \mathrm{k}\left(\mathrm{C}_{2} \mathrm{H}_{5}+\mathrm{O}_{2}\right)=0,36 \pm 0,05
$$

Mit einer unter gleichen Bedingungen gültigen Geschwindigkeitskonstante $k_{\text {ref }}=6,27 \cdot 10^{11}$ $\mathrm{cm}^{3} /(\mathrm{mol} \cdot \mathrm{s})$ nach [SLA84] für die Referenzreaktion Ethyl $+\mathrm{O}_{2}$ erhält man:

$$
\mathrm{k}^{298 \mathrm{~K}}\left(\mathrm{c}-\mathrm{C}_{5} \mathrm{H}_{5}+\mathrm{O}_{2}\right)=(2,25 \pm 0,31) \cdot 10^{11} \mathrm{~cm}^{3} /(\mathrm{mol} \cdot \mathrm{s}) \text {. }
$$

Es liegt keine Literatur über die Geschwindigkeit der Reaktion c-Pentadienyl $+\mathrm{O}_{2}$ vor. 


\subsubsection{2-Chlor-2-Methyl-Propyl $+\mathrm{O}_{2}$}

\section{Produkte:}

Es wurden keine Produktuntersuchungen durchgeführt. Literatur zu Produkten liegt nicht vor.

$\underline{\text { Reaktionsgeschwindigkeit: }}$

Messergebnisse: Die Geschwindigkeit der Reaktion iso- $\mathrm{C}_{4} \mathrm{H}_{8} \mathrm{Cl}+\mathrm{O}_{2}$ wurde bei Zimmertemperatur relativ zur Reaktion Methoxymethyl $+\mathrm{O}_{2}$ mit Versuchsanordnung A (REMPI) bei $\lambda=440 \mathrm{~nm}$ bestimmt. Die Radikale wurden gemäß Abschnitt 3.1.11 und 3.1.14 erzeugt und auf ihren Muttermassen $\mathrm{m} / \mathrm{z}=91$ und $\mathrm{m} / \mathrm{z}=45$ nachgewiesen. Zur Bestimmung des Geschwindigkeitskoeffizienten wurden die Signalintensitäten bei An- und Abwesenheit von $\mathrm{O}_{2}$ gemessen. Durch Variation der Sauerstoffkonzentration wurden verschiedene Umsätze erzielt. Die folgende Abbildung zeigt in doppeltlogarithmischer Auftragung die Messpunkte. Die zugehörigen Werte finden sich in Abschnitt 5 (Messtabellen). Die gewählten Versuchsbedingungen sind dem Text in der Abbildung oder bei den Messtabellen zu entnehmen.

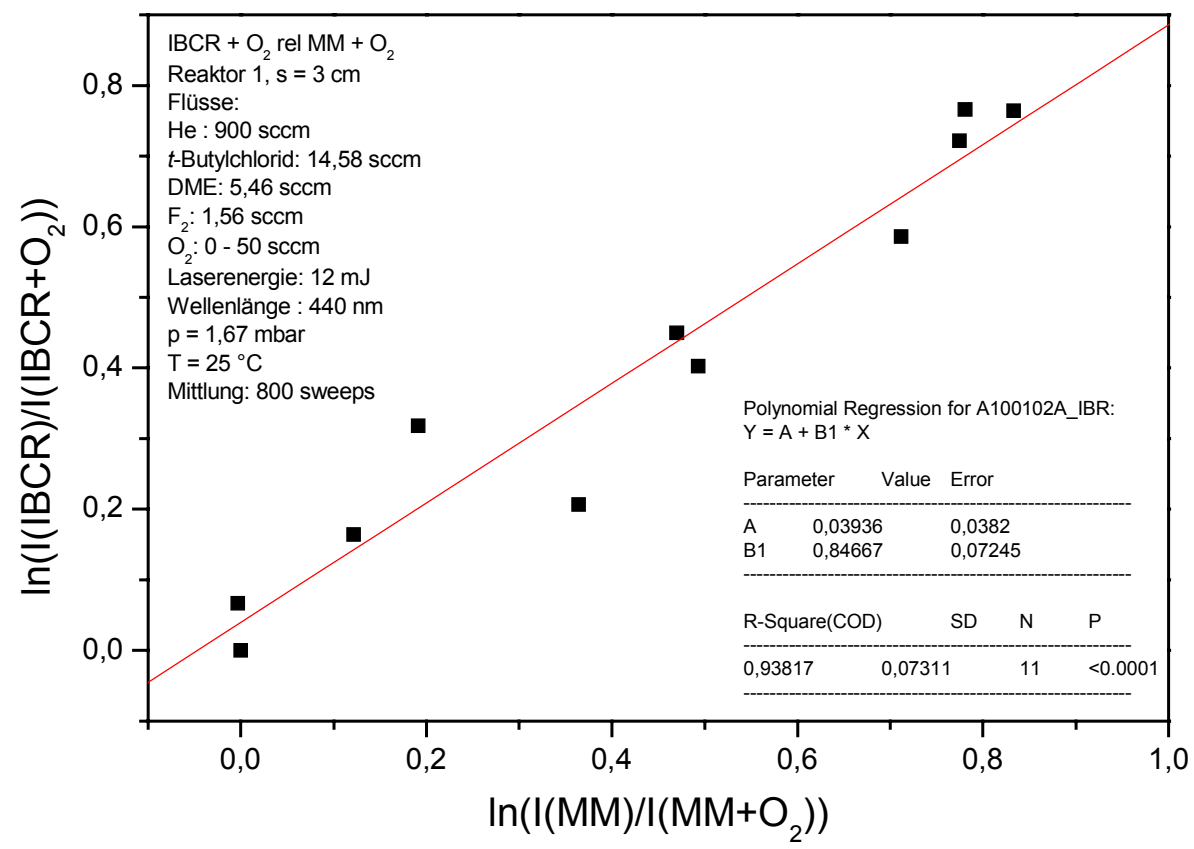

Abbildung 154: iso- $\mathrm{C}_{4} \mathrm{H}_{8} \mathrm{Cl}+\mathrm{O}_{2}$ relativ zu Methoxymethyl $+\mathrm{O}_{2}, \mathrm{~T}=25^{\circ} \mathrm{C}$

Aus der Auftragung ergibt sich:

$$
\mathrm{k}_{\text {rel }}=\mathrm{k}\left(\text { iso- } \mathrm{C}_{4} \mathrm{H}_{8} \mathrm{Cl}+\mathrm{O}_{2}\right) / \mathrm{k}\left(\mathrm{CH}_{3} \mathrm{OCH}_{2}+\mathrm{O}_{2}\right)=0,85 \pm 0,08 \text {. }
$$

Mit der in Abschnitt 3.4.1 erläuterten Geschwindigkeitskonstante $k_{\text {ref }}=5,97 \cdot 10^{12} \mathrm{~cm}^{3} /(\mathrm{mol} \cdot \mathrm{s})$ für die Referenzreaktion Methoxymethyl $+\mathrm{O}_{2}$ erhält man:

$$
\mathrm{k}^{298 \mathrm{~K}}\left(\text { iso- } \mathrm{C}_{4} \mathrm{H}_{8} \mathrm{Cl}+\mathrm{O}_{2}\right)=(5,07 \pm 0,48) \cdot 10^{12} \mathrm{~cm}^{3} /(\mathrm{mol} \cdot \mathrm{s})
$$

Es liegt keine Literatur über die Geschwindigkeit der Reaktion iso- $\mathrm{C}_{4} \mathrm{H}_{8} \mathrm{Cl}+\mathrm{O}_{2}$ vor. 


\subsection{Reaktionen von Radikalen mit $\mathrm{O}_{3}$}

Reaktionen von Kohlenwasserstoffradikalen mit Ozon gelten als O-Atom-Übertragungsreaktionen. Es wird von einer Adduktbildung unter Abspaltung von $\mathrm{O}_{2}$ und anschließendem Zerfall gemäß folgendem allgemeinen Mechanismus ausgegangen:

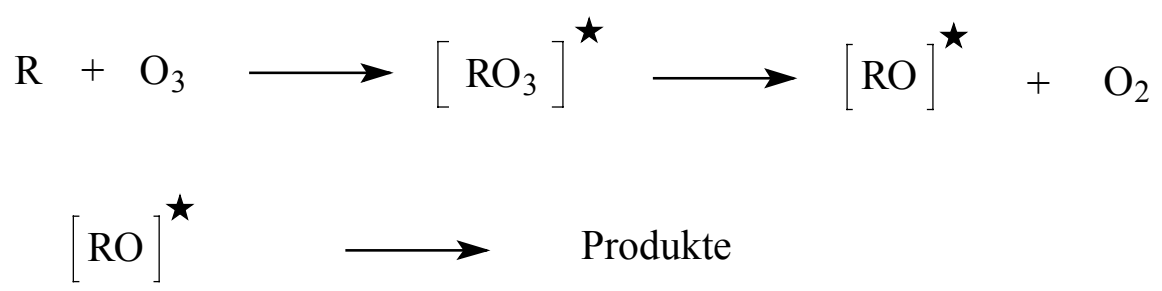

$\mathrm{Ob}$ es tatsächlich zur Bildung eines Zwischenzustands $\mathrm{RO}_{3}$ kommt, wie hier dargestellt, oder ob es sich um einen bloßen Abstreifvorgang der O-Atome handelt, soll hier offen gelassen werden. Für den Zerfall des über (1) gebildeten Alkoxyradikals RO* gilt im Prinzip das, was in Abschnitt 3.3 für die Reaktion Radikal + O gesagt wurde. Allerdings unterscheiden sich die Standardbildungsenthalpien von O-Atomen und $\mathrm{O}_{3}$-Molekülen um mehr als $100 \mathrm{~kJ} / \mathrm{mol}$. Dadurch sind die gebildeten Alkoxyradikale bei den Reaktionen $\mathrm{R}+\mathrm{O}_{3}$ nicht so stark angeregt wie bei $\mathrm{R}+\mathrm{O}$, was sich in veränderte Zerfallswege auswirken kann ([HOY99], [SEE94]). Außerdem kann das durch (1) entstehende $\mathrm{O}_{2}$-Molekül im Falle einer Adduktbildung zu $\mathrm{RO}_{3}$ eine gewisse Restenergie mitnehmen, was die Anregungsenergie des Alkoxyradikals weiter herabsetzt. Neben ihrer großen Bedeutung für die Chemie der Troposphäre und der Stratosphäre sind die Reaktionen von Radikalen mit $\mathrm{O}_{3}$ damit auch für Aufklärung des unimolekularen Charakters der Reaktionen $\mathrm{R}+\mathrm{O}$ wichtig, soweit dadurch mechanistische Informationen gewonnen werden. Im folgenden werden die Untersuchungen zur Reaktionsgeschwindigkeit der Reaktionen von t-Butyl, c-Heptyl, c-Oktyl und 2-Chlor-2-Methyl-Propyl mit Ozon dargestellt.

\subsection{1 t-Butyl $+\mathrm{O}_{3}$}

Reaktionsgeschwindigkeit:

Messergebnisse: Die Geschwindigkeit der Reaktion t-Butyl $+\mathrm{O}_{3}$ wurde bei Zimmertemperatur relativ zur Reaktion Methoxymethyl $+\mathrm{O}_{3}$ mit Versuchsanordnung A (REMPI) bei $\lambda=505 \mathrm{~nm}$ bestimmt. Die Radikale wurden gemäß Abschnitt 3.1.5 und 3.1.14 erzeugt und auf ihren Muttermassen $\mathrm{m} / \mathrm{z}=57$ und $\mathrm{m} / \mathrm{z}=45$ in eindeutiger Weise nachgewiesen. Zur Bestimmung des Geschwindigkeitskoeffizienten wurden die Signalintensitäten bei An- und Abwesenheit von $\mathrm{O}_{3}$ gemessen. Durch Variation der Ozonkonzentration wurden verschiedene Umsätze erzielt. Die folgende Abbildung zeigt in doppeltlogarithmischer Auftragung die Messpunkte. Die zugehörigen Werte finden sich in 
Abschnitt 5 (Messtabellen). Die gewählten Versuchsbedingungen sind dem Text in der Abbildung oder bei den Messtabellen zu entnehmen.

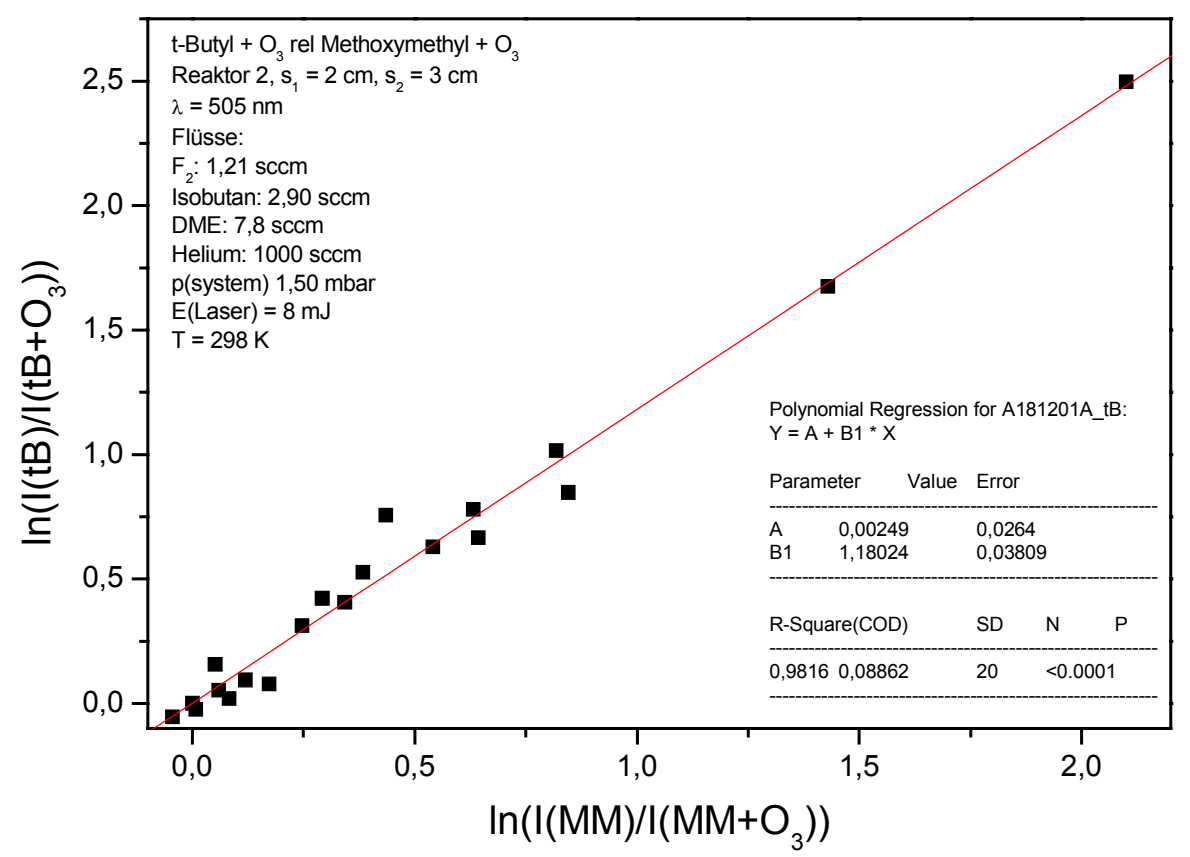

Abbildung 155: t-Butyl $+\mathrm{O}_{3}$ relativ zu Methoxymethyl $+\mathrm{O}_{3}, \mathrm{~T}=25^{\circ} \mathrm{C}$

Aus der Auftragung ergibt sich:

$$
\mathrm{k}_{\mathrm{rel}}=\mathrm{k}\left(\mathrm{t}-\mathrm{C}_{4} \mathrm{H}_{9}+\mathrm{O}_{3}\right) / \mathrm{k}\left(\mathrm{CH}_{3} \mathrm{OCH}_{2}+\mathrm{O}_{3}\right)=1,18 \pm 0,04 \text {. }
$$

Mit der bei [HAC00] und [NAC98] erläuterten Geschwindigkeitskonstante $\mathrm{k}_{\text {ref }}=2,6 \cdot 10^{13}$ $\mathrm{cm}^{3} /$ (mol·s) für die Referenzreaktion Methoxymethyl $+\mathrm{O}_{3}$ erhält man:

$$
\mathrm{k}^{298 \mathrm{~K}}\left(\mathrm{t}-\mathrm{C}_{4} \mathrm{H}_{9}+\mathrm{O}_{3}\right)=(3,06 \pm 0,11) \cdot 10^{13} \mathrm{~cm}^{3} /(\mathrm{mol} \cdot \mathrm{s})
$$

Diskussion: Die Geschwindigkeit der Reaktion t-Butyl $+\mathrm{O}_{3}$ bei Zimmertemperatur und einem Druck von 2 Torr wurde bereits von Paltenghi, Ogryzlo und Bayes gemessen [PAL84]. Die Radikale wurden photolytisch aus 2,4,4-Trimethyl-1-Penten erzeugt, ihre Konzentration wurde mit Hilfe eines Photoionisationsmassenspektrometer als Funktion der Zeit verfolgt. Das Ergebnis $\mathrm{k}^{298 \mathrm{~K}}\left(\mathrm{t}-\mathrm{C}_{4} \mathrm{H}_{9}+\mathrm{O}_{3}\right)=(3,28 \pm 0,69) \cdot 10^{13} \mathrm{~cm}^{3} /(\mathrm{mol} \cdot \mathrm{s})$ stimmt mit dem hier vorgestellten Wert sehr gut überein. Weitere Literatur zu dieser Reaktion liegt nicht vor. 


\subsubsection{Cycloheptyl $+\mathrm{O}_{3}$}

\section{Reaktionsgeschwindigkeit:}

Messergebnisse: Die Geschwindigkeit der Reaktion c-Heptyl $+\mathrm{O}_{3}$ wurde bei Zimmertemperatur relativ zur Reaktion Methoxymethyl $+\mathrm{O}_{3}$ mit Versuchsanordnung A (REMPI) bei $\lambda=440 \mathrm{~nm}$ bestimmt. Die Radikale wurden gemäß Abschnitt 3.1.8 und 3.1.14 erzeugt und auf ihren Muttermassen $\mathrm{m} / \mathrm{z}=97$ und $\mathrm{m} / \mathrm{z}=45$ nachgewiesen. Zur Bestimmung des Geschwindigkeitskoeffizienten wurden die Signalintensitäten bei An- und Abwesenheit von $\mathrm{O}_{3}$ gemessen. Durch Variation der Ozonkonzentration wurden verschiedene Umsätze erzielt. Die folgende Abbildung zeigt in doppeltlogarithmischer Auftragung die Messpunkte. Die zugehörigen Werte finden sich in Abschnitt 5 (Messtabellen). Die gewählten Versuchsbedingungen sind dem Text in der Abbildung oder bei den Messtabellen zu entnehmen.

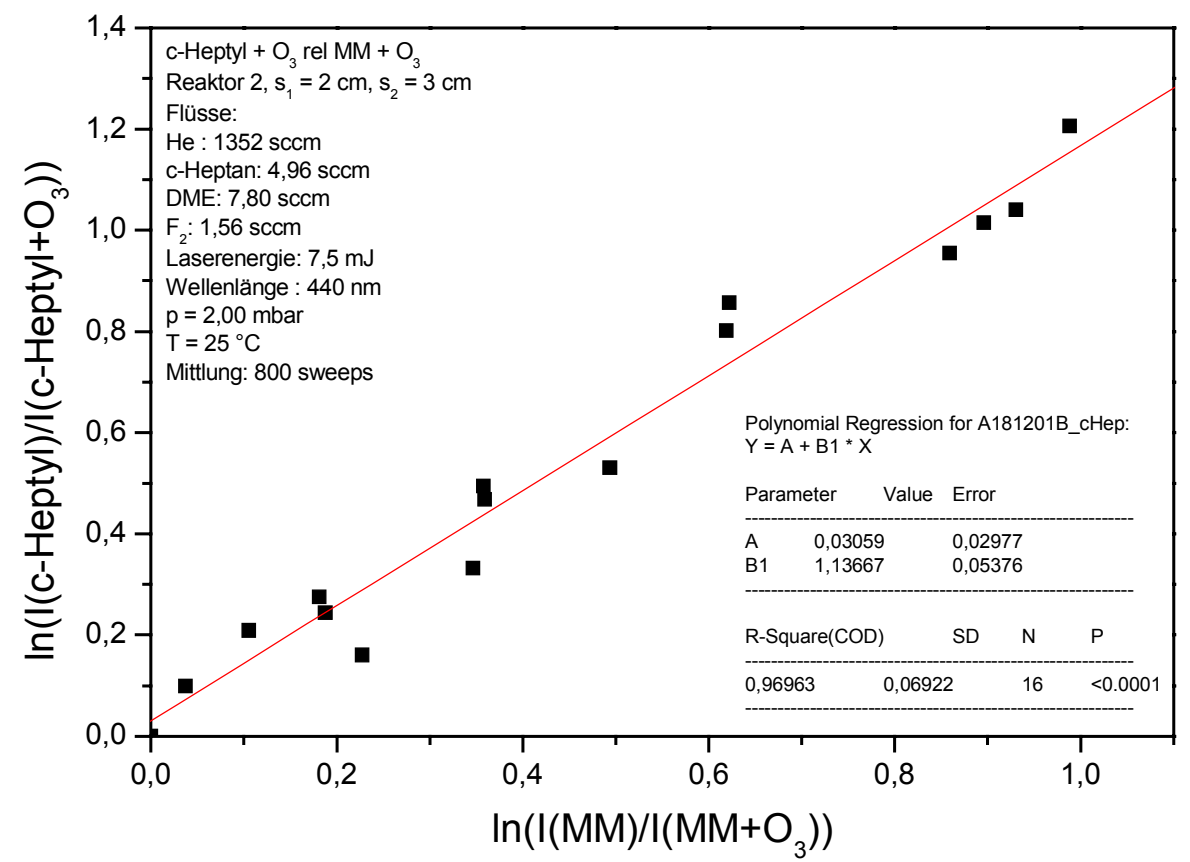

Abbildung 156: c-Heptyl $+\mathrm{O}_{3}$ relativ zu Methoxymethyl $+\mathrm{O}_{3}, \mathrm{~T}=25^{\circ} \mathrm{C}$

Aus der Auftragung ergibt sich:

$$
\mathrm{k}_{\mathrm{rel}}=\mathrm{k}\left(\mathrm{c}-\mathrm{C}_{7} \mathrm{H}_{13}+\mathrm{O}_{3}\right) / \mathrm{k}\left(\mathrm{CH}_{3} \mathrm{OCH}_{2}+\mathrm{O}_{3}\right)=1,14 \pm 0,06 \text {. }
$$

Mit der bei [HAC00] und [NAC98] erläuterten Geschwindigkeitskonstante $k_{\text {ref }}=2,6 \cdot 10^{13}$ $\mathrm{cm}^{3} /(\mathrm{mol} \cdot \mathrm{s})$ für die Referenzreaktion Methoxymethyl $+\mathrm{O}_{3}$ erhält man:

$$
\mathrm{k}^{298 \mathrm{~K}}\left(\mathrm{c}-\mathrm{C}_{7} \mathrm{H}_{13}+\mathrm{O}_{3}\right)=(2,96 \pm 0,16) \cdot 10^{13} \mathrm{~cm}^{3} /(\mathrm{mol} \cdot \mathrm{s})
$$

Es liegt keine Literatur über die Geschwindigkeit der Reaktion c-Heptyl $+\mathrm{O}_{3}$ vor. 


\subsubsection{Cyclooktyl $+\mathrm{O}_{3}$}

\section{$\underline{\text { Reaktionsgeschwindigkeit: }}$}

Messergebnisse: Die Geschwindigkeit der Reaktion c-Oktyl $+\mathrm{O}_{3}$ wurde bei Zimmertemperatur relativ zur Reaktion Methoxymethyl $+\mathrm{O}_{3}$ mit Versuchsanordnung A (REMPI) bei $\lambda=440 \mathrm{~nm}$ bestimmt. Die Radikale wurden gemäß Abschnitt 3.1.9 und 3.1.14 erzeugt und auf ihren Muttermassen $\mathrm{m} / \mathrm{z}=111$ und $\mathrm{m} / \mathrm{z}=45$ nachgewiesen. Zur Bestimmung des Geschwindigkeitskoeffizienten wurden die Signalintensitäten bei An- und Abwesenheit von $\mathrm{O}_{3}$ gemessen. Durch Variation der Ozonkonzentration wurden verschiedene Umsätze erzielt. Die folgende Abbildung zeigt in doppeltlogarithmischer Auftragung die Messpunkte. Die zugehörigen Werte finden sich in Abschnitt 5 (Messtabellen). Die gewählten Versuchsbedingungen sind dem Text in der Abbildung oder bei den Messtabellen zu entnehmen.

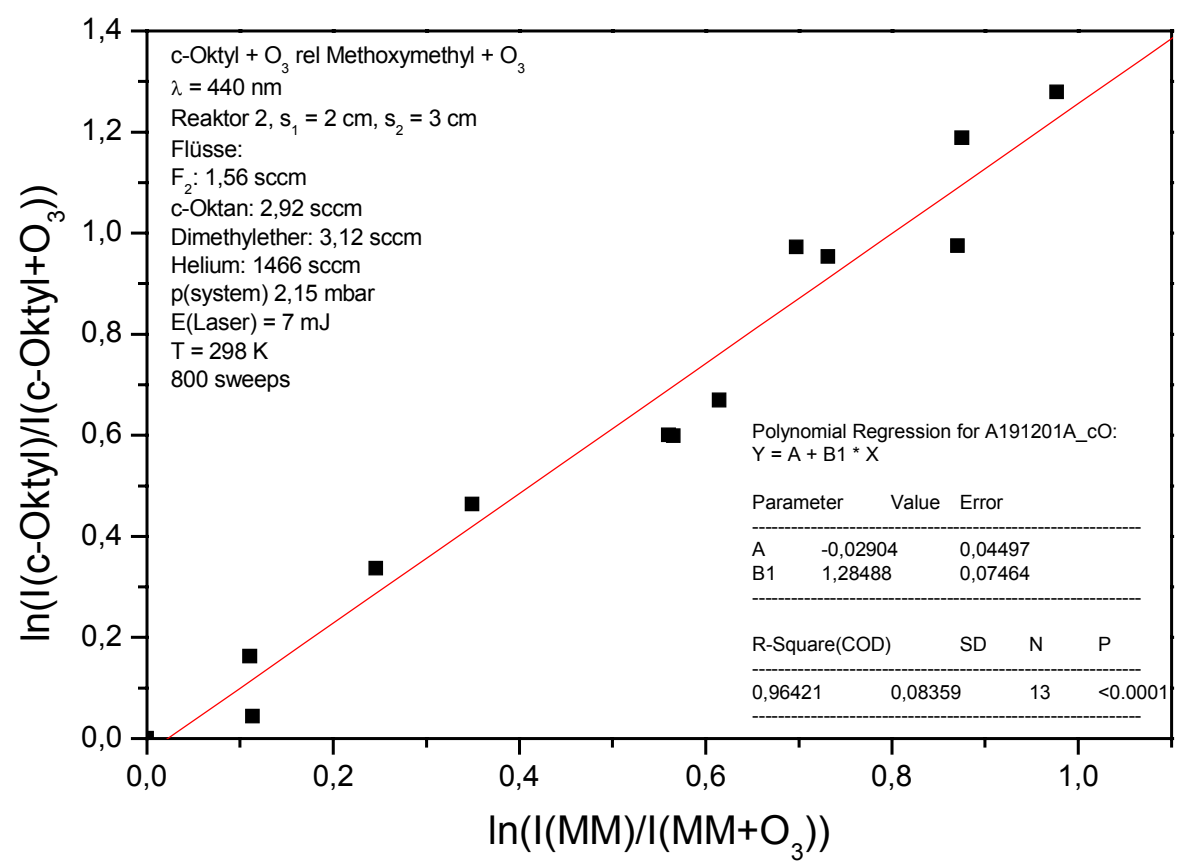

Abbildung 157: c-Oktyl $+\mathrm{O}_{3}$ relativ zu Methoxymethyl $+\mathrm{O}_{3}, \mathrm{~T}=25^{\circ} \mathrm{C}$

Aus der Auftragung ergibt sich:

$$
\mathrm{k}_{\text {rel }}=\mathrm{k}\left(\mathrm{c}-\mathrm{C}_{8} \mathrm{H}_{15}+\mathrm{O}_{3}\right) / \mathrm{k}\left(\mathrm{CH}_{3} \mathrm{OCH}_{2}+\mathrm{O}_{3}\right)=1,28 \pm 0,08 \text {. }
$$

Mit der in [HAC00] und [NAC98] erläuterten Geschwindigkeitskonstante von $\mathrm{k}_{\mathrm{ref}}=2,6 \cdot 10^{13}$ $\mathrm{cm}^{3} /(\mathrm{mol} \cdot \mathrm{s})$ für die Referenzreaktion Methoxymethyl $+\mathrm{O}_{3}$ erhält man:

$$
\mathrm{k}^{298 \mathrm{~K}}\left(\mathrm{c}-\mathrm{C}_{8} \mathrm{H}_{15}+\mathrm{O}_{3}\right)=(3,33 \pm 0,21) \cdot 10^{13} \mathrm{~cm}^{3} /(\mathrm{mol} \cdot \mathrm{s}) .
$$

Es liegt keine Literatur über die Geschwindigkeit der Reaktion c-Oktyl + $\mathrm{O}_{3}$ vor. 


\subsubsection{2-Chlor-2-Methyl-Propyl $+\mathrm{O}_{3}$}

\section{Reaktionsgeschwindigkeit:}

Messergebnisse: Die Geschwindigkeit der Reaktion iso- $\mathrm{C}_{4} \mathrm{H}_{8} \mathrm{Cl}+\mathrm{O}_{3}$ wurde bei Zimmertemperatur relativ zur Reaktion Methoxymethyl $+\mathrm{O}_{3}$ mit Versuchsanordnung A (REMPI) bei $\lambda=440 \mathrm{~nm}$ bestimmt. Die Radikale wurden gemäß Abschnitt 3.1.11 und 3.1.14 erzeugt und auf ihren Muttermassen $\mathrm{m} / \mathrm{z}=91$ und $\mathrm{m} / \mathrm{z}=45$ nachgewiesen. Zur Bestimmung des Geschwindigkeitskoeffizienten wurden die Signalintensitäten bei An- und Abwesenheit von $\mathrm{O}_{3}$ gemessen. Durch Variation der Ozonkonzentration wurden verschiedene Umsätze erzielt. Die folgende Abbildung zeigt in doppeltlogarithmischer Auftragung die Messpunkte. Die zugehörigen Werte finden sich in Abschnitt 5 (Messtabellen). Die gewählten Versuchsbedingungen sind dem Text in der Abbildung oder bei den Messtabellen zu entnehmen.

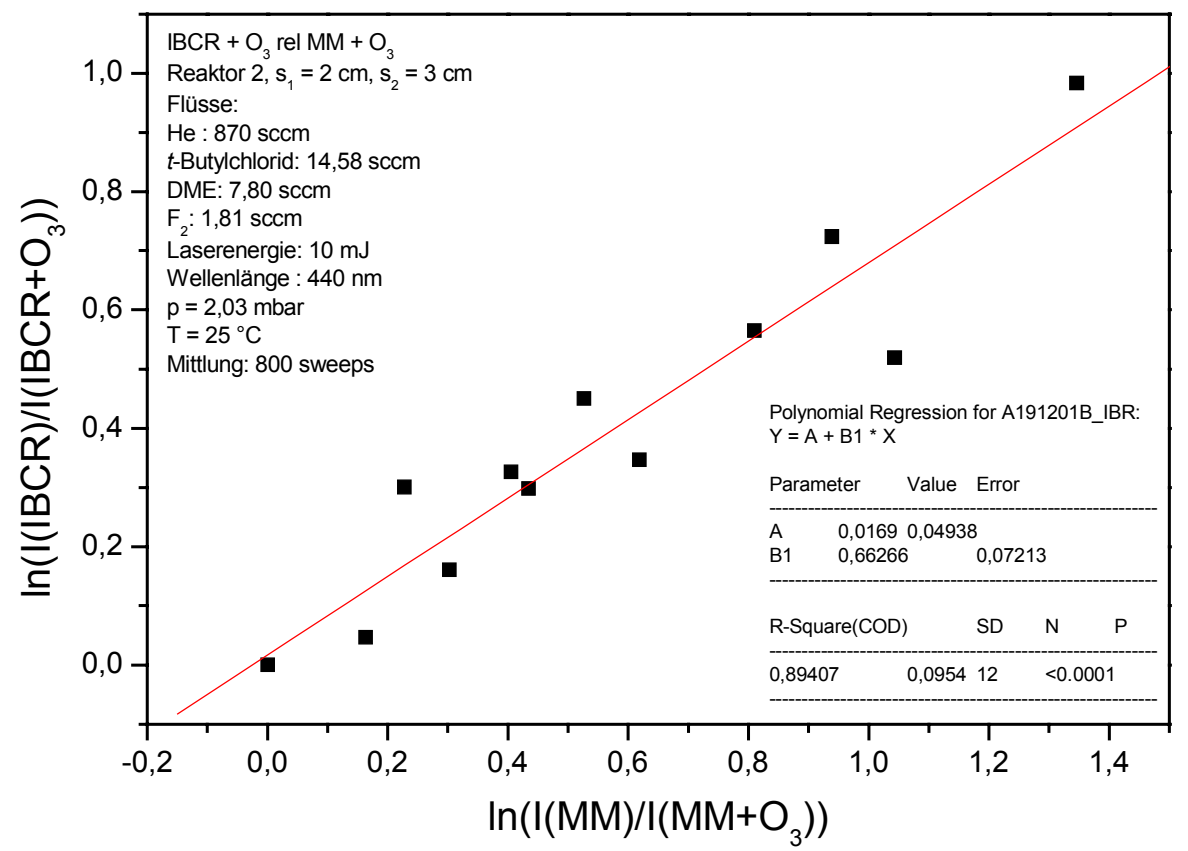

Abbildung 158: iso- $\mathrm{C}_{4} \mathrm{H}_{8} \mathrm{Cl}+\mathrm{O}_{3}$ relativ zu Methoxymethyl $+\mathrm{O}_{3}, \mathrm{~T}=25^{\circ} \mathrm{C}$

Aus der Auftragung ergibt sich:

$$
\mathrm{k}_{\text {rel }}=\mathrm{k}\left(\text { iso- } \mathrm{C}_{4} \mathrm{H}_{8} \mathrm{Cl}+\mathrm{O}_{3}\right) / \mathrm{k}\left(\mathrm{CH}_{3} \mathrm{OCH}_{2}+\mathrm{O}_{3}\right)=0,66 \pm 0,08 \text {. }
$$

Mit der in [HAC00] und [NAC98] erläuterten Geschwindigkeitskonstante von $\mathrm{k}_{\mathrm{ref}}=2,6 \cdot 10^{13}$ $\mathrm{cm}^{3} /(\mathrm{mol} \cdot \mathrm{s})$ für die Referenzreaktion Methoxymethyl $+\mathrm{O}_{3}$ erhält man:

$$
\mathrm{k}^{298 \mathrm{~K}}\left(\text { iso- } \mathrm{C}_{4} \mathrm{H}_{8} \mathrm{Cl}+\mathrm{O}_{3}\right)=(1,71 \pm 0,21) \cdot 10^{13} \mathrm{~cm}^{3} /(\mathrm{mol} \cdot \mathrm{s})
$$

Es liegt keine Literatur über die Geschwindigkeit der Reaktion iso- $\mathrm{C}_{4} \mathrm{H}_{8} \mathrm{Cl}+\mathrm{O}_{3}$ vor. 


\subsubsection{Vergleich der Geschwindigkeiten der Reaktionen Radikal $+\mathrm{O}_{3}$}

Für die Reaktionen Radikal $+\mathrm{O}_{3}$ wurde von Paltenghi, Ogryzlo und Bayes eine Korrelation vorgeschlagen, nach welcher der Logarithmus der Geschwindigkeitskoeffizienten umgekehrt proportional zum Ionisierungspotenzial ist ([PAL84], vgl. auch Abschnitt 3.3.17). Diese Wechselbeziehung ist durch die Geschwindigkeitskonstanten weiterer Reaktionen von Radikalen mit Ozon von Seeba verfestigt worden [SEE94]. Der bei [PAL84] angegebene Geschwindigkeitskoeffizient der Reaktion t-Butyl $+\mathrm{O}_{3}$ fügt sich gut in dieses System ein. Dieser Wert kann durch die Messungen dieser Arbeit bestätigt werden. Die Geschwindigkeitskoeffizienten der anderen drei hier erstmalig vorgestellten Radikal-Ozon-Reaktionen lassen sich nicht in den genannten Zusammenhang einordnen, da die Ionisierungspotenziale der Radikale nicht bekannt sind.

Die Geschwindigkeit der reinen ungesättigten zyklischen Kohlenwasserstoffradikale mit Ozon steigt mit wachsender C-Atom-Zahl an: $\mathrm{k}^{298 \mathrm{~K}}\left(\mathrm{c}-\mathrm{C}_{5} \mathrm{H}_{9}+\mathrm{O}_{3}\right)=1,7 \cdot 10^{13} \mathrm{~cm} /(\mathrm{mol} \cdot \mathrm{s}) \quad$ [NAC98], $\mathrm{k}^{298 \mathrm{~K}}\left(\mathrm{c}-\mathrm{C}_{7} \mathrm{H}_{13}+\mathrm{O}_{3}\right)=2,96 \cdot 10^{13} \mathrm{~cm}^{3} /(\mathrm{mol} \cdot \mathrm{s}) \quad\left(\right.$ diese Arbeit), $\mathrm{k}^{298 \mathrm{~K}}\left(\mathrm{c}-\mathrm{C}_{8} \mathrm{H}_{15}+\mathrm{O}_{3}\right)=3,33 \cdot 10^{13}$ $\mathrm{cm}^{3} /$ (mol·s) (diese Arbeit). Nur der von Nacke gemessene Wert für die Reaktion c-Hexan $+\mathrm{O}_{3}$ scheint sich nicht in diese Reihe einzufügen: $\mathrm{k}^{298 \mathrm{~K}}\left(\mathrm{c}-\mathrm{C}_{5} \mathrm{H}_{9}+\mathrm{O}_{3}\right)=1,1 \cdot 10^{13} \mathrm{~cm}^{3} /(\mathrm{mol} \cdot \mathrm{s})$ [NAC98]. Er fügt sich auch nicht so gut in die oben angesprochene Korrelation zwischen $\ln (\mathrm{k})$ und dem Ionisierungspotenzial der Radikale ein. Eine Überprüfung dieser Geschwindigkeitskonstanten wäre deshalb wünschenswert.

\subsection{Reaktionen von Radikalen mit NO}

Die Reaktionen von Radikalen mit NO gelten als typische Assoziationsreaktionen. Sie können durch folgenden allgemeinen Mechanismus beschrieben werden:

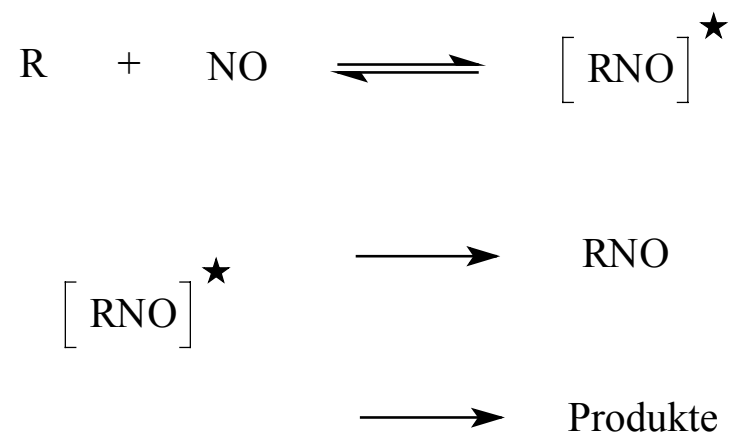

Die durch (1) hin erzeugten energetisch angeregten RNO*-Addukte können redissoziieren $\left\{(1)_{\text {rück }}\right\}$, sich durch Stöße oder interne Energieverteilung stabilisieren $\{(2 \mathrm{a})\}$ oder sich zu anderen Produkten umlagern oder zerfallen $\{(2 \mathrm{~b})\}$. Für kleinere Radikale wie etwa das $\mathrm{CH}_{3}$ ist die Zustandsdichte des RNO-Komplexes klein. Dadurch ist eine intramolekulare Stabilisierung erschwert. Die 
Redissoziation (1)rück ist für diese Radikale bis in höhere Druckbereiche von 1 bar und mehr bedeutsam, was sich darin äußert, dass der Hochdruckgrenzwert der Geschwindigkeitskonstanten kges erst bei solchen Drücken erreicht wird. Für größere Radikale mit einer größeren Zustandsdichte hingegen ist die Druckabhängigkeit auf einen entsprechend kleineren Bereich beschränkt.

Im folgenden werden die Untersuchungen zur Reaktionsgeschwindigkeit der Reaktionen von t-Butyl und von Allyl mit Stickstoffmonoxid dargestellt.

\subsection{1 t-Butyl + NO}

$\underline{\text { Reaktionsgeschwindigkeit: }}$

Messergebnisse: Die Geschwindigkeit der Reaktion t-Butyl + NO wurde bei Zimmertemperatur relativ zur Reaktion Methoxymethyl + NO mit Versuchsanordnung A (REMPI) bei $\lambda=510 \mathrm{~nm}$ bestimmt. Die Radikale wurden gemäß Abschnitt 3.1.5 und 3.1.14 erzeugt und auf ihren Muttermassen $\mathrm{m} / \mathrm{z}=57$ und $\mathrm{m} / \mathrm{z}=45$ in eindeutiger Weise nachgewiesen. Zur Bestimmung des Geschwindigkeitskoeffizienten wurden die Signalintensitäten bei An- und Abwesenheit von NO gemessen. Durch Variation der Stickstoffmonoxidkonzentration wurden verschiedene Umsätze erzielt. Die folgende Abbildung zeigt in doppeltlogarithmischer Auftragung die Messpunkte. Die zugehörigen Werte finden sich in Abschnitt 5 (Messtabellen). Die gewählten Versuchsbedingungen sind dem Text in der Abbildung oder bei den Messtabellen zu entnehmen.

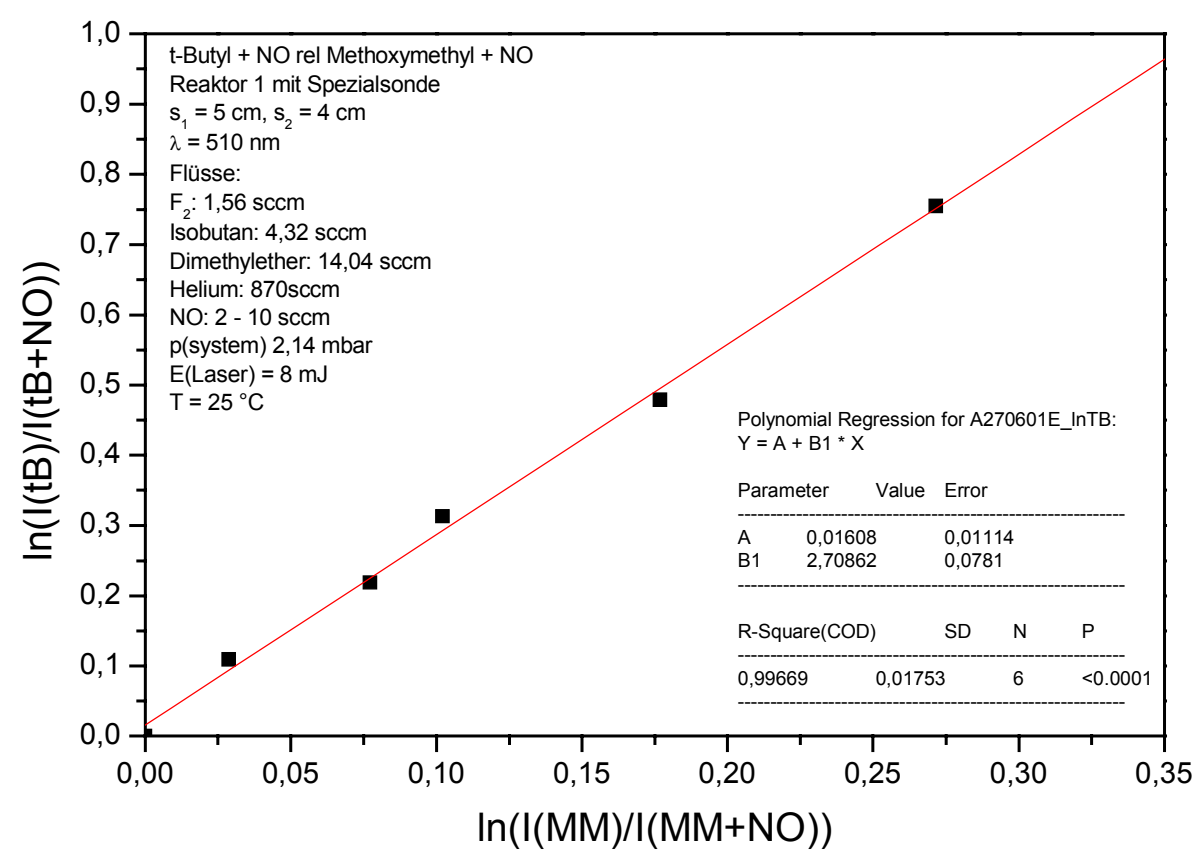

Abbildung 159: t-Butyl + NO relativ zu Methoxymethyl + NO, $\mathrm{T}=25^{\circ} \mathrm{C}$ 
Aus der Auftragung ergibt sich:

$$
\mathrm{k}_{\mathrm{rel}}=\mathrm{k}\left(\mathrm{t}-\mathrm{C}_{4} \mathrm{H}_{9}+\mathrm{NO}\right) / \mathrm{k}\left(\mathrm{CH}_{3} \mathrm{OCH}_{2}+\mathrm{NO}\right)=2,71 \pm 0,08 \text {. }
$$

Mit der bei [NAC98] erläuterten Geschwindigkeitskonstante $k_{\text {ref }}=1,8 \cdot 10^{11} \mathrm{~cm}^{3} /(\mathrm{mol} \cdot \mathrm{s})$ für die Referenzreaktion Methoxymethyl + NO ergibt sich:

$$
\mathrm{k}^{298 \mathrm{~K}}\left(\mathrm{t}-\mathrm{C}_{4} \mathrm{H}_{9}+\mathrm{NO}\right)=(4,88 \pm 0,15) \cdot 10^{11} \mathrm{~cm}^{3} /(\mathrm{mol} \cdot \mathrm{s})
$$

Temperaturabhängigkeit:

Messergebnisse: Die Temperaturabhängigkeit der Reaktion t-Butyl $+\mathrm{NO}$ wurde im Intervall $-30{ }^{\circ} \mathrm{C}$ bis $+90{ }^{\circ} \mathrm{C}$ nach dem selben Verfahren untersucht. Die Temperierung erfolgte mit der in Abschnitt 2.1.2 beschriebenen Methode für Reaktor 1. Die nachfolgenden Abbildungen zeigen die Ergebnisse. Die zugehörigen Werte finden sich in Abschnitt 5 (Messtabellen). Die gewählten Versuchsbedingungen sind dem Text in der Abbildung oder bei den Messtabellen zu entnehmen.

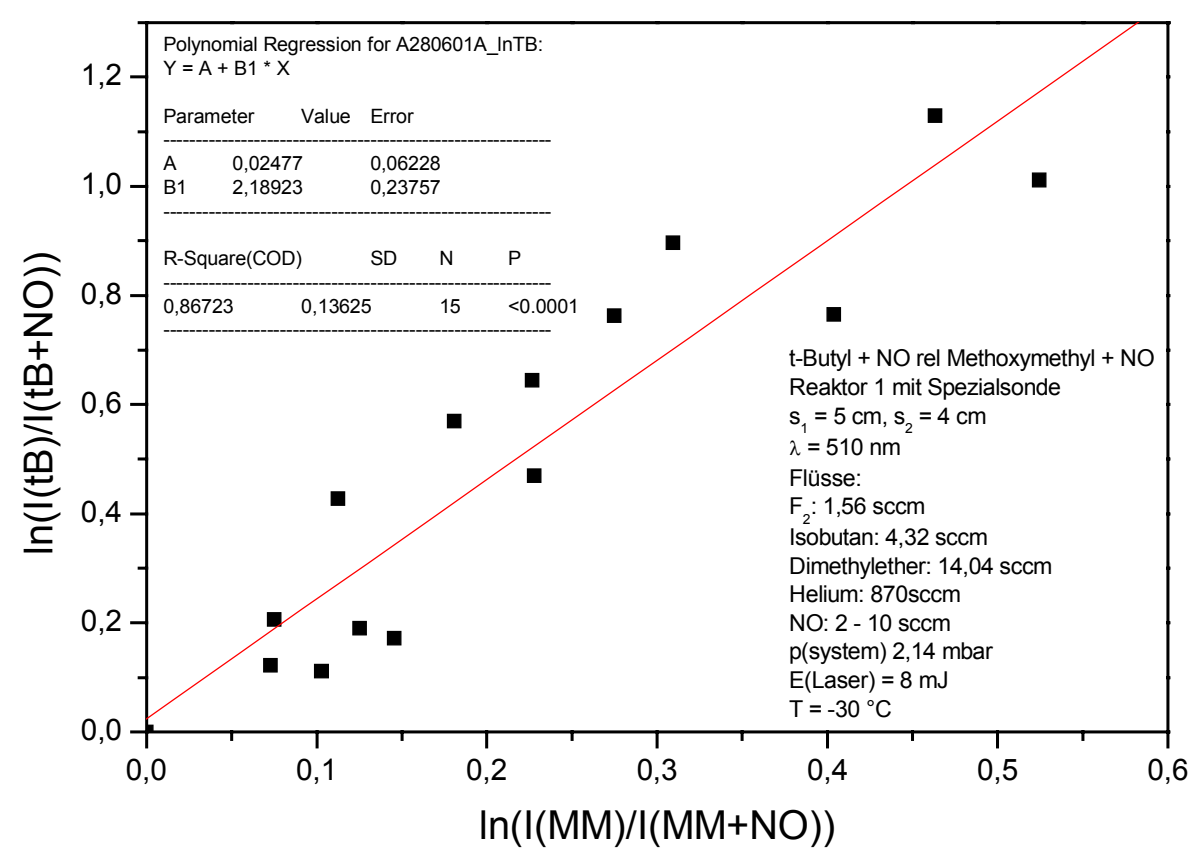

Abbildung 160: t-Butyl + NO relativ zu Methoxymethyl $+\mathrm{NO}, \mathrm{T}=-30^{\circ} \mathrm{C}$ 


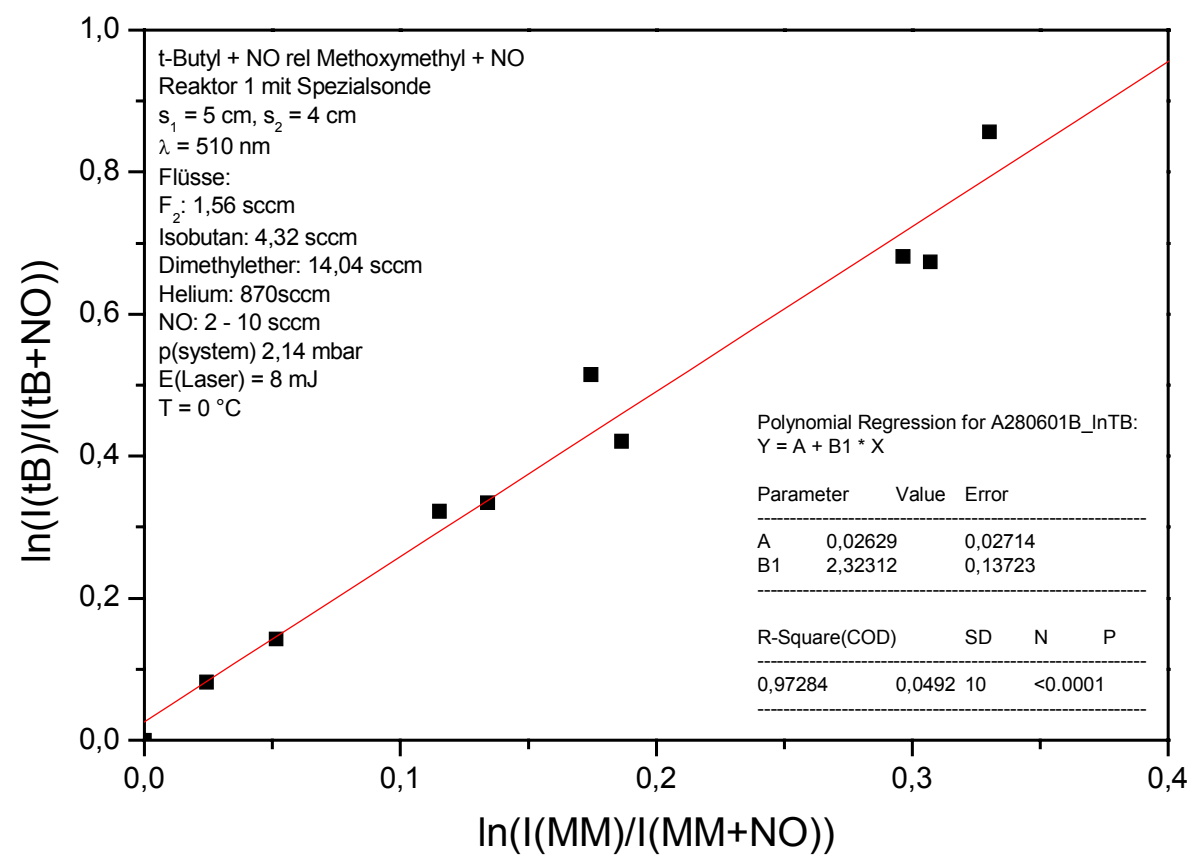

Abbildung 161: t-Butyl + NO relativ zu Methoxymethyl + NO, $\mathrm{T}=0^{\circ} \mathrm{C}$

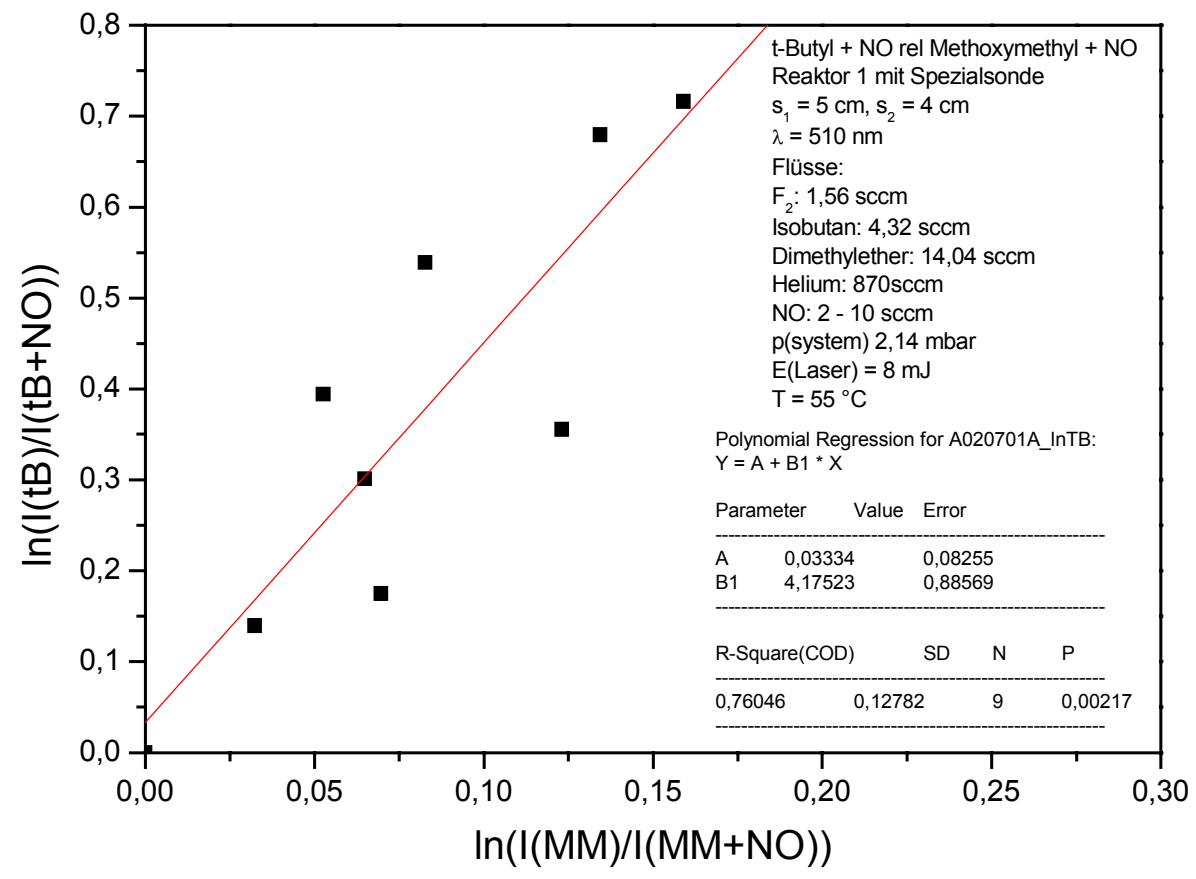

Abbildung 162: t-Butyl + NO relativ zu Methoxymethyl $+\mathrm{NO}, \mathrm{T}=55^{\circ} \mathrm{C}$ 


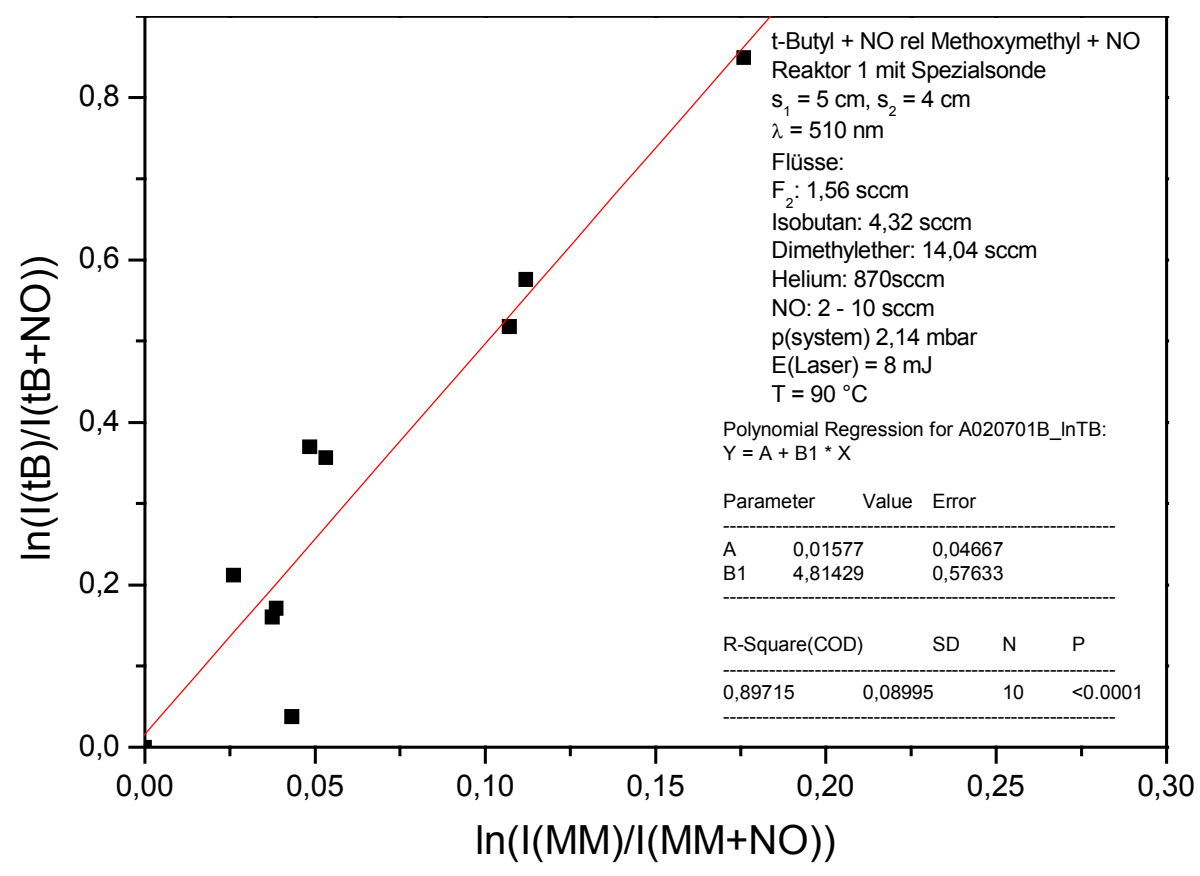

Abbildung 163: t-Butyl + NO relativ zu Methoxymethyl $+\mathrm{NO}, \mathrm{T}=90^{\circ} \mathrm{C}$

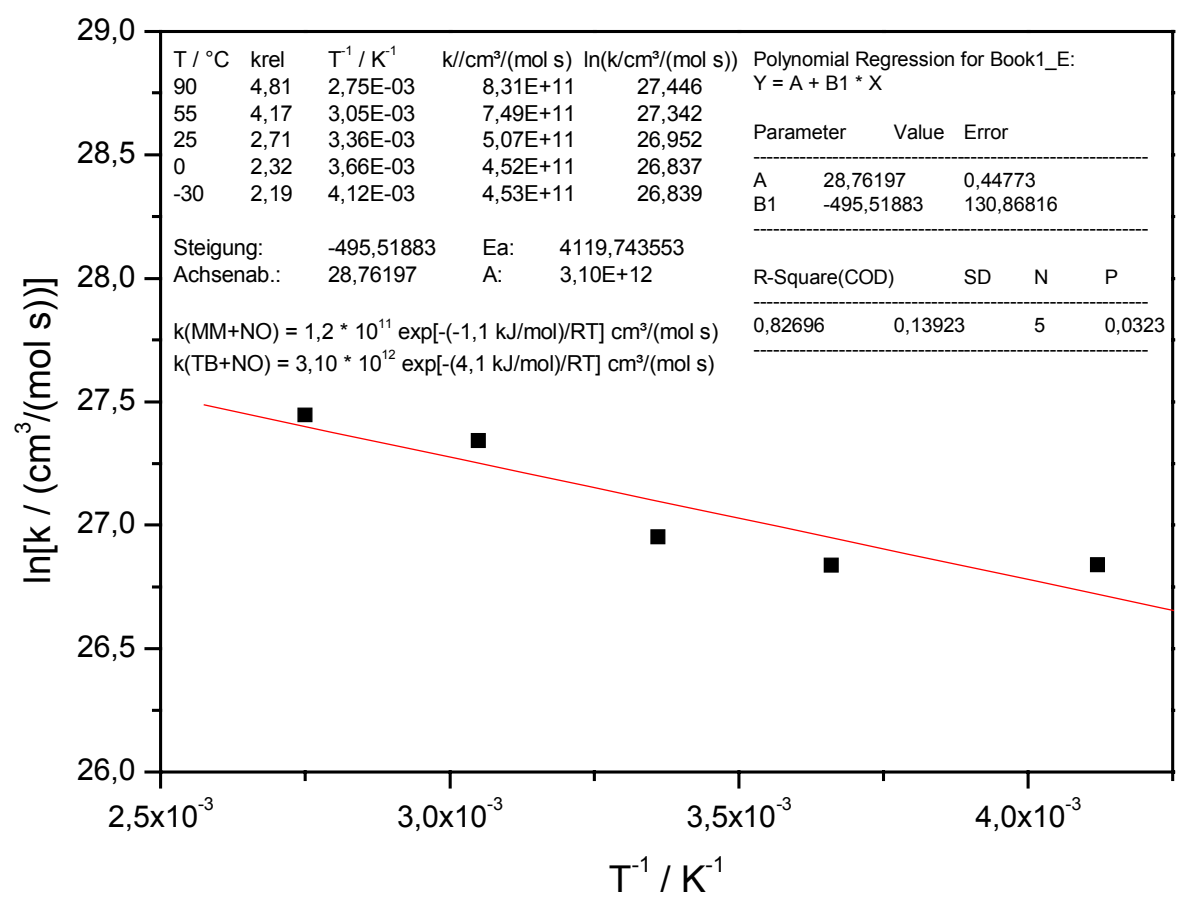

Abbildung 164: t-Butyl + NO relativ zu Methoxymethyl + NO, Arrheniusauftragung

Aus der Arrheniusauftragung ergibt sich:

$$
\begin{gathered}
\mathrm{k}=(3,1 \pm 1,8) \cdot 10^{12} \exp \left\{-(4,1 \pm 1,1) /(\mathrm{RT}) \mathrm{kJ} \cdot \mathrm{mol}^{-1}\right\} \mathrm{cm}^{3} \cdot \mathrm{mol}^{-1} \cdot \mathrm{s}^{-1} \\
\mathrm{k}^{298}=(5,92 \pm 5,0) \cdot 10^{11} \mathrm{~cm}^{3} \cdot \mathrm{mol}^{-1} \cdot \mathrm{s}^{-1}
\end{gathered}
$$


Diskussion: Im Gegensatz zur Reaktion $\mathrm{CH}_{3} \mathrm{OCH}_{2}+\mathrm{NO}$ wird für die Reaktion t-Butyl $+\mathrm{NO}$ in dieser Arbeit eine positive Aktivierungsenergie ermittelt. In der Literatur existiert eine Geschwindigkeitskonstante der Reaktion t-Butyl + NO bei einer Temperatur von $\mathrm{T}=600 \mathrm{~K}$ : $\mathrm{k}^{600 \mathrm{~K}}(\mathrm{t}-\mathrm{Butyl}+\mathrm{NO})=(3,5 \pm 1,7) \cdot 10^{12} \mathrm{~cm}^{3} \cdot \mathrm{mol}^{-1} \cdot \mathrm{s}^{-1}$ [CHO74]. Dieser Wert wurde von Benson et al. aus der Anpassung von Messdaten an einen komplexen Reaktionsmechanismus ermittelt. Die Messdaten wurden durch Reaktionen in einer Niedrigdruckzelle massenspektrometrisch gewonnen. Der Geschwindigkeitskoeffizient liegt sehr nahe beim hier vorgestellten präexponentiellen Faktor von $A=(3,1 \pm 1,8) \cdot 10^{12} \mathrm{~cm}^{3} \cdot \mathrm{mol}^{-1} \cdot \mathrm{s}^{-1}$. Die Richtigkeit beider Datensätze vorausgesetzt, hätte die Reaktion t-Butyl + NO bei $\mathrm{T}=600 \mathrm{~K}$ ihren Hochtemperaturgrenzwert bereits erreicht.

\subsubsection{Allyl + NO}

\section{Reaktionsgeschwindigkeit:}

Messergebnisse: Die Geschwindigkeit der Reaktion Allyl + NO wurde bei Zimmertemperatur relativ zur Reaktion Methoxymethyl + NO mit Versuchsanordnung A (REMPI) bei $\lambda=499,2 \mathrm{~nm}$ bestimmt. Die Radikale wurden gemäß Abschnitt 3.1.5 und 3.1.14 erzeugt und auf ihren Muttermassen $\mathrm{m} / \mathrm{z}=41$ und $\mathrm{m} / \mathrm{z}=45$ in eindeutiger Weise nachgewiesen. Zur Bestimmung des Geschwindigkeitskoeffizienten wurden die Signalintensitäten bei An- und Abwesenheit von NO gemessen. Durch Variation der Stickstoffmonoxidkonzentration wurden verschiedene Umsätze erzielt. Die folgende Abbildung zeigt in doppeltlogarithmischer Auftragung die Messpunkte. Die zugehörigen Werte finden sich in Abschnitt 5 (Messtabellen). Die gewählten Versuchsbedingungen sind dem Text in der Abbildung oder bei den Messtabellen zu entnehmen.

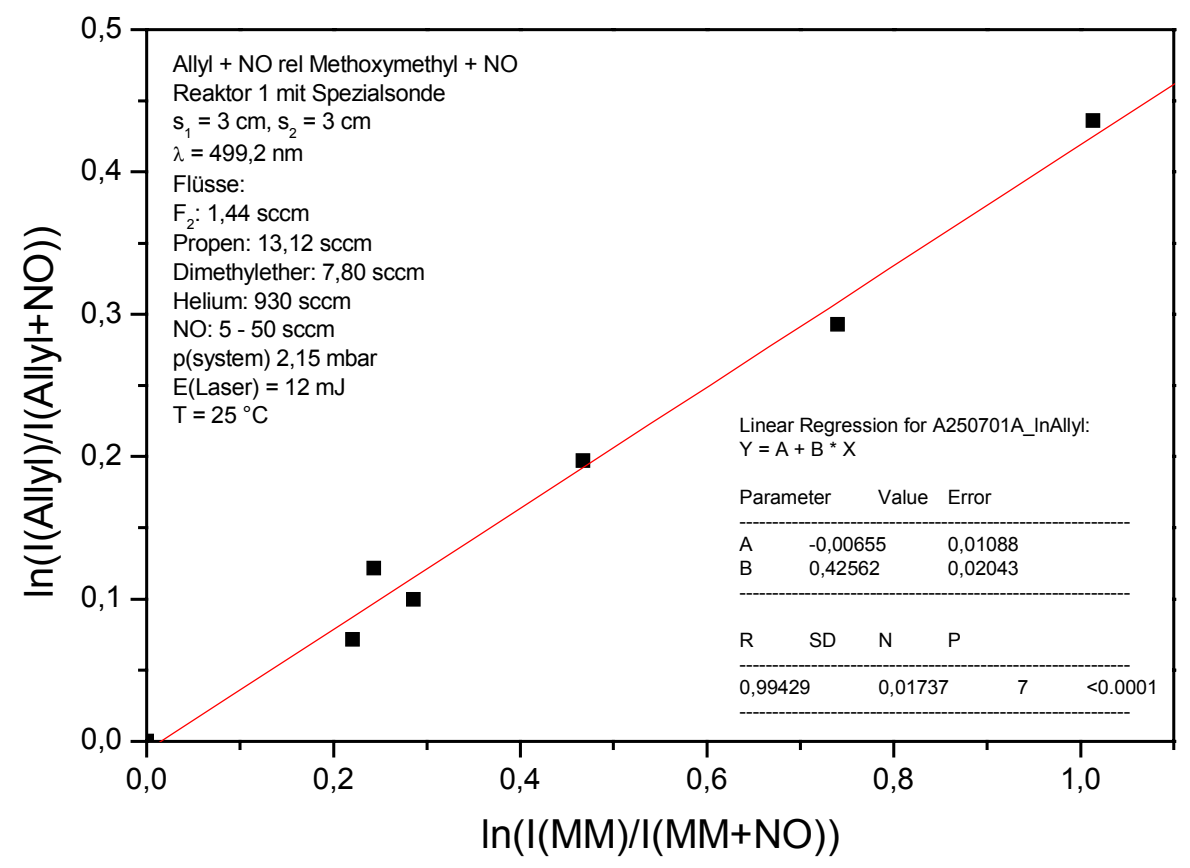

Abbildung 165: Allyl + NO relativ zu Methoxymethyl $+\mathrm{NO}, \mathrm{T}=25^{\circ} \mathrm{C}$ 
Aus der Auftragung ergibt sich:

$$
\mathrm{k}_{\text {rel }}=\mathrm{k}\left(\mathrm{C}_{3} \mathrm{H}_{5}+\mathrm{NO}\right) / \mathrm{k}\left(\mathrm{CH}_{3} \mathrm{OCH}_{2}+\mathrm{NO}\right)=0,43 \pm 0,02 .
$$

Mit der bei [NAC98] erläuterten Geschwindigkeitskonstante $k_{\text {ref }}=1,8 \cdot 10^{11} \mathrm{~cm}^{3} /(\mathrm{mol} \cdot \mathrm{s})$ für die Referenzreaktion Methoxymethyl + NO erhält man:

$$
\mathrm{k}^{298 \mathrm{~K}}\left(\mathrm{C}_{3} \mathrm{H}_{5}+\mathrm{NO}\right)=(7,74 \pm 0,36) \cdot 10^{10} \mathrm{~cm}^{3} /(\mathrm{mol} \cdot \mathrm{s})
$$

Temperaturabhängigkeit:

Messergebnisse: Die Temperaturabhängigkeit der Reaktion Allyl $+\mathrm{NO}$ wurde im Intervall $-30{ }^{\circ} \mathrm{C}$ bis $+90{ }^{\circ} \mathrm{C}$ nach dem selben Verfahren untersucht. Die Temperierung erfolgte mit der in Abschnitt 2.1.2 beschriebenen Methode für Reaktor 1. Die nachfolgenden Abbildungen zeigen die Ergebnisse. Die zugehörigen Werte finden sich in Abschnitt 5 (Messtabellen). Die gewählten Versuchsbedingungen sind dem Text in der Abbildung oder bei den Messtabellen zu entnehmen.

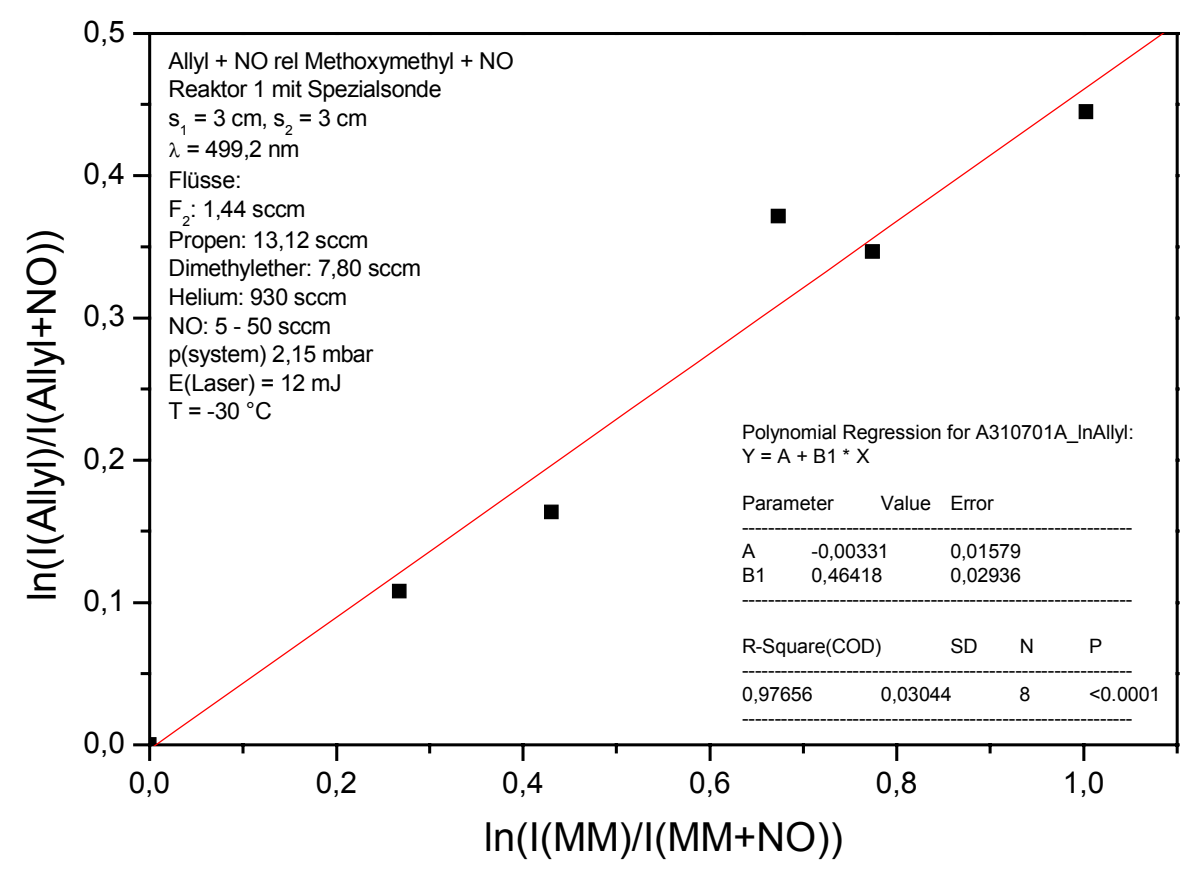

Abbildung 166: Allyl + NO relativ zu Methoxymethyl + NO, $\mathrm{T}=-30^{\circ} \mathrm{C}$ 


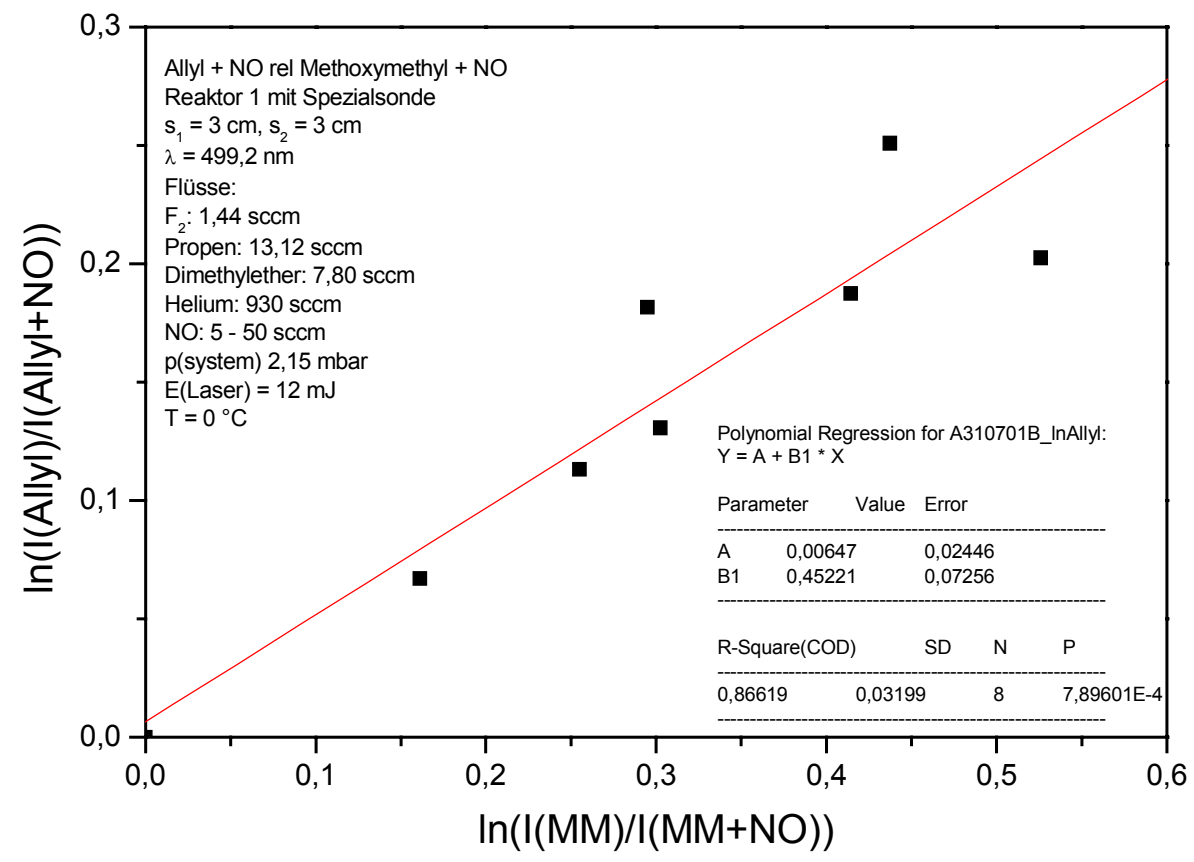

Abbildung 167: Allyl + NO relativ zu Methoxymethyl + NO, $\mathrm{T}=0^{\circ} \mathrm{C}$

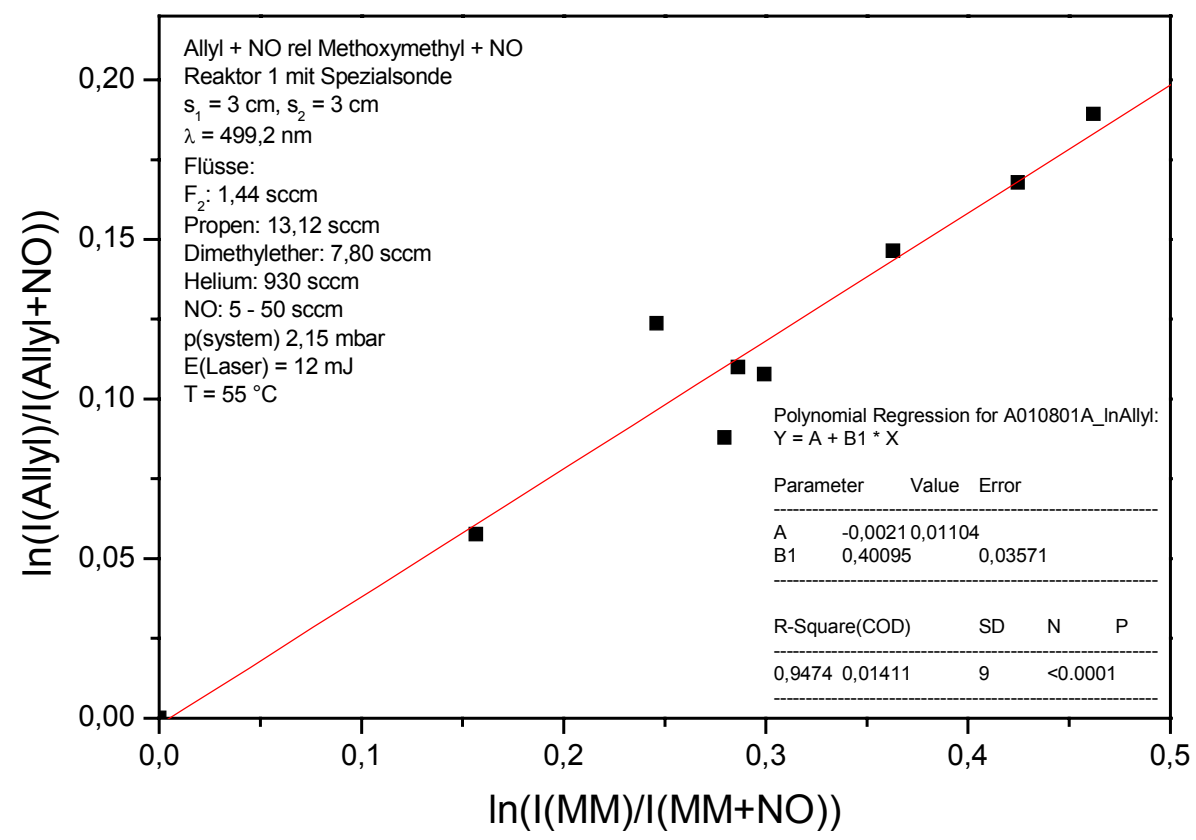

Abbildung 168: Allyl + NO relativ zu Methoxymethyl $+\mathrm{NO}, \mathrm{T}=55^{\circ} \mathrm{C}$ 


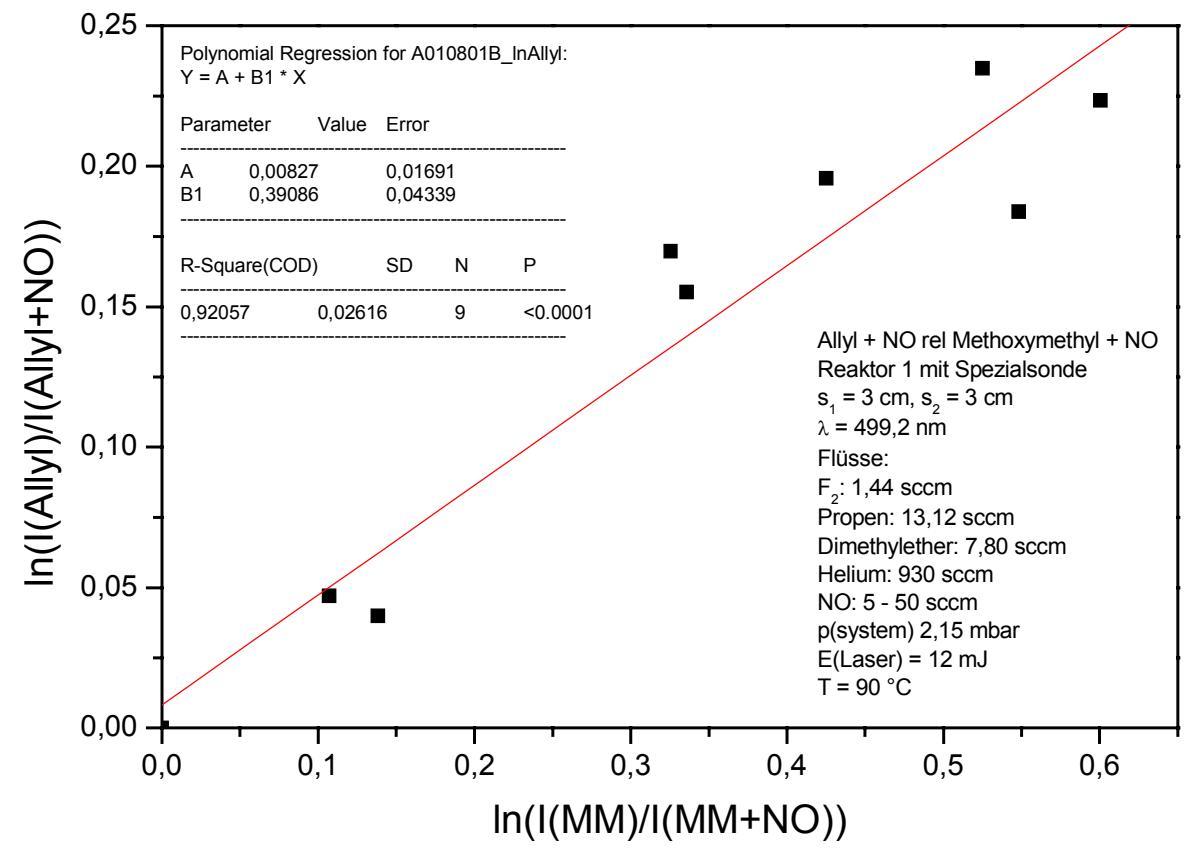

Abbildung 169: Allyl + NO relativ zu Methoxymethyl $+\mathrm{NO}, \mathrm{T}=90^{\circ} \mathrm{C}$

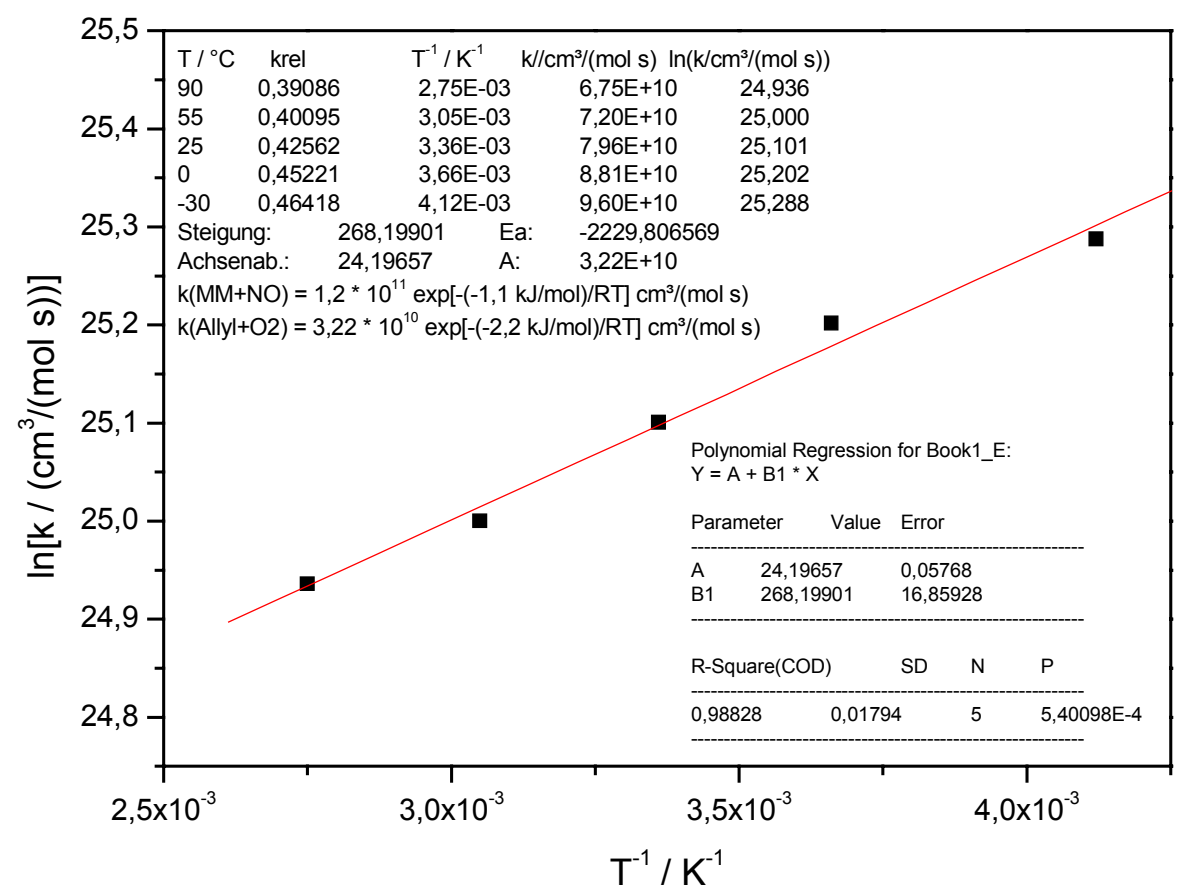

Abbildung 170: Allyl + NO relativ zu Methoxymethyl + NO, Arrheniusauftragung

Aus der Arrheniusauftragung ergibt sich:

$$
\begin{gathered}
\mathrm{k}=(3,22 \pm 0,4) \cdot 10^{10} \exp \left\{-(-2,2 \pm 0,2) /(\mathrm{RT}) \mathrm{kJ} \cdot \mathrm{mol}^{-1}\right\} \mathrm{cm}^{3} \cdot \mathrm{mol}^{-1} \cdot \mathrm{s}^{-1} \\
\mathrm{k}^{298}=(7,82 \pm 1,8) \cdot 10^{11} \mathrm{~cm}^{3} \cdot \mathrm{mol}^{-1} \cdot \mathrm{s}^{-1}
\end{gathered}
$$


Diskussion: In der Literatur finden sich zwei Messungen zur Geschwindigkeit der Reaktion Allyl + NO: (1) $\mathrm{k}^{403 \mathrm{~K}}=(4,28 \pm 0,26) \cdot 10^{12} \mathrm{~cm}^{3} /(\mathrm{mol} \cdot \mathrm{s}), \quad(\mathrm{p}=760$ Torr $)$, [BOY95]; (2) $\mathrm{k}=(2,11 \pm 0,36) \cdot 10^{12} \exp \left\{-(-3,4 \pm 0,1) /(\mathrm{RT}) \mathrm{kJ} \cdot \mathrm{mol}^{-1}\right\} \quad \mathrm{cm}^{3} /(\mathrm{mol} \cdot \mathrm{s}), \mathrm{k}^{298 \mathrm{~K}}=8,32 \cdot 10^{12}$ $\mathrm{cm}^{3} /(\mathrm{mol} \cdot \mathrm{s})$ ( $\mathrm{p}=50-500$ Torr) [TUL82]. Beide Ausdrücke resultieren in Geschwindigkeitskoeffizienten, die um eine Zehnerpotenz größer sind als hier gemessen, sind jedoch wegen des erheblich höheren Drucks nur sehr eingeschränkt vergleichbar mit dem hier bei ca. 2 Torr ermittelten Koeffizienten. Die bei (2) angegebene Aktivierungsenergie ist jedoch vorzeichengleich und vom Betrag her ähnlich wie die in dieser Arbeit bestimmte.

\subsection{Reaktionen von Radikalen mit H-Atomen}

Reaktionen von Kohlenwasserstoffradikalen mit atomarem Wasserstoff stellen Assoziationsreaktionen dar, die durch folgenden Mechanismus allgemein beschrieben werden können:

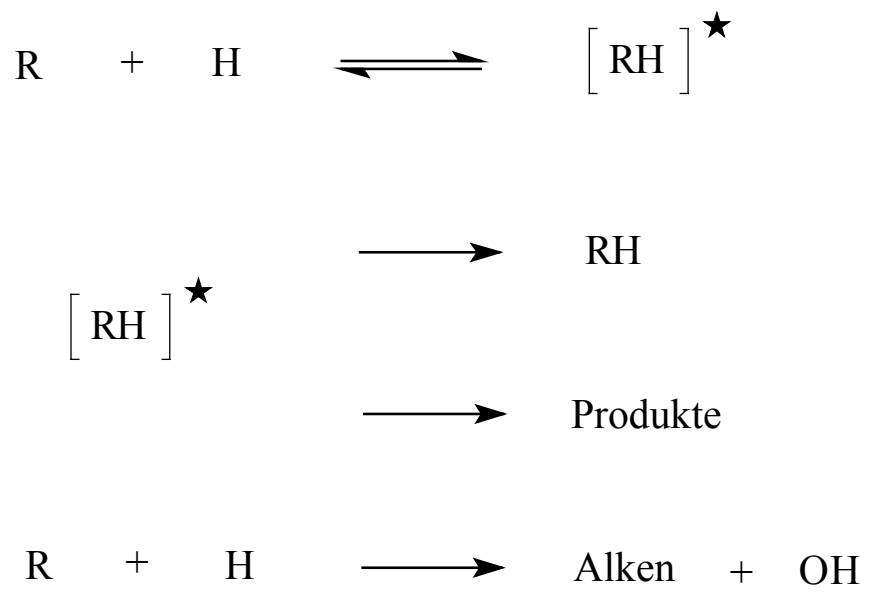

Die durch (1)hin erzeugten energetisch angeregten $\mathrm{RH}^{*}$-Addukte können redissoziieren $\left\{(1)_{\text {rück }}\right\}$ sich durch Stöße oder interne Energieverteilung stabilisieren $\{(2 \mathrm{a})\}$ und sich zu anderen Produkten umlagern oder zerfallen $\{(2 \mathrm{~b})\}$. Wie in den Abschnitten 3.4 und 3.6 erläutert, ist für größere Radikale eine molekülinterne Stabilisierung durch Verteilung der frei werdenden Bindungsenergie im Adduktkomplex RH eher möglich. Für kleinere Radikale ist jedoch eine Stoßstabilisierung erforderlich, was sich in einer beachtlichen Druckabhängigkeit äußert. Die Reaktion $\mathrm{CH}_{3}+\mathrm{H}$ ist in einem weiten Temperatur und Druckbereich intensiv untersucht und mit Hilfe theoretischer Modelle beschrieben worden [BRO85], wobei eine komplizierte Abhängigkeit der Reaktionsgeschwindigkeit von beiden Parametern festgestellt wurde.

Bei Existenz eines besonderen Produktbildungskanals (2b) aus dem Adduktkomplex heraus kann die Redissoziation (1b) an Bedeutung verlieren. Ein Sonderfall einer solchen Reaktion ist im 
H/D-Austausch bei der Reaktion von Methyl mit dem Wasserstoffisotop Deuterium zu sehen. Aufgrund der Unterschiede in den C-H- und C-D-Nullpunktsenergien, ist die Reaktion

$$
\mathrm{CH}_{3}+\mathrm{D} \rightarrow \mathrm{CH}_{2} \mathrm{D}+\mathrm{H}
$$

begünstigt und tritt damit in Konkurrenz zur Redissoziation. Entsprechend charakterisiert die Geschwindigkeitskonstante der Reaktion $\mathrm{CH}_{3}+\mathrm{D}$ den Hochdruckgrenzwert [BRO86].

Üblicherweise ist der Abstraktionskanal (3) wenig relevant. Für die Reaktion von Ethylradikalen mit $\mathrm{H}$-Atomen wurde das durch Harding und Klippenstein auf Basis einer ab initio quantenchemischen Simulation gezeigt [HAR98].

Im folgenden werden die Untersuchungen zur Reaktionsgeschwindigkeit der Reaktionen von 2-Propyl, 2-Butyl, t-Butyl, Allyl, c-Heptyl, c-Oktyl und 2-Chlor-2-Methyl-Propyl mit atomarem Wasserstoff vorgestellt.

\subsubsection{2-Propyl + H}

\section{$\underline{\text { Reaktionsgeschwindigkeit: }}$}

Messergebnisse: Die Geschwindigkeit der Reaktion 2-Propyl + H wurde bei Zimmertemperatur relativ zur Reaktion Methoxymethyl $+\mathrm{H}$ mit Versuchsanordnung A (REMPI) bei $\lambda=465 \mathrm{~nm}$ bestimmt. Die Radikale wurden gemäß Abschnitt 3.1.3 und 3.1.14 erzeugt und auf ihren Muttermassen $\mathrm{m} / \mathrm{z}=43$ und $\mathrm{m} / \mathrm{z}=45$ in eindeutiger Weise nachgewiesen. Zur Bestimmung des Geschwindigkeitskoeffizienten wurden die Signalintensitäten bei An- und Abwesenheit von Wasserstoffatomen gemessen. Durch Variation der Wasserstoffkonzentration wurden verschiedene Umsätze erzielt. Die folgende Abbildung zeigt in doppeltlogarithmischer Auftragung die Messpunkte. Die zugehörigen Werte finden sich in Abschnitt 5 (Messtabellen). Die gewählten Versuchsbedingungen sind dem Text in der Abbildung oder bei den Messtabellen zu entnehmen. 


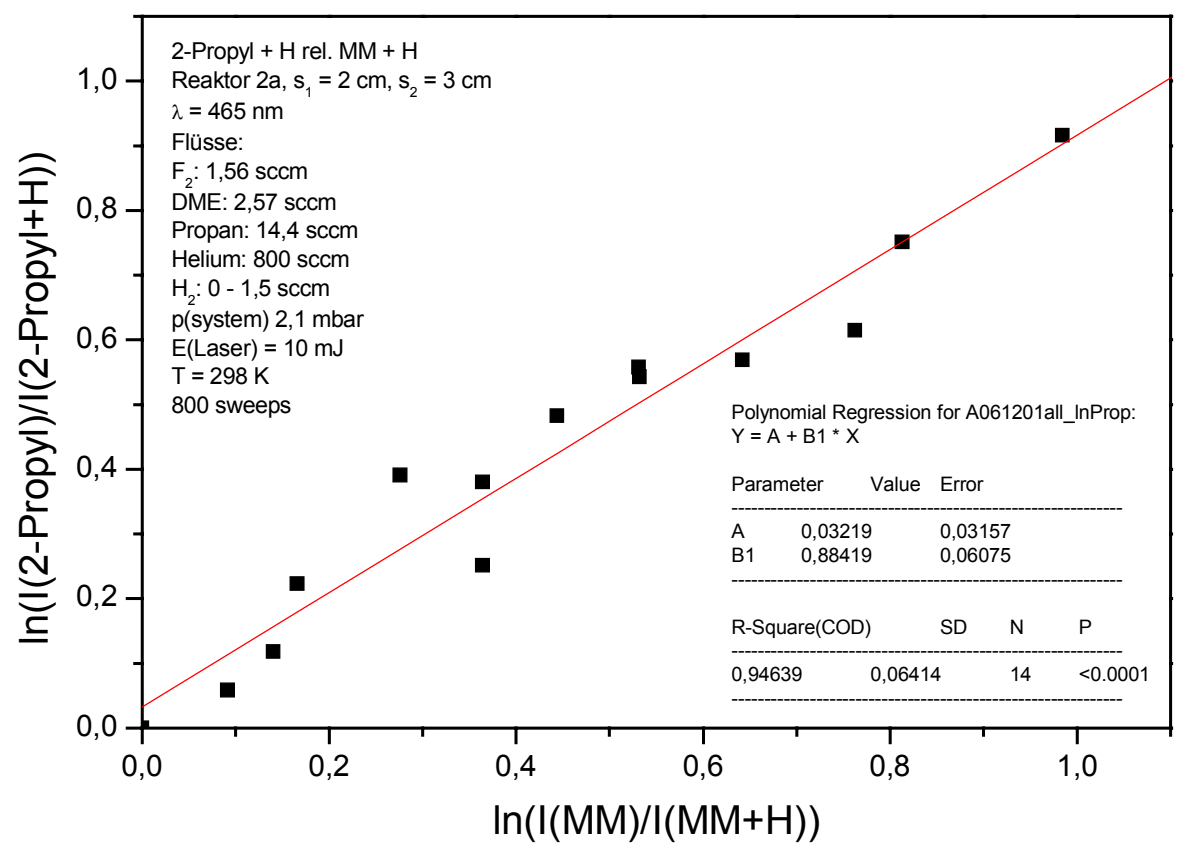

Abbildung 171: 2-Propyl $+\mathrm{H}$ relativ zu Methoxymethyl $+\mathrm{H}, \mathrm{T}=25^{\circ} \mathrm{C}$

Aus der Auftragung ergibt sich:

$$
\mathrm{k}_{\text {rel }}=\mathrm{k}\left(\mathrm{iso}-\mathrm{C}_{3} \mathrm{H}_{7}+\mathrm{H}\right) / \mathrm{k}\left(\mathrm{CH}_{3} \mathrm{OCH}_{2}+\mathrm{H}\right)=0,88 \pm 0,06 \text {. }
$$

Mit der bei [NAC94] erläuterten Geschwindigkeitskonstante $\mathrm{k}_{\mathrm{ref}}=3,5 \cdot 10^{13} \mathrm{~cm}^{3} /(\mathrm{mol} \cdot \mathrm{s})$ für die Referenzreaktion Methoxymethyl + H erhält man:

$$
\mathrm{k}^{298 \mathrm{~K}}\left(\text { iso- } \mathrm{C}_{3} \mathrm{H}_{7}+\mathrm{H}\right)=(3,1 \pm 0,2) \cdot 10^{13} \mathrm{~cm}^{3} /(\mathrm{mol} \cdot \mathrm{s})
$$

Diskussion: In der Literatur gibt es bisher nur eine Abschätzung des Geschwindigkeitskoeffizienten der Reaktion 2-Propyl $+\mathrm{H}$ von Tsang: $\mathrm{k}^{298 \mathrm{~K}}\left(\right.$ iso- $\left.\mathrm{C}_{3} \mathrm{H}_{7}+\mathrm{H}\right)=1,8 \cdot 10^{12} \mathrm{~cm}^{3} /(\mathrm{mol} \cdot \mathrm{s})$ [TSA88]. Die Richtigkeit des Geschwindigkeitskoeffizienten der Referenzreaktion $\mathrm{CH}_{3} \mathrm{OCH}_{2}+\mathrm{H}$ vorausgesetzt, muss dieser Wert in Anbetracht der hier gewonnenen Messwerte um mehr als den Faktor 10 nach oben korrigiert werden.

\subsubsection{2-Butyl + H}

$\underline{\text { Reaktionsgeschwindigkeit: }}$

Messergebnisse: Die Geschwindigkeit der Reaktion 2-Butyl + H wurde bei Zimmertemperatur relativ zur Reaktion Methoxymethyl $+\mathrm{H}$ mit Versuchsanordnung A (REMPI) bei $\lambda=465 \mathrm{~nm}$ bestimmt. Die Radikale wurden gemäß Abschnitt 3.1.4 und 3.1.14 erzeugt und auf ihren Muttermassen $\mathrm{m} / \mathrm{z}=57$ und $\mathrm{m} / \mathrm{z}=45$ in eindeutiger Weise nachgewiesen. Zur Bestimmung des Geschwindigkeitskoeffizienten wurden die Signalintensitäten bei An- und Abwesenheit von Wasserstoffatomen 
gemessen. Durch Variation der Wasserstoffkonzentration wurden verschiedene Umsätze erzielt. Die folgende Abbildung zeigt in doppeltlogarithmischer Auftragung die Messpunkte. Die zugehörigen Werte finden sich in Abschnitt 5 (Messtabellen). Die gewählten Versuchsbedingungen sind dem Text in der Abbildung oder bei den Messtabellen zu entnehmen.

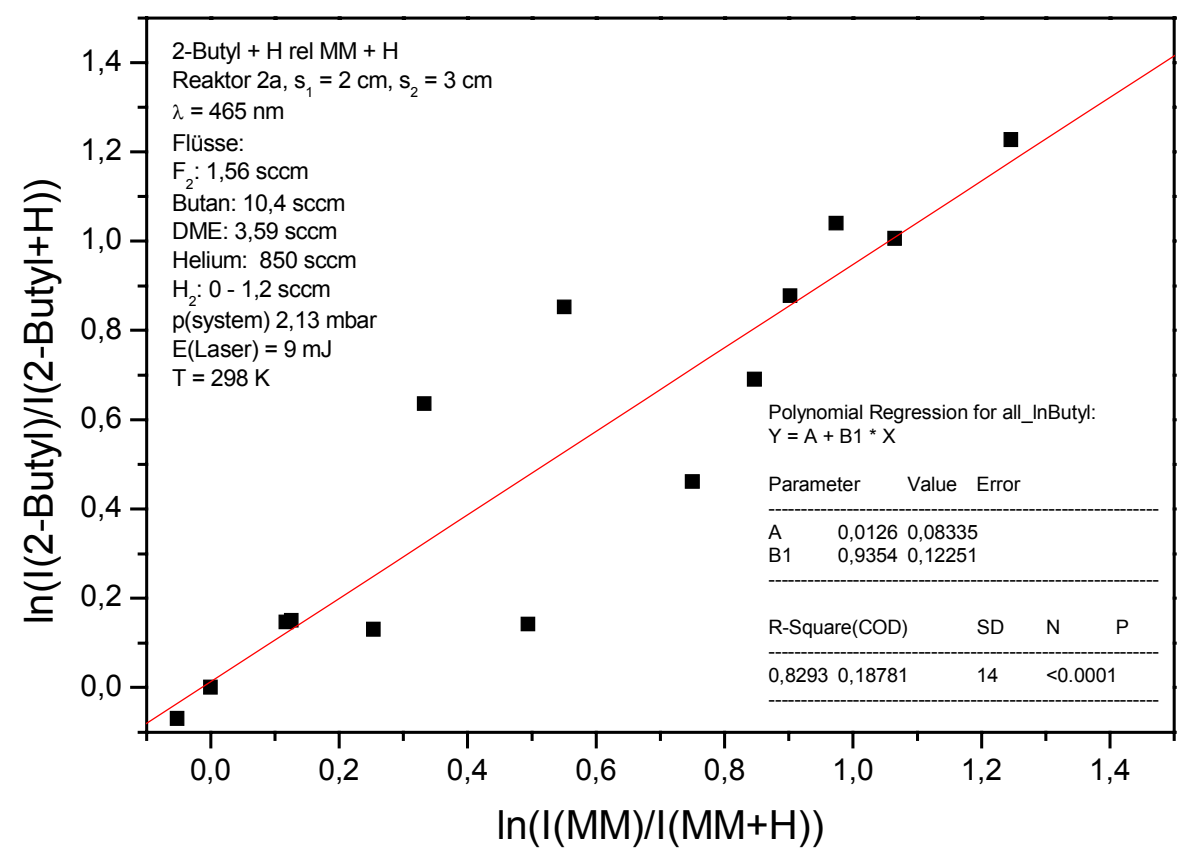

Abbildung 172: 2-Butyl $+\mathrm{H}$ relativ zu Methoxymethyl $+\mathrm{H}, \mathrm{T}=25^{\circ} \mathrm{C}$

Aus der Auftragung ergibt sich:

$$
\mathrm{k}_{\text {rel }}=\mathrm{k}\left(2-\mathrm{C}_{4} \mathrm{H}_{9}+\mathrm{H}\right) / \mathrm{k}\left(\mathrm{CH}_{3} \mathrm{OCH}_{2}+\mathrm{H}\right)=0,94 \pm 0,13
$$

Mit der bei [NAC94] erläuterten Geschwindigkeitskonstante $k_{\text {ref }}=3,5 \cdot 10^{13} \mathrm{~cm}^{3} /(\mathrm{mol} \cdot \mathrm{s})$ für die Referenzreaktion Methoxymethyl + H erhält man:

$$
\mathrm{k}^{298 \mathrm{~K}}\left(2-\mathrm{C}_{4} \mathrm{H}_{9}+\mathrm{H}\right)=(3,3 \pm 0,5) \cdot 10^{13} \mathrm{~cm}^{3} /(\mathrm{mol} \cdot \mathrm{s}) .
$$

Es liegt keine Literatur über die Reaktion 2-Butyl + H vor.

\subsection{3 t-Butyl + H}

\section{$\underline{\text { Reaktionsgeschwindigkeit: }}$}

Messergebnisse: Die Geschwindigkeit der Reaktion t-Butyl + H wurde bei Zimmertemperatur relativ zur Reaktion Methoxymethyl $+\mathrm{H}$ mit Versuchsanordnung A (REMPI) bei $\lambda=500 \mathrm{~nm}$ bestimmt. Die Radikale wurden gemäß Abschnitt 3.1.5 und 3.1.14 erzeugt und auf ihren Muttermassen $\mathrm{m} / \mathrm{z}=57$ und $\mathrm{m} / \mathrm{z}=45$ in eindeutiger Weise nachgewiesen. Zur Bestimmung des Geschwindigkeitskoeffizienten wurden die Signalintensitäten bei An- und Abwesenheit von Wasserstoffatomen 
gemessen. Durch Variation der Wasserstoffkonzentration wurden verschiedene Umsätze erzielt. Die folgende Abbildung zeigt in doppeltlogarithmischer Auftragung die Messpunkte. Die zugehörigen Werte finden sich in Abschnitt 5 (Messtabellen). Die gewählten Versuchsbedingungen sind dem Text in der Abbildung oder bei den Messtabellen zu entnehmen.

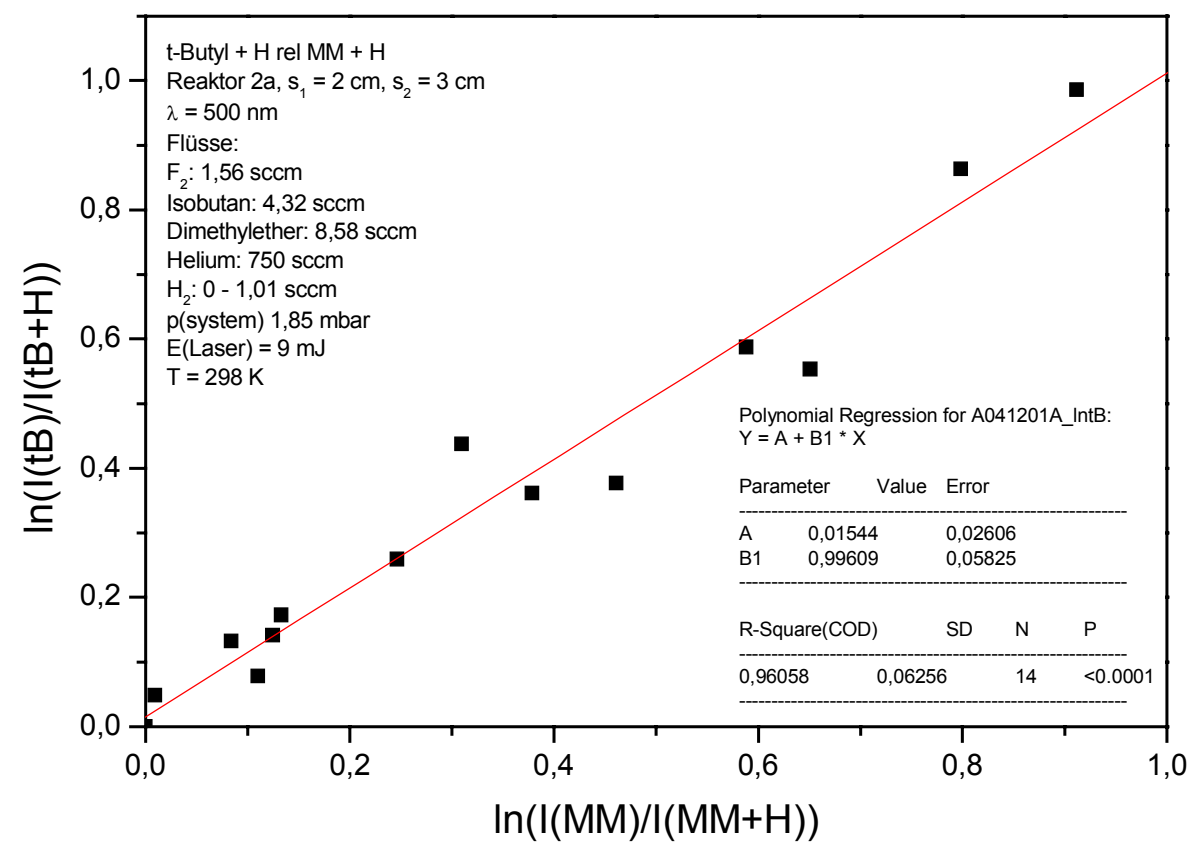

Abbildung 173: t-Butyl $+\mathrm{H}$ relativ zu Methoxymethyl $+\mathrm{H}, \mathrm{T}=25^{\circ} \mathrm{C}$

Aus der Auftragung ergibt sich:

$$
\mathrm{k}_{\mathrm{rel}}=\mathrm{k}\left(\mathrm{t}-\mathrm{C}_{4} \mathrm{H}_{9}+\mathrm{H}\right) / \mathrm{k}\left(\mathrm{CH}_{3} \mathrm{OCH}_{2}+\mathrm{H}\right)=1,00 \pm 0,06
$$

Mit der bei [NAC94] erläuterten Geschwindigkeitskonstante $k_{\text {ref }}=3,5 \cdot 10^{13} \mathrm{~cm}^{3} /(\mathrm{mol} \cdot \mathrm{s})$ für die Referenzreaktion Methoxymethyl + H erhält man:

$$
\mathrm{k}^{298 \mathrm{~K}}\left(\mathrm{t}-\mathrm{C}_{4} \mathrm{H}_{9}+\mathrm{H}\right)=(3,5 \pm 0,2) \cdot 10^{13} \mathrm{~cm}^{3} /(\mathrm{mol} \cdot \mathrm{s}) .
$$

Diskussion: In der Literatur gibt es bisher nur eine Abschätzung des Geschwindigkeitskoeffizienten der Reaktion t-Butyl $+\mathrm{H}$ von Tsang: $\mathrm{k}^{298 \mathrm{~K}}\left(\mathrm{t}-\mathrm{C}_{4} \mathrm{H}_{9}+\mathrm{H}\right)=2,41 \cdot 10^{12} \mathrm{~cm}^{3} /(\mathrm{mol} \cdot \mathrm{s})$ [TSA90]. Der in dieser Arbeit bestimmte Wert liegt um den Faktor 1,5 höher.

\subsubsection{Allyl + H}

Reaktionsgeschwindigkeit:

Messergebnisse: Die Geschwindigkeit der Reaktion Allyl + H wurde bei Zimmertemperatur relativ zur Reaktion Methoxymethyl $+\mathrm{H}$ mit Versuchsanordnung A (REMPI) bei $\lambda=499,37 \mathrm{~nm}$ bestimmt.

Die Radikale wurden gemäß Abschnitt 3.1.5 und 3.1.14 erzeugt und auf ihren Muttermassen 
$\mathrm{m} / \mathrm{z}=41$ und $\mathrm{m} / \mathrm{z}=45$ Weise nachgewiesen. Zur Bestimmung des Geschwindigkeitskoeffizienten wurden die Signalintensitäten bei An- und Abwesenheit von Wasserstoffatomen gemessen. Durch Variation der Wasserstoffkonzentration wurden verschiedene Umsätze erzielt. Die folgende Abbildung zeigt in doppeltlogarithmischer Auftragung die Messpunkte. Die zugehörigen Werte finden sich in Abschnitt 5 (Messtabellen). Die gewählten Versuchsbedingungen sind dem Text in der Abbildung oder bei den Messtabellen zu entnehmen.

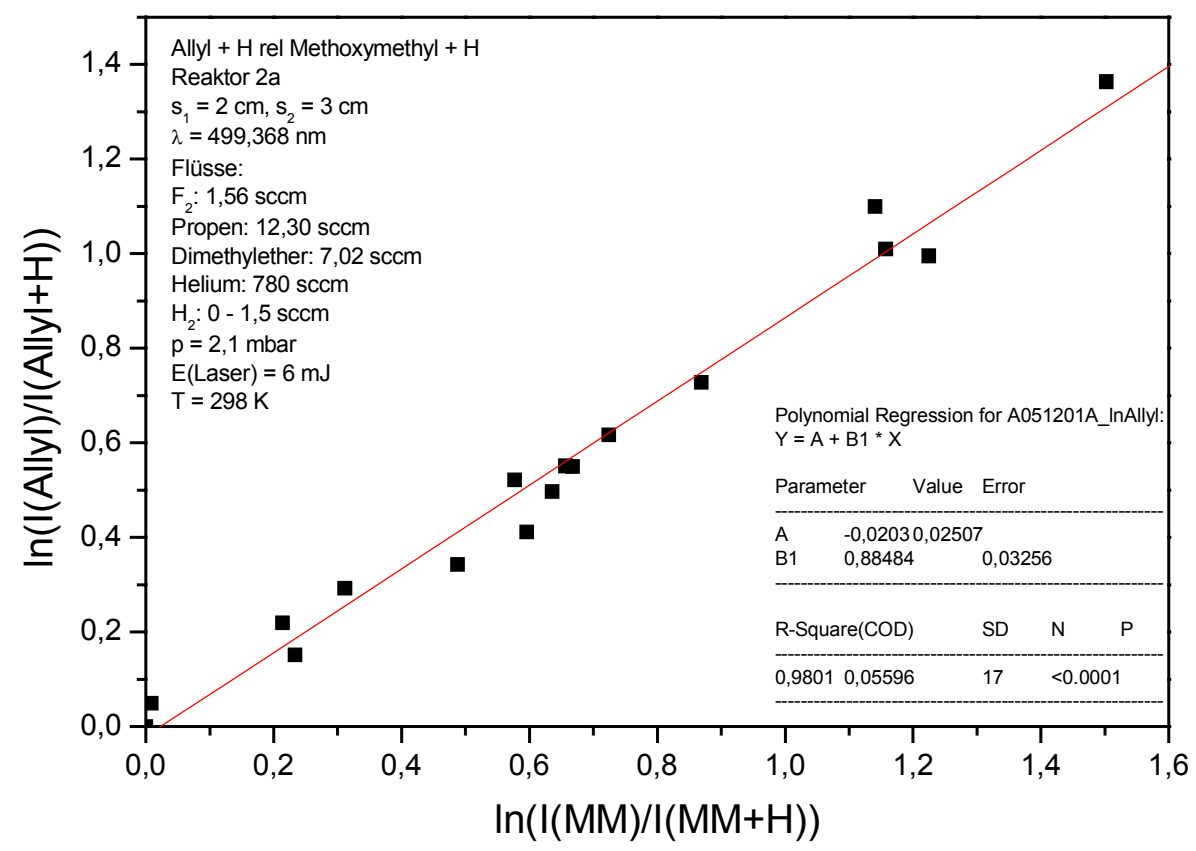

Abbildung 174: Allyl $+\mathrm{H}$ relativ zu Methoxymethyl $+\mathrm{H}, \mathrm{T}=25^{\circ} \mathrm{C}$

Aus der Auftragung ergibt sich:

$$
\mathrm{k}_{\mathrm{rel}}=\mathrm{k}\left(\mathrm{C}_{3} \mathrm{H}_{5}+\mathrm{H}\right) / \mathrm{k}\left(\mathrm{CH}_{3} \mathrm{OCH}_{2}+\mathrm{H}\right)=0,88 \pm 0,03 \text {. }
$$

Mit der bei [NAC94] erläuterten Geschwindigkeitskonstante $\mathrm{k}_{\mathrm{ref}}=3,5 \cdot 10^{13} \mathrm{~cm}^{3} /(\mathrm{mol} \cdot \mathrm{s})$ für die Referenzreaktion Methoxymethyl + H erhält man:

$$
\mathrm{k}^{298 \mathrm{~K}}\left(\mathrm{C}_{3} \mathrm{H}_{5}+\mathrm{H}\right)=(3,1 \pm 0,1) \cdot 10^{13} \mathrm{~cm}^{3} /(\mathrm{mol} \cdot \mathrm{s})
$$

Diskussion: In der Literatur findet sich eine Messung zur Geschwindigkeit der Reaktion Allyl $+\mathrm{H}$ : $\mathrm{k}^{291 \mathrm{~K}}=(1,71 \pm 0,6) \cdot 10^{14} \mathrm{~cm}^{3} \cdot \mathrm{mol}^{-1} \cdot \mathrm{s}^{-1},(\mathrm{p}=98-400$ Torr $)$, [HAN92]. Dieser Geschwindigkeitskoeffizient ist um den Faktor 5,5 größer als hier gemessen, ist jedoch wegen des erheblich höheren Drucks nur eingeschränkt vergleichbar mit der hier bei ca. 2 Torr ermittelten Konstanten. Es ist anzunehmen, dass sich die Reaktion Allyl $+\mathrm{H}$ bei 2 Torr noch im Niederdruckbereich befindet. 


\subsubsection{Cycloheptyl + H}

\section{Reaktionsgeschwindigkeit:}

Messergebnisse: Die Geschwindigkeit der Reaktion c-Heptyl + H wurde bei Zimmertemperatur relativ zur Reaktion Methoxymethyl $+\mathrm{H}$ mit Versuchsanordnung A (REMPI) bei $\lambda=440 \mathrm{~nm}$ bestimmt. Die Radikale wurden gemäß Abschnitt 3.1.8 und 3.1.14 erzeugt und auf ihren Muttermassen $\mathrm{m} / \mathrm{z}=97$ und $\mathrm{m} / \mathrm{z}=45$ nachgewiesen. Zur Bestimmung des Geschwindigkeitskoeffizienten wurden die Signalintensitäten bei An- und Abwesenheit von H-Atomen gemessen. Durch Variation der Wasserstoffkonzentration wurden verschiedene Umsätze erzielt. Die folgende Abbildung zeigt in doppeltlogarithmischer Auftragung die Messpunkte. Die zugehörigen Werte finden sich in Abschnitt 5 (Messtabellen). Die gewählten Versuchsbedingungen sind dem Text in der Abbildung oder bei den Messtabellen zu entnehmen.

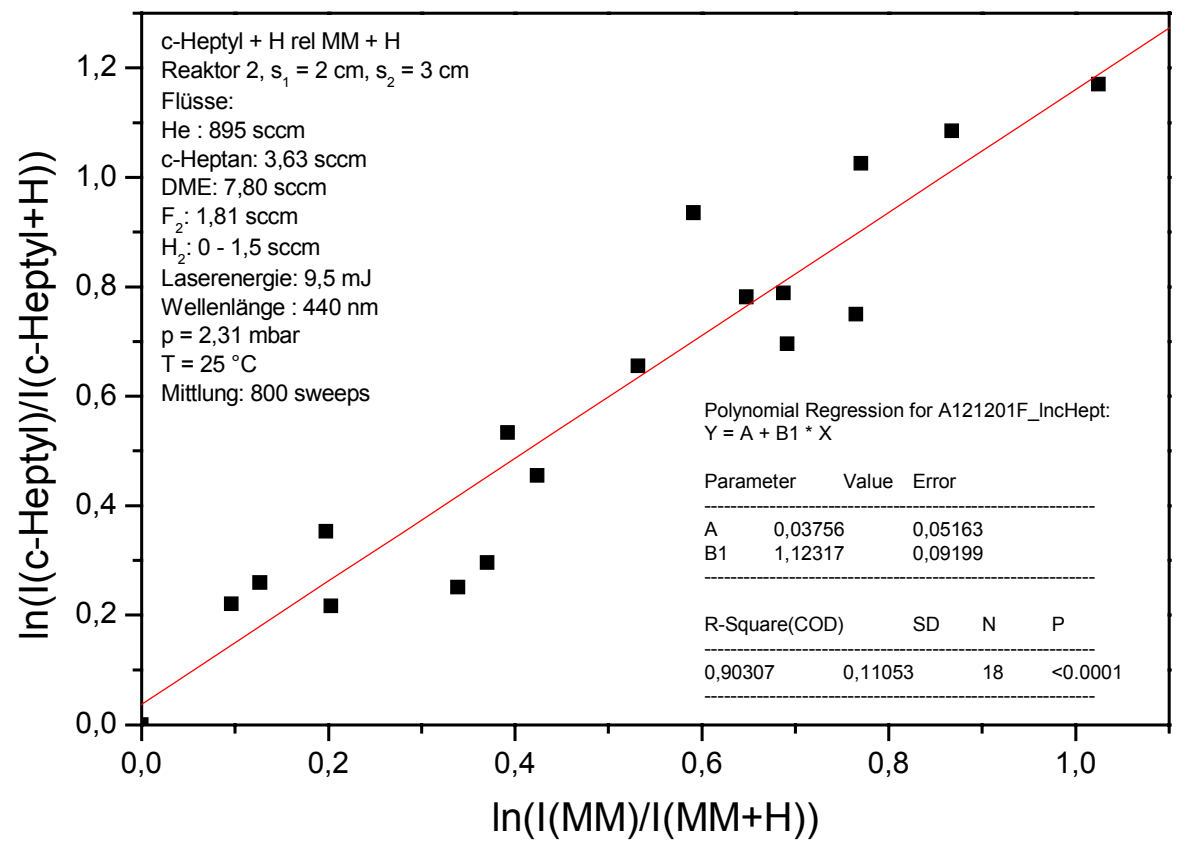

Abbildung 175: c-Heptyl $+\mathrm{H}$ relativ zu Methoxymethyl $+\mathrm{H}, \mathrm{T}=25^{\circ} \mathrm{C}$

Aus der Auftragung ergibt sich:

$$
\mathrm{k}_{\mathrm{rel}}=\mathrm{k}\left(\mathrm{c}-\mathrm{C}_{7} \mathrm{H}_{13}+\mathrm{H}\right) / \mathrm{k}\left(\mathrm{CH}_{3} \mathrm{OCH} \mathrm{H}_{2}+\mathrm{H}\right)=1,12 \pm 0,10 \text {. }
$$

Mit der bei [NAC94] erläuterten Geschwindigkeitskonstante $k_{\mathrm{ref}}=3,5 \cdot 10^{13} \mathrm{~cm}^{3} /(\mathrm{mol} \cdot \mathrm{s})$ für die Referenzreaktion Methoxymethyl + H erhält man:

$$
\mathrm{k}^{298 \mathrm{~K}}\left(\mathrm{c}-\mathrm{C}_{7} \mathrm{H}_{13}+\mathrm{H}\right)=(3,9 \pm 0,4) \cdot 10^{13} \mathrm{~cm}^{3} /(\mathrm{mol} \cdot \mathrm{s})
$$

Es liegt keine Literatur über die Reaktion c-Heptyl + H vor. 


\subsubsection{Cyclooktyl $+\mathbf{H}$}

Reaktionsgeschwindigkeit:

Messergebnisse: Die Geschwindigkeit der Reaktion c-Oktyl + H wurde bei Zimmertemperatur relativ zur Reaktion Methoxymethyl $+\mathrm{H}$ mit Versuchsanordnung A (REMPI) bei $\lambda=440 \mathrm{~nm}$ bestimmt. Die Radikale wurden gemäß Abschnitt 3.1.9 und 3.1.14 erzeugt und auf ihren Muttermassen $\mathrm{m} / \mathrm{z}=111$ und $\mathrm{m} / \mathrm{z}=45$ nachgewiesen. Zur Bestimmung des Geschwindigkeitskoeffizienten wurden die Signalintensitäten bei An- und Abwesenheit von H-Atomen gemessen. Durch Variation der Wasserstoffkonzentration wurden verschiedene Umsätze erzielt. Die folgende Abbildung zeigt in doppeltlogarithmischer Auftragung die Messpunkte. Die zugehörigen Werte finden sich in Abschnitt 5 (Messtabellen). Die gewählten Versuchsbedingungen sind dem Text in der Abbildung oder bei den Messtabellen zu entnehmen.

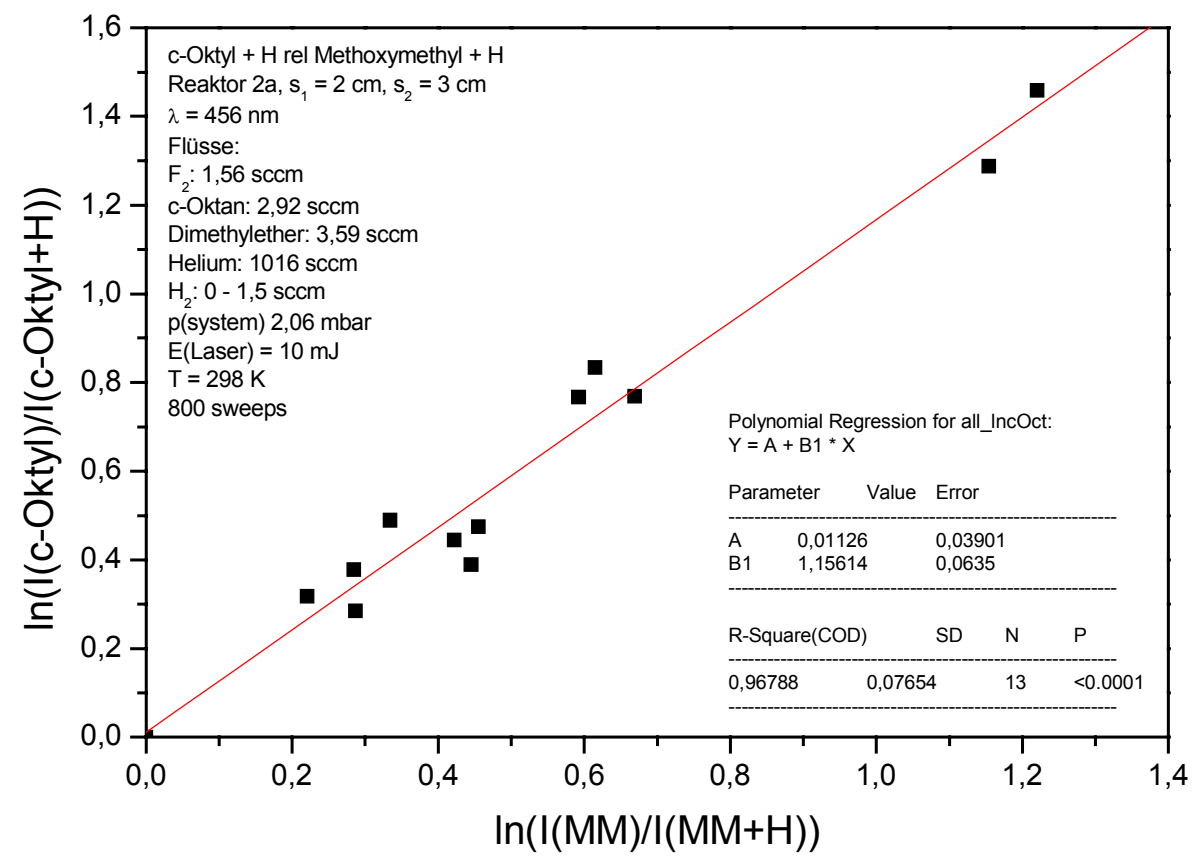

Abbildung 176: c-Oktyl $+\mathrm{H}$ relativ zu Methoxymethyl $+\mathrm{H}, \mathrm{T}=25^{\circ} \mathrm{C}$

Aus der Auftragung ergibt sich:

$$
\mathrm{k}_{\mathrm{rel}}=\mathrm{k}\left(\mathrm{c}-\mathrm{C}_{8} \mathrm{H}_{15}+\mathrm{H}\right) / \mathrm{k}\left(\mathrm{CH}_{3} \mathrm{OCH}_{2}+\mathrm{H}\right)=1,16 \pm 0,07 \text {. }
$$

Mit der bei [NAC94] erläuterten Geschwindigkeitskonstante $k_{\mathrm{ref}}=3,5 \cdot 10^{13} \mathrm{~cm}^{3} /(\mathrm{mol} \cdot \mathrm{s})$ für die Referenzreaktion Methoxymethyl + H ergibt sich:

$$
\mathrm{k}^{298 \mathrm{~K}}\left(\mathrm{c}-\mathrm{C}_{8} \mathrm{H}_{15}+\mathrm{H}\right)=(4,1 \pm 0,3) \cdot 10^{13} \mathrm{~cm}^{3} /(\mathrm{mol} \cdot \mathrm{s})
$$

Es liegt keine Literatur über die Reaktion c-Oktyl + H vor. 


\subsubsection{2-Chlor-2-Methyl-Propyl + H}

Reaktionsgeschwindigkeit:

Messergebnisse: Die Geschwindigkeit der Reaktion iso- $\mathrm{C}_{4} \mathrm{H}_{8} \mathrm{Cl}+\mathrm{H}$ wurde bei Zimmertemperatur relativ zur Reaktion Methoxymethyl $+\mathrm{H}$ mit Versuchsanordnung A (REMPI) bei $\lambda=440 \mathrm{~nm}$ bestimmt. Die Radikale wurden gemäß Abschnitt 3.1.11 und 3.1.14 erzeugt und auf ihren Muttermassen $\mathrm{m} / \mathrm{z}=91$ und $\mathrm{m} / \mathrm{z}=45$ nachgewiesen. Zur Bestimmung des Geschwindigkeitskoeffizienten wurden die Signalintensitäten bei An- und Abwesenheit von H-Atomen gemessen. Durch Variation der Wasserstoffkonzentration wurden verschiedene Umsätze erzielt. Die folgende Abbildung zeigt in doppeltlogarithmischer Auftragung die Messpunkte. Die zugehörigen Werte finden sich in Abschnitt 5 (Messtabellen). Die gewählten Versuchsbedingungen sind dem Text in der Abbildung oder bei den Messtabellen zu entnehmen.

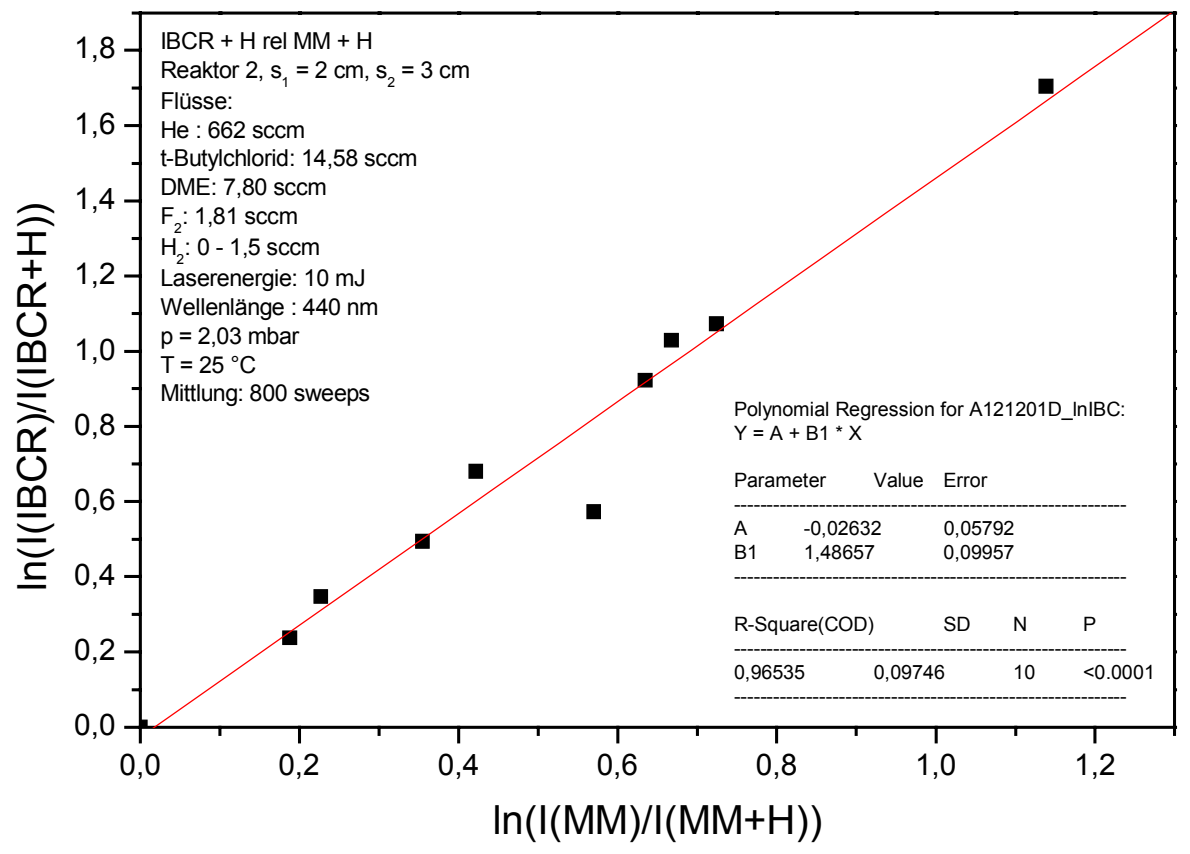

Abbildung 177: iso- $\mathrm{C}_{4} \mathrm{H}_{8} \mathrm{Cl}+\mathrm{H}$ relativ zu Methoxymethyl $+\mathrm{H}, \mathrm{T}=25^{\circ} \mathrm{C}$

Aus der Auftragung ergibt sich:

$$
\mathrm{k}_{\text {rel }}=\mathrm{k}\left(\text { iso- } \mathrm{C}_{4} \mathrm{H}_{8} \mathrm{Cl}+\mathrm{H}\right) / \mathrm{k}\left(\mathrm{CH}_{3} \mathrm{OCH}_{2}+\mathrm{H}\right)=1,49 \pm 0,10 \text {. }
$$

Mit der bei [NAC94] erläuterten Geschwindigkeitskonstante von $\mathrm{k}_{\mathrm{ref}}=3,5 \cdot 10^{13} \mathrm{~cm}^{3} /(\mathrm{mol} \cdot \mathrm{s})$ für die Referenzreaktion Methoxymethyl + H erhält man:

$$
\mathrm{k}^{298 \mathrm{~K}}\left(\mathrm{iso}-\mathrm{C}_{4} \mathrm{H}_{8} \mathrm{Cl}+\mathrm{H}\right)=(5,2 \pm 0,4) \cdot 10^{13} \mathrm{~cm}^{3} /(\mathrm{mol} \cdot \mathrm{s}) \text {. }
$$

Es liegt keine Literatur über die Reaktion iso- $\mathrm{C}_{4} \mathrm{H}_{8} \mathrm{Cl}+\mathrm{H}$ vor. 


\subsubsection{Vergleich der Geschwindigkeiten der Reaktionen Radikal + H}

Die Geschwindigkeitskoeffizienten der untersuchten Reaktionen von Kohlenwasserstoffradikalen mit H-Atomen sind im Abschnitt 4.7 tabellarisch zusammengefasst. Die Ähnlichkeit der Werte ist auffällig. Die Tatsache, dass bei Zimmertemperatur alle Reaktionen nahezu gleich schnell ablaufen, führt zu der Vermutung, dass aufgrund der Größe der Radikale die Geschwindigkeiten der Reaktionen bereits ihren Hochdruckgrenzwert erreicht haben. Die Reaktionen von Kohlenwasserstoffradikalen mit H-Atomen, stellen bei Verbrennungsprozessen eine Verlangsamung des Geschehens dar. Kommt es zu einer Stabilisierung der entstehenden Kohlenwasserstoffe, so würde sowohl ein reaktives Radikal als auch reaktives H-Atom dem Verbrennungsprozess entzogen. Es muss allerdings bedacht werden, dass Verbrennungen im allgemeinen bei höheren Temperaturen ablaufen. Somit wäre sowohl eine Untersuchung der Temperaturabhängigkeit der genannten Reaktionen als auch eine Bestimmung der Geschwindigkeitskonstanten im Temperaturbereich über $500 \mathrm{~K}$ wünschenswert. Die Verwendung des Strömungsreaktors 3 mit einem elektrischen Ofen (vgl. Abschnitt 2.1.2) wäre hierzu eventuell ein geeigneter Ansatz. 


\section{Zusammenfassung der Ergebnisse}

\subsection{Erzeugung und Nachweis von Kohlenwasserstoffradikalen}

Alle im folgenden aufgeführten Kohlenwasserstoffradikale wurden in der Gasphase in einem isothermen Strömungssystem mit kontinuierlicher Molekularstrahlprobennahme massenspektrometrischem Nachweis bei einem Druck von 1-2 mbar dargestellt.

In der vorliegenden Arbeit wurden die Radikale c-Heptyl, c-Oktyl, 2-Chlor-2-Methyl-Propyl und c-Pentadienyl durch Wasserstoffabstraktion aus den jeweiligen Kohlenwasserstoffen c-Heptan, c-Oktan, t-Butylchlorid und c-Pentadien dargestellt und erstmalig mit resonanzverstärkter Mehrphotonenionisation (REMPI) und gekoppeltem Flugzeitmassenspektrometer (TOF) spezifisch nachgewiesen. Der Nachweis des c-Pentadienylradikals zeigt im Bereich $\lambda=423-430 \mathrm{~nm}$ eine charakteristische Wellenlängenabhängigkeit. c-Heptyl, c-Oktyl, 2-Chlor-2-Methyl-Propyl sind wenig wellenlängenselektiv, ihr Signal folgt im Bereich $\lambda=420-530 \mathrm{~nm}$ im wesentlichen der Energie des Nachweislasers. Bei der Darstellung des c-Pentadienylradikals aus einer Abstraktionsreaktion von cPentadien und Fluoratomen wurde als Nebenprodukt auch das aus einer Additionsreaktion hervorgehende Fluoropentenylradikal im Bereich 440-460 $\mathrm{nm}$ in schwacher Intensität nachgewiesen.

Die Radikale 1-Propyl (n-Propyl), 2-Propyl (Isopropyl), 1-Butyl und 2-Butyl wurden durch Wasserstoffabstraktion aus den jeweiligen Kohlenwasserstoffen Propan und n-Butan dargestellt. Durch den Einsatz der deuterierten Vorläufersubstanzen $\mathrm{CH}_{3} \mathrm{CD}_{2} \mathrm{CH}_{3}$ und $\mathrm{CD}_{3} \mathrm{CH}_{2} \mathrm{CH}_{2} \mathrm{CD}_{3}$ konnte für den REMPI-Nachweis zum ersten Mal eine Grenzwellenlänge ( $\lambda=465 \mathrm{~nm}$ ) gefunden werden, ab der nur noch die 2-Alkylradikale aufgrund eines niedrigeren Ionisierungspotenzials massenspektrometrisch signalgebend sind. Die Untersuchung der Reaktionen des 2-Propyl- und des 2-Butylradikals kann bei $\lambda \geq 465 \mathrm{~nm}$ erfolgen, die Untersuchung der Reaktionen des 1-Propyl- und des 1-Butylradikals ist bei dem verwendeten Nachweis nur über den Einsatz der deuterierten Radikalvorläufer möglich.

Die Radikale t-Butyl und Isobutyl wurden durch Wasserstoffabstraktion aus Isobutan dargestellt. Durch den Einsatz der deuterierten Vorläufersubstanz t- $\mathrm{C}_{4} \mathrm{H}_{9}-\mathrm{D}$ wurde für den REMPI-Nachweis erstmalig eine Grenzwellenlänge $(\lambda=470 \mathrm{~nm})$ gefunden, ab der nur noch das t-Butyl signalgebend ist. Die Intensität des t-Butylradikals ist wegen des niedrigen Ionisierungspotenzials bis $\lambda=540 \mathrm{~nm}$ sehr hoch. Die Untersuchung der Reaktionen des t-Butylradikals kann im Wellenlängenbereich 
$\lambda \geq 470 \mathrm{~nm}$ erfolgen, die Untersuchung der Reaktionen des Isobutylradikals ist nur über den Einsatz des deuterierten Radikalvorläufers möglich.

Der REMPI-Nachweis des Ethylradikal im Bereich 397-500 nm ist bereits seit längerem bekannt (vgl. Abschnitte 3.1.2 und 6.1). In der vorliegenden Arbeit wurde $\mathrm{C}_{2} \mathrm{H}_{5}$ auch bei der Wellenlänge $\lambda=340,8$ eindeutig nachgewiesen. Das deuterierte Ethylradikal $\mathrm{C}_{2} \mathrm{D}_{5}$ ist in der selben Weise signalgebend. Untersuchungen bei benachbarten Wellenlängen ließen wie im langwelligen Bereich keine besondere Wellenlängenselektivität erkennen. Bei längeren Wellenlängen als $443 \mathrm{~nm}$ kommt es zu einem drastischen Einbruch der Signalintensität, der wahrscheinlich auf einen Wechsel vom 2+1 zum 3+1 REMPI-Mechanismus, also dem Benötigen eines weiteren Photons zur Ionisierung, zurückzuführen ist.

Das 1,1,1-Trichlorethyl wurde aus einer H-Atom Abstraktionsreaktion aus dem 1,1,1-Trichlorethan erzeugt und mit Elektronenstoßionisation bei $\mathrm{IE}=29,5 \mathrm{eV}$ massenspektrometrisch erstmalig nachgewiesen.

Die Radikale Methyl, c-Pentyl, c-Hexyl, Allyl und Methoxymethyl wurden durch H-AtomAbstraktionsreaktion aus den Vorläufermolekülen Methan, c-Pentan, c-Hexan, Propen und Dimethylether dargestellt und mittels Mehrphotonenionisation spezifisch nachgewiesen. Die Nachweise waren bereits bekannt.

\subsection{Reaktionen von Kohlenwasserstoffen mit F-Atomen}

Alle im folgenden aufgeführten Reaktionen von Kohlenwasserstoffen mit F-Atomen wurden in der Gasphase in einem isothermen Strömungssystem mit kontinuierlicher Molekularstrahlprobennahme und massenspektrometrischem Nachweis bei einem Druck von 1-2 mbar untersucht.

Die Reaktion von Trichlorethan mit F-Atomen wurde in bezug auf Produkte und Geschwindigkeit in einem Sektorfeldmassenspektrometer mit Elektronenstoßionisation erforscht. Es wird folgender Reaktionsmechanismus aufgestellt:

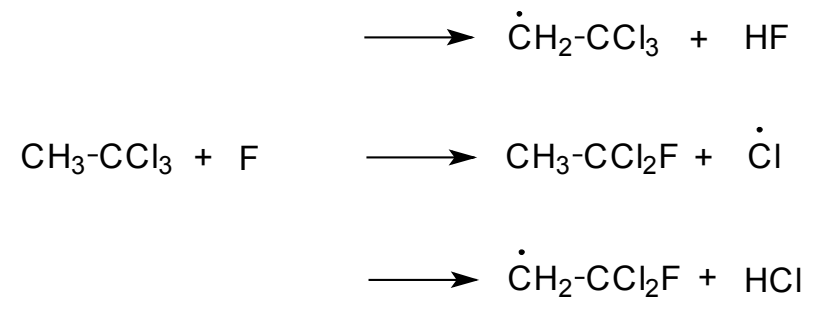


Die Reaktionskanäle (a) und (c) können klar nachgewiesen werden, für Kanal (b) gibt es Anzeichen. Die Geschwindigkeit der Reaktion $\mathrm{CH}_{3} \mathrm{CCl}_{3}+\mathrm{F}$ wurde im Temperaturintervall $\mathrm{T}=-20{ }^{\circ} \mathrm{C}$ bis $+85^{\circ} \mathrm{C}$ ermittelt zu:

$$
\begin{gathered}
\mathrm{k}=(8,94 \pm 0,49) \cdot 10^{12} \exp \left\{-479 \pm 132 /(\mathrm{RT}) \mathrm{J} \cdot \mathrm{mol}^{-1}\right\} \mathrm{cm}^{3} \cdot \mathrm{mol}^{-1} \cdot \mathrm{s}^{-1} \\
\mathrm{k}^{298}=(7,37 \pm 0,76) \cdot 10^{12} \mathrm{~cm}^{3} \cdot \mathrm{mol}^{-1} \cdot \mathrm{s}^{-1}
\end{gathered}
$$

Die Reaktion des Isobutylchlorids (1-Chlor-2-Methyl-Propan) mit F-Atomen wurde mit Hilfe eines Flugzeitmassenspektrometer mit resonanzverstärkter Mehrphotonenionisation untersucht. Es wurde eine H-Atom-Abstraktionsreaktion festgestellt, die in drei Reaktionskanäle münden kann:<smiles>CCC(C)(C)CCl</smiles><smiles>CC([CH]C(C)CCl)[CH]C(C)CC(C)CCl</smiles>

Welche der drei isomeren Radikale in welchen Anteilen signalgebend war, konnte mit den vorhandenen Mitteln nicht entschieden werden.

Die Untersuchung der Reaktion t-Butylalkohol (tBAl) $+\mathrm{F}$ wurde anhand des an der Hydroxylgruppe deuterierten Kohlenwasserstoffs $\mathrm{t}-\mathrm{C}_{4} \mathrm{H}_{9} \mathrm{OD}$ mit Hilfe eines Flugzeitmassenspektrometers mit resonanzverstärkter Mehrphotonenionisation durchgeführt. Aufgrund der Ergebnisse bei der Ionisierungswellenlänge $\lambda=440 \mathrm{~nm}$ können die zwei folgenden Reaktionskanäle angegeben werden:<smiles>CC(C)(C)[O+]</smiles> 
Das Ionensignal bei $\mathrm{m} / \mathrm{z}=57$ wurde dem t-Butoxyradikal nach Kanal (a) zugeordnet. Bei $\lambda=520 \mathrm{~nm}$ konnte lediglich dieses Signal beobachtet werden. Ein Ansteigen der Signalintensität bei zusätzlicher Zugabe von O-Atomen wird für das t-Butoxyradikal als charakteristisch angesehen.

Die Reaktion Di-t-Butyl-Peroxid (DTBP) mit F-Atomen wurde vom Autor bereits in einer Staatsexamensarbeit untersucht [WEH97]. Es wurde folgender Mechanismus vorgeschlagen:<smiles>CC(C)(C)OOC(C)(C)C</smiles><smiles>CC(C)(C)[OH+]</smiles>

Die in dieser Arbeit dem t-Butoxyradikal zugeordnete Signaltätigkeit bei $\mathrm{m} / \mathrm{z}=57$ in einem REMPI-Flugzeitmassenspektrometer bei $\lambda=520 \mathrm{~nm}$ wird in Kombination mit dem besonderen Signalverhalten bei O-Atomzugabe als Bestätigung dieses Mechanismus angesehen.

Auch die Reaktion des t-Butyl-Peroxy-Pivalats (tBPP) mit F-Atomen wurde vom Autor bereits im Rahmen einer Staatsexamensarbeit untersucht [WEH97]. Der Peroxy-Ester eröffnet aufgrund seines unsymmetrischen Molekülbaus zwei Möglichkeiten einer H-Atom Abstraktion. Folgende Reaktionskanäle wurden auf Basis der Messergebnisse vorgeschlagen:<smiles>CCC(C)(C)OOC(=O)C(C)(C)[In]F</smiles><smiles>CC(C)(C)[C+]C(=O)[OH+]</smiles> 


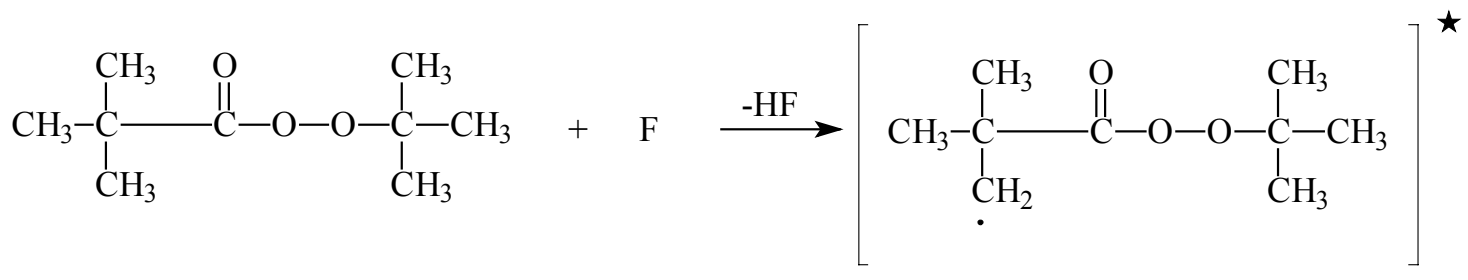<smiles>C=C(C)CCCCCC(=O)OC(C)(C)C</smiles>

Die Interpretation ist aufgrund der Komplexität der Verhältnisse erschwert. Sowohl das t-Butyl als auch das t-Butoxyradikal sind bei $\mathrm{m} / \mathrm{z}=57$ signalerzeugend. Eine Abschätzung des Signalverhaltens bei zusätzlicher O-Atom-Zugabe wird als Bestätigung für beide Kanäle angesehen.

Die Reaktion des Dimethoxymethans mit F-Atomen wurde mit einem REMPI-Flugzeitmassenspektrometer und mit einem EI-Sektorfeldmassenspektrometer erstmalig untersucht. Es wurden für folgende Kanäle Hinweise gefunden.<smiles>COCOC</smiles><smiles>CCOCCOC</smiles>

Für einen schnellen Zerfall des endständig radikalisierten Dimethoxymethylradikals gemäß folgender Reaktionsgleichung wurden Anzeichen gefunden:<smiles>CCCCOCCOC</smiles>

Die Geschwindigkeit der Reaktion $\mathrm{CH}_{3} \mathrm{OCH}_{2} \mathrm{OCH}_{3}+\mathrm{F}$ wurde im Temperaturintervall $\mathrm{T}=-20{ }^{\circ} \mathrm{C}$ bis $+85^{\circ} \mathrm{C}$ ermittelt zu:

$$
\begin{gathered}
\mathrm{k}=(1,78 \pm 0,05) \cdot 10^{14} \exp \left\{-2332 \pm 77 /(\mathrm{RT}) \mathrm{J} \cdot \mathrm{mol}^{-1}\right\} \mathrm{cm}^{3} \cdot \mathrm{mol}^{-1} \cdot \mathrm{s}^{-1} \\
\mathrm{k}^{298}=(6,95 \pm 0,65) \cdot 10^{13} \mathrm{~cm}^{3} \cdot \mathrm{mol}^{-1} \cdot \mathrm{s}^{-1}
\end{gathered}
$$




\subsection{Reaktionen von Kohlenwasserstoffradikalen mit O-Atomen}

Zur Bestimmung der Geschwindigkeit wurden die Reaktionen in einem isothermen Strömungsreaktor mit kontinuierlicher Molekularstrahlprobennahme durchgeführt. Der Nachweis erfolgte massenspektrometrisch mit resonanzverstärkter Mehrphotonenionisation (REMPI) und mit Elektronenstoßionisation (EI). Zur Aufstellung eines in sich konsistenten Systems von Geschwindigkeitskoeffizienten für die Reaktionsklasse Radikal $+\mathrm{O}$, wurden einzelne Reaktionen relativ zueinander vermessen. Die folgende Tabelle gibt die Ergebnisse in kompakter Form wieder. Falls eine Temperaturabhängigkeit angeben ist, bezieht sie sich auf den Bereich $-30 /+5{ }^{\circ} \mathrm{C}$ bis $+90{ }^{\circ} \mathrm{C}$. Einige der erwähnten Reaktionen wurden durch die vorliegende Arbeit erstmalig untersucht.

\begin{tabular}{|c|c|c|}
\hline+0 & $\mathrm{k}^{298 \mathrm{~K}} / \mathrm{cm}^{3}(\mathrm{~mol} \cdot \mathrm{s})^{-1}$ & $\mathrm{k}(\mathrm{T}) / \mathrm{cm}^{3}(\mathrm{~mol} \cdot \mathrm{s})^{-1}$ \\
\hline Methyl & $(6,14 \pm 0,2) \cdot 10^{13}$ & \\
\hline 2-Propyl & $(1,14 \pm 0,04) \cdot 10^{14}$ & \\
\hline 1-Propyl & $(8,43 \pm 0,09) \cdot 10^{13}$ & \\
\hline 2-Butyl & $(9,69 \pm 0,07) \cdot 10^{13}$ & \\
\hline 1-Butyl & $(9,40 \pm 0,12) \cdot 10^{13}$ & \\
\hline t-Butyl & $(1,38 \pm 0,08) \cdot 10^{14}$ & $\mathrm{k} \neq \mathrm{f}(\mathrm{T})$ \\
\hline Isobutyl & $(9,11 \pm 0,09) \cdot 10^{13}$ & \\
\hline c-Pentyl & $(1,73 \pm 0,05) \cdot 10^{14}$ & \\
\hline c-Heptyl & $(2,01 \pm 0,10) \cdot 10^{14}$ & \\
\hline c-Oktyl & $(1,70 \pm 0,4) \cdot 10^{14}$ & $(3,4 \pm 0,30) \cdot 10^{14} \exp \left(-\frac{(1,7 \pm 0,3) \mathrm{kJ} \cdot \mathrm{mol}^{-1}}{\mathrm{R} \cdot \mathrm{T}}\right)$ \\
\hline Allyl & $(1,11 \pm 0,2) \cdot 10^{14}$ & \\
\hline c-Pentadienyl & $(1,34 \pm 0,09) \cdot 10^{14}$ & \\
\hline Methoxymethyl & $(1,31 \pm 0,08) \cdot 10^{14}$ & \\
\hline Trichlorethyl & $(2,83 \pm 0,5) \cdot 10^{13}$ & $(3,47 \pm 0,30) \cdot 10^{13} \exp \left(-\frac{(0,5 \pm 0,2) \mathrm{kJ} \cdot \mathrm{mol}^{-1}}{\mathrm{R} \cdot \mathrm{T}}\right)$ \\
\hline $\mathrm{t}-\mathrm{C}_{4} \mathrm{H}_{8} \mathrm{Cl}$ & $(1,86 \pm 0,19) \cdot 10^{14}$ & \\
\hline
\end{tabular}

Tabelle 7: Zusammenfassung der Geschwindigkeitskoeffizienten der Reaktionen Radikal + O 
Als Basisreaktion wurde die eingehend untersuchte Reaktion Ethyl + O gesetzt. Der zugehörige Geschwindigkeitskoeffizient $\mathrm{k}^{298 \mathrm{~K}}\left(\mathrm{C}_{2} \mathrm{H}_{5}+\mathrm{O}\right)=1,04 \cdot 10^{14} \mathrm{~cm}^{3} /(\mathrm{mol} \cdot \mathrm{s})$ ([HAC02], [ZEU02]) wird für die Angabe der folgenden absoluten Geschwindigkeitskonstanten als richtig vorausgesetzt. Er ist in weitgehender Übereinstimmung mit anderen Literaturstellen (vgl. Abschnitt 3.3.1). Sollte sich dieser Wert dennoch ändern, so behalten die in den einzelnen Abschnitten angegebenen relativen Geschwindigkeitskonstanten ihre Gültigkeit. Eine Neuausrichtung der Werte ist problemlos möglich. Für einige der genannten Reaktionen wurden umfangreiche Vergleichsmessungen durchgeführt, die eine Konsistenz der Werte ergaben. Ein vermuteter Zusammenhang zwischen den Geschwindigkeitskonstanten der Reaktionen Radikal + O und den Ionisierungspotenzialen der Radikale kann aufgrund der Messergebnisse bestätigt werden.

Die Geschwindigkeiten der Reaktionen Methyl + O und Ethyl + O wurden auf einen Isotopeneffekt hin untersucht. Es ergab sich: $\mathrm{k}_{\text {rel }}=\mathrm{k}\left(\mathrm{CD}_{3}+\mathrm{O}\right) / \mathrm{k}\left(\mathrm{CH}_{3}+\mathrm{O}\right)=1,01 \pm 0,12$, $\mathrm{k}_{\text {rel }}=\mathrm{k}\left(\mathrm{C}_{2} \mathrm{D}_{5}+\mathrm{O}\right) / \mathrm{k}\left(\mathrm{C}_{2} \mathrm{H}_{5}+\mathrm{O}\right)=1,01 \pm 0,07$. Für beide Reaktionen ist ein kinetischer Isotopeneffekt demnach nicht nachzuweisen.

Die Reaktion t-Butyl $+\mathrm{O}$ wurde in einer statischen Photolysezelle mit FTIR-Nachweis nach Produkten untersucht. Als Radikalvorläufer wurde Di-t-Butylketon eingesetzt. Es wurden deutliche Hinweise für folgenden Mechanismus gefunden:

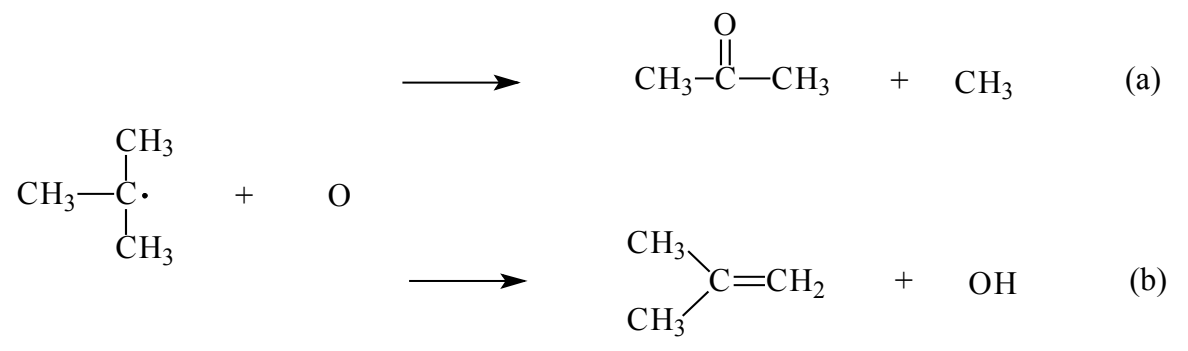

\subsection{Reaktionen von Kohlenwasserstoffradikalen mit $\mathrm{O}_{2}$}

Zur Bestimmung der Geschwindigkeit wurden die Reaktionen in einem isothermen Strömungsreaktor mit kontinuierlicher Molekularstrahlprobennahme durchgeführt. Der Nachweis erfolgte massenspektrometrisch mit resonanzverstärkter Mehrphotonenionisation (REMPI) und mit Elektronenstoßionisation (EI). Die folgende Tabelle gibt die Ergebnisse wieder. Falls eine Temperaturabhängigkeit angeben ist, bezieht sie sich auf den Bereich $-30 /+5{ }^{\circ} \mathrm{C}$ bis $+90{ }^{\circ} \mathrm{C}$. Bis auf die Reaktion t-Butyl $+\mathrm{O}_{2}$ wurden alle aufgeführten Reaktionen durch diese Arbeit erstmalig untersucht. 


\begin{tabular}{|c|c|c|}
\hline$+\mathrm{O}_{2}$ & $\mathrm{k}^{298 \mathrm{~K}} / \mathrm{cm}^{3}(\mathrm{~mol} \cdot \mathrm{s})^{-1}$ & $\mathrm{k}(\mathrm{T}) / \mathrm{cm}^{3}(\mathrm{~mol} \cdot \mathrm{s})^{-1}$ \\
\hline t-Butyl & $(7,4 \pm 2,6) \cdot 10^{12}$ & $(2,2 \pm 0,4) \cdot 10^{11} \exp \left(-\frac{(-8,7 \pm 0,4) \mathrm{kJ} \cdot \mathrm{mol}^{-1}}{\mathrm{R} \cdot \mathrm{T}}\right)$ \\
\hline c-Heptyl & $(1,31 \pm 0,12) \cdot 10^{13}$ & \\
\hline c-Oktyl & $(8,72 \pm 4,9) \cdot 10^{12}$ & $(1,46 \pm 0,36) \cdot 10^{11} \exp \left(-\frac{(-10,14 \pm 0,57) \mathrm{kJ} \cdot \mathrm{mol}^{-1}}{\mathrm{R} \cdot \mathrm{T}}\right)$ \\
\hline c-Pentadienyl & $(2,25 \pm 0,31) \cdot 10^{11}$ & \\
\hline $\mathrm{t}-\mathrm{C}_{4} \mathrm{H}_{8} \mathrm{Cl}$ & $(5,07 \pm 0,48) \cdot 10^{12}$ & \\
\hline
\end{tabular}

Tabelle 8: Zusammenfassung der Geschwindigkeitskoeffizienten der Reaktionen Radikal $+\mathrm{O}_{2}$

Die Reaktion t-Butyl $+\mathrm{O}_{2}$ wurde in einer statischen Photolysezelle mit FTIR-Nachweis nach Produkten untersucht. Als Radikalvorläufer wurde Di-t-Butylketon eingesetzt. Bei niedrigem Druck ( $p=4$ mbar) können Aceton, Formaldehyd und Isobuten als Produkte nachgewiesen werden. Bei einem Druck von 1 bar ist Isobuten nicht mehr nachzuweisen, sondern nur noch Aceton und Formaldehyd.

\subsection{Reaktionen von Kohlenwasserstoffradikalen mit $\mathbf{O}_{3}$}

Zur Bestimmung der Geschwindigkeit wurden die Reaktionen in einem isothermen Strömungsreaktor mit kontinuierlicher Molekularstrahlprobennahme durchgeführt. Der Nachweis erfolgte massenspektrometrisch mit resonanzverstärkter Mehrphotonenionisation (REMPI). Bis auf die Reaktion t-Butyl $+\mathrm{O}_{3}$ wurden alle aufgeführten Reaktionen durch diese Arbeit erstmalig untersucht.

\begin{tabular}{|c||r|}
\hline $\mathbf{+} \mathbf{O}_{\mathbf{3}}$ & $\mathbf{k}^{\mathbf{2 9 8 K}} / \mathbf{c m}^{\mathbf{3}} \mathbf{( \mathbf { m o l } \cdot \mathbf { s } ) ^ { - \mathbf { 1 } }}$ \\
\hline $\mathbf{t}-$ Butyl & $(3,06 \pm 0,11) \cdot 10^{13}$ \\
\hline $\mathbf{c}-$ Heptyl & $(2,96 \pm 0,16) \cdot 10^{13}$ \\
\hline $\mathbf{c}-\mathbf{O k t y l}$ & $(3,33 \pm 0,21) \cdot 10^{13}$ \\
\hline $\mathbf{t}-\mathbf{C}_{4} \mathbf{H}_{\mathbf{8}} \mathbf{C l}$ & $(1,71 \pm 0,21) \cdot 10^{13}$ \\
\hline
\end{tabular}

Tabelle 9: Zusammenfassung der Geschwindigkeitskoeffizienten der Reaktionen Radikal $+\mathrm{O}_{3}$ 


\subsection{Reaktionen von Kohlenwasserstoffradikalen mit NO}

Die Geschwindigkeiten der Reaktionen t-Butyl + NO und Allyl + NO wurden in einem isothermen Strömungsreaktor mit kontinuierlicher Molekularstrahlprobennahme im Temperaturintervall $-30{ }^{\circ} \mathrm{C}$ bis $+90{ }^{\circ} \mathrm{C}$ untersucht. Der Nachweis erfolgte massenspektrometrisch mit resonanzverstärkter Mehrphotonenionisation (REMPI).

$$
\begin{gathered}
\mathrm{t}-\text { Butyl }+\mathrm{NO}: \\
\mathrm{k}=(3,1 \pm 1,8) \cdot 10^{12} \exp \left\{-(4,1 \pm 1,1) /(\mathrm{RT}) \mathrm{kJ} \cdot \mathrm{mol}^{-1}\right\} \mathrm{cm}^{3} \cdot \mathrm{mol}^{-1} \cdot \mathrm{s}^{-1} \\
\mathrm{k}^{298}=(5,92 \pm 5,0) \cdot 10^{11} \mathrm{~cm}^{3} \cdot \mathrm{mol}^{-1} \cdot \mathrm{s}^{-1} \\
\text { Allyl }+\mathrm{NO}: \\
\mathrm{k}=(3,22 \pm 0,4) \cdot 10^{10} \exp \left\{-(-2,2 \pm 0,2) /(\mathrm{RT}) \mathrm{kJ} \cdot \mathrm{mol}^{-1}\right\} \mathrm{cm}^{3} \cdot \mathrm{mol}^{-1} \cdot \mathrm{s}^{-1} \\
\mathrm{k}^{298}=(7,82 \pm 1,8) \cdot 10^{11} \mathrm{~cm}^{3} \cdot \mathrm{mol}^{-1} \cdot \mathrm{s}^{-1} .
\end{gathered}
$$

\subsection{Reaktionen von Kohlenwasserstoffradikalen mit H-Atomen}

Zur Bestimmung der Geschwindigkeit wurden die Reaktionen in einem isothermen Strömungsreaktor mit kontinuierlicher Molekularstrahlprobennahme durchgeführt. Der Nachweis erfolgte massenspektrometrisch mit resonanzverstärkter Mehrphotonenionisation (REMPI).

\begin{tabular}{|c||c|}
\hline $\mathbf{H}$ & $\mathbf{k}^{298 \mathrm{~K}} / \mathbf{c m}^{\mathbf{3}} \mathbf{( \mathbf { m o l } \cdot \mathbf { s } ) ^ { \mathbf { 1 } }}$ \\
\hline 2-Propyl & $(3,1 \pm 0,2) \cdot 10^{13}$ \\
\hline 2-Butyl & $(3,3 \pm 0,5) \cdot 10^{13}$ \\
\hline t-Butyl & $(3,5 \pm 0,2) \cdot 10^{13}$ \\
\hline c-Heptyl & $(3,9 \pm 0,4) \cdot 10^{13}$ \\
\hline c-Oktyl & $(4,1 \pm 0,3) \cdot 10^{13}$ \\
\hline Allyl & $(3,1 \pm 0,1) \cdot 10^{13}$ \\
\hline t-C $\mathbf{H}_{4} \mathbf{C l}$ & $(5,2 \pm 0,4) \cdot 10^{13}$ \\
\hline
\end{tabular}

Tabelle 10: Zusammenfassung der Geschwindigkeitskoeffizienten der Reaktionen Radikal + H

Bis auf die Reaktion Allyl $+\mathrm{H}$ lagen für die aufgeführten Reaktionen bisher nur Abschätzungen in der Literatur vor. 


\subsection{Ausblick}

Die Ergebnisse der Arbeit zur Unterscheidung der isomeren Radikale 1-Propyl/2-Propyl, t-Butyl/Isobutyl und 1-Butyl/2-Butyl mittels massenspektrometrischem Nachweis mit resonanzverstärkter Mehrphotonenionisation (REMPI) geben Anlass zur Hoffnung, dass auch für die isomeren Pentyl- und Hexylradikale eine Grenzwellenlänge zu finden ist, bei der nur jeweils eines der isomeren Radikale in eindeutiger Weise signalgebend ist. Voraussetzung für eine solche Untersuchung ist jedoch die Verfügbarkeit teildeuterierter Kohlenwasserstoffe, so dass eine H-Abstraktionsreaktion mittels Fluor- oder Chloratome zu Radikalen unterschiedlicher Masse führt. Bisher sind nach Wissen des Autors solche Substanzen kommerziell nicht zu erwerben. Für $\mathrm{CH}_{3} \mathrm{CD}_{2} \mathrm{CH}_{3}$ und $\mathrm{t}-\mathrm{C}_{4} \mathrm{H}_{9} \mathrm{D}$ galt aber bis vor kurzem das gleiche.

In der vorliegenden Arbeit wurden nur die Reaktionen von 1-Propyl, 1-Butyl und Isobutyl mit atomarem Sauerstoff untersucht. Wie in den Abschnitten 3.1.3, 3.1.4 und 3.1.5 erläutert wurde, sind solche Experimente, die in Strömungsreaktoren mittels REMPI-MS-Nachweis durchgeführt werden, nur durch den Einsatz der sehr teuren teildeuterierten Radikalvorläuferkohlenwasserstoffe möglich. Der hohe Preis limitierte die Anzahl der Versuche in der vorliegenden Arbeit. Die Erforschung der Geschwindigkeiten von Reaktionen dieser Radikale mit weiteren Reaktionspartnern und der zugehörigen Temperaturabhängigkeit sollte jedoch nicht unterbleiben. Ein Absinken der Preise für die teildeuterierten Substanzen wäre für ein solches Vorhaben sehr förderlich.

In der vorliegenden Arbeit wurde die Temperaturabhängigkeit der Geschwindigkeit einiger Reaktionen Radikal $+\mathrm{X}\left(\mathrm{X}=\mathrm{O}, \mathrm{O}_{2}, \mathrm{O}_{3}, \mathrm{NO}, \mathrm{H}\right)$ im Temperaturintervall $-30 /+5$ bis $+90{ }^{\circ} \mathrm{C}$ untersucht. Die Kinetik der meisten hier dargestellten Reaktionen wurde jedoch nur bei Zimmertemperatur vermessen. Eine Ausdehnung der Untersuchungen zur Temperaturabhängigkeit der Geschwindigkeitskoeffizienten auch auf diese Reaktionen wäre wünschenswert. Auch eine Vergrößerung des Temperaturintervalls insbesondere hin zu höheren Temperaturen (500-700 K) sollte erfolgen. Der Einsatz eines elektrischen Ofens zum Erreichen dieser Temperaturen in der Reaktionszone lässt mit mäßigem Aufwand dieses Ziel erreichbar erscheinen. In Abschnitt 2.1.2 ist ein solcher Aufbau beschrieben. Auch der thermische Zerfall einiger Kohlenwasserstoffradikale könnte gegebenenfalls damit erforscht werden.

$\mathrm{Zu}$ den in dieser Arbeit nachgewiesenen Radikalen könnten weitere Reaktionspartner wie $\mathrm{NO}_{2}$ und $\mathrm{NO}_{3}$ zugegeben werden. Diese Reaktionspartner addieren ähnlich wie das in das in dieser Arbeit verwendete Ozon Sauerstoffatome an das Radikal, so das es wie bei der direkten Addition von O-Atomen zur Ausbildung eines energetisch angeregten Komplexes kommt. Je nach Reaktions 
partner ist die Anregungsenergie unterschiedlich hoch [HOY99]. Aus der Aufklärung der Reaktionsgeschwindigkeiten und Produktverteilungen kann durch entsprechende Rechnungen auf anregungsenergieabhängige Geschwindigkeitskonstanten $\mathrm{k}(\mathrm{E})$ geschlossen werden und somit ein Beitrag zur Erforschung unimolekularer Prozesse geleistet werden.

Die Ähnlichkeit der Geschwindigkeitskonstanten der Reaktionsklasse Radikal + $\mathrm{H}$ bei Zimmertemperatur (vgl. Abschnitt 3.7.8) lässt vermuten, dass aufgrund der Größe der Radikale der Hochdruckgrenzwert der Geschwindigkeitskoeffizienten bei dem hier angelegten Druck von 1,5 bis 2 mbar bereits erreicht ist. Eine Messreihe zur Druckabhängigkeit könnte diese Vermutung bestätigen. Mit Hilfe von Strömungsreaktoren und massenspektrometrischem Nachweis ist eine solche Untersuchung wegen des drohenden Verlustes der erforderlichen strömungsmechanischen und nachweistechnischen Eigenschaften jedoch auf einen Bereich bis zu 5 mbar begrenzt. Eine Untersuchung der Temperaturabhängigkeit der Reaktionen Radikal + H und eine Bestimmung der Geschwindigkeitskonstanten in dem für Verbrennungsprozesse sehr bedeutsamen Temperaturbereich über $500 \mathrm{~K}$ ist anzustreben. Die Verwendung des Strömungsreaktors 3 mit einem elektrischen Ofen (vgl. Abschnitt 2.1.2) wäre hierzu eventuell ein geeigneter Ansatz.

In der vorliegenden Arbeit wurden Produktuntersuchungen in einer statischen Reaktionszelle mit Photolysemöglichkeit und FTIR-Nachweis für die Reaktionen t-Butyl $+\mathrm{O}$ und t-Butyl $+\mathrm{O}_{2}$ durchgeführt. Als Radikalvorläufer wurde Di-t-Butylketon eingesetzt. Die Verwendung anderer Radikalvorläufer ist aus Gründen, wie sie in Abschnitt 3.3.9 erläutert wurden, angebracht. Die Ausdehnung dieser Produktanalysemethode auch auf die anderen in dieser Arbeit untersuchten Reaktionen wäre wünschenswert. 


\section{Messtabellen}

Messtabelle 1: $\mathrm{m} / \mathrm{z}=73$ und 119 mit wachsendem Umsatz der Reaktion DMM + F

Versuchsanordnung B, Reaktor $1, \mathrm{~s}=10 \mathrm{~cm}$, Substanz/Rxn.: $\mathrm{CH}_{3} \mathrm{OCH}_{2} \mathrm{OCH}_{3}+\mathrm{F}, \mathrm{T}=25{ }^{\circ} \mathrm{C}$, $\mathrm{p}=174,4 \mathrm{~Pa}$, Zählzeit $=3 \cdot 10 \mathrm{~s}$, Fluss $(\mathrm{He})=600 \mathrm{SCCM}$, Fluss $(\mathrm{DMM})=0,12 \mathrm{~cm}^{3} / \mathrm{s}, \mathrm{IP}=29,5 \mathrm{eV}$

\begin{tabular}{|c|c|c|c|c|}
\hline Umsatz(DMM+F) & $\mathrm{I}_{73}(-\mathrm{F})$ & $\mathrm{I}_{73}(-\mathrm{F})$ & $\mathrm{I}_{119}(-\mathrm{F})$ & $\mathrm{I}_{119}(+\mathrm{F})$ \\
\hline \hline 2,88 & 72 & 459 & 518 & 2880 \\
\hline 3,43 & 79 & 568 & 529 & 3650 \\
\hline 13,87 & 79 & 1212 & 518 & 10300 \\
\hline 27,47 & 74 & 1374 & 525 & 14580 \\
\hline
\end{tabular}

\section{Messtabelle 2: $\mathrm{CH}_{3} \mathrm{CCl}_{3}+\mathrm{F}+\mathrm{O}$}

Versuchsanordnung B, Reaktor 1, s $=3 \mathrm{~cm}$, Badgas: He, $\mathrm{T}=298 \mathrm{~K}, \mathrm{p}=147 \mathrm{~Pa}$, $\Phi(\mathrm{He})=458 \mathrm{sccm}, \Phi(\mathrm{KW})=8,06 \mathrm{sccm}, \Phi\left(\mathrm{F}_{2}\right)=1,45 \mathrm{sccm}, \Phi\left(\mathrm{O}_{2}\right)=8 \mathrm{sccm}$, Zählzeit $=3 \cdot 10 \mathrm{~s}, \mathrm{IE}=29,5 \mathrm{eV}$

\begin{tabular}{|c|c|c|c|}
\hline $\mathrm{m} / \mathrm{z}$ & I $(K W-F) /$ w.E. & $\mathrm{I}(\mathrm{KW}+\mathrm{F}) / \mathrm{w} . \mathrm{E}$. & $\mathrm{I}(\mathrm{KW}+\mathrm{F}+\mathrm{O}) / \mathrm{w} \cdot \mathrm{E}$. \\
\hline 131 & 251 & 2800 & 969 \\
\hline 133 & 250 & 2303 & 880 \\
\hline 120 & 1753 & 1710 & 2290 \\
\hline 118 & 1477 & 3933 & 3437 \\
\hline 116 & 34 & 14330 & 8317 \\
\hline 115 & 25 & 1443 & 874 \\
\hline 114 & 17 & 21130 & 12170 \\
\hline 111 & 54 & 819 & 3713 \\
\hline 110 & 19 & 140 & 932 \\
\hline 109 & 434 & 1367 & 562 \\
\hline 103 & 460 & 1014 & 863 \\
\hline 100 & 19000 & 20970 & 18470 \\
\hline 98 & 41570 & 58370 & 46230 \\
\hline 96 & 21230 & 43570 & 29570 \\
\hline 95 & 20200 & 27400 & 19900 \\
\hline 94 & 938 & 1893 & 1100 \\
\hline 85 & 622 & 3613 & 4483 \\
\hline 84 & 5990 & 5910 & 12270 \\
\hline 83 & 824 & 2460 & 4410 \\
\hline 82 & 3723 & 7933 & 17000 \\
\hline 81 & 60 & 2603 & 1353 \\
\hline 80 & 48 & 14330 & 10200 \\
\hline 79 & 264 & 8223 & 4203 \\
\hline 77 & 1213 & 1597 & 1610 \\
\hline 76 & 223 & 364 & 830 \\
\hline 72 & 113 & 4793 & 2957 \\
\hline 71 & 3197 & 1717 & 1733 \\
\hline 70 & 171 & 6810 & 4273 \\
\hline
\end{tabular}




\begin{tabular}{|c|c|c|c|}
\hline 67 & 65 & 2257 & 1380 \\
\hline 65 & 448 & 459 & 1917 \\
\hline 64 & 7830 & 9840 & 9387 \\
\hline 62 & 26500 & 25370 & 24270 \\
\hline 61 & 334300 & 304700 & 299300 \\
\hline 60 & 6120 & 10370 & 7403 \\
\hline 58 & 4993 & 1002 & 990 \\
\hline 54 & 23 & 6637 & 2280 \\
\hline 51 & 1590 & 2417 & 1960 \\
\hline 50 & 214 & 1933 & 852 \\
\hline 47 & 898 & 2673 & 4670 \\
\hline 45 & 625 & 11800 & 8440 \\
\hline 44 & 1437 & 3793 & 11830 \\
\hline 42 & 5757 & 983 & 1213 \\
\hline 41 & 4880 & 2570 & 2917 \\
\hline 38 & 2877 & 12600 & 18500 \\
\hline 37 & 54 & 8430 & 20000 \\
\hline 36 & 4023 & 37830 & 56700 \\
\hline 35 & 122 & 26200 & 62900 \\
\hline 33 & 181 & 1327 & 893 \\
\hline 32 & 17070 & 17130 & 84100 \\
\hline 31 & 884 & 1993 & 896 \\
\hline 30 & 2190 & 2593 & 4660 \\
\hline 29 & 1273 & 1767 & 6000 \\
\hline 28 & 51800 & 54530 & 77370 \\
\hline 27 & 23270 & 19770 & 18630 \\
\hline 26 & 4663 & 6850 & 5580 \\
\hline 20 & 1223 & 11170 & 13600 \\
\hline 16 & 184 & 781 & 95070 \\
\hline 63 & 77700 & 70930 & 72400 \\
\hline 97 & 943000 & 788700 & 752000 \\
\hline 99 & 612300 & 510700 & 494300 \\
\hline 101 & 95670 & 80800 & 77670 \\
\hline 117 & 85400 & 73770 & 71400 \\
\hline 119 & 86370 & 74670 & 71700 \\
\hline 121 & 26900 & 22870 & 22170 \\
\hline 130 & 5833 & 16170 & 7737 \\
\hline 132 & 5957 & 16530 & 7890 \\
\hline 134 & 1897 & 5510 & 2587 \\
\hline 135 & 87 & 918 & 312 \\
\hline 136 & 140 & 310 & 190 \\
\hline 146 & -- & 180 & 1143 \\
\hline 147 & 14 & 142 & 73 \\
\hline 148 & 11 & 346 & 1153 \\
\hline 149 & -- & 2093 & 2137 \\
\hline 150 & -- & 371 & 387 \\
\hline
\end{tabular}


Messtabelle 3: DMM + F relativ zu Methan + F (zu Abbildung 85 bis Abbildung 89)

Versuchsanordnung B, Reaktor 1, s $=4 \mathrm{~cm}$, Badgas: He, $\mathrm{p}=161 \mathrm{~Pa}, \Phi(\mathrm{He})=640 \mathrm{sccm}$, $\Phi(\mathrm{DMM})=7,2 \mathrm{sccm}, \Phi\left(\mathrm{CH}_{4}\right)=7,2 \mathrm{sccm}$, Zählzeit $=3.10 \mathrm{~s}, \quad$ IP $=29,5 \mathrm{eV}$, Auffängerstrom $=22 \cdot 10^{-6} \mathrm{~A}$

\begin{tabular}{|c|c|c|c|}
\hline \multicolumn{2}{|c|}{$\mathrm{T}=-20^{\circ} \mathrm{C}$} & \multicolumn{2}{|c|}{$\mathrm{T}=0^{\circ} \mathrm{C}$} \\
\hline$\overline{l n} \ln \left(\mathrm{I}_{16}(-\mathrm{F}) / \mathrm{I}_{16}(+\mathrm{F})\right)$ & 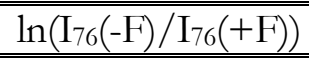 & $\overline{l n}\left(\mathrm{I}_{16}(-\mathrm{F}) / \mathrm{I}_{16}(+\mathrm{F})\right)$ & $e^{\prime \ln \left(\mathrm{I}_{76}(-\mathrm{F}) / \mathrm{I}_{76}(+\mathrm{F})\right)}$ \\
\hline 0,2129 & 0,31915 & 0,04245 & 0,08172 \\
\hline 0,29778 & 0,48146 & 0,02413 & 0,0449 \\
\hline 0,08999 & 0,15654 & 0,07159 & 0,12058 \\
\hline 0,06141 & 0,10356 & 0,08923 & 0,11864 \\
\hline \multirow[t]{2}{*}{0,04327} & 0,06333 & 0,24116 & 0,35882 \\
\hline & & 0,37853 & 0,59605 \\
\hline \multicolumn{2}{|c|}{$\mathrm{T}=25^{\circ} \mathrm{C}$} & \multicolumn{2}{|c|}{$\mathrm{T}=55^{\circ} \mathrm{C}$} \\
\hline $\ln \left(\mathrm{I}_{16}(-\mathrm{F}) / \mathrm{I}_{16}(+\mathrm{F})\right)$ & $\ln \left(\mathrm{I}_{76}(-\mathrm{F}) / \mathrm{I}_{76}(+\mathrm{F})\right)$ & $2 \ln \left(\mathrm{I}_{16}(-\mathrm{F}) / \mathrm{I}_{16}(+\mathrm{F})\right)$ & $\ln \left(\mathrm{I}_{76}(-\mathrm{F}) / \mathrm{I}_{76}(+\mathrm{F})\right)$ \\
\hline 0,00887 & 0,02223 & 0,0128 & 0,01424 \\
\hline 0,04082 & 0,05443 & 0,02301 & 0,04967 \\
\hline 0,05663 & 0,05859 & 0,03433 & 0,05097 \\
\hline 0,05011 & 0,11753 & 0,06854 & 0,07978 \\
\hline 0,13441 & 0,1729 & 0,11469 & 0,15703 \\
\hline 0,24548 & 0,35211 & 0,24194 & 0,34497 \\
\hline 0,38642 & 0,57725 & & \\
\hline \multicolumn{2}{|c|}{$\overline{\mathrm{T}}=90^{\circ} \mathrm{C}$} & & \\
\hline 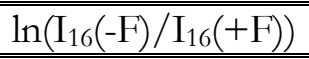 & $2 \ln \left(\mathrm{I}_{76}(-\mathrm{F}) / \mathrm{I}_{76}(+\mathrm{F})\right)$ & & \\
\hline 0,00621 & 0,00715 & & \\
\hline 0,01866 & 0,04022 & & \\
\hline 0,02271 & 0,03338 & & \\
\hline 0,07714 & 0,07014 & & \\
\hline 0,10308 & 0,12058 & & \\
\hline 0,18693 & 0,25423 & & \\
\hline 0,28957 & 0,40653 & & \\
\hline
\end{tabular}


Messtabelle 4: $\mathrm{CH}_{3} \mathrm{CCl}_{3}+\mathrm{F}$ relativ zu $\mathrm{CH}_{2} \mathrm{Cl}_{2}+\mathrm{F}$ (zu Abbildung 68 bis Abbildung 72)

Versuchsanordnung B, Reaktor 1, s $=3 \mathrm{~cm}$, Badgas: He, $\mathrm{p}=150 \mathrm{~Pa}, \Phi(\mathrm{He})=553$ sccm, $\Phi\left(\mathrm{CH}_{3} \mathrm{CCl}_{3}\right)=8,04 \mathrm{sccm}, \Phi\left(\mathrm{CH}_{2} \mathrm{Cl}_{2}\right)=8,04 \mathrm{sccm}$, Zählzeit $=3.10 \mathrm{~s}$, IP $=29,5 \mathrm{eV}$, Auffängerstrom $=20 \cdot 1 \mathrm{E}-06 \mathrm{~A}$

\begin{tabular}{|c|c|c|c|}
\hline \multicolumn{2}{|c|}{$\mathrm{T}=-20^{\circ} \mathrm{C}$} & \multicolumn{2}{|c|}{$\mathrm{T}=0^{\circ} \mathrm{C}$} \\
\hline $\ln \left(\mathrm{I}_{84}(-\mathrm{F}) / \mathrm{I}_{84}(+\mathrm{F})\right)$ & $\ln \left(\mathrm{I}_{97}(-\mathrm{F}) / \mathrm{I}_{97}(+\mathrm{F})\right)$ & $\ln \left(\mathrm{I}_{84}(-\mathrm{F}) / \mathrm{I}_{84}(+\mathrm{F})\right)$ & $\ln \left(\mathrm{I}_{97}(-\mathrm{F}) / \mathrm{I}_{97}(+\mathrm{F})\right)$ \\
\hline 0,13192 & 0,10024 & 0,0696 & 0,06735 \\
\hline 0,17012 & 0,15766 & 0,11722 & 0,10086 \\
\hline 0,26236 & 0,22983 & 0,16115 & 0,1598 \\
\hline 0,39659 & 0,34952 & 0,21969 & 0,20047 \\
\hline \multirow[t]{2}{*}{0,06885} & 0,05057 & 0,28241 & 0,25993 \\
\hline & & 0,3397 & 0,31335 \\
\hline \multicolumn{2}{|c|}{$\mathrm{T}=25^{\circ} \mathrm{C}$} & \multicolumn{2}{|c|}{$\mathrm{T}=55^{\circ} \mathrm{C}$} \\
\hline $\ln \left(\mathrm{I}_{84}(-\mathrm{F}) / \mathrm{I}_{84}(+\mathrm{F})\right)$ & $\ln \left(\mathrm{I}_{97}(-\mathrm{F}) / \mathrm{I}_{97}(+\mathrm{F})\right)$ & $\ln \left(\mathrm{I}_{84}(-\mathrm{F}) / \mathrm{I}_{84}(+\mathrm{F})\right)$ & $\ln \left(\mathrm{I}_{97}(-\mathrm{F}) / \mathrm{I}_{97}(+\mathrm{F})\right)$ \\
\hline 0,06464 & 0,06203 & 0,05404 & 0,06331 \\
\hline 0,11235 & 0,11644 & 0,08383 & 0,07964 \\
\hline 0,1305 & 0,11845 & 0,11619 & 0,12105 \\
\hline 0,27488 & 0,24892 & 0,14854 & 0,14538 \\
\hline 0,32601 & 0,30177 & 0,1975 & 0,18496 \\
\hline 0,24907 & 0,22489 & 0,24961 & 0,23458 \\
\hline 0,19332 & 0,18267 & 0,28504 & 0,27094 \\
\hline \multicolumn{2}{|c|}{$\mathrm{T}=85^{\circ} \mathrm{C}$} & & \\
\hline $2 \ln \left(\mathrm{I}_{84}(-\mathrm{F}) / \mathrm{I}_{84}(+\mathrm{F})\right)$ & 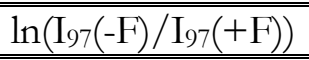 & & \\
\hline 0,03805 & 0,05352 & & \\
\hline 0,06945 & 0,0674 & & \\
\hline 0,13901 & 0,12236 & & \\
\hline 0,1698 & 0,15887 & & \\
\hline 0,20844 & 0,19739 & & \\
\hline 0,24211 & 0,24072 & & \\
\hline 0,11305 & 0,10661 & & \\
\hline 0,04853 & 0,03555 & & \\
\hline
\end{tabular}


Messtabelle 5: $\mathrm{C}_{2} \mathrm{D}_{5}+\mathrm{O}$ relativ zu $\mathrm{C}_{2} \mathrm{H}_{5}+\mathrm{O}$ (zu Abbildung 94)

Versuchsanordnung A, Reaktor 1, $\lambda=435 \mathrm{~nm}, \mathrm{~s}=3 \mathrm{~cm}$, Badgas: He, T = $298 \mathrm{~K}, \mathrm{p}=1,73$ mbar, $\Phi(\mathrm{He})=1100 \mathrm{sccm}, \Phi\left(\mathrm{C}_{2} \mathrm{D}_{6}\right)=7 \mathrm{sccm}, \Phi\left(\mathrm{C}_{2} \mathrm{H}_{6}\right)=7 \mathrm{sccm}, \Phi\left(\mathrm{F}_{2}\right)=1,56 \mathrm{sccm}$, $\Phi\left(\mathrm{O}_{2}\right)=0-1,2 \mathrm{sccm}, \mathrm{E}($ Laser $)=13 \mathrm{~mJ}, 800$ sweeps

\begin{tabular}{|c|c|}
\hline $\ln \left(\mathrm{I}_{29}(-\mathrm{O}) / \mathrm{I}_{29}(+\mathrm{O})\right)$ & $\ln \left(\mathrm{I}_{34}(-\mathrm{O}) / \mathrm{I}_{34}(+\mathrm{O})\right)$ \\
\hline \hline 0.67548 & 0.75124 \\
\hline 0.29844 & 0.328 \\
\hline 0.64455 & 0.55226 \\
\hline 0.70692 & 0.88521 \\
\hline 0.99984 & 1.0924 \\
\hline 1.1631 & 1.1609 \\
\hline 1.1681 & 1.1648 \\
\hline 0.68967 & 0.6671 \\
\hline 0.58731 & 0.47093 \\
\hline 0.69687 & 0.61351 \\
\hline 0.73849 & 0.80828 \\
\hline 0.5932 & 0.4705 \\
\hline 0.24895 & 0.31545 \\
\hline-0.02797 & -0.01635 \\
\hline
\end{tabular}

\section{Messtabelle 6: Methoxymethyl + O relativ zu Ethyl + O (zu Abbildung 95)}

Versuchsanordnung A, Reaktor 1, $\lambda=433 \mathrm{~nm}, \mathrm{~s}=3 \mathrm{~cm}$, Badgas: He, T $=298 \mathrm{~K}, \mathrm{p}=1,8 \mathrm{mbar}$, $\Phi(\mathrm{He})=900 \mathrm{sccm}, \Phi(\mathrm{DME})=2,34 \mathrm{sccm}, \Phi($ Ethan $)=12 \mathrm{sccm}, \Phi\left(\mathrm{F}_{2}\right)=2,13 \mathrm{sccm}$, $\Phi\left(\mathrm{O}_{2}\right)=0-1,2 \mathrm{sccm}, \mathrm{E}($ Laser $)=12 \mathrm{~mJ}, 600$ und 800 sweeps

\begin{tabular}{|c|c|}
\hline $\ln \left(\mathrm{I}_{29}(-\mathrm{O}) / \mathrm{I}_{29}(+\mathrm{O})\right)$ & $\ln \left(\mathrm{I}_{45}(-\mathrm{O}) / \mathrm{I}_{45}(+\mathrm{O})\right)$ \\
\hline \hline 0,36561 & 0,47989 \\
\hline 0,42346 & 0,68976 \\
\hline 0,59746 & 0,76941 \\
\hline 0,7855 & 0,98883 \\
\hline 0,18756 & 0,16155 \\
\hline 0,25993 & 0,23619 \\
\hline 0,50564 & 0,52624 \\
\hline 0,8448 & 1,0551 \\
\hline 0,12439 & 0,13544 \\
\hline 0,102 & 0,19274 \\
\hline 0,22397 & 0,28974 \\
\hline
\end{tabular}


Messtabelle 7: Cyclopentyl + O relativ zu Ethyl + O (zu Abbildung 96)

Versuchsanordnung A, Reaktor 1, $\lambda=433 \mathrm{~nm}, \mathrm{~s}=3 \mathrm{~cm}$, Badgas: He, $\mathrm{T}=298 \mathrm{~K}, \mathrm{p}=2,04$ mbar, $\Phi(\mathrm{He})=739 \mathrm{sccm}, \Phi(\mathrm{c}-$ Pentan $)=6,15 \mathrm{sccm}, \Phi($ Ethan $)=14 \mathrm{sccm}, \Phi\left(\mathrm{F}_{2}\right)=1,56 \mathrm{sccm}$, $\Phi\left(\mathrm{O}_{2}\right)=0-1,2 \mathrm{sccm}, \mathrm{E}($ Laser $)=10 \mathrm{~mJ}, 800$ sweeps

\begin{tabular}{|c|c|}
\hline $\ln \left(\mathrm{I}_{29}(-\mathrm{O}) / \mathrm{I}_{29}(+\mathrm{O})\right)$ & $\ln \left(\mathrm{I}_{69}(-\mathrm{O}) / \mathrm{I}_{69}(+\mathrm{O})\right)$ \\
\hline \hline 0,44995 & 0,70219 \\
\hline 0,37356 & 0,70346 \\
\hline 0,02577 & 0,08494 \\
\hline 0,3407 & 0,55697 \\
\hline 0,35463 & 0,56892 \\
\hline 0,14955 & 0,11667 \\
\hline 0,07622 & 0,15921 \\
\hline 0,26391 & 0,3856 \\
\hline 0,33752 & 0,64841 \\
\hline
\end{tabular}

Messtabelle 8: Cyclopentyl + O relativ zu Methoxymethyl + O (zu Abbildung 97)

Versuchsanordnung A, Reaktor 1, $\lambda=433 \mathrm{~nm}, \mathrm{~s}=3 \mathrm{~cm}$, Badgas: He, T $=298 \mathrm{~K}, \mathrm{p}=1,90 \mathrm{mbar}$, $\Phi(\mathrm{He})=849 \mathrm{sccm}, \Phi(\mathrm{c}-$ Pentan $)=7,64 \mathrm{sccm}, \Phi(\mathrm{DME})=3,64 \mathrm{sccm}, \Phi\left(\mathrm{F}_{2}\right)=2,13 \mathrm{sccm}$, $\Phi\left(\mathrm{O}_{2}\right)=0-1,2 \mathrm{sccm}, \mathrm{E}($ Laser $)=10 \mathrm{~mJ}, 800$ sweeps

\begin{tabular}{|c|c|}
\hline $\ln \left(\mathrm{I}_{45}(-\mathrm{O}) / \mathrm{I}_{45}(+\mathrm{O})\right)$ & $\ln \left(\mathrm{I}_{69}(-\mathrm{O}) / \mathrm{I}_{69}(+\mathrm{O})\right)$ \\
\hline \hline 0,2984 & 0,42739 \\
\hline 0,11537 & 0,16477 \\
\hline 0,34893 & 0,43471 \\
\hline 0,48448 & 0,59939 \\
\hline 0,5767 & 0,77466 \\
\hline 0,69068 & 0,92788 \\
\hline 0,0519 & 0,06594 \\
\hline
\end{tabular}

\section{Messtabelle 9: $\mathrm{CH}_{3}+\mathrm{O}$ relativ zu $\mathrm{C}_{2} \mathrm{D}_{5}+\mathrm{O}$, (zu Abbildung 101)}

Versuchsanordnung A, Reaktor $1, \lambda=340,85 \mathrm{~nm}, \mathrm{~s}=3 \mathrm{~cm}$, Badgas: He, $\mathrm{T}=298 \mathrm{~K}$, $\mathrm{p}=1,73 \mathrm{mbar}, \Phi(\mathrm{He})=1000 \mathrm{sccm}, \Phi\left(\mathrm{CH}_{4}\right)=5,76 \mathrm{sccm}, \Phi\left(\mathrm{C}_{2} \mathrm{D}_{6}\right)=7 \mathrm{sccm}, \Phi\left(\mathrm{F}_{2}\right)=1,56 \mathrm{sccm}$, $\Phi\left(\mathrm{O}_{2}\right)=0-2 \mathrm{sccm}, \mathrm{E}($ Laser $)=8,5 \mathrm{~mJ}, 800$ sweeps

\begin{tabular}{|c|c|}
\hline $\ln \left(\mathrm{I}_{15}(-\mathrm{O}) / \mathrm{I}_{15}(+\mathrm{O})\right)$ & $\ln \left(\mathrm{I}_{34}(-\mathrm{O}) / \mathrm{I}_{34}(+\mathrm{O})\right)$ \\
\hline \hline 0,02647 & 0,05413 \\
\hline 0,50812 & 0,88683 \\
\hline 0,58243 & 1,0099 \\
\hline 0,38782 & 0,65854 \\
\hline 0,27118 & 0,41128 \\
\hline 0,15702 & 0,31136 \\
\hline 0,68343 & 1,132 \\
\hline
\end{tabular}


Messtabelle 10: Methyl + O relativ zu Methoxymethyl + O, (zu Abbildung 102)

Versuchsanordnung A, Reaktor $1, \lambda=450,73 \mathrm{~nm}, \mathrm{~s}=3 \mathrm{~cm}$, Badgas: He, $\mathrm{T}=298 \mathrm{~K}, \mathrm{p}=1,80$ mbar, $\Phi(\mathrm{He})=1000 \mathrm{sccm}, \Phi\left(\mathrm{CH}_{4}\right)=11,52 \mathrm{sccm}, \Phi(\mathrm{DME})=4,68 \mathrm{sccm}, \Phi\left(\mathrm{F}_{2}\right)=1,56 \mathrm{sccm}$, $\Phi\left(\mathrm{O}_{2}\right)=0-1,2 \mathrm{sccm}, \mathrm{E}($ Laser $)=12 \mathrm{~mJ}, 600$ und 800 sweeps

\begin{tabular}{|c|c|}
\hline $\ln \left(\mathrm{I}_{15}(-\mathrm{O}) / \mathrm{I}_{15}(+\mathrm{O})\right)$ & $\ln \left(\mathrm{I}_{45}(-\mathrm{O}) / \mathrm{I}_{45}(+\mathrm{O})\right)$ \\
\hline \hline 0,18928 & 0,3412 \\
\hline 0,32398 & 0,55872 \\
\hline 0,3199 & 0,7922 \\
\hline 0,1066 & 0,20097 \\
\hline 0,25526 & 0,61857 \\
\hline 0,39124 & 0,93029 \\
\hline
\end{tabular}

Messtabelle 11: Methyl + O relativ zu Cyclopentyl + O (zu Abbildung 103)

Versuchsanordnung A, Reaktor $1, \lambda=450,7 \mathrm{~nm}, \mathrm{~s}=3 \mathrm{~cm}$, Badgas: He, T $=298 \mathrm{~K}, \mathrm{p}=1,80 \mathrm{mbar}$, $\Phi(\mathrm{He})=849 \mathrm{~cm}, \Phi(\mathrm{c}-$ Pentan $)=7,64 \mathrm{sccm}, \Phi($ Methan $)=11,52 \mathrm{sccm}, \Phi\left(\mathrm{F}_{2}\right)=2,13 \mathrm{sccm}$, $\Phi\left(\mathrm{O}_{2}\right)=0-1,2 \mathrm{sccm}, \mathrm{E}($ Laser $)=12 \mathrm{~mJ}, 800$ sweeps

\begin{tabular}{|c|c|}
\hline $\ln \left(\mathrm{I}_{15}(-\mathrm{O}) / \mathrm{I}_{15}(+\mathrm{O})\right)$ & $\ln \left(\mathrm{I}_{69}(-\mathrm{O}) / \mathrm{I}_{69}(+\mathrm{O})\right)$ \\
\hline \hline 0,18036 & 0,35729 \\
\hline 0,24368 & 0,54696 \\
\hline 0,30191 & 0,79615 \\
\hline 0,44004 & 1,0366 \\
\hline 0,12473 & 0,17324 \\
\hline 0,06404 & 0,23723 \\
\hline 0,20228 & 0,54385 \\
\hline 0,23045 & 0,71065 \\
\hline 0,35278 & 1,0226 \\
\hline 0,39012 & 0,96519 \\
\hline
\end{tabular}


Messtabelle 12: $\mathrm{CD}_{3}+\mathrm{O}$ relativ zu $\mathrm{CH}_{3}+\mathrm{O}$ (zu Abbildung 104)

Versuchsanordnung A, Reaktor 1, $\lambda=345 \mathrm{~nm}, \mathrm{~s}=3 \mathrm{~cm}$, Badgas: He, $\mathrm{T}=298 \mathrm{~K}, \mathrm{p}=1,76 \mathrm{mbar}$, $\Phi(\mathrm{He})=1100 \mathrm{~cm}, \Phi\left(\mathrm{CD}_{4}\right)=10,08 \mathrm{sccm}, \Phi\left(\mathrm{CH}_{4}\right)=10,08 \mathrm{sccm}, \Phi\left(\mathrm{F}_{2}\right)=1,56 \mathrm{sccm}$, $\Phi\left(\mathrm{O}_{2}\right)=0-3 \mathrm{sccm}, \mathrm{E}($ Laser $)=5,5 \mathrm{~mJ}, 800$ sweeps

\begin{tabular}{|c|c|}
\hline $\ln \left(\mathrm{I}_{15}(-\mathrm{O}) / \mathrm{I}_{15}(+\mathrm{O})\right)$ & $\ln \left(\mathrm{I}_{18}(-\mathrm{O}) / \mathrm{I}_{18}(+\mathrm{O})\right)$ \\
\hline 0,17419 & 0,10027 \\
\hline 0,32823 & 0,26502 \\
\hline 0,3556 & 0,43027 \\
\hline 0,32186 & 0,52719 \\
\hline 0,51184 & 0,44735 \\
\hline 0,62511 & 0,50417 \\
\hline 0,93869 & 1,0709 \\
\hline 0,89765 & 0,98339 \\
\hline 0,76072 & 0,73419 \\
\hline 0,66654 & 0,87762 \\
\hline 0,58202 & 0,61169 \\
\hline
\end{tabular}

Messtabelle 13: 2-Propyl + O relativ zu Methoxymethyl + O (zu Abbildung 105)

Versuchsanordnung A, Reaktor 1, $\lambda=465 \mathrm{~nm}, \mathrm{~s}=4 \mathrm{~cm}$, Badgas: He, T $=298 \mathrm{~K}, \mathrm{p}=1,7 \mathrm{mbar}$, $\Phi(\mathrm{He})=1000 \mathrm{sccm}, \Phi(\mathrm{DME})=1,56 \mathrm{sccm}, \Phi($ Propan $)=11,52 \mathrm{sccm}, \Phi\left(\mathrm{F}_{2}\right)=1,53 \mathrm{sccm}$, $\Phi\left(\mathrm{O}_{2}\right)=0,25$ bis $1 \mathrm{sccm}, \mathrm{E}($ Laser $)=7 \mathrm{~mJ}, 600$ sweeps

\begin{tabular}{|c|c|}
\hline $\ln \left(\mathrm{I}_{43}(-\mathrm{O}) / \mathrm{I}_{43}(+\mathrm{O})\right)$ & $\ln \left(\mathrm{I}_{45}(-\mathrm{O}) / \mathrm{I}_{45}(+\mathrm{O})\right)$ \\
\hline \hline 0,30132 & 0,32326 \\
\hline 0,29777 & 0,35507 \\
\hline 0,44639 & 0,53173 \\
\hline 0,44651 & 0,55287 \\
\hline 0,49402 & 0,62645 \\
\hline 0,82023 & 0,96902 \\
\hline 0,91706 & 1,0755 \\
\hline$-0,04908$ & 0,018 \\
\hline 0,03429 & 0,0514 \\
\hline 0,50623 & 0,5592 \\
\hline 0,69413 & 0,74543 \\
\hline 0,1691 & 0,20912 \\
\hline
\end{tabular}


Messtabelle 14: 1-Propyl + O relativ zu 2-Propyl + O (zu Abbildung 106)

Versuchsanordnung A, Reaktor 1, $\lambda=430$ und $450 \mathrm{~nm}, \mathrm{~s}=4 \mathrm{~cm}$, Badgas: He, T $=298 \mathrm{~K}, \mathrm{p}=1,48$ und 1,6 mbar, $\Phi(\mathrm{He})=831$ und $1000 \mathrm{sccm}, \Phi\left(\right.$ Propan- $\left.\mathrm{d}_{2}\right)=5,76 \mathrm{sccm}, \Phi\left(\mathrm{F}_{2}\right)=1,56 \mathrm{sccm}$, $\Phi\left(\mathrm{O}_{2}\right)=0,25-1 \mathrm{sccm}, \mathrm{E}($ Laser $)=6 \mathrm{~mJ}, 400$ und 600 sweeps

\begin{tabular}{|c|c|}
\hline $\ln \left(\mathrm{I}_{44}(-\mathrm{O}) / \mathrm{I}_{44}(+\mathrm{O})\right)$ & $\ln \left(\mathrm{I}_{45}(-\mathrm{O}) / \mathrm{I}_{45}(+\mathrm{O})\right)$ \\
\hline 0,75535 & 0,57597 \\
\hline 0,5322 & 0,20133 \\
\hline 0,26039 & 0,25475 \\
\hline 0,91481 & 0,64191 \\
\hline 1,1852 & 0,81656 \\
\hline 0,70111 & 0,51395 \\
\hline 0,48829 & 0,34919 \\
\hline 0,57443 & 0,41064 \\
\hline 0,65017 & 0,54846 \\
\hline 0,55124 & 0,42991 \\
\hline
\end{tabular}

\section{Messtabelle 15: 2-Butyl + O relativ zu c-Pentyl + O (zu Abbildung 107)}

Versuchsanordnung A, Reaktor 1, $\lambda=470 \mathrm{~nm}, \mathrm{~s}=3 \mathrm{~cm}$, Badgas: He, T $=298 \mathrm{~K}, \mathrm{p}=2,1 \mathrm{mbar}$, $\Phi(\mathrm{He})=735 \mathrm{sccm}, \Phi($ Butan $)=6,55 \mathrm{sccm}, \Phi(\mathrm{c}-$ Pentan $)=5,56 \mathrm{sccm}, \Phi\left(\mathrm{F}_{2}\right)=1,56 \mathrm{sccm}$, $\Phi\left(\mathrm{O}_{2}\right)=0-1,2 \mathrm{sccm}, \mathrm{E}($ Laser $)=9 \mathrm{~mJ}, 800$ sweeps

\begin{tabular}{|c|c|}
\hline $\ln \left(\mathrm{I}_{57}(-\mathrm{O}) / \mathrm{I}_{57}(+\mathrm{O})\right)$ & $\ln \left(\mathrm{I}_{69}(-\mathrm{O}) / \mathrm{I}_{69}(+\mathrm{O})\right)$ \\
\hline \hline 0,19511 & 0,25779 \\
\hline 0,32914 & 0,46267 \\
\hline 0,55906 & 0,89254 \\
\hline 0,35007 & 0,64677 \\
\hline 0,25467 & 0,41121 \\
\hline 0,24463 & 0,35379 \\
\hline 0,21162 & 0,378 \\
\hline 0,15098 & 0,17085 \\
\hline
\end{tabular}


Messtabelle 16: 1-Butyl + O relativ zu 2-Butyl + O (zu Abbildung 108)

Versuchsanordnung A, Reaktor 1, $\lambda=430 \mathrm{~nm}, \mathrm{~s}=4 \mathrm{~cm}$, Badgas: He, $\mathrm{T}=298 \mathrm{~K}, \mathrm{p}=1,43 \mathrm{mbar}$, $\Phi(\mathrm{He})=831 \mathrm{sccm}, \Phi\left(\right.$ Butan $\left._{-} \mathrm{d}_{6}\right)=4,10 \mathrm{sccm}, \Phi\left(\mathrm{F}_{2}\right)=1,56 \mathrm{sccm}, \Phi\left(\mathrm{O}_{2}\right)=0-1,2 \mathrm{sccm}$, $\mathrm{E}($ Laser $)=6,5 \mathrm{~mJ}, 300$ sweeps

\begin{tabular}{|c|c|}
\hline $\ln \left(\mathrm{I}_{62}(-\mathrm{O}) / \mathrm{I}_{62}(+\mathrm{O})\right)$ & $\ln \left(\mathrm{I}_{63}(-\mathrm{O}) / \mathrm{I}_{63}(+\mathrm{O})\right)$ \\
\hline \hline 0,39848 & 0,20622 \\
\hline 0,60094 & 0,51283 \\
\hline 0,41066 & 0,45171 \\
\hline 0,54955 & 0,62906 \\
\hline 0,82061 & 0,53438 \\
\hline 0,67326 & 0,81647 \\
\hline 0,96453 & 0,95035 \\
\hline 0,67812 & 0,59381 \\
\hline 0,32491 & 0,4549 \\
\hline 0,14275 & 0,04622 \\
\hline 0,33479 & 0,2253 \\
\hline 1,4408 & 1,2062 \\
\hline 0,62349 & 0,76156 \\
\hline
\end{tabular}

Messtabelle 17: t-Butyl + O relativ zu Methoxymethyl + O (zu Abbildung 112 bis Abbildung 118)

Versuchsanordnung A, Reaktor $1, \lambda=510 \mathrm{~nm}, \mathrm{~s}=3 \mathrm{~cm}$, Badgas: He, $\mathrm{p}=1,50 \mathrm{mbar}$, $\Phi(\mathrm{He})=832 \mathrm{sccm}, \Phi($ Isobutan $)=4,10 \mathrm{sccm}, \Phi(\mathrm{DME})=7,85 \mathrm{sccm}, \Phi\left(\mathrm{F}_{2}\right)=1,69 \mathrm{sccm}$, $\Phi\left(\mathrm{O}_{2}\right)=0-1,8 \mathrm{sccm}, \mathrm{E}($ Laser $)=10 \mathrm{~mJ}, 400$ sweeps

\begin{tabular}{|c|c|c|c|}
\hline \multicolumn{2}{|c|}{$\mathrm{T}=-30{ }^{\circ} \mathrm{C}$} & \multicolumn{2}{c|}{$\mathrm{T}=-10{ }^{\circ} \mathrm{C}$} \\
\hline \hline $\ln \left(\mathrm{I}_{45}(-\mathrm{O}) / \mathrm{I}_{45}(+\mathrm{O})\right)$ & $\ln \left(\mathrm{I}_{57}(-\mathrm{O}) / \mathrm{I}_{57}(+\mathrm{O})\right)$ & $\ln \left(\mathrm{I}_{45}(-\mathrm{O}) / \mathrm{I}_{45}(+\mathrm{O})\right)$ & $\ln \left(\mathrm{I}_{57}(-\mathrm{O}) / \mathrm{I}_{57}(+\mathrm{O})\right)$ \\
\hline 0,27439 & 0,23682 & 0,33221 & 0,20157 \\
\hline 0,49517 & 0,60942 & 0,22016 & 0,35094 \\
\hline 0,61899 & 0,67221 & 0,38005 & 0,62831 \\
\hline 0,8506 & 0,86099 & 0,41883 & 0,65007 \\
\hline 0,68671 & 0,98172 & 0,60924 & 0,78921 \\
\hline 1,08752 & 1,11145 & 0,83315 & 0,87129 \\
\hline 1,12253 & 1,2912 & 0,93392 & 1,10621 \\
\hline 1,36855 & 1,65417 & 1,0394 & 1,30276 \\
\hline 1,58319 & 1,7578 & 1,42304 & 1,48479 \\
\hline 0,35427 & 0,33204 & 1,2815 & 1,66223 \\
\hline \hline \multicolumn{2}{|c|}{$\mathrm{T}=0{ }^{\circ} \mathrm{C}$} & \multicolumn{2}{c|}{$\mathrm{T}=25{ }^{\circ} \mathrm{C}$} \\
\hline \hline $\ln \left(\mathrm{I}_{45}(-\mathrm{O}) / \mathrm{I}_{45}(+\mathrm{O})\right)$ & $\ln \left(\mathrm{I}_{57}(-\mathrm{O}) / \mathrm{I}_{57}(+\mathrm{O})\right)$ & $\ln \left(\mathrm{I}_{45}(-\mathrm{O}) / \mathrm{I}_{45}(+\mathrm{O})\right)$ & $\ln \left(\mathrm{I}_{57}(-\mathrm{O}) / \mathrm{I}_{57}(+\mathrm{O})\right)$ \\
\hline \hline 0,11639 & 0,10469 & 0,8825 & 1,05471 \\
\hline 0,45613 & 0,50683 & 0,63343 & 0,77342 \\
\hline 0,54168 & 0,7074 & 0,37897 & 0,52565 \\
\hline 0,6825 & 0,84604 & 1,38263 & 1,46003 \\
\hline 0,68177 & 0,89821 & 1,41467 & 1,46003 \\
\hline 0,89855 & 0,93775 & 1,71534 & 1,89154 \\
\hline 1,0767 & 1,17477 & 0,36493 & 0,38189 \\
\hline
\end{tabular}




\begin{tabular}{|c|c|c|c|}
\hline 1,15319 & 1,28957 & 0,5175 & 0,77342 \\
\hline 1,11441 & 1,32542 & 0,3266 & 0,32079 \\
\hline 1,52249 & 1,7815 & 0,29517 & 0,27957 \\
\hline 0,41883 & 0,39236 & & \\
\hline 1,46602 & 1,52422 & & \\
\hline \multicolumn{2}{|c|}{$\overline{\mathrm{T}}=55^{\circ} \mathrm{C}$} & \multicolumn{2}{|c|}{$\bar{T}=90 \mathrm{C}^{\circ}$} \\
\hline $\ln \left(\mathrm{I}_{45}(-\mathrm{O}) / \mathrm{I}_{45}(+\mathrm{O})\right)$ & $\ln \left(\mathrm{I}_{57}(-\mathrm{O}) / \mathrm{I}_{57}(+\mathrm{O})\right)$ & $\ln \left(\mathrm{I}_{45}(-\mathrm{O}) / \mathrm{I}_{45}(+\mathrm{O})\right)$ & $\ln \left(\mathrm{I}_{57}(-\mathrm{O}) / \mathrm{I}_{57}(+\mathrm{O})\right)$ \\
\hline 0,14678 & 0,35946 & 0,27473 & 0,30982 \\
\hline 0,50421 & 0,50169 & 0,77763 & 0,75867 \\
\hline 0,36695 & 0,58335 & 0,71572 & 0,70631 \\
\hline 0,68381 & 0,6876 & 0,74715 & 0,90813 \\
\hline 0,86789 & 0,97797 & 0,94731 & 0,89821 \\
\hline 1,04533 & 1,20976 & 1,17328 & 1,22149 \\
\hline 0,93392 & 1,28483 & & \\
\hline
\end{tabular}

\section{Messtabelle 18: t-Butyl + O relativ zu Allyl + O (zu Abbildung 119)}

Versuchsanordnung A, Reaktor $1, \lambda=498,8 \mathrm{~nm}, \mathrm{~s}=3 \mathrm{~cm}$, Badgas: He, T $=298 \mathrm{~K}, \mathrm{p}=156 \mathrm{~Pa}$, $\Phi(\mathrm{He})=1020 \mathrm{sccm}, \Phi($ Isobutan $)=6,48 \mathrm{sccm}, \Phi($ Propen $)=13,12 \mathrm{sccm}, \Phi\left(\mathrm{F}_{2}\right)=1,6 \mathrm{sccm}$, $\Phi\left(\mathrm{O}_{2}\right)=0,25$ bis $1 \mathrm{sccm}, \mathrm{E}($ Laser $)=9 \mathrm{~mJ}$

\begin{tabular}{|c|c|}
\hline $\ln \left(\mathrm{I}_{41}(-\mathrm{O}) / \mathrm{I}_{41}(+\mathrm{O})\right)$ & $\ln \left(\mathrm{I}_{57}(-\mathrm{O}) / \mathrm{I}_{57}(+\mathrm{O})\right)$ \\
\hline \hline 0,31656 & 0,37485 \\
\hline 0,50309 & 0,52989 \\
\hline 0,59524 & 0,79014 \\
\hline 0,18086 & 0,22767 \\
\hline 0,32682 & 0,3188 \\
\hline 0,35001 & 0,44338 \\
\hline 0,54126 & 0,57464 \\
\hline 0,31656 & 0,37485 \\
\hline
\end{tabular}

\section{Messtabelle 19: Methoxymethyl + O relativ zu Allyl + O (zu Abbildung 120)}

Versuchsanordnung A, Reaktor 1, $\lambda=498,8 \mathrm{~nm}, \mathrm{~s}=3 \mathrm{~cm}$, Badgas: He, T $=298 \mathrm{~K}, \mathrm{p}=156 \mathrm{~Pa}$, $\Phi(\mathrm{He})=1020 \mathrm{sccm}, \Phi(\mathrm{DME})=$ 9,36 sccm, $\Phi($ Propen $)=13,12 \mathrm{sccm}, \Phi\left(\mathrm{F}_{2}\right)=1,6 \mathrm{sccm}$, $\Phi\left(\mathrm{O}_{2}\right)=0,25$ bis $1 \mathrm{sccm}, \mathrm{E}($ Laser $)=9 \mathrm{~mJ}$

\begin{tabular}{|c|c|}
\hline $\ln \left(\mathrm{I}_{41}(-\mathrm{O}) / \mathrm{I}_{41}(+\mathrm{O})\right)$ & $\ln \left(\mathrm{I}_{45}(-\mathrm{O}) / \mathrm{I}_{45}(+\mathrm{O})\right)$ \\
\hline \hline 0,24218 & 0,36142 \\
\hline 0,40767 & 0,4729 \\
\hline 0,61189 & 0,68675 \\
\hline 0,15628 & 0,23395 \\
\hline 0,09556 & 0,06202 \\
\hline
\end{tabular}


Messtabelle 20: Isobutyl + O relativ zu t-Butyl + O (zu Abbildung 121)

Versuchsanordnung A, Reaktor 1, $\lambda=430 \mathrm{~nm}, \mathrm{~s}=3 \mathrm{~cm}$, Badgas: He, T $=298 \mathrm{~K}, \mathrm{p}=146 \mathrm{~Pa}$, $\Phi(\mathrm{He})=831 \mathrm{sccm}, \Phi($ Isobutan $-2 d)=4,32 \mathrm{sccm}, \Phi\left(\mathrm{F}_{2}\right)=1,56 \mathrm{sccm}, \Phi\left(\mathrm{O}_{2}\right)=0-1,2 \mathrm{sccm}$, $\mathrm{E}($ Laser $)=8 \mathrm{~mJ}$

\begin{tabular}{|c|c|}
\hline $\ln \left(\mathrm{I}_{57}(-\mathrm{O}) / \mathrm{I}_{57}(+\mathrm{O})\right)$ & $\ln \left(\mathrm{I}_{58}(-\mathrm{O}) / \mathrm{I}_{58}(+\mathrm{O})\right)$ \\
\hline \hline 0,48073 & 0,30578 \\
\hline 0,77637 & 0,48042 \\
\hline 0,19171 & 0,09381 \\
\hline 0,35834 & 0,19647 \\
\hline 0,55813 & 0,43801 \\
\hline 0,86136 & 0,53363 \\
\hline 0,93178 & 0,61252 \\
\hline 1,063 & 0,70794 \\
\hline 0,52399 & 0,41023 \\
\hline 0,63786 & 0,30898 \\
\hline
\end{tabular}

\section{Messtabelle 21: c-Heptyl + O relativ zu Methoxymethyl + O (zu Abbildung 122)}

Versuchsanordnung A, Reaktor $1, \lambda=440 \mathrm{~nm}, \mathrm{~s}=3 \mathrm{~cm}, \mathrm{~T}=25^{\circ} \mathrm{C}$, Badgas: He, $\mathrm{p}=1,79$ mbar, $\Phi(\mathrm{He})=1252 \mathrm{sccm}, \Phi(\mathrm{c}-\mathrm{Heptan})=4,96 \mathrm{sccm}, \Phi(\mathrm{DME})=6,24 \mathrm{sccm}, \Phi\left(\mathrm{F}_{2}\right)=1,56 \mathrm{sccm}$, $\Phi\left(\mathrm{O}_{2}\right)=0-0,8 \mathrm{sccm}, \mathrm{E}($ Laser $)=11 \mathrm{~mJ}, 800$ sweeps

\begin{tabular}{|c|c|}
\hline $\ln \left(\mathrm{I}_{45}(-\mathrm{O}) / \mathrm{I}_{45}(+\mathrm{O})\right)$ & $\ln \left(\mathrm{I}_{97}(-\mathrm{O}) / \mathrm{I}_{97}(+\mathrm{O})\right)$ \\
\hline \hline 0,78994 & 1,2896 \\
\hline 0,13141 & 0,14846 \\
\hline 0,22121 & 0,22148 \\
\hline 0,56595 & 0,985 \\
\hline 0,83937 & 1,2337 \\
\hline$-0,01909$ & 0,01666 \\
\hline 0,8483 & 1,2721 \\
\hline 0,01261 & $-0,02617$ \\
\hline 0,04714 & 0,01197 \\
\hline 0,63212 & 0,83529 \\
\hline 0,54708 & 0,96428 \\
\hline 0,45921 & 0,74018 \\
\hline 0,02996 & 0,01087 \\
\hline 0,42664 & 0,64264 \\
\hline 0,25765 & 0,51408 \\
\hline 0,14674 & 0,2177 \\
\hline 0,21266 & 0,44384 \\
\hline 0,0649 & 0,2226 \\
\hline
\end{tabular}


Messtabelle 22: c-Oktyl + O relativ zu Methoxymethyl + O (zu Abbildung 123 bis Abbildung 126)

Versuchsanordnung A, Reaktor 1, $\lambda=456 \mathrm{~nm}, \mathrm{~s}=4 \mathrm{~cm}$, Badgas: He, $\mathrm{p}=$ 1,96 mbar, $\Phi(\mathrm{He})=1600 \mathrm{sccm}, \Phi(\mathrm{c}-$ Oktan $)=2,92 \mathrm{sccm}, \Phi(\mathrm{DME})=3,12 \mathrm{sccm}, \Phi\left(\mathrm{F}_{2}\right)=1,56 \mathrm{sccm}$, $\Phi\left(\mathrm{O}_{2}\right)=0-0,8 \mathrm{sccm}, \mathrm{E}($ Laser $)=10 \mathrm{~mJ}, 600$ sweeps

\begin{tabular}{|c|c|c|c|}
\hline \multicolumn{2}{|c|}{$\mathrm{T}=5^{\circ} \mathrm{C}$} & \multicolumn{2}{c|}{$\mathrm{T}=25^{\circ} \mathrm{C}$} \\
\hline \hline $\ln \left(\mathrm{I}_{45}(-\mathrm{O}) / \mathrm{I}_{45}(+\mathrm{O})\right)$ & $\ln \left(\mathrm{I}_{111}(-\mathrm{O}) / \mathrm{I}_{111}(+\mathrm{O})\right)$ & $\ln \left(\mathrm{I}_{45}(-\mathrm{O}) / \mathrm{I}_{45}(+\mathrm{O})\right)$ & $\ln \left(\mathrm{I}_{111}(-\mathrm{O}) / \mathrm{I}_{111}(+\mathrm{O})\right)$ \\
\hline \hline 0,44539 & 0,50475 & 0,52686 & 0,58796 \\
\hline 0,73494 & 0,92047 & 0,77496 & 0,9993 \\
\hline 0,52706 & 0,62625 & 0,18847 & 0,20335 \\
\hline 0,18286 & 0,17728 & 0,29066 & 0,44085 \\
\hline 0,35673 & 0,33141 & 0,58449 & 0,79087 \\
\hline \hline \multicolumn{2}{|c|}{$\mathrm{T}=55 \mathrm{C}^{\circ}$} & \multicolumn{2}{|c|}{$\mathrm{T}=90{ }^{\circ} \mathrm{C}$} \\
\hline \hline $\ln \left(\mathrm{I}_{45}(-\mathrm{O}) / \mathrm{I}_{45}(+\mathrm{O})\right)$ & $\ln \left(\mathrm{I}_{111}(-\mathrm{O}) / \mathrm{I}_{111}(+\mathrm{O})\right)$ & $\ln \left(\mathrm{I}_{45}(-\mathrm{O}) / \mathrm{I}_{45}(+\mathrm{O})\right)$ & $\ln \left(\mathrm{I}_{111}(-\mathrm{O}) / \mathrm{I}_{111}(+\mathrm{O})\right)$ \\
\hline \hline 0,33737 & 0,48826 & 0,14094 & 0,2517 \\
\hline 0,43771 & 0,56301 & 0,28829 & 0,46454 \\
\hline 0,48123 & 0,66053 & 0,29713 & 0,47644 \\
\hline 0,47393 & 0,75202 & 0,42221 & 0,72761 \\
\hline 0,6821 & 0,97396 & 0,51504 & 0,95449 \\
\hline 0,58453 & 0,76491 & 0,57346 & 0,78086 \\
\hline 0,2154 & 0,31999 & 0,72679 & 0,97765 \\
\hline 0,33737 & 0,48826 & 0,45266 & 0,68965 \\
\hline & & 0,05242 & 0,07642 \\
\hline & & 0,47497 & 0,70372 \\
\hline & & 0,64821 & 0,86142 \\
\hline & & 0,67928 & 1,1157 \\
\hline
\end{tabular}

\section{Messtabelle 23: Cyclopentadienyl + O relativ zu Methoxymethyl + O (zu Abbildung 128)}

Versuchsanordnung A, Reaktor 1, $\lambda=424,87 \mathrm{~nm}, \mathrm{~s}=3 \mathrm{~cm}$, Badgas: He, $\mathrm{p}=2,10 \mathrm{mbar}$, $\Phi(\mathrm{He})=837 \mathrm{sccm}, \Phi($ Cyclopentadien $)=9,07 \mathrm{sccm}, \Phi(\mathrm{DME})=8,58 \mathrm{sccm}, \Phi\left(\mathrm{F}_{2}\right)=1,56 \mathrm{sccm}$, $\Phi\left(\mathrm{O}_{2}\right)=0-3 \mathrm{sccm}, \mathrm{E}($ Laser $)=8 \mathrm{~mJ}, 800$ sweeps

\begin{tabular}{|c|c|}
\hline $\ln \left(\mathrm{I}_{45}(-\mathrm{O}) / \mathrm{I}_{45}(+\mathrm{O})\right)$ & $\ln \left(\mathrm{I}_{65}(-\mathrm{O}) / \mathrm{I}_{65}(+\mathrm{O})\right)$ \\
\hline \hline 0,57175 & 0,60934 \\
\hline 0,45752 & 0,46219 \\
\hline 0,26847 & 0,234 \\
\hline 0,66197 & 0,69153 \\
\hline 0,41386 & 0,36265 \\
\hline 0,15021 & 0,19722 \\
\hline
\end{tabular}


Messtabelle 24: $\mathrm{CH}_{2} \mathrm{CCl}_{3}$ relativ zu $\mathrm{CHCl}_{2}+\mathrm{O}$ (zu Abbildung 129 bis Abbildung 134)

Versuchsanordnung B, Reaktor 1, s $=3 \mathrm{~cm}$, Badgas: He, $\mathrm{p}=1,51 \mathrm{mbar}, \Phi(\mathrm{He})=9,21 \mathrm{~cm}^{3} / \mathrm{s}$, $\Phi\left(\mathrm{CH}_{3} \mathrm{CCl}_{3}\right)=0,134 \mathrm{~cm}^{3} / \mathrm{s}, \Phi\left(\mathrm{CH}_{2} \mathrm{Cl}_{2}\right)=0,134 \mathrm{~cm}^{3} / \mathrm{s}, \Phi\left(\mathrm{F}_{2}\right)=0,019 \mathrm{~cm}^{3} / \mathrm{s}$, Zählzeit $=3 \cdot 10 \mathrm{~s}$, $\mathrm{IE}=29,5 \mathrm{eV}$, Emissionsstrom $=20 \cdot 10^{-6} \mathrm{~A}$

\begin{tabular}{|c|c|c|c|}
\hline \multicolumn{2}{|c|}{$\mathrm{T}=-20 \mathrm{C}^{\circ}$} & \multicolumn{2}{|c|}{$\mathrm{T}=0^{\circ} \mathrm{C}$} \\
\hline $\ln \left(\mathrm{I}_{83}(-\mathrm{O}) / \mathrm{I}_{83}(+\mathrm{O})\right)$ & $\ln \left(\mathrm{I}_{131}(-\mathrm{O}) / \mathrm{I}_{131}(+\mathrm{O})\right)$ & $\ln \left(\mathrm{I}_{83}(-\mathrm{O}) / \mathrm{I}_{83}(+\mathrm{O})\right)$ & $\ln \left(\mathrm{I}_{131}(-\mathrm{O}) / \mathrm{I}_{131}(+\mathrm{O})\right)$ \\
\hline 0,59237 & 0,52765 & 0,69574 & 0,618 \\
\hline 0,60742 & 0,68876 & 0,68181 & 0,60222 \\
\hline 0,6842 & 0,71266 & 0,55601 & 0,51748 \\
\hline 0,57795 & 0,46565 & 0,49453 & 0,37757 \\
\hline 0,47513 & 0,41518 & 0,43022 & 0,33797 \\
\hline 0,40043 & 0,36637 & 0,22752 & 0,16121 \\
\hline 0,30931 & 0,24665 & & \\
\hline 0,2226 & 0,20996 & & \\
\hline \multicolumn{2}{|c|}{$\mathrm{T}=25^{\circ} \mathrm{C}$} & \multicolumn{2}{|c|}{$\mathrm{T}=55^{\circ} \mathrm{C}$} \\
\hline $\ln \left(\mathrm{I}_{83}(-\mathrm{O}) / \mathrm{I}_{83}(+\mathrm{O})\right)$ & $\ln \left(\mathrm{I}_{131}(-\mathrm{O}) / \mathrm{I}_{131}(+\mathrm{O})\right)$ & $\bar{l} \ln \left(\mathrm{I}_{83}(-\mathrm{O}) / \mathrm{I}_{83}(+\mathrm{O})\right)$ & $\ln \left(\mathrm{I}_{131}(-\mathrm{O}) / \mathrm{I}_{131}(+\mathrm{O})\right)$ \\
\hline 0,78011 & 0,77252 & 0,90234 & 0,76269 \\
\hline 0,74152 & 0,68203 & 0,79329 & 0,67743 \\
\hline 0,52016 & 0,52096 & 0,70613 & 0,63318 \\
\hline 0,48524 & 0,43754 & 0,59414 & 0,51283 \\
\hline 0,4137 & 0,3131 & 0,46063 & 0,38932 \\
\hline 0,12612 & 0,20581 & 0,28114 & 0,19245 \\
\hline 0,11613 & 0,03856 & & \\
\hline 0,31213 & 0,25752 & & \\
\hline 0,7615 & 0,5953 & & \\
\hline \multicolumn{2}{|c|}{$\mathrm{T}=85^{\circ} \mathrm{C}$} & & \\
\hline $\ln \left(\mathrm{I}_{83}(-\mathrm{O}) / \mathrm{I}_{83}(+\mathrm{O})\right)$ & $\ln \left(\mathrm{I}_{131}(-\mathrm{O}) / \mathrm{I}_{131}(+\mathrm{O})\right)$ & & \\
\hline 1,126 & 0,96032 & & \\
\hline 0,88764 & 0,82167 & & \\
\hline 0,92001 & 0,77707 & & \\
\hline 0,79789 & 0,61772 & & \\
\hline 0,44468 & 0,4304 & & \\
\hline 0,21413 & 0,24216 & & \\
\hline
\end{tabular}


Messtabelle 25: Iso- $\mathrm{C}_{4} \mathrm{H}_{8} \mathrm{Cl}+\mathrm{O}$ relativ zu Methoxymethyl + $\mathrm{O}$ (zu Abbildung 135)

Versuchsanordnung A, Reaktor 1, $\lambda=440 \mathrm{~nm}, \mathrm{~s}=3 \mathrm{~cm}, \mathrm{~T}=25^{\circ} \mathrm{C}$, Badgas: He, $\mathrm{p}=2,10 \mathrm{mbar}$, $\Phi(\mathrm{He})=900 \mathrm{sccm}, \Phi($ Isobutylchlorid $)=14,58 \mathrm{sccm}, \Phi(\mathrm{DME})=5,46 \mathrm{sccm}, \Phi\left(\mathrm{F}_{2}\right)=1,56 \mathrm{sccm}$, $\Phi\left(\mathrm{O}_{2}\right)=0-1,5 \mathrm{sccm}, \mathrm{E}($ Laser $)=12 \mathrm{~mJ}, 600$ sweeps

\begin{tabular}{|c|c|}
\hline $\ln \left(\mathrm{I}_{45}(-\mathrm{O}) / \mathrm{I}_{45}(+\mathrm{O})\right)$ & $\ln \left(\mathrm{I}_{91}(-\mathrm{O}) / \mathrm{I}_{91}(+\mathrm{O})\right)$ \\
\hline 0,36856 & 0,49407 \\
\hline 0,40201 & 0,64304 \\
\hline 0,69945 & 0,95703 \\
\hline 0,15638 & 0,20716 \\
\hline 0,45291 & 0,80605 \\
\hline 0,19312 & 0,38776 \\
\hline 0,24374 & 0,39518 \\
\hline
\end{tabular}

Messtabelle 26: t-Butyl $+\mathrm{O}_{2}$ relativ zu $\mathrm{CH}_{2} \mathrm{OCH}_{3}+\mathrm{O}_{2}$ (zu Abbildung 141 bis Abbildung 146)

Versuchsanordnung A, Reaktor $1, \lambda=510 \mathrm{~nm}, \mathrm{~s}=3 \mathrm{~cm}$, Badgas: He, $\mathrm{p}=1,50 \mathrm{mbar}$, $\Phi(\mathrm{He})=871 \mathrm{sccm}, \Phi($ Isobutan $)=8,64 \mathrm{sccm}, \Phi(\mathrm{DME})=12,48 \mathrm{sccm}, \Phi\left(\mathrm{F}_{2}\right)=2,06 \mathrm{sccm}$, $\Phi\left(\mathrm{O}_{2}\right)=1-20 \mathrm{sccm}, \mathrm{E}($ Laser $)=10 \mathrm{~mJ}, 400$ sweeps

\begin{tabular}{|c|c|c|c|}
\hline \multicolumn{2}{|c|}{$\mathrm{T}=-30^{\circ} \mathrm{C}$} & \multicolumn{2}{|c|}{$\mathrm{T}=0^{\circ} \mathrm{C}$} \\
\hline $\ln \left(\mathrm{I}_{45}\left(-\mathrm{O}_{2}\right) / \mathrm{I}_{45}\left(+\mathrm{O}_{2}\right)\right)$ & $\ln \left(\mathrm{I}_{57}\left(-\mathrm{O}_{2}\right) / \mathrm{I}_{57}\left(+\mathrm{O}_{2}\right)\right)$ & $\ln \left(\mathrm{I}_{45}\left(-\mathrm{O}_{2}\right) / \mathrm{I}_{45}\left(+\mathrm{O}_{2}\right)\right)$ & $\mid \ln \left(\mathrm{I}_{57}\left(-\mathrm{O}_{2}\right) / \mathrm{I}_{57}\left(+\mathrm{O}_{2}\right)\right)$ \\
\hline 0,19957 & 0,47517 & 0,58163 & 0,67488 \\
\hline 0,49923 & 0,63207 & 0,2775 & 0,53613 \\
\hline 0,47269 & 0,75896 & 0,58753 & 0,80619 \\
\hline 0,5943 & 1,00637 & 0,61875 & 0,78817 \\
\hline 0,61854 & 1,0981 & 0,63876 & 0,92461 \\
\hline 0,82612 & 1,0981 & 0,74681 & 0,96901 \\
\hline 1,04071 & 1,3305 & 0,74991 & 1,02923 \\
\hline 1,04071 & 1,50318 & 0,89045 & 1,09814 \\
\hline 0,98011 & 1,48448 & 0,08222 & 0,09528 \\
\hline 0,06451 & 0,15079 & 0,14949 & 0,19 \\
\hline 0,37936 & 0,35129 & 0,23044 & 0,1743 \\
\hline \multirow[t]{7}{*}{0,16984} & 0,18653 & 0,22303 & 0,35927 \\
\hline & & 0,24676 & 0,52307 \\
\hline & & 0,51961 & 0,74695 \\
\hline & & 0,46406 & 0,57316 \\
\hline & & 0,87497 & 1,17806 \\
\hline & & 1,01793 & 1,36753 \\
\hline & & 0,9979 & 1,5524 \\
\hline \multicolumn{2}{|c|}{$\mathrm{T}=25^{\circ} \mathrm{C}$} & \multicolumn{2}{|c|}{$\mathrm{T}=55^{\circ} \mathrm{C}$} \\
\hline $\ln \left(\mathrm{I}_{45}\left(-\mathrm{O}_{2}\right) / \mathrm{I}_{45}\left(+\mathrm{O}_{2}\right)\right)$ & $\ln \left(\mathrm{I}_{57}\left(-\mathrm{O}_{2}\right) / \mathrm{I}_{57}\left(+\mathrm{O}_{2}\right)\right)$ & $\ln \left(\mathrm{I}_{45}\left(-\mathrm{O}_{2}\right) / \mathrm{I}_{45}\left(+\mathrm{O}_{2}\right)\right)$ & $\ln \left(\mathrm{I}_{57}\left(-\mathrm{O}_{2}\right) / \mathrm{I}_{57}\left(+\mathrm{O}_{2}\right)\right)$ \\
\hline 0,40531 & 0,46649 & 0,35009 & 0,29326 \\
\hline 0,41854 & 0,51066 & 0,22306 & 0,29842 \\
\hline 0,39069 & 0,65902 & 0,37254 & 0,4211 \\
\hline 0,49223 & 0,69293 & 0,33634 & 0,45412 \\
\hline 0,61059 & 0,72927 & 0,4053 & 0,51069 \\
\hline
\end{tabular}




\begin{tabular}{|c|c|c|c|}
\hline 0,61059 & 0,80619 & 0,4053 & 0,52203 \\
\hline 0,79808 & 0,9159 & 0,60193 & 0,67668 \\
\hline 0,02818 & 0 & 0,56612 & 0,82211 \\
\hline 0,19773 & 0,30413 & 0,74409 & 0,8264 \\
\hline 0,08956 & 0,20248 & 0,8799 & 1,07006 \\
\hline 0,74681 & 0,96901 & 0,94044 & 1,07006 \\
\hline 0,58753 & 0,8715 & 0,6282 & 0,82633 \\
\hline 0,76876 & 0,9159 & 0,10002 & 0,12774 \\
\hline 0,80395 & 1,16595 & & \\
\hline 0,78227 & 1,26058 & & \\
\hline 1,0453 & 1,29336 & & \\
\hline 0,91568 & 1,40215 & & \\
\hline \multicolumn{2}{|c|}{$\mathrm{T}=90^{\circ} \mathrm{C}$} & & \\
\hline $\ln \left(\mathrm{I}_{45}\left(-\mathrm{O}_{2}\right) / \mathrm{I}_{45}\left(+\mathrm{O}_{2}\right)\right)$ & $\ln \left(\mathrm{I}_{57}\left(-\mathrm{O}_{2}\right) / \mathrm{I}_{57}\left(+\mathrm{O}_{2}\right)\right)$ & & \\
\hline 0,30736 & 0,27435 & & \\
\hline 0,25579 & 0,20287 & & \\
\hline 0,3745 & 0,36532 & & \\
\hline 0,36084 & 0,46983 & & \\
\hline 0,20466 & 0,47788 & & \\
\hline 0,48928 & 0,49043 & & \\
\hline 0,52103 & 0,51773 & & \\
\hline 0,56766 & 0,57035 & & \\
\hline 0,60042 & 0,47941 & & \\
\hline 0,51052 & 0,60953 & & \\
\hline 0,66291 & 0,6718 & & \\
\hline 0,69271 & 0,65208 & & \\
\hline 0,51052 & 0,78379 & & \\
\hline 0,69272 & 0,91593 & & \\
\hline 0,81027 & 1,0581 & & \\
\hline 1,13055 & 1,12 & & \\
\hline 0,03276 & 0,09306 & & \\
\hline 0,06666 & 0,15759 & & \\
\hline 0,22303 & 0,09761 & & \\
\hline 0,16421 & 0,11505 & & \\
\hline 0,26818 & 0,28019 & & \\
\hline
\end{tabular}

Messtabelle 27: c-Heptyl $+\mathrm{O}_{2}$ relativ zu Methoxymethyl $+\mathrm{O}_{2}$ (zu Abbildung 147)

Versuchsanordnung A, Reaktor $1, \lambda=440 \mathrm{~nm}, \mathrm{~s}=3 \mathrm{~cm}, \mathrm{~T}=25^{\circ} \mathrm{C}$, Badgas: He, $\mathrm{p}=1,79 \mathrm{mbar}$, $\Phi(\mathrm{He})=1252 \mathrm{sccm}, \Phi(\mathrm{c}-\mathrm{Heptan})=4,96 \mathrm{sccm}, \Phi(\mathrm{DME})=6,24 \mathrm{sccm}, \Phi\left(\mathrm{F}_{2}\right)=1,56 \mathrm{sccm}$, $\Phi\left(\mathrm{O}_{2}\right)=0-20 \mathrm{sccm}, \mathrm{E}($ Laser $)=11 \mathrm{~mJ}, 800$ sweeps

\begin{tabular}{|c|c|}
\hline $\ln \left(\mathrm{I}_{45}\left(-\mathrm{O}_{2}\right) / \mathrm{I}_{45}\left(+\mathrm{O}_{2}\right)\right)$ & $\ln \left(\mathrm{I}_{97}\left(-\mathrm{O}_{2}\right) / \mathrm{I}_{97}\left(+\mathrm{O}_{2}\right)\right)$ \\
\hline \hline 0,06621 & 0,23964 \\
\hline 0,20758 & 0,40597 \\
\hline 0,22171 & 0,53695 \\
\hline 0,30796 & 0,78904 \\
\hline 0,47795 & 1,0593 \\
\hline 0,06621 & 0,23964 \\
\hline
\end{tabular}


Messtabelle 28: c-Oktyl $+\mathrm{O}_{2}$ relativ zu $\mathrm{CH}_{2} \mathrm{OCH}_{3}+\mathrm{O}_{2}$ (zu Abbildung 148 bis Abbildung 151)

Versuchsanordnung A, Reaktor 1, $\lambda=456 \mathrm{~nm}, \mathrm{~s}=3 \mathrm{~cm}$, Badgas: He, $\mathrm{p}=1,90 \mathrm{mbar}$, $\Phi(\mathrm{He})=1220 \mathrm{sccm}, \Phi(\mathrm{c}-$ Oktan $)=2,92 \mathrm{sccm}, \Phi(\mathrm{DME})=2,3 \mathrm{sccm}, \Phi\left(\mathrm{F}_{2}\right)=1,56 \mathrm{sccm}$, $\Phi\left(\mathrm{O}_{2}\right)=0-20$ sccm, $\mathrm{E}($ Laser $)=10 \mathrm{~mJ}, 800$ sweeps

\begin{tabular}{|c|c|c|c|}
\hline \multicolumn{2}{|c|}{$\mathrm{T}=5^{\circ} \mathrm{C}$} & \multicolumn{2}{|c|}{$\mathrm{T}=25^{\circ} \mathrm{C}$} \\
\hline $\ln \left(\mathrm{I}_{45}\left(-\mathrm{O}_{2}\right) / \mathrm{I}_{45}\left(+\mathrm{O}_{2}\right)\right)$ & $\overline{\ln \left(\mathrm{I}_{111}\left(-\mathrm{O}_{2}\right) / \mathrm{I}_{111}\left(+\mathrm{O}_{2}\right)\right)}$ & $\bar{l} \ln \left(\mathrm{I}_{45}\left(-\mathrm{O}_{2}\right) / \mathrm{I}_{45}\left(+\mathrm{O}_{2}\right)\right)$ & $\bar{l} \ln \left(\mathrm{I}_{111}\left(-\mathrm{O}_{2}\right) / \mathrm{I}_{111}\left(+\mathrm{O}_{2}\right)\right)$ \\
\hline 0,33185 & 0,52117 & 0,37192 & 0,45647 \\
\hline 0,51035 & 0,69794 & 0,89711 & 1,2774 \\
\hline 0,64705 & 1,093 & 0,44978 & 0,65057 \\
\hline 0,61 & 0,8529 & 0,6395 & 0,94964 \\
\hline 0,45435 & 0,57406 & 0,26835 & 0,31134 \\
\hline 0,19745 & 0,32829 & 0,12819 & 0,12305 \\
\hline 0,14684 & 0,2238 & 0,71481 & 1,1125 \\
\hline 0,5839 & 1,0016 & 0,43417 & 0,61419 \\
\hline \multirow[t]{5}{*}{0,64705} & 1,093 & 0,58107 & 0,78706 \\
\hline & & 0,32641 & 0,41412 \\
\hline & & 0,24044 & 0,35336 \\
\hline & & 0,147 & 0,1526 \\
\hline & & 0,1977 & 0,26076 \\
\hline \multicolumn{2}{|c|}{$\mathrm{T}=55 \mathrm{C}^{\circ}$} & \multicolumn{2}{|c|}{$\mathrm{T}=90^{\circ} \mathrm{C}$} \\
\hline $\ln \left(\mathrm{I}_{45}\left(-\mathrm{O}_{2}\right) / \mathrm{I}_{45}\left(+\mathrm{O}_{2}\right)\right)$ & $\overline{l n}\left(\mathrm{I}_{111}\left(-\mathrm{O}_{2}\right) / \mathrm{I}_{111}\left(+\mathrm{O}_{2}\right)\right)$ & $\ln \left(\mathrm{I}_{45}\left(-\mathrm{O}_{2}\right) / \mathrm{I}_{45}\left(+\mathrm{O}_{2}\right)\right)$ & $\overline{l n}\left(\mathrm{I}_{111}\left(-\mathrm{O}_{2}\right) / \mathrm{I}_{111}\left(+\mathrm{O}_{2}\right)\right)$ \\
\hline 0,56851 & 0,67786 & 0,0611 & 0,11194 \\
\hline 0,24585 & 0,3106 & 0,12296 & 0,15036 \\
\hline 0,33425 & 0,56654 & 0,28367 & 0,26322 \\
\hline 0,5496 & 0,70567 & 0,20615 & 0,17008 \\
\hline 0,54964 & 0,83154 & 0,36265 & 0,43647 \\
\hline 0,59379 & 0,78094 & 0,31392 & 0,35321 \\
\hline 0,75009 & 1,0587 & 0,53725 & 0,63924 \\
\hline 0,08093 & 0,10208 & & \\
\hline 0,22343 & 0,26144 & & \\
\hline 0,24033 & 0,46318 & & \\
\hline 0,32088 & 0,42009 & & \\
\hline 0,26616 & 0,3898 & & \\
\hline 0,37982 & 0,54342 & & \\
\hline
\end{tabular}

Messtabelle 29: Cyclopentadienyl $+\mathrm{O}_{2}$ relativ zu Ethyl $+\mathrm{O}_{2}$ (zu Abbildung 153)

Versuchsanordnung A, Reaktor 1, $\lambda=424,87 \mathrm{~nm}, \mathrm{~s}=3 \mathrm{~cm}$, Badgas: He, $\mathrm{p}=1,8 \mathrm{mbar}$, $\Phi(\mathrm{He})=904 \mathrm{sccm}, \Phi($ Cyclopentadien $)=6,91 \mathrm{sccm}, \Phi($ Ethan $)=20 \mathrm{sccm}, \Phi\left(\mathrm{F}_{2}\right)=2,5 \mathrm{sccm}$, $\Phi\left(\mathrm{O}_{2}\right)=10-70 \mathrm{sccm}, \mathrm{E}($ Laser $)=5 \mathrm{~mJ}, 800$ sweeps, $\mathrm{T}=298 \mathrm{~K}$

\begin{tabular}{|c|c|}
\hline $\ln \left(\mathrm{I}_{29}\left(-\mathrm{O}_{2}\right) / \mathrm{I}_{29}\left(+\mathrm{O}_{2}\right)\right)$ & $\ln \left(\mathrm{I}_{65}\left(-\mathrm{O}_{2}\right) / \mathrm{I}_{65}\left(+\mathrm{O}_{2}\right)\right)$ \\
\hline \hline 0,43552 & 0,14114 \\
\hline 0,27971 & 0,04883 \\
\hline 0,26101 & 0,0825 \\
\hline 0,39121 & 0,09679 \\
\hline 0,74718 & 0,26798 \\
\hline
\end{tabular}


Messtabelle 30: Iso- $\mathrm{C}_{4} \mathrm{H}_{8} \mathrm{Cl}+\mathrm{O}_{2}$ relativ zu Methoxymethyl $+\mathrm{O}_{2}$ (zu Abbildung 154)

Versuchsanordnung A, Reaktor $1, \lambda=440 \mathrm{~nm}, \mathrm{~s}=3 \mathrm{~cm}, \mathrm{~T}=25^{\circ} \mathrm{C}$, Badgas: He, $\mathrm{p}=1,67$ mbar, $\Phi(\mathrm{He})=900 \mathrm{sccm}, \Phi($ Isobutylchlorid $)=14,58 \mathrm{sccm}, \Phi(\mathrm{DME})=5,46 \mathrm{sccm}, \Phi\left(\mathrm{F}_{2}\right)=1,56 \mathrm{sccm}$, $\Phi\left(\mathrm{O}_{2}\right)=0-50$ sccm, $\mathrm{E}($ Laser $)=12 \mathrm{~mJ}, 800$ sweeps

\begin{tabular}{|c|c|}
\hline $\ln \left(\mathrm{I}_{45}\left(-\mathrm{O}_{2}\right) / \mathrm{I}_{45}\left(+\mathrm{O}_{2}\right)\right)$ & $\ln \left(\mathrm{I}_{91}\left(-\mathrm{O}_{2}\right) / \mathrm{I}_{91}\left(+\mathrm{O}_{2}\right)\right)$ \\
\hline 0,77461 & 0,72175 \\
\hline 0,71194 & 0,58588 \\
\hline 0,78057 & 0,76587 \\
\hline 0,83325 & 0,76398 \\
\hline 0,36414 & 0,20628 \\
\hline 0,46975 & 0,44932 \\
\hline 0,4932 & 0,40268 \\
\hline$-0,00284$ & 0,06656 \\
\hline 0,19117 & 0,31777 \\
\hline 0,12141 & 0,16371 \\
\hline
\end{tabular}

Messtabelle 31: t-Butyl $+\mathrm{O}_{3}$ relativ zu Methoxymethyl $+\mathrm{O}_{3}$ (zu Abbildung 155)

Versuchsanordnung A, Reaktor $2 \mathrm{a}, \lambda=505 \mathrm{~nm}, \mathrm{~s}_{1}=2 \mathrm{~cm}, \mathrm{~s}_{2}=3 \mathrm{~cm}$, Badgas: He, $\mathrm{p}=1,50$ mbar, $\Phi(\mathrm{He})=1000 \mathrm{sccm}, \Phi($ Isobutan $)=2,90 \mathrm{sccm}, \Phi(\mathrm{DME})=7,8 \mathrm{sccm}$, $\Phi\left(\mathrm{F}_{2}\right)=1,21 \mathrm{sccm}, \mathrm{E}($ Laser $)=8 \mathrm{~mJ}, 400$ sweeps, $\mathrm{T}=25^{\circ} \mathrm{C}$

\begin{tabular}{|c|c|}
\hline $\ln \left(\mathrm{I}_{45}\left(-\mathrm{O}_{3}\right) / \mathrm{I}_{45}\left(+\mathrm{O}_{3}\right)\right)$ & $\ln \left(\mathrm{I}_{57}\left(-\mathrm{O}_{3}\right) / \mathrm{I}_{57}\left(+\mathrm{O}_{3}\right)\right)$ \\
\hline \hline$-0,04437$ & $-0,05393$ \\
\hline 0,0516 & 0,1574 \\
\hline 0,11921 & 0,09405 \\
\hline 0,3833 & 0,52609 \\
\hline 0,24646 & 0,31365 \\
\hline 0,29225 & 0,42323 \\
\hline 0,05947 & 0,0534 \\
\hline 0,08239 & 0,02047 \\
\hline 0,1733 & 0,07802 \\
\hline 0,00761 & $-0,02424$ \\
\hline 0,64343 & 0,66505 \\
\hline 2,1005 & 2,4973 \\
\hline 1,4296 & 1,6755 \\
\hline 0,81901 & 1,0165 \\
\hline 0,5407 & 0,62874 \\
\hline 0,34255 & 0,40694 \\
\hline 0,84645 & 0,84754 \\
\hline 0,43495 & 0,75529 \\
\hline 0,6321 & 0,78033 \\
\hline & \\
\hline
\end{tabular}


Messtabelle 32: c-Heptyl $+\mathrm{O}_{3}$ relativ zu Methoxymethyl + $\mathrm{O}_{3}$ (zu Abbildung 156)

Versuchsanordnung A, Reaktor $2 \mathrm{a}, \lambda=440 \mathrm{~nm}, \mathrm{~s}_{1}=2 \mathrm{~cm}, \mathrm{~s}_{2}=3 \mathrm{~cm}, \mathrm{~T}=25^{\circ} \mathrm{C}$, Badgas: He, $\mathrm{p}=2,00 \mathrm{mbar}, \Phi(\mathrm{He})=1352 \mathrm{sccm}, \Phi(\mathrm{c}-$ Heptan $)=4,96 \mathrm{sccm}, \Phi(\mathrm{DME})=7,80 \mathrm{sccm}$, $\Phi\left(\mathrm{F}_{2}\right)=1,56 \mathrm{sccm}, \mathrm{E}($ Laser $)=7,5 \mathrm{~mJ}, 800$ sweeps

\begin{tabular}{|c|c|}
\hline $\ln \left(\mathrm{I}_{45}\left(-\mathrm{O}_{3}\right) / \mathrm{I}_{45}\left(+\mathrm{O}_{3}\right)\right)$ & $\ln \left(\mathrm{I}_{97}\left(-\mathrm{O}_{3}\right) / \mathrm{I}_{97}\left(+\mathrm{O}_{3}\right)\right)$ \\
\hline 0,22698 & 0,16055 \\
\hline 0,34643 & 0,33179 \\
\hline 0,6192 & 0,80122 \\
\hline 0,98849 & 1,206 \\
\hline 0,93023 & 1,0401 \\
\hline 0,89628 & 1,0147 \\
\hline 0,35756 & 0,49433 \\
\hline 0,10559 & 0,20853 \\
\hline 0,18768 & 0,2441 \\
\hline 0,18108 & 0,27513 \\
\hline 0,03721 & 0,0989 \\
\hline 0,35886 & 0,46786 \\
\hline 0,49386 & 0,53016 \\
\hline 0,85942 & 0,95523 \\
\hline 0,6218 & 0,85699 \\
\hline
\end{tabular}

Messtabelle 33: c-Oktyl $+\mathrm{O}_{3}$ relativ zu Methoxymethyl $+\mathrm{O}_{3}$ (zu Abbildung 158)

Versuchsanordnung A, Reaktor 2a, $\lambda=440 \mathrm{~nm}, \mathrm{~s}_{1}=2 \mathrm{~cm}, \mathrm{~s}_{2}=3 \mathrm{~cm}$, Badgas: He, $\mathrm{p}=2,15$ mbar, $\Phi(\mathrm{He})=1466 \mathrm{sccm}, \Phi(\mathrm{c}-$ Oktan $)=2,92 \mathrm{sccm}, \Phi(\mathrm{DME})=3,12 \mathrm{sccm}$, $\Phi\left(\mathrm{F}_{2}\right)=1,56 \mathrm{sccm}, \mathrm{E}($ Laser $)=7 \mathrm{~mJ}, 800$ sweeps, $\mathrm{T}=25^{\circ} \mathrm{C}$

\begin{tabular}{|c|c|}
\hline $\ln \left(\mathrm{I}_{45}\left(-\mathrm{O}_{3}\right) / \mathrm{I}_{45}\left(+\mathrm{O}_{3}\right)\right)$ & $\ln \left(\mathrm{I}_{111}\left(-\mathrm{O}_{3}\right) / \mathrm{I}_{111}\left(+\mathrm{O}_{3}\right)\right)$ \\
\hline \hline 0,11327 & 0,04443 \\
\hline 0,2458 & 0,33695 \\
\hline 0,34946 & 0,46375 \\
\hline 0,56033 & 0,60059 \\
\hline 0,87057 & 0,97423 \\
\hline 0,97703 & 1,2788 \\
\hline 0,87513 & 1,1883 \\
\hline 0,6973 & 0,97233 \\
\hline 0,61478 & 0,66963 \\
\hline 0,11033 & 0,16305 \\
\hline 0,7311 & 0,95363 \\
\hline 0,56532 & 0,5988 \\
\hline
\end{tabular}


Messtabelle 34: Iso- $\mathrm{C}_{4} \mathrm{H}_{8} \mathrm{Cl}+\mathrm{O}_{3}$ relativ zu Methoxymethyl $+\mathrm{O}_{3}$ (zu Abbildung 158)

Versuchsanordnung A, Reaktor $2 \mathrm{a}, \lambda=440 \mathrm{~nm}, \mathrm{~s}_{1}=2 \mathrm{~cm}, \mathrm{~s}_{2}=3 \mathrm{~cm}, \mathrm{~T}=25^{\circ} \mathrm{C}$, Badgas: He, $\mathrm{p}=2,03 \mathrm{mbar}, \Phi(\mathrm{He})=870 \mathrm{sccm}, \Phi($ Isobutylchlorid $)=14,58 \mathrm{sccm}, \Phi(\mathrm{DME})=7,80 \mathrm{sccm}$, $\Phi\left(\mathrm{F}_{2}\right)=1,81 \mathrm{sccm}, \mathrm{E}($ Laser $)=10 \mathrm{~mJ}, 800$ sweeps

\begin{tabular}{|c|c|}
\hline $\ln \left(\mathrm{I}_{45}\left(-\mathrm{O}_{2}\right) / \mathrm{I}_{45}\left(+\mathrm{O}_{2}\right)\right)$ & $\ln \left(\mathrm{I}_{91}\left(-\mathrm{O}_{2}\right) / \mathrm{I}_{91}\left(+\mathrm{O}_{2}\right)\right)$ \\
\hline 0,77461 & 0,72175 \\
\hline 0,71194 & 0,58588 \\
\hline 0,78057 & 0,76587 \\
\hline 0,83325 & 0,76398 \\
\hline 0,36414 & 0,20628 \\
\hline 0,46975 & 0,44932 \\
\hline 0,4932 & 0,40268 \\
\hline$-0,00284$ & 0,06656 \\
\hline 0,19117 & 0,31777 \\
\hline 0,12141 & 0,16371 \\
\hline
\end{tabular}

Messtabelle 35: t-Butyl + NO relativ zu $\mathrm{CH}_{2} \mathrm{OCH}_{3}+\mathrm{NO}$ (zu Abbildung 159 bis Abbildung 163)

Versuchsanordnung A, Reaktor 1 mit Spezialsonde, $\lambda=510 \mathrm{~nm}, \mathrm{~s}_{1}=5 \mathrm{~cm}, \mathrm{~s}_{2}=4 \mathrm{~cm}$, Badgas: He, $\mathrm{p}=2,14 \mathrm{mbar}, \Phi(\mathrm{He})=870 \mathrm{sccm}, \Phi($ Isobutan $)=4,32 \mathrm{sccm}, \Phi(\mathrm{DME})=14,04 \mathrm{sccm}$, $\Phi\left(\mathrm{F}_{2}\right)=1,56 \mathrm{sccm}, \Phi(\mathrm{NO})=2-10 \mathrm{sccm}, \mathrm{E}($ Laser $)=8 \mathrm{~mJ}, 600$ sweeps

\begin{tabular}{|c|c|c|c|}
\hline \multicolumn{2}{|c|}{$\mathrm{T}=-30^{\circ} \mathrm{C}$} & \multicolumn{2}{|c|}{$\mathrm{T}=0^{\circ} \mathrm{C}$} \\
\hline $\ln \left(\mathrm{I}_{45} / \mathrm{I}_{45}(+\mathrm{NO})\right)$ & $\ln \left(\mathrm{I}_{57} / \mathrm{I}_{57}(+\mathrm{NO})\right)$ & $\ln \left(\mathrm{I}_{45} / \mathrm{I}_{45}(+\mathrm{NO})\right)$ & $\ln \left(\mathrm{I}_{57} / \mathrm{I}_{57}(+\mathrm{NO})\right)$ \\
\hline 0,07493 & 0,20602 & 0,13407 & 0,33445 \\
\hline 0,10285 & 0,11172 & 0,29652 & 0,68062 \\
\hline 0,52449 & 1,0107 & 0,33016 & 0,8562 \\
\hline 0,46307 & 1,1292 & 0,30705 & 0,67336 \\
\hline 0,3094 & 0,89604 & 0,18645 & 0,42085 \\
\hline 0,40383 & 0,76468 & 0,05157 & 0,14269 \\
\hline 0,1808 & 0,56897 & 0,02429 & 0,08177 \\
\hline 0,1125 & 0,42751 & 0,11545 & 0,322 \\
\hline 0,1251 & 0,19009 & 0,17453 & 0,5146 \\
\hline 0,14577 & 0,17212 & & \\
\hline 0,22777 & 0,46932 & & \\
\hline 0,22642 & 0,64433 & & \\
\hline 0,27485 & 0,76223 & & \\
\hline 0,07291 & 0,12206 & & \\
\hline \multicolumn{2}{|c|}{$\mathrm{T}=25^{\circ} \mathrm{C}$} & \multicolumn{2}{|c|}{$\mathrm{T}=55^{\circ} \mathrm{C}$} \\
\hline $\ln \left(\mathrm{I}_{45} / \mathrm{I}_{45}(+\mathrm{NO})\right)$ & $\ln \left(\mathrm{I}_{57} / \mathrm{I}_{57}(+\mathrm{NO})\right)$ & $\ln \left(\mathrm{I}_{45} / \mathrm{I}_{45}(+\mathrm{NO})\right)$ & $\overline{l n}\left(\mathrm{I}_{57} / \mathrm{I}_{57}(+\mathrm{NO})\right)$ \\
\hline 0,07708 & 0,21838 & 0,03237 & 0,13981 \\
\hline 0,10211 & 0,3129 & 0,15907 & 0,71613 \\
\hline 0,27148 & 0,7547 & 0,13434 & 0,67931 \\
\hline 0,02865 & 0,10915 & 0,08258 & 0,53898 \\
\hline \multirow[t]{3}{*}{0,17674} & 0,47839 & 0,12312 & 0,35542 \\
\hline & & 0,06953 & 0,17473 \\
\hline & & 0,05261 & 0,3942 \\
\hline
\end{tabular}




\begin{tabular}{|c|c|c|c|}
\hline & & 0,06486 & 0,30132 \\
\hline \multicolumn{2}{|c|}{$\mathrm{T}=90^{\circ} \mathrm{C}$} & & \\
\hline $\ln \left(\mathrm{I}_{45} / \mathrm{I}_{45}(+\mathrm{NO})\right)$ & $\ln \left(\mathrm{I}_{57} / \mathrm{I}_{57}(+\mathrm{NO})\right)$ & & \\
\hline 0,03858 & 0,17075 & & \\
\hline 0,11193 & 0,57551 & & \\
\hline 0,04843 & 0,36962 & & \\
\hline 0,02605 & 0,21161 & & \\
\hline 0,04328 & 0,03758 & & \\
\hline 0,03747 & 0,16048 & & \\
\hline 0,05321 & 0,35622 & & \\
\hline 0,10707 & 0,51758 & & \\
\hline 0,17594 & 0,84892 & & \\
\hline
\end{tabular}

Messtabelle 36: Allyl + NO relativ zu $\mathrm{CH}_{2} \mathrm{OCH}_{3}$ + NO (zu Abbildung 165 bis Abbildung 169)

Versuchsanordnung A, Reaktor 1 mit Spezialsonde, $\lambda=499,2 \mathrm{~nm}, \mathrm{~s}_{1}=3 \mathrm{~cm}, \mathrm{~s}_{2}=2 \mathrm{~cm}$, Badgas: He, $\mathrm{T}=298 \mathrm{~K}, \mathrm{p}=2,15 \mathrm{mbar}, \Phi(\mathrm{He})=930 \mathrm{sccm}, \Phi(\mathrm{DME})=7,8 \mathrm{sccm}, \Phi($ Propen $)=$ 13,12 sccm, $\Phi\left(\mathrm{F}_{2}\right)=1,44 \mathrm{sccm}, \Phi(\mathrm{NO})=5-50 \mathrm{sccm}, \mathrm{E}($ Laser $)=12 \mathrm{~mJ}$

\begin{tabular}{|c|c|c|c|}
\hline \multicolumn{2}{|c|}{$\mathrm{T}=-30^{\circ} \mathrm{C}$} & \multicolumn{2}{|c|}{$\mathrm{T}=0^{\circ} \mathrm{C}$} \\
\hline $\ln \left(\mathrm{I}_{45} / \mathrm{I}_{45}(+\mathrm{NO})\right)$ & $\ln \left(\mathrm{I}_{41} / \mathrm{I}_{41}(+\mathrm{NO})\right)$ & $2 \ln \left(\mathrm{I}_{45} / \mathrm{I}_{45}(+\mathrm{NO})\right)$ & $\ln \left(\mathrm{I}_{41} / \mathrm{I}_{41}(+\mathrm{NO})\right)$ \\
\hline 0,10791 & 0,26761 & 0,18161 & 0,29497 \\
\hline 0,16338 & 0,43003 & 0,13062 & 0,30244 \\
\hline 0,34651 & 0,7738 & 0,0671 & 0,16109 \\
\hline 0,37146 & 0,67291 & 0,11303 & 0,255 \\
\hline \multirow{3}{*}{0,4449} & 1,0024 & 0,2024 & 0,5259 \\
\hline & & 0,18742 & 0,41432 \\
\hline & & 0,25087 & 0,43738 \\
\hline \multicolumn{2}{|c|}{$\mathrm{T}=25^{\circ} \mathrm{C}$} & \multicolumn{2}{|c|}{$\mathrm{T}=55^{\circ} \mathrm{C}$} \\
\hline $\ln \left(\mathrm{I}_{45} / \mathrm{I}_{45}(+\mathrm{NO})\right)$ & $\overline{l n}\left(\mathrm{I}_{41} / \mathrm{I}_{41}(+\mathrm{NO})\right)$ & $\ln \left(\mathrm{I}_{45} / \mathrm{I}_{45}(+\mathrm{NO})\right)$ & $\ln \left(\mathrm{I}_{41} / \mathrm{I}_{41}(+\mathrm{NO})\right)$ \\
\hline 0,43613 & 1,0138 & 0,12369 & 0,24607 \\
\hline 0,1214 & 0,24342 & 0,05755 & 0,15657 \\
\hline 0,09972 & 0,28564 & 0,08795 & 0,27961 \\
\hline 0,19691 & 0,46722 & 0,10777 & 0,29922 \\
\hline 0,2928 & 0,74008 & 0,10995 & 0,28642 \\
\hline \multirow[t]{3}{*}{0,07158} & 0,22053 & 0,14641 & 0,36302 \\
\hline & & 0,16784 & 0,42471 \\
\hline & & 0,18937 & 0,46204 \\
\hline \multicolumn{2}{|c|}{$\mathrm{T}=90^{\circ} \mathrm{C}$} & & \\
\hline $\ln \left(\mathrm{I}_{45} / \mathrm{I}_{45}(+\mathrm{NO})\right)$ & $\ln \left(\mathrm{I}_{41} / \mathrm{I}_{41}(+\mathrm{NO})\right)$ & & \\
\hline 0,03994 & 0,13837 & & \\
\hline 0,04703 & 0,10708 & & \\
\hline 0,16974 & 0,32566 & & \\
\hline 0,15509 & 0,33563 & & \\
\hline 0,18382 & 0,5485 & & \\
\hline 0,19567 & 0,42508 & & \\
\hline 0,22336 & 0,60062 & & \\
\hline 0,23472 & 0,52515 & & \\
\hline
\end{tabular}


Messtabelle 37: 2-Propyl + H relativ zu Methoxymethyl + H (zu Abbildung 171)

Versuchsanordnung A, Reaktor $2 \mathrm{a}, \lambda=465 \mathrm{~nm}, \mathrm{~s}_{1}=2 \mathrm{~cm}, \mathrm{~s}_{2}=3 \mathrm{~cm}$, Badgas: He, $\mathrm{T}=298 \mathrm{~K}$, $\mathrm{p}=2,1 \mathrm{mbar}, \Phi(\mathrm{He})=800 \mathrm{sccm}, \Phi(\mathrm{DME})=2,57 \mathrm{sccm}, \Phi($ Propan $)=14,4 \mathrm{sccm}$, $\Phi\left(\mathrm{F}_{2}\right)=1,56 \mathrm{sccm}, \Phi\left(\mathrm{H}_{2}\right)=0-1,5 \mathrm{sccm}, \mathrm{E}($ Laser $)=10 \mathrm{~mJ}, 800$ sweeps

\begin{tabular}{|c|c|}
\hline $\ln \left(\mathrm{I}_{43}(-\mathrm{H}) / \mathrm{I}_{43}(+\mathrm{H})\right)$ & $\ln \left(\mathrm{I}_{45}(-\mathrm{H}) / \mathrm{I}_{45}(+\mathrm{H})\right)$ \\
\hline 0,61435 & 0,76238 \\
\hline 0,55715 & 0,53099 \\
\hline 0,91558 & 0,98427 \\
\hline 0,56872 & 0,64212 \\
\hline 0,54282 & 0,53207 \\
\hline 0,25116 & 0,36411 \\
\hline 0,3906 & 0,27608 \\
\hline 0,38015 & 0,36408 \\
\hline 0,11813 & 0,1405 \\
\hline 0,22302 & 0,16594 \\
\hline 0,05842 & 0,09141 \\
\hline 0,48201 & 0,44369 \\
\hline 0,75129 & 0,81274 \\
\hline
\end{tabular}

\section{Messtabelle 38: 2-Butyl + H relativ zu Methoxymethyl + H (zu Abbildung 172)}

Versuchsanordnung A, Reaktor $2 \mathrm{a}, \lambda=465 \mathrm{~nm}, \mathrm{~s}_{1}=2 \mathrm{~cm}, \mathrm{~s}_{2}=3 \mathrm{~cm}$, Badgas: He, $\mathrm{T}=298 \mathrm{~K}$, $\mathrm{p}=2,1$ mbar, $\Phi(\mathrm{He})=850 \mathrm{sccm}, \Phi($ Butan $)=10,4 \mathrm{sccm}, \Phi(\mathrm{DME})=3,59 \mathrm{sccm}$, $\Phi\left(\mathrm{F}_{2}\right)=1,56 \mathrm{sccm}, \Phi\left(\mathrm{H}_{2}\right)=0-1,2 \mathrm{sccm}, \mathrm{E}($ Laser $)=9 \mathrm{~mJ}, 800$ sweeps

\begin{tabular}{|c|c|}
\hline $\ln \left(\mathrm{I}_{45}(-\mathrm{H}) / \mathrm{I}_{57}(+\mathrm{H})\right)$ & $\ln \left(\mathrm{I}_{57}(-\mathrm{H}) / \mathrm{I}_{57}(+\mathrm{H})\right)$ \\
\hline 0,12578 & 0,15077 \\
\hline 0,25333 & 0,13039 \\
\hline 0,11752 & 0,14646 \\
\hline 1,2457 & 1,2271 \\
\hline$-0,05205$ & $-0,06978$ \\
\hline 0,97362 & 1,0399 \\
\hline 0,75023 & 0,46079 \\
\hline 0,49372 & 0,14185 \\
\hline 0,84637 & 0,6907 \\
\hline 1,0646 & 1,0061 \\
\hline 0,90233 & 0,87755 \\
\hline 0,55056 & 0,85218 \\
\hline 0,33242 & 0,63521 \\
\hline
\end{tabular}


Messtabelle 39: t-Butyl + H relativ zu Methoxymethyl + H (zu Abbildung 173)

Versuchsanordnung A, Reaktor 2a, $\lambda=500 \mathrm{~nm}, \mathrm{~s}_{1}=2 \mathrm{~cm}, \mathrm{~s}_{2}=3 \mathrm{~cm}$, Badgas: He, $\mathrm{p}=1,85$ mbar, $\Phi(\mathrm{He})=750 \mathrm{sccm}, \Phi($ Isobutan $)=4,32 \mathrm{sccm}, \Phi(\mathrm{DME})=8,58 \mathrm{sccm}$, $\Phi\left(\mathrm{F}_{2}\right)=1,56 \mathrm{sccm}, \Phi\left(\mathrm{H}_{2}\right)=0-1,01 \mathrm{sccm}, \mathrm{E}($ Laser $)=9 \mathrm{~mJ}, 400$ sweeps, $\mathrm{T}=25^{\circ} \mathrm{C}$

\begin{tabular}{|c|c|}
\hline $\ln \left(\mathrm{I}_{45}(-\mathrm{H}) / \mathrm{I}_{45}(+\mathrm{H})\right)$ & $\ln \left(\mathrm{I}_{57}(-\mathrm{H}) / \mathrm{I}_{57}(+\mathrm{H})\right)$ \\
\hline 0,00944 & 0,04908 \\
\hline 0,91185 & 0,98613 \\
\hline 0,30969 & 0,43738 \\
\hline 0,08386 & 0,13249 \\
\hline 0,24604 & 0,25932 \\
\hline 0,65061 & 0,55367 \\
\hline 0,5881 & 0,58765 \\
\hline 0,79826 & 0,86378 \\
\hline 0,12459 & 0,14167 \\
\hline 0,11001 & 0,07849 \\
\hline 0,13286 & 0,17313 \\
\hline 0,46085 & 0,37748 \\
\hline 0,3783 & 0,36152 \\
\hline
\end{tabular}

\section{Messtabelle 40: Allyl + H relativ zu Methoxymethyl + H (zu Abbildung 174)}

Versuchsanordnung A, Reaktor 2a, $\lambda=499,368 \mathrm{~nm}, \mathrm{~s}_{1}=2 \mathrm{~cm}, \mathrm{~s}_{2}=3 \mathrm{~cm}$, Badgas: He, $\mathrm{T}=298 \mathrm{~K}, \mathrm{p}=2,1 \mathrm{mbar}, \Phi(\mathrm{He})=780 \mathrm{sccm}, \Phi(\mathrm{DME})=7,02 \mathrm{sccm}, \Phi($ Propen $)=12,30 \mathrm{sccm}$, $\Phi\left(\mathrm{F}_{2}\right)=1,56 \mathrm{sccm}, \Phi\left(\mathrm{H}_{2}\right)=0-1,5 \mathrm{sccm}, \mathrm{E}($ Laser $)=6 \mathrm{~mJ}$

\begin{tabular}{|c|c|}
\hline $\ln \left(\mathrm{I}_{41}(-\mathrm{H}) / \mathrm{I}_{41}(+\mathrm{H})\right)$ & $\ln \left(\mathrm{I}_{45}(-\mathrm{H}) / \mathrm{I}_{45}(+\mathrm{H})\right)$ \\
\hline 0,04924 & 0,00911 \\
\hline 0,29203 & 0,31138 \\
\hline 0,49706 & 0,63573 \\
\hline 0,9947 & 1,2251 \\
\hline 1,0092 & 1,1572 \\
\hline 0,52153 & 0,57718 \\
\hline 1,3631 & 1,5027 \\
\hline 1,0991 & 1,1408 \\
\hline 0,41117 & 0,59601 \\
\hline 0,54967 & 0,6674 \\
\hline 0,61646 & 0,72454 \\
\hline 0,72719 & 0,86869 \\
\hline 0,55128 & 0,65645 \\
\hline 0,15146 & 0,23398 \\
\hline 0,21969 & 0,21446 \\
\hline 0,34272 & 0,48778 \\
\hline
\end{tabular}


Messtabelle 41: c-Heptyl $+\mathbf{H}$ relativ zu Methoxymethyl + H (zu Abbildung 175)

Versuchsanordnung A, Reaktor $2 \mathrm{a}, \lambda=440 \mathrm{~nm}, \mathrm{~s}_{1}=2 \mathrm{~cm}, \mathrm{~s}_{2}=3 \mathrm{~cm}, \mathrm{~T}=25{ }^{\circ} \mathrm{C}$, Badgas: He, $\mathrm{p}=2,31 \mathrm{mbar}, \Phi(\mathrm{He})=895 \mathrm{sccm}, \Phi(\mathrm{c}-$ Heptan $)=3,63 \mathrm{sccm}, \Phi(\mathrm{DME})=7,80 \mathrm{sccm}$, $\Phi\left(\mathrm{F}_{2}\right)=1,81 \mathrm{sccm}, \Phi\left(\mathrm{H}_{2}\right)=0-1,5 \mathrm{sccm}, \mathrm{E}($ Laser $)=9,5 \mathrm{~mJ}, 800$ sweeps

\begin{tabular}{|c|c|}
\hline $\ln \left(\mathrm{I}_{45}(-\mathrm{H}) / \mathrm{I}_{45}(+\mathrm{H})\right)$ & $\ln \left(\mathrm{I}_{97}(-\mathrm{H}) / \mathrm{I}_{97}(+\mathrm{H})\right)$ \\
\hline 0,39207 & 0,53335 \\
\hline 0,69128 & 0,69599 \\
\hline 0,53155 & 0,65574 \\
\hline 0,68687 & 0,78833 \\
\hline 0,7646 & 0,74989 \\
\hline 0,59085 & 0,93532 \\
\hline 0,64778 & 0,78156 \\
\hline 0,77026 & 1,0252 \\
\hline 0,19735 & 0,35289 \\
\hline 0,09604 & 0,22103 \\
\hline 0,12704 & 0,2598 \\
\hline 0,20288 & 0,21672 \\
\hline 0,37009 & 0,29639 \\
\hline 0,33862 & 0,25113 \\
\hline 0,42378 & 0,4553 \\
\hline 1,0244 & 1,17 \\
\hline 0,86707 & 1,0844 \\
\hline
\end{tabular}

Messtabelle 42: c-Oktyl + H relativ zu Methoxymethyl + H (zu Abbildung 176)

Versuchsanordnung A, Reaktor $2 \mathrm{a}, \lambda=456 \mathrm{~nm}, \mathrm{~s}_{1}=2 \mathrm{~cm}, \mathrm{~s}_{2}=3 \mathrm{~cm}$, Badgas: He, $\mathrm{p}=2,06$ mbar, $\Phi(\mathrm{He})=1016 \mathrm{sccm}, \Phi(\mathrm{c}-$ Oktan $)=2,92 \mathrm{sccm}, \Phi(\mathrm{DME})=3,59 \mathrm{sccm}$, $\Phi\left(\mathrm{F}_{2}\right)=1,56 \mathrm{sccm}, \Phi\left(\mathrm{H}_{2}\right)=0-1,5 \mathrm{sccm}, \mathrm{E}($ Laser $)=10 \mathrm{~mJ}, 800$ sweeps, $\mathrm{T}=25^{\circ} \mathrm{C}$

\begin{tabular}{|c|c|}
\hline $\ln \left(\mathrm{I}_{45}(-\mathrm{H}) / \mathrm{I}_{45}(+\mathrm{H})\right)$ & $\ln \left(\mathrm{I}_{111}(-\mathrm{H}) / \mathrm{I}_{111}(+\mathrm{H})\right)$ \\
\hline \hline 0,6151 & 0,83337 \\
\hline 0,45537 & 0,47425 \\
\hline 1,2202 & 1,4591 \\
\hline 1,1543 & 1,2876 \\
\hline 0,44532 & 0,38884 \\
\hline 0,28675 & 0,28421 \\
\hline 0,33383 & 0,48927 \\
\hline 0,42222 & 0,44395 \\
\hline 0,66923 & 0,76869 \\
\hline 0,59233 & 0,7667 \\
\hline 0,2205 & 0,31807 \\
\hline 0,28445 & 0,37804 \\
\hline
\end{tabular}


Messtabelle 43: Iso- $\mathrm{C}_{4} \mathrm{H}_{8} \mathrm{Cl}+\mathrm{H}$ relativ zu Methoxymethyl $+\mathrm{H}$ (zu Abbildung 177)

Versuchsanordnung A, Reaktor 2a, $\lambda=440 \mathrm{~nm}, \mathrm{~s}_{1}=2 \mathrm{~cm}, \mathrm{~s}_{2}=3 \mathrm{~cm}, \mathrm{~T}=25^{\circ} \mathrm{C}$, Badgas: He, $\mathrm{p}=2,03 \mathrm{mbar}, \Phi(\mathrm{He})=662 \mathrm{sccm}, \Phi($ Isobutylchlorid $)=14,58 \mathrm{sccm}, \Phi(\mathrm{DME})=7,80 \mathrm{sccm}$, $\Phi\left(\mathrm{F}_{2}\right)=1,81 \mathrm{sccm}, \Phi\left(\mathrm{H}_{2}\right)=0-1,5 \mathrm{sccm}, \mathrm{E}($ Laser $)=10 \mathrm{~mJ}, 800$ sweeps

\begin{tabular}{|c|c|}
\hline $\ln \left(\mathrm{I}_{45}(-\mathrm{H}) / \mathrm{I}_{45}(+\mathrm{H})\right)$ & $\ln \left(\mathrm{I}_{91}(-\mathrm{H}) / \mathrm{I}_{91}(+\mathrm{H})\right)$ \\
\hline 0,63459 & 0,92261 \\
\hline 1,1387 & 1,7046 \\
\hline 0,66736 & 1,0288 \\
\hline 0,56982 & 0,57273 \\
\hline 0,7241 & 1,0722 \\
\hline 0,42176 & 0,67984 \\
\hline 0,18768 & 0,23707 \\
\hline 0,22679 & 0,34658 \\
\hline 0,35442 & 0,49406 \\
\hline
\end{tabular}




\section{Anhang}

\subsection{Radikale mit REMPI-Signal: eine Übersicht}

Die hier erstellte Auflistung ist für den Kinetiker als Hilfestellung gedacht beim schnellen Auffinden von Referenzradikalen zur Produktuntersuchung und zur Bestimmung von Geschwindigkeitskoeffizienten. Sie erhebt keinen Anspruch auf Vollständigkeit. Es wurde bei der Auflistung ein Schwerpunkt auf die bei Reaktionen von Kohlenwasserstoffradikalen in der Gasphase relevanten Teilchen gelegt. Weitere, vor allem diatomare Radikale, die nicht Kohlenstoff enthalten, finden sich bei [HUD87] und [ASH93]. Auf Details zu spektroskopischen Prozessen wurde im Sinne des geäußerten Anwendungsbereichs verzichtet. Auch wurde zum Zweck der Übersichtlichkeit auf eine eingehende Literaturangabe verzichtet. Als Quelle diente in erster Linie [HUD87], [ASH93] sowie an der Universität Göttingen erstellte Dissertationen. Informationen zur Spektroskopie und genauere Angaben zu den Referenzen, die hier nur mit dem Autorennamen und dem Erscheinungsjahr vielfach sekundär zitiert wurden, finden sich dort.

\begin{tabular}{|c|c|c|c|c|c|}
\hline Radikal & $\mathrm{m} / \mathrm{z}$ & $\lambda / \mathrm{nm}$ & Mech. & Erzeugung & Quelle \\
\hline $\mathrm{CH}$ & 13 & $\begin{array}{l}311,9 \\
310,8\end{array}$ & $2+1$ & $\begin{array}{l}\text { Flamme } \\
\text { Keten + F }\end{array}$ & $\begin{array}{c}\text { Chen, Chupka, Colson (1985) } \\
\text { Tjossem, } \\
\text { Smyth (1987) }\end{array}$ \\
\hline $\mathrm{CH}_{2}$ & 14 & $\begin{array}{l}269,3 \\
311,9 \\
391,9 \\
419,0 \\
423,0 \\
424,7\end{array}$ & $\begin{array}{l}2+x \\
3+x\end{array}$ & & $\begin{array}{l}\text { Johnson, Hudgens (1992) } \\
\text { Irikura, Hudgens (1992) }\end{array}$ \\
\hline $\begin{array}{l}\mathbf{C H}_{3} \\
\text { Methyl }\end{array}$ & 15 & $\begin{array}{c}214,4 \\
264,7 \\
267,0 \\
271,6 \\
276,1 \\
286,3 \\
333,4 \\
335-349 \\
450-451\end{array}$ & $\begin{array}{l}1+1 \\
2+1\end{array}$ & $\begin{array}{l}\text { Photolyse } \\
\text { Pyrolyse }\end{array}$ & $\begin{array}{l}\text { Danon et al (1982) } \\
\text { DiGuiseppe, Hudgens, Lin } \\
\text { (1981) } \\
\text { Smyth, Taylor (1985), Wehmeyer } \\
\text { (2002) } \\
\text { Hudgens et al (1982) } \\
\text { Heinemann, Hoyermann (1988) }\end{array}$ \\
\hline $\mathrm{C}_{2} \mathrm{H}$ & 29 & 277 & $2+1$ & & Cool, Goodwin (1991) \\
\hline $\begin{array}{l}\mathrm{C}_{2} \mathbf{H}_{5} \\
\text { Ethyl }\end{array}$ & 29 & $\begin{array}{c}337-349 \\
398-410 \\
420-442.8 \\
442,8-530\end{array}$ & $\begin{array}{l}2+1 \\
3+1 \\
2+2\end{array}$ & $\begin{array}{l}\mathrm{C}_{2} \mathrm{H}_{6}+\mathrm{F} \\
\text { Photolyse } \\
\mathrm{C}_{2} \mathrm{H}_{6}+\mathrm{F}\end{array}$ & $\begin{array}{c}\text { Wehmeyer (2002) } \\
\text { Sappey, Weishaar (1987), } \\
\text { Edelbüttel-Einhaus (1988), } \\
\text { Rohde (1991), Nacke (1998) }\end{array}$ \\
\hline $\begin{array}{c}\mathbf{C}_{3} \mathbf{H}_{7} \\
\text { 1-Propyl, } \\
\text { 2-Propyl }\end{array}$ & 43 & $\begin{array}{l}397-465 \\
397-500\end{array}$ & & $\begin{array}{c}\text { Propan + F } \\
\mathrm{CH}_{3} \mathrm{CD}_{2} \mathrm{CH}_{3}+\mathrm{F}\end{array}$ & $\begin{array}{l}\text { Edelbüttel-Einhaus (1988) } \\
\text { Wehmeyer (2002) }\end{array}$ \\
\hline $\mathrm{c}-\mathrm{C}_{3} \mathrm{H}_{5}$ & 41 & 498,8 & & $\begin{array}{c}{\mathrm{c}-\mathrm{C}_{3} \mathrm{H}_{6}} \\
(\mathrm{c}-\mathrm{Propan}) \\
+\mathrm{F}\end{array}$ & $\begin{array}{l}\text { Heinemann } \\
\text { (1986) }\end{array}$ \\
\hline $\begin{array}{l}\mathrm{C}_{3} \mathrm{H}_{5} \\
\text { Allyl }\end{array}$ & 41 & $\begin{array}{c}410 \\
492-495\end{array}$ & $\begin{array}{l}2+1 \\
2+2\end{array}$ & $\begin{array}{c}\text { Photolyse } \\
\mathrm{C}_{3} \mathrm{H}_{6} \text { (Propen) }\end{array}$ & $\begin{array}{l}\text { Sappey, Weisshaar (1987), } \\
\text { Hudgens, Dulcey (1985), }\end{array}$ \\
\hline
\end{tabular}




\begin{tabular}{|c|c|c|c|c|c|}
\hline & & $\begin{array}{c}497-506 \\
\max 499,2\end{array}$ & & $+\mathrm{F}, \mathrm{Cl}$ & $\begin{array}{l}\text { Hoyermann, Heinemann (1988), } \\
\text { Rohde (1991) }\end{array}$ \\
\hline $\begin{array}{c}\mathbf{C}_{4} \mathbf{H}_{7} \\
\text { 2-Methylallyl }\end{array}$ & 53 & 521,1 & $2+2$ & Isobuten $+\mathrm{F}$ & Dulcey, Hudgens (1985) \\
\hline $\begin{array}{c}\mathbf{C}_{4} \mathbf{H}_{7} \\
\text { 2-Buten-1-yl }\end{array}$ & 53 & $470-473$ & $2+x$ & & Hudgens et al (1988) \\
\hline $\begin{array}{c}\mathbf{C}_{4} \mathbf{H}_{9} \\
\text { 1-Butyl, } \\
\text { 2-Butyl }\end{array}$ & 57 & $\begin{array}{l}397-465 \\
397-500\end{array}$ & $2+x$ & n-Butan + F & $\begin{array}{l}\text { Edelbüttel-Einhaus (1988) } \\
\text { Wehmeyer (2002) }\end{array}$ \\
\hline $\begin{array}{c}\mathbf{C}_{4} \mathbf{H}_{9} \\
\text { Isobutyl } \\
\text { t-Butyl }\end{array}$ & 57 & $\begin{array}{l}360-470 \\
360-540\end{array}$ & $2+1$ & Isobutan $+\mathrm{F}$ & $\begin{array}{l}\text { Edelbüttel-Einhaus (1988) } \\
\text { Wehmeyer (2002) }\end{array}$ \\
\hline $\begin{array}{c}\mathbf{C}_{5} \mathbf{H}_{6} \\
\text { c-Pentadienyl }\end{array}$ & 65 & $426-428$ & & c-Pentadien + F & Wehmeyer (2002) \\
\hline $\begin{array}{l}\text { neo- } \mathbf{C}_{5} \mathbf{H}_{11} \\
\text { neo-Pentyl }\end{array}$ & 73 & $400-530$ & $2+1$ & neo-Pentan + F & $\begin{array}{l}\text { Edelbüttel-Einhaus } \\
\text { (1988) }\end{array}$ \\
\hline $\begin{array}{l}\text { c- } \mathrm{C}_{5} \mathrm{H}_{9} \\
\text { c-Pentyl }\end{array}$ & 69 & $\begin{array}{c}420-507 \\
\max 437,5\end{array}$ & $\begin{array}{c}3 \\
\text { Photonen }\end{array}$ & $\mathrm{c}-\mathrm{C}_{5} \mathrm{H}_{10}+\mathrm{Cl}, \mathrm{F}$ & $\begin{array}{l}\text { Edelbüttel-Einhaus (1988) } \\
\text { Rohde (1991), Nacke (1998) }\end{array}$ \\
\hline $\begin{array}{l}\text { c- } \mathbf{C}_{6} \mathbf{H}_{11} \\
\text { c-Hexyl }\end{array}$ & 83 & $420-510$ & $\begin{array}{c}3 \\
\text { Photonen }\end{array}$ & $\mathrm{c}-\mathrm{C}_{6} \mathrm{H}_{12}+\mathrm{F}, \mathrm{Cl}$ & $\begin{array}{c}\text { Hudgens et al. (1987) } \\
\text { Nacke (1998) }\end{array}$ \\
\hline $\begin{array}{l}\text { c- } \mathbf{C}_{7} \mathbf{H}_{7} \\
\text { Tropyl }\end{array}$ & 83 & $417-588$ & $2+x$ & & Johnson (1991) \\
\hline $\begin{array}{l}\text { c- } \mathbf{C}_{7} \mathbf{H}_{13} \\
\text { c-Heptyl }\end{array}$ & 97 & $420-460$ & & $\mathrm{c}-\mathrm{C}_{7} \mathrm{H}_{14}+\mathrm{F}$ & Wehmeyer (2002) \\
\hline $\begin{array}{l}\text { c- } \mathbf{C}_{8} \mathbf{H}_{15} \\
\text { c-Oktyl }\end{array}$ & 111 & $440-530$ & & $\mathrm{c}-\mathrm{C}_{8} \mathrm{H}_{16}+\mathrm{F}$ & Wehmeyer (2002) \\
\hline $\begin{array}{c}\mathrm{C}_{6} \mathbf{H}_{5} \mathrm{CH}_{2} \\
\text { Benzyl }\end{array}$ & 91 & $\begin{array}{l}454,6 \\
502.5\end{array}$ & $\begin{array}{l}1+x \\
2+2\end{array}$ & $\begin{array}{c}\mathrm{C}_{6} \mathrm{H}_{5} \mathrm{CH}_{2} \text { (Toluol) } \\
+\mathrm{F}, \underline{\mathrm{Cl}}\end{array}$ & $\begin{array}{c}\text { Im, Bernstein (1991) } \\
\text { Hoffbauer, Hudgens (1987) }\end{array}$ \\
\hline $\mathrm{CO}$ & 28 & 454,3 & $\begin{array}{l}3+2 \\
3+3\end{array}$ & Flamme & Tjossem, Cool (1984) \\
\hline $\mathrm{C}_{2} \mathrm{O}$ & 40 & $443-453$ & $3+1$ & Flamme & Tjossem, Cool (1984) \\
\hline $\begin{array}{l}\text { HCO } \\
\text { Formyl }\end{array}$ & 29 & $\begin{array}{c}373-427 \\
\max 391,7\end{array}$ & $2+1$ & $\begin{array}{l}\text { Photolyse von } \\
\text { Ethanal }\end{array}$ & $\begin{array}{l}\text { Tjossem et al (1986), } \\
\text { Bernstein et al (1988) }\end{array}$ \\
\hline $\begin{array}{l}\mathbf{C H}_{3} \mathbf{O} \\
\text { Methoxy }\end{array}$ & 31 & 320,6 & & $\mathrm{CH}_{3} \mathrm{OH}+\mathrm{F}$ & Long, Johnson, Hudgens (1986) \\
\hline $\begin{array}{c}\mathbf{C H}_{2} \mathbf{O H} \\
\text { Hydroxymethyl }\end{array}$ & 31 & 486.9 & $2+1$ & $\mathrm{CH}_{3} \mathrm{OH}+\mathrm{F}$ & $\begin{array}{l}\text { Dulcey, Hudgens (1983), } \\
\text { Heinemann (1986) }\end{array}$ \\
\hline $\mathrm{CH}_{3} \mathrm{CO}$ & 43 & $397-415$ & & $\mathrm{CH}_{3} \mathrm{CHO}+\mathrm{F}, \mathrm{Cl}$ & Edelbüttel-Einhaus (1988) \\
\hline $\mathrm{C}_{2} \mathrm{H}_{5} \mathrm{O}$ & 15 & $\begin{array}{c}333-340 \\
\max 335,5\end{array}$ & $1+2$ & $\mathrm{C}_{2} \mathrm{H}_{5} \mathrm{OH}+\mathrm{F}$ & Edelbüttel-Einhaus (1988) \\
\hline $\mathrm{C}_{2} \mathrm{H}_{4} \mathrm{OH}$ & 45 & $422-463$ & & $\mathrm{C}_{2} \mathrm{H}_{5} \mathrm{OH}+\mathrm{Cl}$ & Edelbüttel-Einhaus (1988) \\
\hline $\begin{array}{c}\mathbf{C H}_{3} \mathbf{O C H}_{2} \\
\text { Methoxymethyl }\end{array}$ & 45 & $420-530$ & $2+1$ & $\mathrm{CH}_{3} \mathrm{OCH}_{2}+\mathrm{F}$ & Nacke (1998) \\
\hline $\begin{array}{l}\mathrm{CH}_{3} \mathrm{OCHOCH} \\
\mathrm{CH}_{2} \mathrm{OCH}_{2} \mathrm{OCH}_{3} \\
\text { Dimethoxymethyl }\end{array}$ & $\begin{array}{c}(75) \\
45\end{array}$ & $460-540$ & & $\begin{array}{c}\mathrm{CH}_{3} \mathrm{OCHOCH}_{3} \\
+\mathrm{F}\end{array}$ & Wehmeyer (2002) \\
\hline $\begin{array}{c}\mathbf{C}_{4} \mathbf{H}_{7} \mathbf{O}_{2} \\
\text { (Dioxanyl) }\end{array}$ & 87 & $420-510$ & $2+1$ & $\begin{array}{c}\mathrm{C}_{4} \mathrm{H}_{7} \mathrm{O}_{2} \\
\text { (Dioxan) }+\mathrm{F}\end{array}$ & Nacke (1998) \\
\hline $\begin{array}{l}\mathbf{t}-\mathrm{C}_{4} \mathrm{H}_{9} \mathrm{OCH}_{2} \\
\text { t-Butoxymethyl }\end{array}$ & $\begin{array}{c}57 \\
(87) \\
\end{array}$ & $420-530$ & & $\begin{array}{l}\mathrm{t}-\mathrm{C}_{4} \mathrm{H}_{9} \mathrm{OCH} \mathrm{H}_{3} \\
(\mathrm{MTBE})+\mathrm{F}\end{array}$ & Nacke (1998) \\
\hline $\begin{array}{c}\mathbf{C}_{4} \mathbf{H}_{9} \mathbf{O C}_{4} \mathbf{H}_{8} \\
\text { t-Butoxy-t-butyl }\end{array}$ & $\begin{array}{c}57,73 \\
(130) \\
\end{array}$ & 440 & & $\begin{array}{l}\mathrm{C}_{4} \mathrm{H}_{9} \mathrm{OC}_{4} \mathrm{H}_{9} \\
(\mathrm{DTBE})+\mathrm{F}\end{array}$ & Nacke (1998) \\
\hline CF & 31 & $\begin{array}{l}342,5 \\
356,7 \\
368,2 \\
380,7\end{array}$ & $2+1$ & $\begin{array}{l}\text { Keten + F } \\
\mathrm{CH}_{3} \mathrm{~F}+\mathrm{F}\end{array}$ & Johnson, Hudgens (1987) \\
\hline $\begin{array}{c}\mathbf{C F}_{3} \\
\text { Trifluormethyl }\end{array}$ & 69,31 & $\begin{array}{l}415-490 \\
\max 455\end{array}$ & $3+1$ & $\begin{array}{c}\text { Pyrolyse von } \mathrm{CF}_{3} \mathrm{I} \\
\mathrm{CF}_{3} \mathrm{Br} \text { durch } \\
\text { Mikrowelle } \\
\end{array}$ & $\begin{array}{c}\text { Duignan, Hudgens, Wyatt (1982), } \\
\text { Rohde (1991) }\end{array}$ \\
\hline $\begin{array}{c}\mathbf{C H}_{2} \mathbf{F} \\
\text { Fluoromethyl } \\
\end{array}$ & 33 & $\begin{array}{c}292-393 \\
378,0 \\
\end{array}$ & $2+1$ & $\begin{array}{l}\text { Keten + F } \\
\mathrm{CH}_{3} \mathrm{~F}+\mathrm{F} \\
\end{array}$ & $\begin{array}{c}\text { Hudgens et al (1987), } \\
\text { Beiderhase (1995) }\end{array}$ \\
\hline
\end{tabular}




\begin{tabular}{|c|c|c|c|c|c|}
\hline & & $\begin{array}{l}367,5 \\
371,0\end{array}$ & & & \\
\hline $\begin{array}{c}\mathbf{C H F}_{2} \\
\text { Difluoromethyl }\end{array}$ & 51 & $\begin{array}{c}367-396 \\
\max 378 \\
430-460 \\
\max 441 \\
330-430\end{array}$ & $\begin{array}{l}2+1 \\
3+1\end{array}$ & $\mathrm{CH}_{2} \mathrm{~F}_{2}+\mathrm{F}$ & $\begin{array}{l}\text { Hudgens et al (1987), } \\
\text { Beiderhase (1995) }\end{array}$ \\
\hline $\begin{array}{c}\mathbf{C}_{5} \mathbf{H}_{6} \mathbf{F} \\
\text { Fluorocyclo- } \\
\text { penten }\end{array}$ & 85 & $435-460$ & & Cyclopentadien + F & Wehmeyer (2002) \\
\hline $\mathrm{CHFCl}$ & 67,69 & $362-406$ & $2+x$ & & Hudgens et al (1993) \\
\hline $\mathrm{CF}_{2} \mathrm{Cl}$ & 85,87 & 406,3 & $2+x$ & & Hudgens et al (1989) \\
\hline $\mathrm{CFCl}_{2}$ & 101,103 & 401,2 & $2+x$ & & Hudgens et al (1989) \\
\hline $\mathrm{CCl}$ & 47,49 & $\begin{array}{c}271,4 \\
318-399\end{array}$ & $\begin{array}{l}1+1 \\
2+x\end{array}$ & $\begin{array}{l}\text { elektrische } \\
\text { Entladung }\end{array}$ & $\begin{array}{l}\text { Sharpe, Johnson (1984) } \\
\text { (1989) }\end{array}$ \\
\hline $\mathrm{CH}_{2} \mathrm{Cl}$ & 49,52 & 376,0 & & $\mathrm{CH}_{3} \mathrm{Cl}+\mathrm{F}$ & $\begin{array}{c}\text { Hudgens, Johnson (1993) } \\
\text { Hold (2001) }\end{array}$ \\
\hline $\mathrm{CHCl}_{2}$ & 83,85 & $\begin{array}{l}360,4 \\
364,4 \\
370,0 \\
388,1 \\
394,7\end{array}$ & $2+1$ & $\mathrm{CH}_{2} \mathrm{Cl}_{2}+\mathrm{F}$ & $\begin{array}{l}\text { Long, Hudgens (1987) } \\
\text { Hold (2001) }\end{array}$ \\
\hline $\mathrm{CCl}_{3}$ & $\begin{array}{c}117,119, \\
121\end{array}$ & $346-424$ & $2+x$ & & Hudgens et al (1990) \\
\hline $\begin{array}{c}\mathbf{C}_{4} \mathbf{H}_{8} \mathbf{C l} \\
\text { 2-Chlor-2-Methyl- } \\
\text { Propyl-Radikal }\end{array}$ & 92,94 & $420-460$ & & $\mathrm{t}-\mathrm{C}_{4} \mathrm{H}_{9} \mathrm{Cl}+\mathrm{F}$ & Wehmeyer (2002) \\
\hline $\begin{array}{c}\mathrm{CF}_{3} \mathbf{C H O H} \\
\mathbf{C F}_{3} \mathbf{C H}_{2} \mathbf{O} \\
\text { (Radikal nicht } \\
\text { eindeutig) }\end{array}$ & 99 & $\begin{array}{c}381,1 \\
\max 388,6 \\
391,2\end{array}$ & & $\mathrm{CF}_{3} \mathrm{CH}_{2} \mathrm{OH}+\mathrm{F}$ & Hold (2001) \\
\hline $\mathrm{C}_{4} \mathrm{~F}_{9} \mathrm{OCH}_{2}$ & 249 & 387,8 & & $\mathrm{C}_{4} \mathrm{~F}_{9} \mathrm{OCH}_{2}+\mathrm{F}$ & Hold (2001) \\
\hline $\begin{array}{c}\mathbf{C}_{4} \mathrm{~F}_{9} \mathbf{O C}_{2} \mathbf{H}_{4} \\
\text { (Radikal nicht } \\
\text { eindeutig) }\end{array}$ & 263 & $\begin{array}{l}386,3 \\
391,4 \\
469,3\end{array}$ & & $\mathrm{C}_{4} \mathrm{~F}_{9} \mathrm{OC}_{2} \mathrm{H}_{5}+\mathrm{F}$ & Hold (2001) \\
\hline $\begin{array}{c}\text { Cyanocyclopenta } \\
\text { dienyl }\end{array}$ & & $357-370$ & $1+x$ & & Im, Bernstein (1991) \\
\hline $\mathrm{ClO}$ & 51,53 & $\begin{array}{c}428-466 \\
\max 437,8\end{array}$ & $3+1$ & $\mathrm{Cl}+\mathrm{O}_{3}$ & Hudgens, Duignan (1985) \\
\hline $\mathrm{BrO}$ & 95,97 & $\begin{array}{c}426-464 \\
\max 444,5\end{array}$ & $3+1$ & $\mathrm{Br}+\mathrm{O}_{3}$ & Hudgens, Duignan (1985) \\
\hline $\mathrm{BrF}$ & 98,100 & 461,5 & $2+x$ & & Hudgens et al (1993) \\
\hline $\mathbf{O H}$ & 17 & $\begin{array}{l}228,2 \\
244,5\end{array}$ & $2+x$ & & Beer et al. (1991) \\
\hline $\mathbf{N H}$ & 15 & 395,6 & $3+1$ & $\begin{array}{l}\text { Photodissoziation } \\
\text { von } \mathrm{NH}_{3} \\
\end{array}$ & Nieman, Colson (1978) \\
\hline $\mathbf{N H}_{2}$ & 16 & 416,4 & $1+3$ & $\begin{array}{l}\text { Photodissoziation } \\
\text { von } \mathrm{NH}_{3}\end{array}$ & Nieman, Colson (1978) \\
\hline NO & 30 & $\begin{array}{l}380-383 \\
429-431 \\
452-454\end{array}$ & $2+1$ & Untergrund & $\begin{array}{c}\text { Cool (1984) } \\
\text { Edelbüttel-Einhaus(1988) } \\
\text { Wehmeyer (2002) }\end{array}$ \\
\hline $\mathrm{NO}_{2}$ & 46 & $\begin{array}{l}511 \\
523 \\
533 \\
540\end{array}$ & & $\begin{array}{c}\text { molekular; } \\
\mathrm{CO}_{2} \text {-Laser induced } \\
\text { MP-Diss. von } \\
\mathrm{CH}_{3} \mathrm{NO}_{2}\end{array}$ & Rockney, Grant (1981) \\
\hline $\mathrm{SiF}$ & 47 & 437,5 & $1+1+1$ & $\mathrm{SiH}_{4}+\mathrm{F}$ & Dulcey, Hudgens (1985) \\
\hline $\mathrm{SiF}_{2}$ & 66 & $\begin{array}{l}321,5 \\
341,9 \\
367,4 \\
483,8 \\
\end{array}$ & $3+x$ & $\begin{array}{l}\mathrm{Si}+\mathrm{F}_{2} \\
+\mathrm{NF}_{3} \\
(800 \mathrm{~K})\end{array}$ & $\begin{array}{c}\text { Lin et al (1987) } \\
\text { Johnson, Hudgens et al (1990) }\end{array}$ \\
\hline $\mathbf{H}$ & 1 & 266 & $2+1$ & Flamme & Goldsmith (1982) \\
\hline
\end{tabular}




\begin{tabular}{|c|c|c|c|c|c|}
\hline & & 224 & $3+1$ & & Tjossem, Cool (1983) \\
\hline $\mathbf{O}$ & 16 & 226 & & Flamme & Goldsmith (1984) \\
\hline $\mathbf{O}_{2}$ & 32 & $284-288$ & $2+1$ & & Tjossem, Smyth (1987) \\
& & 312 & & & \\
& & 321 & & & \\
\hline PH & 32 & 388,9 & $2+1$ & Photolyse et al (1987) \\
\hline PO & 47 & 320,4 & $1+1$ & Flamme $\mathrm{PH}_{3}$ & Ashfold et al (1985) \\
\hline
\end{tabular}

\subsection{Verzeichnis der verwendeten Standardbildungsenthalpien}

\begin{tabular}{|c|c|c|}
\hline Verbindung & $\mathrm{H}_{\mathrm{f}, 298 \mathrm{~K}}^{0_{1}} /(\mathrm{kJ} / \mathrm{mol})$ & Referenz \\
\hline $\mathrm{CH}_{3}$ (Methyl) & 145,6 & [STE94] \\
\hline $\mathrm{CH}_{4}$ (Methan) & $-74,5$ & [STE94] \\
\hline $\mathrm{C}_{2} \mathrm{H}_{4}$ (Ethen) & 52,3 & [STE94] \\
\hline $\mathrm{C}_{2} \mathrm{H}_{5}$ (Ethyl) & 117,2 & [STE94] \\
\hline $\mathrm{C}_{2} \mathrm{H}_{6}$ (Ethan) & $-84,1$ & [STE94] \\
\hline $\mathrm{C}_{3} \mathrm{H}_{5}$ (Allyl) & 163,2 & [STE94] \\
\hline $\mathrm{C}_{3} \mathrm{H}_{6}$ (Propen) & 20,1 & [STE94] \\
\hline $\mathrm{n}-\mathrm{C}_{3} \mathrm{H}_{7}$ (1-Propyl) & 100,4 & [STE94] \\
\hline iso- $_{3} \mathrm{H}_{7}$ (2-Propyl) & 93,3 & [STE94] \\
\hline $\mathrm{C}_{3} \mathrm{H}_{8}$ (Propan) & $-104,6$ & [STE94] \\
\hline $1-\mathrm{C}_{4} \mathrm{H}_{8}$ (1-Buten) & $-0,4$ & [STE94] \\
\hline 2- $\mathrm{C}_{4} \mathrm{H}_{8}$ (2-Buten) & $-7,9$ & [STE94] \\
\hline Iso- $\mathrm{C}_{4} \mathrm{H}_{8}$ (Isobuten) & $-16,7$ & [STE94] \\
\hline n- $\mathrm{C}_{4} \mathrm{H}_{9}$ (1-Butyl) & 75,3 & [STE94] \\
\hline $2-\mathrm{C}_{4} \mathrm{H}_{9}(2-\mathrm{Butyl})$ & 71,1 & [STE94] \\
\hline $\mathrm{t}-\mathrm{C}_{4} \mathrm{H}_{9}$ (t-Butyl) & 46,0 & [STE94] \\
\hline 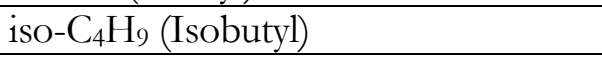 & 66,9 & [STE94] \\
\hline n- $\mathrm{C}_{4} \mathrm{H}_{10}$ (n-Butan) & $-126,4$ & [STE94] \\
\hline iso- $\mathrm{C}_{4} \mathrm{H}_{10}$ (Isobutan) & $-134,3$ & [STE94] \\
\hline $\mathrm{C}_{5} \mathrm{H}_{6}$ (Cyclopentadien) & 129,7 & [STE94] \\
\hline $\mathrm{C}_{5} \mathrm{H}_{5}$ (Cyclopentadienyl) & 242 & [STE94] \\
\hline c- $\mathrm{C}_{5} \mathrm{H}_{8}$ (Cyclopenten) & 36,0 & [STE94] \\
\hline c- $\mathrm{C}_{5} \mathrm{H}_{9}$ (Cyclopentyl) & 101,7 & [STE94] \\
\hline c- $\mathrm{C}_{7} \mathrm{H}_{12}$ (Cyclohepten) & $-9,2$ & [STE94] \\
\hline c-C ${ }_{7} \mathrm{H}_{13}$ (Cycloheptyl) & 74,4 & [STE94], est. \\
\hline c- $\mathrm{C}_{7} \mathrm{H}_{14}$ (Cycloheptan) & $-118,4$ & [STE94] \\
\hline 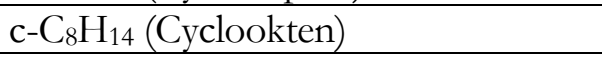 & $-27,2$ & [STE94] \\
\hline c- $\mathrm{C}_{8} \mathrm{H}_{15}$ (Cyclooktyl) & 68,5 & [STE94], est. \\
\hline c- $\mathrm{C}_{8} \mathrm{H}_{16}($ Cyclooktan $)$ & $-124,3$ & [STE94] \\
\hline CO (Kohlenmonoxid) & $-110,5$ & [STE94] \\
\hline HCHO (Formaldehyd) & $-108,8$ & [STE94] \\
\hline $\mathrm{CH}_{3} \mathrm{CHO}$ (Acetaldehyd) & $-165,7$ & [STE94] \\
\hline $\mathrm{CH}_{3} \mathrm{OCH}_{2}$ (Methoxymethyl) & $-11,7$ & [STE94] \\
\hline $\mathrm{CH}_{3} \mathrm{COCH}_{3}$ (Aceton) & $-217,1$ & [STE94] \\
\hline $\mathrm{CH}_{3} \mathrm{CH}_{2} \mathrm{CHO}$ (Propanal) & $-187,4$ & [STE94] \\
\hline$\left(\mathrm{CH}_{3}\right)_{2} \mathrm{CHCHO}$ (2-Methyl-Propanal) & $-215,5$ & [STE94] \\
\hline $\mathrm{CH}_{3} \mathrm{CH}_{2} \mathrm{CH}_{2} \mathrm{CHO}$ (Butanal) & $-207,5$ & [STE94] \\
\hline $\mathrm{CH}_{3} \mathrm{COCH}_{2} \mathrm{CH}_{3}$ (Butanon) & $-240,6$ & [STE94] \\
\hline
\end{tabular}




\begin{tabular}{|c|c|c|}
\hline $\mathrm{C}_{4} \mathrm{H}_{8} \mathrm{O}$ (2,2-Dimethyloxiran) & $-133,9$ & [STE94] \\
\hline $\mathrm{t}-\mathrm{C}_{4} \mathrm{H}_{9} \mathrm{O}$ (t-Butoxy) & $-90,4$ & [STE94] \\
\hline $\mathrm{t}-\mathrm{C}_{4} \mathrm{H}_{9} \mathrm{OH}$ (t-Butylalkohol) & $-312,5$ & [STE94], est. \\
\hline c- $\mathrm{C}_{5} \mathrm{H}_{8} \mathrm{O}$ (Cyclopentanon) & $-192,5$ & [STE94] \\
\hline c- $\mathrm{C}_{7} \mathrm{H}_{12} \mathrm{O}$ (Cycloheptanon) & $-247,3$ & [STE94] \\
\hline $\mathrm{c}-\mathrm{C}_{8} \mathrm{H}_{14} \mathrm{O}$ (Cyclooktanon) & $-272,0$ & [STE94] \\
\hline $\mathrm{CH}_{3} \mathrm{OCH}_{2} \mathrm{OCH}_{2}$ (Dimethoxymethyl) & $-163,0$ & [STE94], est. \\
\hline $\mathrm{CH}_{3} \mathrm{OCH}_{2} \mathrm{OCH}_{3}$ (Dimethoxymethan) & $-348,1$ & [STE94] \\
\hline $\mathrm{t}-\mathrm{C}_{4} \mathrm{H}_{9} \mathrm{O}_{2}$ (t-Butylperoxy) & $-80,3$ & [STE94] \\
\hline $\mathrm{CCl}_{3}$ (Trichlormethyl) & 79,5 & [STE94] \\
\hline $\mathrm{CH}_{2} \mathrm{CCl}_{2}$ (1,1-Dichlorethen) & 2,1 & [STE94] \\
\hline $\mathrm{CH}_{2} \mathrm{CCl}_{3}(1,1,1$-Trichlorethyl) & 44,9 & [STE94] \\
\hline $\mathrm{CHOCCl}_{3}(2,2,2-$-Trichlorethanal $)$ & $-196,6$ & [STE94] \\
\hline $\mathrm{CH}_{3} \mathrm{CCl}_{3}$ (Trichlorethan) & $-144,8$ & [STE94] \\
\hline $\mathrm{CH}_{3} \mathrm{CCl}_{2} \mathrm{~F}$ (1,1-Dichlor-1-Fluorethan) & $-333,1$ & [MEL96], est. \\
\hline $\mathrm{CH}_{2} \mathrm{CCl}_{2} \mathrm{~F}$ (1,1-Dichlor-1-Fluorethyl) & $-108,0$ & [MEL96], est. \\
\hline $\mathrm{C}_{5} \mathrm{H}_{5} \mathrm{~F}$ (Fluorocyclopentadien) & $-51,3$ & [STE94], est. \\
\hline F (Fluoratom) & 79,5 & [STE94] \\
\hline O (Sauerstoffatom) & 249,4 & [STE94] \\
\hline HF (Fluorwasserstoff) & $-272,4$ & [STE94] \\
\hline $\mathrm{Cl}$ (Chloratom) & 121,3 & [STE94] \\
\hline $\mathrm{HCl}$ (Chlorwasserstoff) & $-92,5$ & [STE94] \\
\hline OH (Hydroxyl) & 38,9 & [STE94] \\
\hline $\mathrm{HO}_{2}$ (Hydroperoxy) & 10,5 & [STE94] \\
\hline $\mathrm{OCl}$ (Chlorsauerstoff) & 102,1 & [STE94] \\
\hline $\mathrm{H}$ (Wasserstoffatom) & 218,0 & [STE94] \\
\hline
\end{tabular}

\subsection{Verzeichnis der verwendeten Massenspektren}

Die Massenspektren sind [STE90] entnommen. Sie beziehen sich auf eine IE von $70 \mathrm{eV}$. Im allgemeinen sind die intensivsten zehn Massen angeben. Nur im für die Interpretation der Messdaten nützlichen Fall werden mehr aufgeführt.

$\mathrm{C}_{4} \mathrm{H}_{10}$ (Isobutan), $\mathrm{M}=58 \mathrm{~g} / \mathrm{mol}$

\begin{tabular}{|l|c|c|c|c|c|c|c|c|c|c|}
\hline $\mathbf{m} / \mathbf{z}$ & 43 & 42 & 41 & 27 & 39 & 29 & 58 & 57 & 44 & 40 \\
\hline $\mathbf{I} / \mathbf{\%}$ & 99,9 & 36,7 & 34,9 & 17,3 & 11,2 & 4,7 & 3,9 & 3,7 & 3,4 & 2,0 \\
\hline
\end{tabular}

$\mathrm{C}_{5} \mathrm{H}_{6}$ (Cyclopentadien), $\mathrm{M}=66 \mathrm{~g} / \mathrm{mol}$

\begin{tabular}{|c|c|c|c|c|c|c|c|c|c|c|}
\hline $\mathbf{m} / \mathbf{z}$ & 66 & 65 & 39 & 40 & 38 & 63 & 64 & 37 & 62 & 31 \\
\hline $\mathbf{I} / \mathbf{0}$ & 99,9 & 42,4 & 37,3 & 31,2 & 11,0 & 8,1 & 6,5 & 6,0 & 6,0 & 5,6 \\
\hline
\end{tabular}

$\mathrm{CH}_{2} \mathrm{O}$ (Formaldehyd), $\mathrm{M}=30 \mathrm{~g} / \mathrm{mol}$

\begin{tabular}{|c|c|c|c|c|c|c|c|c|c|c|}
\hline $\mathbf{m} / \mathbf{z}$ & 29 & 30 & 28 & 26 & 27 & 15 & 12 & 13 & 14 & 31 \\
\hline $\mathbf{I} / \mathbf{0} \mathbf{0}$ & 99,9 & 58,0 & 24,0 & 12,0 & 12,0 & 4,0 & 1,0 & 1,0 & 1,0 & 0,5 \\
\hline
\end{tabular}


$\mathrm{CH}_{3} \mathrm{CHO}$ (Acetaldehyd), $\mathrm{M}=44 \mathrm{~g} / \mathrm{mol}$

\begin{tabular}{|c|c|c|c|c|c|c|c|c|c|c|}
\hline $\mathbf{m} / \mathbf{z}$ & 29 & 44 & 43 & 42 & 26 & 28 & 27 & 41 & 25 & 45 \\
\hline $\mathbf{I} / \mathbf{\%}$ & 99,9 & 81,4 & 32,8 & 12,4 & 5,7 & 5,6 & 4,1 & 3,5 & 2,2 & 1,7 \\
\hline
\end{tabular}

$\mathrm{CH}_{3} \mathrm{OCH}_{3}$ (Dimethylether), $\mathrm{M}=46 \mathrm{~g} / \mathrm{mol}$

\begin{tabular}{|c|c|c|c|c|c|c|c|c|c|c|}
\hline $\mathbf{m} / \mathbf{z}$ & 45 & 29 & 15 & 46 & 14 & 31 & 13 & 30 & 28 & 12 \\
\hline $\mathbf{I} / \mathbf{\%}$ & 99,9 & 78,8 & 57,3 & 45,6 & 12,5 & 7,0 & 5,4 & 3,6 & 3,0 & 2,4 \\
\hline
\end{tabular}

$\mathrm{CH}_{3} \mathrm{OCH}_{2} \mathrm{OCH}_{3}$ (Dimethoxymethan), $\mathrm{M}=76 \mathrm{~g} / \mathrm{mol}$

\begin{tabular}{|c|c|c|c|c|c|c|c|c|c|c|}
\hline $\mathbf{m} / \mathbf{z}$ & 45 & 75 & 29 & 31 & 15 & 30 & 47 & 46 & 76 & 44 \\
\hline $\mathbf{I} / \mathbf{\%}$ & 99.9 & 61,1 & 59,0 & 13,3 & 6,0 & 5,7 & 4,9 & 2,0 & 2,0 & 1,8 \\
\hline
\end{tabular}

$\mathrm{t}-\mathrm{C}_{4} \mathrm{H}_{9} \mathrm{OH}$ (t-Butylalkohol), $\mathrm{M}=74 \mathrm{~g} / \mathrm{mol}$

\begin{tabular}{|c|c|c|c|c|c|c|c|c|c|c|}
\hline $\mathbf{m} / \mathbf{z}$ & 59 & 31 & 41 & 43 & 29 & 27 & 57 & 42 & 60 & 28 \\
\hline $\mathbf{I} / \mathbf{\%}$ & 99,9 & 32,5 & 21,6 & 17,9 & 12,5 & 11,0 & 10,2 & 4,2 & 3,3 & 2,8 \\
\hline
\end{tabular}

$\mathrm{CCl}_{2} \mathrm{CH}_{2}$ (1,1-Dichlorethen), $\mathrm{M}=96 \mathrm{~g} / \mathrm{mol}$

\begin{tabular}{|c|c|c|c|c|c|c|c|c|c|c|}
\hline $\mathbf{m} / \mathbf{z}$ & 61 & 96 & 98 & 63 & 26 & 60 & 25 & 62 & 100 & 35 \\
\hline $\mathbf{I} / \mathbf{~} \mathbf{0}$ & 99,9 & 61,3 & 38,4 & 32,0 & 16,2 & 14,6 & 7,2 & 6,9 & 6,1 & 6,0 \\
\hline
\end{tabular}

$\mathrm{C}_{6} \mathrm{H}_{14} \mathrm{O}_{14}$ (1,1,2,2-Tetra-Methoxy-Ethan), $\mathrm{M}=150 \mathrm{~g} / \mathrm{mol}$

\begin{tabular}{|c|c|c|c|c|c|c|c|c|c|c|}
\hline $\mathbf{~} / \mathbf{z}$ & 75 & 73 & 47 & 45 & 119 & 31 & 76 & 29 & 28 & 43 \\
\hline $\mathbf{I} / \mathbf{~}$ & 99,9 & 17,4 & 14,6 & 11,8 & 10,9 & 5,0 & 3,1 & 2,3 & 2,1 & 1,7 \\
\hline
\end{tabular}

$\mathrm{CH}_{3} \mathrm{CCl}_{3}$ (1,1,1-Trichlorethan), $\mathrm{M}=132 \mathrm{~g} / \mathrm{mol}$

\begin{tabular}{|c|c|c|c|c|c|c|c|c|c|c|}
\hline $\mathbf{m} / \mathbf{z}$ & 97 & 99 & 61 & 26 & 27 & 63 & 117 & 119 & 35 & 62 \\
\hline $\mathbf{I} / \mathbf{~} \mathbf{0}$ & 99,9 & 63,6 & 58,2 & 30,8 & 23,8 & 19,2 & 18,7 & 18,2 & 17,0 & 11,1 \\
\hline
\end{tabular}

$\mathrm{CHOCCl}_{3}$ (Trichlorethanal), $\mathrm{M}=146 \mathrm{~g} / \mathrm{mol}$

\begin{tabular}{|l|c|c|c|c|c|c|c|c|c|c|c|c|c|c|c|}
\hline $\mathbf{m} / \mathbf{z}$ & 150 & 148 & 146 & 128 & 122 & 121 & 120 & 119 & 118 & 117 & 115 & 114 & 113 & 112 & 111 \\
\hline $\mathbf{I} / \mathbf{~}$ & 1,5 & 4,4 & 4,6 & 1,3 & 4,5 & 4,3 & 14,0 & 13,6 & 14,6 & 13,9 & 3,8 & 1,8 & 22,5 & 7,6 & 35,3 \\
\hline
\end{tabular}

\begin{tabular}{|l|c|c|c|c|c|c|c|c|c|c|c|c|c|c|c|c|}
\hline $\mathbf{m} / \mathbf{z}$ & 110 & 94 & 87 & 86 & 85 & 84 & 83 & 82 & 62 & 60 & 51 & 50 & 49 & 48 & 47 & 39 \\
\hline $\mathbf{I} / \mathbf{\%}$ & 9,8 & 0,7 & 3,5 & 11,6 & 20,6 & 66,4 & 31,9 & 99,9 & 0,7 & 1,7 & 0,9 & 3,7 & 11,6 & 10,3 & 18,3 & 0,7 \\
\hline
\end{tabular}

\begin{tabular}{|l|c|c|c|c|c|c|c|c|c|c|}
\hline $\mathbf{m} / \mathbf{z}$ & 37 & 36 & 35 & 29 & 28 & 25 & 18 & 17 & 13 & 12 \\
\hline $\mathbf{I} / \mathbf{~ \%}$ & 1,9 & 1,8 & 5,5 & 31,0 & 3,1 & 1,2 & 5,4 & 1,2 & 1,8 & 15 \\
\hline
\end{tabular}




\section{Literaturverzeichnis}

[AKZ92] AKZO NobEL ChEMICALs: Initiators for Polymer Production (Product Catalogue), (1992).

[ARI56] ARIS, R.: On the Dispersion of a Solute in Fluid Flowing through a Tube, Proc. Roy. Soc. A, 235, 67 (1956).

[ASH93] Ashfold, M.N.R.; Clement, S.G.; Howe, J.D.; WeSTERn, C.M.: Multiphoton Ionisation Spectroscopy of Free Radicals, J. Chem. Soc. Faraday Trans., 89(8), 1153-1172 (1993).

[ATK97] AtKInson, R; BAulch, D.C.; Cox, R.A.; HAMPSOn, R.F., JR.; KerR, J.A.; Rossi, M.J.; Troe, J.: Evaluated Kinetic, Photochemical and Heterogeneous Data for Atmospheric Chemistry: supplement V, IUPAC subcommittee on gas kinetic data evaluation for atmospheric chemistry, J. Phys. Chem. Ref. Data, 26, 521-1011 (1997).

[BAR82] BARTELs, M.; HOYERMANN; SIEVERT, R.: Elementary Reactions in the Oxidation of Ethylene: The Reaction of $\mathrm{OH}$ Radicals with Ethylene and the Reaction of $\mathrm{C}_{2} \mathrm{H}_{4} \mathrm{OH}$ Radicals with $\mathrm{H}$ Atoms, 19th Symposium (Intern.) on Combustion, The Combustion Institute, 61 (1982).

[BAR85] BARTELs, M.; HOYERMANN; SIEVERT, R.: The Reactions of Hydrogen Atoms with Cyclo- $\mathrm{C}_{3} \mathrm{H}_{5}$ and Cyclo- $\mathrm{C}_{5} \mathrm{H}_{9}$ Radicals: Rates and Mechanisms at Low Pressure, 20 ${ }^{\text {th }}$ Symposium (Intern.) on Combustion, The Combustion Institute, 723 (1985).

[BAT94] BATT, L.; KHAN, M.A.; MitCHELL, T.J.: Reaction of Hydroperoxy t-Butyl Radicals and $t$-Butyl Peroxy t-Butyl Radicals in Relation to Chain Propagation Combustion, 25 th Symposium (Intern.) on Combustion, The Combustion Institute, 793-800 (1994).

[BAU92] Baulch, D.L.; Cobos, C.J.; Cox, R.A.; Esser, C.; FranK, P.; Just, T.; KerR, J.A.; Pilling, M.J.; Troe, J.; WALKer, R.W.; WARNATZ, J.: Evaluated kinetic data for combustion modelling, J. Phys. Chem. Ref. Data, 21, 411 (1992).

[BEC90] Becker, H; Domschke, G.; FAnghänel, E.; Fischer, M.; GeWALD, K.; MAYER, R.; Pavel, D.; SChMidt, H.; SChWETLick, K.; Berger, W.; FAust, J.; GentZ, F.; Gluch, R.; MÜLler, K.; SCHOlLberG, K.; SeILER, E.; ZePPENFELD, G.: Organikum, Berlin (1990).

[BEI91] BEIDERHASE, T.: Untersuchungen der Reaktionen $\mathrm{F}+\mathrm{CH}_{3} \mathrm{~F}, \mathrm{~F}+\mathrm{CH}_{4}, \mathrm{~F}+\mathrm{C}_{2} \mathrm{H}_{6}$ zur Erzengung der Radikale $\mathrm{CH}_{2} \mathrm{~F}$ und $\mathrm{CH}_{3}$, der Radikalkombinationsreaktion $\mathrm{CH}_{2} \mathrm{~F}+\mathrm{CH}_{2} \mathrm{~F}$ und der Radikalreaktionen $\mathrm{CH}_{2} \mathrm{~F}+\mathrm{F}, \mathrm{CH}_{3}+\mathrm{F}$ Diplomarbeit, Göttingen (1991).

[BEN76] BENSON, S.W.: Thermochemical Kinetics: Methods for the Estimation of Thermochemical Data and Rate Parameters, John Wiley, New York, 1, (1976).

[BEN86] BENSON, S.W.: Combustion, A Chemical and Kinetic View, 21 ${ }^{\text {st }}$ Symposium (Intern.) on Combustion, The Combustion Institute, 703-711 (1986).

[BEN98] BENSON, S.W.; DOBIS, O.: Existence of Negative Activation Energy in Simple Bimolecular Metathesis Reactions and Some Observations on Too-Fast Reactions, J. Phys. Chem. A, 102, 5175-5181 (1998). 
[BIE95] BIEHL, H.; BITTNer, J.; BOHN, B.; GeERS-MÜlLer, R.; STUHL, F.: Temperature Dependence of the Rate Constants of the Reactions of Oxygen Atoms with Trans-2-Butene, Cis-2Butene, 2-Methylpropene, 2-Methyl-2-Butene, and 2,3-Dimethyl-2-Butene, Int. J. Chem. Kinet., 27, 277-285 (1995).

[BOY95] BOYD, A.A.; NOZIERE, B.; LESClAux, R.: Kinetics and Thermochemistry of the Reversible Combination Reactions of the Allyl and Benayl Radicals with NO, J. Phys. Chem., 99, 1081510820 (1995).

[BRO86] Brouard, M.; Pilling, M.J.: An Apparent Isotope Anomaly in the Reaction $\mathrm{CH}_{3}+\mathrm{H} / \mathrm{D}$, Chem. Phys. Lett., 129, 439 (1986).

[BRO85] Bround, M.; MacPherson, M.T.; Pilling, M.J.: A Time-Resolved Technique for Measuring Rates of Alkyl Radical-Atom Reactions: The Pressure Dependence of the $\mathrm{CH}_{3}+\mathrm{H}$ Reaction at 504 K, Chem. Phys. Lett., 113, 413 (1985).

[BUT93] BUTH, R.: Massenspektrometrische Untersuchungen der Reaktionen von cyclischen Koblenwasserstoffen in der Gasphase, Dissertation, Göttingen (1993).

[CAT95] CATF REVIEW: Dimethyl Ether offers Diesels ULEV Performance, Centre for Alternative Transportation Fuels, 21, Vancouver (1995).

[CHO74] Choo, K.Y.; Mendenhall, G.D.; Golden, D.M.; Benson, S.W.: The Pyrolysis of 2Nitrosoisobutane and the Bond Dissociation Energies of Nitroso Compounds, Int. J. Chem. Kinet., 6, 813 (1974).

[COH83] COHEn, N.; WestBerg, K.R.: Chemical Kinetic Data Sheets for High-Temperature Chemical Reactions J. Phys. Chem. Ref. Data, 12, 531 (1983).

[COR75] CORnu, A.; Massot, R.: Compilation of Mass Spectral Data, Heyden \& Son, London (1975).

[CHR88] CHRISTEN, H.-R.: Grundlagen der allgemeinen und anorganischen Chemie, Frankfurt a. M., (1988).

[DEM97] DeMore, W.B.; SANDER, S.P.; GOLDEN, R.F.; HAMPSOn; KurYlo, M.J.; Howard, C.J.; RAvishanKarA, A.R.; KolB, C.E.; MolinA, M.J.: Chemical Kinetics and Photochemical Data for Use in Stratospheric Modelling. Evaluation number 12, JPL Publication 97-4, (1997).

[DIG81] DiGiuseppe, T.G.; Hudgens, J.W.; Lin, M.C.: Detection of Gas-Phase Methyl Radicals Using Multiphoton Ionization, Chem. Phys. Lett., 82, 267 (1981).

[DIL97] Dilger, H.; Stolmar, M; Tragenna-PiggotT, P.L.W.; Roduner, E.: Gas Phase Addition Kinetics of the Tert-Butyl Radical to Oxygen, Ber. Bunsenges. Phys. Chem., 101, 956960 (1997).

[EDE80] EDELSON, D.; Allara, D.L.: A Computational Analysis of Alkane Pyrolysis Mechanism: Sensivity Analysis of Individual Reaction steps, Int. J. Chem. Kinet., 12, 605 (1980).

[EDE88] EDELBÜTTEL-EINHAus, J.: Die Multiphotonenionisation von Radikalen und ibre Anwendung auf chemische Elementarreaktionen in der Gasphase, Dissertation, Göttingen (1988). 
[FAL91] FAlBE, J.; RegitZ, M. (Hrsg.): Römpp Chemie Lexikon, Stuttgart (1991).

[FET60] FetTis, G.C.; KnOx, J.H.; Trotman-Dickenson, A.F.: The Reaction of Fluorine Atoms with Alkanes, J. Chem. Soc., (1960).

[FOC99] Fockenberg, C.; Hall, G.E.; Preses, J.M.; Sears, T.J.; Muckerman; J.T.: Kinetics and Product Study of the Reaction of $\mathrm{CH}_{3}$ Radicals with $\mathrm{O}\left({ }^{3} \mathrm{P}\right)$ Atoms Using Time Resolved Timeof-Flight Spectrometry, J. Phys. Chem. A, 103, 5722-5731 (1999).

[FRA94] Frank, P.; HerZler, J.; Just, T.; WAHL, C.: High-Temperature Reactions of Phenyl Oxidation, 25 $5^{\text {th }}$ Symposium (Intern.) on Combustion, The Combustion Institute, 25, 833-840 (1994).

[GIL96] Gilles, M.K.; Turnipseed, A.A.; TALukdar, R.K.; Rudich, Y; Villalta, P.W.; HueY, L.G.; BurkHOlDer, J.B.; RAvishanKarA, A.R.: Reactions of $O(3 P)$ with Alk.yl Iodides: Rate Coefficients and Reaction Products, J. Phys. Chem., 100, 14005-14010 (1996).

[GRI95] GrifFith, J.F.; BARNARD, J.A.: Flame and Combustion, Blackie Academic \& Professional, Glasgow (1995).

[GRO88] GROTEMEYER, J.; SCHLAG, E.W.: Multiphoton-Ionisations-Massenspektrometrie; ein neues Hilfsmittel in der Analytik, Angew. Chem., 100, 461 (1988).

[HAC00] HACK, W.; HOYERMANN, K.; NACKE, F.: The $t-\mathrm{C}_{4} \mathrm{H}_{9} \mathrm{OCH}_{2}$ Radical in the Gas Phase: Detection by Multiphoton Ionization and the Reactions with $\mathrm{O}, \mathrm{O}_{2}, \mathrm{O}_{3}, \mathrm{H}$ and NO, Proc. Comb. Inst., 28, 1487-1494 (2000).

[HAC01] Hack, W.; Hoyermann, K.; Kersten, C.; Olzmann, M; Viskolcz, H.: Mechanism of the 1- $\mathrm{C}_{4} \mathrm{H}_{9}+\mathrm{O}$ Reaction and the Kinetics of the Intermediate 1- $\mathrm{C}_{4} \mathrm{H}_{9} \mathrm{O}$ Radical, Phys. Chem. Chem. Phys., 3, 2365-2371 (2001).

[HAC02] Hack, W.; Hoyermann, K.; Olzmann, M; Zeuch, T.: Mechanism and Rates of the Reactions $\mathrm{C}_{2} \mathrm{H}_{5}+\mathrm{O}$ and $1-\mathrm{C}_{3} \mathrm{H}_{7}+\mathrm{O}, 29^{\text {th }}$ Symposium (Intern.) on Combustion, The Combustion Institute, zur Veröffentlichung angenommen.

[HAN92] Hanning-LeE, M.A.; Pilling, M.J.: Kinetics of the Reaction between H-Atoms and Allyl Radicals, Int. J. Kinet., 24, 271-278 (1992).

[HAR98] Harding, L.B.; KLIPPENSTEIn, S.J.: A Theoretical Analysis of the Reaction of $\mathrm{H}$ with $\mathrm{C}_{2} \mathrm{H}_{5}$, $27^{\text {th }}$ Symp. (Int.) on Combustion, The Combustion Institute, 151-157 (1998).

[HED85] HEDIGER, H.J.: Quantitative Spektroskopie, Heidelberg (1985).

[HEI86] HeINEMANN, P.: Untersuchungen von $\mathrm{CH}_{3}$, $\mathrm{CH}_{2} \mathrm{OH}$-, $\mathrm{C}_{3} \mathrm{H}_{5}$-Radikalen durch Laserphotoionisation / Massenspektrometrie, Dissertation, Göttingen (1986).

[HEI90] Heinemann-Fiedler, P.; Hoyermann, K.; RoHDe, G: The Reactions of the Cyclic Hydrocarbon Radicals $c-C_{3} H_{5}, c-C_{5} H_{9}$, and $c-C_{6} H_{11}$ with $O-$ Atoms in the Gasphase, Ber. Bunsenges. Phys. Chem., 94, 1400 (1990).

[HER88] HERRON, J.T.: Evaluated Chemical Kinetic Data for the Reactions of Atomic Oxygen O(3P) with Saturated Organic Compounds in the Gas Phase, J. Phys. Chem. Ref. Data, 17, 967, (1988). 
[HOL97] HOLD, M: Erzeugung und Reaktionen von Chlormethyl- und Dichlormethylradikalen, sowie von Radikalen teiffluorierter Ether in der Gasphase, Diplomarbeit, Göttingen (1997).

[HOL01] HOLD, M: Erzeugung von Radikalen aus teilhalogenierten Methan-, Alkohol- und Etherderivaten und deren Reaktionen sowie thermische und chemische Aktivierung von 1-Ethinyl-1methylcyclopropan, Dissertation, Göttingen (2001).

[HOW79] Howard, C.J.: Kinetic Measurements Using Flow Tubes, J. Phys. Chem., 83, 3 (1979).

[HOY75] HOYERMAnN, K.: Interactions of Chemical Reactions, Transport Processes and Flow, in Physical Chemistry, An Advanced Treatise, (Jost, W., Ed.), Vol. VI B, 931, Academic Press (1975).

[HOY79] HOYERMANN, K.: Primärprodukte von Elementarreaktionen aus der Koblenwasserstoffoxidation, Habilitationsschrift, Göttingen (1979).

[HOY79a] HOYERMAnN, K.; SIEVERT, R.: The Reactions of Alkyl Radicals with Oxygen Atoms: Identification of Primary Products at Low Pressure, 17th Symp. (Int.) on Combustion, The Combustion Institute, 17, 517 (1979).

[HOY79b] Hoyermann, K.; SIEverT, R.: Die Reaktionen von neo-Pentyl- und n-Pentyl-Radikalen mit Sauerstoffatomen in der Gasphase bei niedrigem Druck: Mechanismus und Reaktionsgeschwindigkeit, Ber. Bunsenges. Phys. Chem., 83, 732 (1979).

[HOY96] Hoyermann, K.; NACKE, F.: Elementary Reactions of the Methoxymethyl Radical in the Gas Phase, 26. Symp. (Int.) on Combustion, The Combustion Institute, 505 (1996).

[HOY99] Hoyermann, K.; Olzmann, M.; Seeba, J.; ViskolCZ, B.: Reactions of $\mathrm{C}_{2} \mathrm{H}_{5}$ Radicals with $\mathrm{O}, \mathrm{O}_{3}$, and $\mathrm{NO}_{3}$ : Decomposition Pathways of the Intermediate $\mathrm{C}_{2} \mathrm{H}_{5} \mathrm{O}$ Radical, J. Phys. Chem. A, 103, 5692-5698 (1999).

[HUC85] HuCKNALL, D.J.: Chemistry of Hydrocarbon Combustion, Chapman and Hall (1985).

[HUD85] Hudgens, J.W., DulCey, C.S.: Observation of the $3 s^{2} A_{1}$ Rydberg States of Allyl and 2Metbylallyl Radicals with Multiphoton Ionization Spectroscopy, J. Phys. Chem., 89, 1505 (1985).

[HUD87] Hudgens, J.W.: Advances in Multi-Photon Processes and Spectroscopy (S.H. Lin, ed.), World Scientific, Singapore (1987).

[ING69] INGOLD, K.U.: Peroxy Radicals, Acc. Chem. Res., 2, 1 (1969)

[KER99] KERSTEN, C.: Spektroskopische Untersuchungen bei Reaktionen von Koblenwasserstoffradikalen mit Sauerstoffatomen in der Gasphase, Dissertation, Göttingen (1999).

[KHA83] KathoOn, T.: Kinetics of Reactions of Flourine Atoms with Alcohols, Dissertation, Göttingen (1983).

[JOS52] Jost, W.: Diffusion in Solids, Liquids and Gases, Academic Press, N.Y. (1952).

[KNU73] KNUTH, E. L.: Direct-Sampling Studies of Combustion Processes, 9, Plenum Press (1973).

[LAI86] LAIDLER, K.J.: Reaktionskinetik 1, S. 86, Mannheim (1986). 
[LAN95] LANGER, S.; LJungSTRÖM, E.; ElLERMANN, T; NIELSEN, O.J.; SEHESTED, J.: UV absorption spectrum of $\mathrm{CH} 3 \mathrm{OCH} 2$ radicals and kinetics of the reaction of $\mathrm{CH} 3 \mathrm{OCH} 2 \mathrm{O} 2$ radicals with NO and NO2 in the gas phase, Chem. Phys. Lett., 240, $53-56$ (1995).

[LEN80] LENHARDT, T.M.; MCDADE, C.E.; BAYES, K.D.: Rates of Reaction of Butyl Radicals with Molecular Oxygen, J. Chem. Phys., 72, 304 (1980).

[LEN84] Lengsfield, B.H.; Siegbahn, P.E.M.; LiU, B.: Ab Initio Assignment of the UV Spectra of the Ethyl, Isopropyl and t-Butyl Radical, J. Chem. Phys., 81, 710-716 (1984).

[LIG90] Lightfoot, P.D.; Roussel, P.; Veyret, B; Lesclaux, R.: Flash Photolysis Study of the Spectra and Self-Reactions of Neopentylperoxy and t-Butylperoxy Radicals, J. Chem. Soc. Faraday Trans., 86, 2927 (1990).

[LIN98] Lindner, J.; LoOmis, R.A.; KLAASSEN, J.J.; LeONE, S.R.: A Laser Photolysis/TimeResolved Fourier Transform Infrared Emission Study of $\mathrm{OH}\left(X^{2} \Pi, v\right)$ Produced in the Reaction of Alkyl Radicals with O( ${ }^{3}$ P), J. Chem. Phys., 108, 1944 (1998).

[MAR97] MARICQ, M.M.; SZENTE, J.J.; HYBL, J.D.: Kinetic Studies of the Oxidation of Dimethyl Ether and its Chain Reaction with Cl2, J. Phys. Chem. A, 101, 5155-5167 (1997).

[MAS74] Mass spectrometric Data Centre: Eight Peaks Index of Mass Spectra, Reading (1974).

[MEI85] MeIER, U., Grotheer, H.H., Riekert, G., Just, T.: Temperature Dependence and Branching Ratio of the $\mathrm{C}_{2} \mathrm{H}_{5} \mathrm{OH}+\mathrm{OH}$ Reaction, Chem. Phys. Lett., 115, 221 (1985).

[MEL96] MeLIUS, C.F., Sandia National Laboratories, n.v., Livermore (1996).

[MOF63] MoffeTt, R.B.: Preparation of Cyclopentadiene, Org. Synth. Coll., Vol IV, 239, (1963).

[NAC94] NACKE, F.: Erzengung und Reaktionen des Methoxymethylradikals in der Gasphase, Diplomarbeit, Göttingen (1994).

[NAC98] NACKE, F: Temperaturabhängige Untersuchungen der Reaktionen von Koblenwasserstoffradikalen in der Gasphase, Dissertation, Göttingen (1998).

[NIE97] Nielsen, O.J.; Wallington, T.J.: Methods of Preparing Organic Peroxy Radicals for Laboratory Studies, in: Peroxyl Radicals, Alfassi, Z. (Editor), Chichester (GB) (1997).

[NOT02] Nothdurft, J.: Staatsexamensarbeit, Veröffentlichung vorgesehen (2002).

[OGR75] OGRen, P.J.: Analytical Results for First-Order Kinetics in Flow Tube Reactors with Wall Reactions, J. Phys. Chem., 79, 1749 (1975).

[OSE91] Oser, H; Walter, D; Stothard, N.D.; Grotheer, O.; Grotheer, H.H.: Application of Flow Reactors to Very Low Pressures by Using $\mathrm{CH}_{3}+\mathrm{O}(3 \mathrm{P})$ as a Test Reaction, Chem. Phys. Lett., 181, 521-525 (1991).

[PAL84] Paltenghi, R.; OGRYZlo, E.A.; BAyes, K.D.: Rates of Reaction of Alkyl Radicals with Orone, J. Phys. Chem., 88, 2595-2599 (1984). 
[PAU67] Pauling, L.: Die Natur der chemischen Bindung, 3. Auflage, Verlag Chemie GmbH, Weinheim (1967).

[PEA73] Pearson, R.K.; Cowles, J.O.; Hermann, G.L.; GregG, D.W.; Creighton, J.R.: Relative Performance of a Variety of NF3 + Hydrogen-Donor Transverse-Discharge HF ChemicalLaser Systems, IEEE J. Quantum Electron., 9, 879 (1973).

[PED77] PEDLEY, J.B.; RYLANCE, J:: Computer Analysed Thermochemical Data: Organic and Organometallic Compounds, University of Sussex, Brighton, 1, (1977).

[Pla95] Platz, J; Nielson, O.J.; Sehested, J.; Wallington, T.J.: Atmospheric Chemistry of 1,1,1-Trichloroethane, J. Phys. Chem., 99, 6570-6579 (1995).

[PIL95] PILling, M.J.; SEAKINS, P.W.: Reaction Kinetics, S. 208 ff., Oxford University Press, Oxford (1995).

[REN95] Rennert, P.; SCHMiedel, H. (Hrsg.): Physik, S. 65, Mannheim (1995).

[ROH88] ROHDE, G.: Massenspektrometrische Untersuchungen der Reaktionen von Cyclopentan, Cyclohexan und 1,4-Dioxan mit F-Atomen und der Reaktionen von Cyclopentyl-, Cyclohexyl und 1,4-DioxanylRadikalen mit $O\left({ }^{3} \mathrm{P}\right)$-Atomen, Diplomarbeit, Göttingen (1988).

[ROH91] RoHDE, G.: Nachweise und Reaktionen von Radikalen in der Gasphase, Dissertation, Göttingen (1991).

[ROY99] ROY, K.: Kinetische Untersuchungen zur Hochtemperaturpyrolyse und -oxidation von Cyclopentadien und Cyclopentadienyl mit Hilfe der Stoßwellentechnik, Dissertation, Stuttgart (1999).

[SAP87] SAPPEY, A.D., WeISSHAAR, J.C.: Vibronic Spectrum of Cold,Gas-Phase Allyl Radicals by Multiphoton Ionization, J. Phys. Chem., 91, 3731-3736 (1987).

[SCH91] SCHLESINGER, W.: Biogeochemistry, An Analysis of Global Change, Academic Press, San Diego, USA (1991).

[SEA92] SEAKINS, P.W.; LEONE, S.R.: A Laser Flash Photolysis/Time-Resolved FTIR-Emission Study of a Nen Channel in the Reaction of $\mathrm{CH}_{3}+\mathrm{O}$ : Production of $\mathrm{CO}(v)$, J. Phys. Chem., 96, 44784485 (1992).

[SEE94] SEEBA, J.: Mechanismus und Geschwindigkeit der Elementarreaktionen von Koblenwasserstoffradikalen in der Gasphase, Dissertation, Göttingen (1994).

[SEH97] Sehested, J.; Sehested, K; Platz, J.; EgsgaArd, H.; Nielson, O.J.: Oxidation of Dimethyl Ether: Absolute Rate Constants for the Self Reaction of $\mathrm{CH}_{3} \mathrm{OCH}_{2}$ Radicals, the Reaction of $\mathrm{CH}_{3} \mathrm{OCH}_{2}$ Radicals with $\mathrm{O}_{2}$, and the thermal Decomposition of $\mathrm{CH}_{3} \mathrm{OCH}_{2}$ Radicals, Int. J. Chem. Kinet., 29, 627-636 (1997).

[SER94] SERES, L.; NACSA, A.; ARTHUR, N.L.: Thermal Decomposition of Di-t-Butyl-Peroxide in the Presence of $\left(\mathrm{CH}_{3}\right)_{2} \mathrm{C}=\mathrm{CH}_{2}$ : Reactions of $\mathrm{CH}_{3},\left(\mathrm{CH}_{3}\right)_{2} \mathrm{CCH}_{2} \mathrm{CH}_{3}$, and $\left(\mathrm{CH}_{3}\right)_{2} \mathrm{CCH}_{2} \mathrm{C}\left(\mathrm{CH}_{3}\right)_{2} \mathrm{CH}_{2} \mathrm{CH}_{3}$ radicals, Int. J. Chem. Kinet., 26, 227-246 (1994).

[SLA84] SLAgLE, I.R.; FENG, Q.; GuTMAN, D.: Kinetics of the Reaction of Ethyl Radicals with Molecular Oxygen from 294 to 1002 K, J. Phys. Chem., 88, 3648 (1984). 
[SLA87] SLAGLE, I.R.; SARZYNSKI, D.; GUTMAN, D.: Kinetics of the Reaction between Methyl Radicals and Oxygen Atoms between 294 and 900 K, J. Phys. Chem., 91, 4375 (1987).

[SLA88] Slagle, I.R.; SARZYNSKI, D.; GutMan, D.; Miller, J.A.; Melius, C.F.: Kinetics of the Reaction between Oxygen Atoms and Ethyl Radicals, J. Chem. Soc. Farad. Trans. 2, 84, 491 503 (1988).

[SLA90] Slagle, I.R.; BernhardT, J.R.; Gutman, D.; Hanning-Lee, M.A.; Pilling, M.J.: Kinetics of the Reaction between Oxygen Atoms and Allyl Radicals, J. Phys. Chem., 94, 36523656 (1990).

[SMY85] SMYTH, K.C.; TAYLOR, H.P.: Detection of the Methyl Radical in a Methane/Air Diffusion Flame by Multiphoton Ionization Spectroscopy, Chem. Phys. Lett., 122, 518 (1985).

[STE90] STEIN, S.E.: The Nist/EPA/MSDC Mass Spectral Data Base, Version 3.01, June (1990).

[STE94] STEIN, S.E.: Nist Standard Reference Data Base, 25, Structures and Properties, Version 2.01 (1994).

[TAK81] TAkagi, H., Washida, N., BAndow, H., Akimoto, H., OkUda, M.: Photooxidation of $\mathrm{C}_{5}-\mathrm{C}_{7}$ Cycloalkanes in the NO- $\mathrm{H}_{2} \mathrm{O}$-Air System, J. Phys. Chem., 85, 2701-2705 (1981).

[TAY53] TAYLOR, G.: Dispersion of Soluble Matter in a Solvent Flowing Slowly through a Tube, Proc. Roy. Soc. A, 219, 186 (1953).

[TAY54] TAYlor, G.: Conditions under which Dispersion of a Solute in a Stream of Solvent Can Be Used to Measure Molecular Diffusion, Proc. Roy. Soc. A, 225, 473 (1954).

[TRO77] Troe, J.: Theory of Thermal Unimolecular Reactions at low Pressures, II. Strong Collision Rate Constants, Applications, J. Chem. Phys., 66, 4758 (1977).

[TRÖ97] TRÖGER, U:: Anwendung der FT-IR-Spektroskopie und Laserphotolyse auf Untersuchungen von Elementarreaktionen mit Radikalen in der Gasphase, Dissertation, Göttingen (1997).

[TSA86] Tsang, W.; Hampson, R.F.: Chemical Kinetic Data Base for Combustion Chemistry. Part I. Methane and Related Compounds, J. Phys. Chem. Ref. Data, 15, 1087 (1986).

[TSA88] Tsang, W.: Chemical Kinetic Data Base for Combustion Chemistry, Part 3. Propane, J. Phys. Chem. Ref. Data, 17, 887 (1988).

[TSA90] Tsang, W.: Chemical Kinetic Data Base for Combustion Chemistry, Part 4. Isobutane, J. Phys. Chem. Ref. Data, 19, 1-68 (1990).

[TSA91] Tsang, W.: Chemical Kinetic Data Base for Combustion Chemistry, Part 5. Propene, J. Phys. Chem. Ref. Data, 20, 221-273 (1991).

[TUl82] Tulloch, J.M.; MacPherson, M.T.; Morgan, C.A.; Pilling, M.J.: Flash-Photolysis Studies of Free-Radical Reactions: $\mathrm{C}_{3} \mathrm{H}_{5}+\mathrm{C}_{3} \mathrm{H}_{5}(293-691 \mathrm{~K})$ and $\mathrm{C}_{3} \mathrm{H}_{5}+\mathrm{NO}$, J. Phys. Chem., 86, 3812 (1982).

[WAL61] WALKER, R.E.: Chemical Reaction and Diffusion in a Catalytic Tubular Reactor, Phys. Fluids, 4, 1211 (1961). 
[WAL88] Waldington, T.J.; SKewes, L.M.; Siegl, W.O.; Wu, C-H.; JAPAR, S.M.: Gas Phase Reaction of Cl Atoms with a Series of Oxygenated Organic Species at $295 \mathrm{~K}$, Int. J. Chem. Kinet., 20, 867 (1988).

[WAL92] Wallington, T.J.; DAGAUT, P.; KuRYlo, M.J.: Ultraviolet Absorption Cross Sections and Reaction Kinetics and Mechanisms for Peroxy Radicals in the Gas Phase, Chem. Rev., 92, 667710 (1992).

[WAL97] Wallington, T.J.; Hurley, M.D.; Ball, J.C.; Straccia, A.M.; Platz, J.; Christensen, L.K.; SeHested, J.; Nielsen, O.J.: Atmospheric Chemistry of Dimethoxymethan $\left(\mathrm{CH}_{3} \mathrm{OCH}_{2} \mathrm{OCH}_{3}\right)$ : Kinetics and Mechanism of its Reaction with $\mathrm{OH}$ Radicals and the Fate of the Alkoxy Radicals $\mathrm{CH}_{3} \mathrm{OCHO}$ (.) $\mathrm{OCH}_{3}$ and $\mathrm{CH}_{3} \mathrm{OCH}_{2} \mathrm{OCH}_{2} \mathrm{O}($.), J. Phys. Chem. A, 101, 5302-5308 (1997).

[WAR84] Warnatz, J.: Rate Coefficients in the C/H/O System, "Combustion Chemistry", ed. W.C. Gardiner, Jr., pub. Springer Verlag, New York, (1984).

[WAS76] WASHIDA, N; BAYES, K.D.: The Reactions of Methyl Radicals with Atomic and Molecular Oxygen, Int. J. Chem. Kinet., 8, 777 (1976).

[WAS80] WASHIDA, N; BAYES, K.D.: Reactions of Isobutane and the Tert.-Butyl-Radical with Atomic and Molecular Oxygen, J. Phys. Chem., 84, 1309 (1980).

[WAS00] Washida, N; InOmota, S: Rate Constants for Reactions of Alkyl Radicals with Atomic Oxygen, $16^{\text {th }}$ Int. Symp. on Gas Kinetics, Cambridge (GB), P[C6], (2000).

[WED87] WeDLER, G.: Lebrbuch der Physikalischen Chemie, S. 167, 3. Auflage, Weinheim (1987).

[WEH97] WEHMEYER, J: Reaktionen von teiloxidierten Koblenwasserstoffen mit Fluoratomen in der Gasphase, Hausarbeit im Rahmen der Ersten Staatsprüfung für das Lehramt an Gymnasien, Göttingen (1997).

[WEN84] WendT, H.R.; HunZIKER, H.E.: The UV Spectra of Primary, Secondary, and Tertiary Alkyl Radicals, J. Chem. Phys., 81 (2), 717 (1984).

[WES84] Westbrook, C.K.; PITZ, W.J.: J. Combust. Sci. Technol., 37, 117 (1984), zitiert nach [NAC98].

[ZEU02] ZEUCH, T.: Dissertation, Göttingen, Veröffentlichung geplant (2002 oder 2003). 



\section{LEBENSLAUF}

Am 24. Juni 1969 wurde ich, Jens Wehmeyer, als viertes Kind des Landmaschinenmechanikermeisters Erich Wehmeyer und seiner Ehefrau, der Arbeiterin Annegret Wehmeyer, geb. Sander, in Osterode am Harz geboren. Ich besitze die deutsche Staatsangehörigkeit.

Von 1975 bis 1979 besuchte ich die Grundschule in Hörden, danach von 1979 bis 1981 die Orientierungsstufe in Wulften. Anschließend besuchte ich von 1981 bis 1988 das Ernst-MoritzArndt-Gymnasium in Herzberg, wo ich im Mai 1988 die allgemeine Hochschulreife mit einer Durchschnittsnote von 1,0 erwarb.

Von Juli 1988 bis Juni 1990 leistete ich meinen Wehrdienst als Soldat auf Zeit für 2 Jahre im Panzergrenadierbataillon 12 in Osterode/Harz. Im Zeitraum 1991 bis 1997 habe ich vier mehrwöchige Wehrübungen als Reserveoffizier geleistet. Zur Zeit trage ich den Dienstgrad Hauptmann der Reserve.

Im Wintersemester 1991/1992 studierte ich Maschinenbau an der Universität-Gesamthochschule Paderborn. Zum Sommersemester 1991 immatrikulierte ich mich an der Georg-August-Universität Göttingen, um das Studium der Chemie aufzunehmen. Ab dem Wintersemester 1991/1992 studierte ich das Lehramt an Gymnasien in der Fächerkombination Chemie/Mathematik. Im Sommersemester 1993 bestand ich die Zwischenprüfungen mit den Noten 1,0 in Mathematik und 1,0 in Chemie. Von September 1993 bis Juli 1994 studierte ich an der University of California San Diego/USA im Rahmen des Austauschprogramms "Education Abroad Program" (EAP) zwischen der Universität Göttingen und der University of California. Anschließend setzte ich mein Studium in Göttingen fort.

Meine Hausarbeit im Rahmen der Ersten Staatsprüfung für das Lehramt an Gymnasien fertigte ich im Fach Chemie zu dem Thema "Reaktionen von teiloxidierten Kohlenwasserstoffen mit Fluoratomen in der Gasphase" unter Anleitung von Herrn Prof. Dr. K. Hoyermann im Institut für Physikalische Chemie der Universität Göttingen an. Im Juni 1997 bestand ich die erste Staatsprüfung für das Lehramt an Gymnasien in der Fächerkombination Chemie/Mathematik mit der Abschlussnote 1,3.

Im Wintersemester 1997/1998 immatrikulierte ich mich für das Studienfach Volkswirtschaftlehre an der Universität Göttingen. Im Juni 2001 erwarb ich das Vordiplom in den Wirtschaftswissenschaften (Note: Gut).

Seit 1997 beschäftige ich mich im Rahmen des Sonderforschungsbereichs 357 "Molekulare Mechanismen unimolekularer Prozesse" mit Reaktionen von Kohlenwasserstoffradikalen in der Gasphase. Im Rahmen dieser Tätigkeit entstand die vorliegenden Arbeit. 
